\title{
TURBULENT BOUNDARY LAYER OVER A PIEZOELECTRICALLY EXCITED TRAVELING WAVE SURFACE
}

\author{
PATRICK F. MUSGRAVE \\ Dissertation submitted to the Faculty of the \\ Virginia Polytechnic Institute and State University \\ in partial fulfillment of the requirements for the degree of
}

\author{
DOCTOR OF PHILOSOPHY \\ IN \\ MECHANICAL ENGINEERING \\ Pablo A. Tarazaga, Chair \\ JEFFREY T. BORGGAARD \\ WILLIAM J. DEVENPORT \\ KEVIN B. KOCHERSBERGER \\ ANDREW J. KURDILA
}

$27^{\text {th }}$ JULY, 2018

Blacksburg, Virginia

Keywords: Turbulent Boundary Layer, Traveling Waves, Piezoelectric, Skin Friction, Two-Mode Excitation 


\title{
TURBULENT BOUNDARY LAYER OVER A \\ PIEZOELECTRICALLY EXCITED TRAVELING WAVE SURFACE
}

\author{
PATRICK F. MUSGRAVE
}

\begin{abstract}
Skin friction drag plays a significant role in determining the fuel efficiency of a vehicle. Reducing the skin friction thus has implications in a number of applications such as aircraft, ships, and automobiles. In recent years, a large amount of studies have researched various active drag reduction methods. One particular method involves active wall motion with the use of spanwise traveling waves. These out-of-plane traveling waves interact with the vortices in the turbulent boundary layer and weaken the bursting events that are responsible for increased skin friction drag. Computational and experimental studies have shown that these spanwise traveling waves are able to reduce the skin friction by upwards of $13 \%$ in turbulent flow. However, previous studies generated traveling waves using bulky actuation setups requiring arrays of discrete actuators that limited the achievable bandwidth and traveling wave patterns. In order for traveling waves to be a practical drag reduction method, a more implementable wave generation method is necessary.

A promising traveling wave generation method is known as two-mode excitation. This technique takes advantages of a surface's inherent structural properties to generate steady-state traveling waves in an open-loop fashion. In addition, the waves can be excited at most frequencies and using a small number of low-profile piezoelectric actuators. Previous research into two-mode excitation has primarily focused on one-dimensional beams. Traveling waves have been generated on a two-dimensional surface, but this was done from a fundamental standpoint with the resultant waves propagating in arbitrary directions. Before the two-mode excitation method can be applied for drag reduction, traveling waves must be generated on two-dimensional surfaces with tailorable propagation patterns.

The goal of this research is the development and testing of an implementable traveling wave generation method that alters the turbulent boundary layer with the aim of reducing skin friction drag. The first objective is to further develop the two-mode excitation method in order to tailor the traveling waves generated on a two-dimensional plate. Then, these tailored traveling waves are experimentally tested to determine their effect on the turbulent boundary layer. By directly investigating the boundary layer, a more fundamental approach is taken than only focusing on the skin friction drag. Finally, the overall effect of the traveling waves on the boundary layer are compared with standing waves.
\end{abstract}


To tailor the traveling wave pattern generated via two-mode excitation, the dependence on the location and number of actuators (actuation configuration) is investigated. An in-house finite element model is developed and updated via experimental modal analysis. Then, piezoelectric actuators are excited at various configurations to determine the effect on the traveling wave pattern. The results show that by varying the location and number of actuators, different traveling wave patterns can be generated at the same frequency. Propagating wave-fronts can be produced over the entire surface or within an isolated region. By modifying the actuation configuration, the traveling wave pattern can be tailored for specific applications, such as spanwise traveling waves for drag reduction.

A low-speed wind tunnel was then refurbished and heavily modified to study the effects of traveling waves on the turbulent boundary layer. Various methods for measuring skin friction drag were considered, but the boundary layer was measured to provide a more fundamental understanding of how traveling waves effect the fluid. The test-section in the wind tunnel was specifically designed to develop a well-characterized turbulent boundary layer that flows over a surface actuated with traveling waves. This boundary layer was then measured using hot-wire anemometry and a three-dimensional traversing system. Finally, this experimental data was analyzed and compared against accepted data from literature. The resultant experimental setup and measurement procedure allows the proper development, accurate measurement, and correct analysis of a turbulent boundary layer.

The tailored spanwise traveling waves were then experimentally tested in the wind tunnel to determine their effect on the turbulent boundary layer. This was investigated by measuring the boundary layer at numerous locations over a single frequency traveling wave. A phase-locked analysis was also conducted to analyze the boundary layer data at specific instances within the traveling wave period: at the crest, trough, and two zero-displacement positions. The results showed that the traveling wave pushes the boundary layer off of the wall and thickens the viscous sublayer. This indicates a lower velocity gradient and thus lower skin friction. Over the wave crest, the viscous sublayer is not thickened as much. These results agree with previous studies which implemented spanwise traveling waves. The phase-locked analysis also showed that that the traveling waves have a phase-lag effect on the boundary layer. When the wall moves downwards away from the flow, the fluid further from the wall lags behind, stretching the viscous sublayer. This suggests the phase-lag effect may contribute to the overall reduction in skin friction. Although the actual reduction could not be quantified due to the experimental setup, the presented results are consistent with a reduction in shear stress. Thus, this low-profile traveling wave generation method holds promise as a practical and implementable method for using spanwise traveling waves to reduce skin friction drag.

The two-mode excitation method generates traveling waves by taking advantage of a structure's 
modal properties. As a result, the traveling waves closely resemble the mode shapes (standing waves) of the surface. Since traveling waves affect the turbulent boundary layer, standing waves too may have an effect. To investigate this, the turbulent boundary layer was measured over multiple traveling and standing waves. These results showed that both wave types effect the boundary layer in a similar manner. The boundary layer is pushed off the wall and the viscous sublayer thickened, and the phase-lag effect was observed. A parametric analysis was then conducted and showed that both traveling and standing waves have a strong dependence on the wall-normal displacement. As the wave displacement increases, so too does the change in the boundary layer. In some traveling wave instances, the viscous sublayer was actually thinned, indicating a drag increase. This was suggested to result from the traveling wave pattern interacting with the turbulent vortices. Comparing the traveling and standing waves directly, the standing waves appear to have a slightly stronger effect on the boundary layer. However, this could not be fully characterized since there were fewer measurement locations over the standing wave. The displacement was also shown to be the dominant parameter, so dependence on other parameters (e.g. frequency) could not be established.

Overall, this research demonstrated that the two-mode excitation method can be used with lowprofile piezoelectric actuators to generate tailorable traveling waves on a two-dimensional surface. These tailorable traveling waves were used to effect the boundary layer, but other applications include propulsion or steady-state locomotion. The tailored spanwise traveling waves were then experimentally tested and shown to affect the turbulent boundary layer in a manner consistent with skin friction reduction. Thus, this wave generation method has promise as an applicable method for reducing skin friction drag. Finally, the effects of standing and traveling waves on the boundary layer were investigated and shown to produce similar results.

Further work is needed before these traveling waves can be implemented. The two-mode excitation method should be explored to more fundamentally understand the relationship between actuation configuration and traveling wave pattern. The effects of both traveling and standing waves on the turbulent boundary layer should be further investigated and the dependence on the wave pattern better established. Finally, the actual drag reduction capability of both traveling and standing waves should be quantified. 


\section{TURBULENT BOUNDARY LAYER OVER A \\ PIEZOELECTRICALLY EXCITED TRAVELING WAVE SURFACE}

PATRICK F. MusgraVE

\section{General Audience Abstract}

Recent studies have utilized spanwise traveling waves to alter the turbulent boundary layer with the aim of reducing skin friction drag. Spanwise traveling waves are a promising active drag reduction technique; however, the wave generation methods used in previous studies are bulky and could not be practically implemented. This research has developed an implementable traveling wave generation method and then fundamentally demonstrated how it changes the turbulent boundary layer, which is in a manner consistent with skin friction/shear stress reduction. Traveling waves were generated on a two-dimensional surface using low-profile piezoelectric actuators, in an open-loop fashion, and with minimal frequency limitations. The wave generation method was developed to generate tailored traveling wave patterns; thus, yielding control over the propagation direction, number of wave-fronts, and regions of the surface containing traveling waves. These tailored traveling waves have the capacity not just for affecting the boundary layer, but also for other applications such as propulsion.

The implementable traveling wave generation method was then tested in a low-speed wind tunnel and shown to alter the structure of the turbulent boundary layer. The boundary layer is pushed off the wall, and the viscous sublayer is thickened, indicating a reduction in shear stress. Analysis of the boundary layer at positions phase-locked to the wave oscillation suggests that the traveling waves induce a phase-lag effect in the flow. This phase-lag produces a stretching of the viscous sublayer and may contribute to the skin friction reduction. The effects of standing waves on the turbulent boundary layer were also investigated and compared with traveling waves. The results indicate that both wave types alter the boundary layer in the same manner. Standing waves are simpler to generate than traveling waves, suggesting that standing waves may be an effective skin friction reduction method. Before traveling or standing waves can be implemented, further research is necessary to investigate the interaction between the wave pattern and the turbulent phenomena and also to quantify the skin friction reduction and overall net energy usage. 


\section{Dedication}

To my family and friends, who made this possible 


\section{Acknowledgements}

I want to start by thanking my advisor, Dr. Pablo Tarazaga; none of this would have been possible without him. He has been a great advisor and mentor and moving forward I hope to have many opportunities to continue working with him. I also want to thank him for the numerous occasions where he came into lab, started an interesting conversation, and then walked off; leaving us distracted and discussing for an hour or more. Those conversations were one of my favorite parts of being in the lab.

I'd also like to thank Dr. William Devenport for being a constant source of guidance in all things fluids and wind tunnel related. I'm also grateful to him for allowing me to borrow his equipment, the boundary layer measurements would not have been possible without it. I want to acknowledge the support of Dr. Jeff Borggaard who provided advice throughout the project and also contributed heavily to its initial direction. I appreciate the role of Dr. Kevin Kochersberger and Dr. Andrew Kurdila for serving on my committee and Dr. Mike Philen for proxying at my final defense. I'd also like to sincerely thank Margaret (Beth) Howell for her support with all things over the last three years.

I want to recognize the support and friendship of all the current and previous members of the VAST and VTSIL labs. Dr. V.V.N. Sriram Malladi, you were a great mentor and provided both professional and personal advice during my time in the lab. You are the original purveyor of traveling waves, for my research built upon the fundamental work you did. I want to acknowledge the collaboration with Dr. Mohammad Albakri. Your expertise in finite element modeling made the work in Chapter 4 possible. I am grateful to Dr Rodrigo (Reaudouge/El Capitan) Sarlo, you were a great colleague, roommate, and friend. I also want to thank Dr Austin Phoenix for providing both distractions and motivation, you helped keep me sane during parts of the degree. Finally, I want to thank Charlie Tenney, Dr. Mico Woolard, Dustin Bales, Dr. Bryan Joyce, and all the other members of the lab. You contributed to the amazing lab environment; graduate school would not have been as enjoyable without all of you.

I also want to acknowledge the help of Dr. Nathan Alexander and his graduate student Agastya Balantrapu. I spent countless hours using your equipment to repair those hot-wire probes. The data for Chapters 6 and 7 directly resulted from that. 
None of this would have been possible without the support of my family. My parents, Robert and Jacqueline, you raised me to be the person I am today. You have always been there with support and encouragement, and I am eternally grateful. I want to thank my brothers, Brian and Robert, and their wives, Katie and Lindsay. You are always there to chat and you provided many late nights of fun. Finally, I want to thank Alexis Gushiken and the endless support she has given. You always know when to motivate, encourage, or even distract me.

I also want to thank all the friends I made in Blacksburg and all those I made before. I'm grateful to Dr. Aaron Meyer for the continual stream of hypothetical situations. Peter Windes, thanks for being a great roommate and introducing me to ultimate frisbee. Dr. A.J. Garcia, you were always there to grab a beer, chat, and blow off steam. Thanks to all of you.

This research was supported by the Air Force Office of Scientific Research (AFOSR) through the 2015 Young Investigator Program under AFOSR grant FA9550-15-1-0198. I want to recognize the support provided by the John R. Jones III faculty fellowship. Additional financial support was provided by Virgnia Tech through the Hord Fellowship. 


\section{Contents}

Page

$\begin{array}{ll}\text { Abstract } & \text { ii }\end{array}$

General Audience Abstract $\quad$ v

Dedication vi vi

Acknowledgements vii

$\begin{array}{lc}\text { List of Figures } & \text { xiii }\end{array}$

$\begin{array}{lr}\text { List of Tables } & \text { xxiii }\end{array}$

1 Introduction 1

1.1 Research Objectives . . . . . . . . . . . . . . . . . . . 3

1.2 Dissertation Structure . . . . . . . . . . . . . . . . . . 4

2 Experimental Considerations and Boundary Layer Theory 6

2.1 Measurement Methods . . . . . . . . . . . . . . . . . . . 7

2.1 .1 Computational Studies . . . . . . . . . . . . . . . . . 7

2.1.2 Direct Measurement/Force Balance . . . . . . . . . . . . . . . 8

2.1.3 Turbulent Boundary Layer Measurement _. . . . . . . . . . . . . . 9

2.1.3.1 Particle Image Velocimetry (PIV) _ . . . . . . . . . . . . 10

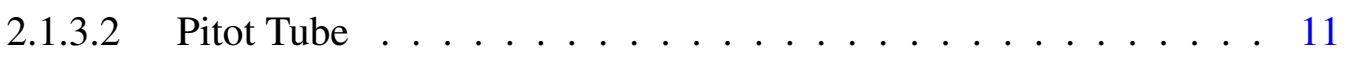

2.1.3.3 Hot-wire Anemometry (HWA) . . . . . . . . . . . 12

2.2 Basic Theory of Turbulent Boundary Layers … . . . . . . . . . . . 13

2.2.1 Turbulent Boundary Layer . . . . . . . . . . . . . . . . . . . . . . . 14

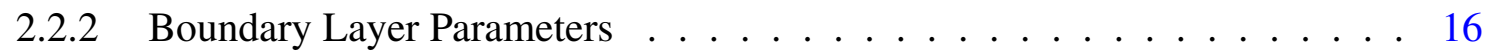

2.2 .3 Turbulence . . . . . . . . . . . . . . . . . . . . 17

2.3 Measurement of Skin Friction/Shear Stress _ . . . . . . . . . . . . . . . 19 
2.4 Summary . . . . . . . . . . . . . . . . . . . . . 22

3 Literature Review $\quad 24$

3.1 Traveling Waves . . . . . . . . . . . . . . . . . . . . . . . . . . . . 24

3.1 .1 Impedance Matching . . . . . . . . . . . . . . . . 25

3.1 .2 Active $\operatorname{Sink} \ldots \ldots \ldots \ldots \ldots \ldots \ldots$

3.1.3 Two-Mode Excitation . . . . . . . . . . . . . . . . . . . 28

3.2 Skin Friction Reduction using Spanwise Traveling Waves with Wall-Normal Motion 34

3.2.1 Passive and active skin friction reduction techniques . . . . . . . . . . 34

3.2.2 Spanwise traveling waves with wall-normal motion . . . . . . . . . 36

3.2.3 Consensus on Skin Friction Reduction . . . . . . . . . . . . . . . 50

4 Steady-State Traveling Wave Generation

4.1 Finite Element Model . . . . . . . . . . . . . . . . . . . . . . . . . . 54

4.2 Modal Analysis and Model Updating . . . . . . . . . . . . . . . . . 58

$4.2 .1 \quad$ Experimental Modal Analysis _ . . . . . . . . . . . . . 58

4.2 .2 Model Updating . . . . . . . . . . . . . . . . . . . . . . . . . 59

4.3 Traveling Waves . . . . . . . . . . . . . . . . . . . . . . 62

4.3.1 Model validation using traveling waves . . . . . . . . . . 63

4.3.2 Traveling Wave Pattern Manipulation _ . . . . . . . . . . . . 67

4.4 Discussion . . . . . . . . . . . . . . . . . . . . . . . 70

5 Wind Tunnel and Boundary Layer Measurement $\quad 72$

5.1 Experimental Setup . . . . . . . . . . . . . . . . . . . . 73

5.1 .1 General Setup . . . . . . . . . . . . . . . . . . 73

5.1 .2 Boundary Layer Development . . . . . . . . . . . . . . . . 77

5.1 .3 Flow Quality Testing . . . . . . . . . . . . . . . . . . 81

5.1.3.1 Flow Visualization . . . . . . . . . . . . . . . . 82

5.1.3.2 Dynamic Pressure Mapping . . . . . . . . . . . . . 83

5.1.3.3 Static Pressure gradient mapping . . . . . . . . . . . . . 84

5.1.3.4 Reference Velocity Calibration . . . . . . . . . . . . 86

5.1.4 Boundary Layer Measurement . . . . . . . . . . . . . . . . . . . 87

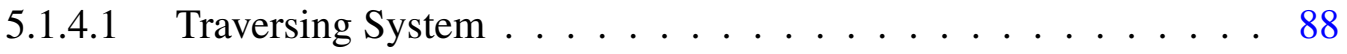

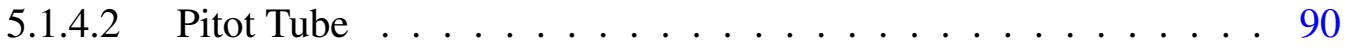

5.1.4.3 Hot Wire Anemometry (HWA) _. . . . . . . . . . . . 93

5.1.4.4 Finding Wall-Normal (y) Position . . . . . . . . . . . . . . . 99

5.1.4.5 Testing Procedure . . . . . . . . . . . . . . 103 
5.2 Data Analysis . . . . . . . . . . . . . . . . . . . . . . 103

5.2.1 Statistical Independence . . . . . . . . . . . . . . . . . . 104

5.2 .2 Non-dimensionalization . . . . . . . . . . . . . . . . . . 106

5.2.3 Wall-Normal (y) Position Adjustment . . . . . . . . . . . . . . . . . . 109

5.2 .4 Boundary Layer Quantities . . . . . . . . . . . . . . . . . . . . . . . . . 1110

5.2.5 Variance, Skewness, and Kurtosis Profiles . . . . . . . . . . . . . . . 112

5.3 Boundary Layer Validation . . . . . . . . . . . . . . . . . . . . . . . . . 114

5.3.1 Laminar boundary layer against Blasius solution . . . . . . . . . . 115

5.3.2 Comparison between Pitot tube and Hot-wire probe . . . . . . . . . . . 116

5.3.3 Validation of boundary layer data against literature . . . . . . . . . . 118

5.3.4 Streamwise Evolution of Boundary Layer . . . . . . . . . . . . . . . 119

6 Turbulent Boundary Layer over Traveling Waves 122

6.1 Traveling Waves in Wind Tunnel . . . . . . . . . . . . . . . . . . . . . . . . . . . . . . . . . . . . . .

6.1 .1 Experimental Setup . . . . . . . . . . . . . . . . . . . . . . 124

6.1 .2 Frequency Response . . . . . . . . . . . . . . . . . . . . . . 125

6.1.3 Traveling Wave Generation . . . . . . . . . . . . . . . . . . . . 129

6.2 Boundary Layer Data Analysis . . . . . . . . . . . . . . . . . . . . . . 134

6.2.1 Measurement Procedure . . . . . . . . . . . . . . . . . . 135

6.2 .2 Phase-locked Analysis . . . . . . . . . . . . . . . . 136

6.3 Effect of Traveling Wave on the Turbulent Boundary Layer . . . . . . . . . . . 140

6.3.1 Streamwise Boundary Layer . . . . . . . . . . . . . . . . . . 140

6.3.2 Phase-Locked Boundary Layers … . . . . . . . . . . . . . . . 142

6.3.3 Variance/Streamwise Reynolds Stress Profile . . . . . . . . . . . . . . 142

6.3.4 Skewness and Kurtosis Profiles _ . . . . . . . . . . . . . . . . . 146

6.3.5 Profiles at Different Spanwise Positions . . . . . . . . . . . . . . . 148

6.3.6 Uncertainty in Boundary Layer Profiles . . . . . . . . . . . . . 150

6.4 Discussion . . . . . . . . . . . . . . . . . . . . . . 153

$7 \quad$ Effect of Traveling and Standing Waves on the Boundary Layer 158

7.1 Traveling and Standing Wave Results . . . . . . . . . . . . . . . . 159

7.1 .1 Traveling Waves . . . . . . . . . . . . . . . . 160

7.1.1.1 607Hz Traveling Wave . . . . . . . . . . . . . 161

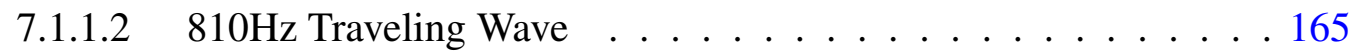

7.1 .2 Standing Waves . . . . . . . . . . . . . . . . . . 167

7.1.2.1 $385 \mathrm{~Hz}$ Standing Wave . . . . . . . . . . . . . 170

7.1.2.2 $630 \mathrm{~Hz}$ Standing Wave . . . . . . . . . . . . . . . . 173 
7.1 .3 Discussion . . . . . . . . . . . . . . . . . . . 177

7.2 Parametric Analysis . . . . . . . . . . . . . . . . . . . . . . 179

7.2 .1 Procedure . . . . . . . . . . . . . . . . . . . . . 179

7.2 .2 Analysis . . . . . . . . . . . . . . . . . 180

7.3 Discussion . . . . . . . . . . . . . . . . . . . . 183

7.3 .1 Traveling Waves $\ldots \ldots \ldots \ldots \ldots \ldots$

7.3.2 Standing versus traveling Waves $\ldots \ldots \ldots \ldots \ldots$

8 Conclusion $\quad 191$

8.1 Summary of Results . . . . . . . . . . . . . . . . . . . . 192

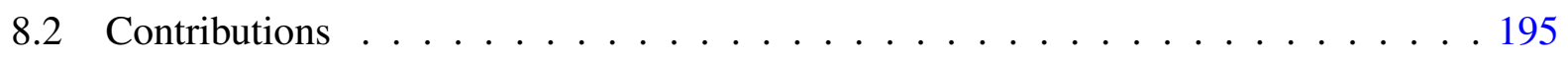

8.3 Future Work . . . . . . . . . . . . . . . . . . . 196

$\begin{array}{lr}\text { References } & 198\end{array}$

$\begin{array}{lr}\text { Appendices } & 208\end{array}$

$\begin{array}{llr}\text { Appendix A Finite Element Model Information } & 209\end{array}$

A.1 Terminology in Finite Element Model . . . . . . . . . . . . . . . . . 209

$\begin{array}{llr}\text { Appendix B Wind Tunnel Correction Factors } & 212\end{array}$

B.1 Pitot Tube Correction Factors . . . . . . . . . . . . . . . . . . . . 212

$\begin{array}{llr}\text { Appendix C Uncertainty Analysis } & 214\end{array}$

C.1 A Brief Review of Uncertainty Theory . . . . . . . . . . . . . . . . . . . 214

C.2 Uncertainty Calculations . . . . . . . . . . . . . . . . . . . 218

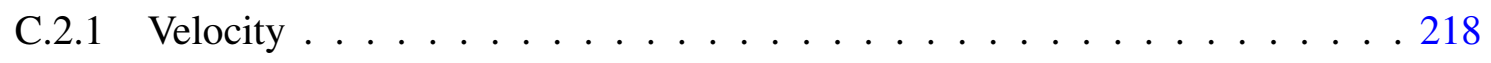

C.2.2 Y Position . . . . . . . . . . . . . . . . . . 222

C.2.3 Momentum Thickness . . . . . . . . . . . . . . . . . . . . 224

Appendix D Additional Traveling and Standing Wave Results 226

D.1 607Hz Traveling Wave . . . . . . . . . . . . . . . . . 226

D.2 810Hz Traveling Wave . . . . . . . . . . . . . . . . . . 227

D.3 $630 \mathrm{~Hz}$ Standing Wave . . . . . . . . . . . . . . . . . . . 228 


\section{List of Figures}

2.1 Front view of a hot-wire probe with the sensor wire welded between the two prongs 12

2.2 General structure of a turbulent boundary layer with velocity $\left(u^{+}\right)$vs. y position in wall units $\left(y^{+}\right) \ldots \ldots \ldots \ldots \ldots \ldots \ldots$

3.1 Modeled beam with mounted piezoelectric (PZT) actuators [1]. Used with permission of SAGE Publishing . . . . . . . . . . . . . . . . 31

3.2 Simulated frequency response of clamped-clamped beam showing resonant and traveling wave responses [1]. Used with permission of SAGE Publishing . . . . . . 32

3.3 Three different methods of visualizing a combined traveling and standing wave (Hybrid) [2]. Used with permission of IOP Publishing . . . . . . . . . . . . . . 32

3.4 (a) Quality of the traveling wave between two modes for different phases where 0 is ideal. (b) Quality of the traveling wave between two modes when the phase is constant at $90^{\circ}$ [2]. Used with permission of IOP Publishing . . . . . . . . . . 33

3.5 Different active drag reduction methods with wall deformation [3]: (a) in plane oscillation, (b) standing wave, (c) streamwise TW with in-plane spanwise oscillation, (d) streamwise TW with wall deformation, (e) spanwise TW with streamwise in-plane motion, (f) spanwise TW with wall deformation. Used with permission of AIP Publishing . . . . . . . . . . . . . . . . . . . . . 36

3.6 Boundary layer profile over the unactuated wall and actuated wall with that over the trough and crest shown separately [4]. Used with permission of Taylor \& Francis 39

3.7 The instantaneous streamwise vorticity fluctuations at $y^{+} \approx 4$ above the unactuated wall (a) and the TW actuated wall (b). The dashed lines represent the contours of the crest region [4]. Used with permission of Taylor \& Francis . . . . . . . . . . 40

3.8 The eccentric motor used to generate spanwise traveling waves in Tamano [5]. Used with permission of Taylor \& Francis . . . . . . . . . . . . . . . . . . . 41

3.9 Channel flow with spanwise traveling waves investigated by Tomiyama and Fukagata [3]. Used with permission of AIP Publishing . . . . . . . . . . . . . . 42 
3.10 Array of piezoelectric cantilever actuators used to generate spanwise traveling waves [6]. Used with permission of Cambridge University Press . . . . . . . . . . 44

3.11 A schematic of the skin friction reduction mechanism with (a) and without (b) traveling waves where flow is into the plane [6]. Used with permission of Cambridge University Press . . . . . . . . . . . . . . . . . . . . . . 45

3.12 Turbulent kinetic energy (TKE) production over a traveling wave for two different drag reductions (a) $11 \%$ and (b) $1 \%$ [7]. Used with permission of Springer Nature . 46

3.13 Array of electromagnetic actuators used to generate spanwise traveling waves [8]. Used with permission of Springer Nature . . . . . . . . . . . . . . . 48

4.1 (a) Underside of the aluminum plate with ten MFC piezoelectric actuators and the frame used for clamping, (b) experimental setup for measuring the modal properties, and (c) the mounting assembly used to ensure clamped boundary conditions . 59

4.2 Comparison between the clamped boundary condition model and experiment showing the (a) natural frequencies plotted with the zero error line, (b) the percent error in natural frequencies, and (c) the MAC values . . . . . . . . . . . . . . 62

4.3 Actuators excited (actuation configuration) for validating traveling wave generation of finite element model. The highlighted actuators $\left(A_{3}-B_{3}\right)$ are excited at $310 \mathrm{~Hz}$ and with a phase difference, $\gamma$, between them . . . . . . . . . 63

4.4 Least squares fit of experimental velocities measured at a single location on the plate for an excitation frequency of $\omega=310 \mathrm{~Hz}$. The amplitude and phase (complex amplitude) calculated by the least squares fit are shown . . . . . . . . . . 64

4.5 (a) the $7^{\text {th }},(3,2)$, experimental mode shape at $282.1 \mathrm{~Hz}$, and (b) the $8^{\text {th }},(1,3)$, experimental mode shape at $353.5 H z \ldots \ldots \ldots$. . . . . . . . . 65

4.6 Comparison between the experimental and model traveling waves generated at $310 \mathrm{~Hz}$ using the actuation configuration in Figure 4.3 and phase differences $\gamma_{\exp }=$ $100^{\circ}$ and $\gamma_{\text {model }}=65^{\circ}$. The instantaneous velocities of experimental (a) and model (b) traveling waves at different time steps in the period, $T$, are shown. The normalized root mean square (RMS) velocities over an entire period are shown for the experiment $(\mathrm{c})$ and model $(\mathrm{d}) \ldots \ldots \ldots 6$

4.7 Time step plots of experimental traveling wave patterns for different actuation configurations excited at $310 \mathrm{~Hz}$. Each traveling wave pattern (a-d) is defined by the actuation configuration at the top of each pattern. Actuators highlighted in blue are excited, where there is a phase difference, $\gamma$ between the top and bottom actuators. Actuators highlighted in red are $180^{\circ}$ out-of-phase with those in blue . . . . . . . . 68 
4.8 Normalized RMS velocity plots of traveling wave patterns for different actuation configurations (a-d) when excited at $310 \mathrm{~Hz}$. The top row is the actuation configuration, the second row is the experimental RMS plot, and the third row is the model RMS plot of the traveling wave pattern. Actuators highlighted in blue are excited, where there is a phase difference, $\gamma$ between the top and bottom actuators. Actuators highlighted in red are $180^{\circ}$ out-of-phase with those in blue . . . . . . . . 69

5.1 Low speed, open return wind tunnel used in the current research with fans, intake nozzle, test section, and traversing section shown . . . . . . . . . . . . . 74

5.2 Experimental setup showing the traveling wave (TW) plate mounted in the floating wall, which is installed in the wind tunnel test section. The top of the test section has been removed. $\mathrm{X}=$ Streamwise, $\mathrm{Y}=$ Wall-normal, $\mathrm{Z}=$ Spanwise direction . . . 78

5.3 Definition of the $x_{p}$ streamwise coordinates, where $p$ refers to the traveling wave plate. $x_{p}=0$ at the upstream edge of the plate. Also shown are the streamwise positions (in $\mathrm{mm}$ ) of the leading edge and the downstream edge of the traveling

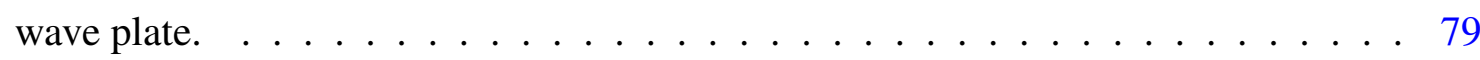

5.4 Downstream view of floating wall with mounted traveling wave plate. Trip strip is visible in foreground with splitter plates running parallel to test section walls. . . . 79

5.5 Flap at trailing edge of floating wall used to adjust the streamwise static pressure

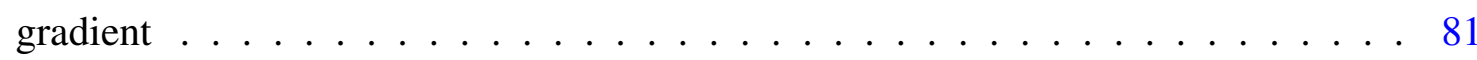

5.6 Top of test section showing seven pairs of static pressure ports and also the velocity probe slots . . . . . . . . . . . . . . . . . . . 81

5.7 China clay flow visualization applied over the traveling wave plate for left to right moving flow with (a) an untripped and (b) a tripped boundary layer . . . . . . . . 84

5.8 Percent variation from the mean dynamic pressure just upstream of the traveling wave plate at $x_{p}=-19 \mathrm{~mm}$. The air flows into the page . . . . . . . 85

5.9 Static pressure gradient in streamwise direction as the trailing edge flap is adjusted. 85

5.10 Reference velocity calibration between the dynamic pressure and static pressure with the linear least squares fit shown. The calibration is split into an upper and lower range due to limitations in how the wind tunnel fans are controlled Table 5.1.

5.11 Three dimensional traversing system used in the current study. The pitot tube is seen attached to the bottom of the $y$ stage and passes into the wind tunnel test section. The hot-wire probe can be attached in a similar way. . . . . . . . . . . . 89

5.12 Hot-wire probe passing through a foam lined slot in the test-section ceiling . . . 90 
5.13 Two different test section ceilings. The black, foam-lined slots allow either velocity probe to traverse through the test section. (a) Used to characterize traveling waves on plate and collect streamwise pressure gradient. (b) Maximizes reachable locations for velocity probe; used during experimental testing . . . . . . . . . . 91

5.14 Small diameter pitot tube positioned close to the wall. The small $y$ distance is confirmed by the reflection of the probe on the wall . . . . . . . . . . . 992

5.15 Hot-wire probe inserted in the wind tunnel and mounted just above the surface. The shadow of the probe is visible on the wall . . . . . . . . . . . . . 94

5.16 Hot-wire probe mounted and passing into the wind tunnel test section. The probe is attached to a support, which is mounted to the $y$ translation stage. . . . . . . . 95

5.17 Data used to calibrate the hot-wire anemometer. The measured voltages are plotted against known flow velocities. The data is fit using a power law, where all three coefficients were variable. . . . . . . . . . . . . . . . . . . . 97

5.18 Mean velocities in the viscous sublayer of the boundary layer showing the nearwall effect when using a hot-wire probe [9]. The non-labeled data is that measured over a non-conducting wall by Hutchins at three different flow velocities $\left[U_{e}=\right.$ $(\nabla) 3 \mathrm{~m} / \mathrm{s},(\bullet) 2.5 \mathrm{~m} / \mathrm{s},(\star) 2 \mathrm{~m} / \mathrm{s}]$. The other lines show the velocity profile and the near-wall effect for conducting and non-conducting walls [10-13]). Used with permission from Elsevier . . . . . . . . . . . . . . . . . . . . . . 99

5.19 Close-up photos of the sensor wire welded onto the faces of the hot-wire probe prongs. (a) shows the wire welded roughly vertically centered and (b) shows the wire welded closer to the bottom, where the prong will contact the wall . . . . . . 101

5.20 View of the setup used to detemine the $y$ absolute reference position. The hot-wire probe is in the wind tunnel test-section and illuminated by the photography backlight. The camera on the left is angled slightly downwards to take a picture of the probe and its shadow. . . . . . . . . . . . . . . . . 101

5.21 (a) The hot-wire probe touching its shadow and making contact with surface of the wall. (b) The probe lifted barely off the surface at $y \approx 0.000,5 \operatorname{in}(12.7 \mu \mathrm{m})$ such that light is visible between the probe and its shadow . . . . . . . . . . 102

5.22 The autocorrelation coefficient function, $\rho_{u}(\tau)$, of the velocity at a $y^{+}=4.8 \ldots 105$

5.23 The intergral time-scale, $T_{I}$, of the velocity signal as a function of distance from

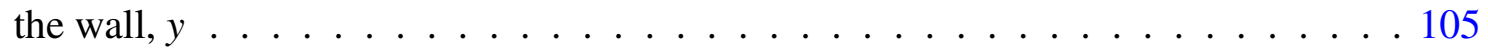

5.24 Mean velocity boundary layer profile in dimensional form with the boundary thickness, $\delta$, shown . . . . . . . . . . . . . . . . . 107

5.25 Mean velocity boundary layer profile normalized by the free-stream velocity, $U_{e}$. Also shown are various fits of the logarithmic region for different $C_{f}$ values $\ldots 108$ 
5.26 Mean velocity boundary layer profiles with and without the $y$ position offset implemented. . . . . . . . . . . . . . . . . . . . . . . . . . . . . . . . 109

5.27 Final mean velocity boundary layer profile with the viscous sublayer and log-law lines shown. The red $\times$ 's are the values used to fit the log-law curve . . . . . . . . 111

5.28 The variance/streamwise Reynolds stress as a function of distance from the wall. The boundary layer thickness, $\delta$, is shown. . . . . . . . . . . . . . . . 113

5.29 The skewness, $S=\frac{\overline{u^{13}}}{\sigma_{u}^{3}}$ of the fluctuating velocity, $u^{\prime}(t)$, as a function of wall posi-

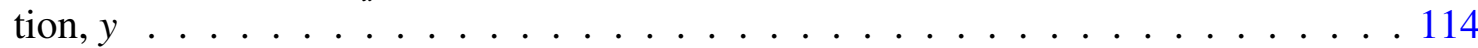

5.30 The kurtosis, $K=\frac{\overline{u^{\prime 4}}}{\sigma_{u}^{4}}$, of the fluctuating velocity, $u^{\prime}(t)$, as a function of wall posi-

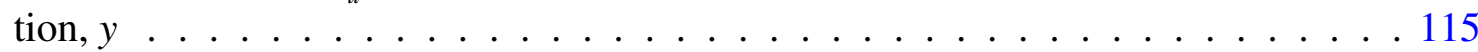

5.31 Comparison between experimentally measured laminar boundary layer and the Blasius solution for $R e_{\theta}=359$ with momentum thicknesses shown . . . . . . . . 117

5.32 Comparison between the turbulent boundary layer measured using the hot-wire

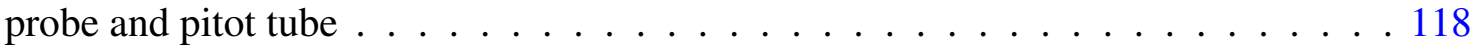

5.33 Comparison of the turbulent boundary layer measured using a hot-wire probe with data from Purtell [14] . . . . . . . . . . . . . . . . . . . . . . . . . . . 119

5.34 Turbulent boundary layer profiles at five different streamwise positions . . . . . . 120

5.35 Evolution of the skin friction coefficient, $C_{f}$, with growth in the boudnary layer (increasing $R e_{\theta}$ ). The values from the streamwise boundary layer profiles are plotted alongside the empirical Coles curve $[15] \ldots$. . . . . . . . . . . . . 121

6.1 Traveling wave plate mounted in the floating wall in the test-section of the wind tunnel. One set of actuator connections is seen passing through the test-section side wall. There is a mirrored connection on the far side of the floating wall. . . . . 125

6.2 Scanning laser doppler vibrometer (SLDV) measuring the out-of-plane velocity of the traveling wave plate in the wind tunnel during operation. . . . . . . . . . 126

6.3 Frequency response of the clamped traveling wave plate for three different cases. This includes the ideal experimental mounting (Chapter 4), in the wind tunnel with no airflow $\left(U_{e}=18.5 \mathrm{~m} / \mathrm{s}\right)$, and in the wind tunnel with air flow $\left(U_{e}=18.5 \mathrm{~m} / \mathrm{s}\right)$. $0 d B=1 \mathrm{~m} / \mathrm{s} / \mathrm{V}$

6.4 MAC values comparing the clamped traveling wave plate in different conditions. (a) compares operational deflection shapes (ODSs) of the plate mounted in the wind tunnel with no airflow $\left(U_{e}=0 \mathrm{~m} / \mathrm{s}\right)$ and the ideal experimental mounting from Chapter 4. (b) compares the plate mounted in the wind tunnel with $\left(U_{e}=18.5 \mathrm{~m} / \mathrm{s}\right.$ and without airflow $\left(U_{e}=0 m / s\right) \ldots \ldots \ldots \ldots \ldots$ 
6.5 (a) First and (b) second mode shape of the ideal experimental mounting described in Chapter 4. Natural frequencies of $f=84.5 \mathrm{~Hz}$ and $f=150.8 \mathrm{~Hz}$, respectively . . 129

6.6 Operational deflection shapes (ODSs) at the first natural frequencies of plate mounted in the wind tunnel. (a) $U_{e}=0 \mathrm{~m} / \mathrm{s}$ and $f=150.6 \mathrm{~Hz}$, (b) $U_{e}=18.5 \mathrm{~m} / \mathrm{s}$ and

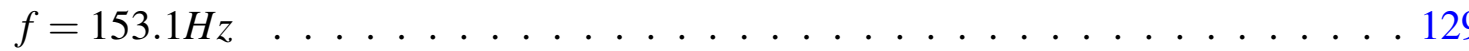

6.7 Operational deflection shapes (ODSs) show the (a) $(5,2)$ mode at $f=423.4 \mathrm{~Hz}$, $T^{+}=113.4$ and (b) the $(3,3)$ mode at $f=438.3 H z, T^{+}=109.4 \ldots \ldots 131$

6.8 Actuation configuration to excite the $430 \mathrm{~Hz}$ traveling wave. The top actuators are $\gamma=-110^{\circ}$ out of phase with the bottom ones. The actuators in red are $180^{\circ}$ offset from those in blue.

6.9 Instantaneous velocities of the $430 \mathrm{~Hz}\left(T^{+}=112\right)$ traveling wave at six different time steps in the period $T$ of the wave. There are three wavefronts propagating in the spanwise direction. The $\times$ symbol follows middle wavefront traveling in the $-z$ direction. The left and right sides travel in the $+z$ direction $\ldots \ldots \ldots . . .132$

6.10 Root-mean-square (RMS) velocity of the traveling wave at $f=430 \mathrm{~Hz}\left(T^{+}=112\right)$. The boundary layer measurement positions are highlighted along with the propagation direction of the traveling waves $\ldots \ldots \ldots 133$

6.11 Time-series data of the (a) traveling wave velocity, (b) traveling wave displacement, and (c) flow velocity. Also shown are indices corresponding to the phaselocked crest and trough positions. 10 periods of the $430 \mathrm{~Hz}\left(T^{+}=112\right)$ traveling wave are shown, where $5 s$ of raw data is collected at each $y$ position. . . . . . . . 137

6.12 Mean velocity boundary layer for the unactuated, time-averaged actuated, and phase-locked crest and trough cases. (a) shows the crest and trough profiles using the $y$ values from the time-averaged. (b) shows the crest and trough with corrected $y$ positions to account for the displacement of the TW surface . . . . . . . . . . 139

6.13 Experimentally measured turbulent boundary layers at six different streamwise locations upstream, over, and downstream of the $430 \mathrm{~Hz}\left(T^{+}=112\right)$ traveling wave surface. The unactuated and time-averaged actuated profiles are shown for each streamwise position. The streamwise position $\left(x_{p}\right)$ and normalized traveling wave displacement $\left(a_{\text {crest }}^{+}\right)$are given for each position. . . . . . . . . . . . . . 141

6.14 Experimentally measured phase-locked, turbulent boundary layers at four streamwise over the $430 \mathrm{~Hz}\left(T^{+}=112\right)$ traveling wave surface. At each location, the time-averaged and four phase-locked positions over the traveling wave surface: the crest and trough and also the zero displacement with both positive and negative wall-normal $(y)$ velocities . . . . . . . . . . . . . . . . . . . 143 
6.15 Experimentally measured turbulent boundary layers at four streamwise over the $430 \mathrm{~Hz}\left(T^{+}=112\right)$ traveling wave surface showing the phase-locked profiles when the traveling wave has zero displacement . . . . . . . . . . . . . . . . 144

6.16 Variance/Streamwise Reynolds stress profile at various streamwise positions over the $430 \mathrm{~Hz}\left(T^{+}=112\right)$ traveling wave surface. The first and last profiles are upstream and downstream of the traveling waves, respectively. The phase-locked profiles at four different points in the traveling wave period are also shown. . . . . 145

6.17 Phase-locked (a) skewness and (b) kurtosis profiles over the $430 \mathrm{~Hz}\left(T^{+}=112\right)$

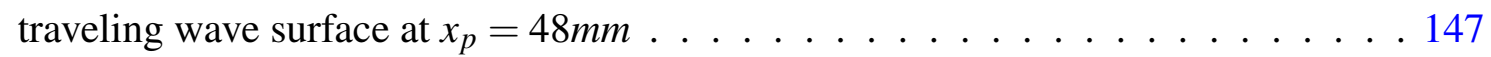

6.18 Experimentally measured phase-locked turbulent boundary layers at five different spanwise positions over the $430 \mathrm{~Hz}\left(T^{+}=112\right)$ traveling wave surface. The spanwise positions are all at $x_{p}=150 \mathrm{~mm}$ and are shown over the traveling wave in Figure $6.10 \ldots \ldots$. . . . . . . . . . . . . . . . . . . . . 149

6.19 Variance/Streamwise Reynolds stress profile at five different spanwise over the $430 \mathrm{~Hz}\left(\mathrm{~T}^{+}=112\right)$ traveling wave surface. The unactuated, time-averaged actuated, and phase-locked crest and trough profiles . . . . . . . . . . . . 150

6.20 Boundary layer profiles over the $430 \mathrm{~Hz}$ wave at $z=-25.4 \mathrm{~mm}$ (Figure 6.18) showing the uncertainty in each measurement point. The phase-locked profiles over the crest and trough of the wave are shown. . . . . . . . . . . . . 151

6.21 Boundary layer profiles over the $430 \mathrm{~Hz}$ wave at $x_{p}=-25.4 \mathrm{~mm}$ (Figure 6.18) showing the uncertainty in each measurement point. The phase-locked profiles over the two zero displacement points (positive and negative velocity) of the wave are shown. . . . . . . . . . . . . . . . . . . . . . . 153

7.1 Actuation configuration to excite the $607 \mathrm{~Hz}\left(T^{+}=78\right)$ traveling wave. The top actuators are $\gamma=-110^{\circ}$ out of phase with the bottom ones. The actuators in red are $180^{\circ}$ offset from those in blue. . . . . . . . . . . . . . . . . . 161

7.2 Root-mean-square (RMS) velocity of the traveling wave at $f=607 \mathrm{~Hz}\left(T^{+}=78\right)$. The boundary layer measurement positions are highlighted along with the propagation direction of the traveling waves $\ldots \ldots \ldots 2 \ldots \ldots$

7.3 Experimentally measured phase-locked, turbulent boundary layers at five streamwise positions for the $607 \mathrm{~Hz}\left(T^{+}=78\right)$ traveling wave surface. At each location, the time-averaged actuated and unactuated profiles are shown. Directly over the moving surface phase-locked profiles are shown at the crest and trough of the wave along with the two points in the wave period with zero displacement. . . . . . . . 164 
7.4 Variance/Streamwise Reynolds stress profile at streamwise positions over the $607 \mathrm{~Hz}\left(\mathrm{~T}^{+}=\right.$ 78) traveling wave surface. The first and last profiles are upstream and downstream of the traveling waves, respectively. The phase-locked extracted profiles are shown for the locations directly over the actuated surface. . . . . . . . . . . . . 165

7.5 Root-mean-square (RMS) velocity of the traveling wave at $f=810 \mathrm{~Hz}\left(T^{+}=59\right)$. The boundary layer measurement positions are highlighted along with the propagation direction of the traveling waves . . . . . . . . . . . 166

7.6 Experimentally measured phase-locked, turbulent boundary layers at five streamwise positions for the $810 \mathrm{~Hz}\left(T^{+}=59\right)$ traveling wave surface. At each location, the time-averaged actuated and unactuated profiles are shown. For the locations directly over the moving surface, the phase-locked actuated profiles are given. . . 168

7.7 Variance/Streamwise Reynolds stress profile at streamwise positions over the $810 \mathrm{~Hz}\left(\mathrm{~T}^{+}=\right.$ 59) traveling wave surface. The first and last profiles are upstream and downstream of the traveling waves, respectively. The phase-locked extracted profiles are shown for the locations directly over the actuated surface. . . . . . . . . . . . . . . 169

7.8 Operational deflection shape (ODS) of the $(2,3)$ mode shape at $385.2 \mathrm{~Hz}\left(\mathrm{~T}^{+}=\right.$ 122) excited using a single piezoelectric actuator . . . . . . . . . . . 170

7.9 Actuation configuration to excite the $385 \mathrm{~Hz}\left(T^{+}=122\right)$ standing wave. The actuators highlighted in blue have $0^{\circ}$ phase and those in red have $180^{\circ}$. The $A_{3}-B_{3}$ actuators are not excited here . . . . . . . . . . . . . . . 171

7.10 Instantaneous velocities of the $385 \mathrm{~Hz}\left(T^{+}=122\right)$ standing wave with $(2,3)$ mode shape. Six different time steps over the period $T$ of the wave are shown. There are no traveling waves, so each anti-node stays at a fixed location. The $\times$ symbol shows how an anti-node oscillates from positive (red) to negative (blue) over the period, but remains at the same location . . . . . . . . . . . . . 172

7.11 Root-mean-square (RMS) velocity of the standing wave at $f=385 \mathrm{~Hz}\left(\mathrm{~T}^{+}=122\right)$. The boundary layer measurement positions are highlighted. . . . . . . . . . . 172

7.12 Experimentally measured phase-locked, turbulent boundary layers at five streamwise positions for the $385 \mathrm{~Hz}\left(T^{+}=122\right)$ standing wave surface. At each location, the time-averaged actuated and unactuated profiles are shown. For the locations directly over the moving surface, the phase-locked actuated profiles are given. . . 174

7.13 Variance/Streamwise Reynolds stress profile at streamwise positions over the $385 \mathrm{~Hz}\left(\mathrm{~T}^{+}=\right.$ 122) traveling wave surface. The first and last profiles are upstream and downstream of the traveling waves, respectively. The phase-locked extracted profiles are shown for the locations directly over the actuated surface. . . . . . . . . . . 175 
7.14 Actuation configuration to excite the $630 \mathrm{~Hz}\left(T^{+}=78\right)$ standing wave. The actuators highlighted in blue have $0^{\circ}$ phase and those in red have $180^{\circ}$. The $A_{2}-B_{2}$ and $A_{4}-B_{4}$ actuators are not excited here . . . . . . . . . . . 176

7.15 Root-mean-square (RMS) velocity of the standing wave at $f=630 \mathrm{~Hz}\left(T^{+}=74\right)$. The boundary layer measurement positions are highlighted. . . . . . . . . . 176

7.16 Experimentally measured phase-locked, turbulent boundary layers at five streamwise positions for the $385 \mathrm{~Hz}\left(T^{+}=74\right)$ standing wave surface. At each location, the time-averaged actuated and unactuated profiles are shown. For the locations directly over the moving surface, the phase-locked actuated profiles are given. . . 178

7.17 $\Delta Y$ offset between the unactuated and time-averaged actuated mean velocity profile at $x_{p}=65 \mathrm{~mm}$ over the $630 \mathrm{~Hz}$ standing wave. Linear scaling is used $\ldots \ldots \ldots$

$7.18 \Delta y$ offset of the time-averaged actuated boundary layer profile as a function of the peak-to-peak, wall-normal displacement. Data from three different frequency traveling waves and two different standing waves is shown. . . . . . . . . . . . 181

7.19 Time-averaged actuated mean velocity profiles over the $630 \mathrm{~Hz}$ standing wave at $x_{p}=150 \mathrm{~mm}$. Four different displacement amplitudes are shown $\ldots \ldots 182$

$7.20 \Delta y$ offset of the time-averaged actuated boundary layer profile as a function of the peak-to-peak, wall-normal displacement. Data from three different frequency traveling waves and two different standing waves is shown. . . . . . . . . . . . 184

C.1 Flow chart detailing the procedure for conducting the hot-wire calibration. The top row are measured quantities and the output is the calibration curve . . . . . . . . 219

C.2 Hot-wire anemometer (HWA) calibration data showing the voltage vs. velocity with the error on each data point. The calibration curve fit to the data is also shown 221

C.3 Example calibration curves generated for the Monte-Carlo simulation along with the actual data and the main calibration curve . . . . . . . . . . . 222

C.4 95\% confidence interval error bounds around the calibration curve determined by the Monte-Carlo simulation . . . . . . . . . . . . . . . . . . . . . 223

C.5 Turbulent boundary layer over the unactuated and actuated surface with the velocity and $y$ position uncertainties shown. . . . . . . . . . . . . 223

D.1 Operational deflection shapes (ODSs) showing the (a) $(2,4)$ mode at $f=579 \mathrm{~Hz}$, $T^{+}=82$ and (b) the $(3,4)$ mode at $f=630 \mathrm{~Hz}, T^{+}=74$. These are the participating mode shapes in the $607 \mathrm{~Hz}\left(\mathrm{~T}^{+}=78\right)$ traveling wave $\ldots \ldots \ldots 226$ 
D.2 Instantaneous velocities of the $607 \mathrm{~Hz}\left(T^{+}=78\right)$ traveling wave at six different time steps in the period $T$ of the wave. There are three wavefronts propagating in the spanwise direction. The propagation direction of these are shown in the first time-step. The $\times$ symbol follows the right wavefront traveling in the $+z$ direction. . 227

D.3 Operational deflection shapes (ODSs) showing the (a) $(5,4)$ mode at $f=792.2 \mathrm{~Hz}$, $T^{+}=60$ and (b) the $(7,3)$ mode at $f=868.8 \mathrm{~Hz}, T^{+}=55$. These are the participating mode shapes in the $810 \mathrm{~Hz}\left(T^{+}=59\right)$ traveling wave . . . . . . . . 227

D.4 Instantaneous velocities of the $810 \mathrm{~Hz}\left(T^{+}=59\right)$ traveling wave at six different time steps in the period $T$ of the wave. There are three wavefronts propagating in the spanwise direction. The propagation direction of these are shown in the first time-step. The $\times$ symbol follows the left wavefront traveling in the $+z$ direction. . 228

D.5 Operational deflection shape (ODS) of the $(3,4)$ mode shape at $630.5 \mathrm{~Hz}\left(T^{+}=\right.$ 74). This is excited using a single actuator, while the standing wave in Section 7.1.2.2 is excited using six actuators . . . . . . . . . . . . . . . . . . 229

D.6 Instantaneous velocities of the $630 \mathrm{~Hz}\left(T^{+}=74\right)$ standing wave with $(2,3)$ mode shape. Six different time steps over the period $T$ of the wave are shown. There are no traveling waves, so each anti-node stays at a fixed location. The $\times$ symbol shows how an anti-node oscillates from positive (red) to negative (blue) over the period, but remains at the same location . . . . . . . . . . . . . . . . 229

D.7 Variance/Streamwise Reynolds stress profile at streamwise positions over the $630 \mathrm{~Hz}\left(\mathrm{~T}^{+}=\right.$ 74) traveling wave surface. The first and last profiles are upstream and downstream of the traveling waves, respectively. The phase-locked extracted profiles are shown for the locations directly over the actuated surface. . . . . . . . . . . . 230 


\section{List of Tables}

4.1 Parameters held constant during the model updating procedure $\ldots \ldots \ldots$

4.2 Initial and final values of the parameters updated to match the model to the exper-

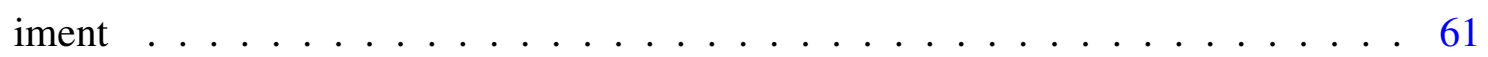

5.1 The two ranges of the air flow speed in the wind tunnel test-section and the states of the two fans (Primary and Secondary) . . . . . . . . . . . . . . . 74

5.2 List of all the equipment used in the wind tunnel . . . . . . . . . . 76

6.1 The streamwise measurement locations for the $430 \mathrm{~Hz}\left(T^{+}=112\right)$ traveling wave along with the maximum and peak to peak displacement amplitude. All positions are at $z=0 \mathrm{~mm}$ and the four positions over the traveling wave surface $\left(a^{+} \neq 0\right)$ are shown in Figure 6.10 . . . . . . . . . . . . . . . . 133

6.2 The peak displacement/amplitude of the $430 \mathrm{~Hz}\left(T^{+}=112\right)$ traveling wave at the spanwise locations shown in Figure 6.10 with $x_{p}=150 \mathrm{~mm} \ldots \ldots \ldots$. . . . 134

6.3 Boundary layer parameters in the wind tunnel upstream and downstream of the traveling wave region . . . . . . . . . . . . . . . . . . . 134

7.1 The streamwise measurement locations for the $607 \mathrm{~Hz}\left(T^{+}=78\right)$ traveling wave along with the maximum and peak to peak displacement amplitude. All positions are at $z=0 \mathrm{~mm}$ and the three positions over the traveling wave surface $\left(a^{+} \neq 0\right)$ are shown in Figure 7.2 . . . . . . . . . . . . . . . . . . 163

7.2 The streamwise measurement locations for the $810 \mathrm{~Hz}\left(T^{+}=59\right)$ traveling wave along with the maximum and peak to peak displacement amplitude. All positions are at $z=0 \mathrm{~mm}$ and the three positions over the traveling wave surface $\left(a^{+} \neq 0\right)$ are shown in Figure $7.5 . \ldots \ldots 167$

7.3 The streamwise measurement locations for the $385 \mathrm{~Hz}\left(T^{+}=122\right)$ standing wave along with the maximum and peak to peak displacement amplitude. All positions are at $z=0 \mathrm{~mm}$ and the three positions over the standing wave surface $\left(a^{+} \neq 0\right)$ are shown in Figure 7.11. . . . . . . . . . . . . . . . . . 173 
7.4 The streamwise measurement locations for the $630 \mathrm{~Hz}\left(T^{+}=74\right)$ standing wave along with the maximum and peak to peak displacement amplitude. All positions are at $z=0 \mathrm{~mm}$ and the three positions over the standing wave surface $\left(a^{+} \neq 0\right)$ are shown in Figure $7.15 \ldots \ldots$. . . . . . . . . . . . . . 177 


\section{Chapter 1}

\section{Introduction}

Environmental concerns currently play a significant role in the advancement of technology. This is evident in the aviation industry with the current drive for reduced emissions, lower noise, and increased fuel efficiency. Within government, NASA is pushing for the development of UltraEfficient Commercial Aircraft [16] and within industry, new environmental standards have placed limitations on fuel burn and emissions [17]. There is also a large economic motivation. The airline industry spends billions of dollars each year on fuel [18], so even small improvements in fuel efficiency would yield substantial benefits. One major step towards achieving these goals is by reducing the drag on the aircraft. This drag reduction would reduce the thrust required by the engines and thus improve fuel burn and decrease emissions. Any advancements in drag reduction would also have huge implications for other applications such as the shipping and automotive industries.

The total drag on an object is the result of several contributing factors. One of these major factors is skin friction drag. Skin friction arises from fluid flowing past an object and imparting shear stresses on the surface. Understanding and reducing the skin friction has been a major research topic for decades. In the 1980s, there was significant interest in geometric features known as using passive riblets [19]. These riblets interacted with the turbulent vortices in the boundary layer in order to reduce the skin friction [20]. Since then, the focus has shifted towards active drag reduction methods. These are advantageous since they can be coupled with passive methods and actively varied depending on the flow conditions. Active drag reduction methods provide continual input to the flow [21] with some examples including gas injection, surface heating and cooling, polymer injection, and external forcing with wall motion.

Of particular interest are those active drag reduction methods using wall motion. Similar to passive riblets, the active wall motion interacts with the vortices in the turbulent boundary layer. The exact method varies between techniques, but in general the turbulence regeneration cycle is disrupted causing weaker bursting events [22]. These bursting events are responsible for increased 
skin friction in turbulent flow [23], so by interacting with the turbulent boundary layer, the active wall motion reduces the skin friction drag. Many of these active wall methods utilize movement of the wall along the in-plane direction [24,25]. While effective at drag reduction, in-plane wall motion would be physically challenging to implement. However, a promsing wall motion technique using spanwise traveling waves with out-of-plane deformation.

The use of spanwise (perpendicular to flow) traveling, surface waves as an active drag reduction mechanism has been researched both computationally and experimentally [3-8,26-32]. These studies have investigated the underlying drag reduction mechanism, the dependence on traveling wave parameters, and also varying flow conditions. Skin friction drag reductions of up $13 \%$ were seen in both experimental [5] and numerical [3] studies. Thus, the use of spanwise traveling waves for drag reduction has been validated. Unfortunately, the experimental methods used to generate the traveling waves in these studies are not realistically implementable. They rely on bulky actuation schemes often using arrays of discrete actuators to deform the surface into a traveling wave. These actuation setups also place limitations on the achievable frequencies and traveling wave patterns. As a result, advancements are needed before spanwise traveling waves can be applied as an active drag reduction method.

A promising method for generating implementable traveling waves is known as two-mode excitation [33]. This method takes advantage of a surface's structural properties to generate steadystate traveling waves without the need for arrays of intrusive actuators. Instead, a small number of low-profile piezoelectric actuators can be used. In addition, the two-mode excitation method is open-loop controlled and has minimal limitations on the achievable frequencies. However, previous studies have primarily used this method on one-dimensional beams [1,2,33,34]. Traveling waves have been excited on a two-dimensional surface [35], but this was done from a fundamental standpoint and was mainly as a proof of concept. Thus, before the two-mode excitation method can be implemented for drag reduction, traveling waves must be generated on a two-dimensional surface with tailorable patterns.

This research aims to develop an implementable traveling wave generation that can be used to interact with the turbulent boundary layer with the goal of reducing skin friction drag. This is done by further developing the two-mode excitation method for generating tailorable traveling waves in a low-profile manner. Once this wave generation method has been developed, its ability to effect the turbulent boundary layer will be investigated. As was discussed, the reduction in skin friction drag occurs via the modification of the turbulent boundary layer. This research takes a fundamental approach by studying the turbulent boundary layer and not focusing directly on the skin friction or quantifying the reduction. Once the effect of traveling waves on the turbulent boundary layer has been investigated, it is compared against the simpler case of standing waves. The effect of these two wave types on the boundary layer is quantified and used to in a parametric analysis to study 
the dependence on amplitude, frequency, and wave pattern.

This section continues by outlining the objectives of this research. Then, the contents of each chapter in this document are outlined.

\subsection{Research Objectives}

The first objective of this research is the development of an implementable traveling wave generation method capable of affecting the turbulent boundary layer. To achieve this, the two-mode excitation method will be used with low-profile piezoelectric actuators to generate traveling waves on a two-dimensional (2D) plate. This method operates in an open-loop fashion, has minimal bandwidth limitations, and does not require a bulky actuation setup. A previous study showed that two-mode excitation can generate traveling waves on a 2D plate, but this was done as a proof of concept and the resultant waves propagated in arbitrary directions. The goal of this study is to generate tailored traveling waves propagating in defined directions. This will be done by understanding the relationship between the actuation configuration (location and number of actuators) and the traveling wave pattern. This relationship can then be leveraged to generate traveling waves tailored to propagate in the spanwise direction in order to effect the turbulent boundary layer.

Second, an experimental setup is necessary to test the effects of the traveling waves on the turbulent boundary layer. As opposed to directly investigating skin friction drag, a more fundamental approach is taken that explores the underlying effect of the traveling waves on the fluid. A low-speed wind tunnel will be used to develop a well-characterized turbulent boundary layer flowing over a traveling wave actuated surface. The boundary layer will then be measured at a number of locations over the traveling wave surface. Finally, these measured boundary layers are analyzed and then validated against accepted data from literature. Based on these requirements, a high-quality wind tunnel setup is necessary to properly study the effects of the actuated surface on the turbulent boundary layer.

The third objective is to investigate the effect of the tailored traveling waves on the turbulent boundary layer. This will be done by generating traveling waves in the wind tunnel and measuring the boundary layer at various locations over the traveling wave surface. In addition, a phase-locked analysis should be conducted to determine the effects at various time steps of the traveling wave, such as the crest and trough. These results can then be compared with those from previous studies into spanwise traveling waves for drag reduction which utilized bulky actuation setups. The goal is to demonstrate that this implementable traveling wave generation method effects the turbulent boundary layer in a manner consistent with skin friction drag reduction.

The fourth and final objective is to compare the effects of standing and traveling waves on the

turbulent boundary layer. The two-mode excitation method generates traveling waves by taking 
advantage of the surface's structural properties. As a result, the excited traveling waves closely resemble the mode shapes (standing waves) of the surface. Due to these similarities in wave pattern, the standing waves may affect the boundary layer in a similar manner as the traveling waves. Thus, the goal is to investigate the effects of traveling and standing waves on the turbulent boundary layer and conduct a parametric analysis to determine the dependence on various wave parameters.

\subsection{Dissertation Structure}

Chapter 1 introduces the research by outlining the motivation and stating the research objectives.

Chapter 2 discusses experimental considerations and the boundary layer theory, which provide a fundamental basis through which to understand the literature review and the overall path taken in the research. The different approaches that could be used to investigate the effect of traveling waves on the turbulent flow are discussed, where the chosen approach experimentally measures the turbulent boundary layer. Then, a brief discussion of turbulent boundary layer theory is presented. Finally, the calculation of skin friction drag from the turbulent boundary layer is presented.

Chapter 3 reviews the literature relevant to this research. This begins by discussing various methods for generating steady-state traveling waves. This primarily focuses on the two-mode excitation method, since it is implemented here. Then, methods for reducing skin friction drag are presented. This includes a general discussion of passive and active methods and then an in-depth review of spanwise traveling waves studies. A consensus is then presented for drag reduction using spanwise traveling waves.

Chapter 4 presents the results of tailoring steady-state traveling waves on a two-dimensional plate using piezoelectric actuators. An in-house finite element model is developed for a plate with mounted actuators. Then, experimental model analysis is conducted and the results used to update the model. The model is then validated using simulated and experimental traveling waves. Finally, the relationship between actuation configuration (location and number of actuators) and traveling wave pattern is investigated.

Chapter 5 explains the wind tunnel setup and turbulent boundary layer measurement. This starts with a discussion of various skin friction measurement methods before settling on boundary layer measurements using hot-wire anemometry. Next, the basic theory of boundary layers and turbulence is outlined. Then, the experimental setup is presented. This includes an explanation of the wind tunnel, development of the boundary layer, ensuring flow quality, and boundary layer measurement. After that, the analysis and post-processing procedure of the boundary layer data is outlined. Finally, the experimental setup is validated.

Chapter 6 presents the results of the turbulent boundary layer flowing over the tailored traveling 
waves developed in Chapter 4. The experimental setup and the effects of fluid loading on the surface are briefly discussed. Next, a spanwise traveling wave pattern is tailored at a single frequency and generated in the wind tunnel. The effect of this traveling wave on the turbulent boundary layer is presented. Finally, the results are discussed and compared with literature.

Chapter 7 compares the effects of traveling and standing waves on the turbulent boundary layer. Two additional traveling waves and two standing waves are generated and their effect on the boundary layer presented. A parametric analysis is then conducted to explore the dependence on wave amplitude, frequency, wave pattern. Finally, the overall effects of traveling and standing waves on the boundary layer are discussed and compared.

Chapter 8 concludes the research presented. The motivation is reiterated and the results summarized. The main contributions are then outlined and suggestions for future work presented.

At the end of the document are several appendices containing supplementary information. Appendix A presents additional information from the finite element model developed in Chapter 4. Appendix B provides information relating to the wind tunnel experimental setup. Appendix C conducts an uncertainty analysis to determine the error in the experimentally measured turbulent boundary layers. Appendix D shows additional figures from the traveling and standing waves investigated in Chapter 7. 


\section{Chapter 2}

\section{Experimental Considerations and Boundary Layer Theory}

The focus of this research is on the development of an implementable traveling wave generation method that alters the turbulent boundary layer, where the application is the reduction of skin friction drag. The development of this novel wave generation method is discussed in Chapter 4. With this traveling wave method, there are several approaches that can be taken to investigate the effect on the turbulent flow. A computational study would provide an in-depth analysis of how the traveling waves affect the turbulent flow; however, this would not align with the aim of experimentally developing an implementable traveling wave generation method. Another approach would involve directly measuring the skin friction/shear stress imparted on the traveling wave surface. But, this method would provide no insight into the fundamental interaction of the traveling waves with the turbulent boundary layer. The approach taken in this research involves experimentally measuring the turbulent boundary layer flowing over the traveling waves. This allows experimental testing of the implementable wave generation method and also yields information about the relationship between the turbulent boundary layer and the spanwise travelings waves.

By taking this approach to measure the turbulent boundary layer, a direct determination of the skin friction/shear stress on the traveling wave surface becomes experimentally challenging. In fact, due to the experimental setup and the sensor choice (hot-wire probe), the skin friction drag is not quantified. However, this was a purposeful trade-off. By measuring the turbulent boundary layer, a fundamental understanding is gained regarding the effect of traveling waves on the turbulent flow. This can then be used to observe the general trend of how traveling waves affect the skin friction. The exact amount cannot be assessed, but increases or decreases in the skin friction drag can be determined.

This chapter begins by outlining the possible approaches that could have been taken in this research. Then, since the chosen approach involves measuring the turbulent boundary layer, a 
brief explanation is given on turbulent boundary layers and the statistical approach to turbulence. Finally, the different methods for calculating the skin friction drag from the boundary layer are discussed. The information in this chapter provides a framework through which to interpret previous literature (Chapter 3) and understand the methodology implemented in this research.

\subsection{Measurement Methods}

To investigate the effect of traveling waves on the turbulent flow for potential skin friction reduction, there are three general approaches that could be taken. These are computational studies, direct drag measurement, and experimental boundary layer measurement. The computational approach yields information about the fluid flow and can also provide changes in the skin friction/shear stress. But, a computational study does not align with main goal of experimentally developing an implementable traveling wave generation method. Directly measuring the shear stress would quantify the skin friction reduction of the travelings waves; however, this is a black-box approach and no information about the underlying mechanism is gathered. Finally, experimental measurements of the boundary layer provide fundamental information about how the traveling waves interact with the turbulent boundary layer. Using this method, the skin friction drag is more challenging to quantify.

Given the available resources and overall problem scope, this research takes the approach of measuring the turbulent boundary layer. As a result, fundamental information regarding the underlying mechanism is gained, and the behavior of the shear stress is observable. However, direct quantifications of the skin friction drag are not possible.

This section discusses the three potential approaches to investigating this research problem: computation studies, direct drag measurements, and experimental boundary layer measurements. Each approach is explained along with its advantages and disadvantages and why it was or was not implemented in this research.

\subsubsection{Computational Studies}

A computational study would yield a significant amount of information showing how a turbulent boundary layer reacts to spanwise travelings waves. The fluid information at all points over the wave surface is known, allowing determination of boundary layer parameters, shear stresses/skin friction, and total drag values. In addition, simulations can be run at various conditions (e.g. Reynolds number). For these reasons, previous literature includes computational studies [3,4,7] investigating the spanwise traveling waves in turbulent flow.

Computational fluid dynamics (CFD) encompasses the modeling of a fluid via the Navier- 
Stokes equations to determine how it flows and interacts with its surroundings. Numerous resources are available that provide an indepth discussion of CFD [36]. In general, there are three types of models within computational fluid dynamics. The first, and simplest, is Reynolds Average Navier-Stokes (RANS). This is the method utilized by most commercial CFD packages and operates by solving the for average quantities in the Navier-Stokes equations. However, since only the average velocities are solved for, this cannot be implemented to analyze coherent structures in turbulence. Thus, Large Eddy Simulations (LES) can be used which is a hybrid approach between direct solution of the Navier-Stokes equations and model of the turbulent phenomena. Turbulence is characterized by the large range of time and length scales involved. As a result, fully resolving the model at the small scale is computationally expensive on the large scale. LES gets around this by directly solving for the Navier-Stokes equations on the large scale and modeling the small scale phenomena. The most computationally expensive method is direct numerical simulation (DNS) and involves directly solving the Navier-Stokes equations down to the smallest scales necessary for turbulence.

However, computational studies were not implemented in the present research. These studies are quite complex and require substantial resources. A significant amount of prior knowledge is necessary to generate a representative model and the model must be processed on a computing cluster. In addition, a computational study does not align with the main research goal of developing an implementable traveling wave generation method. Chapter 4 presents the experimental development of a practical traveling wave generation method, so the effect of this method on turbulent flow should be experimentally investigated.

\subsubsection{Direct Measurement/Force Balance}

The main motivation for using spanwise traveling waves in turbulent flow is to reduce the skin friction drag. Thus, an approach to this research would involve the direct measurement of the skin friction/shear stresses on the traveling wave actuated surface. The skin friction reduction produced by the waved could then be directly quantified. The predominant method for directly measuring drag is with a force balance. While other direct methods such as oil-film interferometry exist [37], they provide results with only limited accuracy. Thus, force balances are preferred with many large-scale wind tunnels utilizing them.

A force balance is a combined system that measures aerodynamic forces in multiple directions. The forces in perpendicular directions are isolated and measured with individual sensors (e.g. load cell). In large wind tunnels, a force balance with measure both lift and total drag (form and friction) experienced by a model placed in the flow. While force balances are an ideal method when measuring both lift and drag, they require an intense amount of time and effort in terms of 
design and calibration [38]. Care must be taken to ensure the forces being measured are along the appropriate axes and that all axes are decoupled from one another. In general, the load cells are positioned outside of the free stream of the tunnel. As a result, the airflow imparts forces upon the mounting between the model and sensors. In order to minimize these unwanted forces, careful design and placement of fairings is required. However, these forces cannot be completely eliminated, so extensive calibration is required to account for and remove these forces from any measurements. In addition, the force balance measures the total force on an object meaning that there is no knowledge of the force distribution across a surface.

Force balances are typically used to measure the total drag on an object: combination of form/pressure drag and skin friction drag. A force balance can be used to measure the skin friction drag alone, but additional factors must be considered. The surface over which the friction drag is measured requires special mounting. It should be isolated from surrounding surfaces so as to minimize any form drag due to non-flush transitions. The gaps between surfaces should also be properly sealed to minimize changes in flow conditions. In addition, the load cell would have to be carefully chosen so as to provide the resolution needed to measure the skin friction. The use of a force balances for skin friction measurements are discussed by Haritonidis [39] and an example is implemented in Gouder [40].

Overall, a force balance to directly measure the skin friction drag would not be an ideal approach for this research. The necessary experimental setup would be complicated and would require significant effort towards calibration and troubleshooting. More importantly, the force balance provides no information regarding the influence of the traveling waves on the fluid. So, the fundamental relationship between the waves and the turbulent boundary layer could not be investigated. In addition, the experimental testing would be tedious and black-box in nature. Traveling waves at certain parameters (i.e. amplitude and frequency) may yield a skin friction reduction, while other parameters may not. Experimental testing at many different parameters would be necessary to establish a relationship.

\subsubsection{Turbulent Boundary Layer Measurement}

The final approach, and the one used in this research, involves experimentally measuring the turbulent boundary layer in a wind tunnel over the traveling waves. This experimental approach provides a representative environment in which to test the implementable traveling wave generation method developed in this research. In addition, measurements of the turbulent boundary layer offers a more fundamental understanding of how the traveling waves interact with the turbulent boundary layer. This fundamental approach will demonstrate the underlying changes in the skin friction/shear stress, but direct quantifications are difficult and often rely on incorrect assumptions. 
This is discussed further in Section 2.3.

Three methods for measuring the boundary layer are discussed here: particle image velocimetry (PIV), pitot tubes, and hot wire anemometers (HWAs). The differences between these methods are not just in terms of capabilities, but also complexity and financial costs. PIV allows for simultaneous measurement of the flow profile along an entire two-dimensional cross-section and provides the most information. In comparison, pitot tubes and HWAs only measure the flow velocity at only a single location in the flow at a time. By traversing the sensor through the flow, the statistically averaged flow profile can be mapped. To map the flow profile, additional equipment (e.g. a three-dimensional traversing system) and considerations are required. Although the capabilities of single point velocity probes are lower then PIV, they require significantly less resources. The advantages and disadvantages of the PIV, pitot tubes, and HWA are discussed here. Both the pitot tube and hot-wire probe are implemented in this research, so they are discussed in more depth; a comparison between the two is found in Section 5.3.2.

\subsubsection{Particle Image Velocimetry (PIV)}

At present, particle image velocimetry (PIV) is one of the most common methods for measuring the turbulent boundary layer. In PIV, a large number of seeding particles are injected into the flow. A laser illuminates particles in a two-dimensional cross-section of the flow. Then, a high-speed camera captures the movement of these particles along the illuminated cross-section. Correlation algorithms and statistical analysis is then applied to the images to determine the velocity field throughout the flow. The velocity fields can then be used to map the turbulent boundary layer or other turbulent phenomena. A thorough discussion of particle image velocimetry can be found in Raffel's book [41]; however, PIV is a heavily researched field and countless other books and publications exist.

For those with the means, PIV provides the most thorough insight into experimental fluids. PIV allows for the measurement of the entire flow field in a two-dimensional (2D) cross-section simultaneously while not altering the flow path by inserting sensors. In addition, the method is quick enough such that the dynamics of the flow can be captured. As a result, PIV can be used to accurately measure both turbulent dynamics and statistically averaged boundary layers while also allowing calculation of the local skin friction/shear stress.

PIV is able to simultaneously provide accurate results at numerous locations in the flow. However, this comes at the cost of an expensive and complex experimental setup with a significant amount of data analysis. The setup requires a system to properly seed the particles, a laser that can illuminate a 2D cross-section, a high-speed camera and viewing window to capture the movement of the particles, and the software and sufficiently powerful computer to analyze the data. It should be noted, this is only the equipment needed to run the experiment. In addition, a significant amount 
of knowledge and experience is required to achieve reliable and accurate results. A proper seeding density using the correct particle sizes is required so as to provide sufficient spatial resolution while not altering the flow. The correlation methods used to extract the velocities from the highspeed images must be thoroughly understood. An extensive knowledge of statistical methods is required so as to properly determine the time- and spatially-averaged velocities and the uncertainties therein. Thus, it is an extremely powerful experimental technique, but significant economic and personnel resources are required to implement it.

Particle image velocimetry (PIV) would have proven advantageous for this research; however, the resources were not available to implement it. Further experimental studies into spanwise traveling waves should utilize PIV since it simultaneously provide information at numerous positions while also allowing calculation of the local skin friction/shear stress.

\subsubsection{Pitot Tube}

A Pitot tube does not directly measure the flow velocity, but rather the dynamic pressure at the tip of the probe. Specifically, the probe measures the total pressure, which is a combination of the dynamic and static pressure. This total pressure, along with the static pressure at the same location, are then sent to a differential pressure transducer. The difference between the total and static pressure is the dynamic pressure, which is directly related to the flow velocity at that location. A pitot-static tube has a total pressure port at the tip of the probe and static pressure ports radially distributed along the diameter of the tube. A pitot tube only consists of a total pressure port and requires static pressure ports located on the wall of the wind tunnel.

Since the pitot tube measures the velocity at only a single location at a time, a traversing system is necessary to move the probe around and map the boundary layer profile. Thus, a pitot tube is at a disadvantage with respect to particle image velocimetry (PIV). Pitot tubes are also large sensors, especially in comparison to the scales seen in a turbulent boundary layer. Even though small diameter pitot tubes can be purchased, these are too large to measure velocities into the inner portion of the boundary layer, such as the viscous sublayer. This region conveys a significant amount of information, especially in regards to the skin friction. As a result, pitot tubes are not able to capture this. Another disadvantage of pitot tubes is that they require correction factors to account for the presence of the probe in the flow itself (Section B.1). Finally, a pitot tube is bandwidth limited and cannot measure fluctuating flow velocities, rather only the mean flow. Further discussion of pitot tubes is given by Chue [42] and MacMillan [43].

Despite all of these disadvantages, pitot tubes have a significant advantage: they are simple. Aside from the traversing system (which is required for any single point sensor), the other main components are a pressure transducer and the pitot tube itself. Both of which are fairly inexpensive and robust. By traversing the pitot tube through the flow, the boundary layer profile can be 
measured and yield a significant amount of information. This includes whether the flow is turbulent, boundary layer thickness, and approximations of the skin friction. While it is not suited for an indepth analysis of skin friction, a pitot tube does allow for an initial analysis of the turbulent boundary layer.

A small diameter pitot tube is utilized in this research as the preliminary approach to investigating the effects of the traveling waves on the boundary layer. IT is discussed further in Section 5.1.4.2. For the final experimental analysis, hot-wire anemometry is used in place of pitot tubes.

\subsubsection{Hot-wire Anemometry (HWA)}

The final boundary layer measurement discussed, and the one utilized for all experimental testing is hot-wire anemometry (HWA). Similar to a pitot tube, HWA measures the fluid velocity at a single location in the flow. A hot-wire probe consists of a small diameter $(\approx 5 \mu \mathrm{m})$ tungsen wire suspended between two prongs, creating an electrical connection (see Figure 2.1). This sensor wire is then resistively heated and submerged into the air flow. The wire loses heat to the air flow via convection, where the rate of heat loss directly relates to the flow velocity. In constant temperature anemometry (CTA), the wire is kept at a constant temperature. As the wire heats up, its resistance increases; thus, to hold the wire at a fixed temperature, the resistance of the wire can be maintained at a specific value. This is done by connecting the hot-wire probe as part of a Wheatstone bridge. The resistance of the bridge is then balanced such that the sensor wire is held at a resistance corresponding to a specific temperature (higher than the air flow). This process and hot-wire anemometry in general are discussed further by Comte-Bellot [44] and Bruun [45].

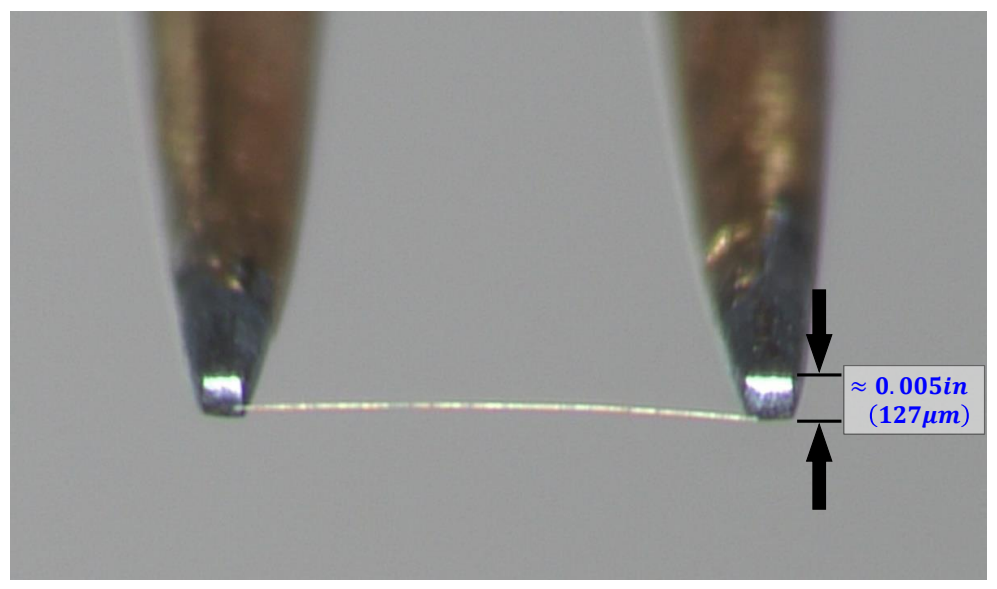

Figure 2.1: Front view of a hot-wire probe with the sensor wire welded between the two prongs

In order to determine the actual flow velocity from the HWA voltage output, a calibration procedure is necessary. This calibration is done by correlating the HWA voltage with known flow 
velocities. In the case of a wind tunnel, the hot-wire probe is placed in the free-stream and the wind tunnel run through different operating speeds. These speeds have been previously calibrated and measured using a pitot-static tube. Through this procedure, the output HWA voltages can be mapped to known wind speeds and then be fit with a calibration curve. This calibration curve is then used during boundary layer testing. Since the HWA operating principle is based on convective heat transfer, the calibration is sensitive to the ambient environmental conditions (e.g. temperature). Temperature corrections can be implemented [45]; however, the calibration must still be rerun fairly often to ensure an accurate correlation between voltage and velocity.

In comparison to the pitot tube, the hot-wire probe is more complex, expensive, and fragile. The operating procedure and underlying principle require a greater degree of understanding. The probe, sensor wire, and controlled wheatstone bridge are considerably more expensive. And the sensor wire is susceptible to breaking with the slightest contact with the probe tip. Although a new sensor wire can be installed, it requires specialized equipment and is a time-consuming endeavor.

Given the resources, hot-wire anemometry provides significantly better capabilities than a pitot tube. The measurement size of a hot-wire probe is significantly smaller than that on a pitot tube; thus, allowing measurement of the viscous sublayer. In addition, HWA is a dynamic measurement with a high enough bandwidth to measure the fluctuating velocity components. As a result, a hotwire probe can be used to investigate turbulent phenomena. A single wire, hot-wire probe can then measure the turbulent boundary layer and map not just the mean velocity profile, but also the higher moment velocity profiles (RMS, skewness, and kurtosis). In addition, hot-wire probes containing multiple wires can measure the three-dimensional flow velocity. This allows the calculation of the Reynolds stresses and an, in general, deeper investigation into the turbulence phenomena.

Hot-wire anemometry is the primary experimental method implemented here to investigate the effect of the traveling waves on the flow. It is discussed further in Section 5.1.4.3.

\subsection{Basic Theory of Turbulent Boundary Layers}

The boundary layer is the region of the flow near a wall where the velocity changes with respect to its distance from the surface. Far away from the surface, the velocity is nominally independent of vertical position and flows at the free-stream velocity, $U_{e}$. The boundary layer height, $\delta$, is defined as the height from the surface to the point in the flow where the free-stream velocity is reached (typically $99 \%$ of this value). The velocity at the surface is the same as the surface itself, which for a stationary surface is zero. Thus, the velocity of the fluid changes from zero to the free stream-velocity over the boundary layer thickness. As the fluid travels further down the surface (streamwise direction), the boundary layer thickness increases. This results from the fluid constantly losing energy to the surface via shearing. This shearing is known as skin friction 
drag and is dependent upon a number of flow properties. The skin friction can then be determined from measurements of the boundary layer profile; however, the accuracy is subject to a number of parameters (Section 2.3).

Depending on the streamwise position and the ambient flow conditions, the boundary layer will be either laminar or turbulent. This research focuses on the effects of traveling waves on the turbulent boundary layer; thus, turbulent boundary layers are the primary focus here. This section provides some basic theory necessary for the measurement and analysis of turbulent boundary layer. The section begins with a discussion of the structure and various regions of the turbulent boundary layer. Next, various boundary layer parameters are explained. Then, a brief discussion of turbulence phenomena is given. For further reading on boundary layers, the reader is directed to Schetz [46] while additional discussions of turbulence can be found in Tennekes and Lumley [47] and Davidson [48].

\subsubsection{Turbulent Boundary Layer}

The structure of the zero-pressure-gradient turbulent boundary layer is shown in Figure 2.2. The $\mathrm{x}$-axis is the logarithmically plotted non-dimensional distance from the wall $\left(y^{+}\right)$while the $\mathrm{y}$ axis is the mean streamwise flow velocity. The non-dimensional position, $y^{+}$is known as wall units and is defined in Equation 2.1, where $y$ is the wall-normal position, $v$ is the kinematic viscosity, and $u_{\tau}$ is the friction velocity. The friction velocity is the main non-dimensionalization factor when discussing turbulent boundary layers. It is defined in Equation 2.2, where $\rho$ is the density and $\tau_{w}$ is the local wall shear stress. The boundary layer is normalized to wall units in order to provide a common reference frame, so that all boundary layers converge to the same profile. Shown in Figure 2.2 are the four regions of the turbulent boundary layer, along with the free-stream region. A properly normalized turbulent boundary layer will converge to the viscous sublayer, buffer layer, and log-law in Figure 2.2.

$$
\begin{gathered}
y^{+}=\frac{y u_{\tau}}{v} \\
u_{\tau}=\sqrt{\frac{\tau_{w}}{\rho}}
\end{gathered}
$$

The four boundary layer regions highlighted in Figure 2.2 are the viscous sublayer, buffer layer, log-law of the wall, and wake region. The viscous sublayer is the region closest to the wall and is characterized by the linear relationship between velocity and wall distance (Figure 2.2 shows a dashed line corresponding to the linear relationship extending into the buffer layer). This linear relationship is defined in terms of the wall units as in Equation 2.3, where $u$ is the local 


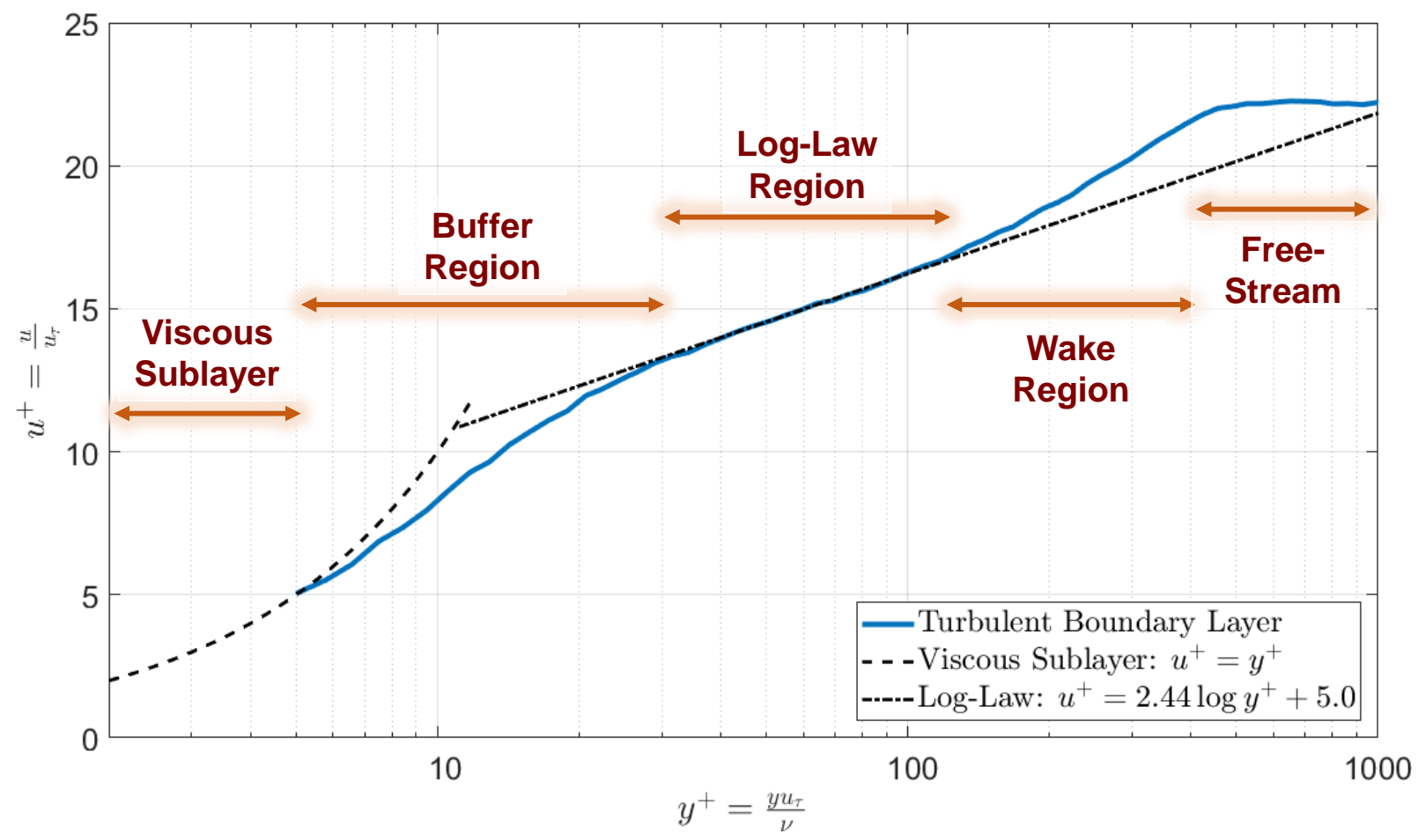

Figure 2.2: General structure of a turbulent boundary layer with velocity $\left(u^{+}\right)$vs. y position in wall units $\left(y^{+}\right)$

mean streamwise velocity. This linear relationship can be rearranged in terms of non-wall units as shown in Equation 2.4, where $\mu$ is the dynamic viscosity. Thus, the slope of the linear relationship is proportional to the shear stress (skin friction) imparted on the wall.

$$
\begin{aligned}
& u^{+}=\frac{u}{u_{\tau}}=y^{+} \\
& u=\frac{\tau_{w}}{\mu} y=\frac{u_{\tau}^{2}}{v} y
\end{aligned}
$$

Also shown in Figure 2.2 is the log-law region. As the name suggests, this region is defined by the fact that it follows a logarithmic relationship. This relationship can be seen as the dashed line in Figure 2.2 which fits the data in the log-law region, with the data diverging away outside of this region. The logarithmic relationship is defined as in Equation 2.5, where $\kappa$ is the von Kármán constant and $B$ is an additive constant. Coles [15] defined these constants as $\kappa=0.41$ and $B=5.0$, however their exact values have been the subject of significant debate [49].

$$
u^{+}=\frac{1}{\kappa} \ln \left(y^{+}\right)+B
$$


The region between the viscous sublayer and the log-law region is known as the buffer layer. As is seen in Figure 2.2, this is the region in which Equations 2.3 and 2.5 overlap. In addition, this is the region of the boundary layer with the largest turbulence production [48]. On the other side of the log-law, is the wake region. This is the transition region between the log-law and the free-stream, and its form is Reynolds number dependent [50]. Far from the wall (large $y^{+}$), the velocity levels off and becomes a constant value, which is the free-stream velocity $\left(U_{e}\right)$. The end of the turbulent boundary layer is defines as $y$ position at which the velocity is $99 \%$ of the free-stream. This is defined as the boundary layer thickness, $\delta$. Further discussion of the different regions in the turbulent boundary layer can be found in Davidson [48].

\subsubsection{Boundary Layer Parameters}

Two parameters commonly used when discussing the boundary layer are the $\operatorname{displacement}\left(\delta^{*}\right)$ and momentum thicknesses $(\theta)$. The displacement thickness is defined in Equation 2.6, where once again $y$ is the position from the surface, and $u$ and $U_{e}$ are the local and free-stream velocities, respectively. This equation arises from separating the flow regime into a boundary layer region and an ideal flow region when solving the Navier-Stokes equations. The displacement thickness is a way of relating the growth of the boundary layer to the ideal flow solution; $\delta^{*}$ is the distance above the surface at which the ideal flow solution can be applied. Another explanation is that the displacement thickness is how much the streamlines of the outer flow have been displaced [51].

$$
\delta^{*}=\int_{0}^{\infty}\left(1-\frac{u}{U_{e}}\right) d y
$$

The momentum thickness, $\theta$, is defined in a similar way using Equation 2.7. As the fluid flows along the object, it loses energy to the surface due to shear stress. The momentum thickness at a location $x=x^{\prime}$ on the surface relates to the amount of momentum lost to the surface from the beginning of the boundary layer, $x=0$. The momentum thickness can then be used to define the momentum-thickness based Reynolds number, $R e_{\theta}$, and in Equation 2.8. The Reynolds number is a non-dimensional parameter that is used to establish a common comparison form. Thus, different fluids or different free-stream velocities can be compared using $\operatorname{Re}_{\theta}$.

$$
\begin{gathered}
\theta(x)=\int_{0}^{\infty} \frac{u(x)}{U_{e}}\left(1-\frac{u(x)}{U_{e}}\right) d y \\
\operatorname{Re}_{\theta}=\frac{\rho \theta U_{e}}{\mu}
\end{gathered}
$$

When discussing the wall shear stress, $\tau_{w}$, it is normalized to the skin friction coefficient as shown in Equation 2.9. The local skin friction coefficient, $C_{f, x}$, defined as the shear stress at a 
specific streamwise positions, $x$. While the average skin friction coefficient, $C_{f, a v g}$, is the mean over a range of streamwise positions.

$$
\frac{C_{f}}{2}=\frac{\tau_{w}}{\rho U_{e}^{2}}
$$

\subsubsection{Turbulence}

Up to this point, only the mean streamwise flow velocity, $u$, has been discussed. This is a timeaveraged mean value. However, the flow velocity fluctuates with time and can be broken into two components, a mean and fluctuating. This is shown in Equation 2.10, where $\tilde{u}(t)$ is the total timedependent flow velocity, $u$ is the time-averaged mean, and $u^{\prime}(t)$ is the fluctuating component with mean value of zero. The time-averaged mean is assumed to be stationary [45], such that the mean remains constant over time. In addition, the flow velocity is erdogodic, such that the mean value remains the same when evaluated over different segments of the time history [47].

$$
\tilde{u}(t)=u+u^{\prime}(t)
$$

Breaking the velocity into a mean and fluctuating component allows for an analysis from a statistical perspective. This analysis can be broken into three separate groupings: amplitude-domain, time-domain, and frequency-domain statistics [45]. Amplitude-domain analyzes the velocity from time-averaged perspective where there is no time-history information. This includes both single and multiple velocity signal analysis; however, the focus here is upon a single velocity signal (streamwise direction), since the experimental setup here utilizes only single wire hot-wire anemometry (HWA). The amplitude-domain analysis will focus on the mean, variance, skewness, and kurtosis. Each of these is a different value to analyze the probability distribution of the velocity. If multi-wire hot-wire probes were employed, multi-directional velocities could be measured, allowing for analysis of Reynolds stresses and joint probability density functions.

The mean of the velocity, $u$ or $\bar{u}$, is defined as shown in Equation 2.11. T is the time length of the signal and $\tilde{u}(t)$ is the time-varying velocity, as introduced in Equation 2.10. The variance of the velocity is defined in Equation 2.12, where $\sigma_{u}^{2}$ is the variance and $\sigma_{u}$ is the standard deviation. In addition, the variance is often non-dimensionalized to wall units $\left(\frac{\overline{u^{\prime 2}}}{u_{\tau}^{2}}\right)$, where $u_{\tau}$ is the the friction velocity (Equation 2.2). When normalized by the friction velocity, it is also known as the streamwise Reynolds stress. In addition, the variance is known as the second moment, where this is the mean of the second power of the velocity [47]. This in turn makes the mean the first moment.

$$
u=\bar{u}=\lim _{T \rightarrow \infty} \frac{1}{T} \int_{0}^{T} u(t) d t
$$




$$
\sigma_{u}^{2}=\overline{u^{\prime 2}}=\lim _{T \rightarrow \infty} \frac{1}{T} \int_{0}^{T}[\tilde{u}(t)-u]^{2} d t=\lim _{T \rightarrow \infty} \frac{1}{T} \int_{0}^{T} u^{\prime 2}(t) d t
$$

The third moment analyzes the asymmetry of the velocity probability distribution, and is defined in Equation 2.13. If the distribution is symmetric, $\overline{u^{\prime 3}}=0$. Non-dimensionalizing the third moment by the standard deviation results in the skewness, $S$, which is shown in Equation 2.14. If the skewness is positive, the signal is more likely to have large positive values than negative ones. For a negative skewness, the opposite is true.

$$
\begin{gathered}
\overline{u^{3}}=\lim _{T \rightarrow \infty} \frac{1}{T} \int_{0}^{T} u^{\prime 3}(t) d t \\
S=\frac{\overline{u^{\prime 3}}}{\sigma_{u}^{3}}
\end{gathered}
$$

The forth moment describes the flatness of the velocity probability distribution. When nondimensionalized by $\sigma_{u}^{4}$, this signal is known as the kurtosis, $K$ (see Equation 2.15). If the probability distribution of the signal is normal/Gaussian, then the kurtosis is $K \approx 3$. Whereas, a signal with small kurtosis $(K<3)$ has more values concentrated about the mean, while that with large kurtosis $(K>3)$ has more values in the tails of the distribution.

$$
K=\frac{\overline{u^{\prime 4}}}{\sigma_{u}^{4}}=\frac{1}{\sigma_{u}^{4}}\left[\lim _{T \rightarrow \infty} \frac{1}{T} \int_{0}^{T} u^{\prime 4}(t) d t\right]
$$

In Section 2.2.1 the mean velocity boundary layer profile was introduced (Figure 2.2), which is the change in mean flow velocity as a function of distance from the surface. Similar to the mean; the variance, skewness, and kurtosis all have a profile that changes as a function of the distance from the surface. By analyzing these profiles, changes imparted by the spanwise traveling waves can be detected.

The assumption when calculating the amplitude-domain statistics, is that every sample in signal is statistically independent. Depending on the sampling rate, that is not always the case with a turbulent velocity signal. The fluctuations in the velocity are caused by varying size vortices in the flow. Since the vortices are a finite size, they take a finite time to pass a location in the flow. As a result, there is a time period over which the velocity values are correlated. Thus, there is a time interval over which the velocity 'remembers' its past history [47]. Since these velocities are correlated, they are not statistically independent. Thus, in order to have a time signal with statistically independent velocity values, consecutive samples must have a large enough time separation between them. This is the basis of time-domain analysis.

The correlation within a velocity signal can be determined by taking the autocorrelation of the fluctuating velocity. The autocorrelation is defined in Equation 2.16, where $u^{\prime}(t)$ and $u^{\prime}(t+\tau)$ are 
the fluctuating velocites with a time difference $\tau$ between them and $R_{u}(\tau)$ is the autocorrelation as a function of $\tau$. This is then normalized by the variance of the signal to define the autocorrelation coefficient, $\rho_{u}(\tau)$ (Equation 2.17). When there is no time difference, $\rho_{u}(\tau=0)=1$, which is the maximum value. This makes intuitive sense, since no time delay indicates that the signal is perfectly correlated with itself. As the time difference grows larger, the correlation decreases until it approaches or crosses zero. A value of $\rho_{u}(\tau)=0$ indicates that the signals are completely uncorrelated.

$$
\begin{gathered}
R_{u}(\tau)=\lim _{T \rightarrow \infty} \frac{1}{T} \int_{0}^{T} u^{\prime}(t) u^{\prime}(t+\tau) d t \\
\rho_{u}(\tau)=\frac{R_{u}(\tau)}{\sigma_{u}^{2}}
\end{gathered}
$$

In order to define the time difference at which the two signals are uncorrelated, the integral time-scale, $T_{I}$, is used. This is defined in Equation 2.18 When $\tau \geq 2 T_{I}$, the signals are considered uncorrelated [47]. This same rule can then be applied to determine statistical independence. If the time interval between samples in a velocity signal is $\geq 2 T_{I}$, then the samples are considered statistically independent and can be used to determine the amplitude-domain statistics. This procedure is explained further and implemented in Section 5.2.1.

$$
T_{I}=\int_{0}^{\infty} \rho_{u}(\tau) d \tau
$$

\subsection{Measurement of Skin Friction/Shear Stress}

There are three general ways to calculate the skin friction coefficient in a turbulent boundary, with each technique varying in accuracy and experimental difficulty. The first method is to measure the velocity gradient in the viscous sublayer. This provides an accurate estimate of the local skin friction, $C_{f, x}$; however, it requires measuring very close to the surface. The second is the Clauser method which involves fitting the log-law region of the boundary layer. This method provides an approximation of the local skin friction, and it is primarily used to find the friction velocity, $u_{\tau}$ and normalize the boundary layer to wall units. The third technique is the momentum balance method. It yields a average skin friction coefficient along the streamwise direction, but it relies on a number of often-violated assumptions. This section presents each of these techniques and discusses their applicability in regards to the present research and the effect of traveling waves on the turbulent boundary layer.

The most straight-forward and accurate way to measure the local skin friction coefficient (Equation 2.9) is by determining the velocity gradient in the viscous sublayer. As was shown in 
Equation 2.4, the profile in this near-wall region is linear and the velocity gradient directly relates to the local shear stress, $\tau_{w}$, imparted by the fluid. As a result, this method relies on no assumptions and could be used to determine the skin friction over a traveling wave excited surface; however, this is experimentally challenging since the viscous sublayer is very thin. The viscous sublayer corresponds to wall positions with $y^{+}<5$ [47]. This is shown for the boundary layer profile in Figure 2.2, which was collected using the experimental setup in this research (Chapter 5). Converting to dimensional units, the viscous sublayer corresponds with wall positions for $y \lesssim 100 \mu \mathrm{m}$. So, to determine the skin friction coefficient, $C_{f, x}$, the flow velocity must be experimentally measured at several locations within this small region. A linear line would then be fit to determine the velocity gradient.

In the current research, the skin friction coefficient cannot be determined by measuring the viscous sublayer. Both a pitot tube and hot-wire probe are used here to measured the turbulent boundary layer, but the pitot tube can be written off immediately since it is too large to measure in the viscous sublayer. A hot-wire probe can measure into the sublayer, but there are several complicating factors that prevent it in the present research. First, there are errors in the hot-wire probe when measuring close to a surface. These are outlined in Section 5.1.4.3, and they prevent accurate measurement of the velocity in the viscous sublayer. The boundary layer profile in Figure 2.2 is measured as close to the wall as possible before errors become visible. Second, even if these errors could be corrected, a traveling wave surface oscillates in the wall-normal direction. So, the hot-wire probe cannot get close enough to the wall (without breaking) to measure the viscous sublayer. If the turbulent boundary layer were measured using particle image velocimetry (PIV), it would be possible to measure the viscous sublayer over the moving wall. But in the current experimental setup, the viscous sublayer and local skin friction cannot be measured using hot wire anemometry (HWA).

The viscous sublayer method can be used to visually observe whether there is a change in skin friction. In this research, the hot-wire probe cannot measure into the viscous sublayer to directly quantify the skin friction. However, comparing two boundary layer profiles, one can visually distinguish which profile has lower skin friction. Due to the no-slip condition of the fluid at the wall, the profile converges to $u^{+}=y^{+}=0$ (no flow at wall). For two profiles plotted on top of each other, if one has a noticeably lower velocity in the buffer layer (Figure 2.2), then then it will converge to the wall $\left(u^{+}=y^{+}=0\right)$ with a lower velocity gradient. Thus, it will have lower skin friction.

The skin friction coefficient, $C_{f, x}$, can also be found using the Clauser method [52]. This method fits the law of the wall (Equation 2.5) to gathered experimental data in order to determine $C_{f}$. Multiple logarithmic profiles are plotted with different $C_{f}$ values, and the $C_{f}$ value that lines up the best with the logarithmic region of the experimental data is the local skin friction value of the 
turbulent boundary layer. This method provides a good estimate of the skin friction coefficient, but it is not the most accurate method. In particular, there is a heavy reliance on the choice of constants $\kappa$ and $B$. As mentioned in Section 2.2, these constants are already the subject of debate. In addition, if the structure of the turbulent boundary layer is changed via surface roughness or a surface excitation such as traveling waves, then these constants vary even more. Wei [53] discusses some limitations of the Clauser method. The Clauser method is implemented in this research (Section 5.2.2) to calculate the friction velocity, $u_{\tau}$, and normalize the boundary layer to wall units. However, it cannot be used to determine an accurate measurement of the skin friction coefficient/shear stress.

The final method for determining the skin friction/shear stress is the momentum balance technique. The momentum balance is derived by conducting a control volume analysis of the turbulent boundary layer for flow that is plane, steady, and incompressible. Through application of the conservation of mass and momentum equations, Equation 2.19 is derived. This is known as the Kármán integral equation [46] or the Momentum-Integral equation [51]. It relates the local skin friction coefficient, $C_{f, x}$, to the streamwise gradients of the momentum thickness and free-stream velocity, $\frac{d \theta}{d x}$ and $\frac{d U_{e}}{d x}$, respectively, where $H=\frac{\delta^{*}}{\theta}$ is the shape factor.

$$
\frac{C_{f, x}}{2}=\frac{d \theta}{d x}+(2+H) \frac{\theta}{U_{e}} \frac{d U_{e}}{d x}
$$

If the flow is assumed to have no steamwise pressure gradient, then the free-stream velocity is constant along the length of the surface and $\frac{d U_{e}}{d x}=0$. This simplifies the momentum-integral equation to the form shown in Equation 2.20. The streamwise gradient of the momentum thickness, $\frac{d \theta}{d x}$, would be time-consuming to calculate, so the momentum balance can be simplified even further to the form shown in Equation 2.21. Using this simplified form, the average skin friction coefficient, $C_{f, a v g}$, between two locations $x_{1}$ and $x_{2}$ is related to the momentum thicknesses at those locations.

$$
\begin{gathered}
\frac{C_{f, x}}{2}=\frac{\tau_{w}}{\rho U_{e}^{2}}=\frac{d \theta}{d x} \\
C_{f, a v g}=2\left[\frac{\theta\left(x_{2}\right)-\theta\left(x_{1}\right)}{x_{2}-x_{1}}\right]
\end{gathered}
$$

At this point, it should be clearly stated: the momentum balance provides an estimate of the skin friction and relies on a number of assumptions. To use the form shown in Equation 2.21, the flow must be plane, steady, incompressible, over a flat surface, and have zero pressure gradient. Even then, the equation is simplified to provide an average skin friction coefficient.

The momentum balance method is not accurate and cannot be applied in the present research to determine the skin friction/shear stress. The momentum balance method assumes that the flow 
is plane (two-dimensional). Anders [54] discusses the momentum balance method for determining the skin friction reduction of outer-layer manipulators. He summarizes several experimental studies and concludes that the momentum balance method tends to overestimate the reductions in skin friction yielded by the manipulators; primarily due to three-dimensional effects. The traveling waves used in this research propagate in the spanwise direction, which induces three-dimensional flow and would yield inaccuracies in the momentum balance method.

To use the simplified form of the momentum balance equation (Equation 2.20), there is assumed to be zero streamwise pressure gradient. For the experimental setup utilized in this research, the streamwise pressure gradient is minimized (Section 5.1.3.3), but it cannot be completely removed. The full momentum balance equation (Equation 2.19), could be implemented to account for the pressure gradient. However, Haritonidis [39] discusses its use, and further shows that it neglects contributions from turbulent quantities. Dutton [55] accounts for the turbulent quantities and demonstrates that they contribute a non-trivial amount to the calculated skin friction coefficient.

In addition, the fundamental control volume analysis and the derivation of the momentum balance equation does not consider the traveling wave surface. The movement of the traveling wave surface injects momentum into the flow which is not accounted for in the control volume analysis $[46,51]$. Thus, even if all the other factors (three-dimensionality, pressure gradient, turbulent effects) were properly accounted for, the fundamental equation (Equation 2.19) would still be incorrect. Thus, the momentum balance equation cannot be accurately implemented over a traveling wave surface.

\subsection{Summary}

This chapter presented some fundamental experimental considerations and the turbulent boundary layer theory necessary to interpret this research. This information also provides a framework to understand the literature review presented in Chapter 3. Three different approaches were presented for investigating the effect of spanwise traveling waves on the turbulent boundary layer and the reduction in skin friction drag. A computational study would provide information about the flow and allow calculation of the skin friction. However, it does not align with the broader scope of this research, which is the experimental development of a practical traveling wave generation method. The second approach involved directly measured the skin friction/shear stresses. But this would provide no information about the underlying effect of the traveling waves on the turbulent flow. The final approach and the one implemented in this research involves experimentally measuring the turbulent boundary layer. This allows both experimental testing of the implementable traveling wave generation method and also investigation of the fundamental interaction between the traveling waves and the turbulent boundary layer. 
Since the approach taken in the research involves measuring the turbulent boundary layer, a brief discussion of the boundary layer was presented. This began with an explanation of the various regions in the turbulent boundary layer (e.g. viscous sublayer). Then, the relevant boundary layer parameters were presented. Finally, the statistical approach to turbulence was outlined.

A main motivation for implementing spanwise traveling waves in turbulent flow is to reduce the skin friction drag. So, the methods for determine the skin friction/shear stress from turbulent boundary layer measurements were discussed. It was shown that the skin friction cannot be directly quantified in this research, but it is demonstrated that relative changes and the overall trend in the skin friction can be seen. The most accurate skin friction measurement technique involves measuring the velocity gradient in the viscous sublayer of the boundary layer; this is not possible since the hot-wire probe cannot accurately measure into the viscous sublayer. The Clauser method was then explained, but it cannot be used over a traveling wave surface and is only implemented here to normalize the boundary layer to wall units. The final method presented was the momentum balance, and it was established to be inaccurate for measuring the skin friction over a traveling wave surface. This method is based on a number of assumptions that are violated (three-dimensionality, non-zero pressure gradient, turbulent effects) and the fundamental derivation does not account for the traveling wave surface. Thus, the momentum balance cannot be used for traveling waves and any studies that rely on it should be cautiously interpreted.

This research develops a traveling wave generation method that is practical and implementable (Chapter 4). The interaction of these traveling waves with the turbulent boundary layer is then presented (Chapters 6 and 7). The underlying mechanisms are investigated and the overall trend of the skin friction drag is addressed (no direct quantifications). 


\section{Chapter 3}

\section{Literature Review}

This research aims to develop an implementable traveling wave generation method that can alter the turbulent boundary layer with the eventual application of reducing skin friction drag. Previous research has demonstrated that spanwise traveling waves can produced skin friction drag reductions, but minimal emphasis was placed on the traveling wave generation method. As a result, previous wave generation techniques relied on bulky actuation setups that placed limitations on the achievable frequencies and traveling wave patterns. For spanwise traveling waves to be applicable drag reduction technique, a more practical (lightweight and variable parameter) wave generation method is necessary. The two-mode excitation method is a promising technique for producing variable parameter traveling waves in a lightweight fashion, but further development is necessary. The traveling waves must be generated on a two-dimensional surface and the traveling wave pattern should be tailorable so that different propagation patterns are possible (e.g. propagation direction and number of wave-fronts).

This chapter presents previous literature that is relevant to the current research. This begins with a discussion of steady-state traveling waves. Several methods for generating these waves are examined, then the two-mode excitation method is presented in depth. Next, various methods for reducing skin friction drag are explained, including general passive and active wall methods. Finally, the the use of spanwise traveling waves to affect the turbulent boundary layer and reduce skin friction drag is discussed at length. This also includes a general summary of the present conclusions from this method.

\subsection{Traveling Waves}

Traveling waves are a fundamental part of vibrations; however, the type most common in vibrations (transient) differ from those employed in this research (steady-state). A transient traveling wave is generated any time an object is excited. Take the case of a beam clamped on both sides 
with an actuator exciting the center of the beam. When the actuator is excited at a single frequency, traveling waves propagate away from the actuator in both directions. These waves continue until they reach the clamped boundaries, they then reflect back towards the actuator. The reflected waves then superimpose with the continually incident waves causing constructive and deconstructive interference. With the actuator still operating, this process settles into a steady-state response. That steady-state response is a standing wave. Within the standing wave, there are nodal points where there is deconstructive interference causing zero amplitude. The location and number of node points is dependent upon the excitation frequency and geometry of the system, hence mode shapes.

The important point to take away is this: when an object is excited, the steady-state response is a standing wave. The natural steady-state response of a system does not include traveling waves. Thus, deliberate effort is required to generate steady-state traveling waves. The exception to this are infinite systems or those with significant damping. In an infinite system, there are no boundaries off of which the initial transient traveling waves can reflect. As a result, the traveling waves propagate forever, giving the impression of a steady-state system. For the case of sizable damping, the transient traveling waves lose energy and decay, preventing development into standing waves. However, a majority of systems do not fall into these categories.

The goal is to generate a steady-state traveling wave that appears to have no reflections at the boundaries. There are three different methods of doing so. They are impedance matching, active sink, and two-mode excitation. There are similarities between the methods, but also subtle differences. The impedance matching and active sink methods utilize two actuators to make a finite structure appear infinite; thus, eliminating reflections. On the other hand, the two-mode excitation method uses two actuators to generate two separate steady-state standing waves that superimpose to yield traveling waves. The two-mode excitation method is discussed at length since it is the method employed in this dissertation. This method was chosen since it can operate with variable parameters, is open-loop controlled, and generates waves with larger amplitudes than the other two methods.

\subsubsection{Impedance Matching}

One method for generating steady-state traveling waves is known as impedance matching. The method works by turning a finite structure into a semi-infinite one such that there are no reflections. Kuribayashi [56] experimentally tested this method with the use of a thin beam, two ultrasonic actuators, and a slider that is propelled along the beam with the waves. Although there are two motors, only one of them is actively excited. The active motor generates the waves, which propagate along the beam and are absorbed by the other passive actuator. To achieve this, the 
impedance of the passive motor is adjusted to match that of the beam. Reflections in a structure result from an impedance change [57]: a wave encountering a free boundary condition sees the end of the structure and the beginning of air and reflects. Thus, by matching the impedance of the passive motor to that of the beam, the wave propagates into the motor.

In order to match the impedance, an inductor and variable resistor are connected to the passive motor. Since it is a motor, the electrical and mechanical impedance are coupled. As a result, adjustments in the resistor yield changes in the mechanical impedance. This also explains what happens when the wave is absorbed by the motor: it is converted to electrical energy. This method can also be implemented with piezoelectric actuators, as was done by Hariri [58] to propel a free beam along a solid surface.

The impedance matching method is useful since it is open-loop controlled and only requires a single active actuator. However, there is a major disadvantage: the actuator location and excitation frequency are coupled. The actuator placement is dependent on the wavelength of the traveling wave, which is in turn related to the excitation frequency. As a result, generating traveling waves at different frequencies requires shifting the actuator location. In addition, the efficiency for generating these traveling waves is low [59], meaning that the traveling wave amplitude is also low.

\subsubsection{Active Sink}

The active sink method was first introduced by Tanaka [60] as a means of vibration suppression. The goal was to eliminate the structural modes of a one-dimensional system to prevent the development of standing waves. As explained earlier, standing waves form due to the interaction between reflected and incident traveling waves. Thus, to prevent standing waves, the reflections should be eliminated. Tanaka explains that since the reflections occur at the boundaries, by controlling the boundaries a semi-infinite media results. On a one-dimensional beam, control of the boundaries translates to an actuator at each of the ends. One actuator emits the wave while the other absorbs it; however, unlike the impedance matching method, the absorption actuator is active. Hence the name active sink.

In order to generate the traveling waves, the amplitude and phase ratio between the two actuators must be precisely chosen. This is found by solving the homogeneous Euler-Bernoulli beam equation. This is shown in Equation 3.1 where $w(x, t)$ is the transverse displacement, $E$ is the elastic modulus, $I$ is the moment of inertia, $\rho$ is the density, and $A$ is the cross-sectional area. Instead of assuming a standing-wave solution, the progressive wave solution shown in Equation 3.2 is used, where $k$ is the wave number. The $j k x$ terms represent traveling waves propagating

in opposing directions, while the $k x$ ones represent near-field (evanscent) waves. By defining the boundary conditions related to the forcing inputs and ignoring the near-field terms, a relationship 
between the forces and $C_{1}$ and $C_{3}$ terms can be found. By then assuming $C_{3}=0$, Equation 3.2 would include only the $C_{1}$ traveling wave term and a relationship between the forcing input results.

$$
\begin{gathered}
E I \frac{\partial^{4} w(x, t)}{\partial x^{4}}+\rho A \frac{\partial^{2} w(x, t)}{\partial t^{2}}=0 \\
w(x)=C_{1} e^{-j k x}+C_{2} e^{-k x}+C_{3} e^{j k x}+C_{4} e^{k x}
\end{gathered}
$$

Minikes [59] presents this relationship for a free-free string of length $\mathrm{L}$ with force $f_{1} e^{j \omega t}$ at $x=0$ and $f_{2} e^{j \omega t}$ at $x=L$, where the forces are complex. The progressive wave solution for the string is shown in Equation 3.3, which is a simplified case of the beam. By applying the forcing boundary conditions and assuming a single direction traveling wave $\left(C_{2}=0\right)$, a relationship between the forces can be found. This relationship is shown in Equation 3.4, where the absorbing actuator's $\left(f_{2}\right)$ magnitude and phase is dependent on the excitation $(k)$ and the excitation force.

$$
\begin{gathered}
w(x)=C_{1} e^{-j k x}+C_{2} e^{j k x} \\
f_{2}=-f_{1} e^{-j k L}
\end{gathered}
$$

The active sink method was then experimentally validated in a follow up study by Tanaka [61]. Pure traveling waves propagated along the beam; however, the amplitude of the waves was quite small. In particular, the traveling wave amplitude was $2.6 \%$ of that for a standing wave excited at the same frequency. This is quite useful from a vibration suppression stand point, but for a traveling wave generation method, it is an issue. Minikes [59] shows that in calculating the forcing ratio, the active sink method is essentially a form of impedance matching. As a result, it suffers from the same issues, namely a low efficiency when generating the waves.

Other work with the active sink method has shown that there is a disconnect between the theory and experimental generation of traveling waves. Gabai [62] found that the force ratios predicted by theory did not yield pure traveling waves. As a result, an online estimation method was implemented to vary the parameters in order to improve the traveling wave quality. Additional work also showed that it is possible to generate traveling waves on two-dimensional surface [63]. But this requires controlling the moments and forces at every single point along the boundary, which would be quite challenging to implement.

Overall, the active sink method is an effective means of generating steady-state traveling waves. In particular it is not limited by the relationship between actuator placement and excitation frequency, as is the case with impedance matching. Unfortunately the active sink method is plagued by the same low efficiency as impedance matching, resulting in low amplitude waves. In addi- 
tion, the theory and experiment don't always match well, requiring extensive manual or automated optimization for generating optimal traveling waves.

\subsubsection{Two-Mode Excitation}

The final method of generating steady-state traveling waves and the one utilized in the present research is known as two-mode excitation. Unlike the previous two methods, the focus is not upon impedance matching. Rather two actuators each excite standing waves which superimpose to produce a traveling wave. The two-mode method was first employed by Tomikawa [34] with the application of transporting objects along a beam. A one-dimensional beam is excited simultaneously at two actuation points. The points are excited with the same magnitude and frequency, but there is a phase difference of $\pi / 2$ between them. In addition, the distance, $d$, between the actuators is such $d=(n \pm 1 / 4) \lambda$, where $n$ is an integer and $\lambda$ is the wavelength. The result is that each actuator excites standing wave where the combination of the two yields a traveling wave.

In Tomikawa's study [34], the excitation frequency is halfway between the $18^{\text {th }}$ and $19^{\text {th }}$ modes of the beam, which is roughly $23.2 \mathrm{kHz}$, hence it is in the ultrasonic range. A mass slider was placed on the beam, and was propelled using the generated traveling waves. The slider mass and input power were varied and resulting in changes in the slider speed.

The next use of the two-mode excitation method was by Loh [33] where the same application as Tomikawa [34] of object transport was studied. Loh further analyzed the theory behind two-mode excitation and began by writing the vibration response as a function of the modal participation factors. This is shown in Equation 3.5, where $P$ is the force amplitude, $\omega$ is the excitation frequency, $\Phi_{n}$ are the mode shapes, $\omega_{n}$ are the natural frequencies, and $l_{1}$ and $l_{2}$ are the actuation locations. The modal participation factor of each mode is defined as in Equation 3.6. As with Tomikawa, the excitation frequency is halfway between two natural frequencies. Thus, a simplification can be made that only the two nearest modes are reasonably excited, which is why the method is called two-mode excitation. The result is that the response only has four terms, each actuator excites two modes. This response has been rearranged and is shown in Equation 3.7 for an excitation frequency between the $n$ and $n+1$ modes, where $C_{1}-C_{4}$ are dependent upon the modal participation factors. The first line is a traveling wave in one direction while the second is a wave propagating opposite. The total propagation direction is dependent on the values and signs of the constants $C_{1}-C_{4}$.

$$
\begin{gathered}
w(x, t)=P \sin (\omega t) \sum_{n=1}^{\infty} \frac{\Phi_{n}(x) \Phi_{n}\left(l_{1}\right)}{\omega_{n}^{2}-\omega^{2}}+P \sin (\omega t-\pi / 2) \sum_{n=1}^{\infty} \frac{\Phi_{n}(x) \Phi_{n}\left(l_{2}\right)}{\omega_{n}^{2}-\omega^{2}} \\
\text { Modal Participation Factor }=\frac{\Phi_{n}(l)}{\omega_{n}^{2}-\omega^{2}}
\end{gathered}
$$




$$
\begin{aligned}
w(x, t)= & {\left[C_{1} \sin \left(\beta_{n} x-\pi / 4\right) \sin (\omega t)-C_{2} \sin \left(\beta_{n+1} x-\pi / 4\right) \cos (\omega t)\right] } \\
& +\left[C_{3} \sin \left(\beta_{n+1} x-\pi / 4\right) \sin (\omega t)-C_{4} \sin \left(\beta_{n} x-\pi / 4\right) \cos (\omega t)\right]
\end{aligned}
$$

Using two Langevin type ultrasonic actuators and exciting between the $30^{\text {th }}$ and $31^{\text {st }}$ modes, Loh found that the maximum speed of a slider occurred for a phase difference of $90^{\circ}$. He also showed that by flipping the phase to $-90^{\circ}$ the slider had a maximum speed in the opposite direction. This shows that the best traveling waves occur at a $\pi / 2$ phase difference with the sign determining the direction. In addition, the slider remained stationary for a phase difference of $180^{\circ}$ while other phases yielded varying amounts of slider movement. Finally, by holding the phase constant at $\pi / 2$ and varying the frequency, the propagation direction changed. Loh surmised that changing the frequency changed the strength of the traveling waves, and that the propagation direction of the total traveling wave corresponded with the term in Equation 3.7 having the larger amplitude.

Kim [64] then expands on the work done by Loh [33], particularly to look at the change in $C_{1}-C_{4}$ for different frequencies. Via modeling, Kim showed that as the excitation frequency is changed, these coefficients changed sign. From that the propagation direction of the total traveling wave can be determined.

While the studies thus far showed that traveling waves were possible, Dehez [65] expanded the fundamental understanding by theoretically analyzed the two-mode excitation method and performed an optimization to generate pure traveling waves. Dehez modeled a simply supported Euler-Bernoilli beam actuated using using two piezoelectric stack actuators symmetrically placed on the beam. Similar to Loh [33], Dehez defines the response to the two excitations ( $\pi / 2$ phase difference) as the summation of all the mode shapes as shown in Equation 3.8. Here $a_{n}$ is a combination of the modal participation factor and the forcing amplitude. Additionally, the mode shape for a simply supported beam is $\sin \left(\beta_{n} x\right)$.

$$
w(x, t)=\sum_{n=1}^{\infty} a_{n} \sin \left(\beta_{n} x\right) \sin (\omega t)+\sum_{n=1}^{\infty}(-1)^{n} a_{n} \sin \left(\beta_{n} x\right) \cos (\omega t)
$$

The full solution can then be simplified to only include the two modes nearest the excitation frequency, which is shown in Equation 3.9 where $n$ and $n+1$ are the modes. In general, a traveling wave is defined as in Equation 3.10, meaning there are two standing waves phase shifted spatially a quarter of a wavelength and temporally a quarter of a period. By rearranging and using trigonometric identities, the solution (Equation 3.9) can be written in a form resembling traveling waves (Equation 3.10). Equation 3.11 shows this result. The first line has the same terms as the travel- 
ing wave trig identity, except that there are amplitude modulation terms $\left(\sin \left(\frac{\pi}{2 L} x\right)\right.$ and $\left.\cos \left(\frac{\pi}{2 L} x\right)\right)$. Thus, the traveling wave is not perfect. In addition, the second line of Equation 3.11 represents a standing wave. The result is that the total response is a traveling wave superimposed with a standing wave. However, the main standing wave component can be removed if $a_{n}=-a_{n+1}$. As a result, the quality of the traveling wave is increased by judicious placement of the actuators and selection of the excitation frequency.

$$
\begin{gathered}
w(x, t)=\left[a_{n} \sin \left(\beta_{n} x\right)+a_{n+1} \sin \left(\beta_{n+1} x\right)\right] \sin (\omega t)+\left[a_{n} \sin \left(\beta_{n} x\right)-a_{n+1} \sin \left(\beta_{n+1} x\right)\right] \cos (\omega t) \\
C \sin (\omega t) \cos (k x)+C \cos (\omega t) \sin (k x)=C \sin (\omega t+k x) \\
\begin{aligned}
w(x, t)= & 2 a_{n} \sin \left(\frac{\pi}{2 L} x\right) \cos \left(\frac{\beta_{n}+\beta_{n+1}}{2} x\right) \sin (\omega t)+2 a_{n} \cos \left(\frac{\pi}{2 L} x\right) \sin \left(\frac{\beta_{n}+\beta_{n+1}}{2} x\right) \cos (\omega t) \\
& +\sqrt{2}\left(a_{n}+a_{n+1}\right) \sin \left(\beta_{n} x\right) \cos \left(\omega t-\frac{\pi}{4}\right)
\end{aligned}
\end{gathered}
$$

The above approach is for a simplified model taking into account only the two nearest modes. Dehez then analyzes the effect of accounting for all modes. What he finds are further conditions on the placement of the actuators which allow the amplitude modulation in Equation 3.11 to be minimized. In essence, the amplitudes of the modes symmetric about the excitation frequency (e.g. 30 and 31, 29 and 32, etc.) sum to form a type of Fourier series. The result is when proper conditions on $a_{n}$ are optimized, the terms sum to equal 1 and the a pure traveling wave results. This also assumes that standing wave component of Equation 3.11 was eliminated. Dehez optimized the solution and was able to generate high quality, but not completely pure, traveling waves. While the quality did improve with this optimization, meeting the condition $a_{n}=-a_{n+1}$ had the largest impact on TW quality.

Up to this point, all experimental setups using the two-mode excitation method have remained relatively the same. A thin beam is attached to two ultrasonic actuators with a slider on top for movement. Hariri [58] then applied the two-mode method using piezoelectric wafers attached to a beam. The traveling waves were still being used for movement, but instead of a slider, the entire beam would be propelled along the ground. Hariri compared the impedance matching and twomode excitation method. He found that the traveling wave amplitude was larger for the two-mode method, which translated to faster movement speeds.

Piezoelectric wafers were also implemented on a beam by Avirovik and Malladi [1] as shown in Figure 3.1. An electromechanical model was then developed in order to match simulated and 
experimental traveling waves. The natural frequencies and mode shapes were experimentally validated for the case of a clamped-clamped, clamped-free, and free-free beam. Experimental and simulated traveling waves were then compared and shown to have a strong match.

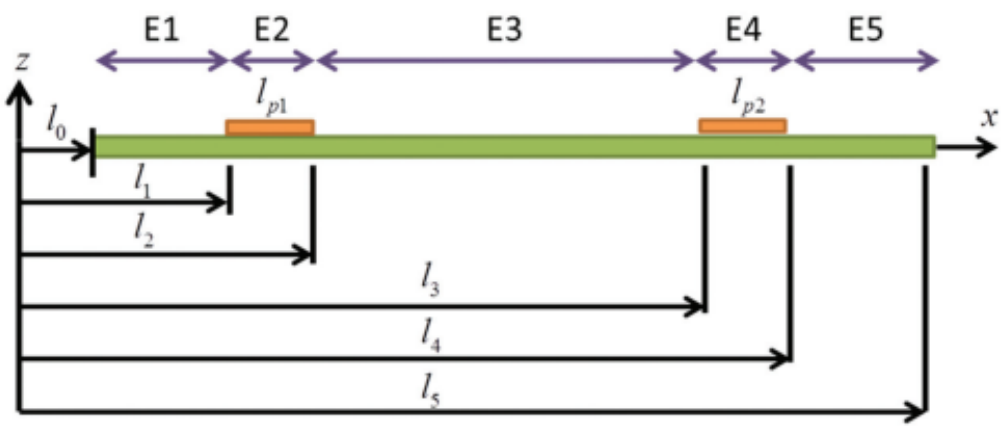

(a)

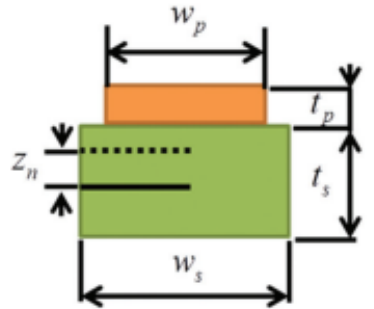

(b)

Figure 3.1: Modeled beam with mounted piezoelectric (PZT) actuators [1]. Used with permission of SAGE Publishing

Using the validated model, Avirovik and Malladi [1] then generated traveling waves between different mode shapes. Figure 3.2 shows a frequency response for a clamped beam. On the top, are the responses of the beam when excited at resonance using a single actuator. The bottom shows traveling waves generated between three different sets of modes. The frequency of each is halfway between the two modes and a $90^{\circ}$ phase difference is employed in all cases. This plot conveys several important points. Firstly, for the same actuator placement, traveling waves can be generated at different frequencies. Secondly, the form of the traveling wave changes between each set of modes with each resembling the nearby mode shapes. Thirdly, these traveling waves were generated at comparatively low frequencies $(<500 \mathrm{~Hz})$. All previous studies generated waves in the $\mathrm{kHz}$ range, with particular emphasis on the ultrasonic regime $(>20 \mathrm{kHz})$.

Malladi and Avirovik [2] also used their model to study the effects of phase difference on the generated traveling waves. As was discussed in Dehez [65], traveling waves generated via twomode excitation are mixed with standing waves and are thus hybrid waves. The quality of the hybrid waves depends upon the amount of standing versus traveling components. Three different ways of visualizing the hybrid waves are shown in Figure 3.3. The wave envelope is the simplest and is the path traced out by the wave over time. The Fourier approach plots the imaginary versus real amplitude response for different points on the beam (see Bucher [66] for a further discussion). For a standing wave, the beam has either $0^{\circ}$ or $180^{\circ}$ phase, resulting in a straight line. Whereas for a pure traveling wave, each point has a different phase, so the result is a circle. A hybrid traveling wave results in an ellipse. The third method is the Hilbert approach. Each point on the beam is plotted as a circle with radius equal to its max amplitude. A standing wave has node points (zero 


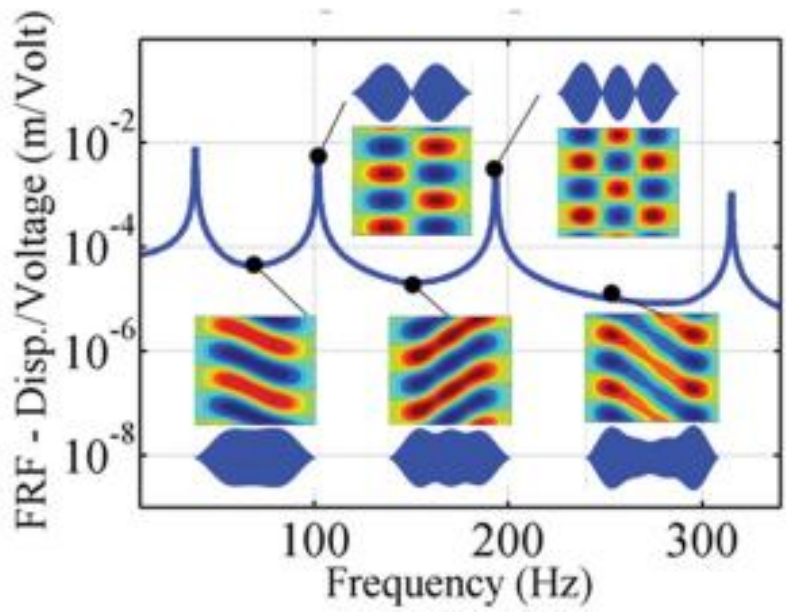

Figure 3.2: Simulated frequency response of clamped-clamped beam showing resonant and traveling wave responses [1]. Used with permission of SAGE Publishing

amplitude) and thus results in a filled in circle. On the other hand a pure traveling wave has equal amplitude along the beam length, yielding a single circle. By using these methods, the quality of the traveling wave is assessed, where a pure traveling wave is the goal.

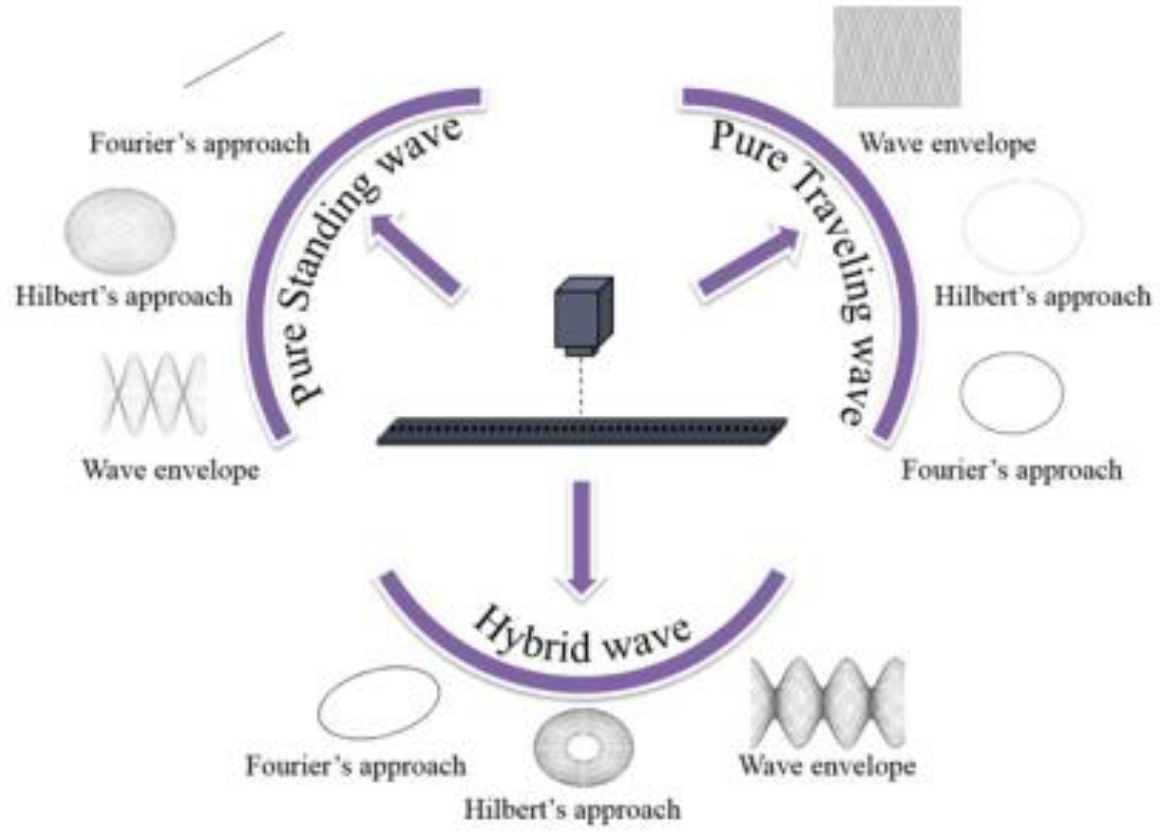

Figure 3.3: Three different methods of visualizing a combined traveling and standing wave (Hybrid) [2]. Used with permission of IOP Publishing

In previous studies, traveling were only generated halfway between two modes and with a phase 
difference of $90^{\circ}$. Via simulation and experimental validation, Malladi found that traveling waves can be generated at different frequencies between two modes. Figure 3.4a shows the quality of the traveling wave between two modes $(\approx 80 \mathrm{~Hz}$ and $\approx 180 \mathrm{~Hz})$ as a function of the phase difference. The cost function value is related to the Hilbert and Fourier approaches, where 1 is a pure standing wave and 0 a pure traveling wave. From the figure, it is clear that there is an optimal phase difference at each frequency and that high quality traveling waves can be generated at a majority of the frequencies. Figure $3.4 \mathrm{~b}$ shows the quality of the wave as a function of frequency when the phase is held constant at $90^{\circ}$. It is clear that as the frequency shifts towards either resonance, the standing wave component increases (also illustrated by the superimposed Hilbert plots). This confirms the observations seen in Tomikawa [34] and Loh [33] where the traveling wave weakened as the frequency shifted with constant phase.
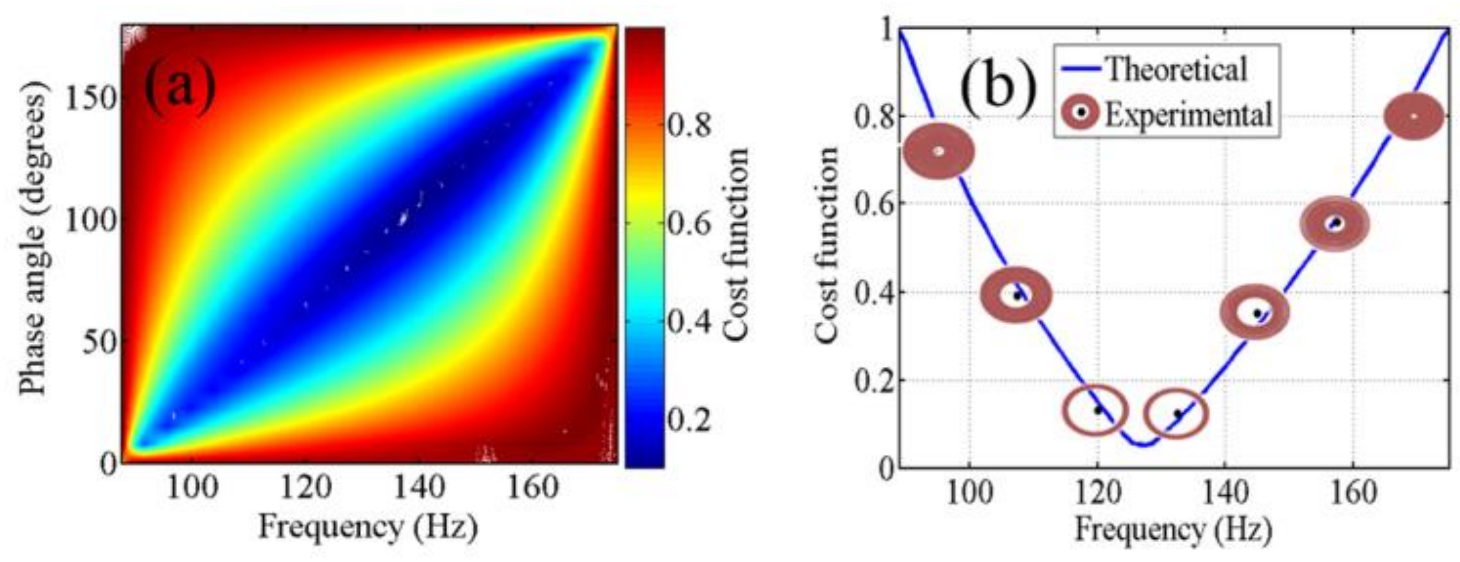

Figure 3.4: (a) Quality of the traveling wave between two modes for different phases where 0 is ideal. (b) Quality of the traveling wave between two modes when the phase is constant at $90^{\circ}$ [2]. Used with permission of IOP Publishing

Malladi [35] then transitioned the two-mode excitation method from 1D to 2D. A model was developed then validated via experimental modal analysis to predict traveling waves on a twodimensional plate (reduced order model developed in [67]). The aim of the study was to determine the effect of actuator location on the resultant traveling waves. Four different macro fiber composite (MFC) piezoeletric actuators were bonded to a free plate and traveling waves were generated by exciting two actuators at a time. Different sets of actuators (two at a time) were then excited to see how the traveling waves changed. The sets of actuators were all excited at the same frequency halfway between the modes and with a $90^{\circ}$ phase difference. Malladi found that exciting different sets of actuators on the plate all generated traveling waves. However, the form/pattern of the wave changed based on which actuators were excited. Thus, when using the two-mode excitation method 
on a $2 \mathrm{D}$ plate, the response changes with actuator location and that different locations can generate different forms of traveling waves.

In addition to traveling waves on a plate, other two-dimensional structures have been tested. Phoenix [68] applied the two-mode excitation method to simulate traveling waves on a thin-walled pipe. By exciting the pipe between two breathing modes and with a $90^{\circ}$ phase difference, circumferential traveling waves resulted.

\subsection{Skin Friction Reduction using Spanwise Traveling Waves with Wall-Normal Motion}

The research presented here focuses upon the effect of spanwise traveling waves on the turbulent boundary layer, specifically with the application of reducing skin friction drag/shear stress. This method falls under the category of active drag reduction since the surface is actively deformed, as opposed to remaining a defined shape. In general, active drag reduction encompasses any technique that involves any controllable procedure. Such examples include blowing and suction, surface heating and cooling, polymer injection, and surface deformation.

Spanwise traveling waves with wall-normal motion are a relatively new active technique, but share some similarities with other surface deformation techniques. This part of the literature review begins by discussing other surface deformation drag reduction techniques and examining their operating mechanisms. Then previous studies using spanwise traveling waves with wallnormal motion are discussed in-depth. These studies are then analyzed as a whole to provide some consensus on the drag reduction mechanism and the relationship with traveling wave parameters.

\subsubsection{Passive and active skin friction reduction techniques}

Active drag reduction techniques can trace their origin back to research into passive techniques, namely riblets. Passive riblets were first investigated at NASA in the late 70s and early 80s by Walsh $[19,69]$. Since then, extensive research has been conducted in this area with the result that riblets were found to yield nominal skin friction reductions of 5-8\% [70] and maxima of 10\% [22]. Investigation into the mechanism behind the reduction determined that the riblets interfere with the turbulence regeneration cycle. The riblets are aligned in the streamwise direction and limit the spanwise movement of the longitudinal vortices that are characteristic in sweep events [20]. The sweep events are a major source of wall shear stress in turbulent flow and result from the down-wash of high-momentum fluid from the outer boundary layer towards the wall in the regeneration cycle [71]. These sweep events are the end result of hairpin vortices, in which two parallel streamwise vortices converge together before ejecting low velocity fluid from the wall and 
sweeping high velocity flow towards it. Since the riblets hamper spanwise motion, the vortices don't fully converge and the sweep events occur prematurely and are weakened [22].

One of the first major applications of riblets was on the U.S. men's rowing boat at the 1984 Summer Olympics in Los Angeles and then on the winning boat in the 1987 America's cup [22]. However, the primary focus was on aircraft. A commercial product was developed by $3 \mathrm{M}$ and a full scale flight test on an Airbus A320 with $70 \%$ coverage of riblets yielded a roughly $2 \%$ total drag reduction [72]. Although these drag reductions were significant, riblets have not been commercially implemented. This is at least in part due to the ineffectiveness or even detriment of the riblets on the skin friction outside of the designed Reynolds numbers.

Active drag reduction techniques, on the other hand, can be varied depending upon the flow condition. While there are many types of active techniques such as blowing and suction, surface heating and cooling, and polymer injection; those discussed here rely on wall motion/surface deformation. The first such paper using wall motion was by Jung [24] in which a computational fluid dynamics (CFD) model using direct numerical simulation (DNS) showed a $40 \%$ skin friction reduction. This technique utilized spanwise, in-plane wall oscillations. Numerous studies have since been conducted using this technique and are reviewed by Quadrio [73]. Similar to the case with riblets, the skin friction decrease stems from the reduction in sweep events; however, the exact mechanism is different. The spanwise oscillation generates a spanwise vorticity that reduces the streamwise velocity. This slows the growth of the longitudinal vortices that produce the sweep events; thus, weakening the sweep events $[22,25]$.

Following the success of Jung [24], the skin friction reduction properties of other wall motions were investigated. Figure 3.5 (modified from Tomiyama [3]) displays some examples, where Figure 3.5a shows the spanwise in-plane wall oscillation like that of Jung [24]. Mito [74] investigated the use of standing waves with wall normal deformation (motion shown in Figure 3.5b) and found no clear decrease in shear stress. Quadrio [75] then simulated the use of in-plane, streamwise traveling waves (Figure 3.5c). Streamwise traveling waves with wall motion (Figure 3.5d) were also examined experimentally by Kendall [76] and computationally by Nakanishi [77] and were found to be an effective means of relaminarizing the turbulent flow.

Spanwise traveling waves for skin friction/shear stress reduction have also been investigated in numerous forms. Du and Karniadakis [78] and then Du et.al [79] computationally analyzed the use of a Lorentz force to generate near-wall body forces in an electrically conducting fluid. They observed that skin friction reductions of up to $30 \%$ are possible. Additionally, they found there is an optimal energy input at which the maximum reduction occurs. When investigating the skin friction reduction mechanism, Karniadakis [22] concluded the mechanism resulted from the interruption of the turbulence regeneration cycle, in particular the stabilization of near-wall streaks discussed by Swearingen [80]. Zhao [81] then conducted a DNS study on the use of in-plane 


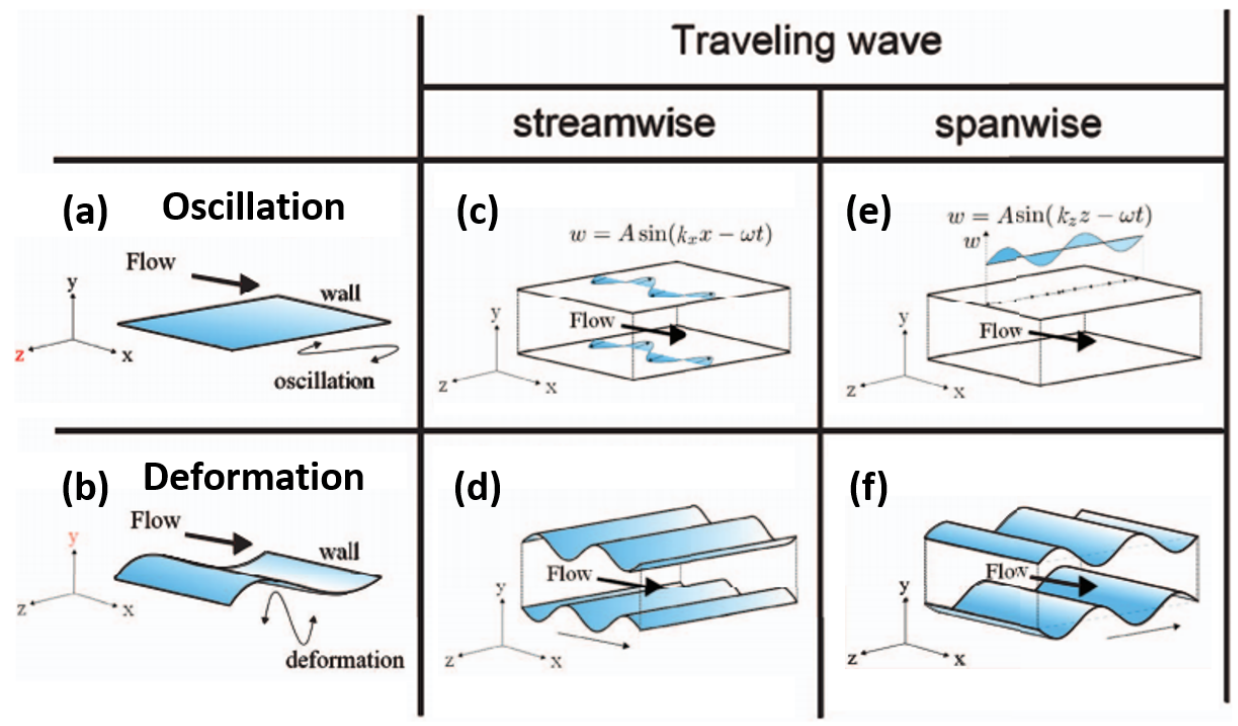

Figure 3.5: Different active drag reduction methods with wall deformation [3]: (a) in plane oscillation, (b) standing wave, (c) streamwise TW with in-plane spanwise oscillation, (d) streamwise TW with wall deformation, (e) spanwise TW with streamwise in-plane motion, (f) spanwise TW with wall deformation. Used with permission of AIP Publishing

spanwise traveling waves (Figure 3.5e) and found them able to suppress the streamwise vortices and thus reduce the wall shear stress.

However, the use of spanwise traveling waves with out of plane deformation are of most interest here. Figure 3.5f shows an example of the spanwise traveling waves investigated by Tomiyama [3]; most studies involve flat plate and not channel flow. The first study was performed by Itoh and Tamano [26] in 2006, and up to present four different research groups have performed their own studies [3-8, 26-29,31,32,82],. Like most other active skin friction reduction techniques involving wall motion, it requires no closed-loop control. Unlike most other techniques, there is no in-plane motion, thus it is easier to implement. As a result multiple techniques have already been tested to experimentally generate spanwise traveling waves with wall deformation. While in their current state these techniques are far from implementable, the results thus far yield the potential for an active skin friction reduction technique that could be realizable in commercial applications.

\subsubsection{Spanwise traveling waves with wall-normal motion}

The first skin friction reduction study using spanwise traveling waves (TWs) with out of plane deformation was an experiment conducted by Itoh and Tamano [26]. The experiment was conducted in a closed loop, low-speed wind tunnel at the Nagoya Institute of Technology in Japan in which zero-pressure gradient turbulent flow was introduced over a flat plate. The flow then passed over 
a flexible polyethylene sheet on which the traveling waves were generated by a speaker mounted flush with the sheet. Upstream of the traveling waves, the momentum thickness based Reynolds number is $R e_{\theta \text {,inlet }} \approx 600$. The traveling wave parameters are defined in Equation 3.12 where $\lambda$ is the wavelength, $a$ the amplitude, $f_{d}$ the frequency of the traveling wave. These have been normalized to wall units (+) using the $v$ the kinematic viscosity and $u_{\tau}$ the friction velocity upstream of the sheet.

$$
\lambda^{+}=\frac{\lambda u_{\tau}}{v} ; \quad a^{+}=\frac{a u_{\tau}}{v} ; \quad f_{d}^{+}=\frac{f_{d} v}{u_{\tau}^{2}}
$$

In order to measure the skin friction reduction, the difference in skin friction coefficients was determined using Equation 3.13, where $C_{f, \text { Rigid }}$ is the average skin friction coefficient across the rigid surface and $C_{f, F l e x, T W}$ is the average coefficient across the flexible sheet actuated with TWs. The rigid surface condition is defined when the flexible sheet is stretched flush across a solid plate. The skin friction coefficients were found by measuring the boundary layer upstream and downstream of the travelings and then implementing the momentum balance method (Section 2.3). For a traveling wave with $\lambda^{+}=2800, a^{+}=16$, and $f_{d}^{+}=0.01\left(\lambda=0.11 \mathrm{~m}, a=0.642 \mathrm{~mm}, f_{d}=\right.$ $100 \mathrm{~Hz}$ ), a skin friction reduction $7.5 \%$ was calculated.

$$
D R \%=\frac{C_{f, \text { Rigid }}-C_{f, \text { Flex }, T W}}{C_{f, \text { Rigid }}} \times 100 \%
$$

However, this reduction should be interpreted understanding the limitations of the measurement method. As was discussed in Section 2.3, the momentum balance relies heavily on a number of assumptions. In particular, it assumes a flat surface and two-dimensional flow. For spanwise traveling waves, the surface is oscillating and thus not flat, and the wall motion likely introduces three-dimensional effects. Anders [54] highlighted that three-dimensional effects yielded increased skin friction reductions when using the momentum balance. Thus, the skin friction reduction values highlighted might not be entirely accurate.

In order to determine the source of the skin friction reduction, Itoh analyzed other flow characteristics. Looking at the boundary layer profile, he found that for the TW actuated case the mean velocity in the laminar sublayer is lower and the log-law region is narrower. For increases in the TW amplitude $\left(a^{+}=7,10,12,16\right)$, he saw that the streamwise velocity fluctuations above the wall increased. Analysis of the power spectra then showed an increase in the low frequency power near the wall $\left(y^{+}=21\right)$. He deduced that the bursting event was affected since the power increase corresponded with the bursting frequency range. Although these flow changes are correlated with the introduction of the traveling waves, no explanation was given for the mechanism behind decreased shear stress.

The next study on these spanwise TWs was a large eddy simulation (LES) performed by 
Klumpp [4]. The study had two purposes: to verify the results of Itoh [26] and to further investigate the near-wall flow field and provide an explanation for the skin friction reduction mechanism. The Reynolds number here was defined in terms of the displacement thickness upstream of the TW region, $\operatorname{Re}_{\delta^{*} \text {,inlet }}=1000$. Since the flow is turbulent, the shape factor is assumed to be $H \approx 1.3$ [51], meaning that the momentum-thickness Reynolds number is $R e_{\theta, \text { inlet }} \approx 770$. The generated traveling wave was defined with $\lambda^{+}=870, a^{+}=30, f_{d}^{+}=0.02$ using the definition from Equation 3.12. Using these parameters, the average wall shear stress was determined over the TW region and when compared to the unactuated wall, the skin friction was reduced by $6 \%$.

Since this study was computational, the instantaneous skin friction coefficient, $C_{f}$, over the actuated surface could be determined. The $6 \%$ reduction was then calculated by taking the timeaverage of the instantaneous values. This reported reduction does not rely on the momentum balance method and the inherent assumptions (Section 2.3); thus, it is not subject to the same level of scrutiny as the $7.5 \%$ reduction reported by Itoh [26]. As a result, Klumpp's result provides confidence that spanwise traveling waves are capable of reducing the skin friction drag. In fact, since both both Klumpp [4] and Itoh [26] yielded similar levels of reduction (6\% and 7.5\%), this allows Itoh's result to be interpreted with greater confidence.

The effect of the traveling waves on the flow and turbulent structure was determined by looking at a number of different quantities. Similar to Itoh [26], the velocity gradient was reduced near the wall for the actuated case. Figure 3.6 from Klumpp [4] shows the boundary layer profile for an unactuated and actuated wall with the trough and crest shown separately for the actuated case. In the figure, there is only a minimal difference between the crest profile and the unactuated one; however, the profile over the trough deviates noticeably from the others. The velocity gradient is smaller in the laminar sublayer $\left(y^{+}<8\right)$, and the profile does not reach the log-law region until further from the wall than the unactuated case $\left(y^{+} \approx 25\right.$ vs 50$)$. The latter is important since it means the buffer layer, where a majority of turbulence production occurs, is pushed further off the wall. Looking at the streamwise velocity fluctuations, in contrast to Itoh [26] the fluctuations actually decrease. Additionally, a spanwise flow was induced near the wall where the flow in the trough propagated with the TW and that above the crest moved opposite.

One of the major changes caused by the traveling wave in Klumpp [4] are those to the flow vorticity. Figure 3.7 shows the streamwise vorticity fluctuations for an unactuated and actuated case. The unactuated case displays the streaky structures characteristic of a turbulent boundary layer, while the structure of the actuated case is markedly different. The traveling waves cause the streamwise vorticity to form a ribbon-like structure near the wall of width roughly equal to the wavelength of the traveling wave. These structures have been seen in $\mathrm{Du}$ [79] and Zhao [81] for Lorentz force and in-plane wall motion spanwise TWs, respectively. Karniadakis [22] supposes these near wall structures act as a protective shield for the surface. In addition to changes in the 


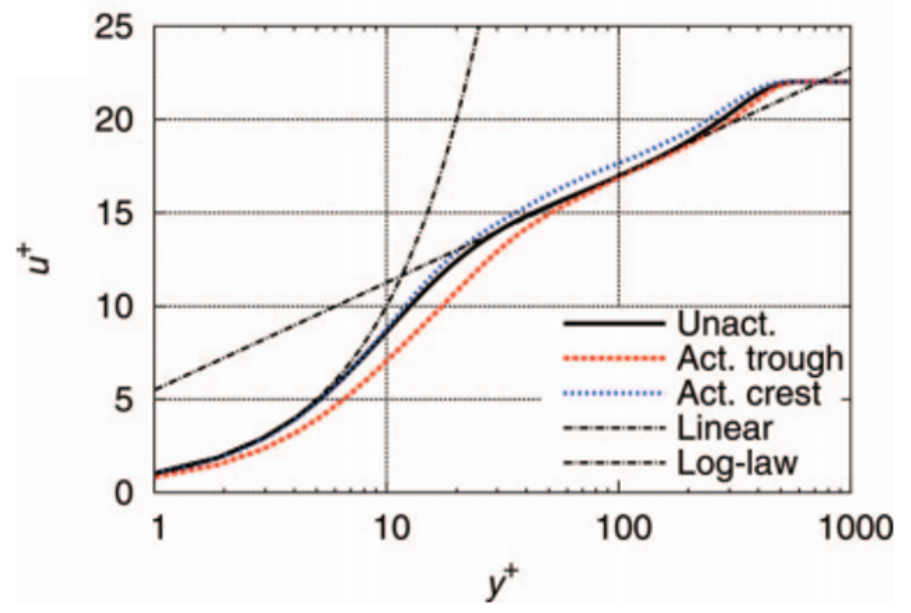

Figure 3.6: Boundary layer profile over the unactuated wall and actuated wall with that over the trough and crest shown separately [4]. Used with permission of Taylor \& Francis

streamwise vorticity fluctuations, the wall-normal vorticity is also heavily damped by the traveling wave actuation, particularly above the wave crest. Klumpp references both Schoppa [83] and Jimenez [84] and how the wall-normal vorticity is a key quantity in the turbulence regeneration cycle. While a clear explanation of the drag reduction mechanism is not given, it is concluded that the traveling waves affect the turbulent regeneration cycle and subsequently reduce the wall friction.

Klumpp [27] then performed a follow up study to determine the relationship between the streamwise vorticity and the drag reduction. The same computational setup was used as before [4]; however, two separate traveling wave cases were tested. One case yielded a skin friction decrease while the other a friction increase. The flow characteristics were compared to isolate which effects signified drag reduction. A reduction in shear stress of $9 \%$ was achieved for $\lambda^{+}=870, a^{+}=30$, $f_{d}^{+}=0.02$ while an increase of $8 \%$ resulted for $\lambda^{+}=174, a^{+}=10, f_{d}^{+}=0.1$.

When looking at the streamwise vorticity for both the skin friction decrease and increase, it was found that in both cases, the structure shown in Figure 3.7 emerged. As a result, this streamwise ribbon-like structure does not correspond with reductions in wall shear stress. On the other hand, the wall-normal vorticity is an indicator of decreased shear stress. For a reduction in skin friction, there was a decrease in the wall normal vorticity, while there was increase in skin friction for increased vorticity. In addition to changes in wall normal vorticity, there were differences in velocity fluctuations and turbulent kinetic energy (TKE) between the drag decrease and increase cases. Quadrant analysis showed that the streamwise velocity fluctuations decrease in the Q2 and Q4 quadrants while the wall normal velocity fluctuation slightly increases; Q2 and Q4 represent ejection and sweep events, respectively. In addition, the incidence rate in the Q1 and Q3 quadrants 


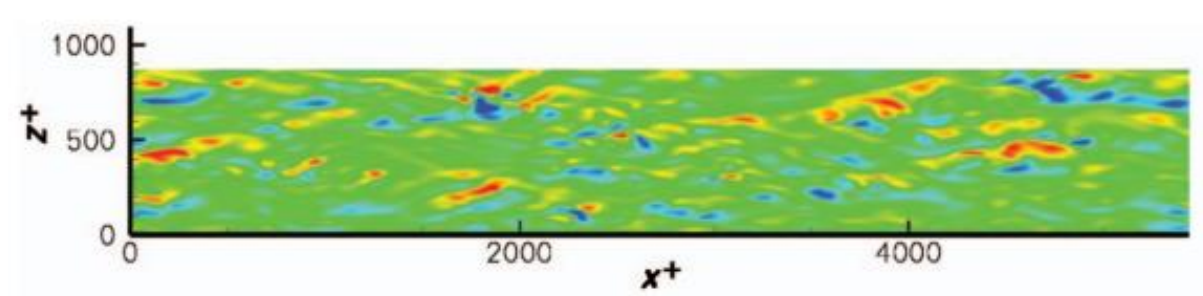

(a)

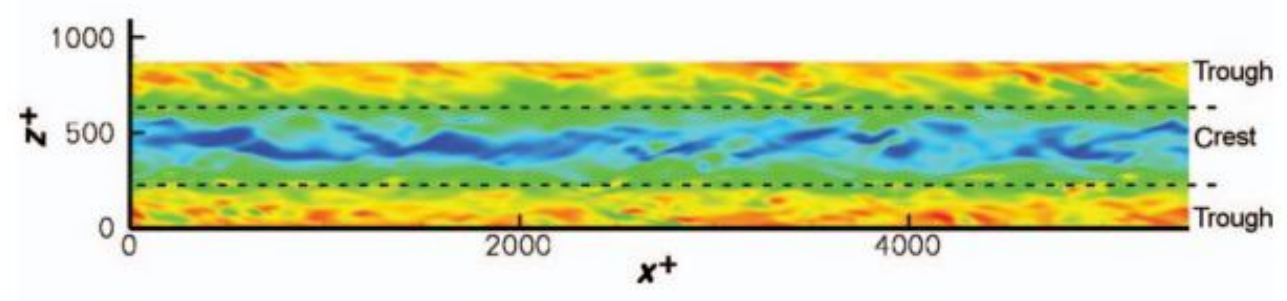

(b)

Figure 3.7: The instantaneous streamwise vorticity fluctuations at $y^{+} \approx 4$ above the unactuated wall (a) and the TW actuated wall (b). The dashed lines represent the contours of the crest region [4]. Used with permission of Taylor \& Francis

increased, which correspond with the outward and inward interactions, respectively. In terms of TKE production, for the skin friction decrease the production is halved above the trough and stays roughly the same above the crest. Whereas for the friction increase, the peak TKE production is increased by $400 \%$.

Tamano and Itoh [5] again investigated the skin friction reduction capability of spanwise traveling waves with out of plan deformation but with an updated wave generation method. The same experimental setup was used as in Itoh [26]; however, the speaker was replaced with the motor and crankshaft shown in Figure 3.8. This allowed for greater control of the wave parameters as compared to the speaker. Following the results of Klumpp [4,27] where changes in wall-normal vorticity and turbulence production were indicators of drag reduction, the goal of Tamano's study was to analyze the turbulent statistics in an experimental setting. Consequently both a single and cross-wire hot wire anemometer were employed.

The skin friction reduction for a variety of wave parameters was measured and the largest reduction of $13 \%$ was yielded for $\lambda^{+} \approx 2600, a^{+}=24, f_{d}^{+}=0.0087\left(T^{+}=115\right)$. This reduction was determined using the momentum balance method, so just as for their previous study [26], this $13 \%$ skin friction reduction should be cautiously interpreted. Analysis of the results showed that the shear stress decreased with decreases in period. Therefore, Tamano concluded that maximum skin friction reductions occurred at or below $T^{+}=100$, where this time scale corresponds well with the averaged bursting period for the wall-bounded turbulent flows [85]. The recovery length 


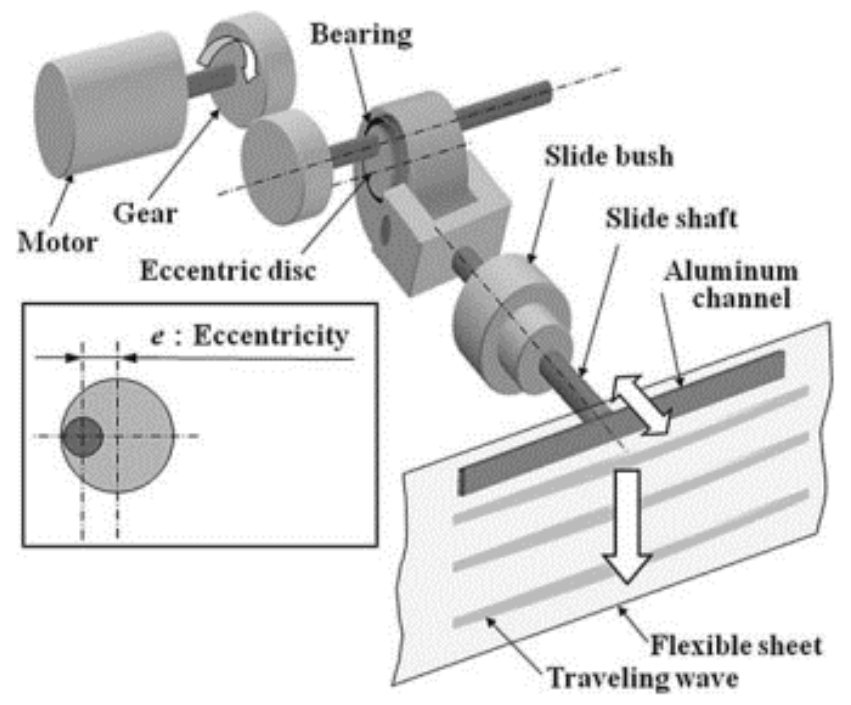

Figure 3.8: The eccentric motor used to generate spanwise traveling waves in Tamano [5]. Used with permission of Taylor \& Francis

downstream of the TW region was also investigated. Tamano determined that by $4 \delta$ downstream of the traveling wave region, where $\delta$ is the boundary layer thickness, the momentum thickness had converged with that from the non-actuated surface. This convergence implies that while the skin fricition is reduced over the TW region, there is actually a skin friction increase directly downstream of it; in effect, the net change in skin friction drag across the traveling wave and downstream region is zero.

When looking at changes in the flow structure due to the TWs, there are a number of differences between the actuated and unactuated case. As with previous TW drag reduction papers, the mean velocity is lower near the wall indicating a lower velocity gradient and an extension of the laminar sublayer. When looking at the turbulence intensity, both the streamwise and wall-normal values are increased directly above the TW region. Concerning turbulent statistics, the Reynolds shear stress across the boundary layer is much lower in the case of a large drag reduction than for the unactuated wall. Quadrant analysis then shows that the magnitude of sweep and ejection events are decreased while the inward and outward interactions are increased, which are the same results achieved by Klumpp [27].

Following the 13\% skin friction reduction achieved by Tamano [5], Tomiyama [3] performed a direct numerical simulation (DNS) using spanwise traveling waves in channel flow. While Klumpp [27] had provided some explanation for the skin friction reduction mechanism, Tomiyama felt that further investigation was required to explain it and also the relationship between reduction and the traveling wave parameters. Unlike previous studies in which flat plate flow was investigated, 
their DNS consisted of flow through a channel with spanwise traveling waves on both the top and bottom, as shown in Figure 3.9. The streamwise pressure gradient was controlled in each time step of the simulation in order to maintain a constant flow rate; thus, the drag reduction between the unactuated and actuated case was determined by looking at the difference in the mean pressure gradient for the two cases.

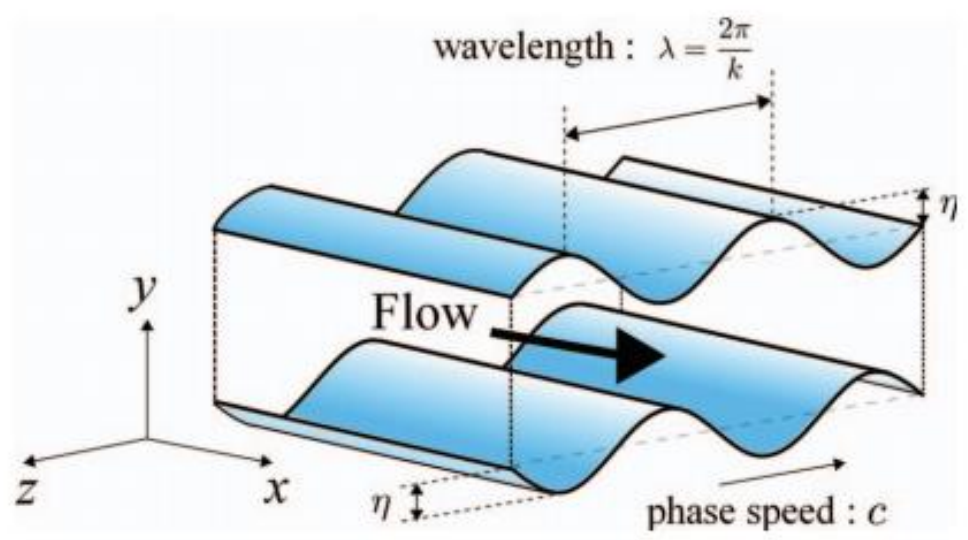

Figure 3.9: Channel flow with spanwise traveling waves investigated by Tomiyama and Fukagata [3]. Used with permission of AIP Publishing

Tomiyama varied the traveling wave parameters in an attempt to discern a relationship between these parameters and the drag reduction. The largest drag reduction yielded was $13.4 \%$. They also found that there existed a correlation between the decreasing drag and increasing amplitude and decreasing wavenumber (increasing wavelength); however, these correlations were not strong enough to claim a relation. Taking inspiration from previous research on passive riblets, Tomiyama looked at the relationship between the drag reduction and scaling factors composed of the TW parameters. For riblets, reasonable success was achieved modeling the DR against two different factors: the groove height to width ratio $\left(2 a^{+} / \lambda^{+}\right.$, in terms of TW parameters used here) and the square-root of the cross sectional area of the groove $\left(\sqrt{A_{g}^{+}}\right)[86]$.

These scaling factors did not translate well to spanwise TWs, but it inspired Tomiyama to propose a new scaling factor using the magnitude of the velocity induced by the traveling wave and the thickness of its effective layer. The scaling factor is $v^{+} / \sqrt{k^{+} c^{+}}$where $v^{+}$is the wall normal velocity (study uses $a^{+}[3]$ ), $k^{+}$is the wave number, $c^{+}$is the wave speed, and the combined term $1 / \sqrt{k^{+} c^{+}}$is the Stokes layer thickness. The wave number and wave speed are defined in Equation 3.14 using the non-dimensional wavelength, $\lambda^{+}$, and period, $T^{+}$. Fitting this scale factor versus the drag reduction showed a fairly good match. As a result, Tomiyama supposed that the spanwise velocity induced by the traveling wave and the resultant turbulence reduction is the source of the 
drag reduction.

$$
k^{+}=\frac{2 \pi}{\lambda^{+}} ; \quad c^{+}=\frac{\lambda^{+}}{T^{+}}
$$

When looking at the turbulent statistics to determine the DR mechanism, Tomiyama [3] observed a reduction of the Reynolds shear stress and that the peaks were pushed further off the wall. In addition, the wall normal and spanwise velocity fluctuations were less than for the actuated case. Passive riblets exhibited the same effect such that the DR mechanism was correlated with the weakening and pushing off the wall of the quasi-streamwise vortices (QSVs) [87,88], which are integral to the turbulence regeneration cycle. However, for riblets the wavelength of the grooves is smaller than the width of the QSVs and thus prevents them from reaching the wall, whereas for the spanwise TWs the wavelength is larger than the QSVs. Regardless, Tomiyama still saw reductions in the streamwise vorticity fluctuations, meaning the QSVs were weakened. Therefore, based on the semi-correlated scaling factor and the reductions in different turbulent phenomena, they believe the spanwise velocity induced by the traveling waves is the source of the drag reduction. The spanwise velocity prevents the QSVs from entering the troughs; thus, the QSVs are pushed further off the wall and weakened leading to reductions in Reynolds shear stresses and subsequently drag.

Thus far, research into spanwise traveling waves focused on waves generated over a continuous surface. In contrast, Bai [6] experimentally investigated the shear stress reduction potential of an array of piezoelectric cantilever actuators as shown in Figure 3.10. The local skin friction was measured directly downstream of the actuators using a hot-wire anemometer and the friction was found to depend heavily on measurement location. A skin friction reduction of $50 \%$ was measured roughly 20 wall units (w.u.) downstream of the actuators, yet that dropped to $25 \%$ at 60 w.u. with $0 \%$ reduction at $160 w . u$. In addition, there was a significant spanwise variation; $50 \%$ was achieved at one spanwise location, but the spanwise average was $23 \%$. When analyzing the dependence upon TW parameters, Bai found that the shear stress reduction increased with increasing amplitude. However, he found that above a certain amplitude, the shear stress reduction dipped off; thus, signifying an optimal amplitude. Bai references Du [79], who found that for a spanwise traveling wave using a Lorentz force, the largest reduction in skin friction occurred when the penetration depth was on the order of the laminar sublayer thickness. Looking at the relationship of the shear stress reduction to the TW parameters, Bai saw that the skin friction reduction increased with increasing traveling wave frequency (decreasing period). This agrees well with Tamano's [5] observation.

Bai [6] then gives a very in-depth analysis of the effects of the TWs on the flow structure. Like Tomiyama [3], he places the manipulation of the quasi-streamwise vortices (QSVs) as the main source of the drag reduction. Using smoke visualization experiments the low and high speed streaks responsible for the ejection and sweep events were replaced with more stable longitudinal 

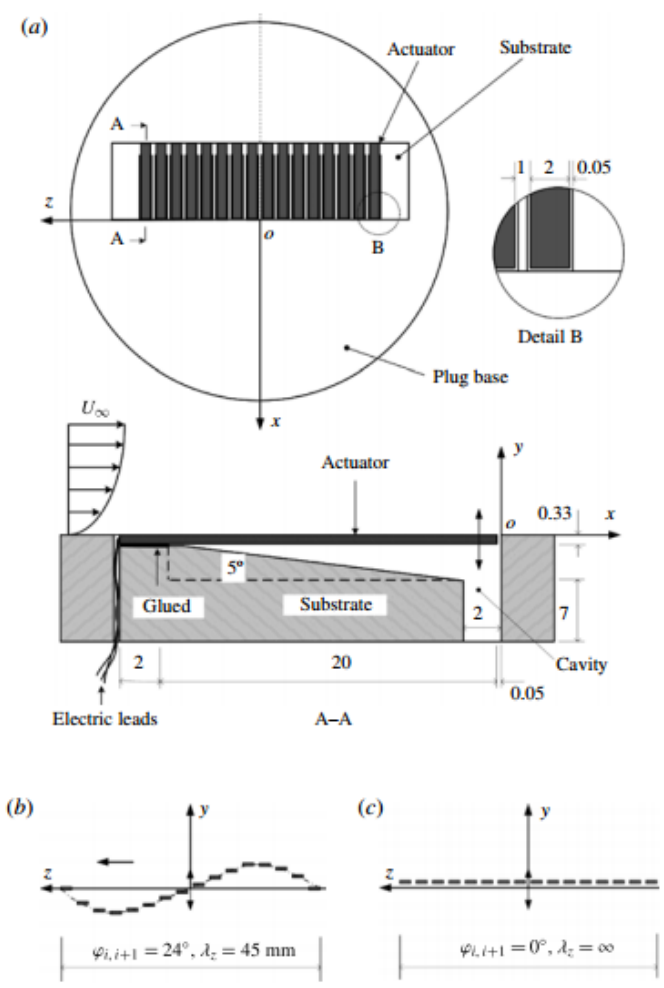

Figure 3.10: Array of piezoelectric cantilever actuators used to generate spanwise traveling waves [6]. Used with permission of Cambridge University Press

structures (streamwise vortices). The effect of the streamwise vortices is shown in Figure 3.11. The actuators generate a ribbon of small scale vortices near the wall which act as a barrier between the wall and the low and high speed streaks produced by larger QSVs. The result is the interruption of the turbulence production cycle. He expands to state that the generated streamwise vortices should be an optimal thickness so as to maximally disrupt the large-scale coherent structures (QSVs). Analysis of the flow structure, showed that this proposed mechanism increased the laminar sublayer thickness and energy dissipation near the wall and weakened the bursting events.

While Bai's paper [6] proposes a fairly comprehensive explanation of the skin friction reduction mechanism, the TW generation method is quite different from the others and thus introduces questions about the comparability of the results. The traveling waves are produced at a roughly discrete location in the streamwise direction and the wall shear stress was only measured downstream. As a result, the wall shear over the surface with the actuators was not considered. Alongside this, the actuators generate the vortex barrier such that the shear stress reduction occurs downstream, while previous studies indicate the skin friction reduction results from the direct interaction of the fluid over the TW surface. These methods are particularly juxtaposed since Tamano [5] observed an increase in wall shear stress downstream of the TWs. While Bai's study produces very promising 


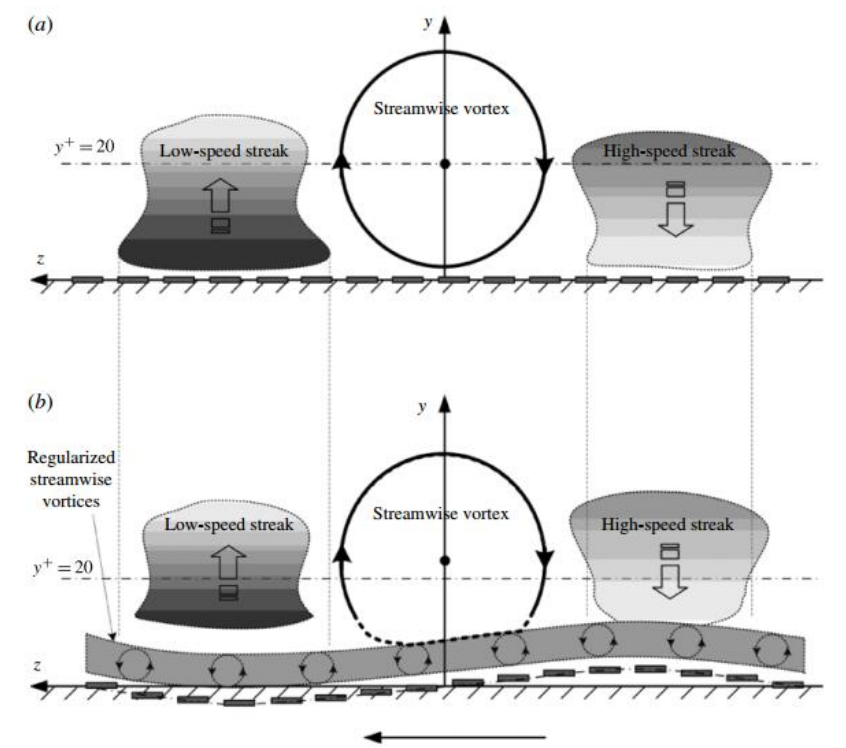

Figure 3.11: A schematic of the skin friction reduction mechanism with (a) and without (b) traveling waves where flow is into the plane [6]. Used with permission of Cambridge University Press

results, the method appears different from previous spanwise traveling wave studies.

After the paper from Bai [6], all the papers up to the present relating to skin friction reduction using traveling waves have emerged from the Aerodynamic Institute at Aachen University in Germany. This is the same group from which the two papers by Klumpp [4,27] were published. Koh and Meysonnat published a study [7] in which the skin frictionreduction capabilities of spanwise traveling waves were computationally tested for different Reynolds number flows. As in the papers by Klumpp, LES was used and the reduction in skin friction was determined by looking at the average shear stress over the actuated region. The reduction was determined for four different momentum thickness Reynolds number (momentum thickness at the inlet of the TW region) while the non-dimensional TW parameters were held constant at $a^{+}=50, \lambda^{+}=500$, and $T^{+}=80$. The Reynolds numbers tested were $R e_{\theta}$,inlet $=1000,2000,5000$, and 7000 and the corresponding skin friction reductions were $11 \%, 7.5 \%, 2 \%$, and $1 \%$. From the results it was quite clear that under constant TW parameters, the shear stress reduction decreases with increasing Reynolds number. However, it is important to note that the dimensional TW parameters changed from test to test, only the non-dimensional forms remained constant

When analyzing the flow structure, Koh and Meysonnat's [7] results concurred with the results from previous studies. Looking at the boundary layer profiles, the velocity gradient of the laminar sublayer above the trough was lower for the TW actuated case. The streamwise velocity and wall normal vorticity fluctuations also decreased with the application of the traveling waves. A spanwise flow was induced and the turbulent kinetic energy (TKE) production decreased in the 
trough of the TW. The turbulent kinetic energy production describes the transfer of energy from the mean flow velocity to the turbulent velocity fluctuations [47]. Of particular interest is the variation in these changes with different levels of drag reduction. Koh and Meysonnat observed that as the Reynolds number increased and the drag reduction decreased, the induced spanwise flow in the trough weakened. Additionally, the TKE production exhibited the same trend. Figure 3.12 shows the TKE production for two different Re. The case with $11 \%$ skin friction reduction produces significantly less TKE in the trough than for the $1 \%$ case. At the same time, the TKE production above the crest remains relatively constant. Based on these flow characteristics, there is a strong correlation between shear stress reduction and changes in spanwise flow and TKE production. Moreover, the flow in the trough plays a paramount role in the skin friction reduction mechanism.
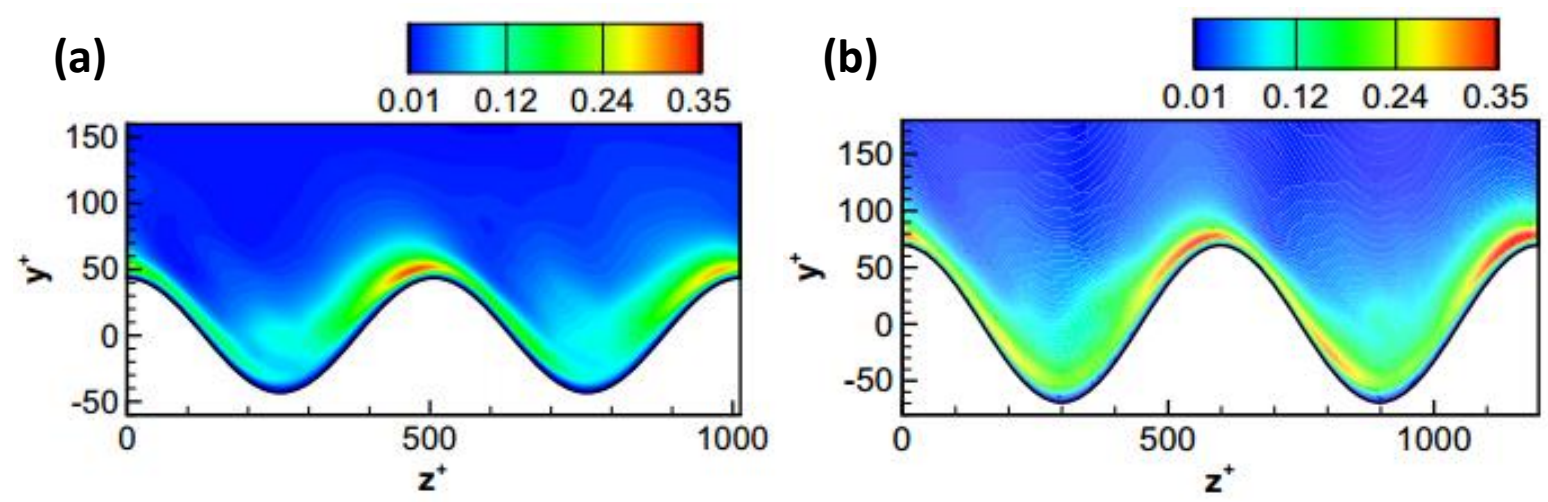

Figure 3.12: Turbulent kinetic energy (TKE) production over a traveling wave for two different drag reductions (a) $11 \%$ and (b) $1 \%$ [7]. Used with permission of Springer Nature

At the same time, Koh and Meysonnat [28] published another study investigating the effect of traveling wave amplitude on the skin friction reduction. Again using LES, they looked at three different TW amplitudes at three different Reynolds numbers $\left(\operatorname{Re}_{\theta}\right.$,inlet $=2000,5000$, and 7000) for nine total cases. The non-dimensional amplitudes for each test were $a^{+}=30,50$, and 70 with the period and wavelength the same as in their previous study [7]. The largest reduction in shear stress was $11.4 \%$ which occurred for $R e_{\theta, \text { inlet }}=2000$ and $a^{+}=50$. Since this is the middle amplitude value, it indicated that there is an optimum amplitude. For $R e_{\theta, \text { inlet }}=7000$ the largest reduction occurred at $a^{+}=70$; as a result, Koh and Meysonnat concluded that the optimum amplitude value is dependent upon the Reynolds number.

In their study, Koh and Meysonnat [28] showed that above the crest there is an increase in the skin friction whereas in the trough the skin friction, velocity gradient, and TKE production all decrease. The reduction in the trough outweighs the increase over the crest resulting in a net shear stress reduction over the surface. They also observed that the traveling waves pushed the peak of 
the wall normal vorticity off the wall and reduced its magnitude. Koh and Meysonnat found that by increasing the TW amplitude, and thus wall normal velocity, the vorticity could be pushed further off the wall. However, increasing the amplitude beyond a certain point yielded smaller reductions in drag. Koh and Meysonnat explain this by the ability of the streamwise flow to transport away the vorticity that is pushed away from the wall. For the $R e_{\theta, \text { inlet }}=2000$ and 5000 case, the optimum amplitude was at $a^{+}=50$, with reduced effectiveness at $a^{+}=70$. For $\operatorname{Re}_{\theta \text {, inlet }}=7000$, the largest $\mathrm{DR}$ occurred at $a^{+}=70$. They presumed that at the highest Re, there was enough streamwise momentum to transport away the vorticity from the largest amplitude, but at the lower Reynolds numbers there is not enough streamwise momentum and thus the skin friction reduction is weaker than at the optimum amplitude. As a means of correlating across Reynolds numbers, Koh and Meysonnat recommended the use of an outer scaling instead of an inner one.

Meysonnat and Koh [31] then performed a final computational study to look at the effects of pressure gradients on the skin friction reduction capabilities of spanwise traveling waves. LES was used to model the shear stress reduction for zero, adverse, and favorable pressure gradients with the same parameters used in their previous studies [7,28]: $R e_{\theta, \text { inlet }}=2000, a^{+}=50, \lambda^{+}=$ $500, T^{+}=80$. Using the same parameters for all three gradients they found a $9.9 \%$ shear stress reduction for the zero pressure gradient, $6 \%$ reduction for the adverse gradient, and $-4 \%$ reduction (increase) for the favorable gradient. Meysonnat and Koh deduced that the TWs were less effective in the adverse gradient since the turbulence production region is already pushed off the wall by the gradient itself. They then surmised the skin friction increased in the favorable flow by equating the gradient to an increase in Reynolds number which, they have previously shown, decreases the effectiveness of the TWs.

Looking at the flow characteristics, they observed, as in previous studies, that the flow in the trough is where the drag reduction occurs. Over both the trough and the crest the wall-normal vorticity magnitude decreases. In the trough the peak was displaced further from the wall while over the crest it encroached more upon the wall. Similarly, the velocity gradient in the laminar sublayer decreased in the trough while it increased above the crest. However, since the reduction in the trough outweighed that over the crest, a net skin friction reduction resulted.

While conducting the computational studies [7,28,31] of spanwise TWs, the group at Aachen University in Germany also began running a number of experimental tests. The first paper by Roggenkamp [8] was conducted in a closed-return, open test-section wind tunnel in which the traveling waves were generated by an array of electro-magnetic actuators. This array is shown in Figure 3.13 and can achieve TWs with $a=0-0.5 \mathrm{~mm}, f=0-160 \mathrm{~Hz}$, and $\lambda>60 \mathrm{~mm}$. Unlike the previous experimental studies by Itoh and Tamano $[5,26]$ in which a flexible sheet was used, the surface here was aluminum. Typical commercial applications do not have surfaces constructed from polyethylene, whereas aluminum is often used. Thus, by testing with aluminum, the use 
of spanwise TWs as an implementable drag reduction technique is displayed. In this experiment, particle image velocimetry (PIV) and $\mu$-PTV were used to measure the boundary layer downstream of the actuated plate. The laminar sublayer was measured using $\mu$-PTV from which the local skin friction coefficient was calculated.
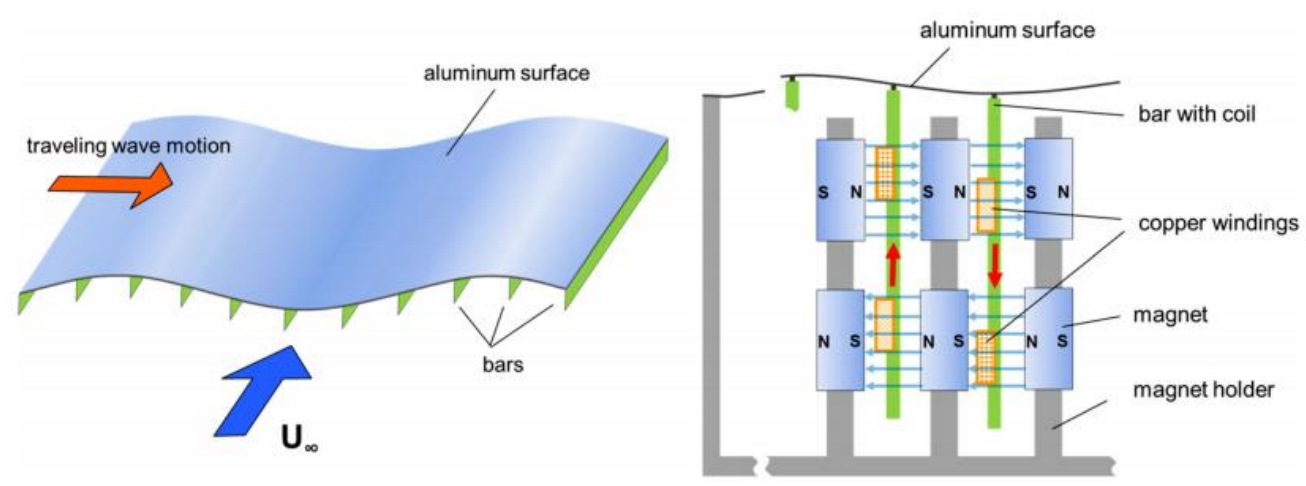

Figure 3.13: Array of electromagnetic actuators used to generate spanwise traveling waves [8]. Used with permission of Springer Nature

Three different Reynolds number flow were tested, each with three different amplitudes. The Reynolds numbers tested were $R e_{\theta, \text { outlet }}=1200,1660$, and 2080. Unlike previous studies, these were defined using the momentum thickness downstream of the actuated plate instead of upstream. For each Reynolds number, the same three dimensional amplitudes were tested; however, due to increases in the Re the non-dimensional values were not constant. The largest reduction in wallshear stress was 3.4\% and it occurred at the lowest Reynolds number and the largest amplitude. With increasing Reynolds number, the reduction in skin friction decreased such that at $\operatorname{Re}_{\theta}=2080$ the reductions ranged from -0.2 to $-0.7 \%$, all indicating a skin friction increase.

However, the decrease in wall-shear stress may have also resulted from increases in the nondimensional period, $T^{+}$, instead of the Reynolds number. The dimensional period remained constant at all $\mathrm{Re}$, but due to the increasing friction velocity with $\mathrm{Re}$, the non-dimensional period increased as well; the periods at the three $R e_{\theta}$ were $T^{+}=100,230$, and 380. Roggenkamp compared the data with Tamano [5] and showed that both have stronger skin friction reduction for decreases in the period; however, the contributions from the Reynolds number could not be separated. Looking at the flow structure Roggenkamp [8] found only minor differences between the TW and unactuated cases. Specifically the streamwise velocity fluctuations and the Reynolds shear stress increased in the logarithmic region $\left(y^{+}>200\right)$ which indicated the displacement of the turbulence off the wall

In order to validate the experimental results of Roggenkamp [8], Meysonnat and Roggenkamp [32] conducted a combined experimental and computational study. Roggenkamp's experimental 
setup [8] and LES were used and the flow conditions between the two were validated by comparing the momentum thickness, boundary layer profiles, and RMS velocity fluctuations. A single Reynolds number $\left(R e_{\theta, \text { outlet }} \approx 1300\right)$ and set of traveling wave parameters $\left(a^{+}=9, \lambda^{+}=3862\right.$, and $T^{+}=115$ ) was tested. The shear stress reductions determined from the computational and experimental methods were $1.27 \%$ and $0.89 \%$, respectively, where the source of these small reductions were the small amplitudes and large wavelengths of the TWs. In addition to computationally validating the experiment, Meysonnat and Roggenkamp confirmed that no wake exists downstream of the traveling waves. Consequently, using the skin friction downstream of the TWs is indeed a valid method for measuring the shear stress reduction.

Li and Roggenkamp [82] then conducted another experimental study to further analyze the underlying skin friction reduction mechanism and its correlation with the TW parameters. For a single Reynolds number $\left(\operatorname{Re}_{\theta, \text { outlet }}=1194\right)$, multiple amplitudes and two different frequencies were tested and directly compared to results from Roggenkamp [8]. They showed that the skin friction reduction increases with increasing frequency (decreasing period) and amplitude. In addition, they showed that the majority of the skin friction reduction occurs over the trough of the traveling wave and that the crest only contributes slightly.

When looking at the flow structure of the TW actuated flow, Li and Roggenkamp [82] found that there was a reduction in streamwise velocity fluctuations (albeit slight) and that this effect increased with corresponding increases in drag reduction. They described this as a damping effect and that its effect was much more pronounced over the crest than the trough. Quadrant analysis also showed additional differences between the unactuated and actuated case. In the Q4 region, there was a reduction of the u' and v' components above both the trough and crest. Consequently the wave motions damp the sweep events and result in a reduction of the Reynolds shear stress, with this effect magnified for larger TW amplitudes. While the change in sweep events was stronger, they also observed a weakening of the ejection events.

In addition to the experiments generating spanwise traveling waves on a smooth surface [8, 32, 82], Li [29] also investigated the combination of spanwise traveling waves and passive riblets. Using the same experimental setup, Li compared the results of the hybrid riblet/TW setup with the results of the traveling wave from Roggenkamp [8]. For $R_{\theta, \text { outlet }}=1200$, the riblets on an unactuated plate yielded a $4.7 \%$ reduction in skin friction drag while the combination of the riblets and TW yielded a reduction of 9.4\%. In comparison, the TWs alone in Roggenkamp achieved only a $3.4 \%$ reduction. At higher Reynolds numbers the hybrid riblet/TW structure was less effective; the skin friction was reduced by only $2.7 \%$. However, this resulted primarily because the riblets were outside the Reynolds number for which they were designed.

When looking at the flow structures near the wall, the hybrid riblet/TW case shows that the near wall velocity gradient is decreased and that the buffer region is pushed further off the wall. 
The displacement of the turbulence was also confirmed by lower wall normal velocity fluctuations near the wall and greater values in the log layer. In addition, the distribution of Reynolds shear stress showed a shifting away from the wall for the TW versus unactuated case.

Despite the low skin friction reductions at higher Reynolds numbers, a near $10 \%$ reduction for a specific case is significant. As a step towards implementing this system in an application, Stille [89] conducted an endurance test of the combined EM actuator system and riblets. This was done in order to determine the fatigue life and failure method of the aluminum plate and riblets. They found that the failure can occur after a relatively low number of cycles $\left(10^{6}\right)$, but if the structure passed this point, the lifetime could reach up to and beyond $10^{8}$ cycles.

\subsubsection{Consensus on Skin Friction Reduction}

The studies performed thus far [3-8, 26-29, 31, 32, 82] have shown that spanwise traveling waves with wall deformation can yield a significant reduction in the skin friction drag. The maximum average reductions were 13\% from Tamano and Itoh's [5] experimental study and 13.4\% from Tomiyama's [3] DNS study, and the local reduction has been shown to be as high as 50\% [6]. Yet the skin friction reduction mechanism and its relationship with the traveling wave parameters is not fully understood. But that is not to say that no progress has been made.

The boundary layer profile for traveling wave actuated surfaces has shown that the velocity gradient in the laminar sublayer is lower than that for the unactuated wall. Since the gradient is directly related to the skin friction coefficient this comes as no surprise, but this decrease in the gradient is not uniform across the entire TW surface. The gradient in the trough of the traveling wave is lower while that in the crest is equal or higher than that of the unactuated wall (see Figure 3.6). In addition, the laminar sublayer is thicker for reductions in skin friction. As a result the buffer layer occurs further off the wall. This is of particular importance since the buffer layer is the largest source of turbulent kinetic energy (TKE) production in the boundary layer [48]. Confirming this, numerous studies $[3,5,27,28]$ observed that the peak of the TKE production and Reynolds shear stress were displaced off the wall and that the magnitude of the peaks decreased, primarily in the TW trough.

Another common thread throughout most of the studies [4, 7, 27, 28,31] was the pushing of the wall normal vorticity further from the wall. Klumpp [4] references both Schoppa [83] and Jimenez's [84] studies in which changes in the wall-normal vorticity propagated to changes in the streamwise vorticity and the turbulent structure. As a result the turbulence regeneration cycle is interrupted. An important component of this regeneration cycle are the bursting events which encompass both ejection and sweep events. Ejections are the violent transport of low-speed fluid away from the wall and sweeps are the in rushes of high-speed fluid towards the wall. These burst- 
ing events occur primarily in the buffer region and are the source for a majority of the turbulence production [23]. As a result, these events contribute heavily to the skin friction drag in turbulent flow. Consequently, since multiple traveling wave studies $[5,27,82]$ have shown weakened ejection and sweep events and reduced shear stress, the traveling waves can be confirmed to interrupt the turbulence regeneration cycle.

Thus far, a consistent thread in all the studies is that the turbulence is displaced further from the wall, specifically the buffer layer, TKE production, wall normal vorticity and bursting events. The reduction in these events near the wall occurs primarily in the trough of the traveling wave, so now the question is, what exactly is pushing the turbulence out of the trough? In the trough, there is an induced spanwise flow that corresponds with the propagation direction of the traveling wave [3, 4, 7, 27]. Tomiyama's [3] explains that this spanwise flow acts as a shield that minimizes the interaction between the trough surface and the quasi-streamwise vortices (QSVs) which are intimately related to the bursting events and the turbulence regeneration cycle [23]. However, Tomiyama also references the wall normal velocity of the traveling wave as a potentially important factor in the drag reduction mechanism. Koh [28] agrees that it is the wall-normal velocity that correlates with the skin friction reduction, stating that this velocity is responsible for the displacement of the turbulence from the wall. In particular, the wall normal velocity pushes the vorticity in the trough further out into the boundary layer where it is then swept downstream by the higher velocity flow. Overall, Koh describes it as a ratio between the wall normal momentum of the traveling wave and the streamwise momentum of the flow. However, what remains unexplained is how the turbulence is weakened once it is displaced. The mechanism could be similar to that suggested by Karniadakis [22] for Lorentz forcing traveling waves. The waves stabilize the near-wall streaks, which are integral to the turbulence regeneration cycle [80], and thus reduce the TKE production and Reynolds shear stresses. However, no explanation has been giving in terms of traveling waves with wall deformation.

This brings into focus the relationship between the skin friction drag reduction and the traveling wave parameters: amplitude, frequency/period, wavelength, and wave speed. There is general agreement that increases in the amplitude yield increases in the skin friction reduction $[3,5,6,8$, $28,82]$; however, it has also been shown $[6,28]$ that there is an optimal amplitude above which further increases are not as effective at reducing the skin friction. Bai [6] references Du [79] wherein a DNS study showed that when using Lorentz force traveling waves, the optimal amplitude is comparable to the laminar sublayer thickness. On the other hand, Koh's [28] discussion of the optimal amplitude relates to his assumed skin friction reduction mechanism in which the displaced vorticity is swept downstream. Investigating the shear stress reduction induced by the TWs at different Reynolds numbers [7,28], Koh observed that the optimal amplitude was higher for larger Re-even though the overall skin friction reduction decreased with increasing Re. As a 
result, he surmised that if the wall normal momentum of the waves was too large when compared to the streamwise momentum of the flow, the turbulence would be displaced from the wall but not transported away quickly enough to efficiently reduce the shear stress. However, only three amplitudes at three different Reynolds numbers were tested here, so further validation is required to verify this relationship between amplitude, Reynolds number, and the skin friction reduction.

Most of the relationships between traveling wave parameters and skin friction reduction focused upon amplitude, but some conclusions were drawn regarding the others. Tamano and Itoh [5] and then Roggenkamp [8] observed that larger reductions in shear stress were possible for smaller periods (larger frequencies). Tamano surmised that $T^{+}<100$ produces the largest reduction since this is on the order of the bursting period for wall bounded canonical flows. The implication is that by having a TW which is quicker than the bursting events, the turbulence regeneration cycle can be more effectively disrupted. Regarding wavelength, Tomiyama [3] found a weak correlation between increases in it and increased skin friction reduction, but further investigation would be required to verify this. In terms of wave speed, other wall-motion techniques investigating skin friction reduction showed a dependence on the speed [22], but no strong correlation has been shown yet for spanwise traveling waves. However, the wave speed in the TWs is dependent upon the frequency and wavelength, so a relationship between wave speed and skin friction reduction may yet be determined. 


\section{Chapter 4}

\section{Steady-State Traveling Wave Generation}

As discussed in Section 3.2, spanwise traveling waves are a promising method for altering the turbulent boundary layer with the aim of reducing skin friction drag. Previous research has shown that reductions of up to $13 \%$ are possible $[3,5]$. However, before this technique can be implemented, a more practical traveling wave generation method is necessary. The method should utilize a lowprofile actuation scheme and have minimal limitations on the achievable frequencies. A promising wave generation method is known as two-mode excitation. Two-mode excitation is capable of generating steady-state traveling waves using low-profile piezoelectric actuators. It is also open-loop controlled and can produce waves at a wide range of frequencies.

In order to apply two-mode excitation for drag reduction purposes, further development of the method is necessary. In particular, traveling waves should be generated on a two-dimensional surface and tailored to propagate in the spanwise direction. Previous research has shown that traveling waves can be generated on a two-dimensional (2D) surface [35], but most studies have investigated one-dimensional systems (see Section 3.1.3). The study into 2D surfaces did not focus on controlling the wave propagation direction, but it did show a dependence on the actuation location. This dependence results because the two-mode excitation method is inherently coupled to the surface's structural properties. Thus, the actuation location affects the modal participation of the nearby modes. Using this same logic, the modal participation also depends on the number of actuators excited. Thus, the location and number of actuators-henceforth known as actuation configuration-plays a key role in the resultant traveling wave pattern. This chapter seeks to better understand this complex relationship and then leverage it to tailor steady-state traveling waves on a $2 \mathrm{D}$ surface. In particular, the waves are tailored for drag reduction and thus propagate in the spanwise direction; however, traveling waves could be tailored for other applications such as propulsion and solid-state locomotion.

This chapter begins by developing an in-house finite element model capable of simulating traveling waves.Next, experimental modal analysis is conducted and the model is updated to match 
the test setup: a thin, clamped plate with mounted piezoelectric actuators. Then, simulated and experimental traveling waves are generated to validate the model. Finally, traveling waves are generated using various actuation configurations (location and number of actuators) for a single frequency test case. The interdependencies between actuation configuration, mode participation, and wave propagation (pattern) are discussed.

\subsection{Finite Element Model}

Consider a thin, isotropic, linear-elastic plate, with $n$ piezoelectric actuators bonded to its surface. The piezoelectric actuators are assumed to be perfectly bonded to the plate's surface. Therefore, those sections of the plate covered with the piezoelectric actuators are treated as composite structures and no additional degrees of freedom are introduced for the piezoelectric actuators, except for the electric displacements. The plate is also assumed to be subjected to large in-plane normal stresses. Such stresses arise from the plate's manufacturing processes, in the form of residual stresses, and when the plate is attached to the clamping frame, as discussed in Section 4.2.1. For the thin plate considered in this study, the first order shear deformation theory is adopted to define longitudinal and lateral deformations, which yields the displacement field in Equation 4.1. $u_{0}, v_{0}$, and $w_{0}$ are the generalized displacements of the point $(x, y)$ on the plate's mid-plane along the $\mathrm{x}$, $\mathrm{y}$, and $\mathrm{z}$ directions, respectively. $\theta_{x}$ and $\theta_{y}$ are the generalized rotations of the normal to the plate's mid-plane in the $\mathrm{xz}$ and the yz planes, respectively.

$$
\begin{aligned}
u(x, y, z, t) & =u_{0}(x, y, t)+z \theta_{x}(x, y, t) \\
v(x, y, z, t) & =v_{0}(x, y, t)+z \theta_{y}(x, y, t) \\
w(x, y, z, t) & =w_{0}(x, y, t)
\end{aligned}
$$

Elastic waves induced in the plate due to piezoelectric actuation are assumed to result in small perturbations of the initial state of the plate. Therefore, the assumption of infinitesimal strains can be adopted and the strain-displacement relations are defined as in Equation 4.2. The strains are split into in-plane strains $\varepsilon_{b}$ and transverse shear strains $\varepsilon_{s}$ to facilitate the finite element formulation. This allows the implementation of a reduced integration rule for the evaluation of $\varepsilon_{s}$ so as to avoid the issue of shear-lock associated with shear deformations, while using a full integration rule for 
$\varepsilon_{b}$.

$$
\begin{aligned}
& \varepsilon_{b}=\left\{\begin{array}{l}
\varepsilon_{x x} \\
\varepsilon_{y y} \\
\gamma_{x y}
\end{array}\right\}=\left[\begin{array}{ccc}
\partial / \partial x & 0 & 0 \\
0 & \partial / \partial y & 0 \\
\partial / \partial y & \partial / \partial x & 0
\end{array}\right]\left\{\begin{array}{l}
u_{0} \\
v_{0} \\
w_{0}
\end{array}\right\}+z\left[\begin{array}{cc}
\partial / \partial x & 0 \\
0 & \partial / \partial y \\
\partial / \partial y & \partial / \partial x
\end{array}\right]\left\{\begin{array}{l}
\theta_{x} \\
\theta_{y}
\end{array}\right\} \\
& \varepsilon_{s}=\left\{\begin{array}{l}
\gamma_{x z} \\
\gamma_{y z}
\end{array}\right\}=\left[\begin{array}{lll}
0 & 0 & \partial / \partial x \\
0 & 0 & \partial / \partial y
\end{array}\right]\left\{\begin{array}{l}
u_{0} \\
v_{0} \\
w_{0}
\end{array}\right\}+\left[\begin{array}{cc}
1 & 0 \\
0 & 1
\end{array}\right]\left\{\begin{array}{l}
\theta_{x} \\
\theta_{y}
\end{array}\right\}
\end{aligned}
$$

Similarly, the state of the stress at any point in the plate is described by the in-plane stresses $\left(\sigma_{b}\right)$ and transverse shear stresses $\left(\sigma_{s}\right)$. For a linear-elastic material, these stresses are defined in Equation 4.3, where $C$ denotes the reduced stiffness matrix obtained with plane-stress assumption as defined in Appendix A.1.

$$
\begin{aligned}
& \sigma_{b}=\left\{\begin{array}{lll}
\sigma_{x x} & \sigma_{y y} & \tau_{x y}
\end{array}\right\}^{T}=C_{b} \varepsilon_{b} \\
& \sigma_{s}=\left\{\begin{array}{ll}
\tau_{x z} & \tau_{y z}
\end{array}\right\}^{T}=C_{s} \varepsilon_{s}
\end{aligned}
$$

For the piezoelectric actuators, linear piezoelectricity is assumed and the constitutive equations can be expressed as in Equation 4.4 [90].

$$
\begin{aligned}
\sigma_{b} & =C_{b}^{D} \varepsilon_{b}-h_{b} D \\
\sigma_{s} & =C_{s} \varepsilon_{s}-h_{s} D \\
E & =-h_{b}^{T} \varepsilon_{b}-h_{s}^{T} \varepsilon_{s}+\beta^{\varepsilon} D
\end{aligned}
$$

where $D$ is the electric displacement, $E$ is the electric field, $h_{b}$ and $h_{s}$ are the piezoelectric coupling coefficients related to bending and shear strains, $C_{b}^{D}$ and $C_{s}^{D}$ are the mechanical stiffness coefficients of the material measured at zero electric displacement, $\beta^{\varepsilon}$ is the inverse permittivity coefficient measured at zero strain. In this study, Macro-fiber composite (MFC) piezoelectric actuators are used to experimentally generate steady-state traveling waves. These actuators operate in the 3-3 mode. However, their electrodes are designed such that the $3-3$ mode of operation results in in-plane deformations [91]. Therefore, MFCs are modeled in this study as monolythic piezoelectric actuators operating in the $3-1$ mode.

In the presence of large initial stresses, finite strains and deformations can be induced in the plate. Thus, geometric nonlinearities have to be taken into consideration. For the plate addressed in this study, only in-plane normal stresses along the $x$ and $y$ directions, denoted respectively by $\sigma_{x x}^{0}$ and $\sigma_{y y}^{0}$, are considered. The corresponding work-conjugate strain components are defined in 
Equation 4.5.

$$
\begin{aligned}
& E_{x x}=\frac{\partial u_{0}}{\partial x}+z \frac{\partial \theta_{x}}{\partial x}+\frac{1}{2}\left[\left(\frac{\partial u_{0}}{\partial x}+z \frac{\partial \theta_{x}}{\partial x}\right)^{2}+\left(\frac{\partial v_{0}}{\partial x}+z \frac{\partial \theta_{y}}{\partial x}\right)^{2}+\left(\frac{\partial w_{0}}{\partial x}\right)^{2}\right] \\
& E_{y y}=\frac{\partial v_{0}}{\partial y}+z \frac{\partial \theta_{y}}{\partial y}+\frac{1}{2}\left[\left(\frac{\partial u_{0}}{\partial y}+z \frac{\partial \theta_{x}}{\partial y}\right)^{2}+\left(\frac{\partial v_{0}}{\partial y}+z \frac{\partial \theta_{y}}{\partial y}\right)^{2}+\left(\frac{\partial w_{0}}{\partial y}\right)^{2}\right]
\end{aligned}
$$

For the FE formulation, eight-node quadrilateral elements are used to discretize the plate. The displacement field at any point within an element can be defined in terms of the nodal displacement vectors, $d_{t}^{e}$ and $d_{r}^{e}, D$, and shape functions matrices, $N_{t}, N_{r}$, and $N_{d}$, as in Equation 4.6.

$$
\begin{gathered}
d_{t}=N_{t} d_{t}^{e} \\
d_{r}=N_{r} d_{r}^{e} \\
D_{3}=N_{d} D^{e}
\end{gathered}
$$

where

$$
\begin{gathered}
d_{t}=\left\{\begin{array}{lll}
u_{0} & v_{0} & w_{0}
\end{array}\right\}^{T} \\
d_{r}=\left\{\begin{array}{lll}
\theta_{x} & \theta_{y}
\end{array}\right\}^{T} \\
d_{t}^{e}=\left\{\begin{array}{llllll}
u_{0}^{1} & v_{0}^{1} & w_{0}^{1} & u_{0}^{2} & \cdots & w_{0}^{8}
\end{array}\right\}^{T} \\
d_{r}^{e}=\left\{\begin{array}{lllll}
\theta_{x}^{1} & \theta_{y}^{1} & \theta_{x}^{2} & \cdots & \theta_{y}^{8}
\end{array}\right\}^{T}
\end{gathered}
$$

the nodal electrical displacements are $D^{e}=\left\{\begin{array}{llll}D_{3}^{1} & D_{3}^{2} & \cdots & D_{3}^{8}\end{array}\right\}^{T}$, and $N_{t}, N_{r}$, and $N_{d}$ are the shape functions matrices. The shape function matrices are defined in Appendix A.1.

Based on the aforementioned assumptions, the kinetic energy of the coupled piezoelectric-plate element is expressed using Equation 4.7, where $\rho_{\text {Plate }}$ is the volumetric mass density of the plate, $\rho_{\text {Piezo }}$ is that of the piezoelectric actuator, $V_{\text {Plate }}$ is the volume of the plate section, $V_{\text {Piezo }}$ is the volume of the piezoelectric actuator section, and $(\cdot)$ denotes the first partial derivative with respect to time.

$$
\begin{aligned}
T_{k}=\frac{1}{2} \int V_{V_{\text {Plate }}} \iint\left(\rho_{\text {Plate }}\left(\dot{d}_{t}^{e}+z \dot{d}_{r}^{e}\right)^{T}\left(\dot{d}_{t}^{e}+z \dot{d}_{r}^{e}\right)\right) \mathrm{d} V \\
\quad+\frac{1}{2} \iiint_{V_{\text {Piezo }}}\left(\rho_{\text {Piezo }}\left(\dot{d}_{t}^{e}+z \dot{d}_{r}^{e}\right)^{T}\left(\dot{d}_{t}^{e}+z \dot{d}_{r}^{e}\right)\right) \mathrm{d} V
\end{aligned}
$$

Following Helmholtz free energy definition for piezoelectric materials, the potential energy functional of the coupled piezoelectric-plate elements, including the strain energy associated with 
the large initial stresses, are expressed in Equation 4.8.

$$
\begin{gathered}
T_{p}=\frac{1}{2} \iiint_{V_{\text {Plate }}}\left(\varepsilon_{b}^{T} \sigma_{b}+\varepsilon_{s}^{T} \sigma_{s}\right) \mathrm{d} V+\iiint_{V_{\text {Plate }}}\left(E_{x x} \sigma_{x x}^{0}+E_{y y} \sigma_{y y}^{0}\right) \mathrm{d} V \\
\quad+\frac{1}{2} \iiint_{V_{\text {Piezo }}}\left(\varepsilon_{b}^{T} \sigma_{b}+\varepsilon_{s}^{T} \sigma_{s}-2 \varepsilon_{b}^{T} h_{b} D+D^{T} \beta^{\varepsilon} D\right) \mathrm{d} V
\end{gathered}
$$

Substituting equations 4.2 to 4.6 into equations 4.7 and 4.8 yields

$$
\begin{aligned}
& T_{k}=\frac{1}{2}\left(\dot{d}_{t}^{e T} M_{t t}^{e} \dot{d}_{t}^{e}+\dot{d}_{r}^{e T} M_{r r}^{e} \dot{d}_{r}^{e}\right) \\
& T_{p}=\frac{1}{2}\left(d_{t}^{e T} K_{t t}^{e} d_{t}^{e}+d_{t}^{e T} K_{t r}^{e} d_{r}^{e}+d_{r}^{e T} K_{r t}^{e} d_{t}^{e}+d_{r}^{e T} K_{r r}^{e} d_{r}^{e}-d_{t}^{e T} K_{t d}^{e} D^{e}+D^{e T} K_{d d}^{e} D^{e}\right)
\end{aligned}
$$

where all the elemental matrices appearing in Equation 4.9 are defined in Appendix A.1.

The work done by external forces and moments, along with the externally applied voltage to the piezoelectric actuators, is defined in Equation 4.10.

$$
W=\iiint_{V_{\text {Piezo }}} \frac{V D_{3}}{t_{\text {Piezo }}} \mathrm{d} V+\iint_{A_{\text {Plate }}} d^{T} f \mathrm{~d} A
$$

where $f$ is the externally applied surface traction vector acting over the plate's surface area $A_{\text {Plate }}$, and $V$ the externally applied electric potential on the piezoelectric actuator is defined as $V=$ $E_{3} t_{\text {Piezo }}$.

Upon the application of Hamilton's principle, the equations of motion for the plate with the piezoelectric actuators are shown in Equation 4.11.

$$
\begin{aligned}
A_{\text {Piezo }} V+K_{t d}^{e T} d_{t}^{e} & =K_{d d}^{e} D^{e} \\
M_{t t}^{e} \ddot{d}_{t}^{e}+K_{t t}^{e} d_{t}^{e}+K_{t r}^{e} d_{r}^{e} & =F_{t}^{e}+K_{t d}^{e} D^{e} \\
M_{r r}^{e} \ddot{d}_{r}^{e}+K_{r t}^{e} d_{t}^{e}+K_{r r}^{e} d_{r}^{e} & =F_{r}^{e}
\end{aligned}
$$

For the thin plate and thin piezoelectric actuators considered in this study, the effect of rotary inertia is negligible. Thus $M_{r r}^{e} \approx 0$. This allows solving for rotational degrees of freedom in terms of the translational ones, and thus, reducing the computational cost the model. Finally, global mass and stiffness matrices are obtained by assembling their corresponding elemental matrices following standard assembly procedures. 


\subsection{Modal Analysis and Model Updating}

The in-house finite element model described in Section 4.1 is updated to match the modal properties of an experimental clamped, thin plate. Experimental modal analysis is conducted on the clamped plate and the modal properties measured using a scanning laser doppler vibrometer (SLDV). The model is then manually updated to match the experimental modal parameters. The updating procedure primarily focuses on matching internal stresses in the plate and non-ideal (i.e. elastic) boundary conditions.

\subsubsection{Experimental Modal Analysis}

The experimental, clamped plate is made of rolled Al6061 and is shown in Figure 4.1a. Epoxied to the underside of the plate is an aluminum frame (frame 1) with threaded holes that allow the entire plate to be clamped. The dimensions of the clamped plate are $292.1 \times 190.5 \times 0.397 \mathrm{~mm}$ $(7.5 \times 11.5 \times 1 / 64 i n)$, where the length and width are defined by the inner dimensions of frame 1. Also mounted to the underside of the plate are ten M4312-P1 macro fiber composite (MFC) piezoelectric actuators [91]. These actuators are used to generate traveling waves in Section 4.3, and actuator $A_{3}$ is excited to determine the modal properties.

In order to measure the modal properties, the plate is mounted as shown in the bottom of Figure 4.1b. Clamped boundary conditions are produced by bolting the plate and frame 1 to frame 2 , as shown in Figure 4.1c. To increase the stiffness even further, the clamped plate is then fixed to a rigid aluminum base (Frame 3$)$. Due to the thinness of the plate $(0.397 \mathrm{~mm})$, precautions are taken to minimize acoustic and mass loading effects. Frames 1-3 all have hollow centers such that the underside of the plate (Figure 4.1a) is visible through the frames. This is done to avoid a sealed air cavity below the plate that would introduce additional mass and stiffness loading. To mitigate any acoustic coupling issues, the frame assembly is placed on a raised 80/20 platform (Figure 4.1b) and acoustic foam is positioned below the plate to minimize acoustic radiation and prevent reflection off of the table.

The experimental modal analysis is then conducted by measuring the velocity using a PSV-400 Polytec scanning laser doppler vibrometer (SLDV). This is shown in the top of Figure 4.1b. The frequency response of the plate is measured by exciting actuator $A_{3}$ with a $150 \mathrm{~V}, 0-1 \mathrm{kHz}$ burst

chirp signal. The mode shapes, natural frequencies, and damping values are then extracted from the $H_{1}$ response of the plate using the Polymax fit in Siemens LMS modal analysis package. 


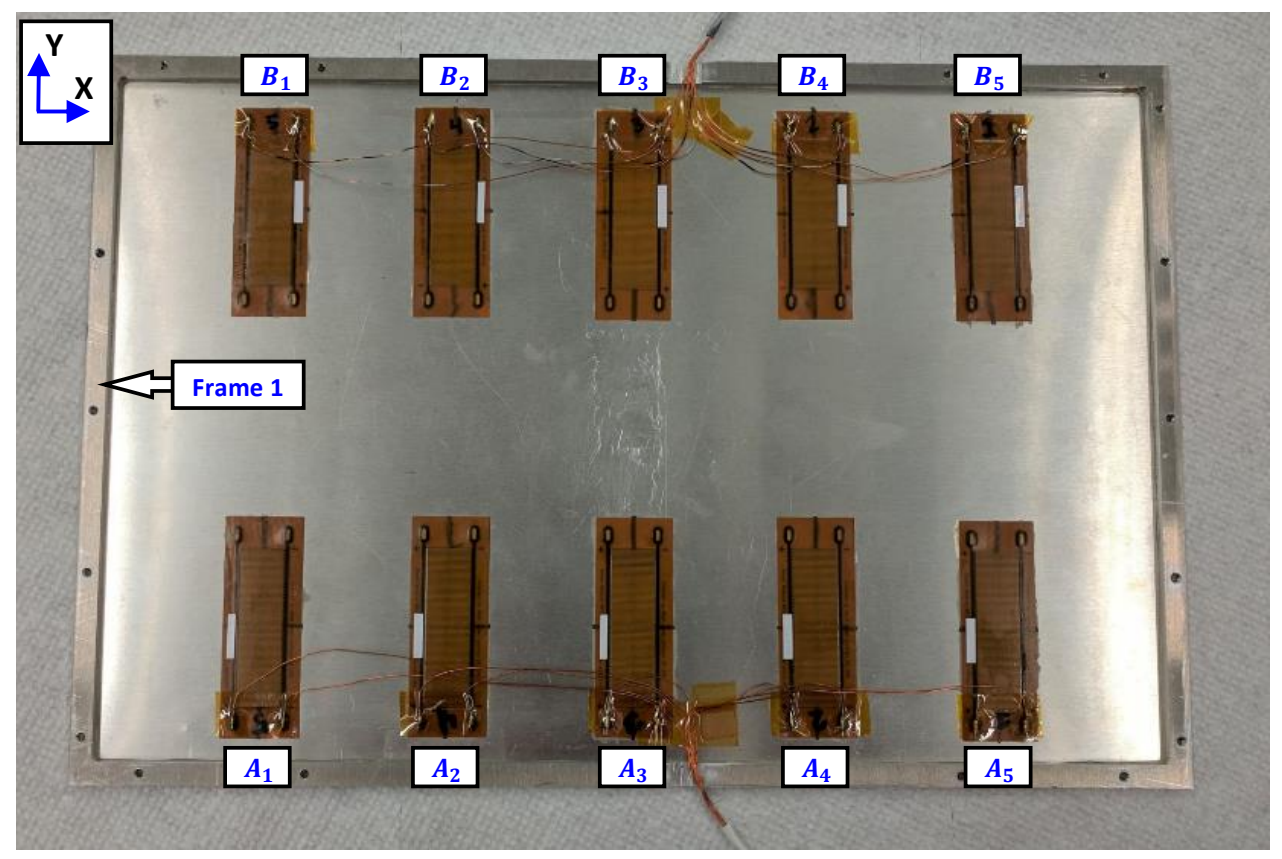

(a)

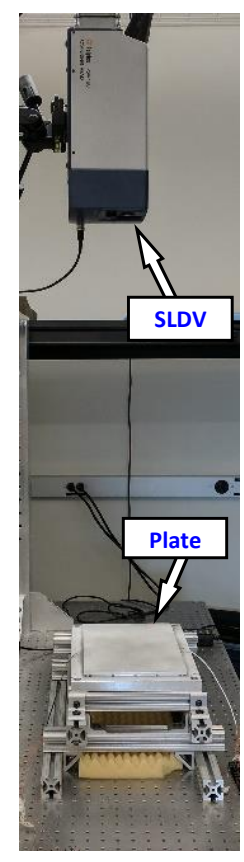

(b)

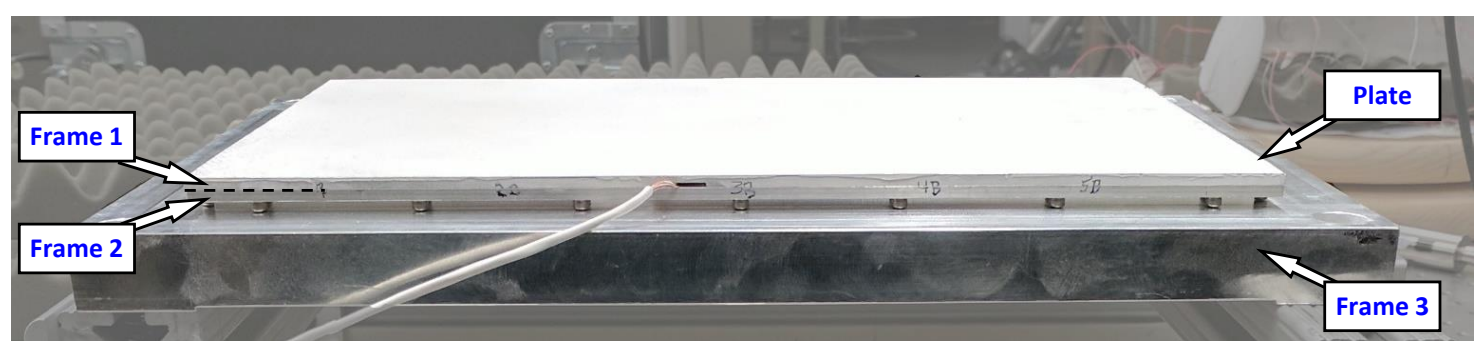

(c)

Figure 4.1: (a) Underside of the aluminum plate with ten MFC piezoelectric actuators and the frame used for clamping, (b) experimental setup for measuring the modal properties, and (c) the mounting assembly used to ensure clamped boundary conditions

\subsubsection{Model Updating}

With the experimental modal data collected, the parameters in the finite element model are manually updated to match the modal properties in the experiment. In the model, a mesh of $46 \times 23$ was chosen, with 46 elements in the $x$ direction (Figure 4.1a). A convergence study was conducted and this was deemed sufficient. In the model, the MFC actuators are defined by choosing specific elements in the mesh. As a result, the final mesh size is chosen so that both the size and position of the MFCs could be defined using an integer number of elements. For this reason, the surface area (length and width) and the position of the MFCs are held constant in the model updating procedure. The MFCs are positioned symmetrically on the plate. In the $x$ direction, there is $50.8 \mathrm{~mm}$ (2in) between each actuator (center to center). In the $y$ direction, the center of each MFCs is $43 \mathrm{~mm}$ 
(1.69in) from the edge of the plate. This $y$ length corresponds with the length of the active area of the MFC.

Other parameters are also held constant during model updating. These are shown in Table 4.1 and include the dimensions $(L, W$, and $h)$, elastic modulus $(E)$, and density $(\rho)$ of the plate and also the thickness $\left(h_{M F C}\right)$ and density $\left(\rho_{M F C}\right)$ of the MFCs. The plate dimensions are defined by the inner dimensions of frame 1 (Figure 4.1a) and the nominal thickness of the plate. The MFC thickness and elastic modulus values are taken from Shahab [92]. In the initial steps of the model updating procedure, these constants were adjusted, but their effect on matching the model was found to be minimal. While the MFC thickness and modulus are held constant, the density of the MFC is updated. This is necessary because the surface area of the MFCs in the model are defined by the MFCs' 'active' area. Looking at Figure 4.1a, the MFC contains a center (active) region composed of piezoelectric material and an outside (passive) region containing the electrodes and kapton. The MFC elements in the finite element model only encompass the active area, since this is the area that is actuated. Thus, the density of the MFC is updated in the model to also account for the passive area of the MFC (Table 4.2).

Table 4.1: Parameters held constant during the model updating procedure

\begin{tabular}{|c|c|c|c|c|c|c|}
\hline \multicolumn{5}{|c|}{ Plate Properties } & \multicolumn{2}{|c|}{ MFC Properties } \\
\hline$L[m m](i n)$ & $W[m m](i n)$ & $h[m m]$ & $E[G P a]$ & $\rho\left[\frac{\mathrm{kg}}{\mathrm{m}^{3}}\right]$ & $h_{M F C}[\mathrm{~mm}]$ & $E_{M F C}[G P a]$ \\
\hline $292.1(11.5)$ & $190.5(7.5)$ & 0.397 & 69 & 2700 & 0.31 & 31 \\
\hline
\end{tabular}

The main parameters of the clamped plate that are updated are the internal stresses and the clamped boundary conditions. The initial and final values are shown in Table 4.2. The internal stresses only arise in the plate due to its small thickness $(0.397 \mathrm{~mm})$. These stresses stem from two separate sources. The first is a pre-stress introduced in the $x$ direction during the rolling process of the Al6061 plate. This was quantified during a preliminary model updating procedure of the plate with free boundary conditions. In order to match the natural frequencies and mode shapes of the free model to the experiment, a tensile stress was necessarily introduced in the $x$ direction of the plate. This correlates with a manufacturing related stress, since there is no stress in the $y$ direction and the grains on the plate indicate that the plate was rolled in the $x$ direction. The second source of the stresses results from clamping the plate. Frames 1-3 (Figure 4.1c) are used to produce boundary conditions as close to clamped as possible; however, since the plate is so thin, the clamping process introduces non-trivial stresses into the plate. The updated stresses are shown in Table 4.2, where the larger stress in the $x$ direction results from those introduced during the rolling process.

Although extra care was taken to stiffen the system, the resultant clamped boundaries are non- 
Table 4.2: Initial and final values of the parameters updated to match the model to the experiment

\begin{tabular}{|c|c|c|c|c|c|}
\hline \multirow[b]{3}{*}{ Initial } & \multirow{2}{*}{$\frac{\text { MFC Properties }}{\rho_{M F C}\left[\frac{\mathrm{kg}}{\mathrm{m}^{3}}\right]}$} & \multicolumn{2}{|c|}{ Plate Stress } & \multicolumn{2}{|c|}{ Boundaries } \\
\hline & & $\sigma_{x}[\mathrm{kPa}]$ & $\sigma_{y}[\mathrm{kPa}]$ & $k_{z}\left[\frac{N}{m}\right]$ & $k_{R}\left[\frac{N}{r a d}\right]$ \\
\hline & 5440 & 0 & 0 & $\infty$ & $\infty$ \\
\hline Final & 4000 & 300 & 100 & 5000 & 10 \\
\hline
\end{tabular}

ideal. A perfect clamped boundary has infinite stiffness; however, the experimental setup here has some finite elasticity. In this case, the boundaries are assumed to have finite out-of-plane $\left(k_{z}\right)$ and rotational stiffnesses $\left(k_{R}\right)$. The rotation stiffness is about the axis of the clamp itself, so it would be about the $x$ axis on the top and bottom of the plate (Figure 4.1a) and the $y$ axis on the left and right. Both the out-of-plane and rotational stiffnesses are assumed homogeneous on all clamped boundaries. The initial and final model stiffnesses are shown in Table 4.2 ( $\infty$ represents ideal clamped boundaries). The clamped boundaries are actually fairly close to ideal. Comparing the natural frequencies of the ideal and non-ideal boundaries, there is only a $3 \%$ difference.

The comparison between the model and the experimental plate are shown in Figure 4.2. Figure 4.2a shows the natural frequencies for the model and experiment along with the zero error line. Figure $4.2 \mathrm{~b}$ plots the error in the natural frequencies for the first 13 modes of the model. From these two plots it is clear that the model provides a better match at higher modes than lower ones. In fact, the error in the natural frequencies for modes 1,2, and 4 are not even shown in Figure $4.2 \mathrm{~b}$. That is because they are $\approx-10,-25$, and $-10 \%$, respectively. Whereas for modes $6-13$, there is a $\pm 2 \%$ error. Looking at the MAC values (Figure 4.2c), it is clear though that the model can accurately represent the mode shapes. This is the case even for the lower modes with large frequency errors.

This discrepancy between the model and experiment stems from issues with the experimental setup. Significant effort was spent pinpointing and mitigating unwanted effects in the experiment: such as fluid loading and acoustic coupling. However, there were consistent issues in mounting the plate without introducing non-uniform or shear stresses. Based on trends observed in updating the model, stresses in the plate tend to disproportionately affect the lower mode shapes. As a result, the larger errors in the clamped model at lower frequencies likely result from unmodeled, non-uniform/shear stresses in the experimental plate.

Overall, these natural frequency errors at lower modes are not an issue in this study. In Section 4.3 traveling waves are generated at higher modes, specifically between modes 7 and 8 . The natural frequency error is within $\pm 2 \%$ in this region, thus the model and experiment match quite well in the region of interest. 


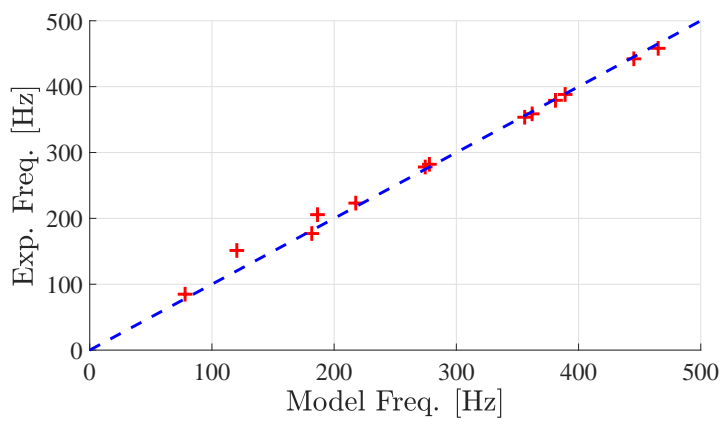

(a)

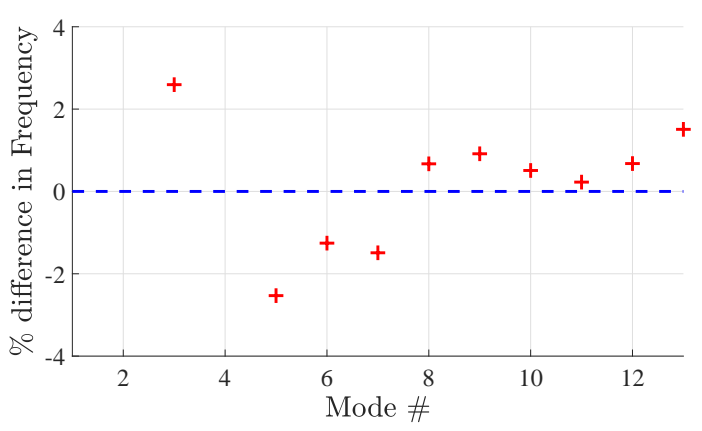

(b)

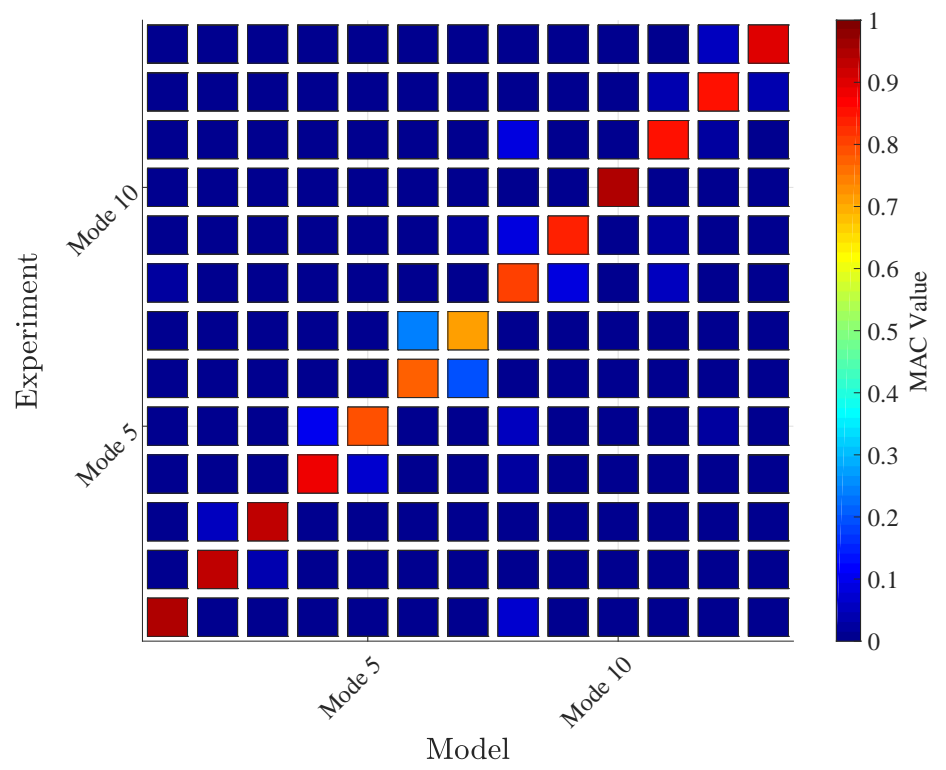

(c)

Figure 4.2: Comparison between the clamped boundary condition model and experiment showing the (a) natural frequencies plotted with the zero error line, (b) the percent error in natural frequencies, and (c) the MAC values

\subsection{Traveling Waves}

Using the updated finite element model, traveling waves are generated with the two-mode excitation method. Initially, traveling waves are generated using a single pair of MFC actuators and then validated against experimental waves. Then, the effects of different actuation configurations are investigated with. These waves were intentionally tailored to propagate in the spanwise direction in order to be used for drag reduction purposes. This includes single actuator pairs at different locations, multiple actuator pairs in unison, and actuator pairs excited $180^{\circ}$ out of phase. Each of these actuation configurations produces a different traveling wave response on the plate. Signifi- 
cantly, this is despite the excitation frequency remaining constant across all cases. These actuation patterns are implemented experimentally and correlated with those simulated in the model.

\subsubsection{Model validation using traveling waves}

To validate the finite element model for traveling wave generation, experimental and modeled waves are compared. Waves are generated with the two-mode excitation method using the middle set of actuators $\left(A_{3}-B_{3}\right)$ on the clamped plate. The excitation actuators are highlighted in Figure 4.3 , where there is a phase difference of $\gamma$ between them. To compare the model and experimental traveling waves, the correlation coefficient defined in Equation 4.12 is used. In the equation, $[\psi]$ is a vector of complex amplitudes for the traveling wave, where $E$ and $M$ correspond to the experiment and model, $T$ is the transpose of the vector, and $\left(^{*}\right)$ represents the complex conjugate. In the vector, $[\psi]$, each element represents a complex amplitude at a physical location on the plate. The complex amplitude defines the relative amplitude and phase of each location to all other locations on the plate. The correlation coefficient has the same definition as the modal assurance criterion (MAC), but was relabeled here as $C_{E M}$ to avoid confusion since MAC is used in Section 4.2.2. As such, a value of 0 signifies no correlation and 1 signifies perfect correlation.

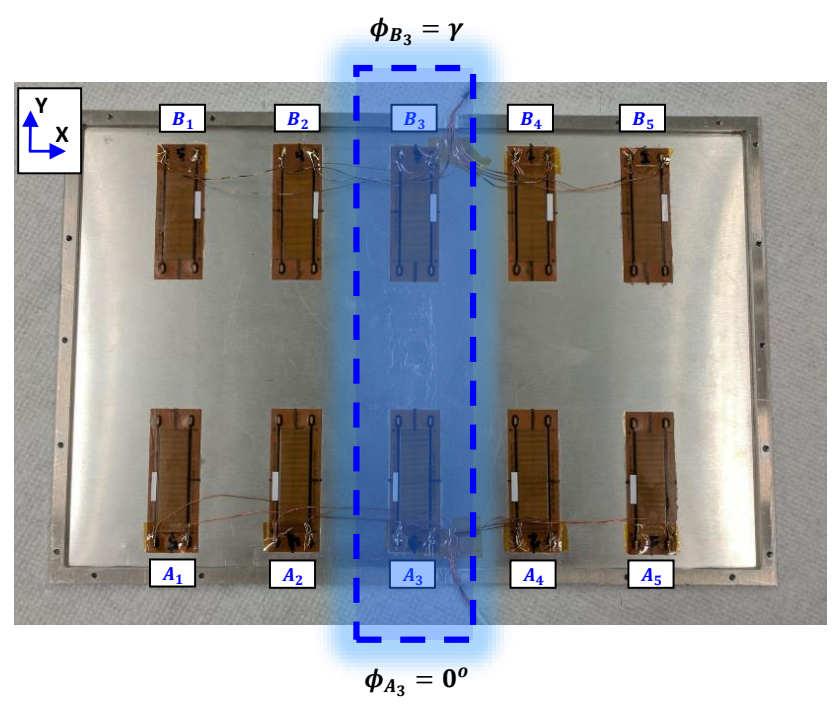

Figure 4.3: Actuators excited (actuation configuration) for validating traveling wave generation of finite element model. The highlighted actuators $\left(A_{3}-B_{3}\right)$ are excited at $310 \mathrm{~Hz}$ and with a phase difference, $\gamma$, between them

$$
C_{E M}=\frac{\left|\left[\psi_{E}\right]^{T}\left[\psi_{M}^{*}\right]\right|^{2}}{\left[\psi_{E}\right]^{T}\left[\psi_{E}^{*}\right]\left[\psi_{M}\right]^{T}\left[\psi_{M}^{*}\right]}
$$


The experimental traveling waves are measured using the experimental setup shown in Figure 4.1b. The two actuators (Figure 4.3) are excited at the same frequency $(\omega)$ and amplitude, but with a phase difference $(\gamma)$ between them. The velocity of the plate as a function of time is then measured using the scanning laser doppler vibrometer (SLDV). In order to compare the experiment and modeled traveling waves, the time data measured by the SLDV must be translated to complex amplitudes $\left(\left[\psi_{M}\right]\right)$. At each measurement location, the SLDV returns a time vector of velocity values. This was converted to a complex amplitude by least squares fitting a sinusoid to the time data. The frequency of the sinusoid is the excitation frequency, $\omega$, so the least squares fit calculates the amplitude and phase (complex amplitude). The result of calculating this for every measurement location is a vector of complex amplitudes defining the traveling wave. Figure 4.4 shows a representative set of experimental velocities measured at a single location on the plate. The experimental data is a least squared fit, resulting in $R^{2}>0.99$. Thus, the experimental velocity at this location can be described by the amplitude and phase (complex amplitude) given in the plot.

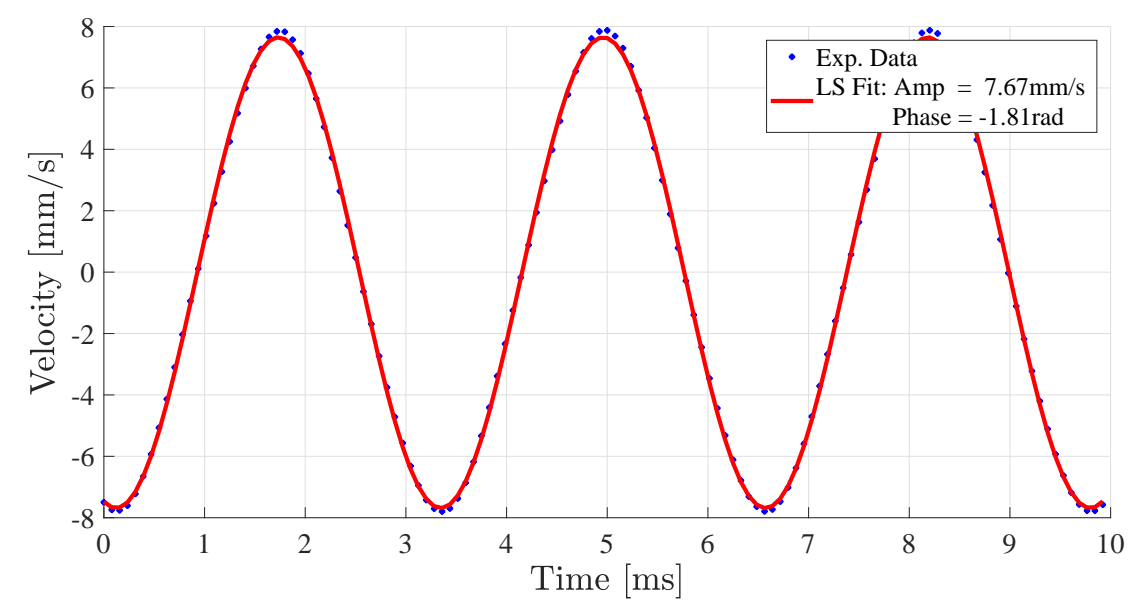

Figure 4.4: Least squares fit of experimental velocities measured at a single location on the plate for an excitation frequency of $\omega=310 \mathrm{~Hz}$. The amplitude and phase (complex amplitude) calculated by the least squares fit are shown

The traveling waves generated here were excited at $310 \mathrm{~Hz}$, which is between the $7^{\text {th }}$ and $8^{\text {th }}$ mode shapes of the clamped plate. These experimental mode shapes are shown in Figures 4.5a and $4.5 \mathrm{~b}$, where the natural frequencies are $282.1 \mathrm{~Hz}$ and $353.5 \mathrm{~Hz}$, respectively. The $7^{\text {th }}$ mode is the $(3,2)$ mode shape, where there are 3 anti-nodes in the $x$ direction and 2 in the $y$ direction. The $8^{\text {th }}$ mode is the $(1,3)$ mode. These modes were chosen since neither mode shape has a vertical node line in the middle of the plate that would overlap with the excitation actuators $\left(A_{3}-B_{3}\right)$.

The excitation frequency was chosen roughly halfway between the two natural frequencies, since it yields the highest quality traveling waves. Traveling waves could be generated at any 


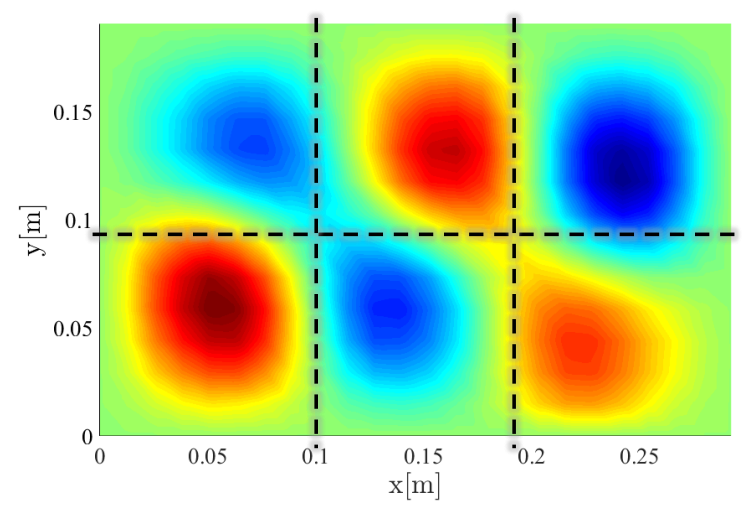

(a)

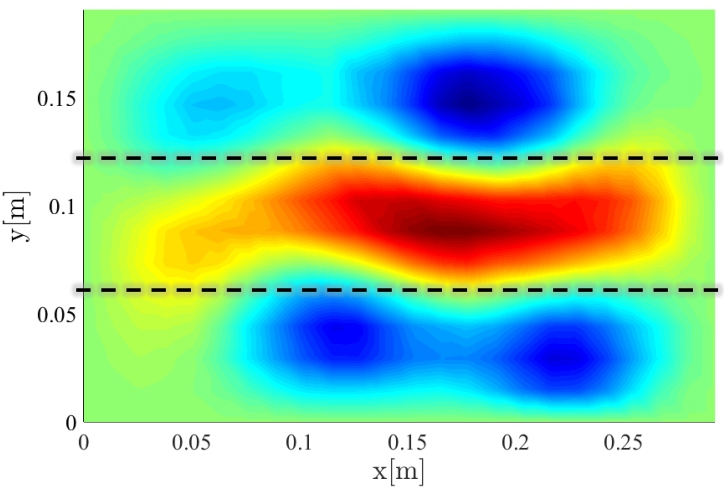

(b)

Figure 4.5: (a) the $7^{\text {th }},(3,2)$, experimental mode shape at $282.1 \mathrm{~Hz}$, and (b) the $8^{\text {th }},(1,3)$, experimental mode shape at $353.5 \mathrm{~Hz}$

frequency between these two modes [2], but the quality of the wave decreases as the excitation frequency approaches the natural frequencies. The quality of the traveling wave is defined by the amount of standing wave component in the traveling wave [2,66]. Ideal steady-state traveling waves have no standing wave component, so higher quality waves have a lower ratio of standing waves.

The experimentally generated and modeled traveling waves are shown in Figure 4.6. Figures 4.6a and 4.6b show the instantaneous velocities of the experimental and model traveling waves, respectively, at different time steps in the period, $T$. There is a traveling wave front in the middle of the plate that propagates in the negative $y$ direction. This can be seen by following a single anti-node through successive time steps in either $4.6 \mathrm{a}$ or $4.6 \mathrm{~b}$. Figures $4.6 \mathrm{c}$ and $4.6 \mathrm{~d}$ show the root-mean-square (RMS) velocities of the experimental and model traveling waves, respectively. The RMS velocity in essence describes the maximum velocity that each location on the plate experiences. The propagation path of the wave in the $-y$ direction has been pointed out in each plot. The path of these traveling waves is visually confirmed by observing that there are no horizontal node lines corresponding to either of the standing waves in Figure 4.5.

The experimental phase difference which generates the best traveling waves in the experiment was determined to be $\gamma_{\exp }=100^{\circ}$. This phase difference was found by comparing the experimental waves measured with different $\gamma_{\text {exp }}$ values. The optimal phase difference produces the highest quality traveling waves, where the highest quality are those that minimize the standing wave component (described above). High quality waves can be visually determined by looking at the consistency in the amplitude and wave speed of the traveling wave along the propagation path. At non-optimal phase differences, the nodal lines of the nearby standing waves (Figure 4.5) become visible in the traveling wave pattern. 

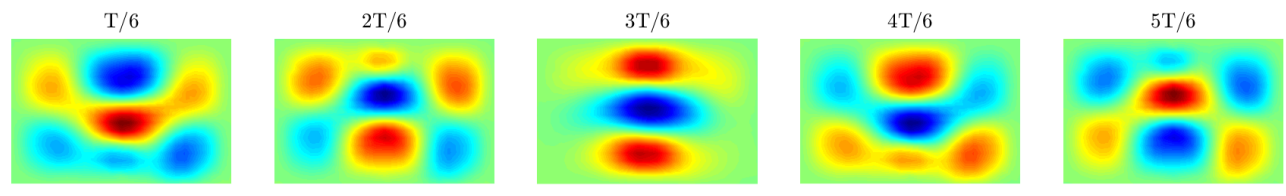

(a)
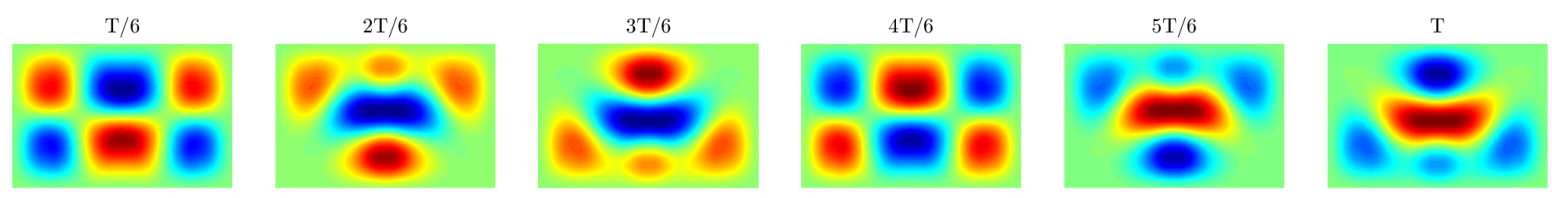

(b)

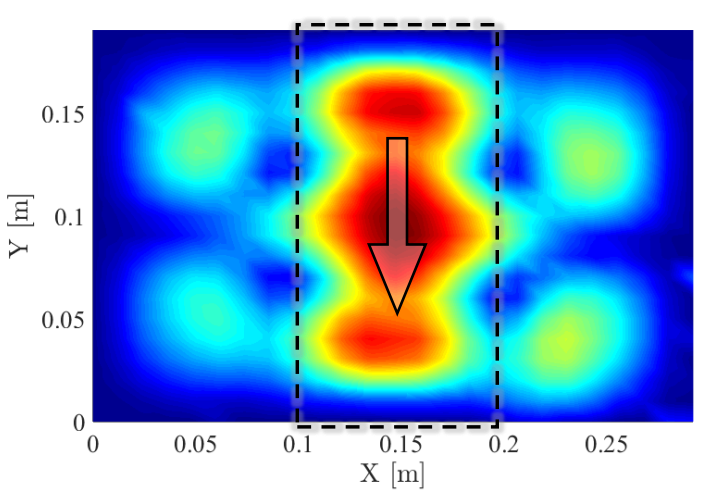

(c)

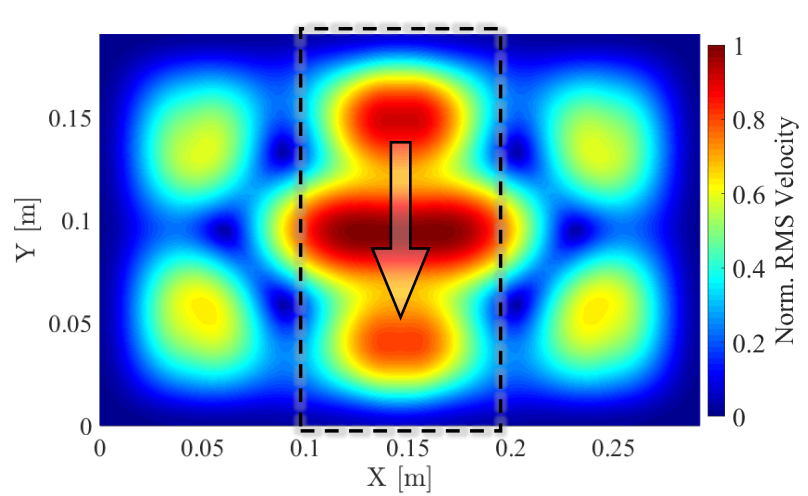

(d)

Figure 4.6: Comparison between the experimental and model traveling waves generated at $310 \mathrm{~Hz}$ using the actuation configuration in Figure 4.3 and phase differences $\gamma_{\text {exp }}=100^{\circ}$ and $\gamma_{\text {model }}=65^{\circ}$. The instantaneous velocities of experimental (a) and model (b) traveling waves at different time steps in the period, $T$, are shown. The normalized root mean square (RMS) velocities over an entire period are shown for the experiment (c) and model (d)

Comparing the model and experimental traveling waves for a phase difference of $\gamma_{\text {exp }}=\gamma_{\text {model }}=$ $100^{\circ}$, the correlation is $C_{E M}=0.760$. However, the correlation can be made even stronger if the phase difference in the model is shifted to $\gamma_{\text {model }}=65^{\circ}$, this results in $C_{E M}=0.846$. The optimal phase difference in the experiment and model are shifted due to the errors between the model and experimental natural frequencies and MAC values (Figures 4.2). However, as evidenced above, there is still a strong correlation between modeled and experimental traveling waves when both have the same phase difference. The experimental traveling waves are shown in Figures 4.6a and $4.6 \mathrm{c}$ and the modeled in Figures 4.6b and 4.6d. Traveling waves propagate in the $-y$ direction only in the middle of the plate, while the left and right sides contain only standing waves. As evidenced by the visual comparison and the strong correlation coefficient $\left(C_{E M}=0.846\right)$, the model is able to accurately represent the experimental traveling waves. 


\subsubsection{Traveling Wave Pattern Manipulation}

This section investigates how the excited traveling wave pattern depends on the actuation configuration (location and number of actuators). Experimentally generated traveling waves are compared for different actuation patterns and then correlated with those simulated using the finite element model.

In Section 4.3.1, a single pair of actuators in the middle of the plate produced a traveling wave front propagating in the $-y$ direction (shown again in Figure 4.7a). The wave front was contained between the two vertical node lines of the $(3,2)$ mode shape. Elsewhere on the plate, a standing wave pattern was observed. When comparing the traveling waves to the standing wave patterns in Figure 4.5 , it is clear that the traveling waves strongly resemble the $(3,2)$ mode shape. Thus, the traveling wave patterns are controlled by the patterns of the nearby mode shapes.

Another actuation configuration is then tested using the same excitation frequency $(310 \mathrm{~Hz})$. This configuration is shown in Figure 4.7b, where a single pair of actuators is used, but only the right side of the plate is excited. The experimental optimal phase difference is $\gamma_{\text {exp }}=100^{\circ}$, while the model optimal was $\gamma_{\text {model }}=125^{\circ}$. Time steps of the experimental traveling waves are shown in Figure 4.7b and the experimental and model RMS plots are shown in Figure 4.8b. The resultant correlation between the model and experiment is $C_{E M}=0.681$.

The result of exciting only the right actuators is a traveling wave front only on the right side of the plate: directly over the excited actuators and confined by the $(3,2)$ mode vertical nodal lines. This can be compared to excitation using only the middle actuators in Figures 4.7a and 4.8a. For both the middle and right actuation patterns (Figures 4.7a and 4.7b), traveling waves only exist directly on top of the actuators. Specifically, the traveling wave region is contained by the vertical node lines of the $(3,2)$ mode shape. Elsewhere on the plate, there are standing waves following the $(3,2)$ mode shape. Thus, the traveling wave pattern depends not only on the standing wave mode shapes, but also on the location of the actuators.

For both of the actuation configurations discussed so far (Figures $4.7 \mathrm{a}$ and $4.7 \mathrm{~b}$ ), the resultant traveling wave patterns resemble the $(3,2)$ mode shape more strongly than the $(1,3)$ mode. The excitation frequency of $310 \mathrm{~Hz}$ is roughly halfway between the $(1,3)$ and $(3,2)$ natural frequencies. So for the $(3,2)$ mode to be preferentially excited, its participation factor must be higher for those excitation locations (Figures $4.7 \mathrm{a}$ and $4.7 \mathrm{~b}$ ). To produce a traveling wave pattern resembling the $(1,3)$ mode shape, the actuation configuration is shifted such that the $(1,3)$ mode is more favorably excited. This is done by simultaneously exciting the plate with multiple actuator pairs.

The $(1,3)$ mode has a single anti-node extending along the $x$ direction (Figure 4.5b). To excite this single anti-node and produce traveling waves in the $y$ direction, all five sets of MFCs mounted on the plate are excited simultaneously. This actuator configuration is shown in Figure 4.7c. A global phase difference $(\gamma)$ is used, such that within each actuator pair, the phase difference is the 

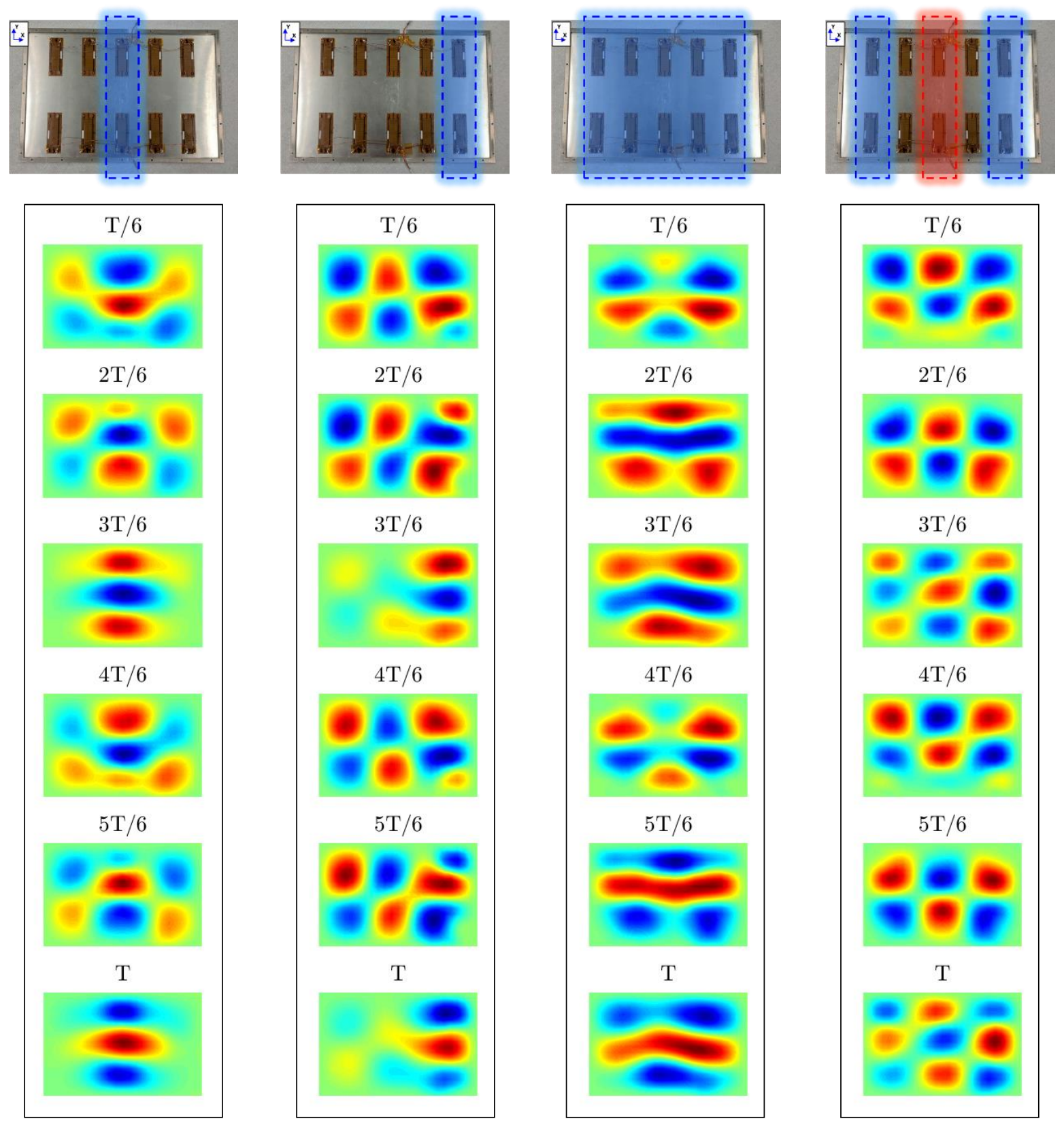

(a)

(b)

(c)

(d)

Figure 4.7: Time step plots of experimental traveling wave patterns for different actuation configurations excited at $310 \mathrm{~Hz}$. Each traveling wave pattern (a-d) is defined by the actuation configuration at the top of each pattern. Actuators highlighted in blue are excited, where there is a phase difference, $\gamma$ between the top and bottom actuators. Actuators highlighted in red are $180^{\circ}$ out-of-phase with those in blue

same $(\gamma)$. The result is that all the $A$ actuators (bottom) in Figure 4.1a are in phase, and all the $B$ actuators (top) are in phase, while there is a phase difference, $\gamma$, between $A$ and $B$. 


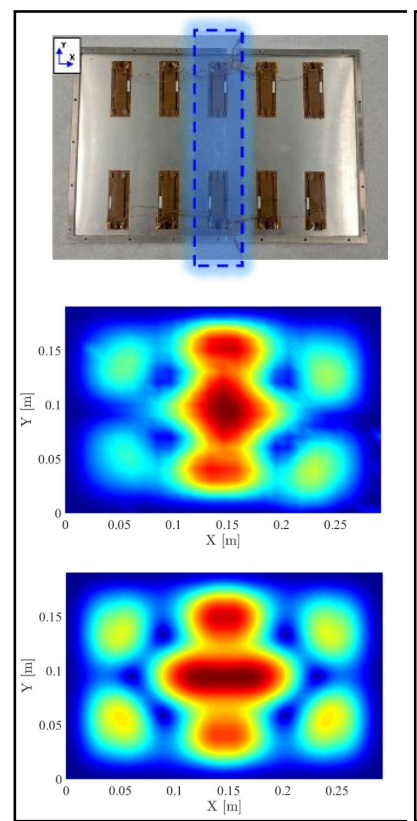

(a)

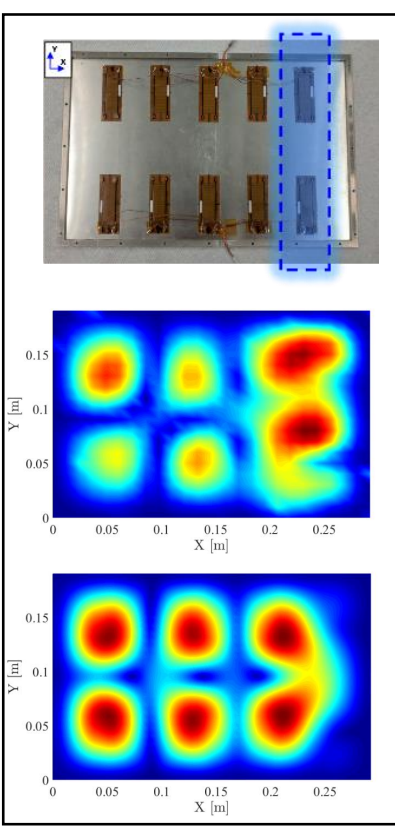

(b)

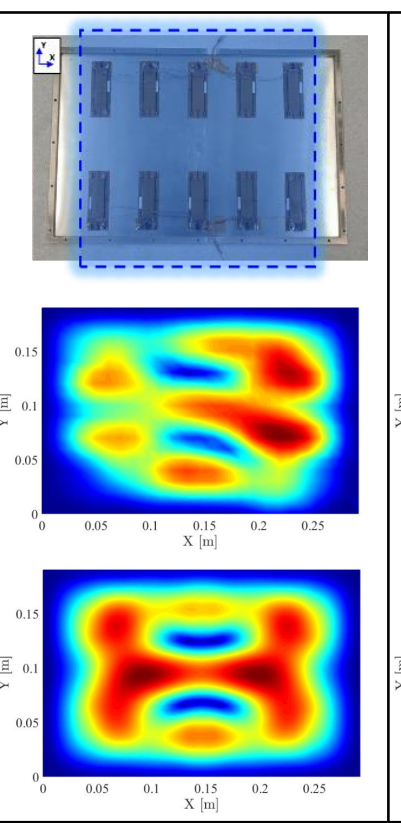

(c)

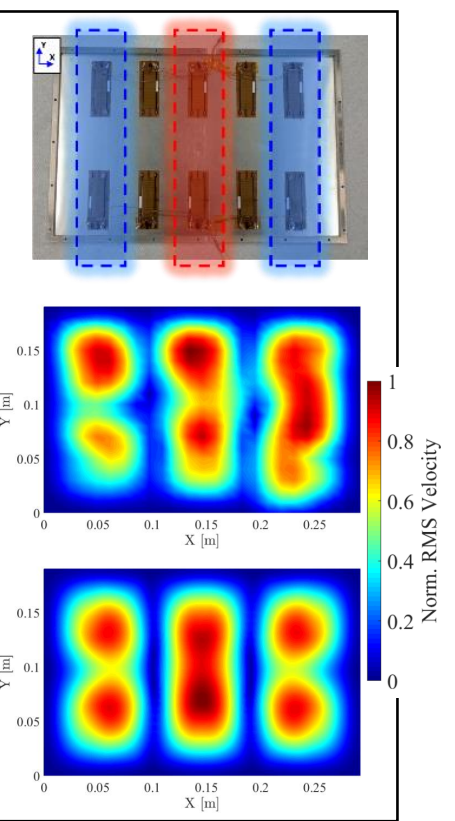

(d)

Figure 4.8: Normalized RMS velocity plots of traveling wave patterns for different actuation configurations (a-d) when excited at $310 \mathrm{~Hz}$. The top row is the actuation configuration, the second row is the experimental RMS plot, and the third row is the model RMS plot of the traveling wave pattern. Actuators highlighted in blue are excited, where there is a phase difference, $\gamma$ between the top and bottom actuators. Actuators highlighted in red are $180^{\circ}$ out-of-phase with those in blue

Using the same excitation frequency $(310 \mathrm{~Hz})$, the time step and RMS plots of the experimental traveling wave patterns are shown in Figures $4.7 \mathrm{c}$ and $4.8 \mathrm{c}$, respectively. The experimental optimal phase difference is $\gamma_{\text {exp }}=140^{\circ}$ and the model optimum is $\gamma_{\text {model }}=100$. The resultant correlation coefficient between the model and experiment is $C_{E M}=0.852$, indicating a strong correlation. Analyzing the time step and RMS plots, the traveling wave pattern has two separate propagation fronts: one on the left and one on the right. There are no traveling waves in the middle of the plate, rather a standing wave with the same nodal lines as the $(1,3)$ mode.

By changing the actuation pattern to include multiple MFC pairs, traveling waves fronts were generated on multiple parts of the plate instead of just in a specific region (as with Figures 4.8a and $4.8 \mathrm{~b}$ ). In addition, the traveling wave pattern resembled the $(1,3)$ mode shape (Figure $4.5 \mathrm{~b}$ ); there are two horizontal nodal lines in the middle of the plate (Figure 4.8c). However, no traveling waves were generated in the middle of the plate, despite exciting actuators there. Looking once again to the mode shapes (Figure 4.5), the middle anti-node of the $(3,2)$ mode shape is $180^{\circ}$ out-of-phase with the left and right anti-nodes. The two-mode excitation method works because the two nearby mode shapes combine to produce a traveling wave. Although the actuation pattern includes five actuator pairs (Figure 4.8c) and the traveling wave pattern is primarily defined by the $(1,3)$ mode, 
the $(3,2)$ mode still contributes. The $180^{\circ}$ out-of-phase anti-node prevents traveling waves from forming in the middle of the plate.

Instead of fighting the $180^{\circ}$ out-of-phase anti-node, it is taken advantage of. The actuation pattern shown in Figure 4.7d is used. Three pairs of MFCs are excited where there is once again a global phase difference within all actuator pairs $(\gamma)$. However, the middle set of actuators (red) are excited $180^{\circ}$ out-of-phase with the left and right pairs (blue). These phase differences are defined in Equation 4.13, where $\phi$ is the global phase of each actuator.

$$
\phi_{A_{1}}=\phi_{A_{5}}=0^{\circ} \quad, \quad \phi_{A_{3}}=180^{\circ} \quad ; \quad \phi_{B_{1}}=\phi_{B_{5}}=\gamma \quad, \quad \phi_{B_{3}}=180^{\circ}+\gamma
$$

The time step and RMS plots of the experimental traveling wave patterns are shown in Figures $4.7 \mathrm{~d}$ and $4.8 \mathrm{~d}$, respectively. The experimental optimal phase difference is $\gamma_{\text {exp }}=60^{\circ}$ and the model optimum is $\gamma_{\text {model }}=55^{\circ}$, where the model and experimental correlation is $C_{E M}=0.732$. Exciting the plate at $310 \mathrm{~Hz}$ and using the actuation pattern described in Equation 4.13 results in three separate traveling wave fronts. The form is clearly dominated by the $(3,2)$ mode shape, given that the three wave fronts are separated by that mode's vertical nodal lines. Additionally, by looking at the time step of the traveling wave (Figure $4.7 \mathrm{~d}$ ) the middle traveling wave front is $180^{\circ}$ outof-phase with the two side wave fronts. This makes sense, given that these middle actuators were excited $180^{\circ}$ out-of-phase.

Overall, four different actuation patterns were tested at the exact same frequency, and four different traveling wave patterns were generated. It is clear that the resultant traveling wave pattern is dependent on the nearby mode shapes, the placement of the actuators, and the number of actuators excited. This results from the fundamental operating principle of the two-mode excitation method: two mode shapes combining to yield traveling waves. As a result, by changing the placement and number of actuators, the participation of different modes in the final traveling wave form is itself changed. Within this, the global phase of specific actuator pairs can be shifted by $180^{\circ}$ to promote a traveling wave pattern resembling a specific mode shape.

\subsection{Discussion}

In order to investigate the spanwise traveling waves as a drag reduction method, previous studies relied on bulky actuation setups. Large arrays of discrete actuators were employed to physically displace the surface into a traveling wave pattern. These methods successfully reduced the skin friction, but they are cost, space, and bandwidth prohibitive. A more practical traveling wave generation method is two-mode excitation. Steady-state travelings can be produced in a solid-state fashion without the need for restrictive actuator arrays. Flush-mounted piezoelectric actuators 
can be used to generate traveling waves at almost any frequency. To be applied for reducing skin friction drag, the method is further developed to excite spanwise traveling waves on a twodimensional surface. Specifically, this study has investigated how the location and number of excited actuators (actuation configuration) can affect the traveling wave patterns generated using two-mode excitation. These results lay the foundation for understanding this complex relationship and leveraging it to tailor the resultant traveling wave pattern.

An in-house finite element model was developed for a thin, clamped plate with ten piezoelectric actuators mounted to the surface. In particular, the model accounted for the composite effects of the mounted piezoelectrics, pre-stresses in the plate, and non-ideal clamped boundary conditions. The parameters of this finite element model were then manually updated to match experimental modal data. The model matched the experimental natural frequencies to within $\pm 2 \%$ in the range of interest. The ability of the model to simulate traveling waves was then experimentally validated.

The effects of different actuation configurations (location and number of actuators) were then experimentally and computationally investigated. It was found that the location of the excited actuators directly controls how traveling waves were generated on the plate. By changing the actuation location but holding the frequency constant, traveling waves could be generated in different, isolated portions of the plate. Traveling waves could then be generated on the entire plate by increasing the number of actuators used. Thus, the traveling wave pattern depends on both the location and the number of actuators. The two-mode excitation method is fundamentally coupled with the plate's structural properties (mode shapes). So, by changing the actuation configuration, the modal participation is effected and traveling wave pattern shifts. This was demonstrated in this chapter by generating four different traveling patterns at the exact same frequency, simply by changing the actuation configuration.

This investigation has extended the understanding of traveling wave generation in two-dimensional systems. The results show that there is a complex relationship between the actuation configuration, the mode participation, and the resultant traveling wave (propagation) pattern. While complex, this relationship allows for significant freedom in variations to the traveling wave pattern, all while holding the frequency constant. Thus, by understanding and manipulating this relationship, traveling wave patterns can be tailored for various applications. This is leveraged in the following chapters to tailor spanwise traveling waves for turbulent boundary layer manipulation with the goal of skin friction drag reduction. 


\section{Chapter 5}

\section{Wind Tunnel and Boundary Layer Measurement}

The main goal of this research is the development of a novel method for generating two-dimensional traveling surface waves and then implementing these waves to modify the turbulent boundary layer to potentially reduce the skin friction drag. The development of these traveling waves was discussed in Chapter 4. The focus now shifts towards implementing these traveling waves to interact with the turbulent boundary layer. As was discussed in Chapter 2, the approach taken in this research involves experimentally measuring the turbulent boundary layer. Specifically, this is done in a low-speed open return wind tunnel. This chapter focuses on the development of the experimental setup and the data analysis necessary to investigate the effect of the traveling waves on the turbulent boundary layer.

The chapter begins by discussing the experimental setup and the measurement of the turbulent boundary layer in the wind tunnel. This begins with a general overview of the wind tunnel and the equipment used in the setup. Then, the development of a zero pressure gradient, turbulent boundary layer over a flat plate is discussed. Next, the flow quality in the wind tunnel is assessed. Finally, the experimental setup and considerations necessary to actually measure the boundary layer are outlined.

With the experimental setup explained, the next two sections focus on analyzing and validating the measured boundary layers. The analysis portion begins with a discussion of statistical independence and then progresses to estimating the skin friction coefficient and normalization to wall units. Then, correction factors are applied and the relevant quantities calculated from the boundary layer. The data analysis concludes with presentation of measured turbulent phenomena. Using this analyzed data, the validity of the measured boundary layers and experimental setup are then investigated. This includes comparing against accepted data from literature and looking at the streamwise boundary layer growth. 


\subsection{Experimental Setup}

This research investigates the effect of spanwise traveling waves on the turbulent boundary layer and for potential drag reduction. The experimental setup for testing this is an open-return, lowspeed, wind tunnel capable of developing a turbulent boundary layer over a flat plate with zero pressure gradient. At the onset of the project, the experimental setup consisted of a disassembled low-speed wind tunnel that had been out of service for several years. As a result, a significant amount of time and effort went into repairing and heavily modifying the tunnel for use with this research. The theory and development of the experimental setup for growing and then measuring a turbulent boundary layer is discussed here.

This section begins with a general overview of the experimental setup. Then the focus turns towards proper development of a turbulent boundary layer. This includes some basic theory of boundary layer growth followed by the design of the wind tunnel test-section to ensure this growth. This is then followed by the discussion of and results from testing the flow quality in the testsection.

Finally, the actual measurement of the boundary layer is discussed in-depth. This begins with an explanation of the three-dimensional traversing system used to translate the velocity probes through the wind tunnel. Then, the merits and implementation of two different velocities probes (pitot tube and hot-wire probe) are discussed. Next, the proper placement of the probe in the air flow is outlined. This subsection then concludes with the actual procedure for measuring the boundary layer profile.

\subsubsection{General Setup}

The wind tunnel used in this research is a $15^{\prime}$ low-speed open-return wind tunnel originally designed and constructed by Bilgen [93]. The tunnel has two 1/3 hp fans connected in series to pull the air through the tunnel and can achieve a roughly $20 \mathrm{~m} / \mathrm{s}$ velocity in the test section with turbulence intensity of $T u \approx 0.8 \%$ (Section 5.2.5). The test section is 14 " wide by 5.35" tall by $36 "$ long and is preceded by a nozzle with a 6 to 1 contraction ratio. The nozzle contains an aluminum honeycomb flow straightener and two fiberglass screens for large eddy breakup. The original design of the tunnel was not capable of measuring the boundary layer; thus, it was modified for this research. The modifications were conducted specifically for measuring the boundary layer over a traveling wave actuated surface. The completed wind tunnel is shown in Figure 5.1.

The air flow in the wind tunnel is generated by the two fans that pull air through the wind tunnel (Figure 5.1). The fans are connected to a relay, allowing for remote start and stop. To adjust the speed of the airflow, the power sent to the fans is controlled. The primary fan is connected to a variable resistance potentiometer (variac) that allows the power pulled by the fan to be adjusted on 


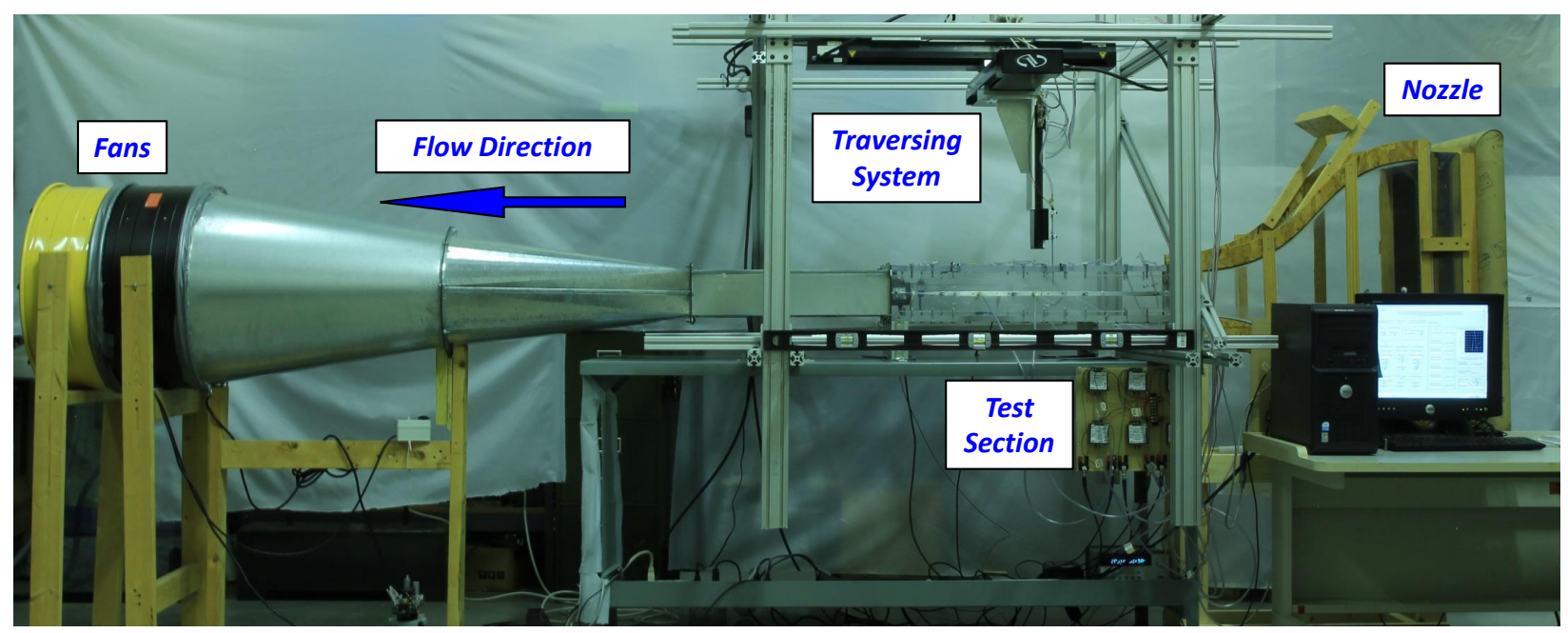

Figure 5.1: Low speed, open return wind tunnel used in the current research with fans, intake nozzle, test section, and traversing section shown

a continuous scale. A stepper motor is embedded in the top of the variac for automated adjustment along this continuous scale. The secondary fan is connected to a switch for manual adjustment of the fan to one of three levels: off, low, and high. Using these two fans, the wind tunnel is operated in two flow ranges. These ranges are outlined in Table 5.1. Rough estimates of the flow speeds capable are shown in the Table, along with the states of the two fans for each range. The full operation range is from $2.5-19.5 \mathrm{~m} / 5 \mathrm{~s}$ with some overlap between the lower and upper ranges.

Table 5.1: The two ranges of the air flow speed in the wind tunnel test-section and the states of the two fans (Primary and Secondary)

\begin{tabular}{cccc}
\hline Speed Range & Primary Fan & Secondary Fan & Flow Speed \\
\hline Lower & Continuous & Off & $2.5-14 \mathrm{~m} / \mathrm{s}$ \\
Upper & Continuous & High & $13.5-19.5 \mathrm{~m} / \mathrm{s}$ \\
\hline
\end{tabular}

During experimental measurements of the boundary layer, the wind tunnel is operated in the upper flow range with a constant speed of $\approx 18.5 \mathrm{~m} / \mathrm{s}$. The full operating range of flow speeds is used for two separate calibration tests. The first is for calibration of the reference velocity (Section 5.1.3.4), which allows the free-stream velocity to be continuously monitored without a dedicated velocity probe in the test-section. The second is for calibration of the hot-wire probe (Section 5.1.4.3), which requires a mapping between the output voltage of the probe and the actual velocity.

The wind tunnel is controlled via a graphic user interface (GUI) written in Labview. This GUI controls every aspect of the tunnel such as the flow speed, movement of the 3D traversing system, data input and output, and automated testing procedures. All of the equipment that is 
connected to the wind tunnel is listed in Table 5.2. The various sensor data is collected using the NI data acquisition unit. These sensors vary based on the test but can include the differential pressure transducers, thermocouple, a flow velocity sensor (pitot tube or hot-wire probe), and a laser vibrometer.

When measuring the boundary layer, the local velocity is collected at various the wall-normal distances $(y)$. The temperature is also collected using the thermocouple and is used in combination with atmospheric pressure to determine the air density (Section 5.2.4). In the current setup, there is no dedicated atmospheric pressure sensor, so the elevation corrected pressure is periodically collected from a local weather station. Finally, the static pressure from a single pressure transducer is used to monitor the reference free-stream velocity (Section 5.1.3.4).

The other equipment listed in Table 5.2 is described in the following sections. This excludes the bottom few rows of the table which are used for traveling wave generation and testing. These items are are discussed further in Chapter 6. 
Table 5.2: List of all the equipment used in the wind tunnel

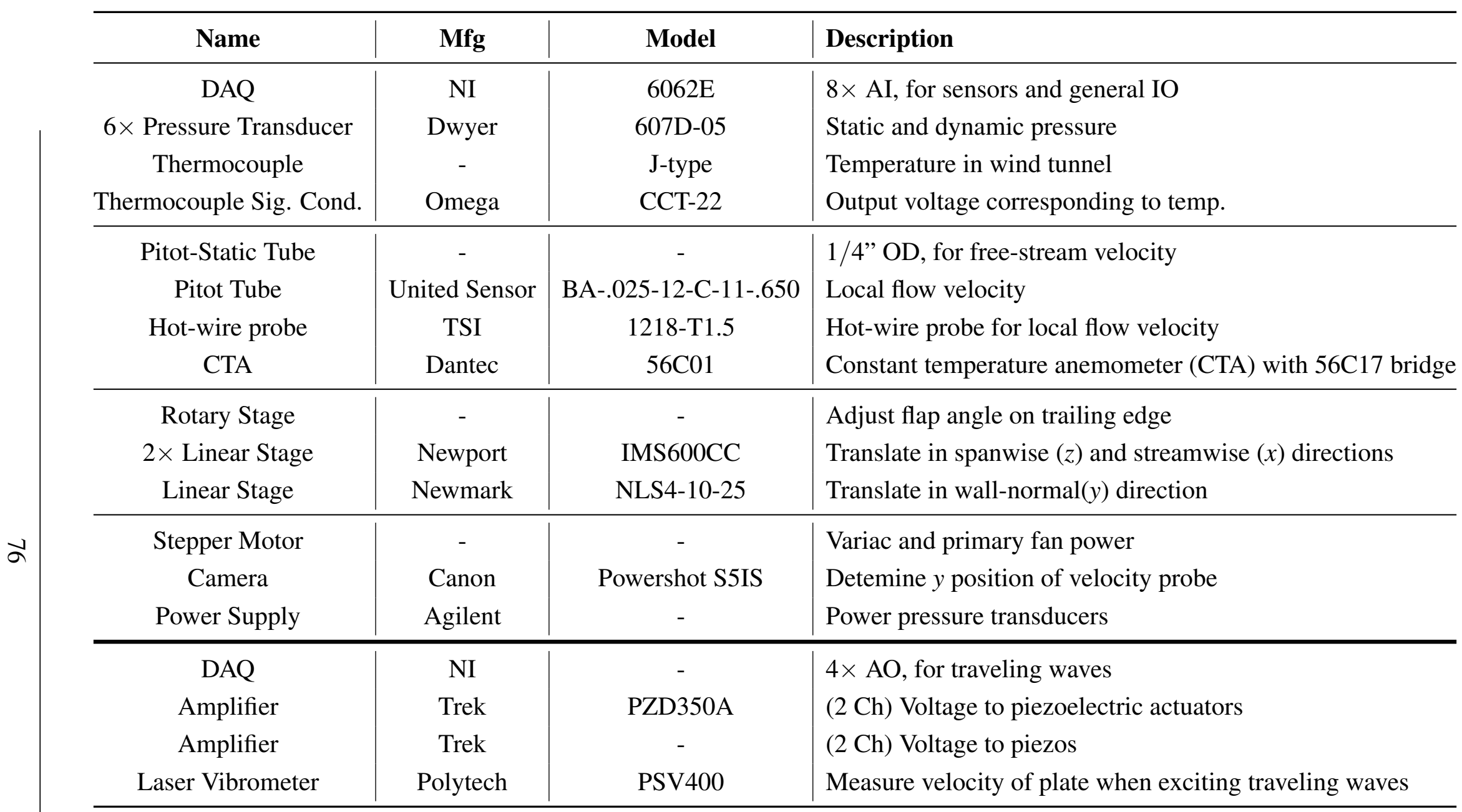




\subsubsection{Boundary Layer Development}

In order to properly study the effects of the traveling waves on a zero pressure gradient turbulent boundary layer, the boundary layer itself must be well-characterized. This requires that the turbulent boundary layer is fully-developed, two-dimensional, and has known properties. In particular, the boundary layer should have a known Reynolds number or thickness directly upstream of the traveling waves region. As a result, the upstream development of the boundary layer is particularly important.

At the leading edge of the surface, the boundary layer has zero thickness and is laminar. In zeropressure gradient flow over a flat plate, the development of a laminar boundary layer follows well the Blasius solution, which is an analytical solution derived from the Navier-Stokes equation [46]. See Section 5.3.1 for further explanation and experimental comparison with the Blasius solution. Using the Blasius solution, the momentum thickness, $\theta$, is defined using Equation 5.1 as a function of the distance downstream from the leading edge, $x . R e_{x}=\frac{x U_{e}}{v}$ is the Reynolds number as a function of $x$, where $U_{e}$ is the free-stream velocity, and $v$ is the kinematic viscosity. The momentum thickness can then be non-dimensionalized and represented as the momentum thickness-based Reynolds number, which is defined in Equation 5.2.

$$
\begin{aligned}
& \theta=\frac{0.664 x}{\sqrt{\operatorname{Re}_{x}}} \\
& \operatorname{Re}_{\theta}=\frac{\theta U_{e}}{v}
\end{aligned}
$$

As the laminar boundary layer develops over the plate, it will eventually transition to turbulence. This transition results from instabilities in the flow and is exacerbated by surface roughness and the turbulence level of the free-stream. Unlike for laminar flow, in turbulence there are no analytical solutions for the boundary layer growth. As a result, empirical solutions must be relied upon. One such empirical estimate is shown in Equation 5.3, where $\delta$ is the turbulent boundary layer thickness, and $x$ and $R e_{x}$ are the same as before. An estimate of the momentum thickness can then be calculated using the relation $\frac{\theta}{\delta}=\frac{7}{72}$ [46]. However, this procedure can only be used to provide an estimate of the momentum thickness. Equation 5.3 assumes that there is no transition from laminar to turbulence and that the boundary layer has been turbulent since the leading edge.

$$
\delta(x)=\frac{0.375 x}{\operatorname{Re}_{x} \frac{1}{5}}
$$

Using these estimates for the boundary layer development, the experimental setup was designed for a certain turbulent boundary layer range. Figure 5.2 shows a top view of the experimental setup in the test section of the wind tunnel. The setup consists of an aluminum wall that is analogous 
to a flat plate in zero pressure gradient flow. The wall is mounted vertically in the center of the tunnel so as to allow roughly equal flow over the top and bottom. Moving forward, it is referred to as a floating wall. A cavity is machined into the wall and the traveling wave plate from Chapter 4 installed. The transition between the plate and the floating wall is then smoothed using wax. The streamwise coordinates, $x_{p}$, are referenced with respect to the upstream edge of the TW plate. This is shown in Figure 5.3 where $x_{p}=0$ at the upstream edge of the TW plate and $x_{p}=305 \mathrm{~mm}(12 \mathrm{in})$ at the downstream edge. The leading edge of the wall is $x_{p}=-375 \mathrm{~mm}$ upstream of the TW plate. Using this distance in Equation 5.3 and assuming a free-stream velocity of $U_{e} \approx 20 \mathrm{~m} / \mathrm{s}$, the turbulent boundary layer directly at the upstream edge of the traveling wave plate is $R e_{\theta} \sim 1000$.

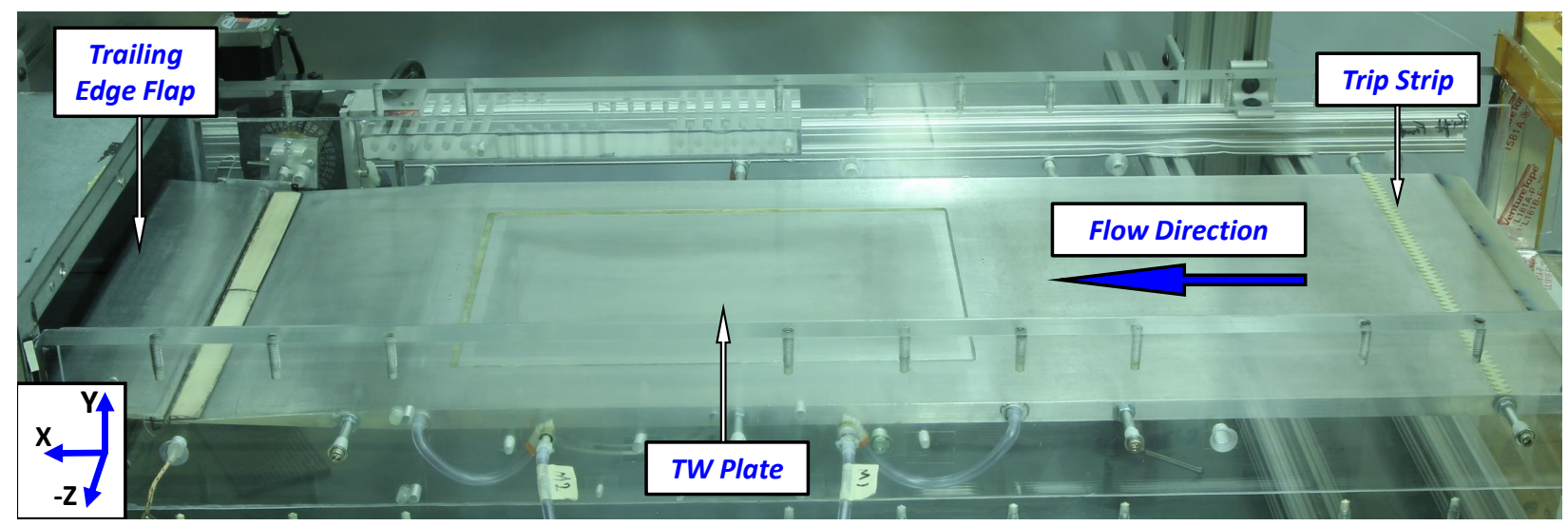

Figure 5.2: Experimental setup showing the traveling wave (TW) plate mounted in the floating wall, which is installed in the wind tunnel test section. The top of the test section has been removed. $\mathrm{X}=$ Streamwise, $\mathrm{Y}=$ Wall-normal, $\mathrm{Z}=$ Spanwise direction

Since the turbulent transition location is difficult to predict, experiments involving turbulent flow intentionally trip the boundary layer so as to generate a known and repeatable transition location. This experiment is no exception. Figure 5.2 shows the white trip strip on the right side of the plate just downstream of the leading edge and Figure 5.4 shows the strip in greater detail. The strip is a $0.85 \mathrm{~mm}$ thick adhesive backed plastic with both edges serrated. The trip strip was placed roughly 2.75 " downstream of the leading edge on the top and bottom of the plate to replicate the same flow conditions on both sides. The height and placement of the trip strip was determined by examining Dryden's plots which analyze the transition of the boundary layer due to single roughness elements [51]. While Dryden's analysis is slightly different than that in this experiment, it does provide an estimate. By using the Blasius solution to estimate the momentum thickness at various streamwise locations, the location of transition to turbulence was estimated for various trip strip heights and placement locations. The height and streamwise location were then chosen so as to initiate the transition as far upstream as possible while preventing flow separation over the trip strip. 


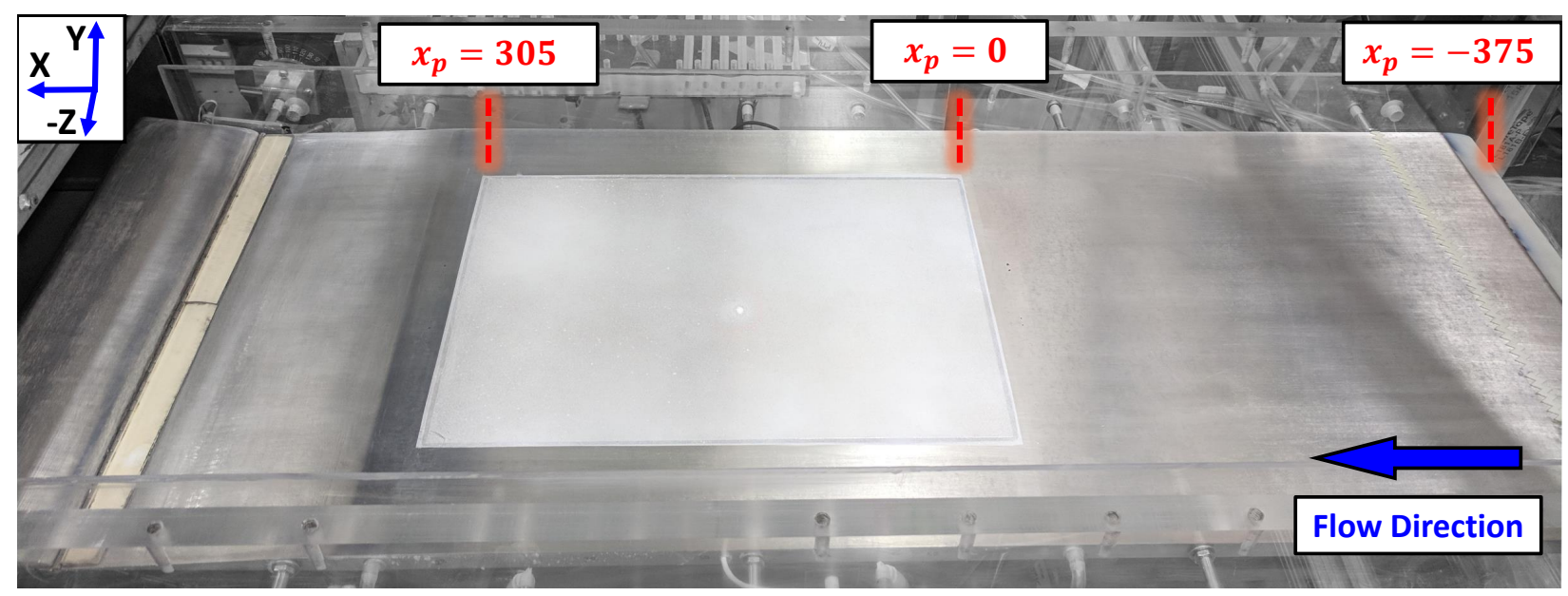

Figure 5.3: Definition of the $x_{p}$ streamwise coordinates, where $p$ refers to the traveling wave plate. $x_{p}=0$ at the upstream edge of the plate. Also shown are the streamwise positions (in $\mathrm{mm}$ ) of the leading edge and the downstream edge of the traveling wave plate.

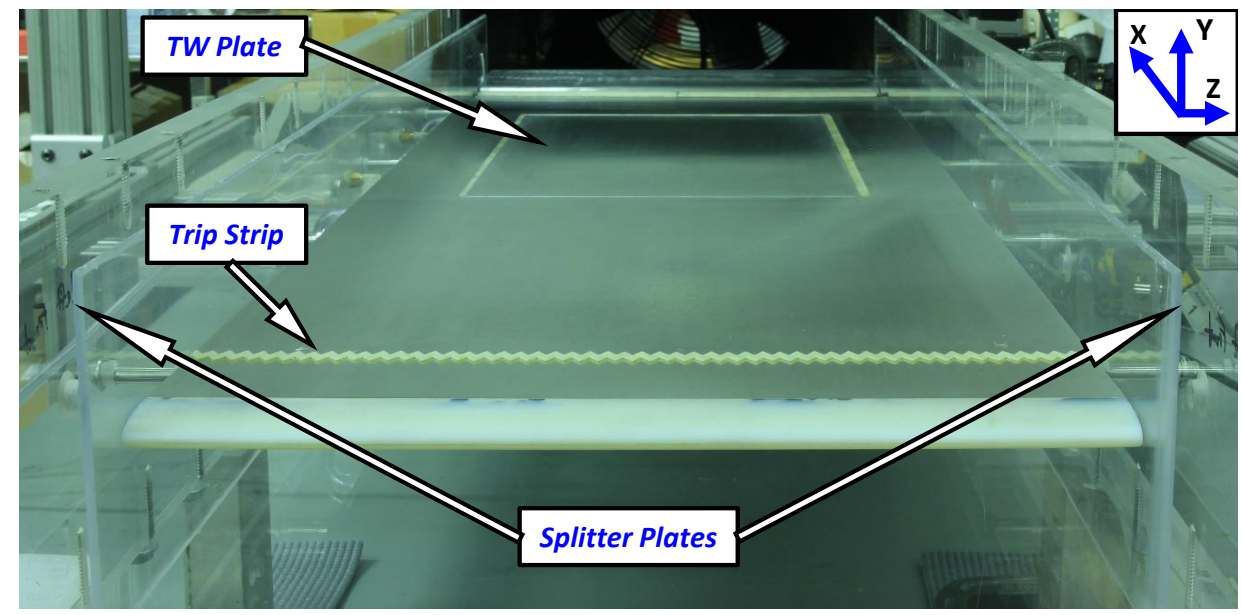

Figure 5.4: Downstream view of floating wall with mounted traveling wave plate. Trip strip is visible in foreground with splitter plates running parallel to test section walls.

Also seen in Figure 5.4 is the leading edge. The floating wall was machined from a single piece of aluminum and has a semicircular leading edge. To avoid flow separtion, a more streamlined leading edge was then 3D printed and adhered over the semicircular edge. The printed edge is an ellipse with a major to minor axis ratio of 4 to 1 . The interface between the printed edge and the aluminum was then smoothed using ascending grits of sandpaper. This process was continued over the entire surface of the floating wall. This was done to ensure that the surface is hydraulically smooth, indicating the surface roughness is well within the viscous sublayer, and that the boundary layer grows regularly. 
One of the other major considerations when developing a boundary layer, is that it is twodimensional. If the boundary layer is two-dimensional, then it does not vary with spanwise position ( $\mathrm{z}$ axis). Looking at Figure 5.4, the floating wall is mounted in the center of the test section with roughly 1 in of clearance between the left and right side walls. This was done so as to prevent the boundary layers on the side walls of the test section from contaminating the flow over the flat plate. However, the hardware mounting the floating wall generates disturbances in the flow that would interact with the central flow and ruin the two-dimensionality. As a result, vertical splitter plates constructed from clear acrylic (shown in Figure 5.4) are installed along the edges of the floating wall that isolate the central flow from that over the mounting hardware. The edges of these splitter plates are smoothed to prevent flow separation, and while a boundary will grow on these plates, the thickness is much smaller than that existing on the test section side walls.

An underlying assumption of the experimental setup is that there is zero streamwise pressure gradient. Unless accounted for, the natural state of flow in a wind tunnel is to have a pressure gradient. This is due to the fact that as flow moves along the streamwise (x) direction, the boundary layer grows and penetrates further into the free-stream. This yields a reduction in the cross section in which there is free-stream velocity: in effect a virtual convergence of the test section walls. As a result, the free-stream velocity increases and there is a favorable (negative) pressure gradient in the streamwise direction. In order to counteract this pressure gradient, a flap is installed at the rear of the test section (Figure 5.2) to provide flow blockage. Figure 5.5 shows a zoomed in view of the flap installed on the rear of the floating wall. The position of the flap is controlled by a servo motor mounted outside of the test section, seen in the background of Figure 5.5. While the flap could be manually maneuvered, the flow is quite sensitive to the flap angle, requiring an automated method.

In the wind tunnel there are seven static pressure ports in the ceiling of the test section. By monitoring these pressure from these ports and adjusting the flap through different angles, the streamwise pressure gradient can be found. While the streamwise pressure gradient cannot be completely eliminated by adjusting the flap angle, the pressure gradient can be minimized enough such that it can be ignored. This is shown in Section 5.1.3.3.

The static pressure ports used in this tunnel are shown in Figure 5.6. The pressure is measured at seven streamwise $(x)$ locations. Each position has two ports manifolded together such that the average pressure is measured and variations local to a single port are minimized. In addition, the dimension of the port is particularly important. If the port is too large the streamlines deflect into the hole. If the hole is too deep, a secondary vortex structure develops in the hole, while if it is too shallow the flow deflects into the cavity behind the hole. In extreme cases, correction factors must be applied to the measured pressures to account for these effects. Chue [42] and Bailey [94] provide a thorough discussion of the effect of hole dimension on the measured pressure. The static ports here have a diameter of $0.05 \mathrm{in}$ and a depth of $0.07 \mathrm{in}$ and the port correction factors assumed 


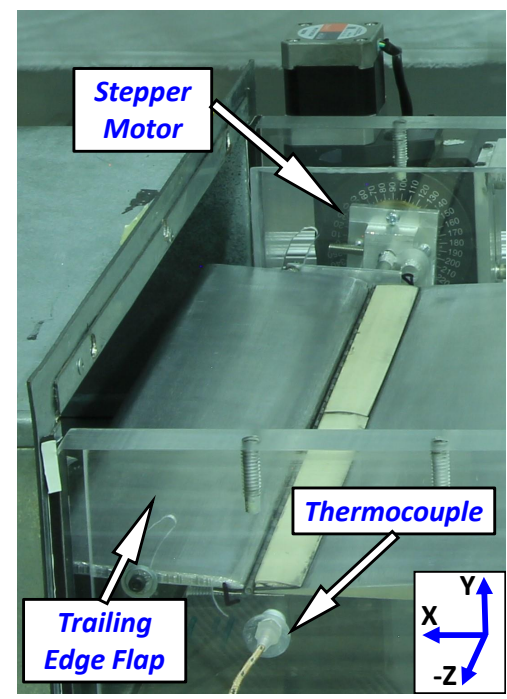

Figure 5.5: Flap at trailing edge of floating wall used to adjust the streamwise static pressure gradient

to be negligible.

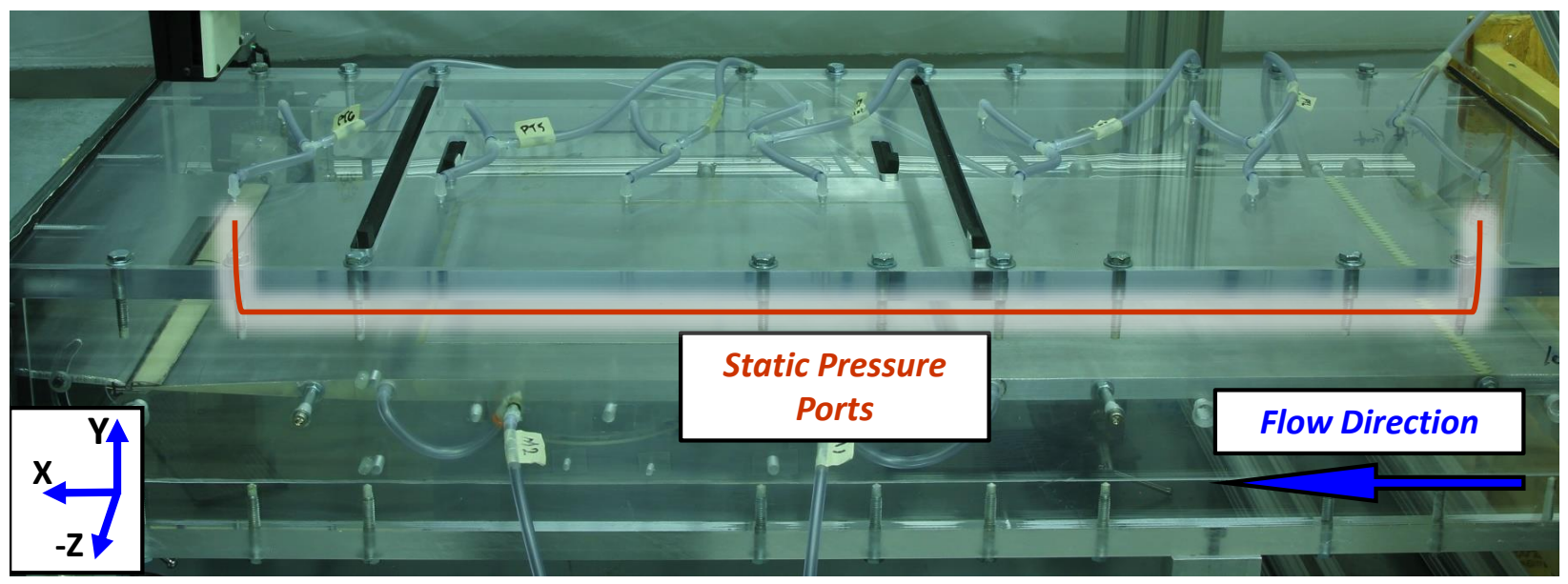

Figure 5.6: Top of test section showing seven pairs of static pressure ports and also the velocity probe slots

\subsubsection{Flow Quality Testing}

As was outlined in Section 5.1.2, numerous design considerations were implemented to ensure a properly developed boundary layer. This section discusses and presents the results of various tests to quantify flow quality in the test section. First, flow visualization was conducted to visualize whether flow separation occurs on the flat plate. Then, the dynamic pressure was mapped over 
a cross-section of the test-section to determine two-dimensionality. Next, the trailing edge of the plate was adjusted to minimize the streamwise pressure gradient. Finally, the reference velocity calibration was performed so that the free-stream velocity can be monitored using only a static pressure port.

\subsubsection{Flow Visualization}

Flow visualization techniques are a qualitative way of testing the flow conditions in a wind tunnel. In particular, they are used to observe the flow direction and where/if separation and transition to turbulence occur. There are a number of different techniques that one can use such as smoke, oil films, tufts, and china clay with Barlow [38] providing a good discussion of all of these methods. In this study, both tufts and the china clay were utilized. The tufts provided a straight forward and easy method of observing the flow, while the china clay yielded a more in depth picture without the need for the specific equipment of smoke or the mess of oil flow visualization.

The tufts used in this study were created using a tuft board [38] with a thin, black sewing thread and scotch tape. The tufts were applied to the floating wall, splitter plates, and test-section walls. This was done as a first observation of the flow conditions to ensure that there were no major problems such as flow separation or highly three dimensional flow. The tufts indicated that for large angles of the trailing edge flap, the flow no longer flowed evenly along the boundary layer and even spilled over the tops of the splitter plates (Figure 5.4).

Since the trailing edge flap would only ever be positioned at moderate to low angles, these effects were considered negligible. The next flow visualization technique employed was the china clay method. This involves the use of a powder suspended in a mixture of kerosene. In addition, small amounts of oleic acid were added to the mixture to act as an anti-clumping agent. The proportions used here by weight were roughly 15 parts kerosene, to 5 parts powder, to 1 part oleic acid. A fine black powder was used to promote the greatest visibility against the aluminum surface. The mixture was then uniformly painted onto the top surface of the floating wall using a foam brush before immediately starting the wind tunnel. As the mixture flowed along the surface and the kerosene evaporated, the black powder formed patterns on the wall which correlate to the local flow conditions.

Figure 5.7 shows the resultant powder/china clay pattern on the plate for two different experimental conditions: an untripped (5.7a) and a tripped (5.7b) boundary layer. The state of the boundary layer can be inferred from these patterns, but they cannot be used to conclusively state what is happening. The untripped boundary layer did not have the trip strip shown in Figure 5.4 installed. On the untripped boundary layer (Figure 5.7a) downstream of the traveling wave plate, the flow separated in the middle of the floating wall where it begins tapering in thickness towards to trailing edge flap. The edges near the splitter plates do not separate. This feature is connected 
to the angular pattern seen over top of the traveling wave plate. This occurred because the edges of the wall near the splitter plates transitioned to fully turbulent flow while the middle had not completely transitioned. This is confirmed since the powder is thinner on the edges due to the higher shear stress in full turbulence. As the flow progressed downstream along the wall, the outer turbulence interacted with the inner flow causing this region to also transition to turbulence. As a result, the width (spanwise) of the inner laminar region decreases as the air flows downstream. Looking at the separation point in the flow, the width of the separation bubble is the same as that of the laminar region just upstream. Laminar flow separates more easily than turbulence; thus, as the flow passes over the taper, the turbulent edges don't separate while he laminar inner region does.

In contrast, Figure $5.7 \mathrm{~b}$ shows the china clay pattern when the flow has been tripped upstream. The flow is turbulent across the entire width of the floating wall and moves uniformly in the streamwise direction. As a result, it is clear that when the flow is tripped, the boundary layer can be considered two dimensional and that no flow separation occurs.

\subsubsection{Dynamic Pressure Mapping}

The flow quality in the wind tunnel should be two-dimensional such that there should be no variation in the boundary layer in the spanwise direction. As such, the free-stream velocity should depend only upon the streamwise direction and be independent of the wall-normal and spanwise positions. This is tested by mapping the dynamic pressure along a cross-section using a pitot-static tube. Barlow provides an indepth discussion of this test [38]. In particular, he recommends that the mean variation in the dynamic pressure should ideally be no larger than $0.50 \%$.

Figure 5.8 shows the variation in the dynamic pressure at $x_{p}=-19 \mathrm{~mm}$ (Figure 5.3). The figure shows the variation from the mean dynamic pressure along a single cross-section, where the air flows into the page. The $\mathrm{x}$ axis is the width of the boundary layer wall (spanwise) with the splitter plates at $z= \pm 152.4 \mathrm{~mm}(6 \mathrm{in})$. The y-axis is the wall-normal direction with the flat plate at $y=0 \mathrm{~mm}$ and the test-section ceiling at $y=62 \mathrm{~mm}(2.43 \mathrm{in})$. The figure shows that in the center of the test-section, the dynamic pressure varies by less than $0.5 \%$. Near the flat plate and the testsection ceiling, the dynamic pressure varies substantially, but this is a result of the boundary layer. The boundary layer is seen to be thicker on the ceiling. This occurs because that layer has been growing since the wind tunnel inlet whereas the wall layer only began at its leading edge. There is also some variation in the dynamic pressure in the upper right corner. This results most likely from the splitter plate. Since the traveling wave only plate extends between $z= \pm 101.6 \mathrm{~mm}(4 \mathrm{in})$, the variation in the upper right corner is ignored. 


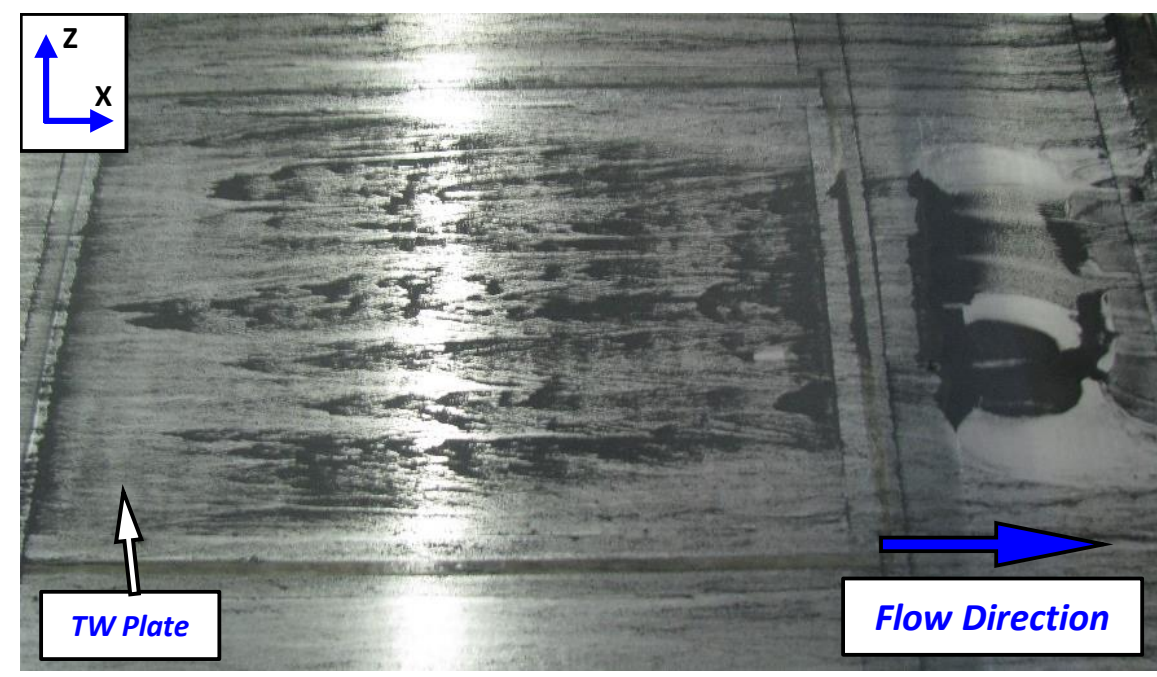

(a) Untripped BL

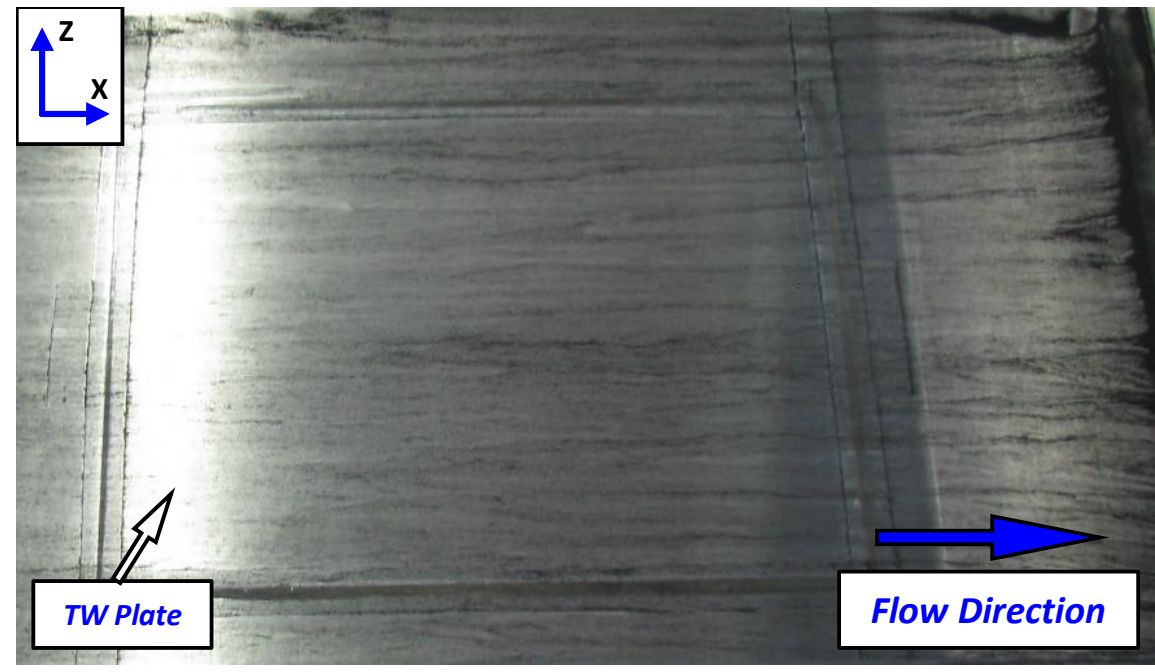

(b) Tripped BL

Figure 5.7: China clay flow visualization applied over the traveling wave plate for left to right moving flow with (a) an untripped and (b) a tripped boundary layer

\subsubsection{Static Pressure gradient mapping}

In order to minimize the streamwise pressure gradient along the test section, there is a flap on the trailing edge of the floating wall to adjust the flow blockage. By monitoring the static pressure ports along the test section (Figure 5.6) and adjusting the angle of the flap, the minimum pressure gradient can be found. Figure 5.9 shows the streamwise pressure gradient along the wall for six different flap angles. The traveling wave plate extends from $x_{p}=0 \mathrm{~mm}$ to $x_{p}=305 \mathrm{~mm}$ (Figure 5.3). The figure shows the static pressure coefficient $C_{p}$ which is defined in Equation 5.4. Here the static pressure difference from atmospheric $\left(P-P_{a t m}\right)$ is normalized by the dynamic pressure 


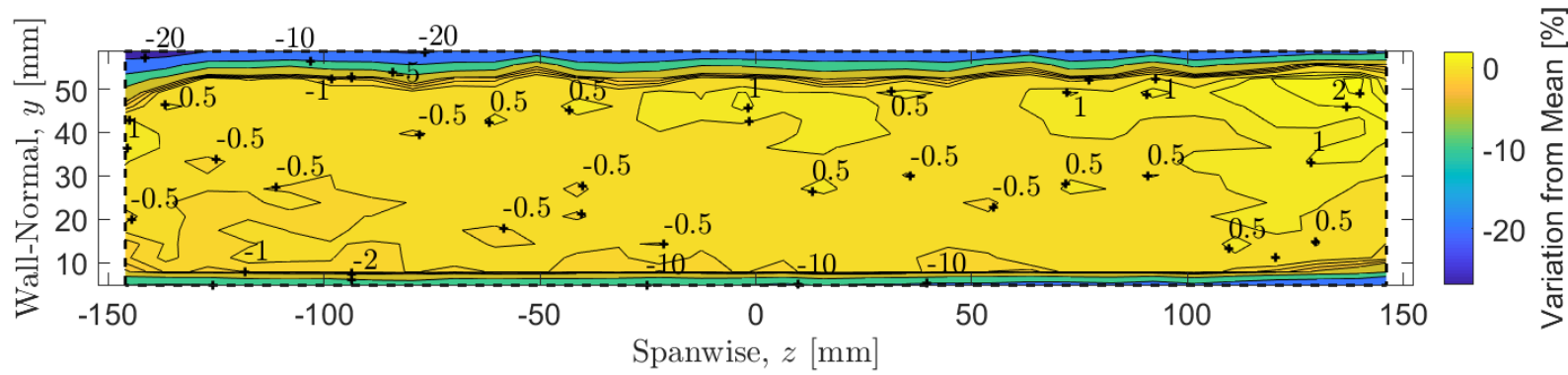

Figure 5.8: Percent variation from the mean dynamic pressure just upstream of the traveling wave plate at $x_{p}=-19 \mathrm{~mm}$. The air flows into the page

$\left(q=\frac{1}{2} \rho u^{2}\right)$ upstream of the traveling wave plate $\left(x_{p}=-19 m m\right)$.

$$
C_{p}=\frac{P-P_{a t m}}{\frac{1}{2} \rho u^{2}}
$$

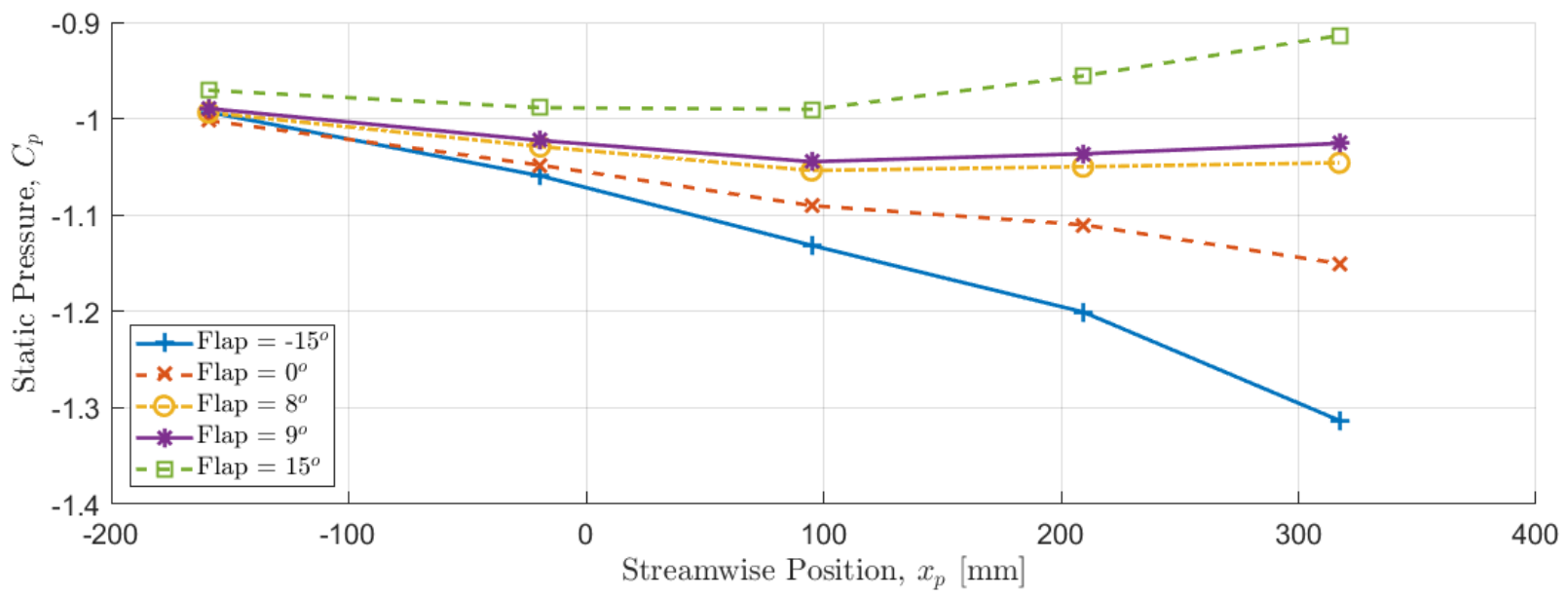

Figure 5.9: Static pressure gradient in streamwise direction as the trailing edge flap is adjusted.

For a large negative angle of $-15^{\circ}$, there is a strong favorable (negative) pressure gradient; whereas for a large positive angle of $15^{\circ}$ there is a small favorable gradient at the front of the plate that transitions to an adverse (positive) gradient downstream. A flap angle of $9^{\circ}$ minimizes the pressure gradient with a variation of $\approx 2 \%$ in the streamwise direction. For this angle there is still a slight favorable pressure gradient upstream, but the pressure is approximately constant directly over the traveling wave region. The flap angle of $0^{\circ}$ shows the case as if there was no flap. There is a strong favorable pressure gradient with a change of $\approx 10 \%$ in the streamwise direction. As a result, the flap is able to reduce the gradient from $10 \%$ to $2 \%$. 


\subsubsection{Reference Velocity Calibration}

In a wind tunnel, the free-stream velocity, $U_{e}$, is on of the main parameters that defines the flow conditions. When running experiments, the free-stream velocity should be continually monitored to ensure that the flow conditions do not change during testing. A pitot-static tube could be used, but this would not be ideal. Placing the pitot-static tube in the wind tunnel during testing would alter the flow conditions during experimental testing. As a result, another method is needed to continuously monitor the free-stream velocity. This method is a calibration performed prior to experimental testing. The free-stream velocity is measured with a pitot-static tube and related to the static pressure at specific locations in the tunnel. The static pressure can then be measured during experimental testing and the calibration applied to determine the free-stream velocity. This is known as the reference velocity calibration. It is summarized here, but is described in depth by Barlow [38].

The theory behind the calibration relies upon the application of Bernoulli's equation. The total pressure at the inlet of the tunnel is related to the total pressure in the test section. This is shown in Equation 5.5 where $\mathrm{P}$ is the static pressure, $\mathrm{q}$ is the dynamic pressure, $K_{1}$ is a constant, and the subscripts $I$ and $T S$ represent the inlet and test section, respectively. The conservation of mass is then applied to express the inlet dynamic pressure as a function of that in the test section. This results in Equation 5.6, where $K_{2}$ is a function of the contraction ratio between the inlet and test section.

$$
\begin{gathered}
P_{I}+q_{I}=P_{T S}+q-K_{1} q_{T S} \\
P_{r e f}=P_{I}-P_{T S}=q\left(1-K_{1}-K_{2}\right)
\end{gathered}
$$

Equation 5.6 can then be used to determine the free-stream velocity at a single location in the test section. This calibration is performed by measuring $q$ with a pitot-static tube and $\left(P_{\text {ref }}=\right.$ $\left.P_{I}-P_{T S}\right)$ with static pressure ports at the inlet, $P_{I}$, and the test section, $P_{T S}$. The pitot-static tube is placed just upstream of the traveling wave plate at $x_{p}=-19 \mathrm{~mm}(-0.75 \mathrm{in})$ (Figure 5.3), where the tube is along the center-line of the test-section $(z=0 \mathrm{~mm})$ and is $y=25.4 \mathrm{~mm}$ off the surface. The wind tunnel is then run through various speeds, to gather the dynamic and static pressures at different flow conditions. The resultant data can be then be linearly fit and a value for $\left(1-K_{1}-K_{2}\right)$ determined. An example calibration is shown in Figure 5.10, where the calibration is split into an upper and lower range (Table 5.1). The $\mathrm{x}$-axis shows the static pressure, and the $\mathrm{y}$-axis the dynamic pressure. A linear trend is then fit to each range using a least squares fit. Although Equation 5.6 does not have a y-offset, one is implemented in the linear fit to account the non-zero offset in the pressure transducers. From Figure 5.10, it is clear that the linear trends are a good fit and that there 
is minimal discrepancy between the upper and lower range.

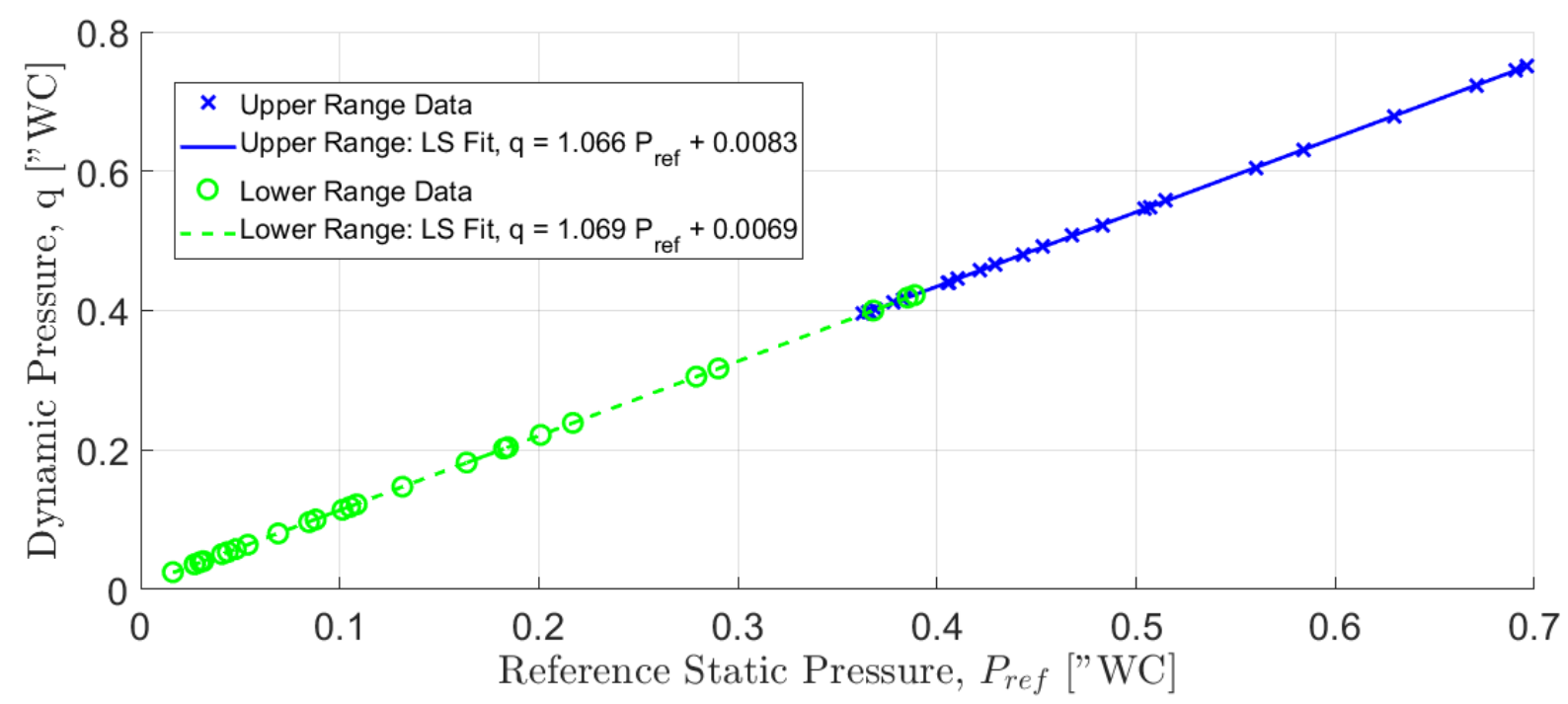

Figure 5.10: Reference velocity calibration between the dynamic pressure and static pressure with the linear least squares fit shown. The calibration is split into an upper and lower range due to limitations in how the wind tunnel fans are controlled Table 5.1.

The linear trend shown in Figure 5.10 can then be used to determine the free-stream velocity during experimental testing. During testing, the static pressure, $\left(P_{I}-P_{T S}\right)$, is continuously monitored. The linear fit from the reference velocity calibration is then used to determine the dynamic pressure $(q)$ in the wind tunnel. Finally the free-stream velocity is calculated by converting the dynamic pressure to velocity using Equation 5.7, where $U_{e}$ is the free-stream velocity and $\rho$ is the air density. The density is calculated in Section 5.2.4.

$$
q_{T S}=\frac{1}{2} \rho U_{e}^{2}
$$

The limitations of the reference velocity calibration should be known. The linear trend in Figure 5.10 can only be used to determine the velocity at the location of the pitot-static tube during the calibration $\left(x_{p}=-19 \mathrm{~mm}\right)$. To continuously monitor the free-stream velocity at multiple streamwise locations, a calibration must be performed for each location. In addition, the calibration is only valid for a single flow condition in the wind tunnel. Thus, if the trailing edge flap angle is changed or the model/plate is reinstalled, the calibration must be redone.

\subsubsection{Boundary Layer Measurement}

As was described in Section 2.1.3, the boundary layer is measured here using single point velocity probes: pitot tube and hot-wire probe. This section describes the experimental setup and compo- 
nents needed to measure the boundary layer with these probes. This begins with a discussion of the traversing system used to translate the probes through the boundary layer. Then, the specifics of the pitot tube and hot-wire probe are described in subsequent sections. Next, the importance of and procedure for accurately placement the probe in test-section is explained. Finally, general information for measuring the boundary layer is discussed.

\subsubsection{Traversing System}

Both the pitot tube and hot-wire probe utilized in this research are only capable of measuring the velocity at a single location in the flow at a time. As a result, a traversing system is necessary both to traverse through the boundary layer profile and to measure the boundary layer at different streamwise and spanwise locations. The traversing system installed on this wind tunnel (Figure 5.1)is composed of three linear drive screw stages, in total allowing movement in three dimensions. A close-up is shown in Figure 5.11 of the traversing system mounted above the wind tunnel test section. The $y$ stage positions the probe in the wall normal direction to measure the boundary layer profile. The $x$ and $z$ stages allow translation in the streamwise and spanwise directions, respectively. The stages are mounted to an 80/20 support structure that is fixed to the wind tunnel; this helps to prevent decoupled motion between the test section and the velocity probe (pitot or hot-wire).

The stages in the $x$ and $z$ direction are both Newport IMS600CC linear stages (Table 5.1). They have a full travel range of $600 \mathrm{~mm}$, an accuracy of $\pm 4 \mu \mathrm{m}$, and a resolution of $1.35 \mu \mathrm{m}$. The $y$ stage is a Newmark NLS4-10-25 linear stage with 10in $(254 \mathrm{~mm})$ of travel, $0.6 \mu \mathrm{m} / \mathrm{mm}$ accuracy, and $0.13 \mu \mathrm{m}$ resolution. The stages are connected to controllers and then coupled to Labview from which commands are issued. Although all three stages have high accuracy, the $y$ stage is the only one requiring this level of accuracy. The $y$ stage is used to translate the velocity probe through the boundary layer, with the distance between some points near the wall as low as $0.000,4 i n=10.2 \mu \mathrm{m}$.

The stages are mounted outside of the wind tunnel and the velocity probe passes through slots in the test-section ceiling. In order to seal the tunnel while still allowing probe movement, the slots are lined with foam. Figure 5.12 shows the hot-wire probe passing though a slot in the test-section ceiling. The foam strips are coated with adhesive on one side and with a low-friction plastic on the other. Once inserted in a slot, the traversing system can be used to translate the probe within the wind tunnel, without exposing the test-section to the ambient environment.

Two separate test-section ceilings were machined, each used for different purposes. The two different ceilings are shown in Figure 5.13. The first test section shown in Figure 5.13a has four independent slots along the streamwise direction and was originally designed for use with the pitot tube. The first and last slot in this ceiling are located just upstream and downstream of the traveling wave section. The streamwise position of these slots is $x_{p}=19 \mathrm{~mm}(-.75 \mathrm{in})$ and $x_{p}=$ $317.5 \mathrm{~mm}(12.5 \mathrm{in})$ for the upstream and downstream, respectively. They extend almost the entire 


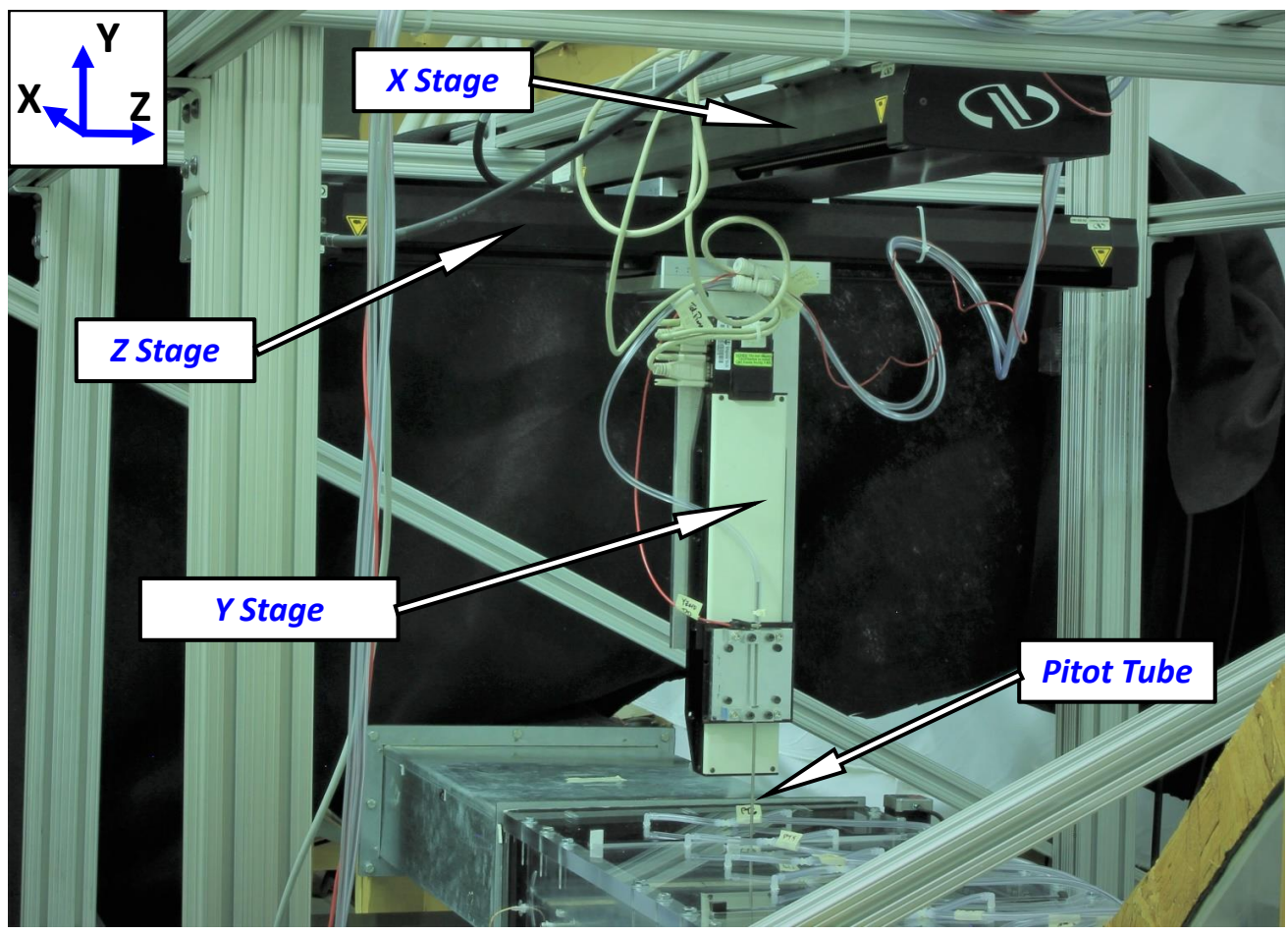

Figure 5.11: Three dimensional traversing system used in the current study. The pitot tube is seen attached to the bottom of the $y$ stage and passes into the wind tunnel test section. The hot-wire probe can be attached in a similar way.

width of the the test-section and allow investigation into the spanwise variation of the boundary layer. The two smaller slots in the ceiling are located over the traveling wave plate and were used for initial applications of the hot-wire probe. Between these smaller slots, there is a large area with no pass-throughs such that either velocity probe cannot reach this region. This is the area directly over the traveling wave plate (white surface in Figure 5.13). This was left open primarily for two reasons. Firstly, there are static pressure ports in the ceiling to allow measurement of the streamwise static pressure gradient (Figure 5.6). Second, and more importantly, this area was left open to have a clear visual of the traveling wave region. The static pressure ports have been removed in Figure 5.13a. When traveling waves are excited on the plate, a scanning laser doppler vibrometer (SLDV) is used to measure how the plate is vibrating at each point on its surface. In order to measure the plate velocity, the SLDV requires a clear line of sight through the test-section to the plate.

The second test-section ceiling is shown in Figure 5.13b. This ceiling has five slots extending in the spanwise direction, with one large slot extending along the streamwise direction to connect them all. The first and last slot in streamwise direction are in the same locations as the large slots in the first ceiling (Figure 5.13a). This ceiling was constructed entirely for the purpose of allowing 


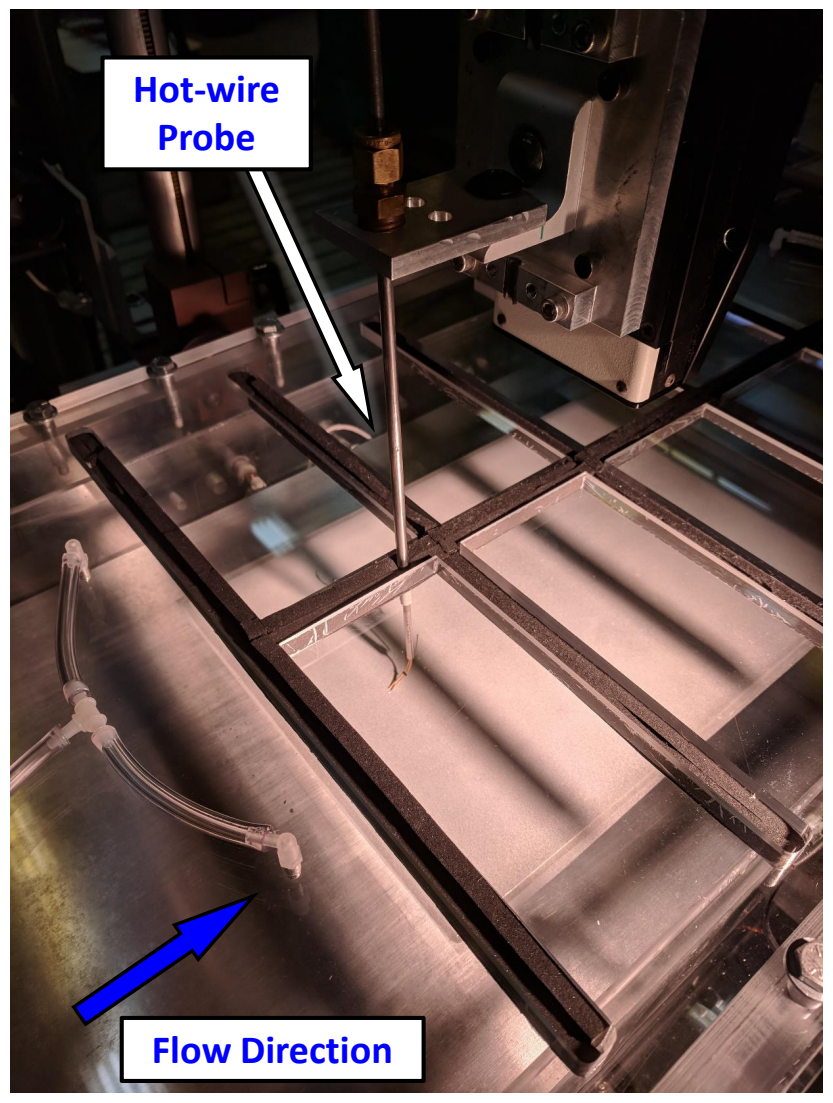

Figure 5.12: Hot-wire probe passing through a foam lined slot in the test-section ceiling

the velocity probe to reach as much of the traveling wave plate as possible. Using this ceiling, the streamwise growth of the boundary layer can be observed and the boundary layer can be measured at multiple spanwise locations directly over top of the traveling wave plate. This test-section ceiling is the one used during all experiments investigating the effects of traveling waves on the boundary layer.

\subsubsection{Pitot Tube}

A pitot tube was the initial velocity probe used in this research to measure the turbulent boundary layer. A description of its operation can be found in Section 2.1.3.2. The pitot tube was initially implemented due to its simplicity. It is quick and easy to setup, and can be physically handled without a major risk of breaking. However, what the pitot tube has in simplicity, it lacks in functionality. Its large size prevents it from measuring velocities very near the wall. In addition, the pitot tube requires external static pressure ports, limiting the measurement locations to only those where there are static ports on the walls. Finally, it has a low bandwidth and thus cannot be used to investigate turbulent phenomena. 


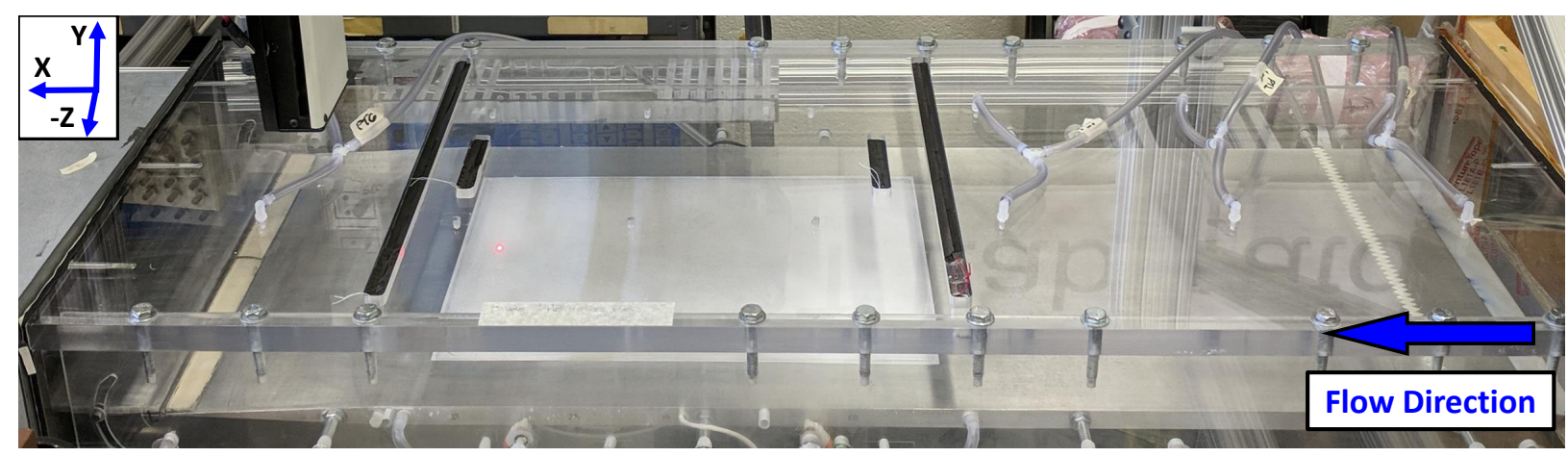

(a)

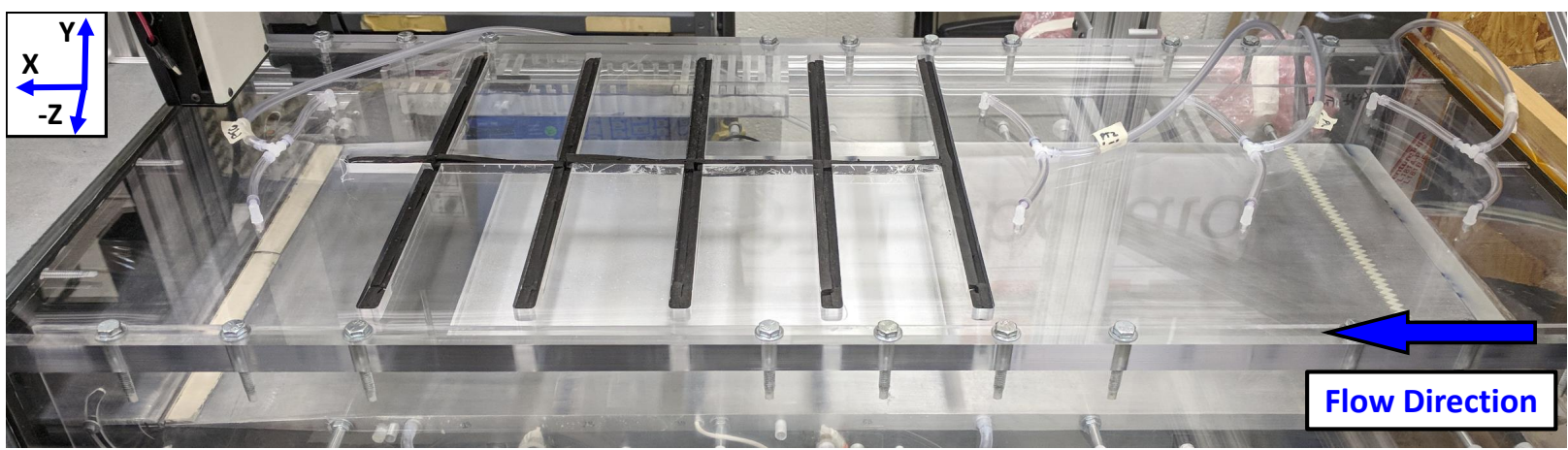

(b)

Figure 5.13: Two different test section ceilings. The black, foam-lined slots allow either velocity probe to traverse through the test section. (a) Used to characterize traveling waves on plate and collect streamwise pressure gradient. (b) Maximizes reachable locations for velocity probe; used during experimental testing

The main consideration for the pitot tube chosen here was its diameter. In general, the smaller the diameter of the tube the better. Since the boundary layer is measured with reference to the distance from the surface ( $y$ position), the smallest achievable y position is when the tube is touching the surface. This corresponds to a $y$ position of half the pitot tube diameter. A smaller diameter means velocity values at $y$ positions closer to the wall.

In addition, the larger the diameter of the pitot tube, the more it alters the local flow around it. Correction factors must be applied to account for viscous shearing, and near-wall effects. In each case, larger corrections are required for larger pitot tube diameters. Thus, a smaller diameter tube can be used to minimize the necessity for the correction factors. A discussion of pitot tube correction factors is found in Section B.1.

Although a smaller diameter tube certainly has an advantage, extra care must be taken to not bend it. Especially in this case since it is mounted to the traversing system outside of the tunnel and extends into the test-section. A small diamter pitot tube could have been constructed using a hyperdermic needle, but for simplicity one was purchased. The pitot tube used in this research was 
purchased from United Sensor Corp (Table 5.2) and can be seen in Figure 5.14. It has a diameter of $0.025 \mathrm{in}(0.635 \mathrm{~mm})$ which flattens to half that at the tip, yielding a rectangular end. The opening of the probe is $0.003 \mathrm{in}$ in height. Thus, the closest position to the wall at which the pitot tube can measure is $y=\frac{1}{2}(0.025 \mathrm{in})=0.0125 \mathrm{in}[317.5 \mu \mathrm{m}]$.

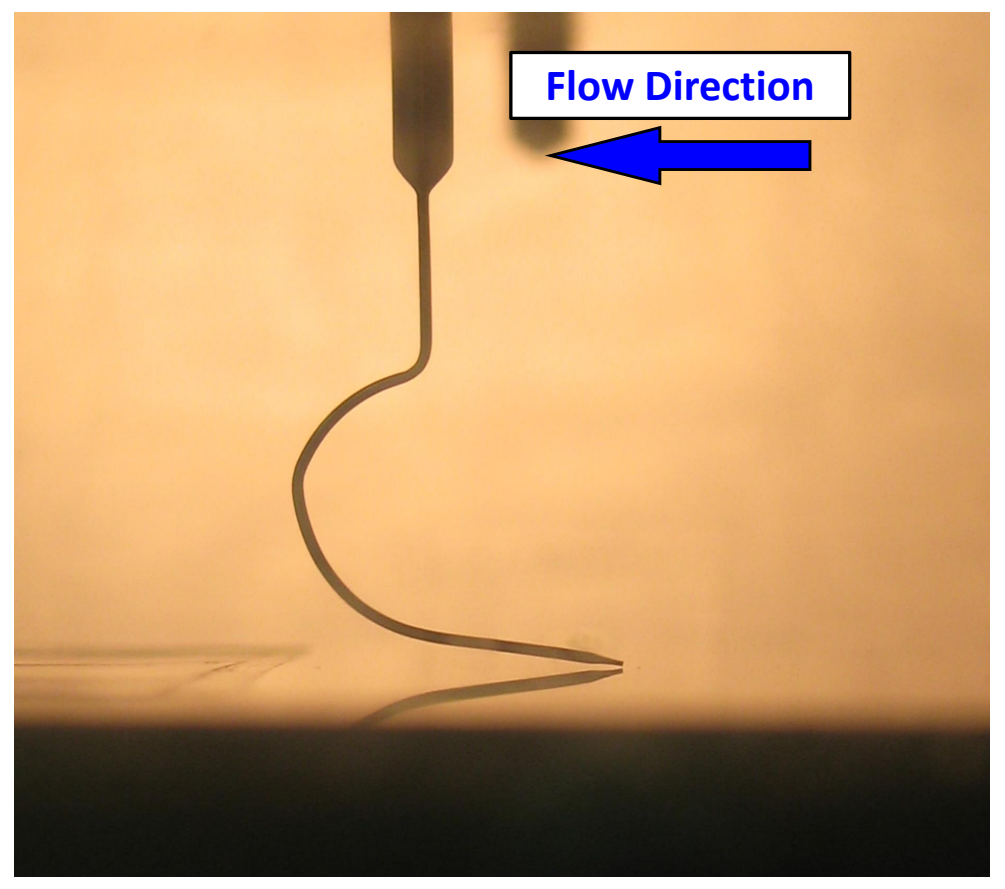

Figure 5.14: Small diameter pitot tube positioned close to the wall. The small $y$ distance is confirmed by the reflection of the probe on the wall

Unlike the pitot-static tube, the pitot tube does not have built in static pressure ports; thus, in order to measure the dynamic pressure, static pressure ports are needed on the surface of the floating wall. Static pressure ports were machined into the aluminum wall at two locations, just upstream and downstream of the traveling wave plate $\left(x_{p}=19 \mathrm{~mm}\right.$ and $\left.x_{p}=317.5 \mathrm{~mm}\right)$. At each location there are two 0.031 in diameter ports symmetrically placed on either side of the pitot tube in Figure 5.14; they are not visible in the figure due to the shallow angle with respect to the wall. The ports at each location are manifolded together by a hole drilled into the floating wall, parallel to the surface. The ports are connected together so as to average the static pressure in the spanwise direction. Each streamwise manifold is then connected to flexible tubing that in turn connects to adapters which pass through the test section wall. The flexible tubing and connections passing through the test section wall can be seen in Figures 5.2 and 5.6, with one upstream and one downstream of the traveling wave plate.

The total pressure from the pitot tube and the static pressure from the floating wall ports are then connected to a differential pressure transducer to measure the dynamic pressure. Six differen- 
tial pressure transducers (Table 5.2) with a range of $0-2.5$ "WC and a $0.25 \%$ full-scale accuracy are used here. During testing one is used for the pitot tube, one is used to monitor the static pressure for the free-stream velocity (Section 5.1.3.4), and four used to monitor the streamwise pressure gradient. These sensors have a sampling rate of $100 \mathrm{~Hz}$ and provide sufficient accuracy to measure the velocity distribution in the boundary layer. The velocity can then be calculated from the dynamic pressure using Equation 5.7.

\subsubsection{Hot Wire Anemometry (HWA)}

Similar to the pitot tube, hot-wire anemometry (HWA) is able measure the flow velocity at a single location in the flow at a time. A general description of HWA and hot-wire probes is given in Section 2.1.3.3. The hot-wire probe is more complex than a pitot tube, but it has significantly greater functionality. A hot-wire probe is smaller and can measure velocities very close to the wall. It also has a large bandwidth, allowing measurement of high frequency velocity fluctuations, which in turn allows investigation of turbulent phenomena. On the downside, the operating principle is more complex, and the hot-wire probe must be calibrated against a pitot-static tube. In addition, the sensor wire is extremely fragile and is easily broken. It can be repaired, but this takes time. Despite these disadvantages, the hot-wire probe is an extremely capable sensor, and is used for all experimental investigation in this research.

The hot-wire (HW) probe used here is a TSI 1218-T1.5 and is shown in Figure 5.15 inserted in the wind tunnel and just above the surface. The HW probe is then mounted to a probe support, which is in turn mounted to the $y$ stage of the traversing system. Figure 5.16 shows this mounting configuration with the probe and probe support passing through a slot in the test-section ceiling. The HW probe is then connected via a BNC cable to a Dantec 56C01 constant temperature anememometer (CTA) with a 56C17 CTA bridge. The CTA and bridge hold the hot-wire probe at a constant temperature with an output voltage related to the power necessary to maintain that temperature (see Section 2.1.3.3 for more details). This voltage output is then fed into the analog input of data acquisition unit (Table 5.2).

As discussed in Section 2.1.3.3, the hot-wire (HW) probe measures the voltage required to maintain the sensor wire at a constant temperature. The temperature is set at a value significantly hotter than the mean temperature of the air. In general, it is set in the neighborhood of $250^{\circ} \mathrm{C}$. The larger the temperature difference between the mean airflow and the sensor wire, the greater the sensitivity of the probe to changes in the flow velocity. However, if the temperature is set too high, then the tungsten sensor wire will oxidize. As a result, the temperature is set as large as possible while remaining below the range at which corrosion occurs $\left(\approx 350^{\circ} \mathrm{C}\right)$ [45].

In practice, the temperature of the sensor wire is defined by the overheat ratio, $a$. This is given in Equation 5.8, where $R_{\text {sensor,hot }}$ and $R_{\text {sensor,cold }}$ are the resistances of the sensor wire at the 


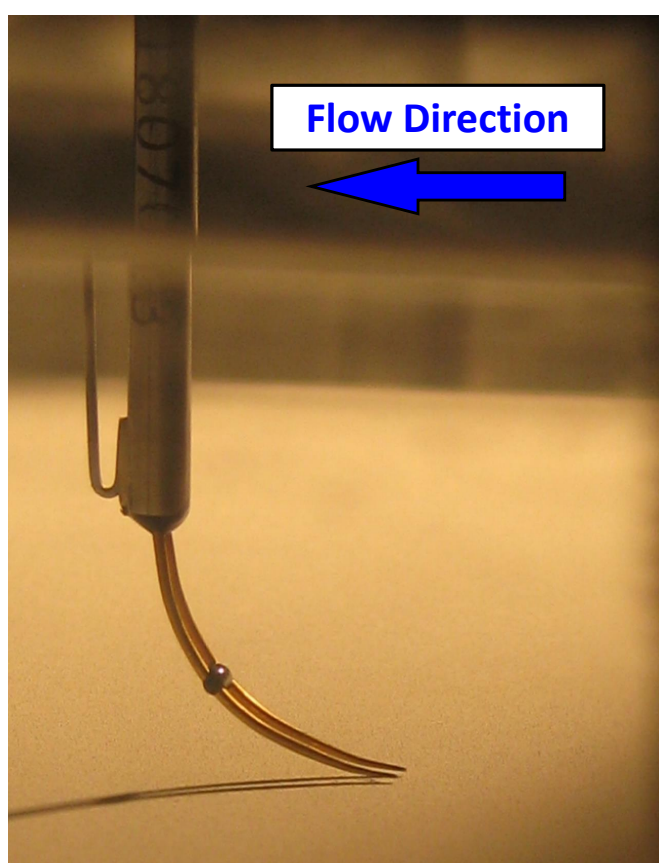

Figure 5.15: Hot-wire probe inserted in the wind tunnel and mounted just above the surface. The shadow of the probe is visible on the wall

hot $\left(T_{h o t}\right)$ and cold $\left(T_{h o t}\right)$ temperatures. The cold temperature is the ambient temperature of the flow, while the hot temperature is the operating temperature $\left(\approx 250^{\circ} \mathrm{C}\right.$ as discussed above $)$. The resistance of the sensor wire increases with temperature, with this change in resistance as a function of temperature $(\alpha)$ given by the manufacturer. The tungsten wire used here was purchased from Dantec and has $\alpha=0.36 \frac{\%}{{ }^{\circ} \mathrm{C}}$. Knowing this constant and the desired temperature, the operating resistance of the sensor wire $\left(R_{\text {sensor,hot }}\right)$ and in turn the overheat ratio $(a)$ can be calculated. The standard overheat ratio of $a=1.8$ was used here.

$$
a=\frac{R_{\text {sensor }, \text { hot }}}{R_{\text {sensor,cold }}}
$$

When connected to the Dantec constant temperature anemometer (CTA), the hot-wire probe becomes one arm in a Wheatstone bridge circuit. The operating temperature is controlled by setting the resistance of this arm to a specific level. The total resistance of this arm is defined in Equation 5.9, where $R_{t o t}$ is the total resistance of the arm, $R_{\text {sensor }}$ is the resistance of the sensor wire, $R_{\text {probe int }}$ is the internal resistance of the HW probe, $R_{\text {support }}$ is the resistance of the probe support, and $R_{\text {cable }}$ is the resistance of the wire connecting the support to the HWA unit. The square brackets separate the resistance of the total hot-wire probe and that of the connecting components. The resistances of the probe, support, and cable are much lower than that of the sensor wire and are fairly insensitive to change. When $R_{\text {tot }}$ is set to different levels, the resistance of sensor is the only 


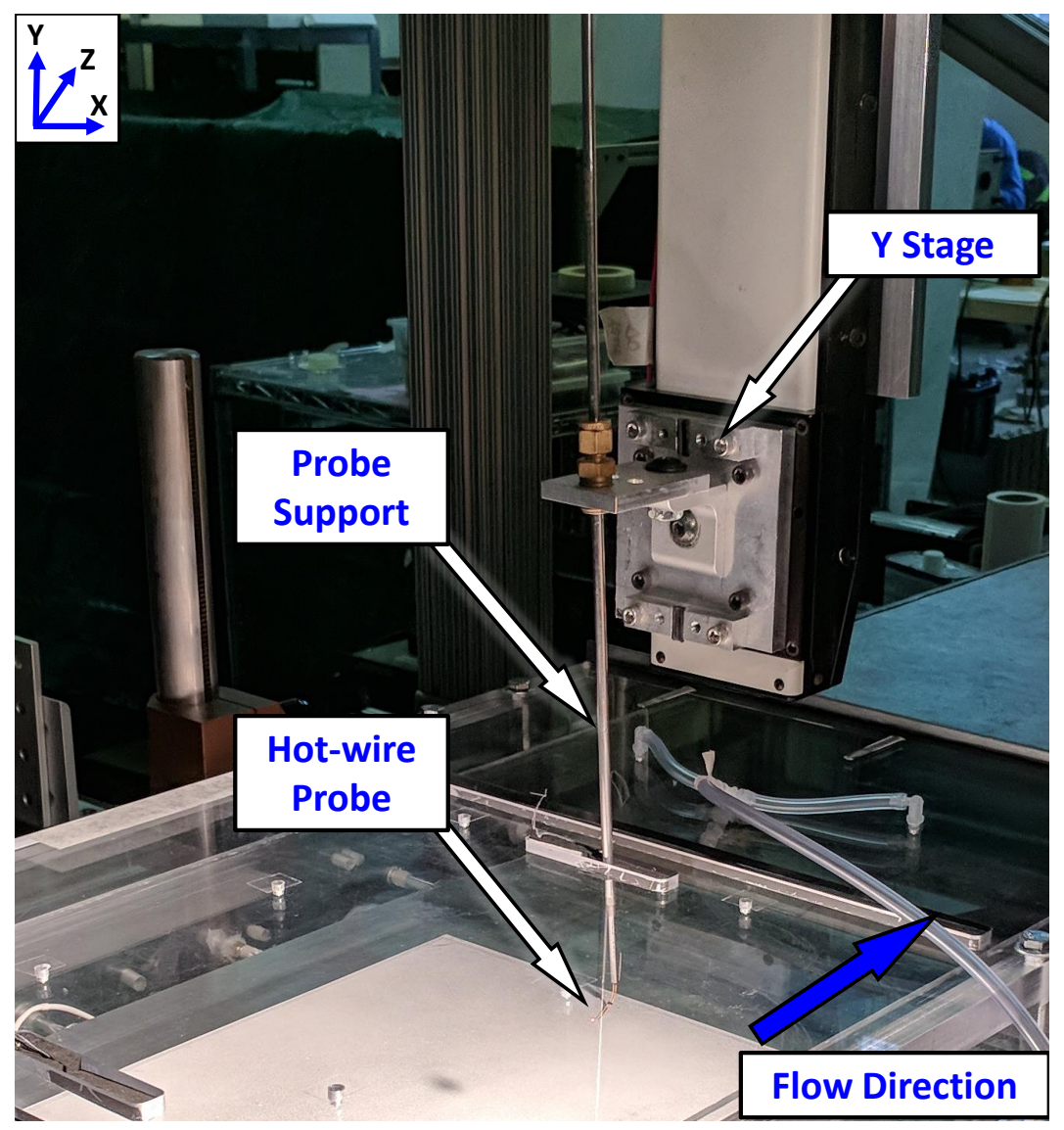

Figure 5.16: Hot-wire probe mounted and passing into the wind tunnel test section. The probe is attached to a support, which is mounted to the $y$ translation stage.

value that changes. Equation 5.9 can be rewritten for the operating (hot) temperature. The result is Equation 5.10, where Equation 5.8 is used to substitute the overheat ratio and the ambient (cold) sensor wire resistance. Knowing the resistances of the cold sensor wire, probe internal, support, and cable; the total resistance $\left(R_{t o t, h o t}\right)$ is then set on the HWA to achieve a specific overheat ratio, $a$, and thus temperature.

$$
\begin{aligned}
R_{\text {tot }} & =\left[R_{\text {sensor }}+R_{\text {probe }, \text { int }}\right]+\left[R_{\text {support }}+R_{\text {cable }}\right] \\
R_{\text {tot }, \text { hot }} & =\left[R_{\text {sensor, }, \text { ot }}+R_{\text {probe }, \text { int }}\right]+\left[R_{\text {support }}+R_{\text {cable }}\right] \\
& =\left[a R_{\text {sensor }, \text { cold }}+R_{\text {probe }, \text { int }}\right]+\left[R_{\text {support }}+R_{\text {cable }}\right]
\end{aligned}
$$

In order to use the hot-wire probe to measure the flow velocity, the output voltage of the HWA must be calibrated against known velocities. The calibration procedure is done by placing the probe in a flow with a known velocity and then recording the voltage at each velocity. These velocity 
versus voltage points are then plotted and fit to create a calibration curve. This curve is then used during experimental testing to translate the measured voltages to flow velocity. The calibration must be redone every so often to ensure that the curve remains accurate. This is because the hotwire probe is sensitive to changes in temperature and other environmental conditions. Here, the calibration is rerun every $2-3$ hours while testing. For large changes in temperature during testing, corrections can be implemented [45]. The temperature fluctuations in the current testing are small and temperature corrections changed the velocities $<0.5 \%$; thus, corrections are not utilized here.

The hot-wire calibration procedure is implemented in the wind tunnel by placing the probe at $x_{p}=-19 m m(-0.75 i n)$, the same location as the reference velocity calibration, and running the tunnel through its full range of operating speeds. In Section 5.1.3.4, the procedure is discussed for conducting a reference velocity calibration, so that the flow velocity can be monitored in the wind tunnel without requiring the permanent installation of a pitot-static tube. The dynamic pressure is measured just upstream of the traveling wave plate using a pitot-static tube (upstream slot in Figure 5.13), while simultaneously collecting the static pressure. The data is then fit with a linear line to map the static pressure to the dynamic pressure at this upstream location.

The actual flow velocity at the hot-wire probe is known by monitoring the static pressure and using the reference velocity fit (Figure 5.10). Thus, the pitot-static tube is not needed during the hot-wire calibration. The voltage from the HWA $(E)$ is then collected as the wind tunnel is incrementally adjusted through its entire operating range. Since the operating range is broken into a lower and upper range (Table 5.1), the data for the hot-wire calibration must be collected in two separate stages. This is similar to the reference velocity calibration; however, only one curve will be fit to all of the data here instead of the two separate lines used in the reference velocity calibration.

An example of the gathered calibration data is shown in Figure 5.17. The velocities were calculated from the dynamic pressures obtained using the reference velocity curve. The calibration points clustered about $\approx 14 \mathrm{~m} / \mathrm{s}$ are at the interface between the lower and upper operating range of the wind tunnel.

There are a number of different curves that can be fit to the calibration data. Two common options are a third-order polynomial in $E^{2}$ [9] and a power law fit. Bruun [45] provides a good discussion comparing the accuracy of each. A power law fit is used here and fit using a non-linear least squares fitting algorithm in Matlab. The resultant fit is shown in Figure 5.17. This power law fit is based off of King's law, which is described in Equation 5.11, where $E$ is the HWA voltage, and $u$ is the known velocity. King's law is analytically based on the convective heat loss of the wire and the voltage required to maintain the sensor wire resistance [45]. The calibration data can be fit with an assumed power of $n=0.45$, but greater accuracy is possible if the power is fit in addition 


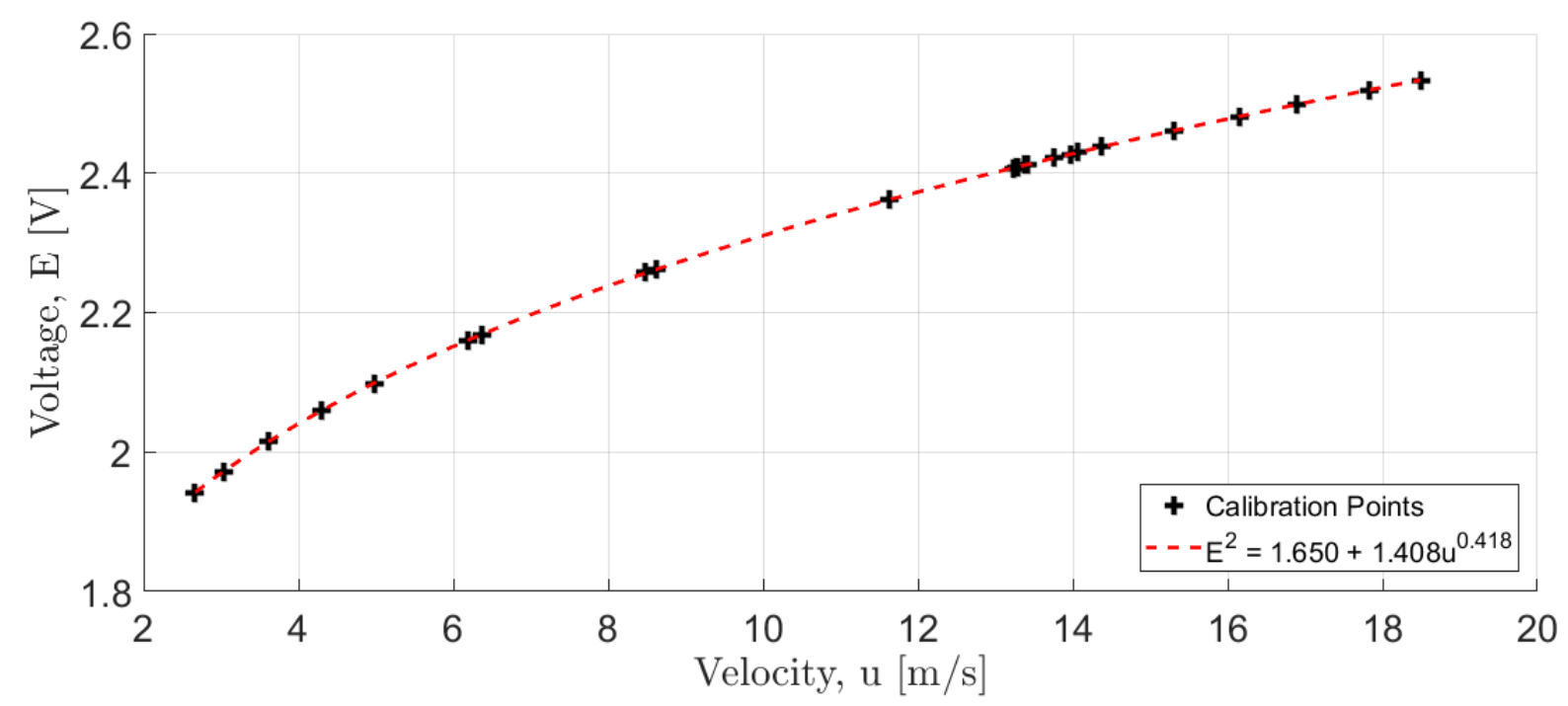

Figure 5.17: Data used to calibrate the hot-wire anemometer. The measured voltages are plotted against known flow velocities. The data is fit using a power law, where all three coefficients were variable.

to the other constants $(A$ and $B)$.

$$
E^{2}=A+B u^{n}
$$

King's law was chosen for the calibration curve, since it is analytically based. This produces a greater confidence in the result and also allows for interpolation of the curve outside of the actual calibration range. In the case of a polynomial fit, it can vary wildly outside of the fit range, and thus cannot be trusted. While the calibration curve can be used for interpolation, its use has been minimized here. The calibration was conducted by sweeping the wind tunnel through as large of a velocity range as possible. The only case where the curve is used outside of the calibration range is at very low velocities. When measuring the boundary layer, the flow velocity very near the wall approaches zero. Depending on the exact range of the calibration, the curve is used at velocities just below the lower edge of the range shown in Figure 5.17.

The quality of the fit is analyzed using the goodness defined by Bruun [45], which is the normalized standard deviation, $\varepsilon_{u}$. This is defined in Equation 5.12, where $N$ is the number of calibration points, $u_{r e f}$ the known reference velocities, and $u_{f i t}$ the velocities fit using the calibration curve. The fit velocities are calculated by using the inverse of the King's law (Equation 5.13). For the calibration shown in Figure 5.17, there is a goodness of $\varepsilon_{u}=0.17 \%$. This low value along with the fact that the fit is analytically based, indicates a strong fit and overall justification for the fitting method. For a discussion of the uncertainties in the velocities obtained with this method, 
see Appendix C

$$
\begin{gathered}
\varepsilon_{u}=\left[\frac{1}{N} \sum_{i=1}^{N}\left(1-\frac{u_{\text {ref }}}{u_{\text {fit }}}\right)^{2}\right]^{1 / 2} \\
u=\left[\frac{E^{2}-A}{B}\right]^{1 / n}
\end{gathered}
$$

With the calibration curve mapping HWA voltages to velocities, the hot-wire probe can be used to measure the velocities along the boundary layer. The hot-wire is placed a known distance away from the wall $(y)$ and the voltages collected. Given the HWA is able to measure voltages at high bandwidths, the voltage is sampled around $20 \mathrm{kHz}$ and for $5 \mathrm{~s}$. This yields 100,000 samples at each $y$ location. The voltages are then converted to velocities using the fit coefficients (Figure 5.17) and the inverse of King's law (Equation 5.13. Despite the large number of samples, not all of them are statistically independent. This is because the fluctuating velocities are caused by turbulent vortices (Section 2.2.3). These vortices have a physical length and time scale; thus, consecutive velocities measured within this time scale are correlated with each other. To achieve independent samples, the velocities must be downsampled to have a time interval between samples greater than the largest turbulent time scale. This is discussed in Section 5.2.1.

A major advantage of the hot-wire probe is its ability to measure velocities much closer to the wall. However, when the HW probe is in the viscous sublayer $\left(y^{+}<5\right)$, the near-wall effect becomes an issue. The operating principle of HWA is that the heat is convectively lost to the flow and this is related to the flow velocity. Once the hot-wire is close to the wall, the sensor wire also starts losing heat to the wall itself, in the form of conduction. This manifests as an increase in the measured flow velocity, since greater heat transfer implies larger velocities. Interestingly, this effect is related to the non-dimensional distance from the wall $\left(y^{+}\right)$, not the absolute distance [45]. In addition, the distance at which this effect occurs is dependent on the material from which the wall is constructed. If the wall is made from a material with high-thermal conductivity, then the near-wall effect occurs further from the wall.

Hutchins [9] shows a comparison of the velocity in the viscous sublayer for different wall materials. This plot is shown in Figure 5.18, where data collected by Hutchins (non-labeled) is compared with that from other studies [10-13]. In this plot, as the hot-wire probe gets closer to the wall, the velocity departs the expected linear trend (Equation 2.3) and has an apparent increase in velocity. It is clear that non-conducting walls although for velocity measurements closer to the wall. In the case of Hutchins [9], he was able to accurately measure the velocity profile down to $y^{+}=3$ before the near-wall effect occurred.

For the case of the conducting wall, the near-wall effect becomes an issue at $y^{+}=4-5$. The 


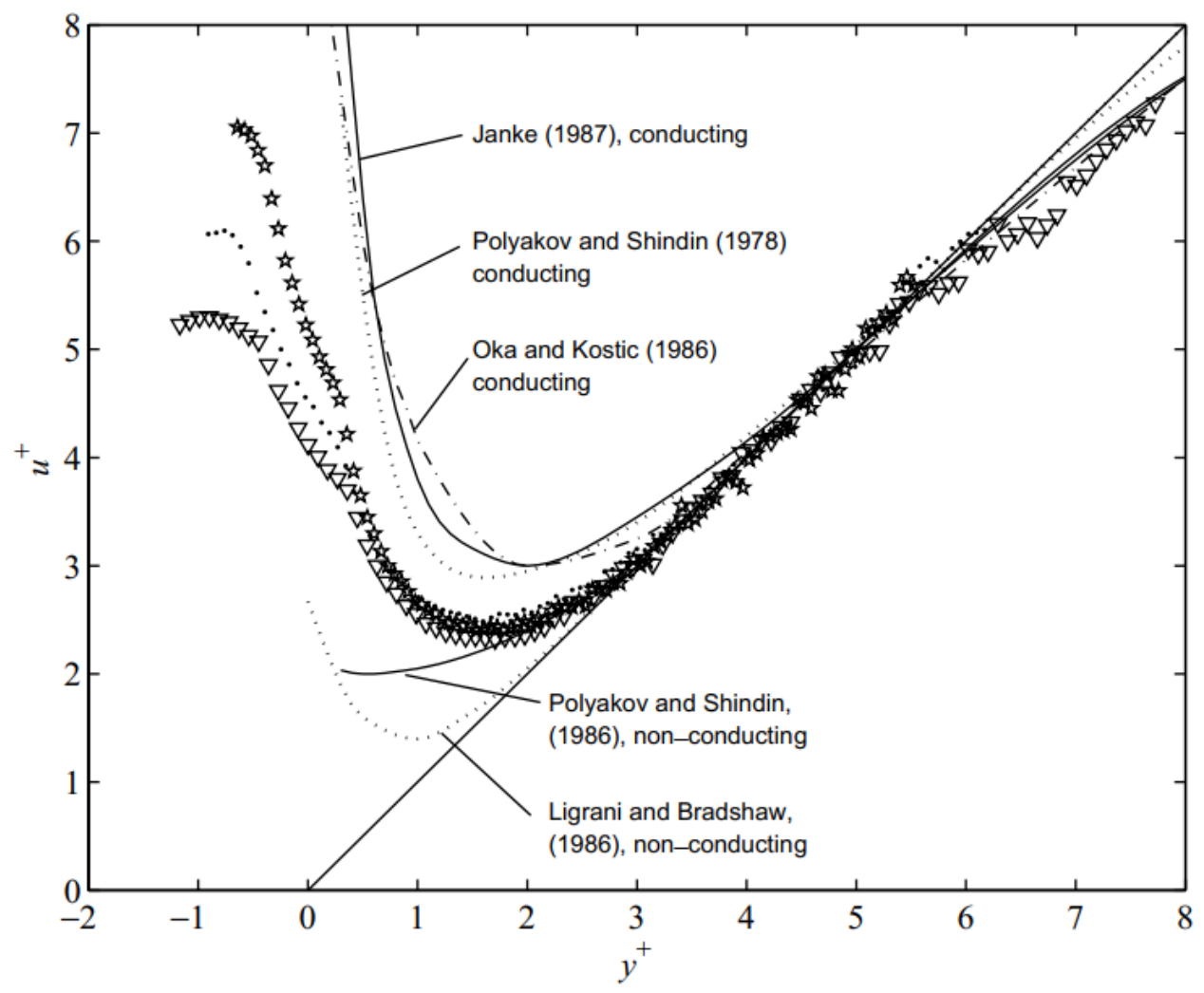

Figure 5.18: Mean velocities in the viscous sublayer of the boundary layer showing the nearwall effect when using a hot-wire probe [9]. The non-labeled data is that measured over a nonconducting wall by Hutchins at three different flow velocities $\left[U_{e}=(\nabla) 3 \mathrm{~m} / \mathrm{s},(\bullet) 2.5 \mathrm{~m} / \mathrm{s},(\star)\right.$ $2 \mathrm{~m} / \mathrm{s}$. The other lines show the velocity profile and the near-wall effect for conducting and nonconducting walls [10-13]). Used with permission from Elsevier

cutoff of the viscous sublayer is at $y^{+}=5$; thus, if the wall is conducting, accurate velocities cannot be measured in the viscous sublayer. The floating wall in this study is made from aluminum, which has a high thermal conductivity. As a result, the flow velocities in the viscous sublayer cannot be measured. This is experimentally shown in Section 5.2.3.

\subsubsection{Finding Wall-Normal (y) Position}

When measuring the boundary layer, it is extremely important to have an accurate measurement of the distance from the wall (the $y$ position). The $y$ position defines the separation between different regions of the boundary layer (Figure 2.2), and the boundary layer properties, such as the momentum thickness (Equation 2.7) are also dependent on the $y$ position. While the $y$ position is adjusted some in post-processing, significant effort is necessary to accurately position the probe during experimental testing. 
The $y$ position is the distance of the velocity measurement probe from the wall. The linear $y$ stage traverses the probe to different positions and as such can be used to track the relative position of the probe. Once an absolute position of the probe with respect to the wall is known, the $y$ position of the probe can be known at all times using the relative position of the linear stage. The absolute position is defined when the velocity probe is touching the wall surface. For a pitot tube, the velocity is measured at the mid point of the opening on the tip of the probe. Hence, when the pitot tube is touching the surface, the $y$ position is half the pitot tube diameter.

The absolute position of the hot-wire probe is slightly more complicated to define. The closest the hot-wire probe can get to the wall is when the prong tips are touching the surface. The $y$ position of the probe is then the distance from the bottom of the probe to the center of the wire. The sensor wire used here is coated tungsten wire from Dantec, and has a diameter of $d_{\text {wire }}=5 \mu \mathrm{m}$ . However, the sensor wire can be placed anywhere on the tips of the prongs. For the TSI 1218T1.5 hot-wire probe used here, the manufacturer quoted dimension of the prong tip diameter is $d_{\text {prong }} \approx 0.005 \operatorname{in}(127 \mu \mathrm{m})$. When the probe is touching the surface, the initial y position could only be defined with an accuracy of $y=0.0025 \pm 0.0025 \mathrm{in}$. The final uncertainty is smaller than this since the $y$ position is adjusted in post-processing by aligning the boundary layer profile (Section $5.2 .3)$.

However, the position of the sensor wire on the prong also affects the measurement capabilities of the HW probe. If the sensor wire is mounted at the very top of the prongs, then the smallest measurable y position is $y=0.005 \operatorname{in}(127 \mu \mathrm{m})$. As discussed in Section 2.3, the viscous sublayer in the current research occurs for $y \lesssim 100 \mu \mathrm{m}$. Thus, if the sensor wire is mounted at the top of the prong, its position is roughly twice the viscous sublayer thickness. To allow for measurements as close to the wall as possible, the hot-wire probe is repaired with the sensor wire is welded near the bottom of the prongs. Figure 5.19 shows a close-up view of two different sensor wire placements. In the Figure 5.19a, the wire is welded at roughly the mid point of the prongs, while in Figure $5.19 \mathrm{~b}$, the wire is closer to the bottom where the prong will touch the surface. From the figures it is also clear that the sensor isn't welded in the same location on both the left and right prong. The true $y$ position would then be somewhere between them.

In order to determine the absolute position where the probe touches the surface, a $y$ offset procedure was developed using a camera and macro-photography. A Canon Powershot S5IS camera is mounted outside of the wind tunnel test-section at roughly the same vertical position as the wall but angled slightly downward. A photography light is placed opposite of the camera and shines down on the probe. This bright light casts a clear shadow of the probe onto the surface of the wall. This setup is shown in Figure 5.20. The probe is then moved incrementally closer to the wall and pictures taken at each step. When the probe's shadow meets the probe itself, the probe is said to be touching the wall. This is shown for the hot-wire probe in Figure 5.21a. However, this is not 


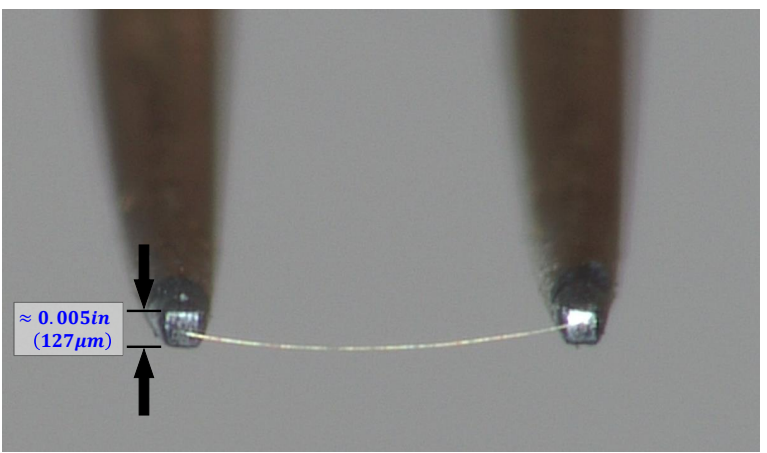

(a)

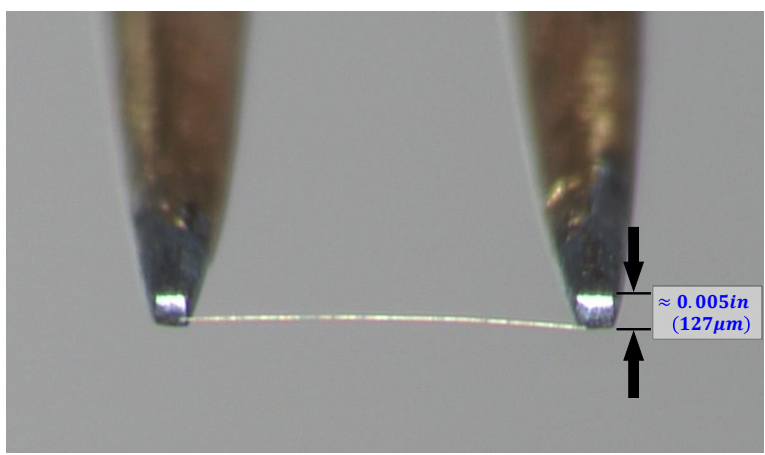

(b)

Figure 5.19: Close-up photos of the sensor wire welded onto the faces of the hot-wire probe prongs. (a) shows the wire welded roughly vertically centered and (b) shows the wire welded closer to the bottom, where the prong will contact the wall

the initial $y$ position. The linear stage has a small amount of backlash it it; thus, changing direction will cause a position error. To remove this, the probe is then slowly lifted off the stage in intervals of $0.000,5 \operatorname{in}(12.7 \mu \mathrm{m})$, while taking pictures. The probe has lifted off the surface once it no longer touches its shadow in Figure 5.21b. This is defined as the absolute $y$ position. In practice, the backlash is $\approx 0.0015 \mathrm{in}(38.1 \mu \mathrm{m})$.

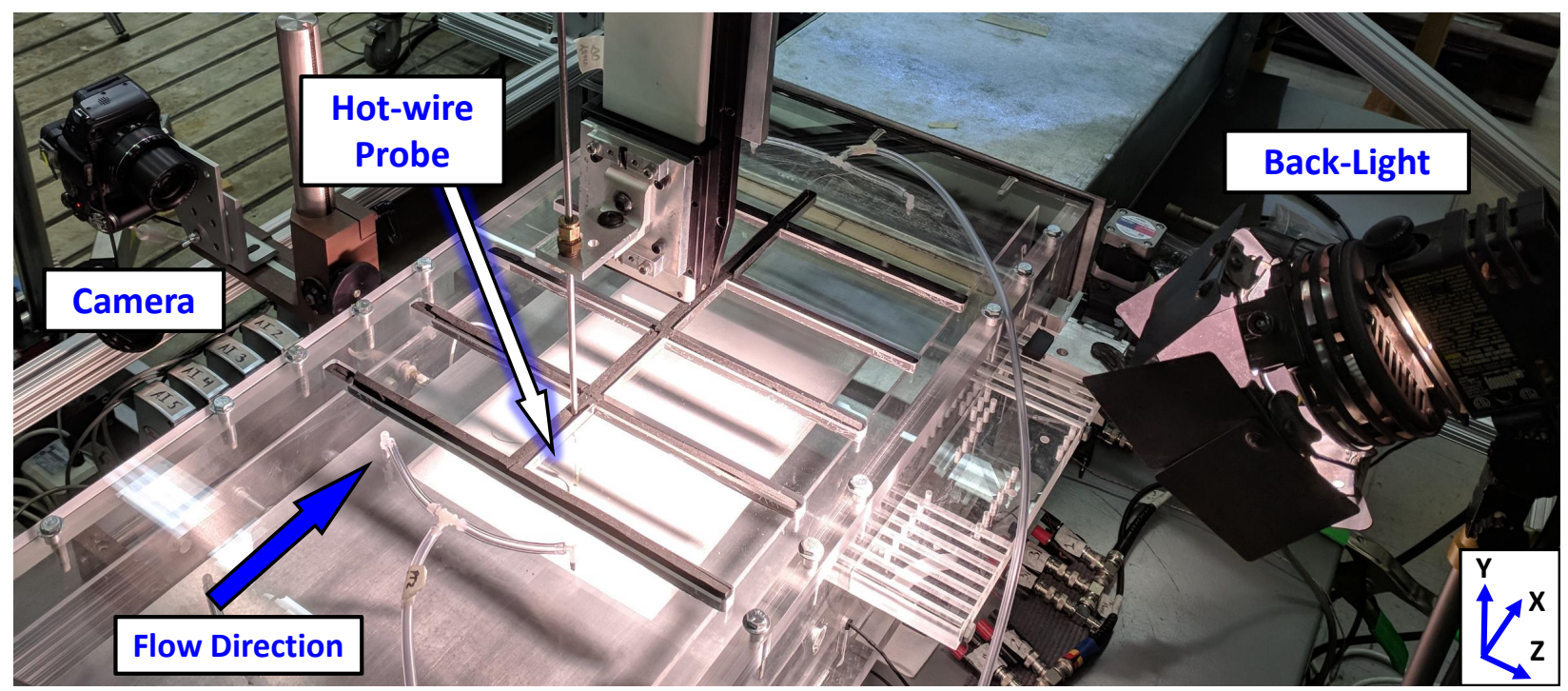

Figure 5.20: View of the setup used to detemine the $y$ absolute reference position. The hot-wire probe is in the wind tunnel test-section and illuminated by the photography back-light. The camera on the left is angled slightly downwards to take a picture of the probe and its shadow.

This procedure is complicated when measuring the boundary layer directly over the traveling wave surface. As will be discussed in Chapter 6, the traveling waves are excited on a thin plate that is flush mounted into the floating wall. Since the traveling wave plate is thin, it is susceptible to 


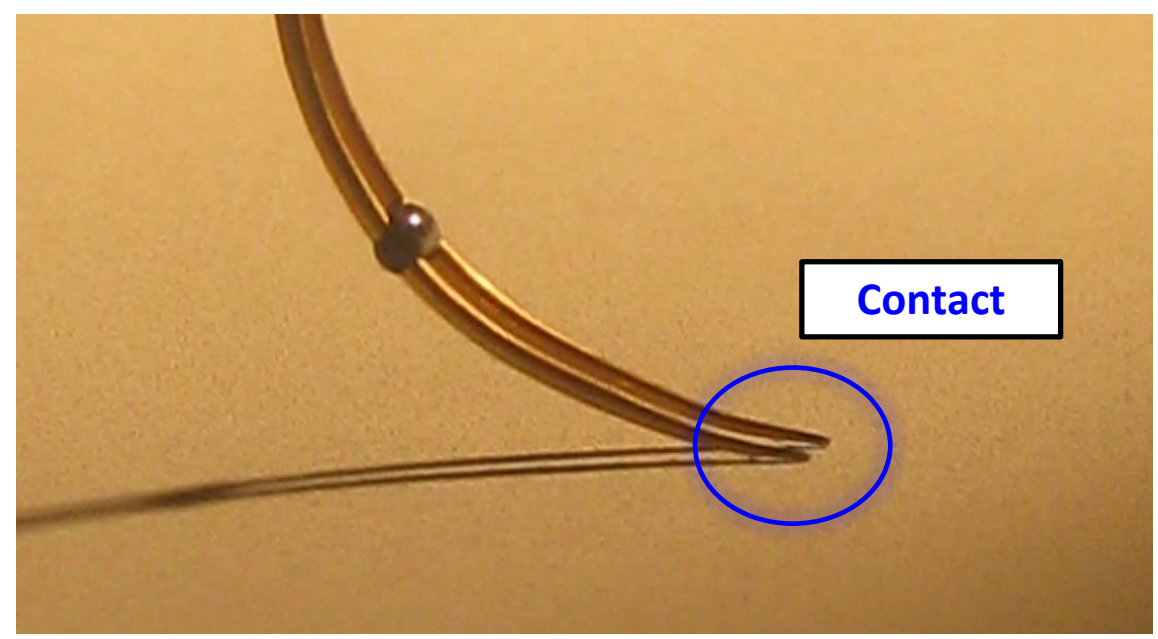

(a)

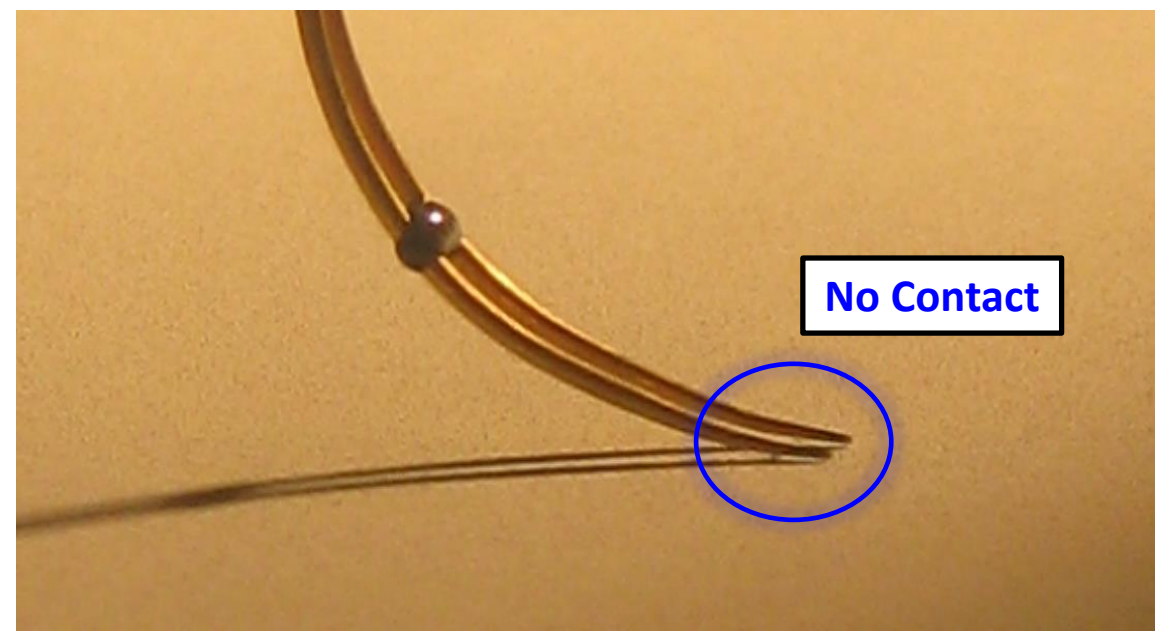

(b)

Figure 5.21: (a) The hot-wire probe touching its shadow and making contact with surface of the wall. (b) The probe lifted barely off the surface at $y \approx 0.000,5 \operatorname{in}(12.7 \mu \mathrm{m})$ such that light is visible between the probe and its shadow

deformations in the wall-normal, $y$, direction. This manifests in two ways during when conducting the $y$ offset procedure while the probe is directly over the traveling wave plate. Firstly, the thin plate deforms upwards by $\Delta y \approx 0.004 i n(101.6 \mu \mathrm{m})$ when the wind tunnel is running. This mostly likely results from a pressure difference under and above the plate. To account for this, the $y$ offset procedure is conducted while the wind tunnel is running at the operating speed of the experimental testing. Secondly, the heat from the photography light causes the thin plate to deform downwards by $\Delta y \approx-0.002 i n(-50.8 \mu m)$. The plate already bows slightly downwards, due to its own weight. Thus, the light most likely heats up the plate and causes it to deform even further in the $-y$ direction. This is corrected by turning the light on only while taking the photo. The plate does not 
deform immediately and the short time the light is on does not cause noticeable deformation. The deformation is observable since the mean output voltage of the HW probe shifts as the plate heats up and deforms (i.e. the probe is measuring at a different position on the boundary layer profile).

\subsubsection{Testing Procedure}

With the $y$ absolute reference position found, the actual measurement of the boundary layer can begin. The entire experimental testing procedure is automated and controlled using a Labview GUI. The time-dependent velocity is measured at a number of $y$ positions. The first measurement position is the absolute reference position found in the previous section. The linear stage then moves incrementally in the $+y$ direction measuring the velocities at each location. The probe only ever moves in the $+y$ direction so as to eliminate backlash errors in the $y$ position associated with the linear stage changing direction. The $y$ positions are logarithmically spaced, since the scaling of the boundary layer is logarithmic in nature. Generally the velocity is measured at $75 y$ locations, with the largest $10-20 y$ values outside of the boundary layer and in the free-stream.

At each $y$ position, a number of different quantities are collected and written to a file specific to each $y$ location. The velocity probe collects data for $5 s$ where the sampling rate is dependent on the probe used. The pressure transducers for the pitot tube are limited to $100 \mathrm{~Hz}$; thus, the velocity is collect at this rate or downsampled in post-processing to $100 \mathrm{~Hz}$. On the other hand, the hotwire probe does not have significant limitation on bandwidth. As discussed in Section 5.1.4.3, the sampling rate is roughly $20 \mathrm{kHz}$. The entire time series of data values is then written to the $y$ specific data file. The static pressures at multiple streamwise locations are also collected. This includes the reference static pressure used to define the free-stream reference velocity (Section 5.1.3.4); these time series are downsampled in post-processing to the $100 \mathrm{~Hz}$ transducer limit. Also written to each $y$ file are the mean and standard deviation of the temperature, the atmospheric pressure, the sampling rate, and number of samples.

This procedure is for collecting a boundary layer at a single streamwise and spanwise location over the flat wall. When collecting the boundary layer for a traveling wave actuated surface, this procedure changes. This is discussed in Chapter 6.

\subsection{Data Analysis}

With the experimental data collected, it is processed and analyzed using Matlab. This section walks through that procedure on a set of boundary layer data collected with a hot-wire probe just upstream of the traveling wave plate $\left(x_{p}=-19 \mathrm{~mm}(-0.75 \mathrm{in})\right.$ in Figure 5.3). Once the raw data has been extracted from the files, the hot-wire voltages are converted to velocities using the calibration 
curve discussed in Section 5.1.4.3. These velocities are not statistically independent, so they are down-sampled to the appropriate rate. An estimate for the skin friction coeffient is then found and the boundary layer normalized to wall units. The $y$ positions are then adjusted to correct for errors from the experimental setup. The skin friction coefficient is then calculated and the boundary layer re-normalized. This is the final boundary layer profile. Using this profile, the boundary layer quantities such as momentum thickness and Reynolds number are calculated. Finally, the other turbulent boundary layer profiles are plotted; these include the variance, skewness, and kurtosis.

\subsubsection{Statistical Independence}

The main method implemented here to analyzing the flow velocities is via amplitude domain statistics. This was discussed in Section 2.2.3 and involves calculating the mean, variance, skewness, and kurtosis of the measured velocity signal. The assumption when calculating these are that each sample in the velocity signal is statistically independent. However, due to the nature of turbulence this is not always the case. The velocities here are measured using a hot-wire probe with a sampling rate of $\approx 20 \mathrm{kHz}$, so every sample is not likely to be independent. Thus, before the mean and variance can be calculated, the velocity signal are down-sampled such that the time interval between each velocity is large enough to ensure statistical independence.

As outlined in Section 2.2.3, samples are independent when the time interval between them is greater than twice the Integral time-scale $\left(\tau \geq T_{I}\right)$. The Integral time-scale is calculated from the autocorrelation coefficient function, $\rho_{u}(\tau)$, of the velocity signal. To determine the appropriate sample rate to down-sample to, the autocorrelation function is determined for the velocity signal at each $y$ position. Figure 5.22 shows the autocorrelation function at $y^{+}=4.8$. At $\tau=0 m s$, the autocorrelation coeffient has a value of one, signifying that the velocity signal is perfectly correlated with itself. As the time difference $(\tau)$ increases, the correlation drops until it crosses zero at $\tau \approx 3.2 \mathrm{~ms}$. From this autocorrelation function, the integral time-scale, $T_{I}$, can then be calculated as in Equation 2.18. The integral is conducted only out to the first zero crossing at $\tau \approx 3.2 \mathrm{~ms}$. The result is an integral time-scale of $T_{I} \approx 0.6 \mathrm{~ms}$, which is shown in Figure 5.22.

Figure 5.22 shows the autocorrelation and integral time-scale at only a single $y$ position. The autocorrelation function is dependent on the turbulence, and the turbulence is dependent on the position in the boundary layer. So the integral time-scale, $T_{I}$, will depend on the $y$ position in the boundary layer. This is shown in Figure 5.23, where the $y$ position is given in wall units $y^{+}$. Near the wall, the integral time-scale is roughly constant at $T_{I} \approx 0.6 \mathrm{~ms}$ and then decreases as the position moves further and further from the wall. $T_{I}$ peaks at $y^{+} \approx 10$, in the buffer layer of the boundary layer. The largest amount of turbulence is produced in this region [47], so it makes sense that $T_{I}$ peaks here. Outside of the boundary layer in the free-stream, $T_{I}$ rapidly increases and then 


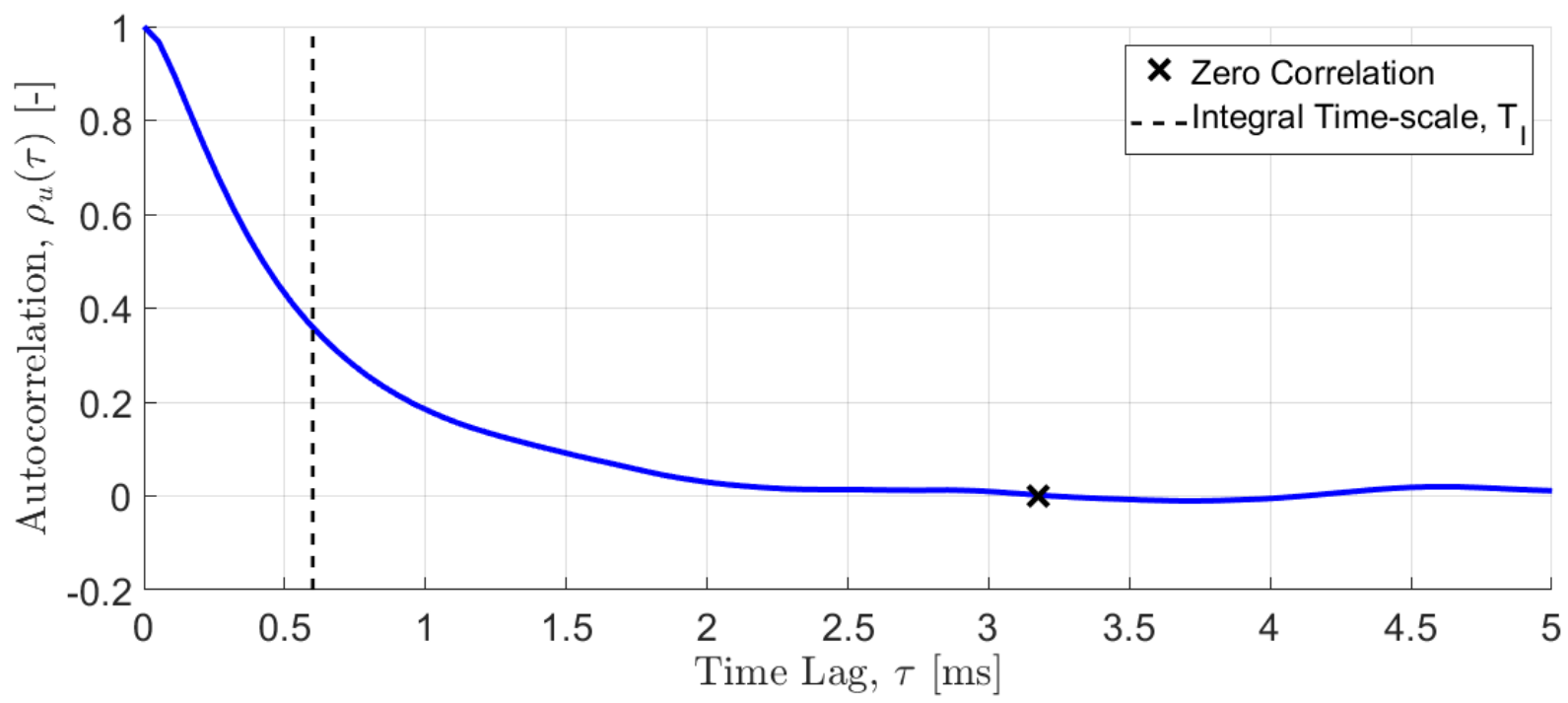

Figure 5.22: The autocorrelation coefficient function, $\rho_{u}(\tau)$, of the velocity at a $y^{+}=4.8$

fluctuates wildly with position. The larger $T_{I}$ values are attributed to the larger vortices and thus slower time-scales that occru int eh free-stream.

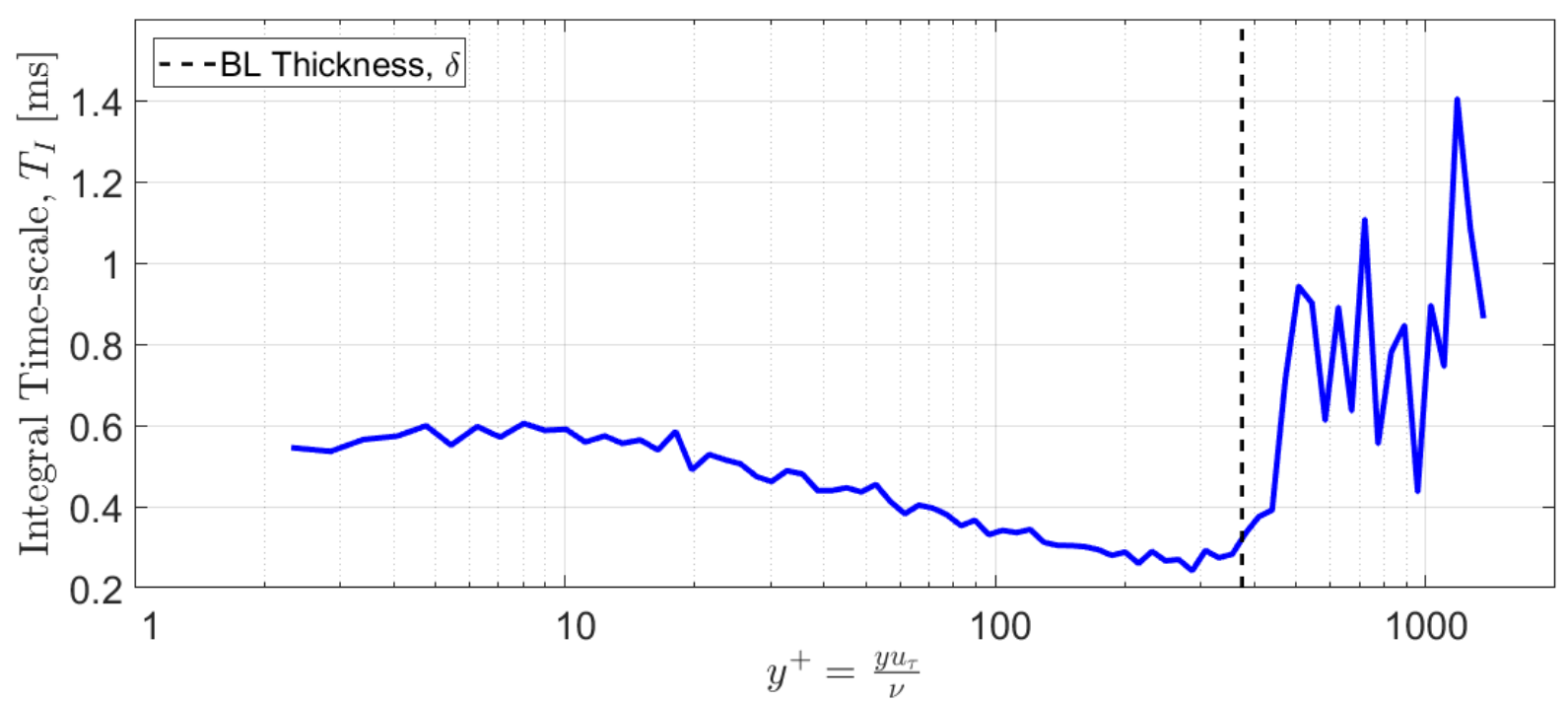

Figure 5.23: The intergral time-scale, $T_{I}$, of the velocity signal as a function of distance from the wall, $y$

In order for the velocity signals to be statistically independent, the time interval between samples must be $\tau \geq 2 T_{I}$. To simplify the data analysis process, all of the velocity signals are downsampled using the same integral time-scale. Since the boundary layer, and not the free-stream, 
is the region of interest here, the signals are down-sampled using the maximum time-scale in the boundary layer: $T_{I} \approx 0.6 \mathrm{~ms}$. The final sampling rate is then chosen so as to be an integer divisor of the initial hot-wire sampling rate $(\approx 20 \mathrm{kHz})$. As a result, the velocity signal at each $y$ value is down-sampled so that there is $5 s$ of data at $\approx 800 \mathrm{~Hz}$.

\subsubsection{Non-dimensionalization}

Using the down-sampled velocity signals, the mean velocity is calculated at each $y$ location. The mean velocity boundary layer profile is then shown in Figure 5.24. Each of these mean velocities has been normalized to account for variations in the free-stream velocity over the length of the test. Using the method described in Section 5.1.3.4, the free-stream velocity is continuously measured during testing. This is known as the reference velocity, $U_{r e f}$, since it is the free-stream velocity at a reference location just upstream of the traveling wave plate $\left(x_{p}=-19 \mathrm{~mm}\right)$. During testing, the reference velocity remains nominally constant, but will very slightly due to changes in the environmental conditions. As such, there is a reference velocity for each $y_{i}$ position, $U_{r e f, i}$. To account for the slight changes, the boundary layer velocities are normalized by the free-stream reference velocity using Equation 5.14. $u_{\text {raw }}\left(y_{i}\right)$ are the local velocities at different $y_{i}$ locations in the boundary layer, $U_{r e f, i}$ is the reference velocity for each $y_{i}$ location, $U_{r e f, a v g}$ is the average over all $y_{i}$ locations, and $u\left(y_{i}\right)$ are the normalized velocities shown in Figure 5.24.

$$
u\left(y_{i}\right)=u_{\text {raw }}\left(y_{i}\right) \frac{U_{\text {ref }, i}}{U_{\text {ref,avg }}}
$$

As mentioned, the reference velocity is the free-stream velocity at a fixed reference location upstream of the traveling wave plate. The boundary layer is measured at various streamwise locations, so the free-stream velocity for a boundary layer is generally different from the reference velocity. The flow has nominally zero streamwise pressure gradient, so the free-stream velocity only varies slightly $(<2 \%)$. The actual free-stream velocity for a boundary layer, $U_{e}$, is found by averaging the velocities measured in the free-stream using the hot-wire probe. This is the region of flat velocities far from the wall in Figure 5.24. The boundary layer thickness, $\delta$, is then defined as the $y$ position where the velocity reaches $99 \%$ of the free-stream velocity. This is shown in Figure 5.24 .

The boundary layer profile shown in Figure 5.24 is given in dimensional units. To fully understand and analyze the profile, it must be normalized/non-dimensionalized to wall units $\left(y^{+}\right.$and $u^{+}$). Non-dimensionalizing yields a definable profile that is similar across all Reynolds numbers. This is the generic profile of Figure 2.2 in Section 2.2 which shows the viscous sublayer, buffer layer, log region. The non-dimensionalized terms, $y^{+}$and $u^{+}$, are defined in Section 2.2 but are given again here in Equations 5.15 and 5.16. $u_{\tau}$ is the friction velocity, which is shown again in 


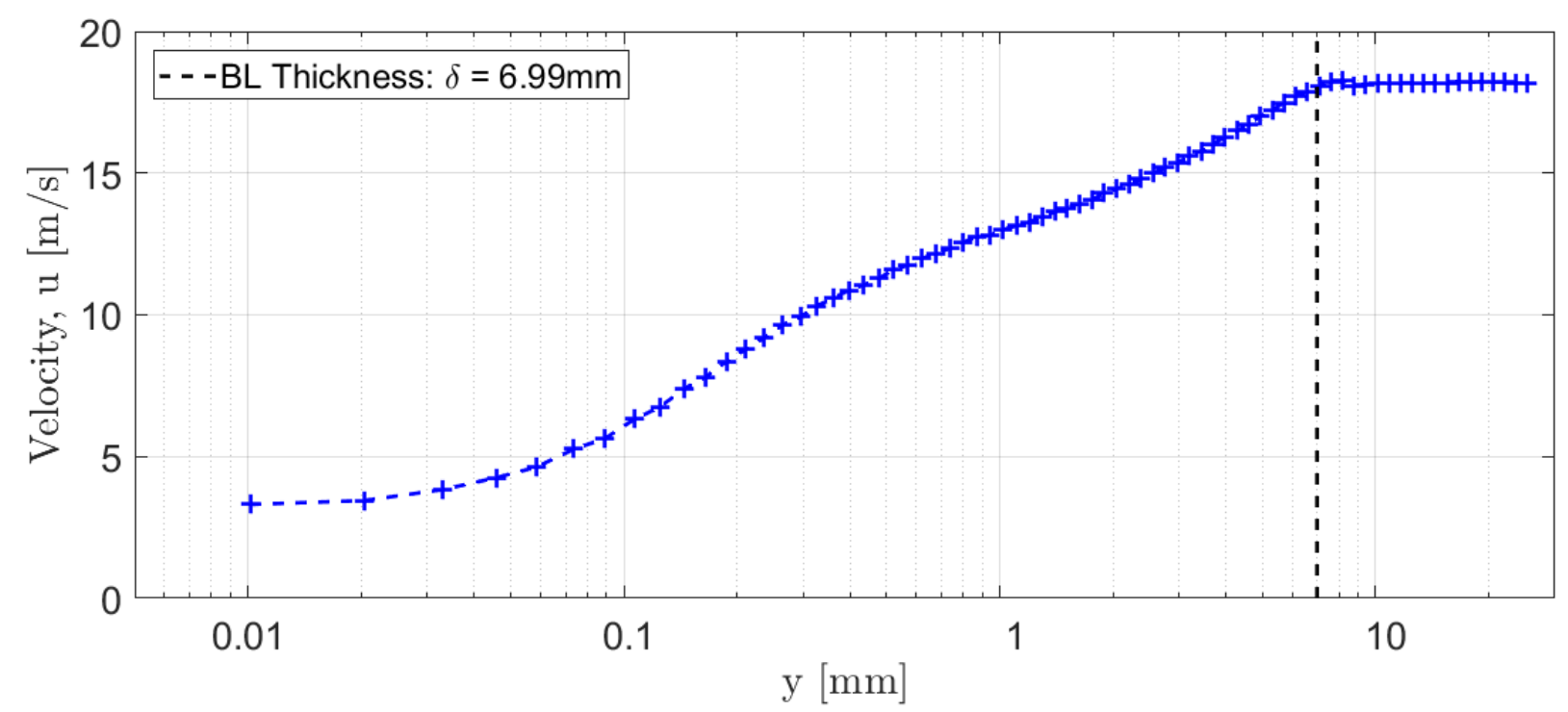

Figure 5.24: Mean velocity boundary layer profile in dimensional form with the boundary thickness, $\delta$, shown

Equation 5.17. Thus, in order to normalize the boundary layer, the friction velocity must be defined using the local skin friction coefficient, $C_{f}$.

$$
\begin{gathered}
y^{+}=\frac{y u_{\tau}}{v} \\
u^{+}=\frac{u}{u_{\tau}} \\
u_{\tau}=\sqrt{\frac{\tau_{w}}{\rho}}=U_{e} \sqrt{\frac{C_{f}}{2}}
\end{gathered}
$$

As discussed in Section 2.3, there are three ways to determine the skin friction coefficient, $C_{f}$. The first is by determining the slope of the boundary layer profile in the viscous sublayer $\left(y^{+}<5\right)$. Unfortunately that method is not possible here. The velocities have been measured in this region, but due to the near wall effect in hot-wire measurements (Section 5.1.4.3) the velocities are not accurate. This is shown more clearly in Section 5.2.3 with experimental data. Next, the momentum balance method was established in Section 2.3 as inaccurate due to the violation of assumptions. As a result, the Clauser method is used to determine $C_{f}$ which involves fitting the logarithmic region of the turbulent boundary layer. The curve defining this region is known as the law of the wall and was defined in Equation 2.5. By rearranging and combining with Equation 5.17, it can be expressed as in Equation 5.18, where $k=0.41, C=5.0$, and the velocities have be 
normalized by the free-stream velocity, $U_{e}$.

$$
\frac{u}{U_{e}}=\sqrt{\frac{C_{f}}{2}}\left[\frac{1}{k} \ln \left(\frac{y U_{e}}{v}\right)+\frac{1}{k} \ln \sqrt{\frac{C_{f}}{2}}+C\right]
$$

The logarithmic portion of the boundary layer is defined as the region with $y^{+}>50$ and $y / \delta<$ 0.2 [48]. The aim is to non-dimensionalize to wall units, but the lower limit for the log region is given in wall units. Thus, an initial estimate of $C_{f}$ must be found so that the boundary layer can be non-dimensionalized. Once it is in wall units, the limits of the log region can be found. Then, a more precise value of $C_{f}$ value can be found by fitting Equation 5.18 to the log region.

A simple way to find an initial estimate for the skin friction coefficient, $C_{f}$, is by generating multiple curves of Equation 5.18 for various values of $C_{f}$. These Clauser curves are then plotted alongside the boundary layer profile as shown in Figure 5.25. All of the velocities have been normalized by the free-stream velocity, similar to the left side of Equation 5.18. The Clauser curve that best fits the $\log$ region $(y \approx 0.0046)$ is then the initial estimate of $C_{f}$.

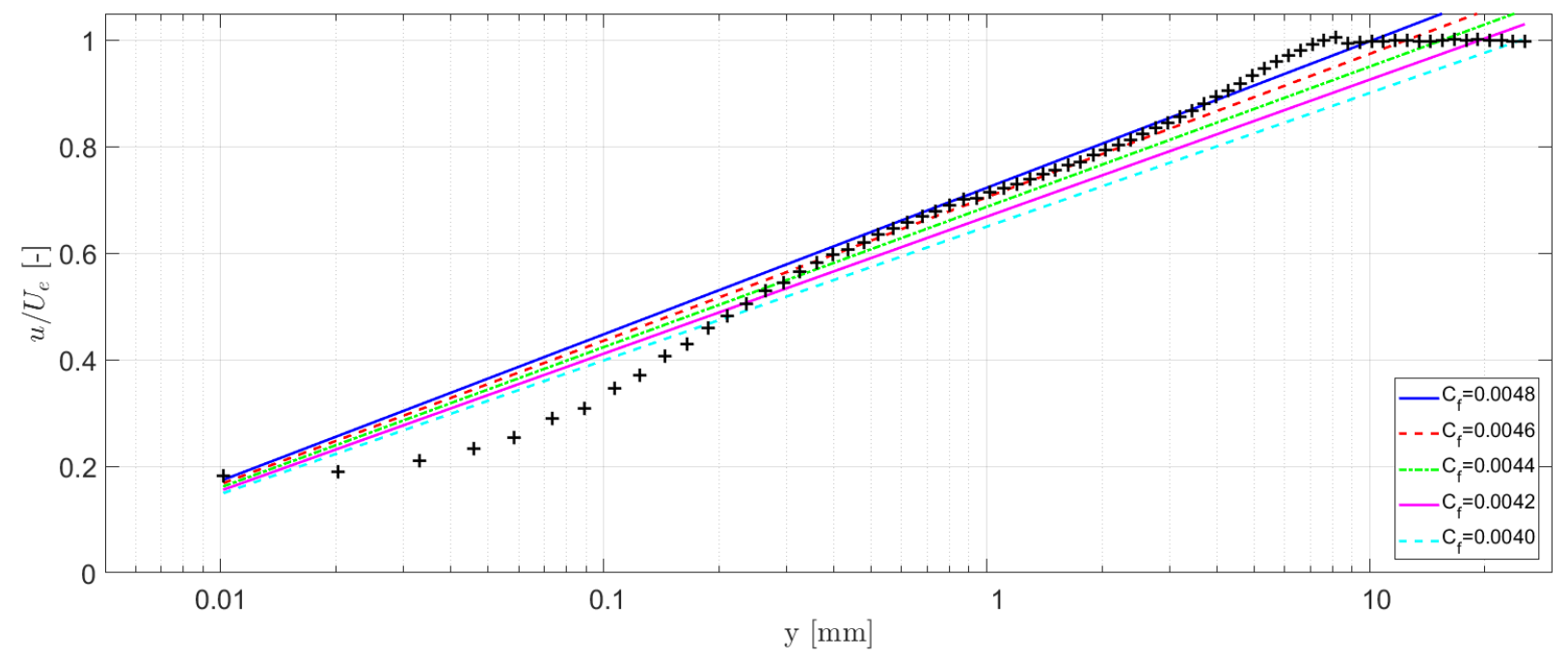

Figure 5.25: Mean velocity boundary layer profile normalized by the free-stream velocity, $U_{e}$. Also shown are various fits of the logarithmic region for different $C_{f}$ values

Another and more automated way to find an initial estimate of $C_{f}$ is by using the Coles curve [15]. The Coles curve is an empirical curve of the skin friction coefficient as a function of the momentum thickness-based Reynolds number for flat plate, zero pressure gradient flow. To implement this, estimates of the momentum thickness, $\theta$, and Reynolds number, $R e_{\theta}$, are calculated from the boundary layer profile (Section 5.2.4). The Reynolds number is then used with the Coles curve to provide an initial estimate of the $C_{f}$. The Coles curve is discussed further in Section 5.3.4 where it is compared to experimental data at various streamwise locations. 
Once the initial estimate of the skin friction is found (either visual or Coles curve), the boundary layer is normalized to wall units with this value. Now the velocities that fall into the log region $\left(y^{+}>50\right.$ and $\left.y / \delta<0.25\right)$ can be pulled out; the upper limit of the log region was extended (normally $y / \delta<0.2$ )to include more points. These values are then fit to Equation 5.18 using a non-linear least squares fit. The boundary layer profile is then non-dimensionalized using this estimate of $C_{f}$. This $C_{f}$ value is still an estimate since the $y$ positions have not yet been corrected to account for errors. This adjustment procedure is discussed in the next section. Once the $y$ positions have have been adjusted, the non-linear fit is run again and to determine the final value of the skin friction coefficient, $C_{f}$.

\subsubsection{Wall-Normal (y) Position Adjustment}

During experimental testing, a great deal of effort was spent to place the hot-wire probe as accurately as possible (Section 5.1.4.4). However, there is still a fair amount of uncertainty in the $y$ position. Figure 5.26 shows the mean velocity boundary layer profile normalized to wall units. Also shown in the figure are the lines representing the viscous sublayer and the log-law. The log-law fits the the unadjusted $y$ profile well, as this was the aim of non-dimensionalizing in the previous section (Section 5.2.2). However, the profile does not converge with the viscous sublayer profile shown at smaller $y^{+}$values. This mismatch is primarily the result of incorrect $y$ values in the experimental boundary layer.

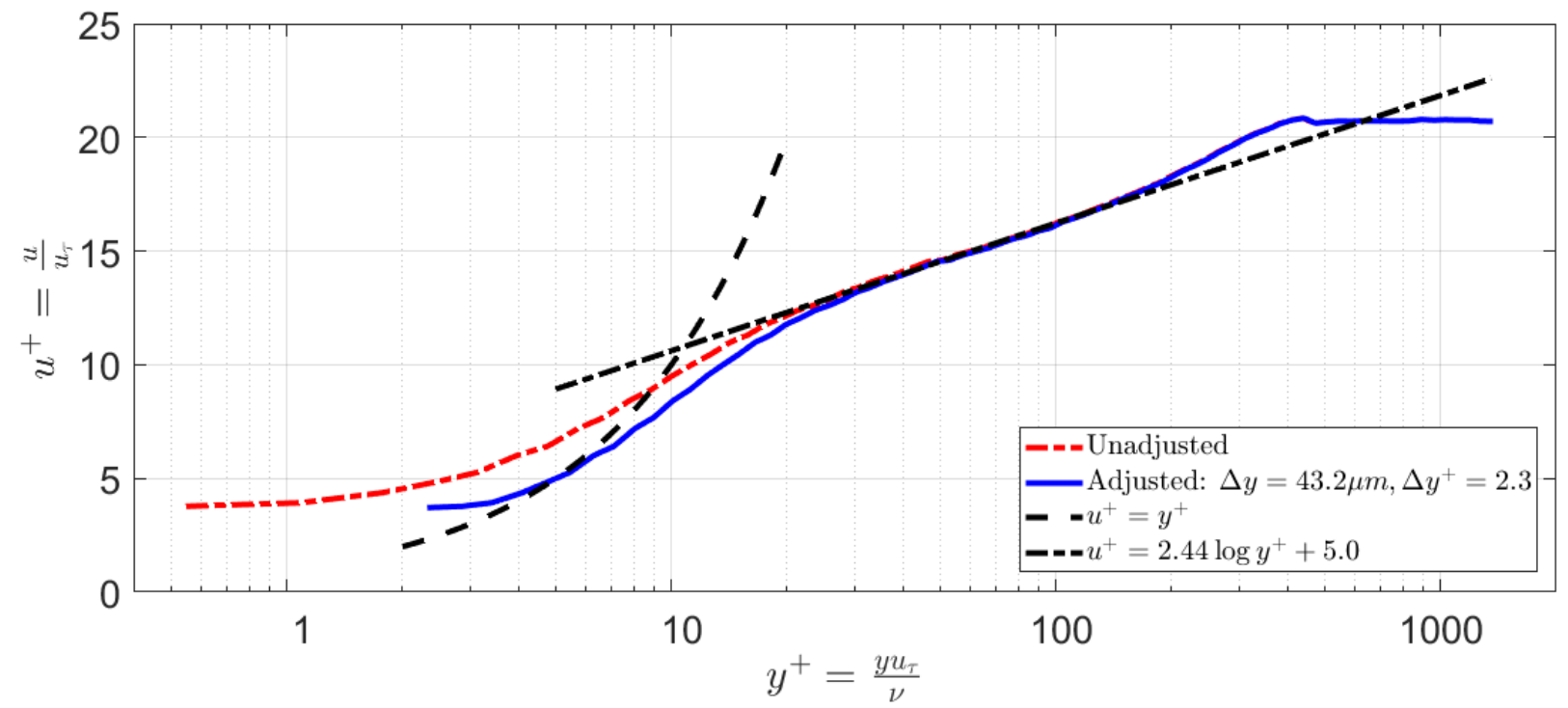

Figure 5.26: Mean velocity boundary layer profiles with and without the $y$ position offset implemented. 
The viscous sublayer line in Figure 5.26 is a linear line representing the curve $u^{+}=y^{+}$. The line is curved in the figure due to the logarithmic scaling. A common way to adjust the $y$ position of the experimental data would be to fit a linear line to the viscous sublayer region [9]. The actual viscous sublayer does not have an intercept since the no-slip condition of the wall requires zero flow velocity at the wall $(y=0)$. However, when fitting a linear line to the experimental data, there will be a non-zero intercept. This intercept results from the offset in the $y$ position, and can be used to correct all of the $y$ values in the boundary layer.

Unfortunately, this method cannot be applied here due to the near-wall effect of the hot-wire probe (Section 5.1.4.3). The measured velocities in the near wall region are artificially increased preventing an accurate linear line from being fit. Instead, the $y$ position must be adjusted manually. Figure 5.26 shows the boundary layer profile where each $y$ position has been adjusted by $\Delta y=$ $43.2 \mu m\left(\Delta y^{+}=2.3\right)$. Looking at this profile and comparing it to those seen in Figure 5.18, the nearwall effect is clearly visible. Instead of converging with the viscous sublayer line and approaching zero, the velocity seems to increase.

The actual $y$ offset value $(\Delta y)$ was determined so that the boundary layer profile passes through $u^{+}=y^{+}=5$. This is the edge of the viscous sublayer and is where the standard boundary layer profile begins diverging from the linear fit line. Figure 5.18 shows boundary layer profiles for varying intensities of the near wall effect. In the case of the conducting wall, even though it does not converge with the viscous sublayer line, it still passes through $u^{+}=y^{+}=5$. Thus, to correct for the $y$ offset, the boundary layer profile is manually adjusted to pass through this point. An uncertainty of $\pm 0.000, \sin (12.7 \mu \mathrm{m})$ is added to this value (Appendix C), which is based on the accuracy to which the $y$ position was manually adjusted in this section.

With the $y$ position adjusted, a final skin friction coefficient, $C_{f}$, is determined and the boundary layer re-normalized to wall units. This boundary layer profile is shown in Figure 5.27. The $C_{f}$ value

was found by fitting the logarithmic region of the boundary layer to Equation 5.18. The values used to fit this curve are highlighted in the figure with the log-law line passing directly through them. In addition, the viscous sublayer line passes directly through the point $u^{+}=y^{+}=5$. As a result, this is the final mean velocity boundary layer profile and the local skin friction coefficient is defined as $C_{f}=0.00460$.

\subsubsection{Boundary Layer Quantities}

Now that all the corrections have been applied to the data, the quantities of interest can be determined. This includes the momentum thickness, $\theta$, and the momentum-thickness based Reynolds number, $\operatorname{Re}_{\theta}$. The skin friction coefficient, $C_{f}$, is also important but was determined in the previous sections during the non-dimensionalization procedure. 


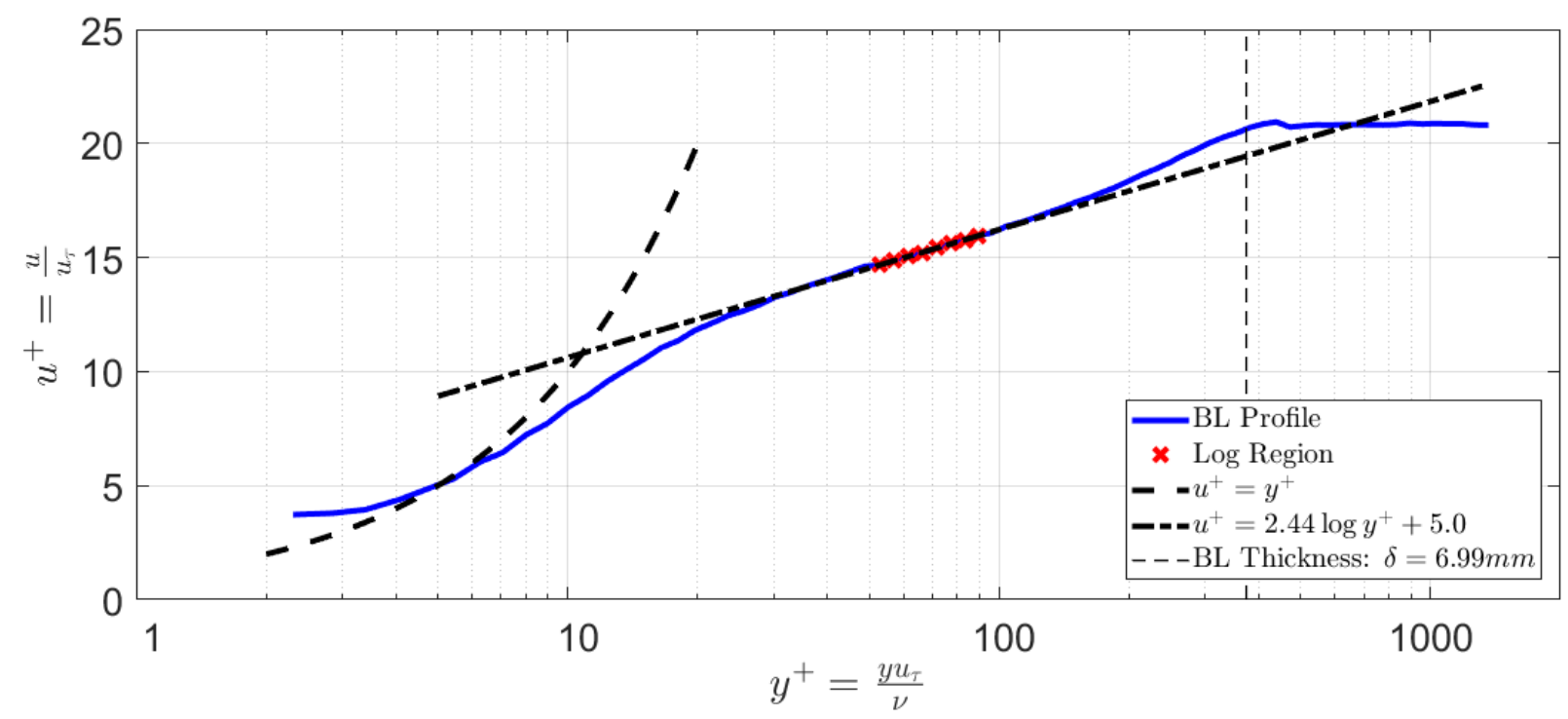

Figure 5.27: Final mean velocity boundary layer profile with the viscous sublayer and log-law lines shown. The red $\times$ 's are the values used to fit the log-law curve

The momentum thickness was initially defined in Equation 2.7 in Section 2.2.2. It is redefined here in Equation 5.19 where once again $u(y)$ is the local velocity, $y$ is the wall position, and $U_{e}$ is the free-stream velocity. In order to apply Equation 5.19, it is modified to the form as shown in Equation 5.20. This is the trapezoidal method of numerical integration, where $N$ is the number of points evaluated in the boundary layer.

$$
\begin{gathered}
\theta(x)=\int_{0}^{\infty} \frac{u(y)}{U_{e}}\left(1-\frac{u(y)}{U_{e}}\right) d y \\
\theta=\frac{1}{2} \sum_{j=1}^{N-1}\left(y_{j+1}-y_{j}\right)\left[\frac{u_{j}}{U_{e}}\left(1-\frac{u_{j}}{U_{e}}\right)+\frac{u_{j+1}}{U_{e}}\left(1-\frac{u_{j+1}}{U_{e}}\right)\right]
\end{gathered}
$$

When using Equation 5.20, not every point in the boundary layer is used. First off, the boundary layer in Figure 5.27, does not have a point at the wall $\left(u^{+}=y^{+}=0\right)$ defining the no-slip condition, so a point is added there. Next, the velocities measured in the viscous sublayer region are not accurate due to the near-wall effect. As a result, all velocities for $y^{+}<5$ are removed. Finally, the momentum thickness is only evaluated up to the limit of the boundary layer thickness, $\delta$ (shown in Figure 5.27). The definition of the momentum thickness (Equation 5.19) integrates until $y=\infty$, which would mean integrating into the free-stream. However, the integrating into the free-stream here would introduce small variations in $\theta$. As a result, the calculation of $\theta$ is cut of at $\delta$ for consistencies sake.

When Equation 5.20 is implemented on the boundary layer shown in Figure 5.27, the resultant 
momentum thickness is $\theta=0.8084 \mathrm{~mm} \pm 0.0101 \mathrm{~mm}$. The uncertainty in this value is very low and is equivalent to $\pm 1.25 \%$. The primary source of this error results from uncertainty in the velocity values, whereas the $y$ value only contributes slightly. This low contribution from $y$ comes from the significant effort spent in experimentally determining the $y$ position (Section 5.1.4.4) and correcting the value during processing (Section 5.2.3). Further discussion of the uncertainty is given in Appendix C.

The other main quantity calculated is this momentum-thickness based Reynolds number, $\operatorname{Re}_{\theta}$. This is defined in Equation 5.21, where $U_{e}$ is the free-stream velocity, $\rho$ is the density, and $\mu$ is the dynamic viscosity. Calculation of the momentum thickness was just described, and the free-stream velocity is the average of the boundary layer profile points that are in the free-stream (Section 5.2.2). Dynamic viscosity and density values are calculated for each point in the boundary layer profile. The dynamic viscosity, $\mu$, is determined using the Sutherland formula in Equation 5.22, where $T$ is temperature measured by the thermocouple in Kelvin. The density is calculated using the ideal gas law in Equation 5.23. $P_{\text {atm }}$ is the atmospheric pressure, which is manually collected from a local weather station, and $R$ is the ideal gas constant. The Reynolds number is then calculated using the mean values of $\mu$ and $\rho$ for the entire profile. This results in $R e_{\theta}=912$ for the boundary layer in Figure 5.27.

$$
\begin{gathered}
\operatorname{Re}_{\theta}=\frac{\rho \theta U_{e}}{\mu} \\
\mu=1.458 \times 10^{-6} \frac{T^{3 / 2}}{T+110.4} \\
\rho=\frac{P_{a t m}}{R T}
\end{gathered}
$$

\subsubsection{Variance, Skewness, and Kurtosis Profiles}

Up to this point in the data analysis procedure, the focus has been on the mean velocity profile. However, when studying turbulent boundary layers, the variance, skewness, and kurtosis should also be studied. These other amplitude-domain quantities were introduced in Section 2.2.3 and are ways of analyzing the distribution of the velocity fluctuation, $u^{\prime}(t)$. For turbulent boundary layers, the time dependent velocity is separated into a mean and fluctuating component: $\tilde{u}(t)=u+u^{\prime}(t)$, where the mean of $u^{\prime}(t)$ is zero. When the variance and other higher order moments are discussed, they only refer to the distribution of the fluctuating velocity component, $u^{\prime}(t)$. The square root of the variance is the standard deviation, $\sigma_{u}$. Since the mean of $u^{\prime}(t)$ is zero, the standard deviation, $\sigma_{u}$, is the same as the RMS (root-mean-square). 
The variance was defined in Equation 2.12 and is annotated as either $\sigma_{u}^{2}$ or $\overline{u^{\prime 2}}$. As is clear from the latter term, the variance here is that of the streamwise velocity component, $u^{\prime}(t)$. This streamwise variance is also known as the streamwise Reynolds stress, and it is generally normalized by the by the friction velocity, $u_{\tau}$. If the wall-normal velocity, $v$, were measured in these experiments, a wall normal Reynolds stress $\left(\overline{v^{\prime 2}} / u_{\tau}\right)$ or the standard Reynolds stress $\left(\overline{u^{\prime} v^{\prime}} / u_{\tau}\right)$ could also be defined.

Figure 5.28 shows the streamwise Reynolds stress $\left(\overline{u^{\prime 2}} / u_{\tau}\right)$ as a function of $y$ position. The Reynolds stress is a measure of the turbulent intensity and thus regions with larger values have more turbulence. The peak Reynolds stress occurs in Figure 5.28 at $y^{+} \approx 15$, which is in the middle of the buffer layer. The buffer layer is the region in the boundary layer with the largest turbulent energy production [47], hence the large streamwise Reynolds stress. In the free-stream, the the Reynolds stress converges to a roughly constant value. This constant value is related to the turbulence intensity of the free-stream. The turbulence intensity, $T u$ is defined in Equation 5.24 and is the ratio of the standard deviation to the mean. The free-stream in the current wind tunnel has a turbulent intensity in percent of $T u \approx 0.8 \%$.

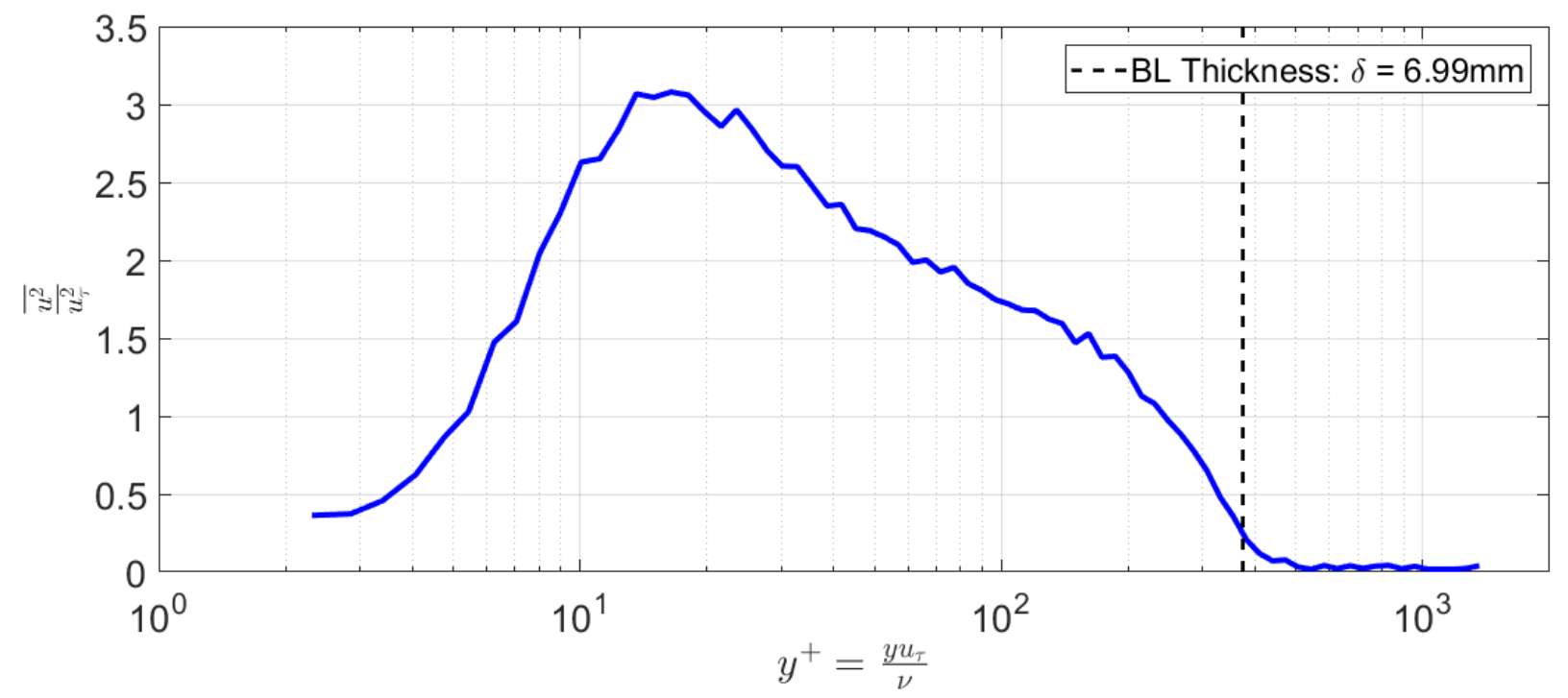

Figure 5.28: The variance/streamwise Reynolds stress as a function of distance from the wall. The boundary layer thickness, $\delta$, is shown.

$$
T u=\frac{\sigma_{u}}{u}
$$

Figures 5.29 and 5.30 show the skewness and kurtosis profiles as a function of the $y$ position. For a Gaussian distribution, the skewness is $S=0$ and the kurtosis is $K=3$. From these profiles, it is clear that the fluctuating velocity, $u^{\prime}(t)$, does not follow a normal distribution at all points in 
the boundary layer. In the free-stream, the skewness and kurtosis values indicate that the velocity is roughly Gaussian. The same can be roughly said for parts of the logarithmic region $(y+=$ $30-100)$. Near the wall, both values increase. The larger skewness indicates an asymmetry towards positive $u^{\prime}$ values and the increase in kurtosis indicates more spikes in velocity. These are likely explained by the bursting events in the buffer layer, which are the violent ejections and in-rushes of fluid [23].

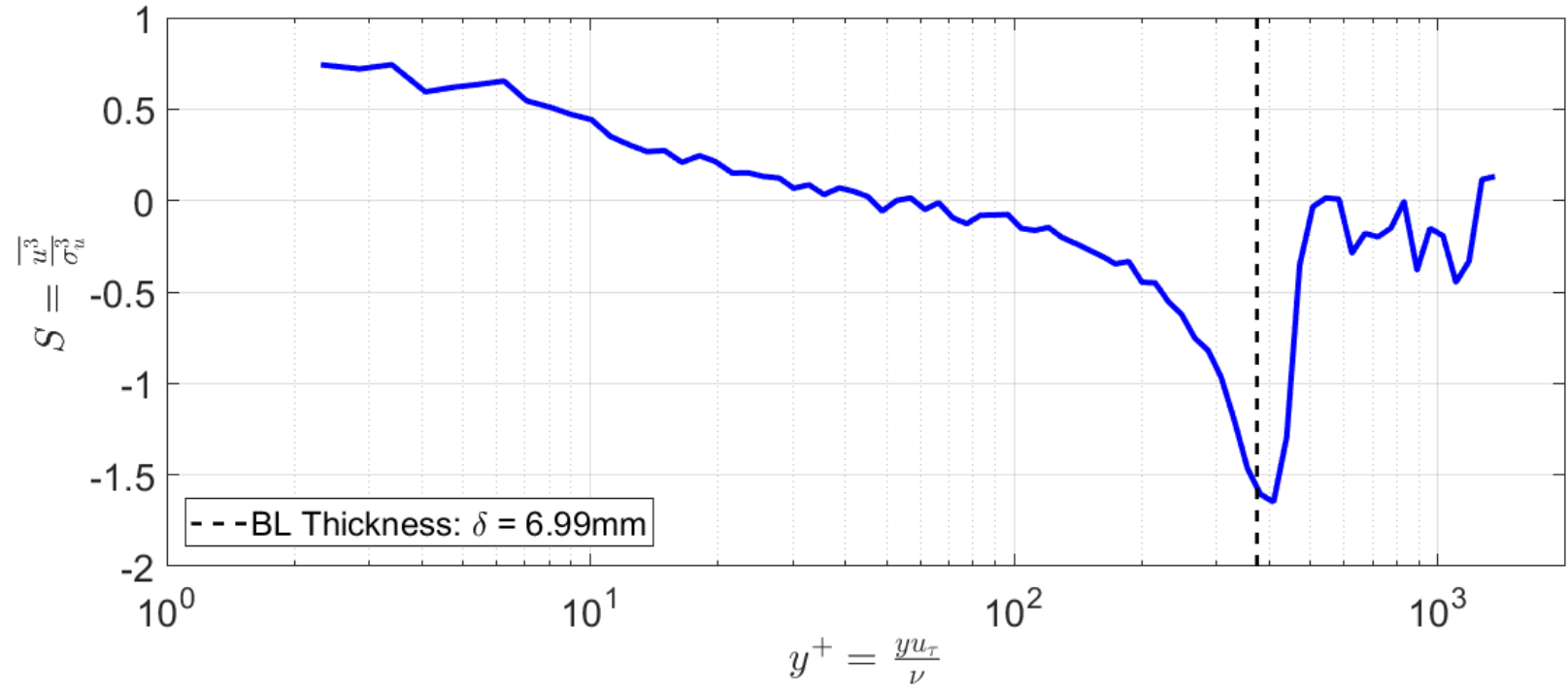

Figure 5.29: The skewness, $S=\frac{\overline{u^{\prime 3}}}{\sigma_{u}^{3}}$ of the fluctuating velocity, $u^{\prime}(t)$, as a function of wall position, $y$

At the edge of the boundary layer, there are sharp changes in the skewness and kurtosis. The large positive kurtosis indicates that there are significant spikes in the fluctuating velocity, $u^{\prime}(t)$. This can be explained by the separation between the boundary layer and the free-stream, or rather that there isn't a clear separation. In this region, there is a turbulent/non-turbulent interface as the flow transitions to the free-stream. However, there is not a clear separation, ration large threedimensional bulges of turbulence with deep irrotational valleys in between which is responsible from entraining fluid into the boundary layer [23]. As a result, the velocity at a given position alternates between turbulence and irrotational flow which yields the large kurtosis. In addition, the vortices tend to flow in a specific direction here, resulting in the skewness spike.

\subsection{Boundary Layer Validation}

Now that the data analysis procedure has been complete, this section validates the procedure and data collection. The first step is the comparison of the laminar boundary layer measured with a 


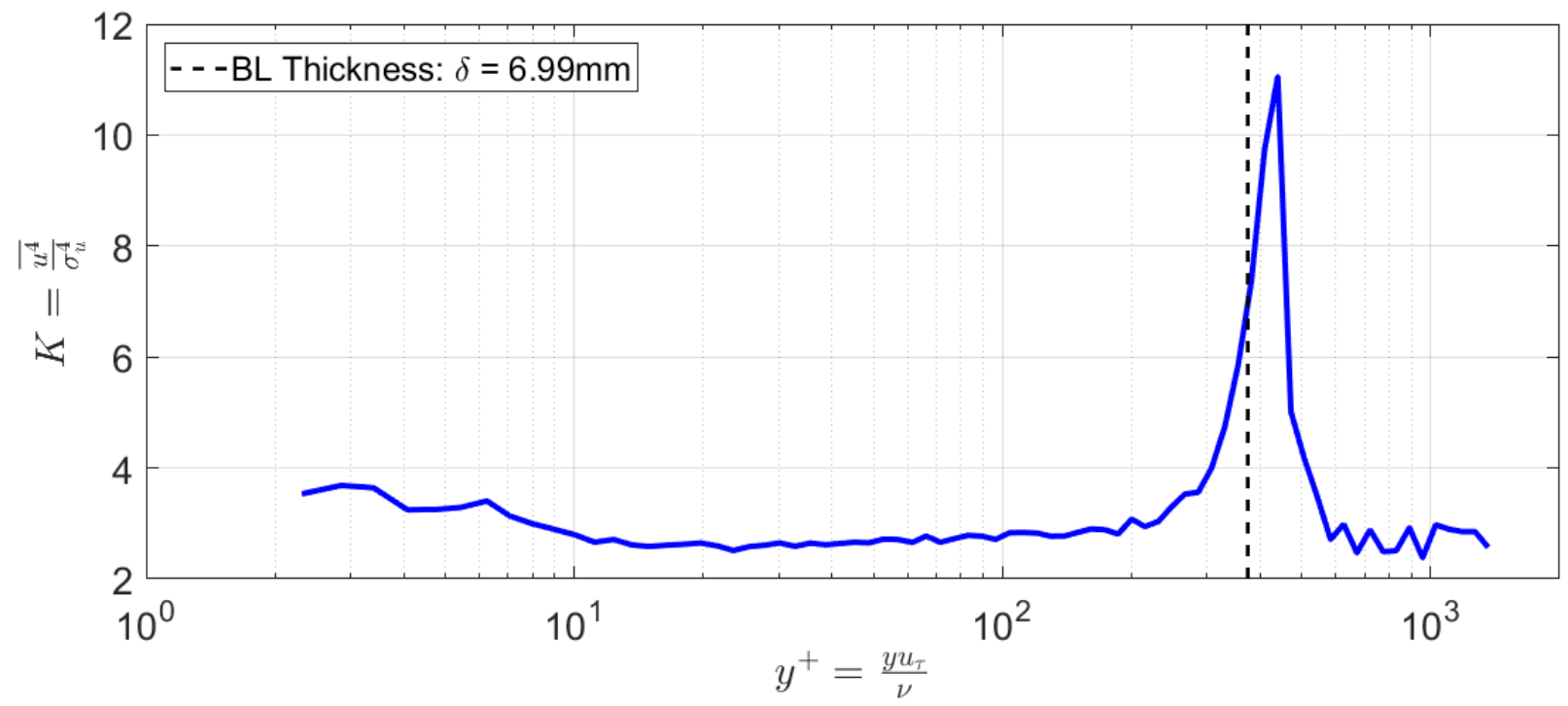

Figure 5.30: The kurtosis, $K=\frac{\overline{u^{4}}}{\sigma_{u}^{4}}$, of the fluctuating velocity, $u^{\prime}(t)$, as a function of wall position, $y$

pitot tube to the Blasius solution. Then, the turbulent boundary layers measured by both the pitot tube and hot-wire probe at the same location are compared. Next, the turbulent boundary layer measured by the the hot-wire probe is compared with accepted data from literature. Finally, the streamwise evolution of the boundary layer is presented.

\subsubsection{Laminar boundary layer against Blasius solution}

Although the turbulent boundary layer is primarily of interest in this research, the initial step is to validate the laminar boundary layer. This is done via comparison to the Blasius solution. The Blasius theory is a solution for the Navier-Stokes equation for the case of zero-pressure gradient flow over a flat plate that allows for the calculation of the boundary layer profile in laminar flow. The theory applies when the following assumption is made: the flow is steady, planar, constantdensity, and constant-property and has a uniform velocity prior to reaching the plate [46]. The basis of the solution is that laminar boundary layers provide similar solutions. Specifically, that when the velocity and $y$ position are normalized, all boundary profiles possess the same form.

The Blasius solution is obtained by non-dimensionalizing the Navier-Stokes equation under the above defined assumptions. In particular, the local velocity is defined as shown in Equation 5.25, where $u$ is the local velocity, $U_{e}$ is the free-stream velocity, $f^{\prime}$ is the form of the velocity profile, and $\eta$ is the non-dimensionalized position. Equation 5.26 shows the definition of eta, where $y$ is the height above the plate, $U_{e}$ the free-stream velocity, $v$ the kinematic viscosity, and $x$ the streamwise 
length from the leading edge of the plate. The profile $f^{\prime}$ is the solution of the nonlinear PDE shown in Equation 5.27, where ()$^{\prime}$ is the derivative with respect to $\eta$. The solution of Equation 5.27 is given as a table giving the value of $f^{\prime}(\eta)$ for different values of $\eta$ [46]. Thus the velocity profile for a laminar boundary layer is plotted by defining values for $U_{e}, v$, and $x$.

$$
\begin{gathered}
\frac{u}{U_{e}}=f^{\prime}(\eta) \\
\eta=y \sqrt{\frac{U_{e}}{2 v x}} \\
f^{\prime \prime \prime}+f f^{\prime \prime}=0
\end{gathered}
$$

The laminar boundary layer was measured in the current setup using a pitot tube at $x_{p}=$ $-19 \mathrm{~mm}$, just just upstream of the traveling wave plate. he boundary layer at this location is laminar, since the trip strip (5.4) forcing transition to turbulence has not been installed. The Blasius solution of the velocity profile was then calculated using the experimentally measured free-stream velocity and $x=356 \mathrm{~mm}$ : the distance from the leading edge to the measurement location (Figure 5.3). Figure 5.31 shows the experimentally measured laminar boundary layer and the calculated Blasius solution. While the measured boundary layer has slightly larger velocities between 0.5 and $1.5 \mathrm{~mm}$, the profiles match each other fairly well. The deviation at the smallest $y$ position results from a slight inaccuracy in defining the $y$ position of the pitot tube. The momentum thicknesses, $\theta$, for each of these boundary layers are also shown in Figure 5.31 and match with a difference of $<0.1 \%$.

\subsubsection{Comparison between Pitot tube and Hot-wire probe}

As was discussed in Section 5.1.4, both the pitot tube and hot-wire probe were used to measure the flow velocity and map the boundary layer. The pitot tube was used initially, primarily due to its simplicity. The hot-wire (HW) probe was implemented later since it can measure closer to the wall and also capture turbulence phenomena. Due to the expanded capability of the HW probe, it was used to collect all experimental results. However, since both the pitot tube and HW probe are capable of measuring the mean velocity profile, they are compared here.

Figure 5.32 shows the turbulent mean velocity profiles measured using the pitot tube and hotwire probe. Both profiles have been normalized to wall units $\left(y^{+}\right.$and $\left.u^{+}\right)$. The local skin friction coefficient, $C_{f}$, for each profile was found by fitting the logarithmic portion of the boundary layer (Section 5.2.2). In both cases, $C_{f} \approx 0.0046$. From Figure 5.32, it is clear that the boundary layers measured using each of the probes match up fairly well. Both profiles diverge from the log region 


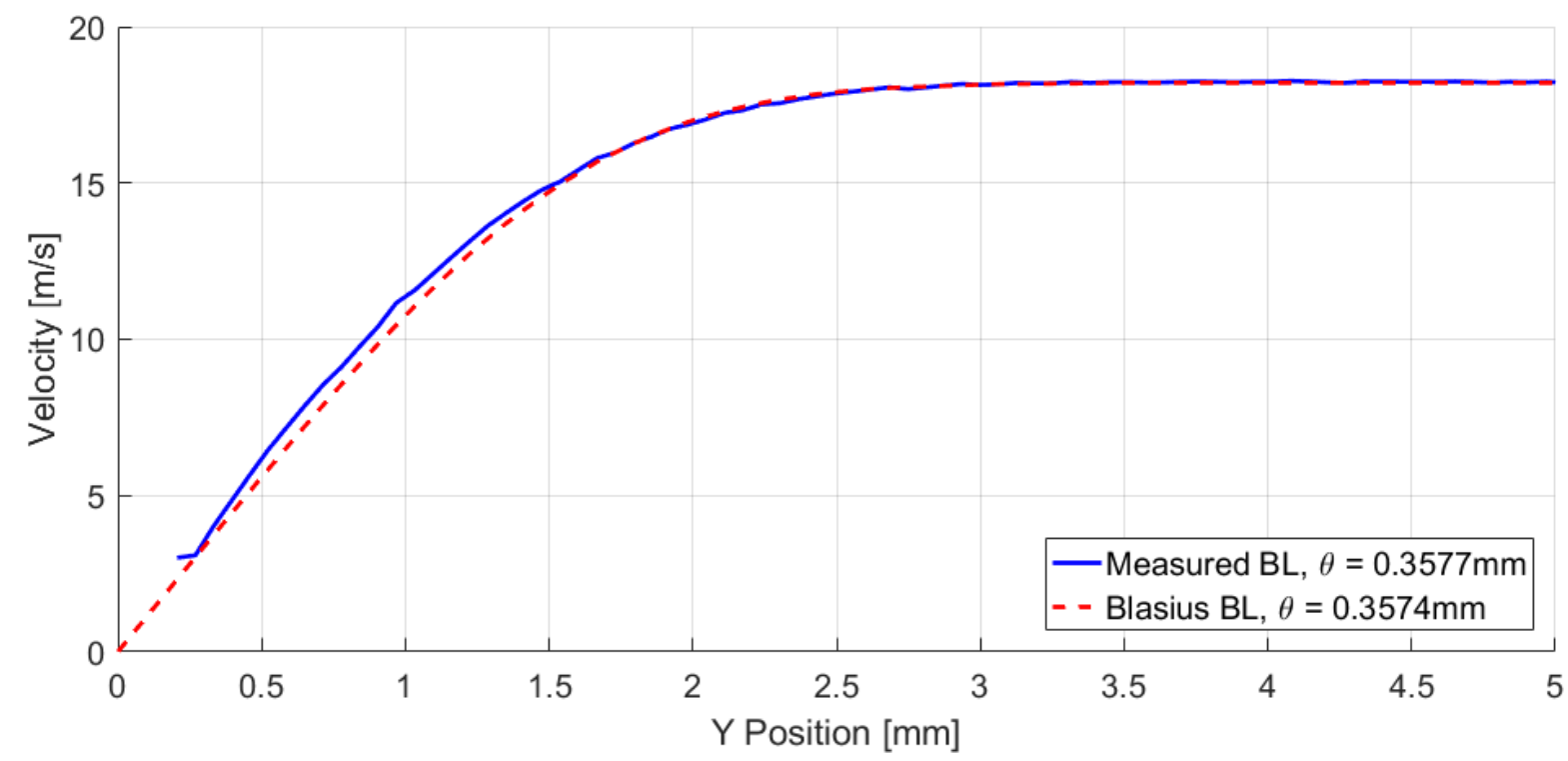

Figure 5.31: Comparison between experimentally measured laminar boundary layer and the Blasius solution for $\operatorname{Re}_{\theta}=359$ with momentum thicknesses shown

together, converge to the same free-stream velocity, and have roughly the same boundary layer thickness. The main difference is that the pitot tube cannot measure as close to the wall as the HW probe. The smallest $y$ position for the pitot tube is $y^{+} \approx 15$, while it is $y^{+} \approx 2$ for the HW probe. As a result, the HW probe can measure all of the buffer layer and into the viscous sublayer; even though near wall effect reduces the usability of these values (Section 5.1.4.3).

The other difference is that the pitot tube velocity values in Figure 5.32 rise above the log-law in the lower log region $\left(y^{+} 25-50\right)$. This could result from errors in the $y$ position of the pitot tube. The $y$ position of the HW probe was found by manually adjusting the boundary layer profile such that it crossed $u^{+}=y^{+}=5$ (Section 5.2.3). However, the smallest wall-normal position for the pitot tube is at $y+=15$, so this alignment method cannot be done. The $y$ position of the pitot tube is adjusted only using only its physical dimensions (i.e. the diameter of the tube where it touches the surface). As a result, there are larger errors in the $y$ position of the pitot tube, that disproportionately affect the profile close to the wall.

An additional factor to consider is that correction factors must be applied to any pitot tube measurement. These are discussed in Appendix B.1, and allow the pitot tube to fairly accurately measure the velocity profile in the boundary layer. Unfortunately, the contribution from these correction factors increase as the the pitot tube nears the wall. This is particularly important since the turbulence intensity is largest in the buffer layer near the wall $\left(y^{+} \approx 15\right)$ and affects the pitot velocity measurements. There is a correction factor, but it requires knowledge of the fluctuating velocity. A hot-wire probe would be needed to measure this quantity, which at that point would 


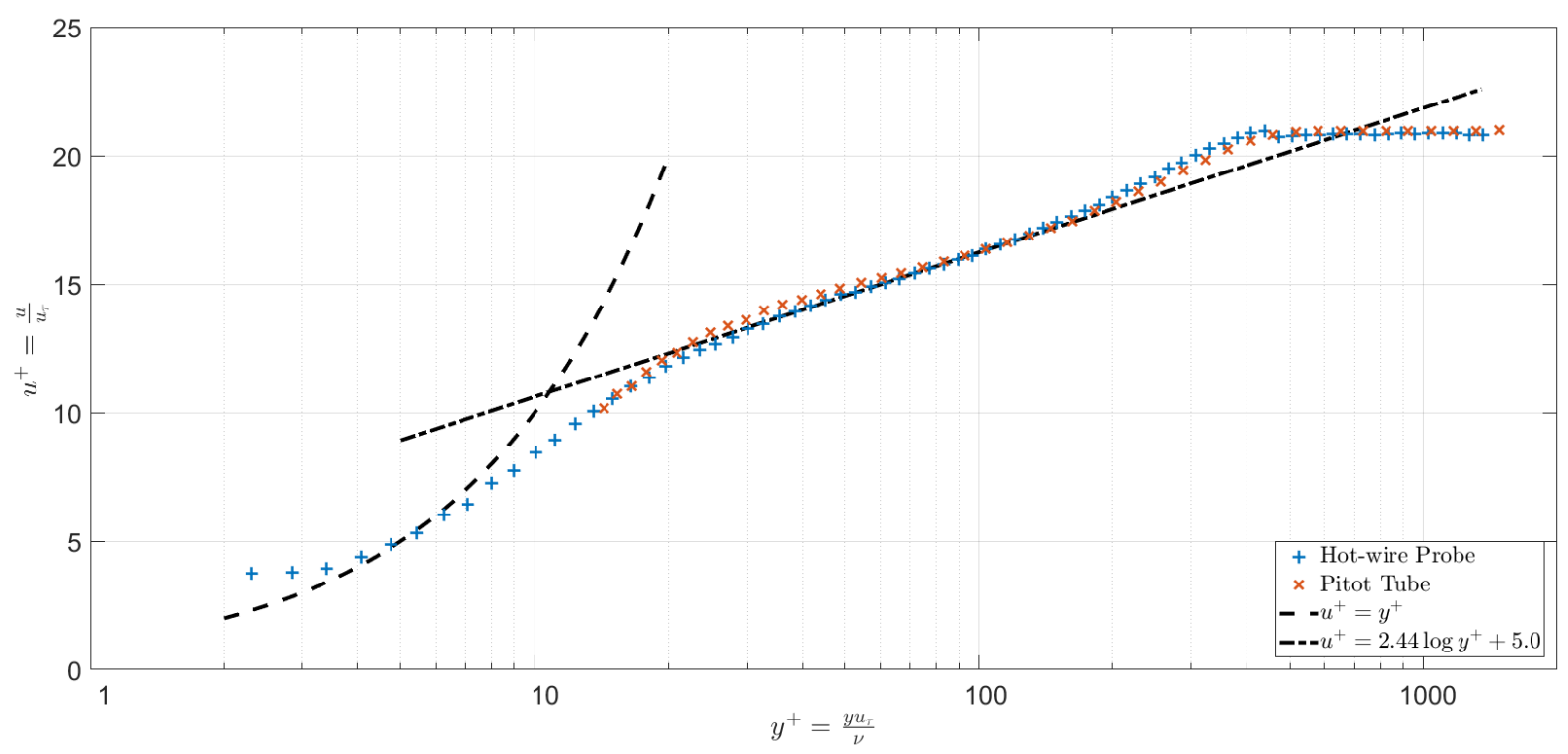

Figure 5.32: Comparison between the turbulent boundary layer measured using the hot-wire probe and pitot tube

defeat the purpose of using the pitot tube in the first place.

Overall, Figure 5.32 shows that both the pitot tube and hot-wire probe do a good job of measuring the mean velocity profile in the turbulent boundary layer. However, the hot-wire probe provides a more accurate measurement. It can measure closer to the wall, the $y$ position error can be minimized, and and it can measure turbulent phenomena. As a result, all experiments are conducted using the hot-wire probe.

\subsubsection{Validation of boundary layer data against literature}

In order to validate the turbulent boundary layer, it is compared here against accepted data from literature. Figure 5.33 shows the boundary layer measured using the hot-wire (HW) probe just upstream of the traveling wave plate at $x_{p}=-19 \mathrm{~mm}$. Also shown in the Figure are various experimental boundary layers from Purtell [14] alongside the viscous sublayer and log-law curves. The profiles have been normalized to wall units and the Reynolds number, $\operatorname{Re}_{\theta}$ and skin friction coefficient, $C_{f}$, are given. All of the profiles follow the log-law line and then diverge in the buffer layer in order to converge with the viscous sublayer line. The HW probe data does not converge with the viscous sublayer, but this is due to the near wall effect of the hot-wire probe (Section 5.1.4.3).

Far from the wall, the boundary layer profiles diverge from the the log-law curve at different $y$ values. This is the wake region and the divergence is Reynolds number dependent. In addition, 


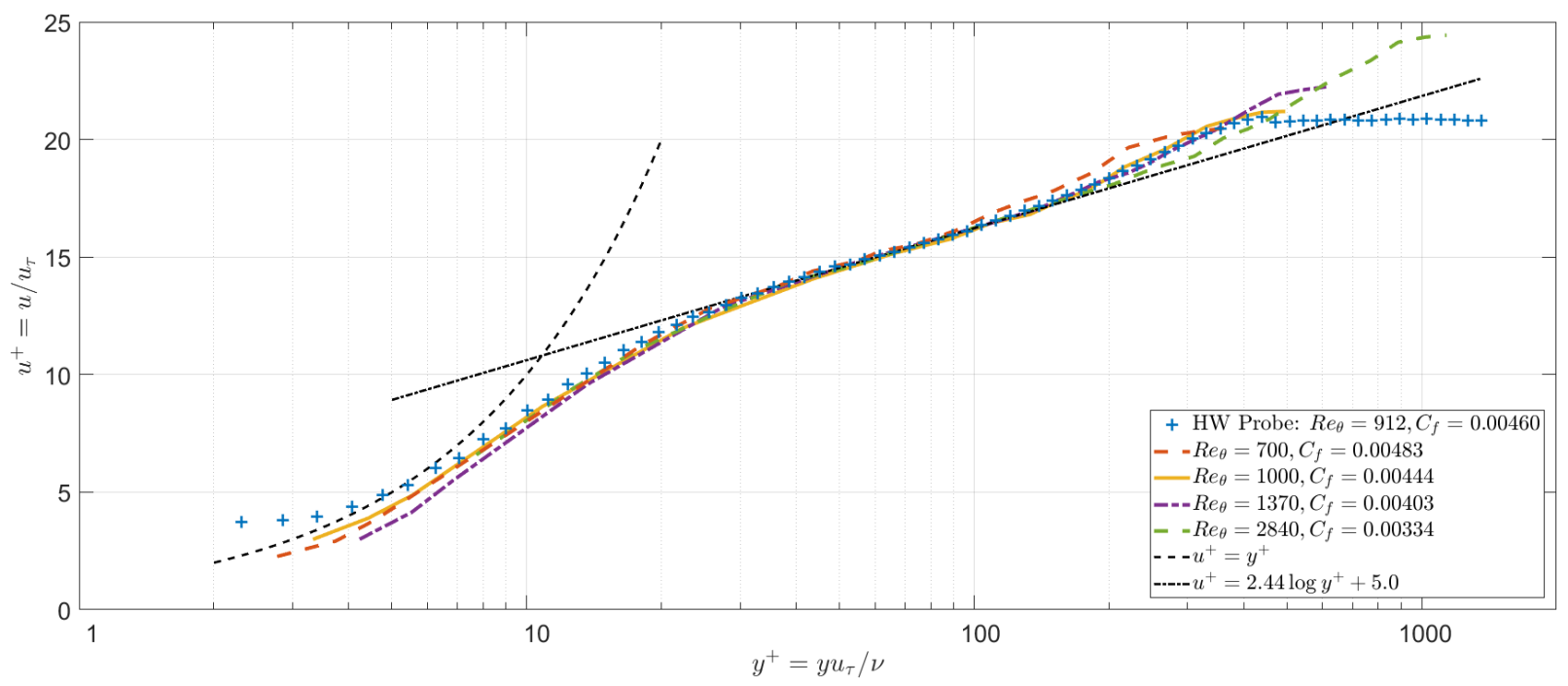

Figure 5.33: Comparison of the turbulent boundary layer measured using a hot-wire probe with data from Purtell [14]

as the Reynolds number increases, both the boundary layer thickness and the non-dimensional free-stream velocity increase. This is a product of non-dimensionalizing to wall units. They are an inner scaling method, so the boundary layer profiles all converge at small $y$ positions, but then diverge at large values. As a result, the turbulent boundary layer follows a profile that is dependent on the Reynolds number, $\operatorname{Re}_{\theta}$.

The experimental boundary layer measured here with the hot-wire probe has $\operatorname{Re}_{\theta}=912$. When comparing this profile to those from literature in Figure 5.33, it matches well the $\operatorname{Re}_{\theta}=1000$ profile, which is that with the closest Reynolds number. The free-stream velocity for the $R e_{\theta}=1000$ profile is slightly larger, but this makes sense since the HW probe Reynolds number is smaller. As a result, Figure 5.33 confirms that the turbulent boundary layer profiles measured in this experimental setup are valid.

\subsubsection{Streamwise Evolution of Boundary Layer}

Now that the experimental boundary layers have been validated against literature, the growth of the boundary layer in the streamwise direction is analyzed. Figure 5.34 shows the boundary layer measured here at five different streamwise locations. The Reynolds number, $\operatorname{Re}_{\theta}$, and streamwise positions, $x_{p}$, are shown in the figure, where the streamwise positions reference the locations in Figure 5.3. For $y^{+}<100$, all of the profiles converge and follow the log-law curve and then approach the viscous sublayer line before diverging due to the near wall effect. Above the logarithmic region, all of the boundary layers diverge from one another. As the $x$ position increases, the boundary layer 
grows, yielding increases in the momentum thickness, $\theta$, and in turn the Reynolds number, $\operatorname{Re}_{\theta}$. Thus, similar to Figure 5.33, as the Reynolds number increases, so does the free-stream velocity and the boundary layer thickness.

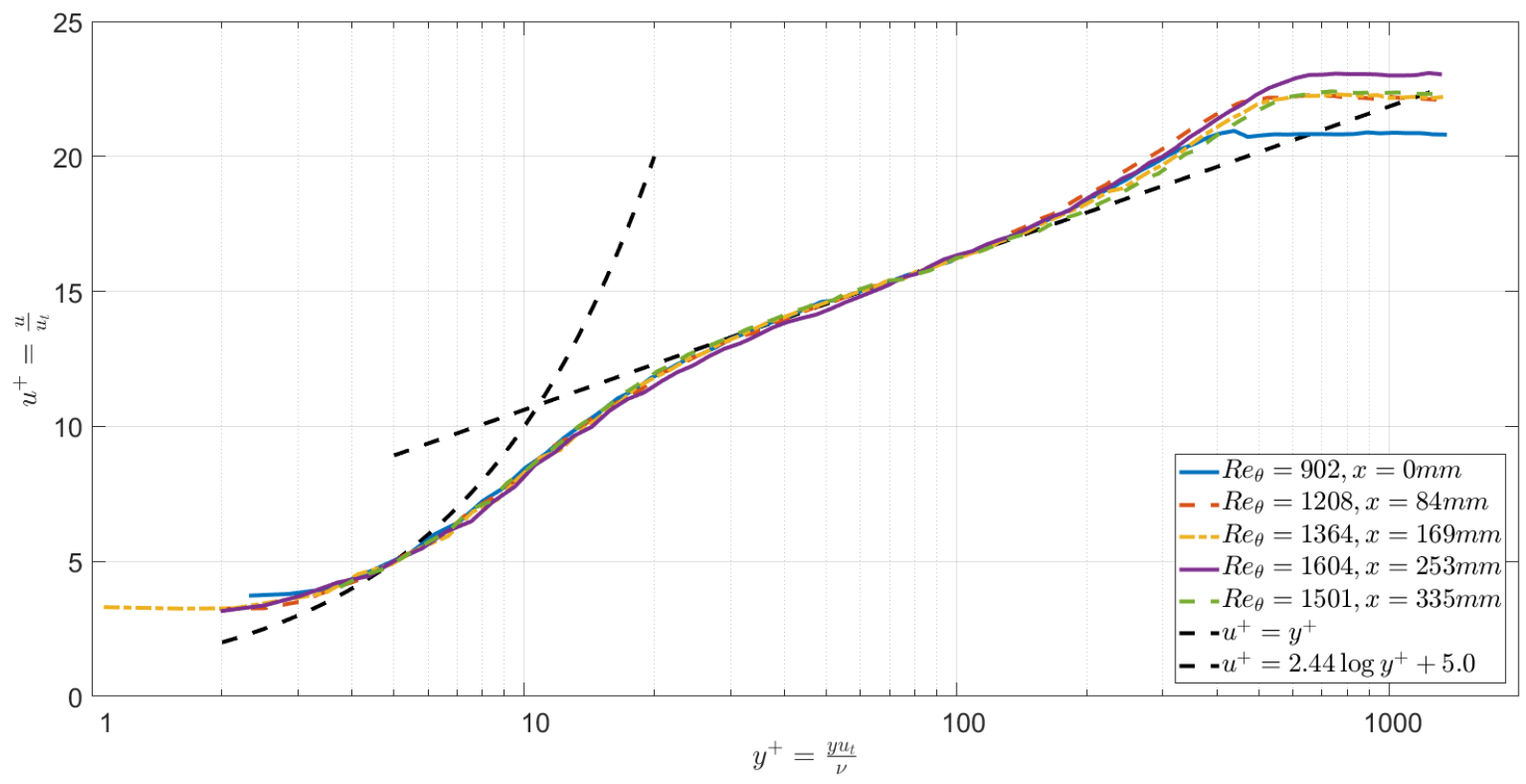

Figure 5.34: Turbulent boundary layer profiles at five different streamwise positions

It should be pointed out here that the Reynolds number actually decreases for the point furthest downstream, $x=335 \mathrm{~mm}$. This decrease downstream is odd, since the momentum thickness should always grow as the boundary layer progresses downstream. However, looking at the streamwise pressure gradient (Figure 5.9), there is a slight favorable gradient at this same streamwise location. The growth of the momentum thickness is affected by the pressure gradient [95]; thus, this pressure gradient most likely affects the momentum thickness growth.

The profiles in Figure 5.34 have all been non-dimensionalized to wall units with a skin friction coefficient found using the Clauser method (Section 5.2.2). As the boundary layer grows, the skin friction coefficient decreases and can be represented using the empirical curve defined by Coles [15]. This curve is shown in Figure 5.35 where the skin friction, $C_{f}$, decreases with increasing Reynolds number, $R e_{\theta}$. Plotted on top of the curve are the skin friction values versus the Reynolds numbers for the streamwise boundary layers (Figure 5.34). The agreement between the data and the curve indicates that the presence of a standard turbulent boundary layer. 


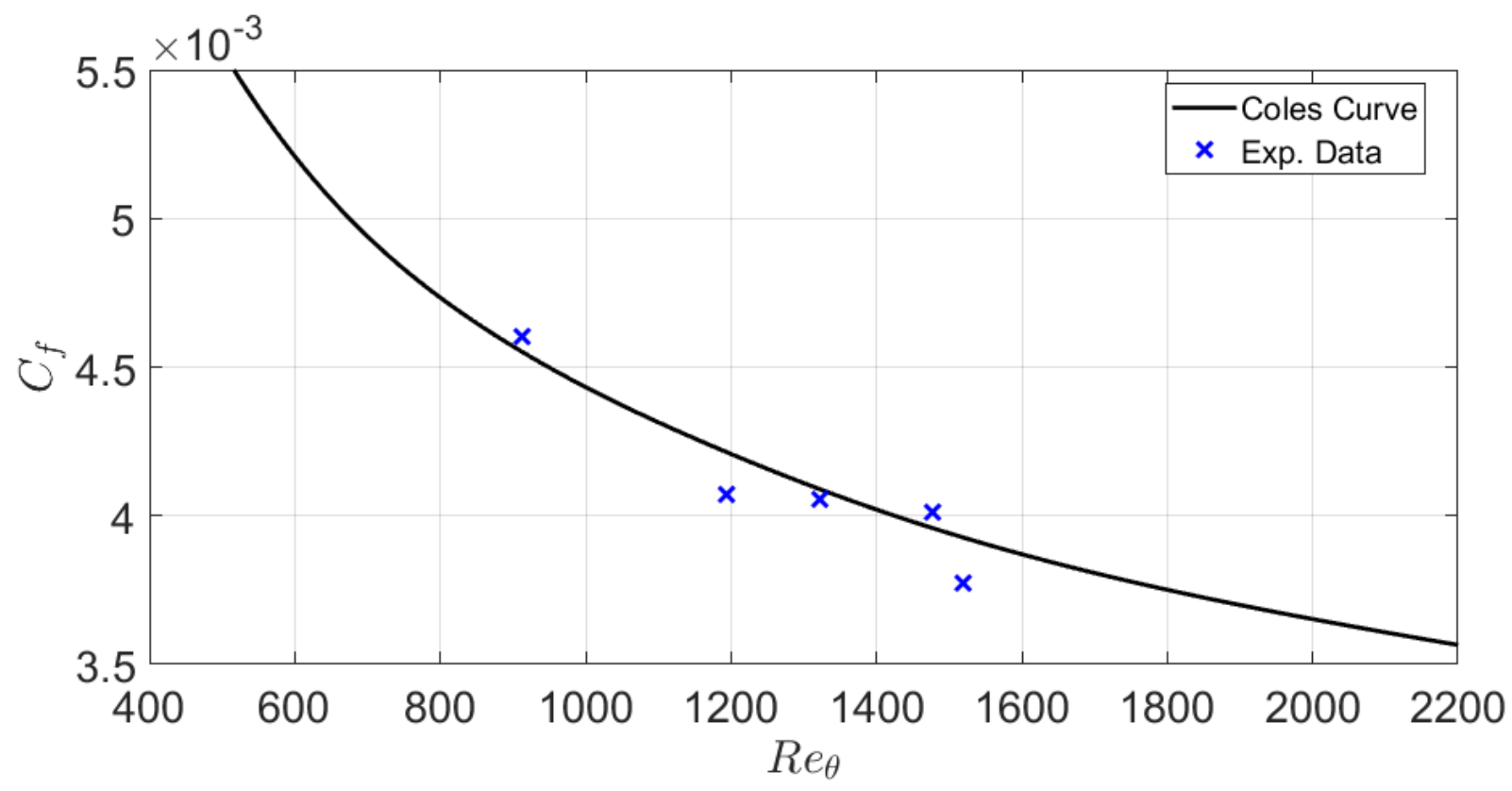

Figure 5.35: Evolution of the skin friction coefficient, $C_{f}$, with growth in the boudnary layer (increasing $R e_{\theta}$ ). The values from the streamwise boundary layer profiles are plotted alongside the empirical Coles curve [15] 


\section{Chapter 6}

\section{Turbulent Boundary Layer over Traveling Waves}

Previous research has shown that spanwise traveling waves are a promising method for manipulating the turbulent boundary layer with the aim reducing skin friction drag/shear stress. However, the experimental setups from these studies are bulky and not practically implementable (see Chapter 3). This research has focused on developing a traveling wave generation method that is low-profile and could be applied in the field for the manipulation of turbulent boundary layers. Chapter 4 introduced a novel method of generating steady-state traveling waves on a two-dimensional surface using flush-mounted piezoelectric actuators. The actuators are excited in an open-loop fashion and the resultant wave propagation pattern can be controlled by varying the number and placement of these actuators. It was shown that two-dimensional traveling waves could be generated and tailored for various applications.

These traveling waves are experimentally tested in this chapter to determine their ability to affect the turbulent boundary layer. The clamped traveling wave plate from Chapter 4 is installed in the low-speed wind tunnel described in Chapter 5. A single frequency traveling wave is then generated and the boundary layer measured at various points along the actuated surface. The resultant data shows that the traveling wave generation method investigated here is capable of of manipulating the turbulent boundary layer and suggests that a reduction of skin friction drag is possible.

The chapter begins by discussing the generation of traveling waves in the wind tunnel with flow over the surface. The installation of the traveling wave plate and the experimental setup necessary to excite and measure the waves is presented. The same plate used in Chapter 4 is installed in the wind tunnel; thus, the variation in the structural response due to the mounting conditions and air flow are investigated. This is primarily done to determine whether the model for generating traveling waves developed in Chapter 4 could be applied to the plate when installed in the wind 
tunnel. Following this, traveling wave generation in the wind tunnel is presented. This includes a discussion of normalizing the traveling wave parameters to boundary layer units and also the optimal wave parameters necessary to affect the boundary layer.

The chapter then explains the experimental setup and analysis procedure necessary for measuring the turbulent boundary layer over the traveling wave actuated surface. The main wind tunnel setup and boundary layer measurement procedure are explained in Chapter 5, but updates to that procedure are provided here. A phase-locked data analysis method is then outlined to examine the boundary layer data at specific time instances over the traveling wave surface. In particular, phase-locked profiles are generated over the crest and trough of oscillating surface as well as at the two points of zero displacement (positive and negative wall-normal velocity). Thus, the turbulent boundary layer is analyzed for the time-averaged case as well as for four phase-locked positions.

The results of exciting the surface with a single frequency traveling wave are then presented. This begins by analyzing the turbulent boundary layer at six streamwise positions: upstream, directly over, and downstream of the traveling waves. The time-averaged mean velocity profiles over the unactuated and traveling wave actuated surface are compared, before analyzing the phaselocked profiles. Then, higher order moments of the fluctuating flow velocity then presented. The variance/streamwise Reynolds stress profiles at multiple streamwise positions are examined for both the time-averaged and phase-locked conditions. Then, the skewness and kurtosis profiles are discussed for a single location. Next, the mean velocity and variance profiles are investigated at multiple spanwise positions. Finally, the changes in the boundary layer profile are discussed while considering the experimental uncertainty.

This chapter finishes by summarizing and comparing the experimental boundary layer results with that seen in literature. These results show that the traveling waves generated in this study affect the turbulent boundary layer in similar ways to what is seen in other studies, but with the advantage of implementation. The experimental procedure utilized here prevents the drag reduction from being quantified; however, the results suggest a reduction in the skin friction drag. Thus, the agreeable comparison with literature and the potential drag reduction demonstrates the implementability of the novel traveling wave generation method investigated in this research.

\subsection{Traveling Waves in Wind Tunnel}

Chapter 4 focused on generating steady-state traveling waves on a two-dimensional plate using piezoelectric actuators. That methodology is implemented here to generate traveling waves in the wind tunnel and investigate their effect on the turbulent boundary layer.

This section begins by explaining the experimental setup necessary to generate and measure traveling waves in the wind tunnel. Then, the frequency response of the plate is analyzed. Since 
the same plate was used in Chapter 4, variations in the plate's structural response are discussed for the change in mounting condition and air flow over the surface. This change denotes whether the model developed in Chapter 4 can be used to generate traveling waves. Finally, traveling waves are generated in the wind tunnel during operation. In this chapter, a single frequency traveling wave is generated to investigate the effect on the turbulent boundary layer. The parameters of this single wave (e.g. frequency, amplitude) are chosen based on results from previous studies so as to maximize the effect on the boundary layer.

\subsubsection{Experimental Setup}

Traveling waves are generated here on a clamped aluminum plate with dimensions of $292.1 \times$ $190.5 \times 0.397 \mathrm{~mm}(7.5 \times 11.5 \times 1 / 64 \mathrm{in})($ Same as in Chapter 4$)$. This plate is mounted into a cavity in the floating wall which is installed in the test-section of the wind tunnel; this was described in Section 5.1.2 and is also shown in Figure 6.1. The top side of the plate is exposed to the air flow while the underside is in a semi-sealed cavity. Mounted to the underside of the plate, in the cavity, are ten macro-fiber composite (MFC) piezoelectric actuators. These excite the plate in the out-ofplane direction and are used to generate the traveling waves that propagate in the spanwise direction (perpendicular to flow). In order to actuate the MFCs, the analog voltage signal is delivered to the positive and negative electrodes for each actuator: for $10 \mathrm{MFCs}$, this is 20 separate connections. This is done by passing 10 thin-gauge wires through each of two holes drilled through through the side of the floating wall and into the cavity (Figure 6.1). These wires then bridge the gap from the floating wall to the test-section side walls and then exit the test-section through foam lined holes. These wires are then broken out based on each actuator. These actuators are controlled via the same Labview GUI that controls the rest of the wind tunnel. Up to four different voltage signals can be generated from a dedicated four channel analog output card. For traveling waves, each output carries the same signal, but has a different phase difference: $\left(0^{\circ}\right),\left(0^{\circ}+\phi\right),\left(180^{\circ}\right)$, and $\left(180^{\circ}+\phi\right)$. Each signal is then amplified $100 \times$ with a separate amplifier channel. Each of the 10 MFC actuators are then excited with one of these signals, where the overall actuation configuration is dependent on the traveling wave generated.

As in Chapter 4, the response of the plate is measured using a Polytec PSV400 scanning laser doppler vibrometer (SLDV). The SLDV is a non-contact method of measuring the out-of-plane velocity of the plate at a single location. The response of the plate is then determined by scanning over the surface and measuring at a grid of locations. The total response of the plate is then visualized by reconstructing all of these points. The traveling waves are measured here by mounting the SLDV outside of the wind tunnel and then shining the laser through the optically clear walls of the test-section. As was explained in Section 5.1.4.1, the ceiling of the test-section was manufactured 


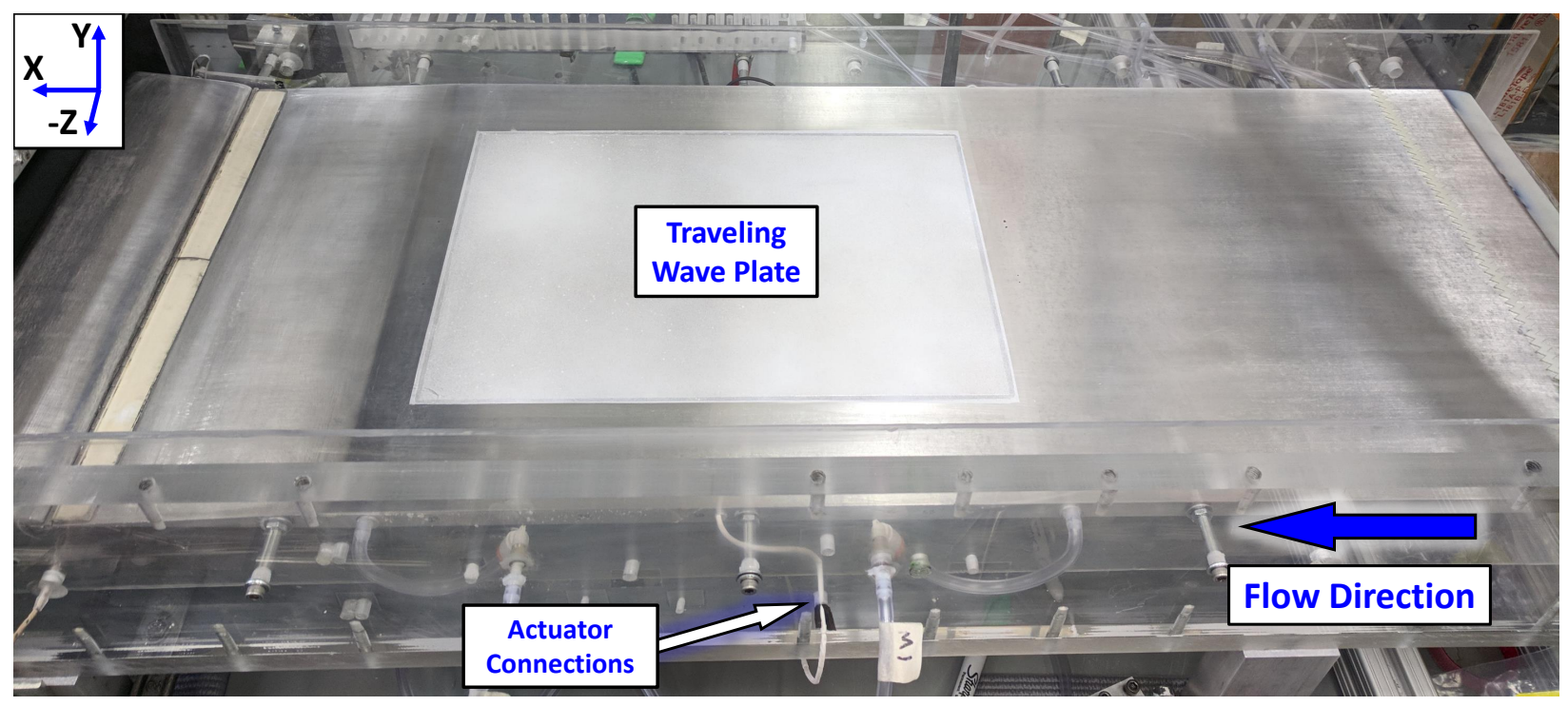

Figure 6.1: Traveling wave plate mounted in the floating wall in the test-section of the wind tunnel. One set of actuator connections is seen passing through the test-section side wall. There is a mirrored connection on the far side of the floating wall.

to provide an unobstructed view of the traveling wave plate. Figure 6.2 shows the SLDV mounted to the $80 / 20$ frame of the wind tunnel and shining through the test-section. For the SLDV to operate properly, the measurement surface must diffuse light in all directions (i.e. not reflective). Thus, a thin coating of white powder (Magnaflux SKD-S2 developer) was sprayed onto the surface. This powder can be seen in Figure 6.1 and remains adhered during wind tunnel operation. The boundary layer was measured both with and without the powder and no differences were noticed. Thus, the roughness of the powder is considered to be well within the viscous sublayer $\left(k^{+}<5\right)$.

\subsubsection{Frequency Response}

The first step in generating traveling waves is to determine the frequency response of the structure. This was conducted in Chapter 4 for this plate and a model was developed that was capable of accurately generating the traveling waves. However, as discussed in Section 4.2.1, this thin plate is sensitive to the mounting conditions. In Chapter 4, great care was taken when clamping the plate such that pre-stresses in the plate were minimized. This was done through repeated comparisons of the experimental and simulated structural response. In addition, the underside of the plate was intentionally left open to eliminate effects from having a sealed air cavity. This includes mass and stiffness loading and also acoustic coupling.

Unfortunately, when the plate is mounted in the wind tunnel, these effects could not be eliminated. For aerodynamic reasons, the underside of the plate is in a semi-sealed cavity with $\approx$ 


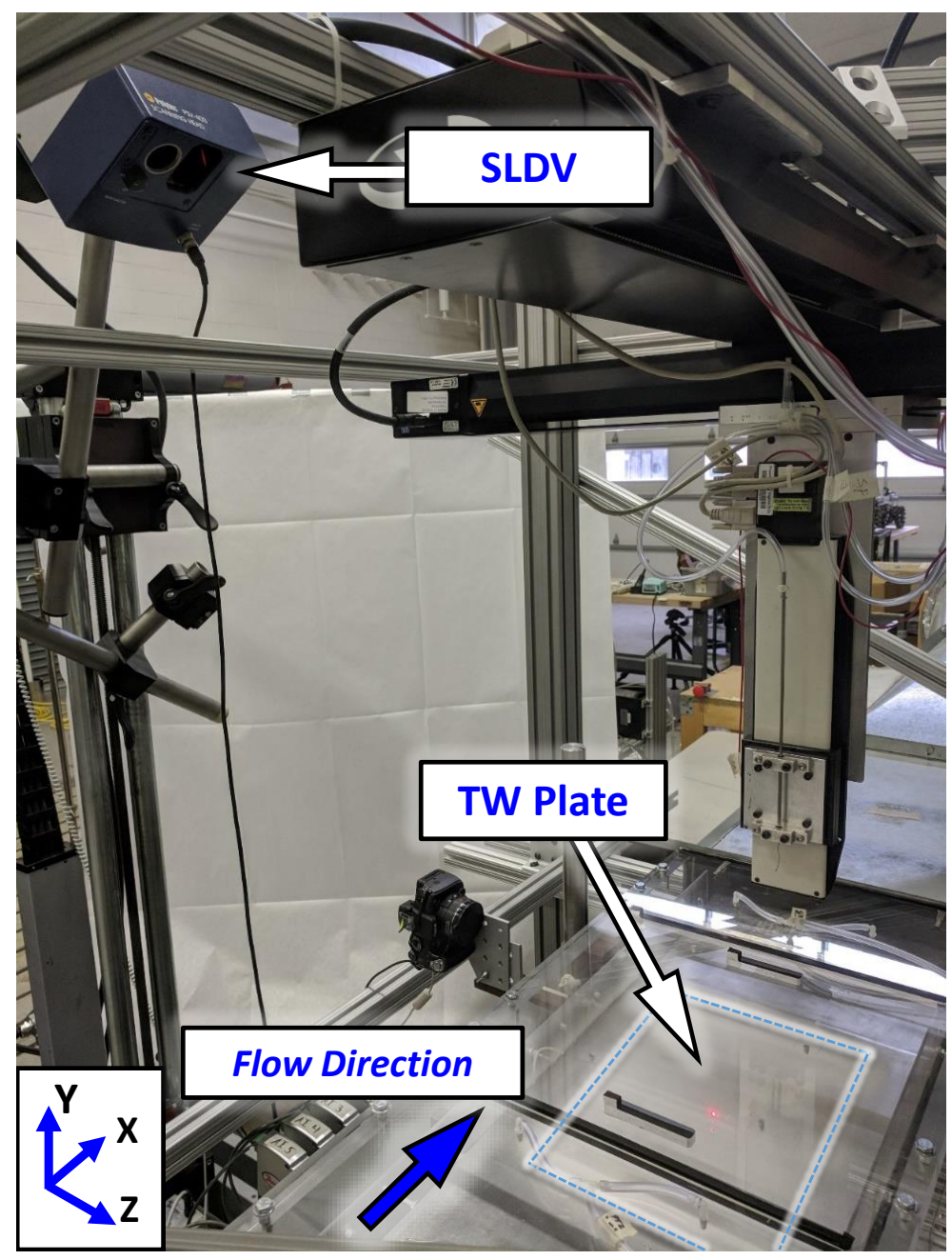

Figure 6.2: Scanning laser doppler vibrometer (SLDV) measuring the out-of-plane velocity of the traveling wave plate in the wind tunnel during operation.

$6 m m(0.25 \mathrm{in})$ between the plate and the surface below. The effects from the cavity then disrupt the accuracy of the model. As a result, the pre-stresses can no longer be minimized through repeated comparison between experiment and simulation. Both of these lead to changes in the structural response of the plate that question the validity of the model developed in Chapter 4. This is even further exacerbated when the wind tunnel is in operation. The air flow over the top of the surface introduces fluid-structure interaction effects that alter the plate's dynamic response.

Figure 6.3 shows the frequency response of the clamped traveling wave plate for three different conditions: the ideal experimental mounting from Chapter 4, in the wind tunnel with no airflow $\left(U_{e}=0 \mathrm{~m} / \mathrm{s}\right)$, and in the wind tunnel with a free-stream velocity of $U_{e}=18.5 \mathrm{~m} / \mathrm{s}$. From this figure it is clear that the response varies for each case. This is further confirmed by looking at the modal assurance criterion (MAC) values between the cases. Figure 6.4a shows the MAC values between 
the no airflow and ideal cases and Figure $6.4 \mathrm{~b}$ compares the $U_{e}=18.5 \mathrm{~m} / \mathrm{s}$ and $U_{e}=0 \mathrm{~m} / \mathrm{s}$ cases.

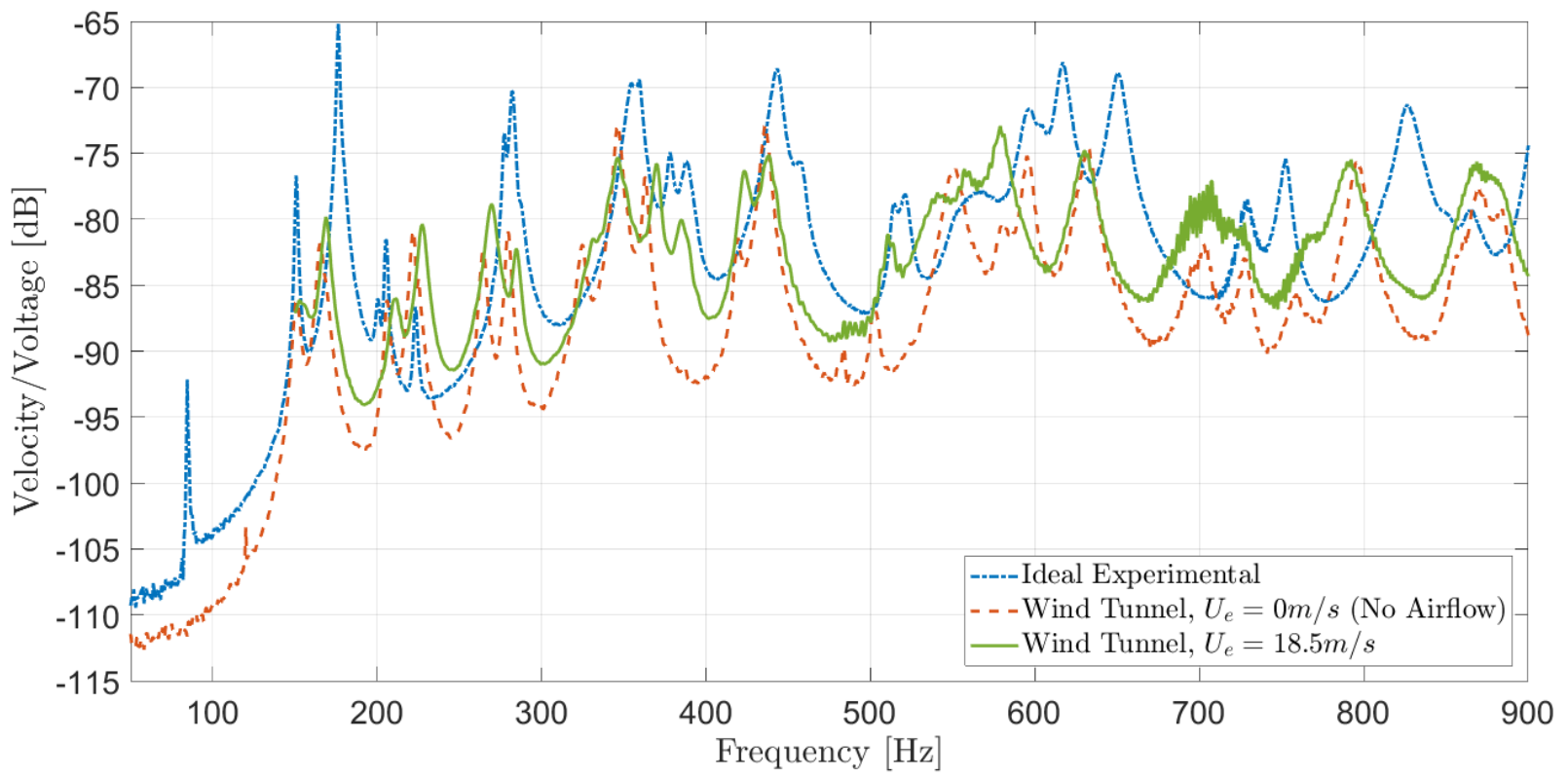

Figure 6.3: Frequency response of the clamped traveling wave plate for three different cases. This includes the ideal experimental mounting (Chapter 4), in the wind tunnel with no airflow $\left(U_{e}=18.5 \mathrm{~m} / \mathrm{s}\right)$, and in the wind tunnel with air flow $\left(U_{e}=18.5 \mathrm{~m} / \mathrm{s}\right) .0 \mathrm{~dB}=1 \mathrm{~m} / \mathrm{s} / \mathrm{V}$

The first natural frequency of the ideal case is the $(1,1)$ mode at $f=84.5 \mathrm{~Hz}$ and is seen in Figure 6.5a. The mode shapes are defined as $(m, n)$, where $m$ is the number of anti-nodes (peaks) in the $x$ direction, and $n$ the number in the $z$ direction. While the $(1,1)$ mode is seen for the ideal case, it is not observed when the plate is mounted in the wind tunnel. Rather the the first observable modes for the wind tunnel cases are shown in Figures 6.6a and 6.6b. These are the $(2,1)$ modes and they occur at $f=150.6 \mathrm{~Hz}$ for $U_{e}=0 \mathrm{~m} / \mathrm{s}$ and $f=153.1 \mathrm{~Hz}$ for $U_{e}=18.5 \mathrm{~m} / \mathrm{s}$. The modes in Figure 6.6 are actually the operational deflection shapes (ODSs) of the plate at the first natural frequencies. The MAC value between the $(2,1)$ wind tunnel ODSs is quite strong (Figure 6.4b), but there is greater noise in Figure $6.6 \mathrm{~b}$ due to the air flow over the surface.

The $(2,1)$ mode for the ideal experimental case is the second observed mode and has a frequency of $f=150.8 \mathrm{~Hz}$. Figure 6.3 of the frequency response shows that $(2,1)$ modes for all three cases have a natural frequency of $f \approx 150 \mathrm{~Hz}$. Based on the MAC values in Figure 6.4 there is also fairly good agreement between the ODSs and mode shapes. The suppressing of the $(1,1)$ mode in the wind tunnel likely results from the interaction with the sealed cavity below the plate. The plate is very thin and thus behaves somewhat like a membrane, so there is acoustic coupling between the plate and the cavity that pushes the natural frequency of the $(1,1)$ mode towards $0 \mathrm{~Hz}$ [96]. A contributing factor could also be the mass and stiffness loading by the air in the cavity, since fluid 


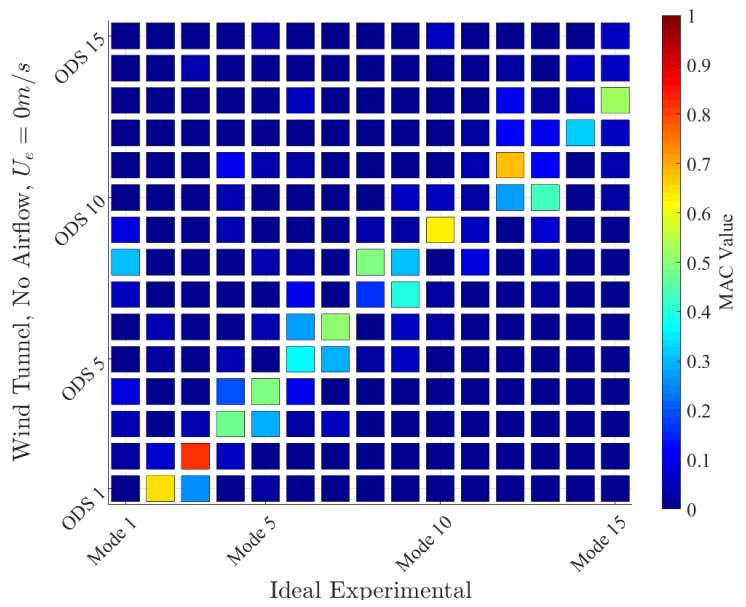

(a)

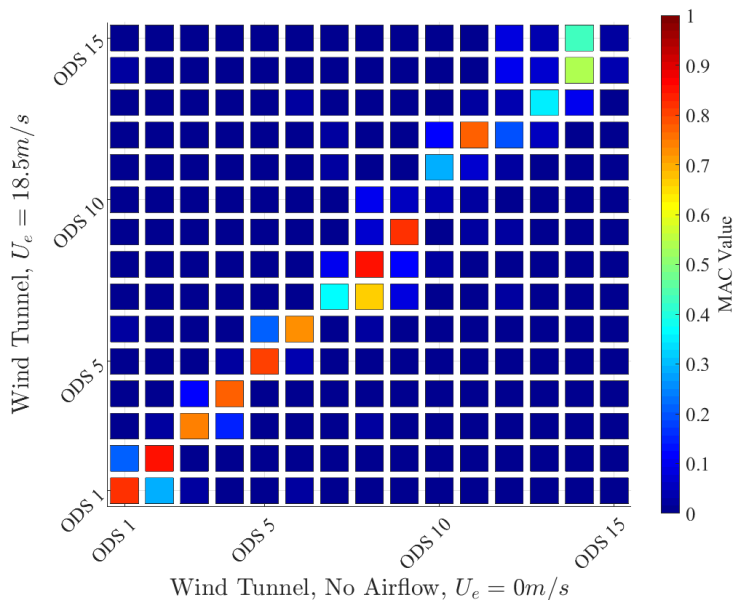

(b)

Figure 6.4: MAC values comparing the clamped traveling wave plate in different conditions. (a) compares operational deflection shapes (ODSs) of the plate mounted in the wind tunnel with no airflow $\left(U_{e}=0 \mathrm{~m} / \mathrm{s}\right)$ and the ideal experimental mounting from Chapter 4. (b) compares the plate mounted in the wind tunnel with $\left(U_{e}=18.5 \mathrm{~m} / \mathrm{s}\right.$ and without airflow $\left(U_{e}=0 \mathrm{~m} / \mathrm{s}\right)$

loading has been shown to disproportionately effect the lower frequency modes $[97,98]$. If the frequency response were captured at lower frequencies6.3, the ODS of the plate would likely be in the form of the $(1,1)$ mode shape. A slight spike in the frequency response at $f \approx 70 \mathrm{~Hz}$ can be seen in Figure 6.3 for the no airflow case; however, this was investigated and found not to be the $(1,1)$ mode.

Further comparing the $U_{e}=0 \mathrm{~m} / \mathrm{s}$ and the ideal case, indicates that the higher natural frequencies and ODSs are also significantly affected by the change in mounting condition. Figure 6.4a shows that there is a poor MAC value for higher than the fourth mode. In addition, the order of occurrence of the modes changes. The $11^{\text {th }}$ mode of the ideal experimental case was not observed when mounting the plate in the wind tunnel.

If the two wind tunnel cases are compared, there is not as significant of a difference as with the ideal case. Figure $6.4 \mathrm{~b}$ shows that the MAC values for the first nine ODSs are strong (aside from the $7^{\text {th }}$ ) and there is no change in the order. However, Figure 6.3 shows that there is a shift in the natural frequencies. When there is airflow over the surface of the plate, the frequencies tend to increase slightly. For the first nine modes, the $u_{e}=18.5 \mathrm{~m} / \mathrm{s}$ airflow yields an increase of roughly $1-3 \%$.

Based on the changes in the natural frequencies and mode shapes, it is clear that both the mounting and the airflow sufficiently alter the structural response of the clamped plate. As a result, the model developed in Chapter 4 no longer accurately represents the plate. The model could be updated to provide a better match; however, this would require accounting for mass and 


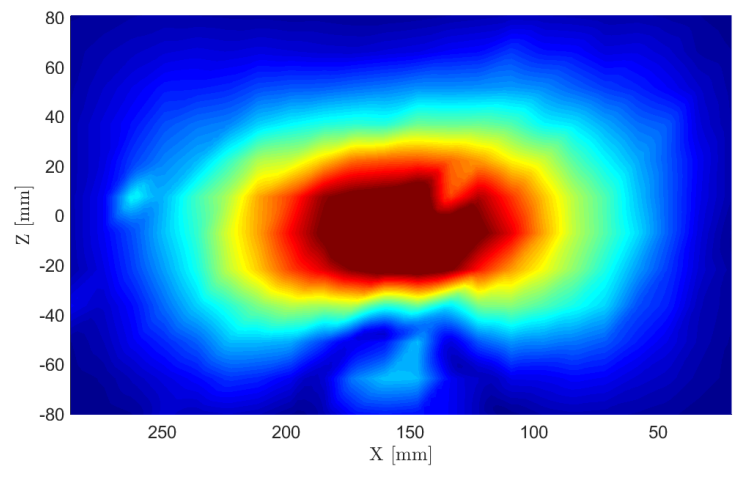

(a)

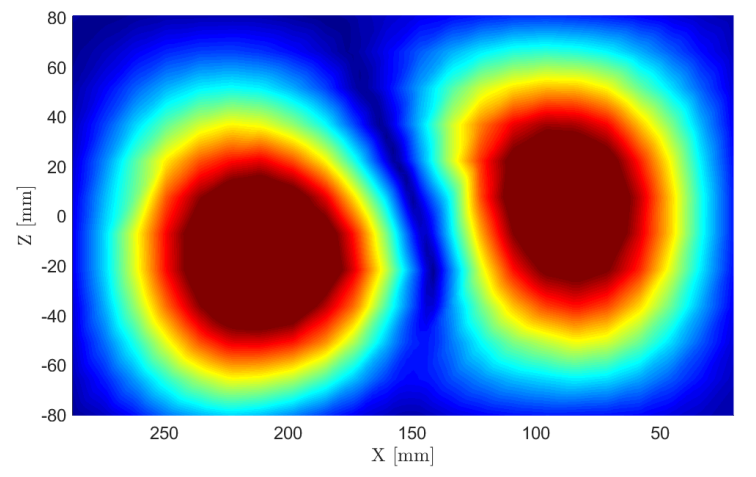

(b)

Figure 6.5: (a) First and (b) second mode shape of the ideal experimental mounting described in Chapter 4. Natural frequencies of $f=84.5 \mathrm{~Hz}$ and $f=150.8 \mathrm{~Hz}$, respectively

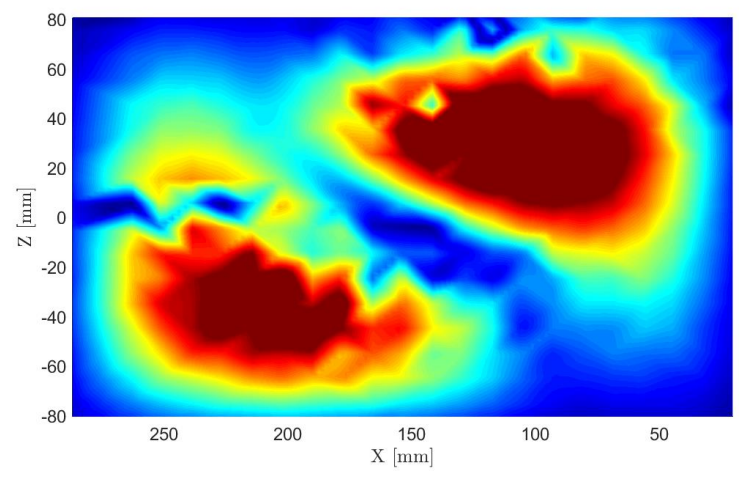

(a)

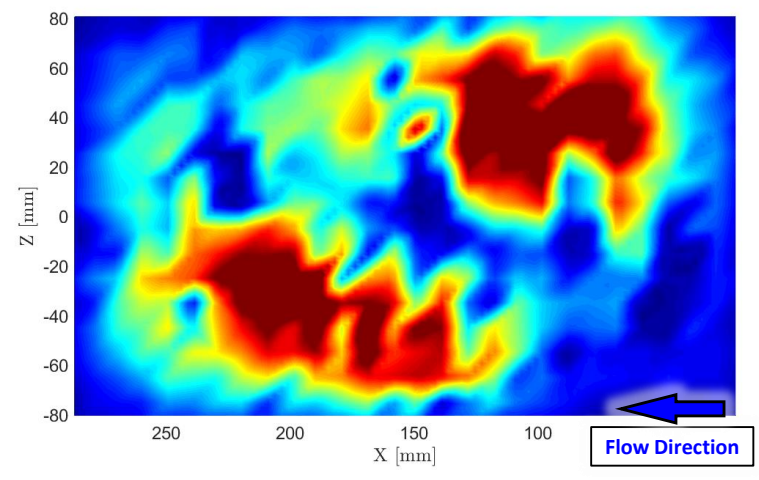

(b)

Figure 6.6: Operational deflection shapes (ODSs) at the first natural frequencies of plate mounted in the wind tunnel. (a) $U_{e}=0 \mathrm{~m} / \mathrm{s}$ and $f=150.6 \mathrm{~Hz}$, (b) $U_{e}=18.5 \mathrm{~m} / \mathrm{s}$ and $f=153.1 \mathrm{~Hz}$

stiffness loading, non-uniform stresses, and fluid loading due to the air flow. This would be quite challenging to implement. Having said this, the model proved its worth in Chapter 4 by confirming the experimental results. Leveraging the relationship established in that chapter, traveling waves can be tailored without the model. As a result, all of the traveling waves generated in the wind tunnel are tailored using experimental data of the natural frequencies and mode shapes.

\subsubsection{Traveling Wave Generation}

In order to validate the traveling wave generation method presented in this research, a single frequency traveling wave is used in this chapter. The parameters of this wave are chosen so as to maximally effect the turbulent boundary layer. These parameters are determined using results from previous studies in literature. 
Before the optimal parameters can be discussed, the wave parameters must be properly scaled. The aim is to have the traveling wave interact with the turbulent boundary layer, so it is appropriate to normalize the wave parameters using the same scaling as in the boundary layer. As discussed in Section 2.2.1, turbulent boundary layers are normalized to wall units using the friction velocity, $u_{\tau}$, and kinematic viscosity, $v$ in order to establish a common structure (e.g. viscous sublayer, buffer layer, log-law). The traveling wave parameters are normalized in the same manner. The amplitude (displacement) is expressed using Equation 6.1, where $a$ is the dimensional amplitude, $a^{+}$is the normalized amplitude, and ${ }^{+}$denotes wall units. The normalized wave length, $\lambda^{+}$, is similarly expressed using Equation 6.2. Finally, the frequency is normalized and given as a normalized period, $T^{+}$, using Equation 6.3, where $f$ is the frequency in $H z$.

$$
\begin{gathered}
a^{+}=\frac{a u_{\tau}}{v} \\
\lambda^{+}=\frac{\lambda u_{\tau}}{v} \\
T^{+}=\frac{u_{\tau}^{2}}{f v}
\end{gathered}
$$

The optimal parameters at which to generate the traveling wave are determined based on previous literature. Multiple studies have suggested that the optimal excitation period for affecting the boundary layer and reducing skin friction drag is $T^{+} \approx 100$. This was shown both for spanwise traveling waves [5,8,30] and also for spanwise in-plane wall motion [24]. This timescale is roughly the same as that of the bursting events [85], which are a major source of turbulence production and responsible for an increase in the skin friction [23]. In terms of amplitude, most studies agree that larger traveling wave amplitudes are more effective $[3,5,8,82]$ and should at least extend into the buffer layer $a^{+} \geq 10$. Regarding the wavelength, there has yet to be shown a significant correlation. Thus, to maximize the effect on the boundary layer, the traveling wave excited here should have $T^{\approx 100}$ and $a^{+} \geq 10$. Since no previous correlation has been shown with wavelength, its effect is not considered here. This is also convenient since the the wavelength cannot be defined independently of the frequency (period).

The traveling wave is then generated using the experimental natural frequencies and mode shapes (Section 6.1.2) and the methods described in Chapter 4. The traveling wave is excited at $430 \mathrm{~Hz}$ and has a normalized period of $T^{+}=111.6$. The friction velocity and viscosity are $u_{\tau}=0.88 \mathrm{~m} / \mathrm{s}$ and $v=1.63 \times 10^{-5} \mathrm{~m}^{2} / \mathrm{s}$, respectively, with the friction velocity found using the unactuated boundary layer upstream of the traveling wave plate (see Table 6.3). The traveling wave is excited between the two mode shapes seen in Figure 6.7. Figure 6.7a shows the $(5,2)$ 
mode shape at $f=423.4 \mathrm{~Hz}\left(T^{+}=113.4\right)$ and Figure $6.7 \mathrm{~b}$ shows the $(3,3)$ mode shape at $f=$ $438.8 \mathrm{~Hz}\left(T^{+}=109.4\right)$.

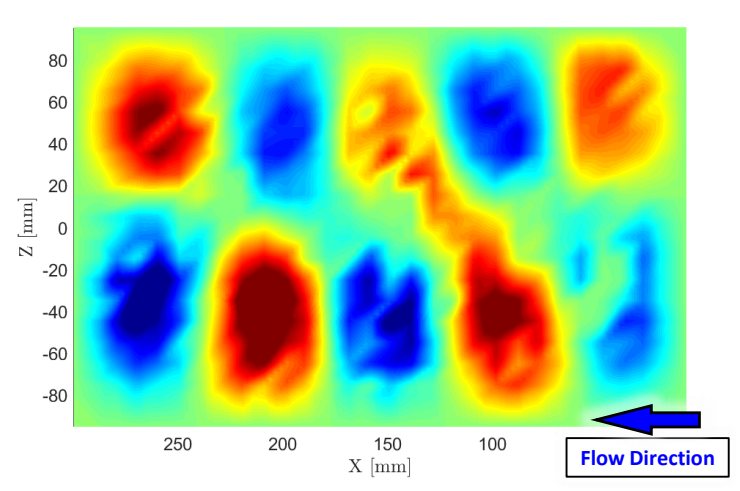

(a)

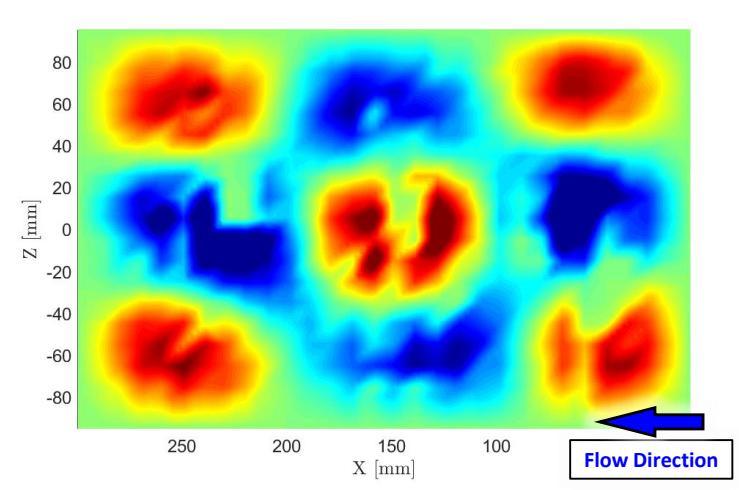

(b)

Figure 6.7: Operational deflection shapes (ODSs) show the (a) $(5,2)$ mode at $f=423.4 \mathrm{~Hz}, \mathrm{~T}^{+}=$ 113.4 and (b) the $(3,3)$ mode at $f=438.3 \mathrm{~Hz}, T^{+}=109.4$

All 10 MFC actuators are excited here using the actuation configuration given in Equation 6.4 and shown in Figure 6.8. There is a phase difference of $\phi=-110^{\circ}$ between the MFCs on the top and bottom of the plate. To excite a traveling wave resembling the $(5,2)$ mode shape, the actuators in red excited $180^{\circ}$ out-of-phase with those in blue; this procedure is explained in Section 4.3.2. The resultant traveling wave, excited in the wind tunnel with a free-stream velocity of $U_{e}=18.5 \mathrm{~m} / \mathrm{s}$, is shown in Figure 6.9. The figure shows the traveling wave at different time steps within a single period, $T$. There are three separate wave fronts propagating in the spanwise direction $( \pm z)$. The wave front in the middle propagates in the $-z$ direction with the position of the peak visible in each subsequent time step. The wave fronts upstream and downstream propagate in the $+z$ direction. This is shown more clearly in Figure 6.10, which shows the root-mean-square (RMS) velocity of the plate over a single period.

$$
\begin{array}{rll}
\phi_{A_{1}}=\phi_{A_{3}}=\phi_{A_{5}}=0^{\circ} \quad & \phi_{B_{1}}=\phi_{B_{3}}=\phi_{B_{5}}=\gamma \\
\phi_{A_{2}}=\phi_{A_{4}}=180^{\circ} \quad, \quad \phi_{B_{2}}=\phi_{B_{4}}=180^{\circ}+\gamma .
\end{array}
$$

The boundary layer is measured at 11 different locations on the plate. This is split into two groups: six streamwise positions along the centerline $(z=0 \mathrm{~mm})$ and five spanwise positions $(x=$ $150 \mathrm{~mm}$ ). The amplitude of the traveling wave at each of these streamwise and spanwise positions are shown in Tables 6.1 and 6.2, respectively. The amplitudes have been normalized to wall units using the friction velocity of the unactuated boundary layer at that location. For the streamwise case, there are four measurement locations directly over the traveling wave and one each upstream and downstream. All of the measurement locations over the traveling wave are highlighted in Figure 6.10. 


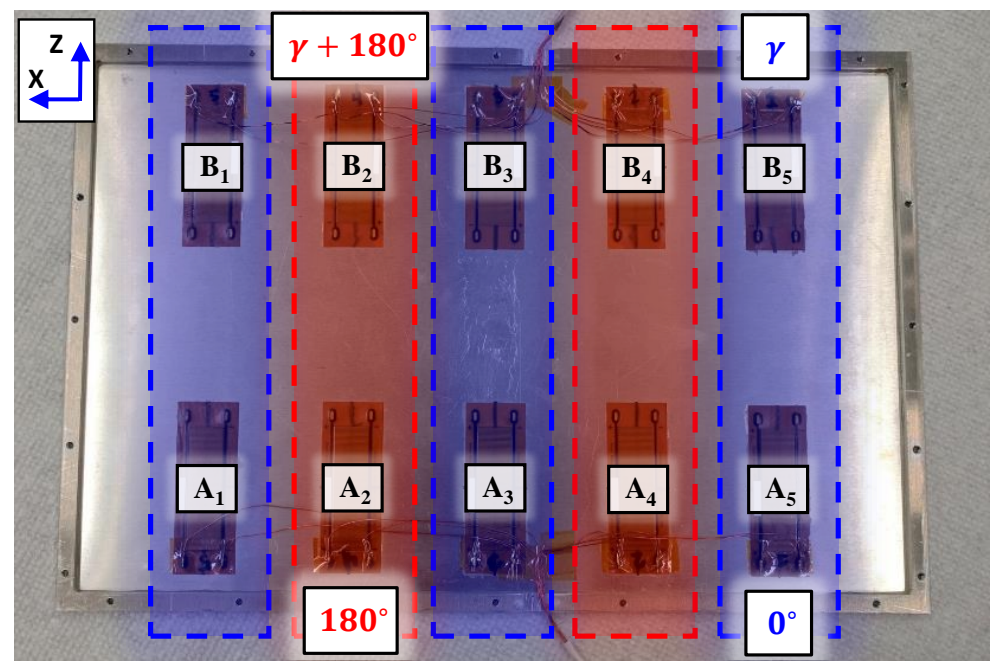

Figure 6.8: Actuation configuration to excite the $430 \mathrm{~Hz}$ traveling wave. The top actuators are $\gamma=-110^{\circ}$ out of phase with the bottom ones. The actuators in red are $180^{\circ}$ offset from those in blue.

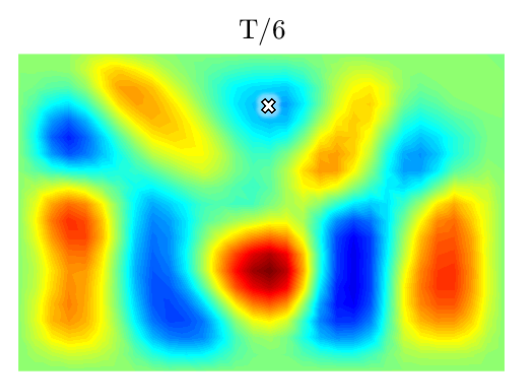

$4 \mathrm{~T} / 6$

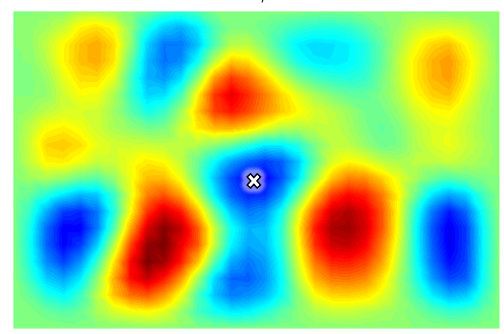

$2 \mathrm{~T} / 6$

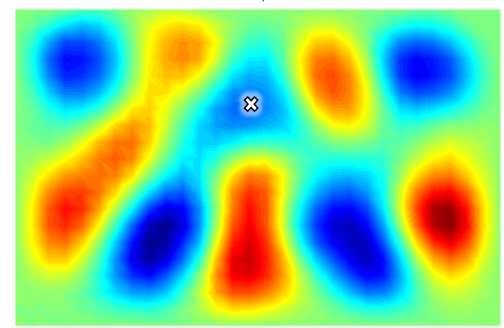

$5 \mathrm{~T} / 6$

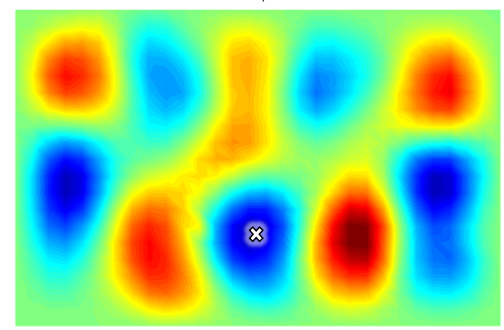

$3 \mathrm{~T} / 6$

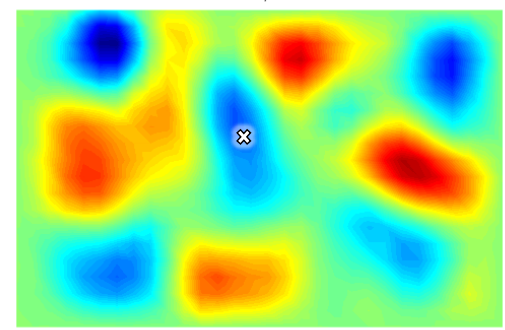

$\mathrm{T}$

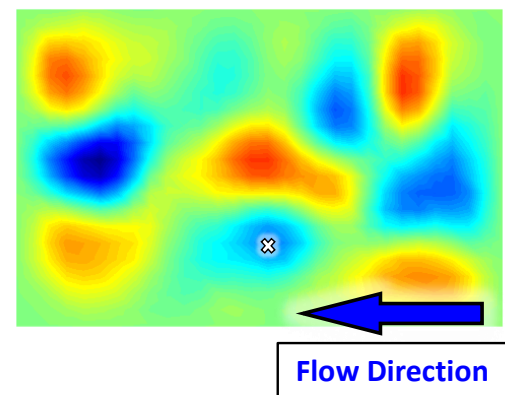

Figure 6.9: Instantaneous velocities of the $430 \mathrm{~Hz}\left(T^{+}=112\right)$ traveling wave at six different time steps in the period $T$ of the wave. There are three wavefronts propagating in the spanwise direction. The $\times$ symbol follows middle wavefront traveling in the $-z$ direction. The left and right sides travel in the $+z$ direction

These displacements result from exciting all the actuators with voltage amplitudes of $\pm 350 \mathrm{~V}$. This input of $\pm 350 \mathrm{~V}$ was the maximum voltage, and thus displacement, that could be achieved 


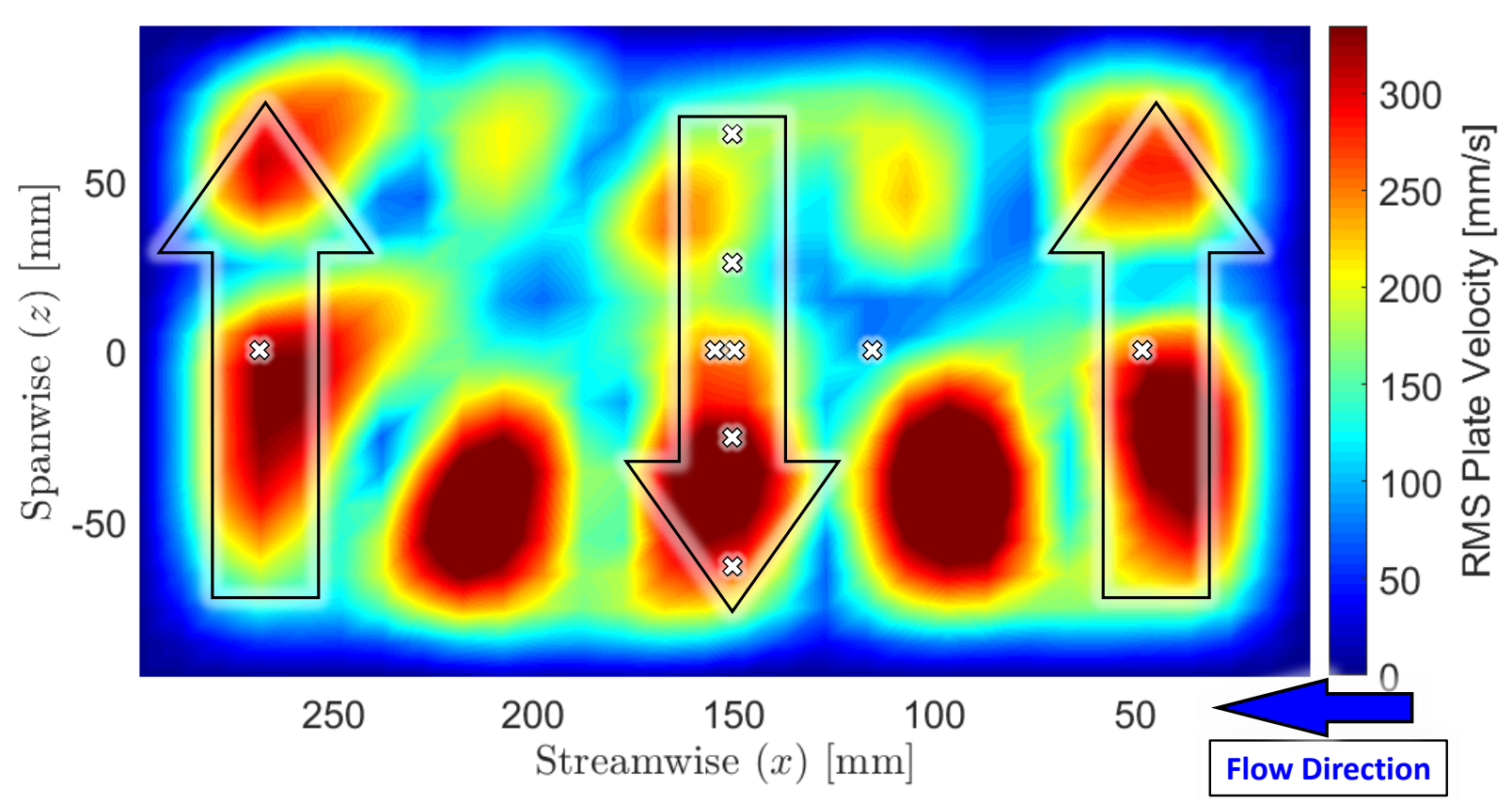

Figure 6.10: Root-mean-square (RMS) velocity of the traveling wave at $f=430 \mathrm{~Hz}\left(\mathrm{~T}^{+}=112\right)$. The boundary layer measurement positions are highlighted along with the propagation direction of the traveling waves

for $430 \mathrm{~Hz}\left(T^{+}=112\right)$ traveling wave. The limiting factor on the voltage is the experimental setup of the plate. For input voltages larger than $\pm 350 \mathrm{~V}$, the response of the plate was nonlinear and the plate audibly rattled. This likely resulted from the MFC electrodes inside the cavity short circuiting and causing discrete jumps in the voltage inputs to the actuators. With properly insulated electrodes, the upper limit of the input voltage is the actuators themselves. The MFCs are rated for $-500 \mathrm{~V}$ to $+1500 \mathrm{~V}$ [91]; thus, the maximum input would be $500 \mathrm{~V} \pm 1000 \mathrm{~V}$.

Table 6.1: The streamwise measurement locations for the $430 \mathrm{~Hz}\left(T^{+}=112\right)$ traveling wave along with the maximum and peak to peak displacement amplitude. All positions are at $z=0 \mathrm{~mm}$ and the four positions over the traveling wave surface $\left(a^{+} \neq 0\right)$ are shown in Figure 6.10

\begin{tabular}{c|cccccc}
\hline Streamwise Position, $x[\mathrm{~mm}]$ & 316 & 268 & 154 & 116 & 48 & -19 \\
\hline$a_{\text {crest }}[\mu \mathrm{m}]$ & 0 & 182 & 249 & 78 & 165 & 0 \\
$a_{\text {crest }}^{+}$ & 0 & 9.2 & 12.5 & 4.0 & 8.5 & 0 \\
$a_{p-p}^{+}$ & 0 & 17.2 & 23.4 & 8.2 & 15.1 & 0 \\
\hline
\end{tabular}


Table 6.2: The peak displacement/amplitude of the $430 \mathrm{~Hz}\left(T^{+}=112\right)$ traveling wave at the spanwise locations shown in Figure 6.10 with $x_{p}=150 \mathrm{~mm}$

\begin{tabular}{c|ccccc}
\hline Spanwise Position, $z[\mathrm{~mm}]$ & 64 & 25 & 0 & -25 & -64 \\
\hline$a_{\text {crest }}[\mu \mathrm{m}]$ & 154 & 94 & 231 & 202 & 137 \\
$a_{c r e s t}^{+}$ & 7.9 & 4.9 & 12.5 & 10.3 & 7.0 \\
$a_{p-p}^{+}$ & 16.0 & 9.5 & 23.8 & 21.5 & 12.7 \\
\hline
\end{tabular}

\subsection{Boundary Layer Data Analysis}

To determine the effect of the traveling wave on the turbulent boundary layer, the boundary layer is experimentally measured in the low-speed wind tunnel described in Chapter 5 . The boundary layer parameters are given in Table 6.3 for locations upstream and downstream of the traveling wave surface. A hot-wire probe is used to measure the profile at different positions along the traveling wave actuated surface. These measurement positions are given in Tables 6.1 and 6.2, where there are six streamwise locations along the plate centerline and five spanwise positions. Two of the streamwise positions are upstream and downstream of the traveling wave surface; the same ones as given in Table 6.3. The other nine measurement positions are directly over the traveling waves and are shown in Figure 6.10 with an $\times$.

Table 6.3: Boundary layer parameters in the wind tunnel upstream and downstream of the traveling wave region

\begin{tabular}{lcccccccc}
\hline & $x_{p}[\mathrm{~mm}]$ & $U_{e}[\mathrm{~m} / \mathrm{s}]$ & $\delta[\mathrm{mm}]$ & $\theta[\mathrm{mm}]$ & $C_{f}$ & $u_{\tau}[\mathrm{m} / \mathrm{s}]$ & $\operatorname{Re}_{\theta}$ & $H$ \\
\hline Upstream & -19 & 17.99 & 6.4 & 0.740 & 0.0048 & 0.882 & 819 & 1.461 \\
Downstream & 316 & 19.43 & 10.7 & 1.275 & 0.0041 & 0.877 & 1507 & 1.405 \\
\hline
\end{tabular}

These actuated boundary layer profiles are then compared with the unactuated profiles measured at the same locations. This comparison is used to determine the effect of the traveling waves on the time-averaged mean velocity profile. At any given position on the traveling wave surface, the wall-normal deflection is periodic; thus, the surface oscillates between a maximum (crest) and minimum (trough) displacement, passing through zero twice each period (positive and negative velocity). The time-averaged boundary layer profile is the mean over the entire periodic motion. However, the turbulent boundary layer profile may vary over this period. To investigate this, a phase-locked analysis is conducted to extract the boundary layer profiles at specific instances in the traveling wave period. Thus, separate boundary layer profiles are generated over the crest and trough of the wave and at each of the two zero displacement positions.

This section begins by outlining the procedures for measuring the boundary layer over the 
traveling wave actuated surface. The general measurement procedure is described in Section 5.1.4, but modifications to account for the actuated surface are explained here. Then the phase-locked analysis is described. This includes a discussion of the experimental methods, along with the processing necessary to extract phase-locked profiles.

\subsubsection{Measurement Procedure}

To determine the effect of the traveling waves on the turbulent boundary layer, the actuated and unactuated profiles are compared. Thus, both profiles are necessary at each measurement location. A combined procedure is implemented to measure both the unactuated and actuated profiles together. This is primarily done to minimize the error in the $y$ position between the two profiles. The combined procedure begins by finding the $y=0$ position of the probe (Section 5.1.4.4). The probe then moves away from the wall in a stepwise fashion to the defined $y$ positions. At each $y$ position, the hot-wire probe measures two separate sets of time-varying velocities; one each for the unactuated and actuated case. The unactuated case is measured first, and $5 s$ of velocity data is collected at $\approx 20 \mathrm{kHz}$. The traveling wave surface is then activated and allowed to settle for $10 \mathrm{~s}$. Next, the time-varying velocity is captured for the actuated case, using the same sample length and sampling rate. The traveling wave is then turned off and the procedure repeated for every $y$ position in the boundary layer profile.

When measuring directly over the traveling wave surface, the actuated profile is not measured at $y$ positions very close to the wall. If the probe is placed too close to the surface, the wall will hit the tip of the probe and break the sensor wire. As a result, the traveling wave is only turned on and the flow velocity measured when the probe is sufficiently clear of the wall. When the probe is in the near wall region, the combined measurement procedure only measures the flow velocity over the unactuated wall.

Once the unactuated and actuated boundary layer profiles have been measured, they are processed. The unactuated profile is processed using the exact same procedure as in Section 5.2: statistically independent samples are found, the $y$ offset is corrected, and the profile is normalized to wall units. Since the actuated profile was captured alongside the unactuated one, its analysis is simplified. Statistical independence is still necessary, so the integral time scale is found at each $y$ position and the time-varying velocities down-sampled. The $y$ positions of the actuated profile are then corrected using the same $y_{a d j}$ value as the unactuated case. Finally, the actuated profile is converted to wall units using the friction velocity, $u_{\tau}$, from the unactuated case at that location. 


\subsubsection{Phase-locked Analysis}

The boundary layer over the actuated wall consists of a set of time-varying velocity values at each $y$ position. To determine the time-averaged profile, the velocities are down-sampled for statistical independence and then the mean, variance, skewness, and kurtosis at each $y$ position is calculated. The data is considered time-averaged since the velocity samples occur at arbitrary positions within the period of the traveling wave. This provides insight into the average response of the boundary layer, but there is no understanding regarding what happens when the surface is at a specific position in its period: e.g. the crest or trough. To capture this information, a phase-locked data analysis is conducted on the actuated boundary layer data. Separate boundary layer profiles are extracted directly over the crest and trough of the moving wall and at the two zero displacement instances over the period (positive and negative wall-normal velocity).

This phase-locked analysis is implemented by simultaneously measuring the displacement of the traveling wave surface and the hot-wire flow velocities. This is experimentally done by using the scanning laser doppler vibrometer (Figure 6.2) to measure the out-of-plane velocity of the actuated surface directly below the hot-wire probe. During data collection, the laser vibrometer and hot-wire probe measure simultaneously at the same sampling rate. The sampling rate is specifically defined as an integer multiple of the traveling wave excitation frequency. For the case of the $430 \mathrm{~Hz}$ wave, the data was collected at $17.2 \mathrm{kHz}$, which is $40 \times$ the traveling wave frequency. As a result, there are an integer number of velocity samples for each period of the traveling wave.

The laser vibrometer measures the out-of-plane velocity of the traveling wave surface, but the displacement is necessary for the phase-locked analysis. So, the out-of-plane velocity signal is numerically integrated to determine the displacement. The displacement is then fed through a high-pass filter to remove the DC offset caused by the integration. A cutoff frequency of $43 \mathrm{~Hz}$ $(10 \%$ of $430 \mathrm{~Hz})$ is used. Figure 6.11 shows an example of the out-of-plane velocity and high-pass filtered displacement of the traveling wave surface along with the instantaneous flow velocity. Only 10 periods are shown in this figure, accounting for a sample length of $\approx 25 \mathrm{~ms}$. At each $y$ position of the boundary layer, $5 \mathrm{~s}$ of data is collected, resulting in 2150 periods of the $430 \mathrm{~Hz}$ traveling wave.

Now, the displacement of the surface is known at each time step of the instantaneous flow velocity. The flow velocities at the various phase-locked instances over the actuated surface can then be extracted to create a separate flow velocity signal for each. For the case of the crest, the point of maximum displacement is found within each period of the traveling wave (shown in Figure $6.11 b)$. Since the sampling rate was defined as an integer multiple of the excitation frequency, the maximum displacement occurs at the same location within each period of the wave. The hot-wire velocities corresponding to these positions are shown in Figure 6.11c and are then extracted. This is implemented for both the maximum and minimum displacements within each period, crest and 


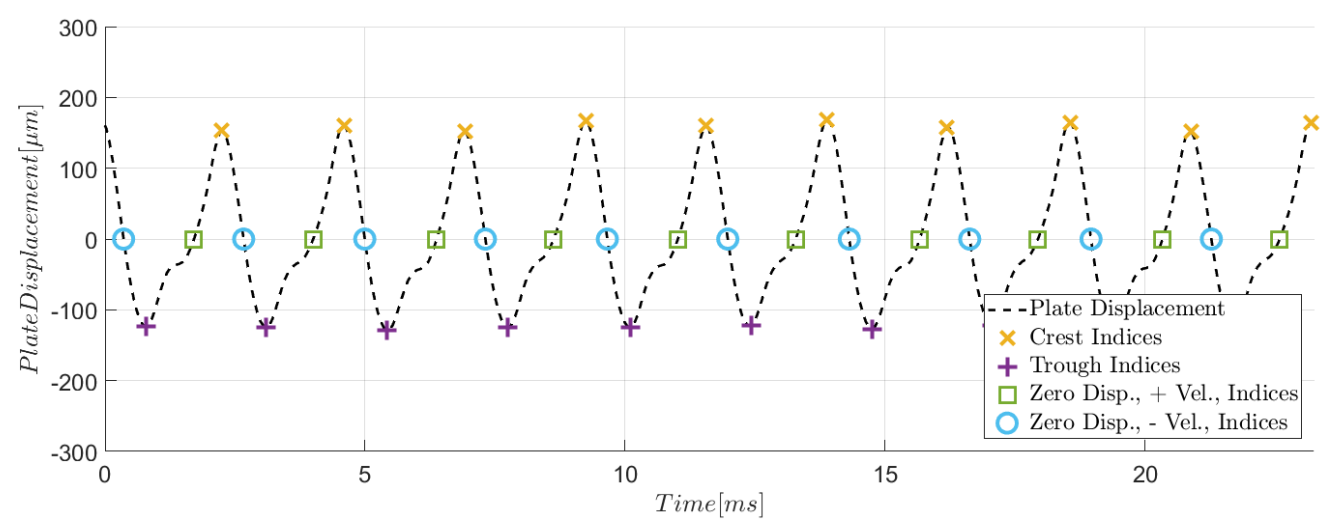

(a)

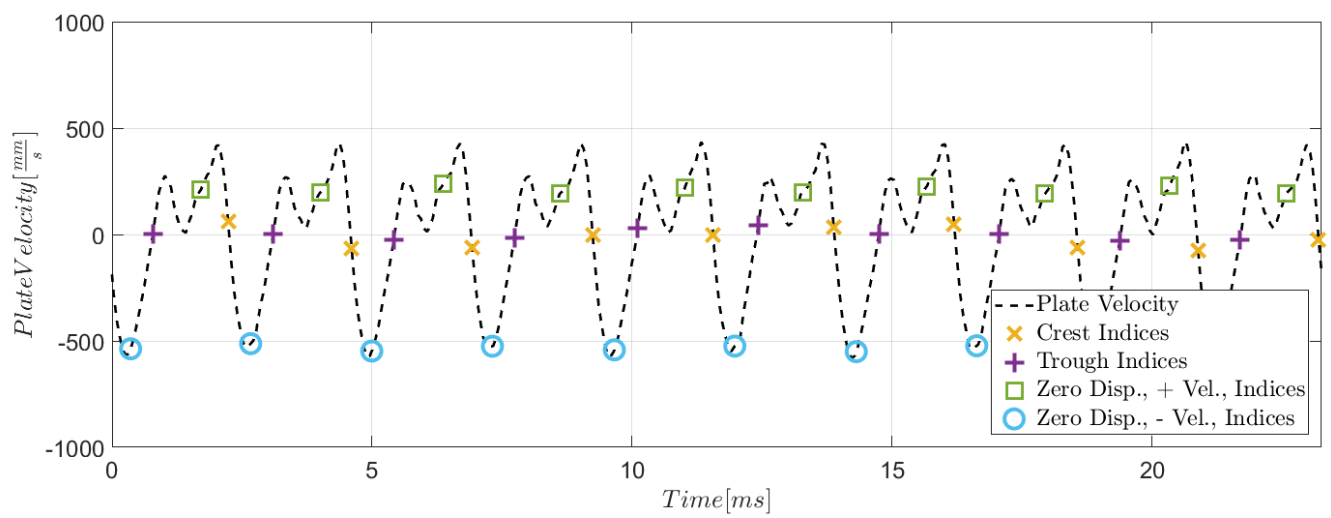

(b)

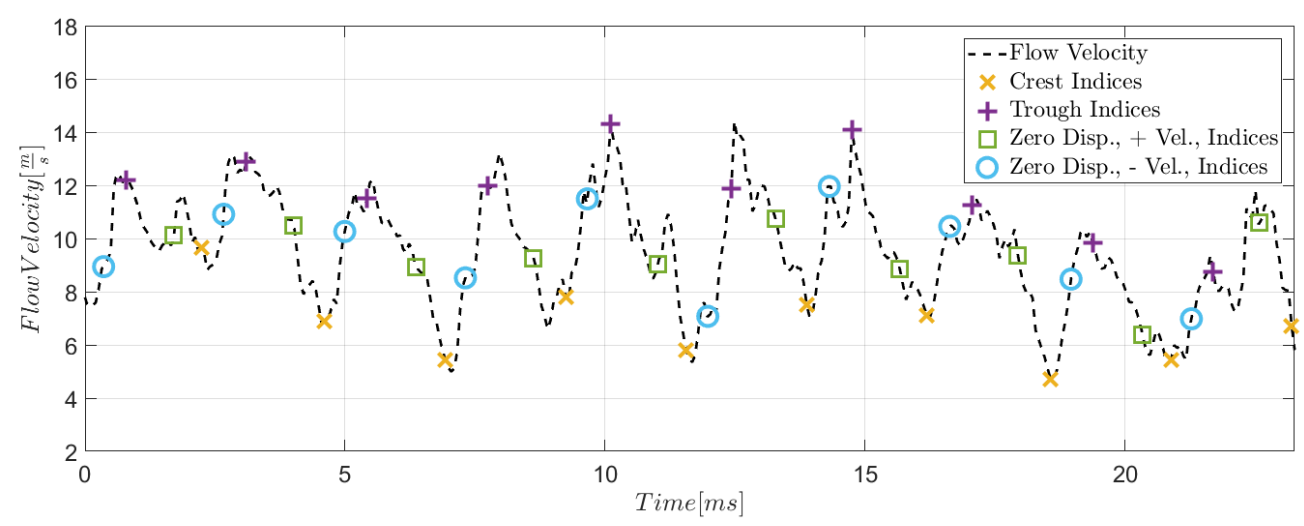

(c)

Figure 6.11: Time-series data of the (a) traveling wave velocity, (b) traveling wave displacement, and (c) flow velocity. Also shown are indices corresponding to the phase-locked crest and trough positions. 10 periods of the $430 \mathrm{~Hz}\left(T^{+}=112\right)$ traveling wave are shown, where $5 \mathrm{~s}$ of raw data is collected at each $y$ position. 
trough respectively. The same procedure is then conducted for the zero displacement positions with positive and negative wall-normal velocity. The points of zero displacement do not exactly line up with the measured samples, so the time instances are linearly interpolated. This hot-wire velocities are then extracted by linearly interpolating at these time values.

As a result, there are four separate fluctuating velocity signals at each $y$ position, corresponding with the four phase-locked positions.For the unactuated and time-averaged actuated boundary layers, the velocity signals were down-sampled to ensure statistical independence (Section 5.2.1). That is not necessary for the phase-locked boundary layers since the crest and trough velocity signals were already down-sampled to the excitation frequency $(430 \mathrm{~Hz})$. The maximum sampling rate based on the integral time scale is $\approx 800 \mathrm{~Hz}$; thus, each sample in the phase-locked velocity signals is statistically independent.

Using the phase-locked velocity signals at each $y$ position, the mean, variance, skewness, and kurtosis are then calculated. Figure 6.12a shows an example of the mean velocity boundary layer profile for the unactuated, time-averaged actuated, and four phase-locked cases. The crest and trough were plotted using the $y$ positions with respect to the unactuated wall. However, the $y$ positions must be adjusted to account for the displacement of the crest and trough with respect to the unactuated position. The crest displacement is the mean maximum displacement of the traveling wave over the $5 s$ sample length. The trough is the mean minimum. Figure $6.11 \mathrm{~b}$ shows the maximum and minimum displacements for 10 of the 2150 total periods. For the case in Figure $6.11 \mathrm{~b}$ the crest displacement is $a_{\text {crest }}=165.4 \pm 0.5 \mu \mathrm{m}$ and the trough is $a_{\text {trough }}=-130.1 \pm 0.5 \mu \mathrm{m}$. This corresponds with displacements in wall units of $a_{\text {crest }}^{+}=8.5$ and $a_{\text {trough }}^{+}=-6.7$. The $y$ positions of the crest and trough boundary layer profiles are then adjusted by these displacements. The $y$ corrected boundary layer is shown in Figure 6.12b. The phase-locked boundary layer profiles at zero displacement do not need to be adjusted, since the $y$ values already correspond to zero displacement.

The $y$ positions of the time-averaged profile must be corrected. The wall-normal displacement is not perfectly symmetric, so the mean displacement is not zero. As a result, the time-averaged profile must be adjusted to account for this slight offset. For the displacement shown in Figure $6.11 \mathrm{~b}$, the mean displacement is $a=17.5 \pm 22.6 \mu \mathrm{m}$, which is $a^{+}=0.89$ in wall units. The timeaveraged profile in Figure 6.12b has been adjusted by this value.

It should also be pointed out that the time-averaged profile has an inherent error. The timeaveraged is the mean flow velocity as the surface oscillates due to the traveling wave. The hot-wire probe is stationary over the moving surface, so the effective $y$ position of the probe varies over the wave period. This is the effect that was corrected in Figure 6.12 to ensure the proper $y$ positions for the phase-locked profiles. Looking at Figure 6.11c, the hot-wire flow velocity also fluctuates periodically with the wall oscillation. This is is seen in the uncorrected $y$ boundary layer profiles in 


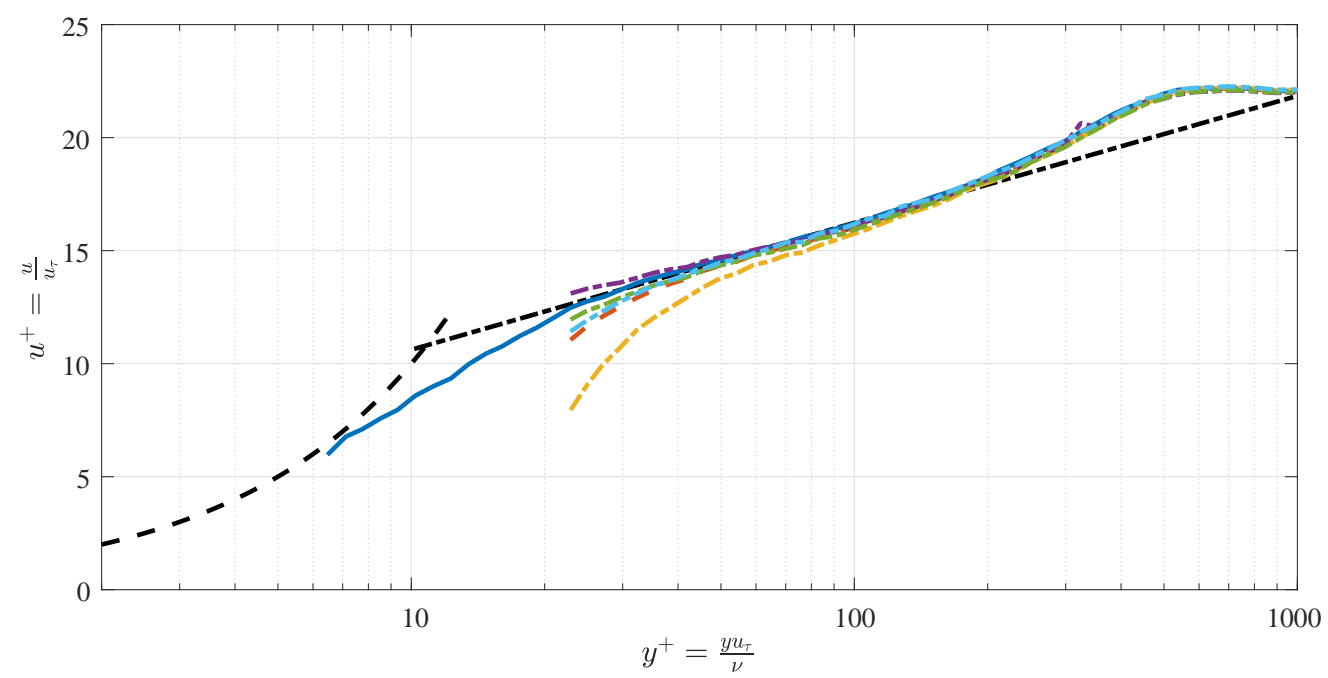

(a)

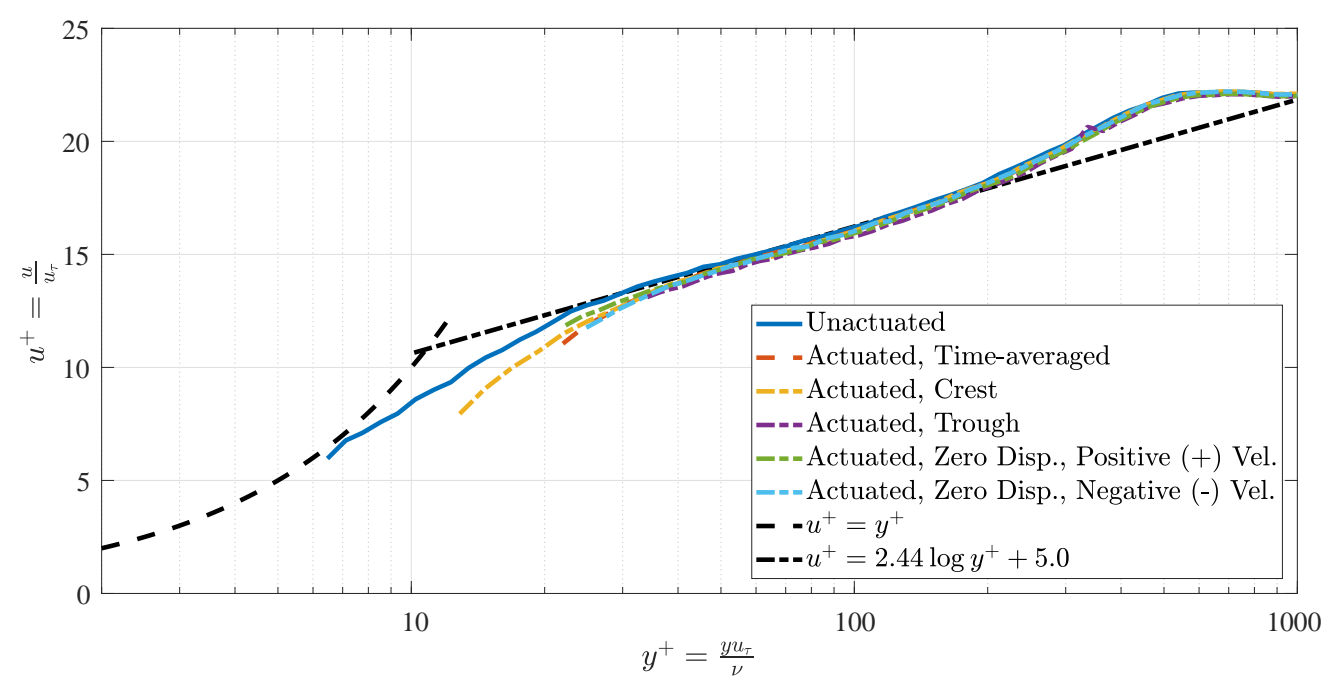

(b)

Figure 6.12: Mean velocity boundary layer for the unactuated, time-averaged actuated, and phaselocked crest and trough cases. (a) shows the crest and trough profiles using the $y$ values from the time-averaged. (b) shows the crest and trough with corrected $y$ positions to account for the displacement of the TW surface

Figure 6.12a. At the closest position to the surface $\left(y^{+} \approx 23\right)$, the oscillation in the flow velocity is evidenced by the spread in the phase-locked profiles. The crest is the minimum velocity at $u^{+} \approx 8$, and the trough is the max at $u^{+} \approx 13$, while the time-averaged is at $u^{+} \approx 11$. But, this oscillation in the velocity $\left(u^{+}\right)$over the wave period is not symmetric, rather it varies non-linearly. This is because the wall-normal oscillation of the surface essentially moves the hot-wire probe to different $y$ positions in the boundary layer profile, and the profile changes rapidly in the buffer layer. As 
a result, the time-averaged boundary layer profile is not the true mean of the flow velocity, rather there is some small error. However, the phase-locked profiles are not subject to this non-linear error.

\subsection{Effect of Traveling Wave on the Turbulent Boundary Layer}

The previous sections described the generation of traveling waves in the wind tunnel, and the procedures necessary to measure the turbulent boundary layer. This section presents the results of exciting a $430 \mathrm{~Hz}\left(T^{+}=112\right)$ traveling wave in the turbulent boundary layer. The unactuated and time-averaged actuated boundary layers are compared for six different streamwise locations: four positions directly over the traveling waves and one each upstream and downstream. Then, the results of the phase-locked analysis are presented for the four streamwise locations directly over the actuated surface. Next, the effect of the traveling waves on the variance/streamwise Reynolds stress are shown, including for the phase-locked data. Changes in the skewness and kurtosis are also presented. Then, the phase-locked boundary layers measured at five different spanwise positions are discussed. Finally, the results and changes in the turbulent boundary layer are discussed with respect to the experimental uncertainties.

\subsubsection{Streamwise Boundary Layer}

Figure 6.13 shows the turbulent boundary layers measured at six different streamwise positions (Table 6.1). The actuated profiles have been normalized to wall units using the friction velocity of the unactuated profile at the same location. The top and bottom profiles in the figure were measured upstream and downstream of the traveling wave surface at $x_{p}=-19 \mathrm{~mm}$ and $x_{p}=316 \mathrm{~mm}$, respectively. The other four profiles were measured directly over the $430 \mathrm{~Hz}\left(T^{+}=112\right)$ traveling wave shown in Figure 6.10. At each location, the unactuated and time-averaged actuated profile are shown. Looking at the four locations measured directly over the traveling waves, the actuated profile diverges from the log-law line at a larger $y^{+}$value than the unactuated. As a result, the actuated profile appears to be pushed away from the wall. Due to the no-slip condition at the wall, the viscous sublayer is thickened and has a smaller slope and thus smaller skin friction coefficient. Unfortunately, the experimental setup prevents measurement of the boundary layer into the viscous sublayer. The hot-wire probe cannot be positioned near the surface due to the out-of-plane motion of the wall. This is also the reason that the actuated boundary layers in Figure 6.13 terminate at different $y^{+}$positions. The amplitude of the wall, $a_{\text {crest }}^{+}$, is different for each streamwise location, so the closest $y^{+}$position is changed to prevent the wall from contacting the probe.

Looking at the upstream and downstream positions in Figure 6.13, the unactuated and actuated 


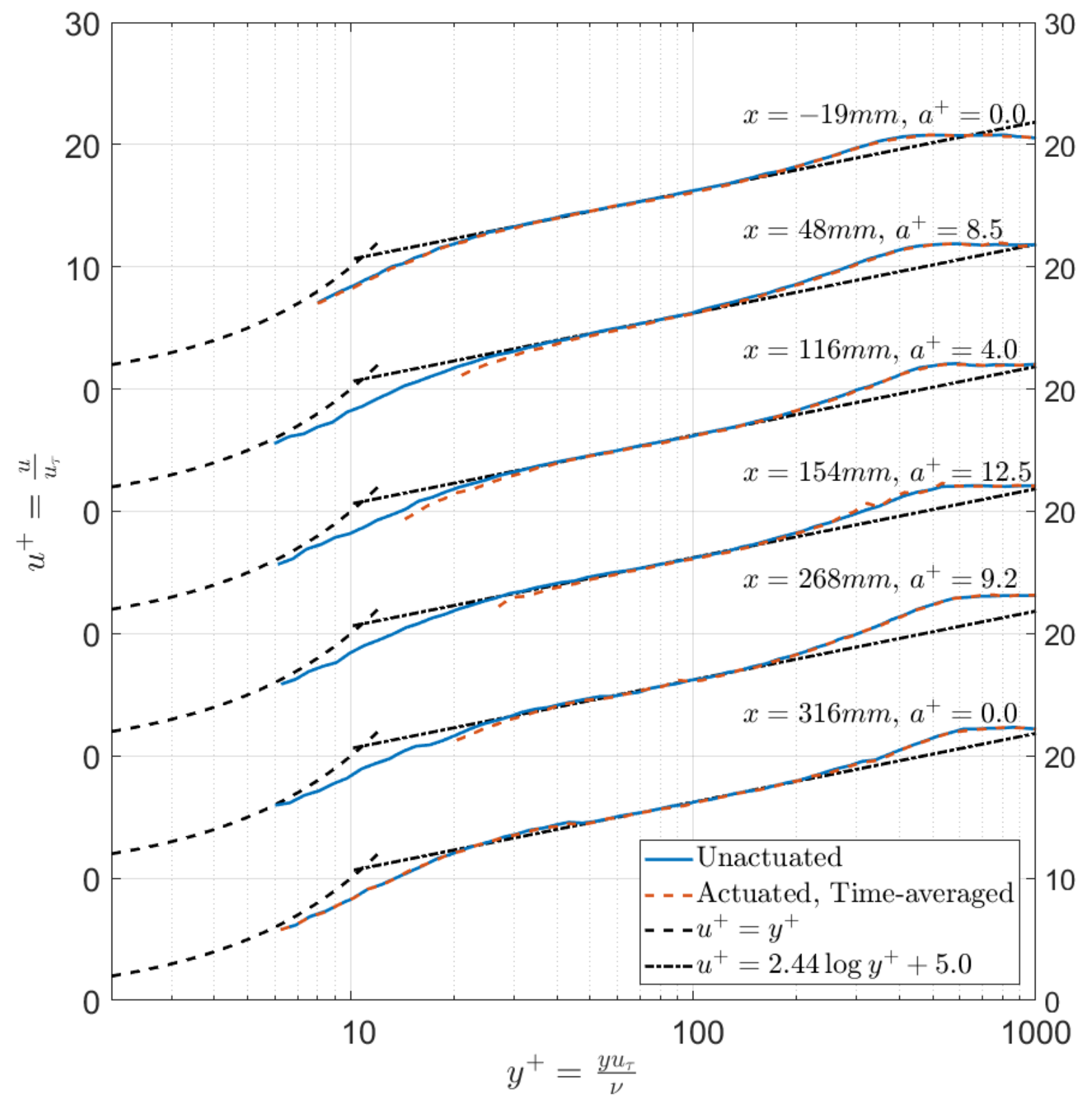

Figure 6.13: Experimentally measured turbulent boundary layers at six different streamwise locations upstream, over, and downstream of the $430 \mathrm{~Hz}\left(T^{+}=112\right)$ traveling wave surface. The unactuated and time-averaged actuated profiles are shown for each streamwise position. The streamwise position $\left(x_{p}\right)$ and normalized traveling wave displacement $\left(a_{\text {crest }}^{+}\right)$are given for each position.

boundary layer profiles are essentially the same. This is somewhat expected for the upstream location, since the flow has not yet encountered the traveling waves. However, the lack of difference at the downstream location implies that the flow is able to recover from the effects of the traveling wave. The downstream measurement location is at $x_{p}=316 \mathrm{~mm}$ which is $10 \mathrm{~mm}$ downstream from 
the end of the traveling waves (Figure 5.3). Looking at Figure 6.10, the closest upstream peak of the traveling wave occurs at $x_{p} \approx 265 \mathrm{~mm}$. Thus, the downstream measurement position is $\approx 50 \mathrm{~mm}$ downstream of the traveling wave peak, corresponding to $\approx 5 \delta$.

\subsubsection{Phase-Locked Boundary Layers}

The phase-locked analysis procedure described in Section 6.2.2 is then conducted on the timeaveraged actuated boundary layer profiles from Figure 6.13. Figure 6.14 shows the boundary layer profiles at four phase-locked positions over the traveling wave surface. From this figure, it is clear that there are differences between the phase-locked positions. Focusing on the profiles at the streamwise position of $x_{p}=154 \mathrm{~mm}$, the profile over the crest of the traveling wave is not affected as strongly as the time-averaged case. The viscous sublayer is still thickened, but not as significantly. On the other hand, the profile over the trough seems to be affected in a similar manner as the time-averaged, but it is not measured close enough to the wall to definitively tell.

Looking at different streamwise positions $\left(x_{p}\right)$, the same trend is not as pronounced. In addition, at $x_{p}=268 \mathrm{~mm}$ the phase-locked profile over the trough of the wave seems to be pushed away from the surface more than any of the other profiles. This is actually due to a change in the freestream velocity $\left(y^{+}>600\right)$. However, these variations between streamwise positions demonstrate the impact of uncertainty on the measurements. The effect of the uncertainty on the confidence in the results and observations is discussed further in Section 6.3.6.

Also shown in Figure 6.14 are the phase-locked profiles when the traveling wave passes through zero displacement. This is separated into two profiles, one with positive wall-normal velocity $(+y$ direction) and the other with negative velocity ( $-y$ direction). To more clearly see the effect, the phase-locked zero displacement profiles are plotted in Figure 6.15 alongside the time-averaged case. At all four streamwise positions, the two phase-locked profiles vary from one another, with the negative velocity case pushed further off the wall. This is even though both boundary layer profiles are measured over a wall with zero displacement, which is the same as the unactuated case. This indicates that the traveling wave has a phase-lagged effect on the turbulent boundary layer. When the surface has negative velocity, the boundary layer is still reacting to when the surface was protruding into the flow (i.e. crest).

\subsubsection{Variance/Streamwise Reynolds Stress Profile}

As described in Section 5.2.5, the variance of the fluctuating flow velocity is akin to the streamwise Reynolds stress. Figure 6.16 shows the streamwise Reynolds stress profiles at the six streamwise measurement positions. The spikes in the data at certain $y^{+}$positions can be ignored (e.g. $y^{+} \approx 200$ for $x_{p}=154 \mathrm{~mm}$ ). They result from experimental errors in the hot-wire probe at those $y$ positions 


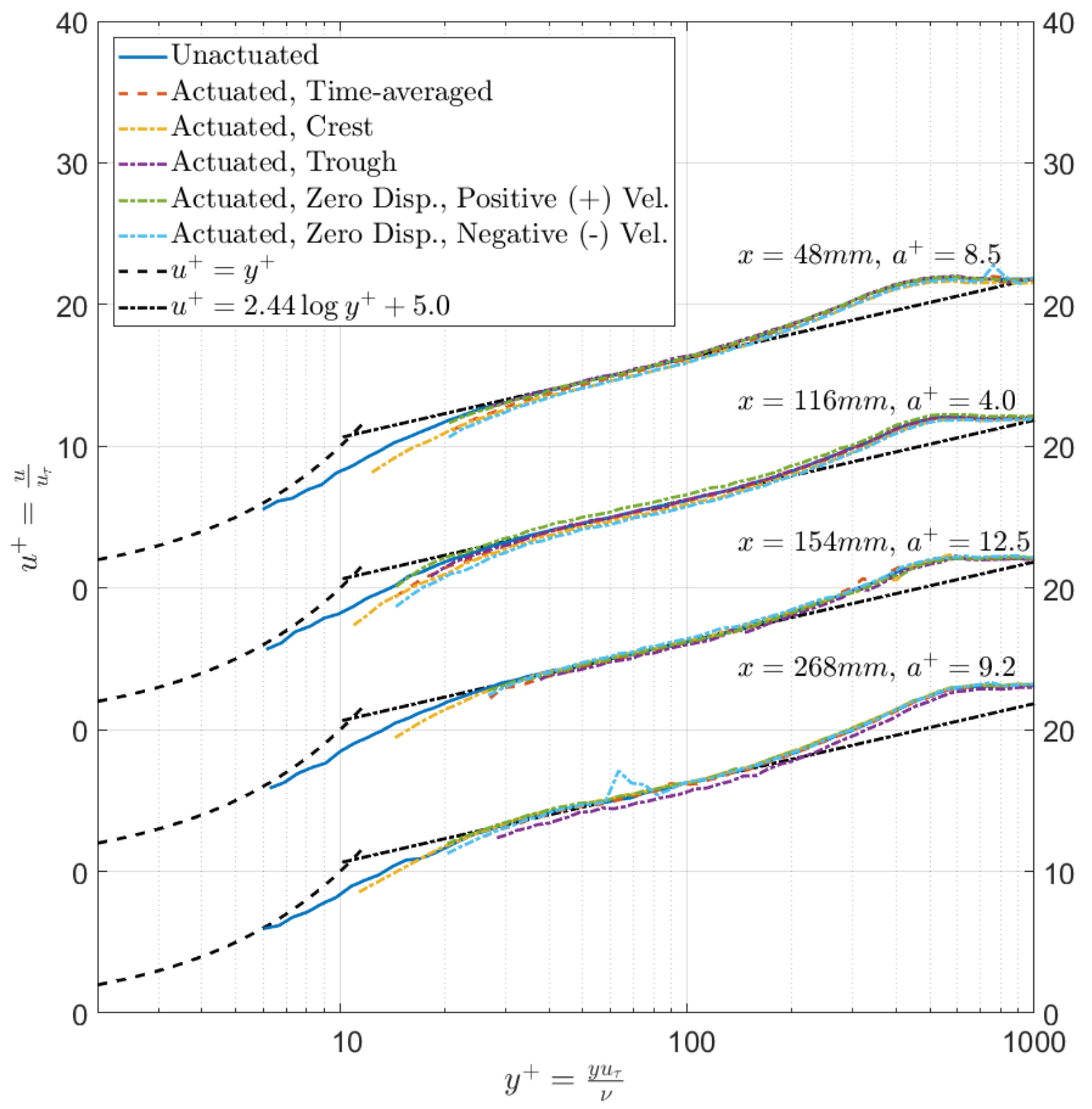

Figure 6.14: Experimentally measured phase-locked, turbulent boundary layers at four streamwise over the $430 \mathrm{~Hz}\left(T^{+}=112\right)$ traveling wave surface. At each location, the time-averaged and four phase-locked positions over the traveling wave surface: the crest and trough and also the zero displacement with both positive and negative wall-normal $(y)$ velocities

(i.e. drop-outs visible in the fluctuating velocity signal). For the upstream and downstream measurement locations, the actuated profile lines up with that over the unactuated plate; similar to the mean velocity profiles in Figure 6.13. For the cases directly over the traveling wave, the timeaveraged actuated profile is significantly larger near the wall. While the variance for the unactuated 


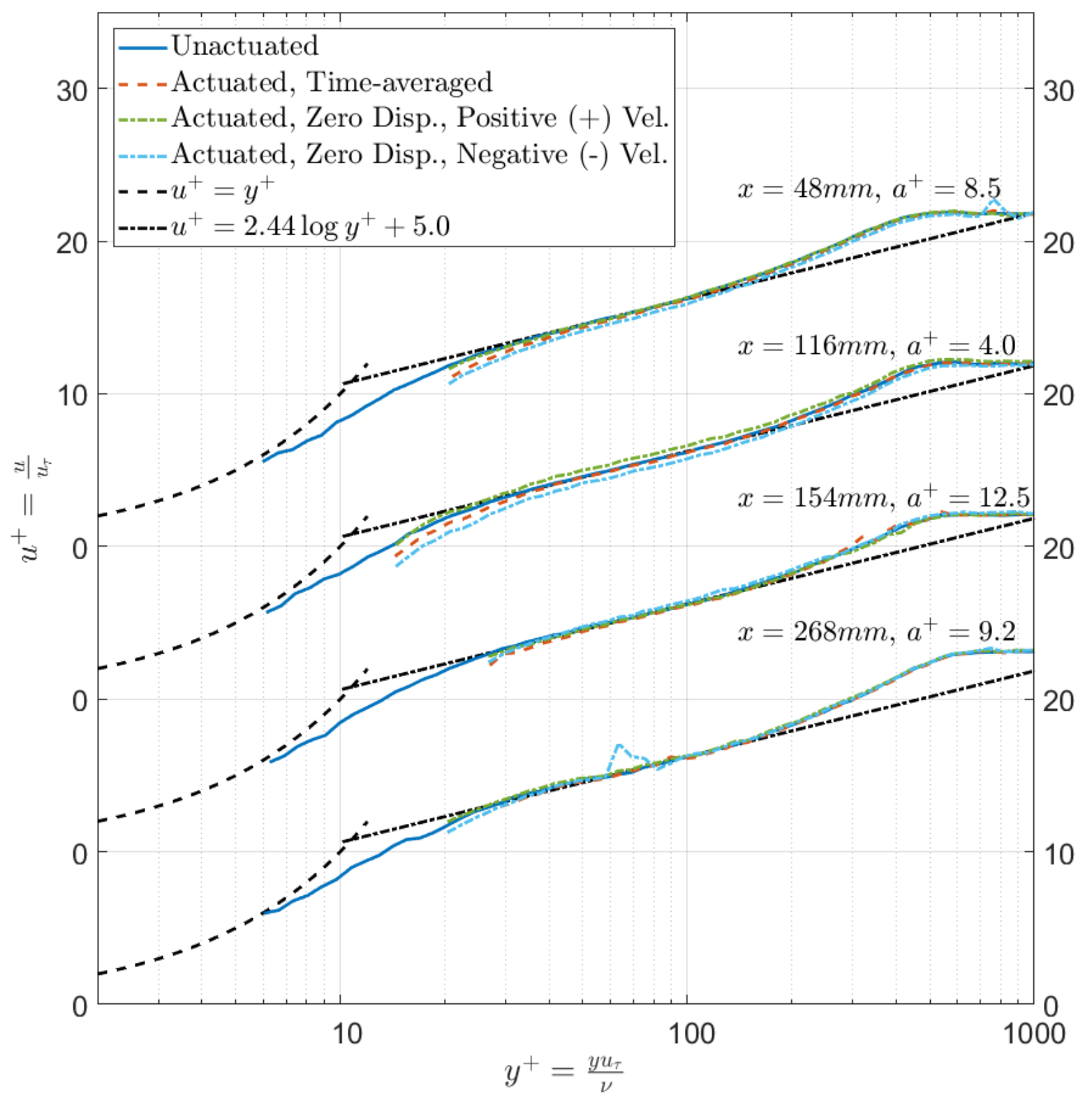

Figure 6.15: Experimentally measured turbulent boundary layers at four streamwise over the $430 \mathrm{~Hz}\left(\mathrm{~T}^{+}=112\right)$ traveling wave surface showing the phase-locked profiles when the traveling wave has zero displacement

case has a peak at $y^{+} \approx 15$, the time-averaged case seems to continually increase closer to the wall.

The streamwise Reynolds stress of the phase-locked profiles (crest and trough) do not see the near-wall increase in variance that occurred for the time-averaged actuated case. Looking at the profile over the crest, it is pushed slightly off the wall in the same way that the mean velocity profiles are pushed off the wall (Figure 6.14). As a result, the peak variance in the crest profile 


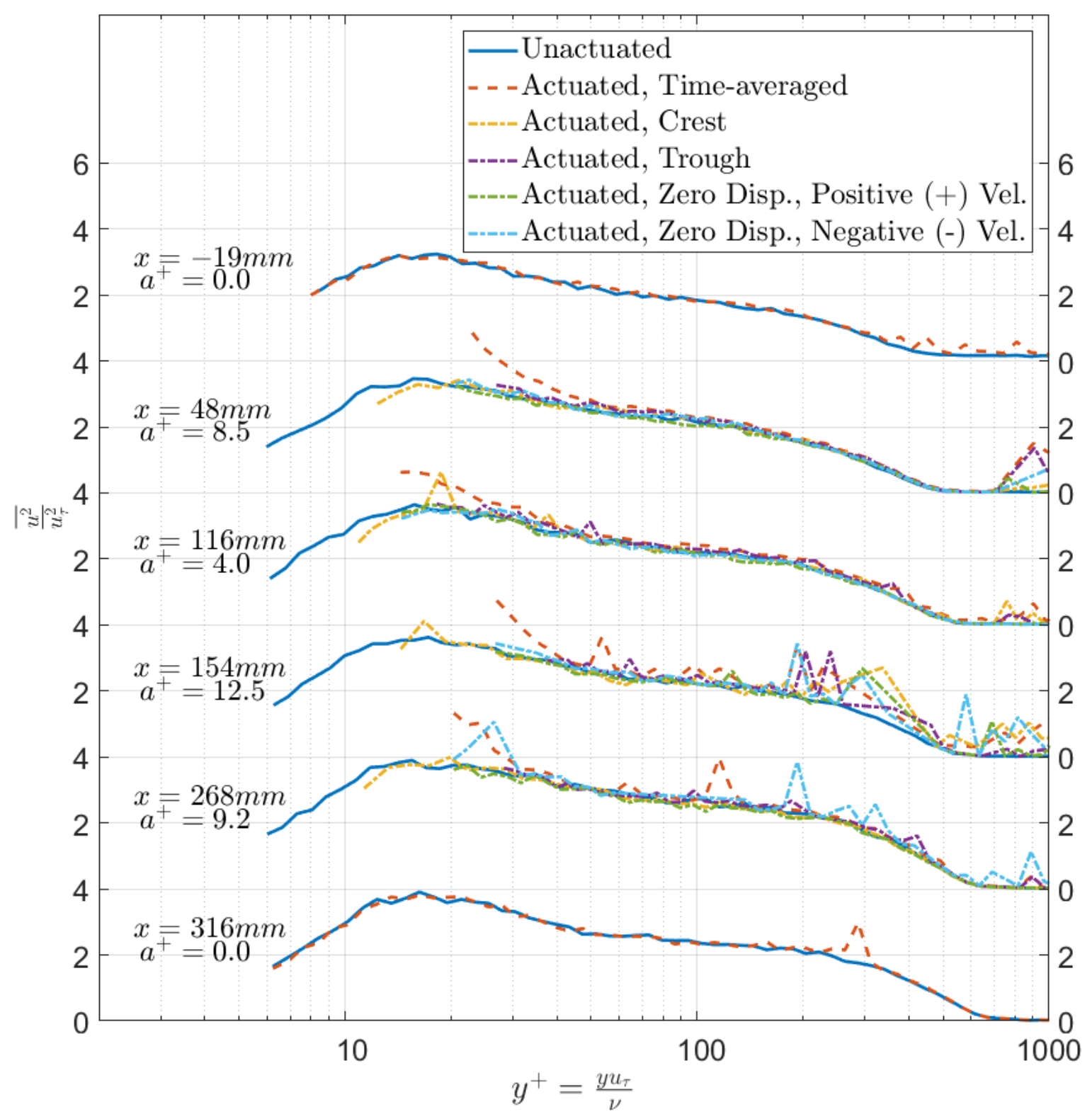

Figure 6.16: Variance/Streamwise Reynolds stress profile at various streamwise positions over the $430 \mathrm{~Hz}\left(\mathrm{~T}^{+}=112\right)$ traveling wave surface. The first and last profiles are upstream and downstream of the traveling waves, respectively. The phase-locked profiles at four different points in the traveling wave period are also shown.

occurs at a slightly larger $y^{+}$value. In addition, the $x_{p}=48 \mathrm{~mm}$ and $x_{p}=268 \mathrm{~mm}$ profiles suggest that the peak variance is reduced versus the unactuated case; however, this is difficult to definitively tell due to the uncertainty in the measurements. The uncertainty in the variance is not directly quantified in this study, but it is assumed large enough to prevent a conclusive statement about the 
effect of the traveling wave on the magnitude of the variance. This is based on the uncertainties in the velocity discussed in Section 6.3.6 in combination with the fact that the uncertainty in the variance is larger than that for the mean velocity [45]. Unfortunately, not much insight can be gained from the other phase-locked profiles. The peak variance occurs at $y^{+} \approx 15-20$ and the profiles could not be measured to $y^{+}$values in this range.

The increase in the time-averaged actuated variance near the wall is a result of the moving wall causing an oscillation in the relative $y$ position of the hot-wire to the wall. While measuring the flow velocity, the hot-wire probe sits at a stationary $y$ position with respect to the unactuated wall. However, when measuring directly over the traveling wave, the wall surface moves with respect to the stationary hot-wire probe. As a result, the relative $y$ position between the surface and probe changes based on the displacement of the plate (Figure 6.11b). This is the same effect that requires the $y$ position to be adjusted in Figure 6.12. This change in relative $y$ position is the same as the probe moving over a stationary wall. The probe is then effectively moving to different $y$ positions on the mean velocity boundary layer profile (Figure 6.13). Thus, the increase in the variance for the time-averaged actuated profile is purely a result of the change in relative $y$ position between the hot-wire probe and traveling wave surface.

\subsubsection{Skewness and Kurtosis Profiles}

The skewness and kurtosis were introduced in Section 2.2.3 and nominal profiles for the unactuated surface were shown in Section 5.2.5. Figure 6.17 shows the time-averaged and phase-locked profiles of the skewness and kurtosis over the $430 \mathrm{~Hz}\left(T^{+}=112\right)$ surface at $x_{p}=48 \mathrm{~mm}$. The effect of the traveling wave on the skewness and kurtosis is minimal, so the profiles are only analyzed here at a single streawise positions. Looking at the skewness profile in Figure 6.17a: far from the wall, all of the actuated profiles align with the unactuated one. The sharp drop of the time-averaged at $y^{+} \approx 450$ is smaller than the other cases, but this likely results from both the measurement density in the $y$ direction and the uncertainty in the results. As discussed by Bruun [45], the uncertainty in the skewness and kurtosis is generally quite large. The main difference occurs closer to the wall where the time-averaged profile diverges from the unactuated and phase-locked cases. Similar to the variance (Section 6.3.3), this is a result of the change in relative $y$ position over the moving wall.

Closer to the wall, the time-averaged profile has a roughly constant skewness of $S=0$, which indicates that the flow velocity fluctuates symmetrically about the mean. The $y$ position of the hot-wire probe oscillates, thus shifting the probe to different $y$ values on the mean velocity profile (Figure 6.12). As a result, there is an oscillation in the fluctuating velocity component resulting in a skewness of $S \approx 0$. When the phase-locked analysis is conducted, this oscillation in the relative 


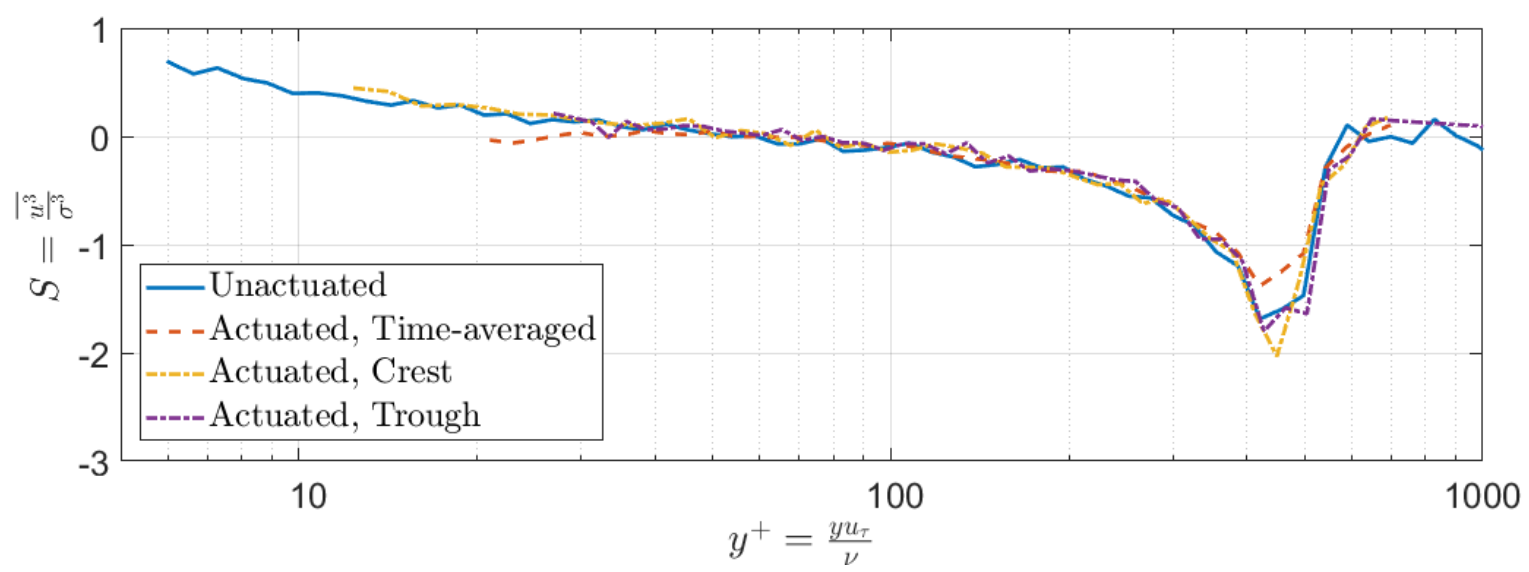

(a)

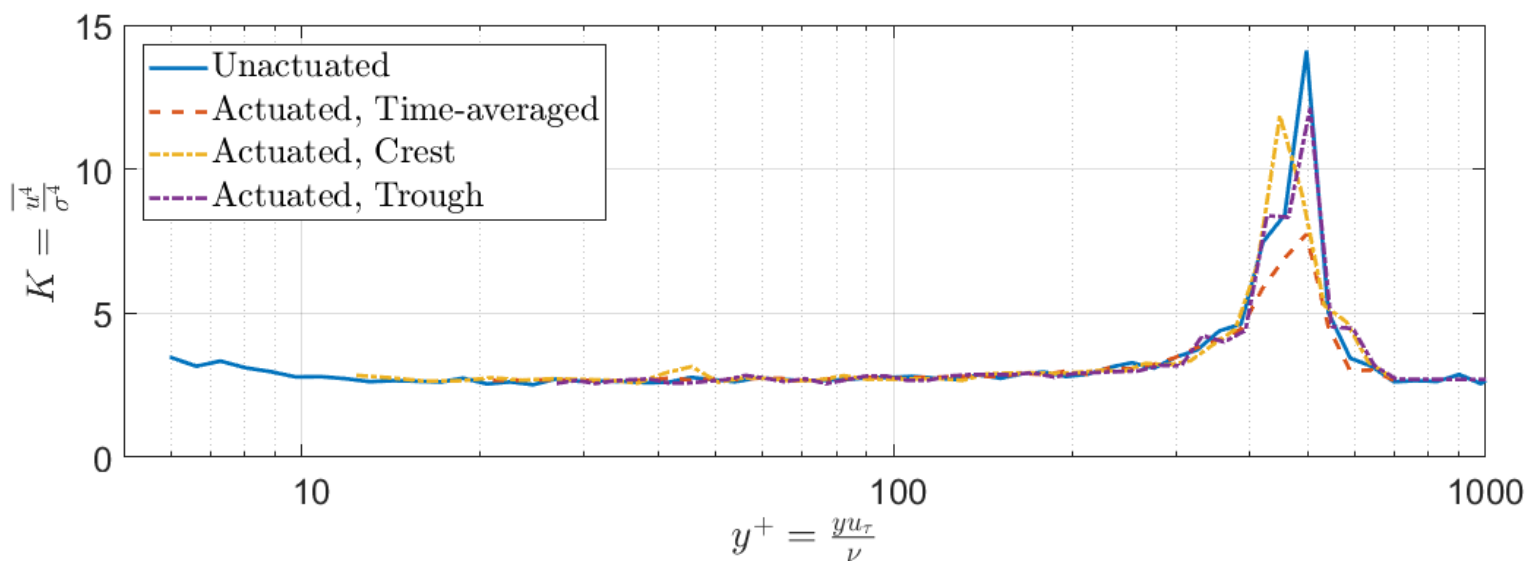

(b)

Figure 6.17: Phase-locked (a) skewness and (b) kurtosis profiles over the $430 \mathrm{~Hz}\left(T^{+}=112\right)$ traveling wave surface at $x_{p}=48 \mathrm{~mm}$

$y$ position is removed, so the crest and trough profiles return to the unactuated case. The phaselocked profiles at zero-displacement are not shown since they are the same as the crest and trough cases.

The kurtosis of the fluctuating flow velocity is shown in Figure 6.17b. All of the profiles converge well together and follow the unactuated profile. Similar to the skewness, the time-averaged actuated profile has a reduced magnitude at the spike at $y^{+} \approx 450$ which likely results from uncertainty in the velocity measurements. Unlike the skewness, there is no variation in the time-averaged profile from the phase-locked profiles. This is because the kurtosis captures the effective flatness of the fluctuating velocity, and the oscillation of the relative $y$ position does not effect this. 


\subsubsection{Profiles at Different Spanwise Positions}

The previous subsections have analyzed the actuated boundary layer over six different streamwise positions. Presented here are the results for boundary layers measured at five different spanwise positions all at $x_{p}=150 \mathrm{~mm}$ (Table 6.2). These positions relative to the traveling wave surface are shown in Figure 6.10. The mean velocity profiles are presented in Figure 6.18 and include the unactuated, time-averaged actuated, and phase-locked profiles. The traveling wave affects these turbulent boundary layers in the same manner as was seen in Figure 6.14. The time-averaged actuated profile is pushed further off the surface and the viscous sublayer is thickened, while the crest profile is pushed off as well, but not as much. In addition, the phase-lag effect demonstrated by the two zero-displacement cases is seen.

In Figure 6.18, the amount each crest boundary is pushed off the surface varies with spanwise positions. The two profiles at $z=63.5 \mathrm{~mm}$ and $z=25.4 \mathrm{~mm}$ are barely affected by the traveling wave or may even be pulled closer to the wall. Thus, there seems to be some underlying parameter(s) that is governing how much the traveling wave affects the boundary layer. The profiles at $z=0.0 \mathrm{~mm}$ and $z=-25.4 \mathrm{~mm}$ have the most significant change, and they also have the largest wall amplitudes at $a_{\text {crest }}^{+}=12.5$ and $a_{\text {crest }}^{+}=10.3$, respectively. Thus, there seems to be some connection to amplitude. However, the wall displacement at the measurement location is not the only parameter that contributes.

The two boundary layers at $z= \pm 63.5 \mathrm{~mm}$ have similar amplitudes of $a_{\text {crest }}^{+}=7.9$ and $a_{\text {crest }}^{+}=$ 7.0 , yet the boundary layers are affected to different degrees by the traveling wave. The crest profile at $z=+63.5 \mathrm{~mm}$ seems to be slightly pulled towards the wall. On the other hand, the profile at $z=-63.5 \mathrm{~mm}$ is pushed away from the wall. If the effect was purely amplitude, there should be a more similar reaction between the two profiles. Looking at the traveling wave itself (Figure 6.10) may give some indication of what is causing the effect. One suggestion is the upstream history of the traveling wave. Comparing the amplitude of the traveling wave upstream of the $z= \pm 63.5 \mathrm{~mm}$ measurement locations, the amplitude is significantly larger for the $z=-63.5 \mathrm{~mm}$ case. As a result, the upstream traveling amplitude may be contributing to the total effect of the traveling wave on the boundary layer. Another contributing parameter could be the propagation direction of the traveling wave. The wave front begins at $z=+63.5 \mathrm{~mm}$ and propagates in the $-z$ direction. Thus, the more strongly affected boundary layer at $z=-63.5 \mathrm{~mm}$ could result from the wave inducing a spanwise flow that is stronger at $z=-63.5 \mathrm{~mm}$ than the positive case.

The variance/streamwise Reynolds stress for the different spanwise positions are shown in Figure 6.19. The variance profiles at these spanwise locations are affected in the same way as for the streamwise positions (Figure 6.16). The time-averaged profile increases sharply near the surface as a result of the moving wall. The crest profile is pushed off of the surface and the peak amplitude is the same or slightly decreased. The other phase-locked profiles are not measured 


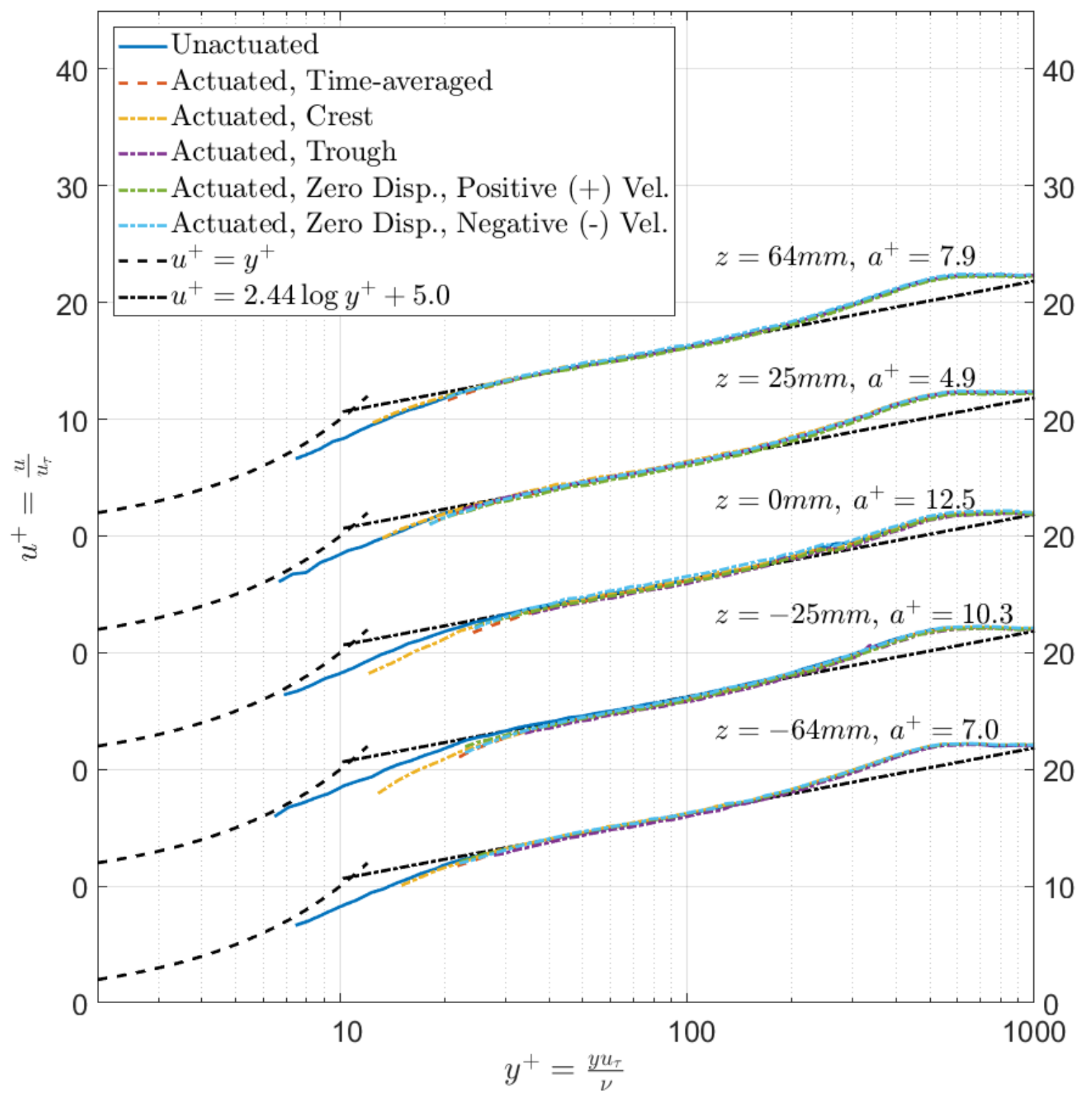

Figure 6.18: Experimentally measured phase-locked turbulent boundary layers at five different spanwise positions over the $430 \mathrm{~Hz}\left(T^{+}=112\right)$ traveling wave surface. The spanwise positions are all at $x_{p}=150 \mathrm{~mm}$ and are shown over the traveling wave in Figure 6.10

close enough to the wall to demonstrate any clear differences. 


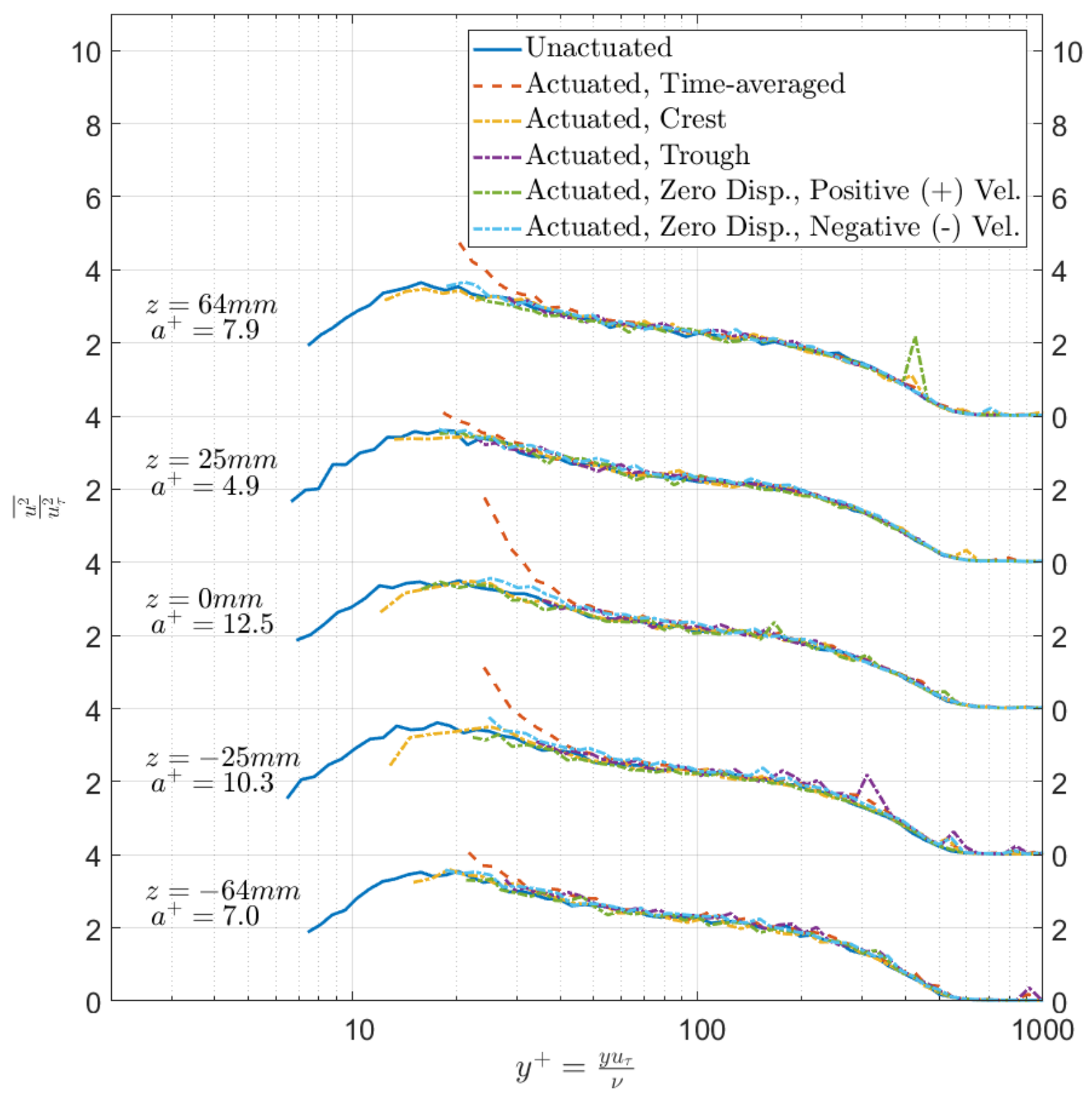

Figure 6.19: Variance/Streamwise Reynolds stress profile at five different spanwise over the $430 \mathrm{~Hz}\left(\mathrm{~T}^{+}=112\right)$ traveling wave surface. The unactuated, time-averaged actuated, and phaselocked crest and trough profiles

\subsubsection{Uncertainty in Boundary Layer Profiles}

The results presented in this chapter have looked at how the traveling waves affect the turbulent boundary layer. It has been shown that the time-averaged profile over the actuated surface is pushed further away from the wall such that the viscous sublayer is thickened. Analysis of phase-locked profiles then showed that the effect on the boundary layer changes based on the position within the 
wave oscillation period. The profile over the crest is still pushed off the wall, but not as strongly as the time-averaged. The zero-displacement positions showed that even when the plate has no deformation (zero-displacement) the profile is shifted, indicating a phase-lag effect. However, these results were all based on visual observations of the relative positions between profiles. As discussed in Appendix C, the experimentally measured boundary layer profiles have uncertainties. Thus, in order to increase the confidence in the above observations, they should be considered in light of the uncertainties in the measurements.

Figure 6.20 shows the mean velocity profiles over the $430 \mathrm{~Hz}$ wave at $z=-25.4 \mathrm{~mm}$ (Shown in Figure 6.18). In the figure are the unactuated, time-averaged actuated, and phase-locked crest and trough profiles. The error bounds in the velocity and $y$ position are given for each measured point. As has been discussed in the previous sections, the main effect of the traveling waves on the turbulent boundary layer is to extend the viscous sublayer and push the profile further from the surface. Looking at the time-averaged profile in Figure 6.20, near the wall there is a clear separation between the time-averaged and unactuated case. From the zoomed in portion of the figure, it is also clear that there is no overlap between the error bounds of the these profiles. Thus, the pushing off of the boundary layer can be attributed to the traveling waves.

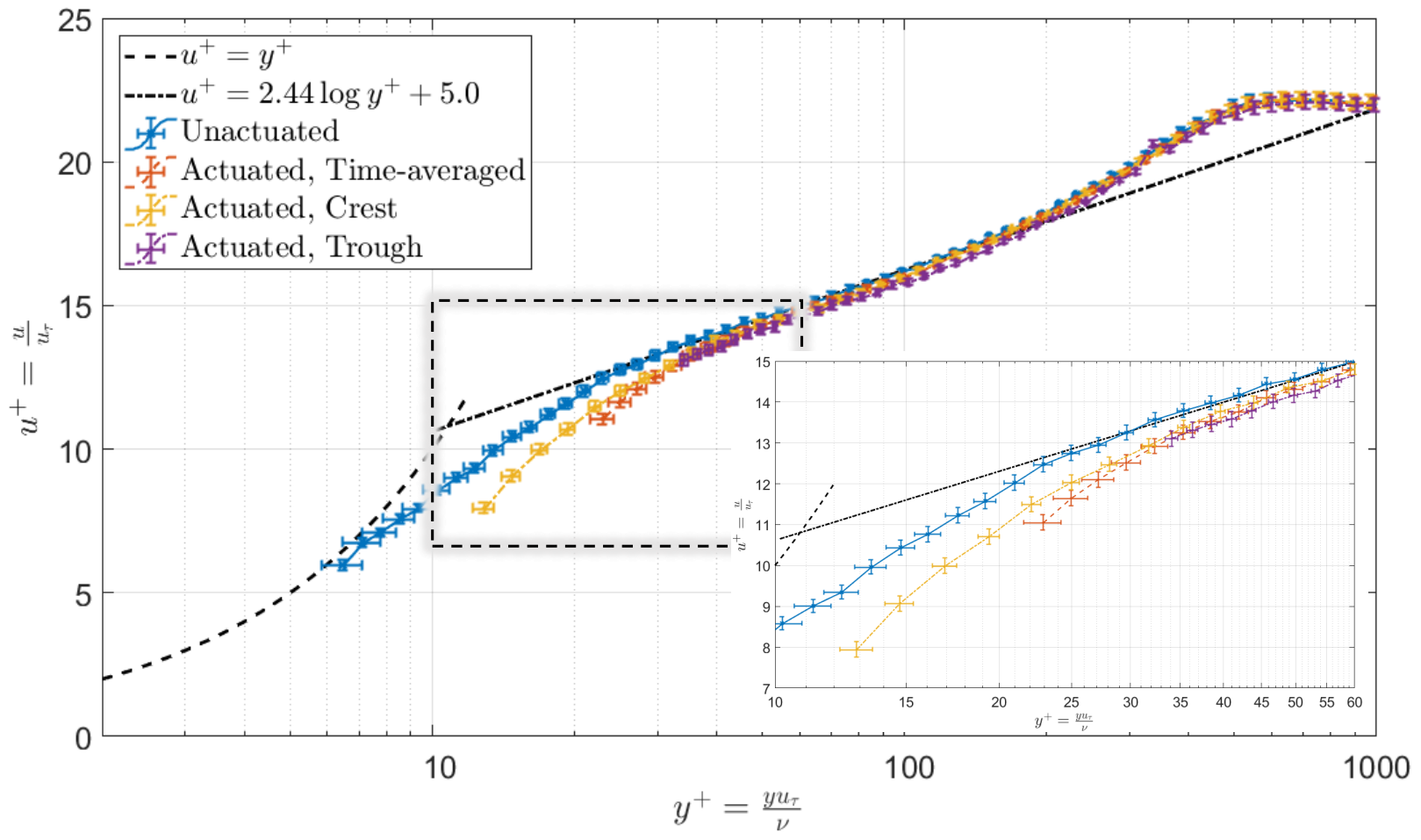

Figure 6.20: Boundary layer profiles over the $430 \mathrm{~Hz}$ wave at $z=-25.4 \mathrm{~mm}$ (Figure 6.18) showing the uncertainty in each measurement point. The phase-locked profiles over the crest and trough of the wave are shown. 
Looking at the phase-locked profiles over the traveling waves, it was observed that the profile over the crest of the wave (peak displacement) was not affected as strongly. This is visible in Figure 6.20 where the crest profile is pushed off the wall, but not as much as the time-averaged case. This difference is confirmed by the error bounds on the profiles. Near the wall $\left(y^{+} \approx 25\right)$, the crest and time-averaged profiles are separated from one another with no overlap of the error bounds. Slightly further from the wall $\left(y^{+}>25\right)$, the error bounds begin to converge and there is no clear separation. Thus, the uncertainties confirm that the crest and time-averaged profiles are affected differently, but that the differences are only visible in the near-wall region $(y+<25)$. Looking at the trough profile in Figure 6.20, its error bounds overlap with both the crest and time-averaged profiles, preventing any statement on differences over the trough. If the trough were measured at $y$ positions closer to the surface, deviations from the other profiles may become visible.

The other phase-locked profiles analyzed were the two zero-displacement positions (positive and negative velocity). Figure 6.21 shows the profiles over the $430 \mathrm{~Hz}$ wave at $x_{p}=48 \mathrm{~mm}$ (Figure $6.15)$ with the error bounds on each measurement point given. The time-averaged actuated profile is once again pushed away from the unactuated case with no overlap in their error bounds. Turning to the phase-locked profiles, the negative velocity case is clearly pushed off the surface and the error bounds do not overlap with the unactuated profile. This difference supports the observation that the traveling wave has a phase-lag effect on the boundary layer (Section 6.3.2). For the other zero-displacement profile, the traveling wave appears to have no effect on the boundary layer for the positive velocity case. The positive velocity profile is within the error bounds of the unactuated profile. Thus, the two zero-displacement positions yield different effects on the turbulent boundary layer.

The profiles shown in Figures 6.20 and 6.21 demonstrate the changes in the turbulent boundary layer caused by the traveling wave. These confidence in these differences is supported by the presentation of the experimental uncertainties. However, the profiles shown in these figures represent the cases where the traveling wave had the largest effect (i.e. largest displacement). For the other cases presented in the previous sections, the change in the turbulent boundary is often not is distinct. So, accounting for the uncertainty, any differences between profiles (e.g. crest and time-averaged) may no longer be clear. While this may be the case, some confidence can be gained by observing the same trend across various cases. As an example, the crest is generally always affected less than the time-averaged (Figures 6.14 and 6.18). For a single case, the uncertainty bounds may cloud any definitive differences between the crest and time-averaged profiles. However, the overall repeating trend combined with the clear results presented in Figure 6.20 give a degree of confidence as to how the crest profile behaves. This same rationale can also be used to gain confidence in the behavior of the zero-displacement profiles and the accompanying phase-lag effect. 


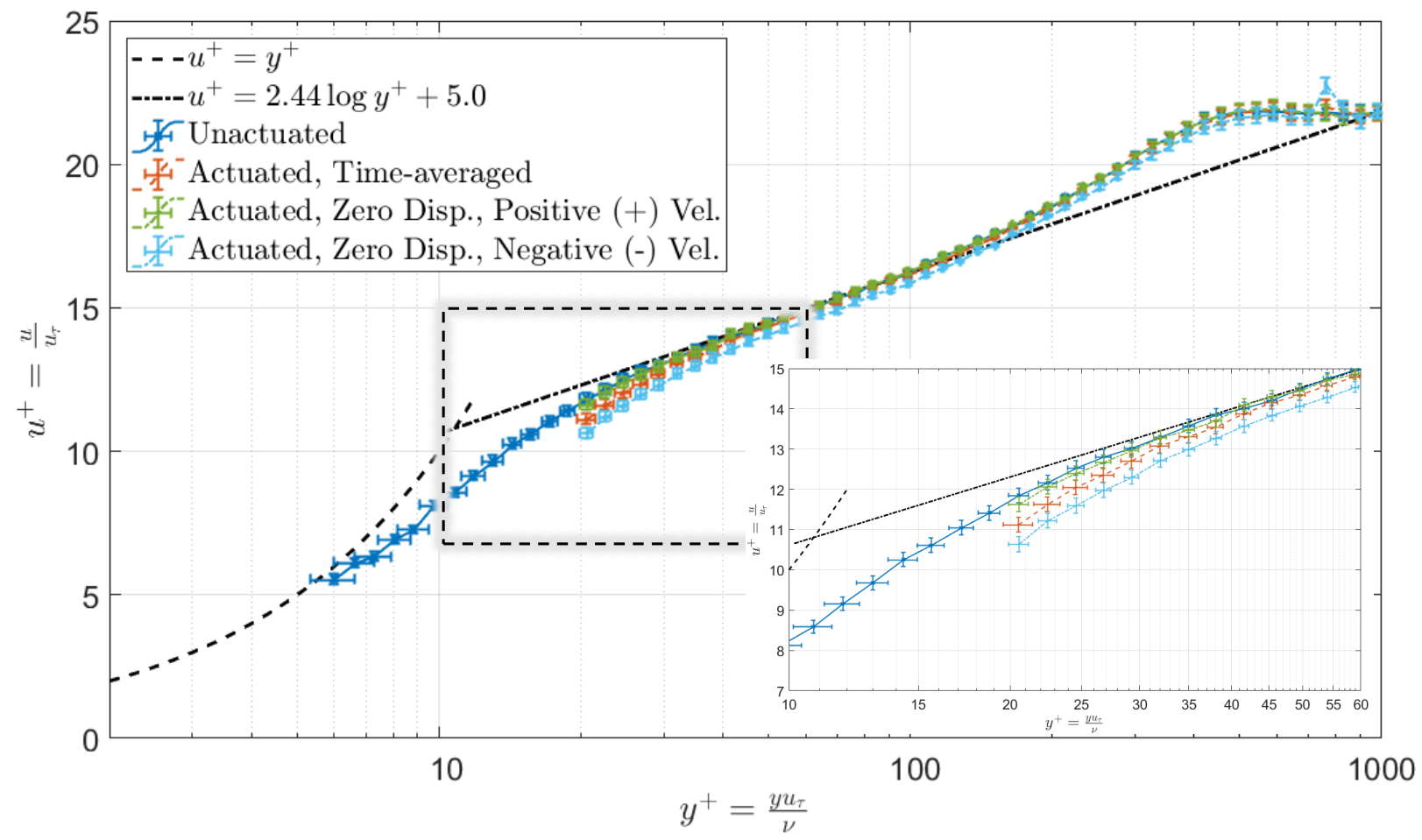

Figure 6.21: Boundary layer profiles over the $430 \mathrm{~Hz}$ wave at $x_{p}=-25.4 \mathrm{~mm}$ (Figure 6.18) showing the uncertainty in each measurement point. The phase-locked profiles over the two zero displacement points (positive and negative velocity) of the wave are shown.

\subsection{Discussion}

The previous section presented the results of how the $430 \mathrm{~Hz}\left(T^{+}=112\right)$ traveling wave in Figures 6.9 and 6.10 affects the turbulent boundary layer. In particular, time-averaged and phase-locked profiles over the traveling wave actuated surface were compared to the profile over the unactuated surface. The mean velocity profiles were analyzed at streamwise positions upstream, directly over, and downstream of the traveling wave region. The variance/streamwise Reynolds stress, skewness, and kurtosis profiles were also presented at these streamwise positions. Then, the mean velocity and variance profiles were analyzed at five different spanwise positions over the surface of the traveling wave. Finally, the results with considered with respect to the experimental uncertainty. In this section, these results are summarized and compared against others in the literature, in order to confirm the effectiveness of the low-profile traveling wave generation method investigated in this research.

The main effect of the traveling wave on the turbulent boundary layer is the thickening of the viscous sublayer and pushing off of the buffer layer from the wall (Figure 6.13). This was consistently observed at different locations over the traveling wave surface and the change in boundary 
layer was seen even when accounting for experimental uncertainty. This thickening of the viscous sublayer is the same effect that was seen in other studies investigating the effect of traveling waves $[4,5,26,30]$. In addition, this effect was also seen when investigating spanwise in-plane wall motion [24] and also compliant wall effects [99]. In each of these cases, the viscous sublayer is thickened and the velocity gradient decreases. As a result, this indicates a reduction in the local skin friction coefficient. The present experimental setup prevents measurements of the profile into the viscous sublayer; thus, the reduction in local skin friction cannot be quantified. However, the thickening of the viscous sublayer seen here suggests that the the skin friction coefficient is reduced.

The thickening of the viscous sublayer was only seen when the boundary layer was measured directly over the traveling wave surface itself. Upstream and downstream of the traveling wave actuated plate, no change in the turbulent boundary layer profile was observed. Tamano [5] observed the same effect and also showed that there is a recovery length downstream of the traveling waves over which the turbulent boundary layer recovers to the unactuated profile. For his experiments, the recovery length was $\approx 4 \delta$ and he compared that to Ricco [100] who saw a recovery length of $2 \delta$ for spanwise wall oscillations. In the present work, the turbulent boundary layer was measured $\approx 5 \delta$ downstream of the traveling wave peak. At this position there is no difference between the unactuated and actuated profiles; thus, the boundary layer has recovered by $5 \delta$ downstream and the recovery length can be said to be on the same scale as seen by Tamano [5].

A phase-locked analysis was also conducted here in which the boundary layer data at a single location over the traveling wave is analyzed and profiles pulled out corresponding to specific instances in the oscillation period. In particular, the profiles over the maximum and minimum displacement (crest and trough) were extracted along with profiles at the two zero displacement points (positive and negative wall-normal velocity. The result showed that the mean velocity profiles varied between these phase-locked locations. The profile over the trough was pushed off the wall and the sublayer thickened, but no clear difference from the time-averaged was seen. The profile over the crest of the wave still had a slight thickening of the sublayer, but not as large as the trough. Klumpp [4] and Koh [28] observed that the boundary layer profiles over the crest and trough of the traveling wave also varied from one another. The viscous sublayer was thickened over the trough, while there was no change or even a thinning of the sublayer over the crest. In the present study, the viscous sublayer appeared to be thinned over the crest in a few instances (Figure 6.18). However, the general effect seen here still appears to be a slight thickening of the sublayer over the crest. The crest profile is consistently affected less than the time-averaged, but the experimental uncertainty in the data (Section 6.3.6) could account this variation in whether it is thinned or thickened.

The phase-locked analysis also demonstrated that the traveling wave may affect the turbulent boundary layer in a non-linear fashion such that there is a phase-lag effect. Over a single period of 
the traveling wave, the displacement passes through zero twice. Once with a positive and once with a negative wall-normal velocity. Figure 6.15 showed that the boundary layer profiles over these zero displacement positions were effected but with different strengths. The fact that the boundary layers at zero displacement were even affected indicates the presence of phase-lag. If there was no phase-lag, then boundary layer profiles over zero displacement would not be changed and would thus converge with the unactuated case, which inherently has zero displacement.

The source of the phase-lag seems to relate to how quickly the turbulent boundary layer reacts to the traveling wave. For positive wall-normal velocity, there was minimal change in the boundary layer profile. While for negative velocity, the profile was substantially pushed off the wall as much or greater than the time-averaged profile. Thus, for positive velocity, the surface is moving upwards into the flow, but the boundary layer has not yet reacted to this movement, so there is minimal change in the boundary layer structure. For negative velocity, the wall is moving downwards and away from the from the flow. The fluid near the surface reacts, but that further from the wall has not yet reacted and lags behind. Thus, the viscous sublayer is stretched and thickened, decreasing the velocity gradient and in turn decreasing the skin friction drag.

This agrees well with that seen by Li [30]. In that study, it was suggested that the inertia of the flow is too high such that it cannot follow the downward motion of the surface. So, the local skin friction reduction is the sum of the reaction to the upwards and downwards movement of the surface. Other literature [3,28] has also suggested that the wall-normal velocity plays a role in how traveling waves decrease the wall-shear stress. However, these studies suggested the wall-normal motion pushes the turbulent vortices away from the surface. Thus, both the phase-lag and pushing of turbulent vortices off the surface may contribute to the mechanism by which traveling waves decrease the skin friction.

The variance/streamwise Reynolds stress of the fluctuating velocities are shown in Figures 6.16 and 6.19 for streamwise and spanwise positions, respectively. For the time-averaged actuated profile, there is a large increase in the variance near the wall. This large increase has also been seen in previous experimental studies $[5,26]$. However, by analyzing the phase-locked profiles, this increase near the wall has been shown to result from the shifting $y$ position over the moving wall. The relative position of the hot-wire probe with respect to the wall fluctuates since the wall itself is moving, so the probe is essentially moving to different $y$ positions on the mean velocity profile (Figure 6.13). This variation in the mean velocity is captured as variance in the velocity. Thus, the increase in the variance for the time-averaged actuated profile increase is purely a function of the $y$ position relative to the moving wall. The increase in the variance seen by Itoh [26] and Tamano [5] can also be attributed to this effect since both studies utilize hot-wire probes and present the variance as a time-average over the moving surface. Thus, the increase in variance near the wall does not result from a change in turbulent boundary layer structure as cited by Tamano [5]. 
The phase-locked variance profiles over the traveling wave, do not have the large increase near the wall observed in the time-averaged case. Thus, these phase-locked locations (crest, trough, zero displacement) better represent the effect of the traveling wave on the variance. Comparing the crest variance profile with the unactuated one (Figure 6.19), the peak in the streamwise Reynolds stress over the crest is pushed off of the wall. This peak occurs in the buffer layer, where the maximum turbulence production occurs [48]. Thus, the shifting of the peak indicates the pushing of the buffer layer and the peak turbulence production further off of the wall. At the peak, the maximum variance remains either constant or is slightly decreased from the unactuated case. However, these observations should be considered knowing that there is some uncertainty in the experimental data. In Koh's study [28], the peak is reduced over both the crest and trough and is shifted off the wall mainly over the trough. In the present study, the variance profile over the trough and other phaselocked positions could not be studied since they are not measured close enough to the surface. Klumpp [4] also showed that the peak variance is reduced over the crest and the trough, but he showed no clear shift in the position of this peak. Thus, there is no clear consensus on the effect of the traveling wave on the variance. Some studies suggest a reduction in magnitude while others present a shifting of the peak position. Further research is necessary to fully quantify the effect.

The results presented for the various spanwise positions in Section 6.3.5 suggest that the effect of the traveling waves on the boundary layer is parameter dependent. As the amplitude increases, the thickening of the viscous sublayer also increases. This agrees well with most previous studies investigating the the effect of traveling waves $[3,5,6,8,28,30,82]$. However, the results presented in Figue 6.18 suggest that the amplitude is not the only dependent factor. These results suggest that the upstream traveling wave amplitude upstream may contribute. Previous studies have also presented a dependence on the excitation frequency [5,8], this is discussed further in Chapter 7.

The results presented in this chapter clearly indicate that traveling waves generated here affect the turbulent boundary layer. The viscous sublayer is thickened and the buffer layer is pushed off of the way suggesting a reduction in local skin friction. The phase-locked analysis shows that the profiles at various points within the traveling wave period react differently. The profile over the crest is affected as much as the time-averaged. In addition, the phase-locked profiles at zero displacement indicate a phase-lag effect which may contribute to mechanism behind the skin friction reduction. The large increase in the streamwise Reynolds stress near the wall was found to be a product of the experimental setup and analysis method. The position of the peak variance was shown to be pushed off the wall. Finally, a dependence on the traveling wave amplitude and upstream effect was also presented.

Overall, these results demonstrate that the traveling wave generation method presented here is a viable method for affecting the turbulent boundary layer and potentially reducing the skin friction. Previous experimental studies have generated traveling waves using a speaker [26], an eccentric 
motor [5], and arrays of electomagnetic actuators [8,30,32]. While they established the effectiveness of traveling waves, these methods are bulky and could not be practically implemented. The traveling wave generation method presented in this research uses low-profile, open-loop controlled piezoelectric actuators. As a result, this research provides an implementable solution for manipulating the turbulent boundary layer with spanwise traveling waves. 


\section{Chapter 7}

\section{Effect of Traveling and Standing Waves on the Boundary Layer}

Steady-state traveling waves have been shown to alter the structure of the turbulent boundary layer. Previous research has pursued this for the aim of skin friction drag reduction. Chapter 6 presented the effects of a novel traveling wave generation method on the boundary layer. The steady-state waves were excited on a two-dimensional plate using open-loop controlled piezoelectric actuators. These results showed that the turbulent boundary layer can be modified by traveling waves without the need for bulky actuation setups as used in previous research. That chapter presented the results of a single frequency traveling wave, but additional frequencies and a parameteric dependence was not discussed. Thus, to better understand the relationship between the turbulent boundary layer and the traveling wave parameters, part of this chapter investigates traveling waves with different frequencies and patterns.

As explained in Chapter 4, the steady-state traveling waves generated in this research are structural borne. This means they are inherently tied to the structure's mode shapes and natural frequencies. As a result, the traveling waves share a number of common features with standing waves (mode shapes). Both have out-of-plane wall motion. Both also have node lines along the width of the surface (spanwise) which create separate peaks along the streamwise direction. Thus, the traveling and standing waves are quite similar and may have similar effects on the turbulent boundary layer. To investigate this, standing waves are generated in the same frequency and amplitude range as the traveling waves. The differences and similarities between the two types of waves are then compared.

This chapter begins by presenting the experimental results of exciting both traveling and standing waves in the wind tunnel described in Chapter 5. The same measurement and analysis procedure described in Chapter 6 is implemented. Two traveling waves are generated at $607 \mathrm{~Hz}$ and $810 \mathrm{~Hz}$, with each having a pattern similar to the $430 \mathrm{~Hz}$ wave from Chapter 6 . For each of these 
waves the traveling wave pattern is presented along with the effects on the mean velocity and variance boundary layer profiles. The results are compared and contrasted to the $430 \mathrm{~Hz}$ wave. Then, the results of standing waves at $385 \mathrm{~Hz}$ and $630 \mathrm{~Hz}$ are presented. Similar to the traveling waves, the standing wave pattern and the resultant effect on the mean velocity and variance boundary layer profiles are shown. Finally, all the results are summarized and briefly discussed. This includes the effects of the traveling waves and also a comparison between the two wave types.

Then, a brief parametric analysis is conducted. This begins by quantifying the change in the turbulent boundary layer due to the traveling and standing wave. The result is a single quantity for each boundary layer measurement. These values are calculated for 26 different actuated boundary layers representing different wave types, frequencies, and amplitudes. This data is then analyzed to determine how the turbulent boundary layer is affected by various parameters. This primarily focuses on the relationship with the wall-normal amplitude due to the strong dependence. However, conclusions are also drawn regarding the correlation with frequency and the differences between traveling and standing waves.

Finally, the chapter ends with an in-depth discussion of both traveling and standing waves. First, the overall effect of traveling waves on the turbulent boundary layer is outlined. A brief explanation of the underlying mechanism is given. Then, the dependence on various parameters is discussed; this includes displacement, frequency, and wave pattern. Second, the standing wave results are summarized and compared with those from the traveling waves. The differences and similarities between the two wave types are presented along with the dependence of various parameters. Recommendations for future traveling and standing wave experiments are also highlighted.

\subsection{Traveling and Standing Wave Results}

This section presents the results of experimentally measuring traveling and standing waves in the wind tunnel. The experimental setup described in Chapters 5 and 6 is used and both types of waves are excited using piezoelectric MFC actuators. The traveling waves are excited using the method outlined in Chapter 4 with a phase difference between the actuators. The standing waves use a similar method, but no phase difference is necessary. These results are used to investigate the overall effect of traveling waves on the turbulent boundary layer and also compare between standing and traveling waves.

The first part of the section presents the results of two different frequency traveling waves. The traveling waves are generated at $607 \mathrm{~Hz}$ and $810 \mathrm{~Hz}$ and compared with the $430 \mathrm{~Hz}$ wave from Chapter 6. For each wave, the traveling wave pattern and the resultant effect on the mean velocity and variance boundary layer profiles are given. Then the results of exciting two standing waves at $385 \mathrm{~Hz}$ and $630 \mathrm{~Hz}$ are presented. Similarly, the standing wave pattern and the resultant effect 
on the boundary layer via the mean velocity and variance profiles are given. The general effect of different traveling waves is presented, then the similarities between the traveling and standing waves are outlined.

\subsubsection{Traveling Waves}

The effects of a $430 \mathrm{~Hz}\left(T^{+} 112\right)$ traveling wave on the turbulent boundary layer was investigated in Chapter 6. Directly over the actuated surface, the boundary layer profile was pushed off the surface and the viscous sublayer thickened. This same trend was also seen in the variance/streamwise Reynolds stress profile. The location of peak variance, which occurs in the buffer layer, was pushed off the wall. Then a phase-locked analysis was conducted and showed that the boundary layer changes as the traveling wave oscillates through its period. In particular, a phase lag is shown such that the near wall flow responds quickly to the motion of the surface while the flow further away lags behind. As a result, the boundary layer is affected more strongly when the surface is moving downwards away from the flow, while the boundary layer at the crest of the wave is not altered as much. These results agreed with literature and demonstrated the effectiveness of the wave generation method, but they did not examine any parametric dependence.

To investigate the parametric dependence two additional traveling waves are generated and their effect on the turbulent boundary layer measured. These traveling waves are excited at $607 \mathrm{~Hz}$ and $810 \mathrm{~Hz}$, having normalized periods of $T^{+}=78$ and $T^{+}=59$, respectively. The traveling waves were excited at these frequencies for two reasons. First off, higher frequencies were used so as to investigate how the effect on the boundary layer varies; higher frequencies correspond to smaller periods $\left(T^{+}\right)$. Second, these frequencies minimize the difference in wave pattern between the three traveling waves. The $430 \mathrm{~Hz}$ traveling wave pattern strongly resembles the $(5,2)$ mode shape (Section 6.1.3). Here, the $607 \mathrm{~Hz}$ and $810 \mathrm{~Hz}$ waves resemble the $(5,3)$ and $(5,4)$ mode shapes, respectively. Thus, each wave has the same number of streamwise anti-nodes (5) and the wavefronts propagate in roughly the same location and direction. This allows the three traveling waves to be more easily compared without significant variations in the wave pattern.

The $607 \mathrm{~Hz}$ traveling wave is discussed first, and begins by presenting the traveling wave pattern. This includes discussion of the nearby mode shapes and the excitation configuration used to excite the piezoelectric actuators. Then the mean velocity boundary layer profile is presented at five streamwise locations over the traveling wave. Three positions are directly over the actuated surface, while one each is measured upstream and downstream. Finally, the variance/streamwise Reynolds stress profiles at these locations are presented. This same procedure is then repeated for the $810 \mathrm{~Hz}$ traveling wave. Some additional figures are presented in Appendix D. 


\subsubsection{607Hz Traveling Wave}

The first traveling wave is generated at $f=607 \mathrm{~Hz}\left(T^{+}=78\right)$ and is between the $(2,4)$ and $(3,4)$ mode shapes at $f=579 \mathrm{~Hz}$ and $f=630 \mathrm{~Hz}$, respectively. The operational deflection shapes (ODSs) for these mode shapes can be seen in Appendix D.1. The traveling wave is excited here using the actuation configuration expressed in Equation 7.1 and shown in Figure 7.1, where $\gamma=-110^{\circ}$. This is the same configuration used by the $430 \mathrm{~Hz}$ wave in Chapter 6, and was used to generate a similar wave pattern here as for the previous traveling wave. Each actuator was excited with $\pm 350 \mathrm{~V}$, which is the maximum allowable voltage due to nonlinear effects in the experimental setup (Section 6.1.3).

$$
\begin{array}{rll}
\phi_{A_{1}}=\phi_{A_{3}}=\phi_{A_{5}}=0^{\circ} & , \quad \phi_{B_{1}}=\phi_{B_{3}}=\phi_{B_{5}}=\gamma \\
\phi_{A_{2}}=\phi_{A_{4}}=180^{\circ} & , \quad \phi_{B_{2}}=\phi_{B_{4}}=180^{\circ}+\gamma
\end{array}
$$

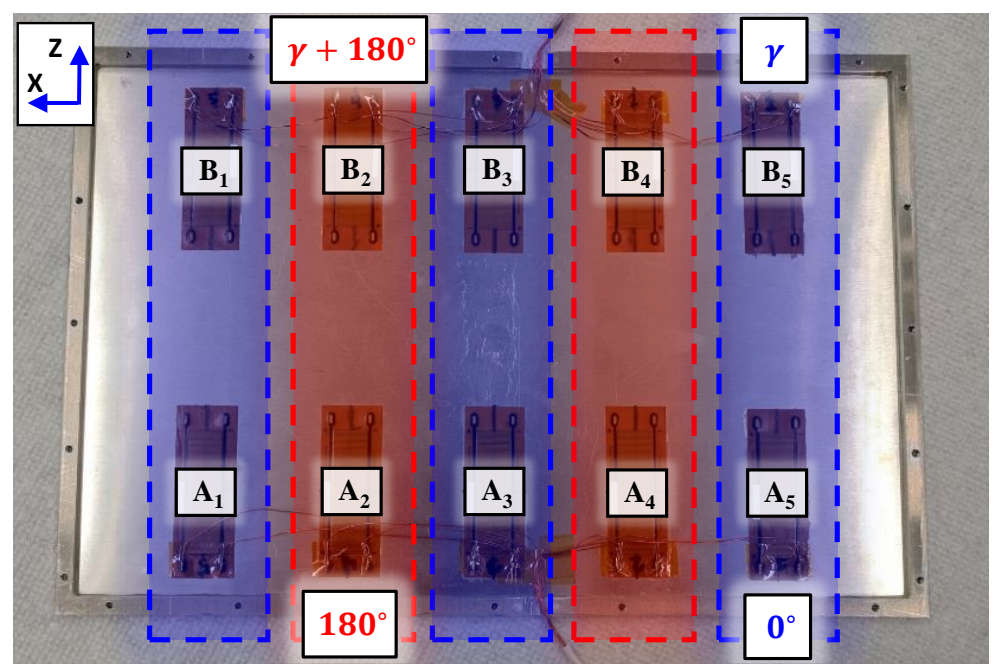

Figure 7.1: Actuation configuration to excite the $607 \mathrm{~Hz}\left(T^{+}=78\right)$ traveling wave. The top actuators are $\gamma=-110^{\circ}$ out of phase with the bottom ones. The actuators in red are $180^{\circ}$ offset from those in blue.

The resultant traveling wave is shown in Figure 7.2, which displays the experimentally measured RMS velocity of the wave. Figures showing the traveling wave over time are found in Figure D.1. The $607 \mathrm{~Hz}$ traveling wave resembles the $(5,3)$ mode shape and has three separate traveling wave fronts. Upstream, there are two waves both propagating in the $+z$ direction with a $180^{\circ}$ phase difference between them. Over the middle of the plate, there is no significant wave propagation. Downstream, there is a wave front propagating in the $+z$ direction, but with some recirculation near the center-line (not-shown in Figure). Interestingly, this traveling wave resembles the $(5,3)$ mode shape even though the nearby natural frequencies are the $(2,4)$ and $(3,4)$ modes. This results from 
the actuation configuration used, which has five actuators along the streamwise direction excited in a similar locations and phase as the $(5,3)$ mode.

This traveling wave pattern has some similarities to the $430 \mathrm{~Hz}$ wave from Chapter 6 (Figure 6.10). The $430 \mathrm{~Hz}$ resembled the $(5,2)$ mode shape, and the $607 \mathrm{~Hz}$ resembles the $(5,3)$ mode. The streamwise wavelengths are the same for each (five antinodes), while the $607 \mathrm{~Hz}$ wave has a smaller spanwise wavelength (three versus two anti-nodes). In addition, they both have similar wavefronts. Both frequencies have a traveling wave propagating the $+z$ direction and the far upstream and downstream edges. The differences lie in the middle of the plate. The $430 \mathrm{~Hz}$ pattern had a wavepropagating in the $-z$ direction, while the $607 \mathrm{~Hz}$ has one slightly upstream in the $+z$ direction. Despite these differences, the patterns are fairly similar, providing a good comparison between the $430 \mathrm{~Hz}\left(T^{+}=112\right)$ and $607 \mathrm{~Hz}\left(T^{+}=78\right)$ traveling waves.

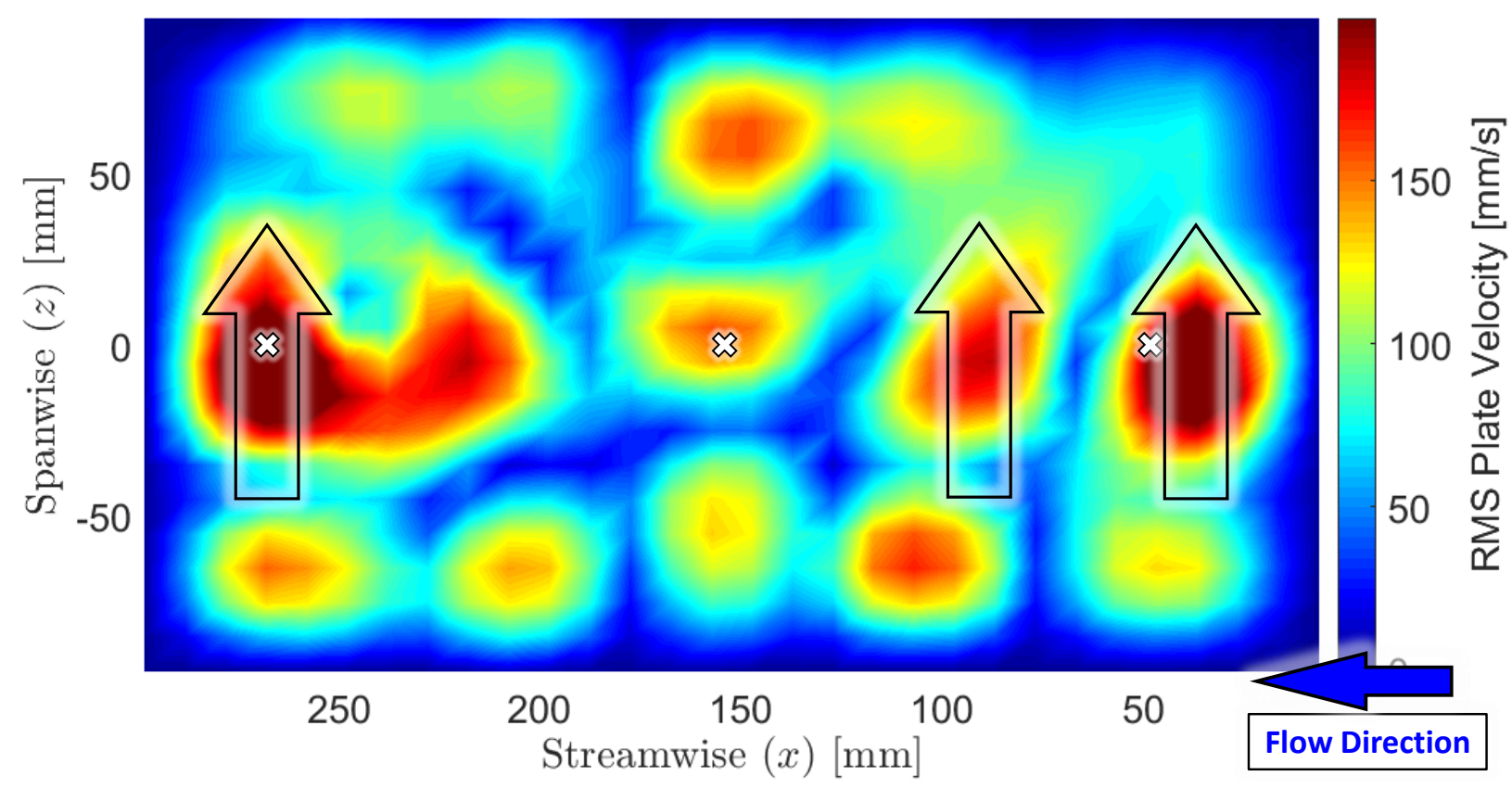

Figure 7.2: Root-mean-square (RMS) velocity of the traveling wave at $f=607 \mathrm{~Hz}\left(T^{+}=78\right)$. The boundary layer measurement positions are highlighted along with the propagation direction of the traveling waves

The turbulent boundary layer was experimentally measured at five streamwise locations for the $607 \mathrm{~Hz}$ wave. These are listed in Table 7.1 and the three positions directly over the wave are shown in Figure 7.2. The displacement amplitude of this traveling wave is smaller than that of the $430 \mathrm{~Hz}$ even though both frequencies were excited with ten actuators at $\pm 350 \mathrm{~V}$. This difference in amplitude is because the traveling waves are inherently tied to the structural response of the plate. So, the response amplitude depends on the magnitude of the response (Figure 6.3) at the excitation 
frequency. The maximum amplitude for the $607 \mathrm{H}$ wave is $a_{\text {crest }}^{+}=5.9$, while it was $a_{\text {crest }}^{+}=12.5$ for the $430 \mathrm{~Hz}$ wave. So, the differences in amplitude will slightly impede a direct comparison.

Table 7.1: The streamwise measurement locations for the $607 \mathrm{~Hz}\left(T^{+}=78\right)$ traveling wave along with the maximum and peak to peak displacement amplitude. All positions are at $z=0 \mathrm{~mm}$ and the three positions over the traveling wave surface $\left(a^{+} \neq 0\right)$ are shown in Figure 7.2

\begin{tabular}{c|ccccc}
\hline Streamwise Position, $z[\mathrm{~mm}]$ & 316 & 268 & 154 & 48 & -19 \\
\hline$a_{\text {crest }}[\mu \mathrm{m}]$ & 0 & 117.2 & 74.4 & 99.6 & 0 \\
$a_{c r e s t}^{+}$ & 0 & 5.9 & 4.0 & 5.2 & 0 \\
$a_{p-p}^{+}$ & 0 & 12.0 & 7.8 & 10.22 & 0 \\
\hline
\end{tabular}

The time-averaged and phase-locked boundary layer profiles at the streamwise positions in Table 7.1 are shown in Figure 7.3. Similar to the $430 \mathrm{~Hz}$ case, upstream and downstream of the traveling waves, there is no difference between the actuated and unactuated profiles. Looking directly over the surface, the traveling waves do not have a significant effect on the boundary layer profile. At $x_{p}=48 \mathrm{~mm}$ and $x_{p}=268 \mathrm{~mm}$, the time-average actuated profile is pushed slightly off of the surface and the viscous sublayer thickened. However, the crest profiles behave differently for each position. At $x_{p}=268 \mathrm{~mm}$, the crest profile responds as expected (Section 6.3.2) with the sublayer thickened but not as much as the time-averaged. For the $x_{p}=48 \mathrm{~mm}$ position, the crest profile seems to be negatively affected by the traveling wave, and is pulled closer to the surface. This same effect is also more significantly seen at $x_{p}=154 \mathrm{~mm}$. No clear differences in the other phase-locked profiles can be seen.

The variance/streamwise Reynolds stress profiles at the streamwise locations are shown in Figure 7.4. As with the mean velocity profile, the traveling wave has no effect on the upstream and downstream profiles. Directly over the surface, the variance of the time-averaged actuated profile increases near the wall. This is once again due to the oscillation in the relative $y$ position over the moving surface (Section 6.3.3). At $x_{p}=154 \mathrm{~mm}$ the profile over the crest suggests that the peak variance is closer to the wall and that the viscous sublayer is thinner. This agrees with the mean velocity profile at that position (Figure 7.3).

Overall, the viscous sublayer of the crest profile appears to thin in some cases. This could be connected to the frequency of the excitation or more likely, the traveling wave pattern itself (see Section 7.3). However, no clear conclusion can be drawn from this. The boundary layer is not measured at small enough $y^{+}$values to sufficiently see a difference between the profiles. In addition, the overall effect on the boundary layer is small. There is only slightly separation between the unactuated and actuated profiles, especially when comparing to the cases seen for the $430 \mathrm{~Hz}$ traveling wave (Figure 6.14). Finally, the inclusion of experimental uncertainties (Section 6.3.6) 


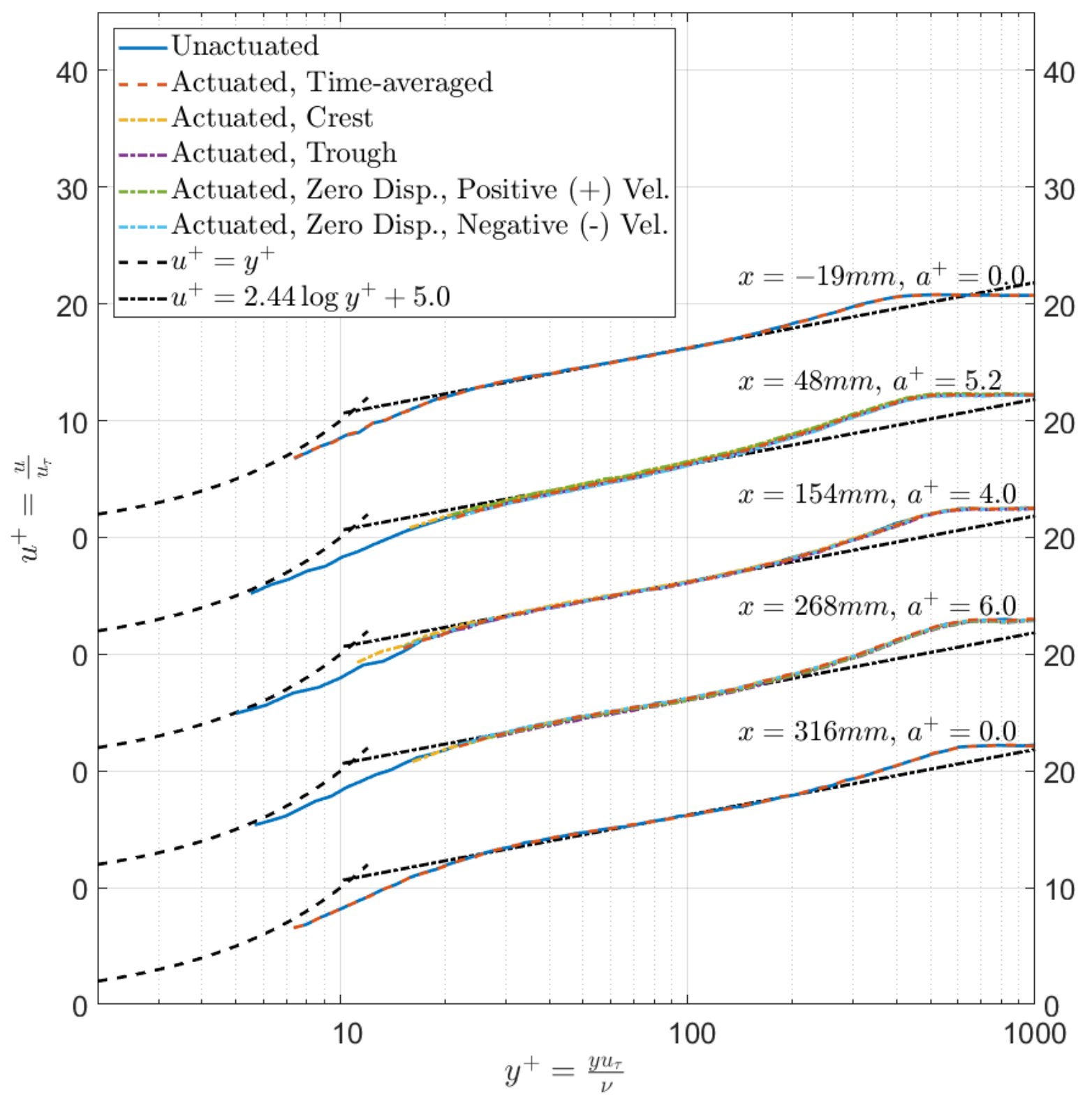

Figure 7.3: Experimentally measured phase-locked, turbulent boundary layers at five streamwise positions for the $607 \mathrm{~Hz}\left(T^{+}=78\right)$ traveling wave surface. At each location, the time-averaged actuated and unactuated profiles are shown. Directly over the moving surface phase-locked profiles are shown at the crest and trough of the wave along with the two points in the wave period with zero displacement.

can further complicates the matter. As a result, the $607 \mathrm{~Hz}$ traveling wave should be excited with larger amplitudes to provide a clearer difference. 


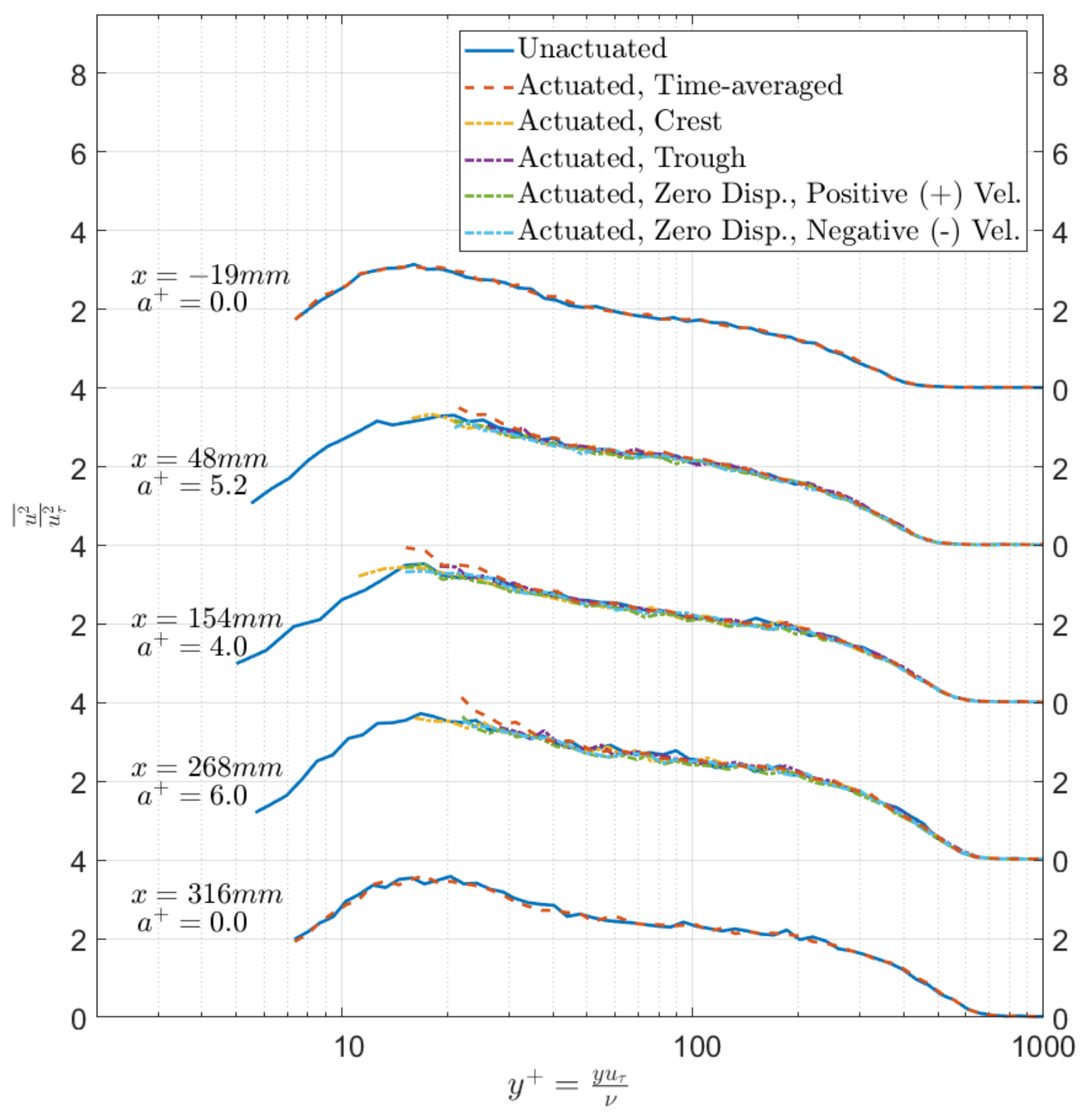

Figure 7.4: Variance/Streamwise Reynolds stress profile at streamwise positions over the $607 \mathrm{~Hz}\left(\mathrm{~T}^{+}=78\right)$ traveling wave surface. The first and last profiles are upstream and downstream of the traveling waves, respectively. The phase-locked extracted profiles are shown for the locations directly over the actuated surface.

\subsubsection{810Hz Traveling Wave}

The second traveling wave is generated at $f=810 \mathrm{~Hz}\left(T^{+}=59\right)$ between the $(5,4)$ and $(7,3)$ modes shapes at $f=792 \mathrm{~Hz}$ and $f=869 \mathrm{~Hz}$, respectively. This traveling wave is excited using the same actuation configuration as the $430 \mathrm{~Hz}$ and $607 \mathrm{~Hz}$ traveling waves (Equation 7.1 and Figure 
7.1) with $\gamma=90^{\circ}$ and $\pm 350 \mathrm{~V}$. This was done to produce a wave resembling the $(5,4)$ mode shape and thus have a traveling wave pattern similar to that of the previous two waves. The $430 \mathrm{~Hz}$ and $607 \mathrm{~Hz}$ waves took the form of the $(5,2)$ and $(5,3)$ mode shapes, respectively. The resultant $810 \mathrm{~Hz}$ traveling wave is shown in the RMS velocity plot in Figure 7.5. The wave pattern here is similar to both previous frequencies. In fact, the $810 \mathrm{~Hz}$ pattern here has wave-fronts propagating in the same location and direction as the $430 \mathrm{~Hz}$ case. The upstream and downstream edges have a wave moving the $+z$ direction while the middle of the plate has one in the $-z$ direction. Figures of the $810 \mathrm{~Hz}$ traveling wave over time a be seen in Appendix D.2.

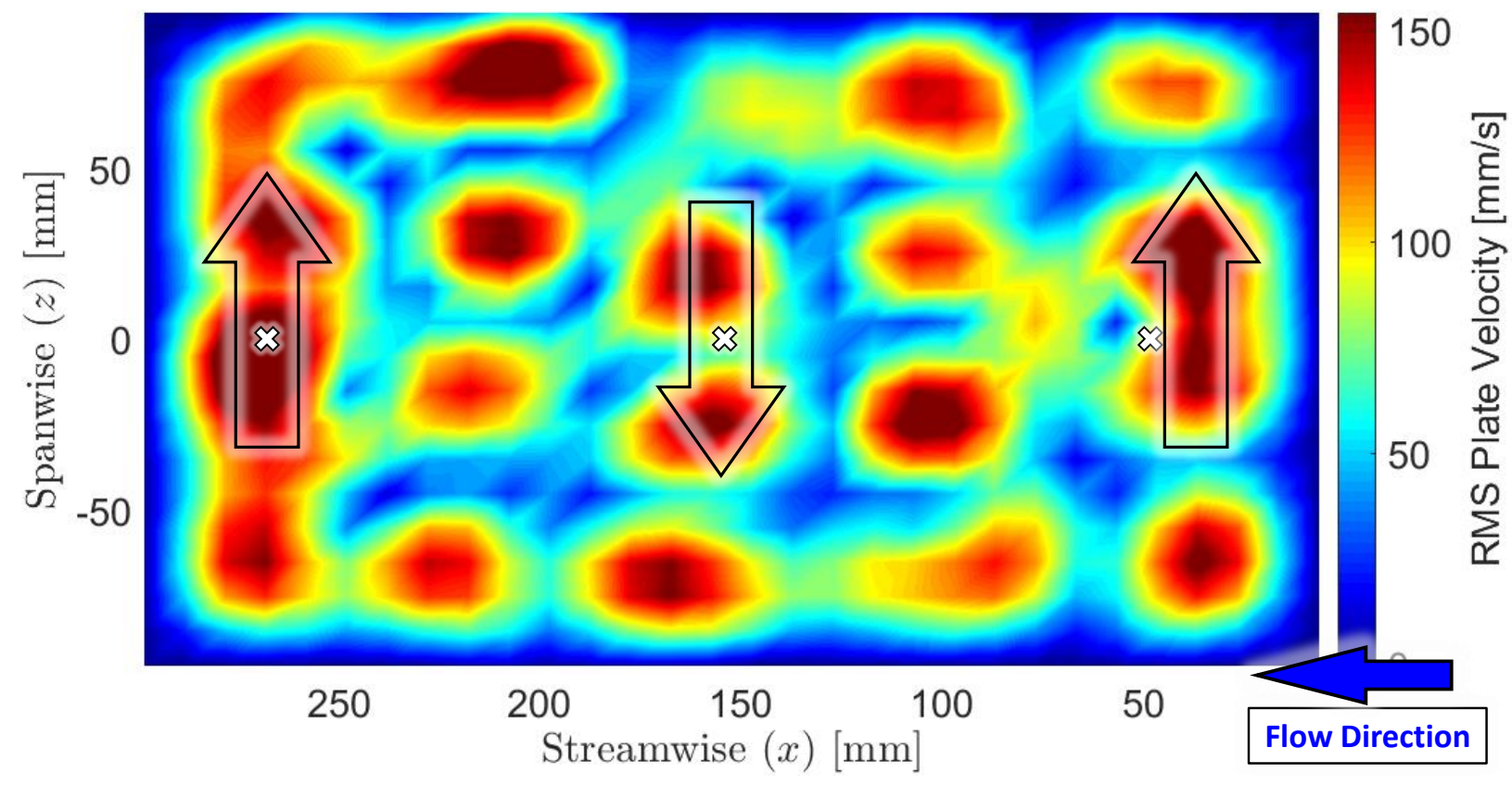

Figure 7.5: Root-mean-square (RMS) velocity of the traveling wave at $f=810 \mathrm{~Hz}\left(\mathrm{~T}^{+}=59\right)$. The boundary layer measurement positions are highlighted along with the propagation direction of the traveling waves

As with the other traveling wave cases, the turbulent boundary layer is measured at five streamwise locations. These are shown in Table 7.2, with the three positions directly over the actuated surface shown in Figure 7.5. The wall-normal amplitudes $\left(a_{c r e s t}^{+}\right)$for this wave are quite low and are actually within the viscous sublayer $\left(y^{+}<5\right)$ of the boundary layer. There were experimental issues at $x_{p}=48 \mathrm{~mm}$, so the amplitude could not be measured and there is visible noise in the mean velocity and variance profiles. The boundary layer profiles at these measurement positions are shown in Figure 7.6. At positions $x_{p}=48 \mathrm{~mm}$ and $x_{p}=268 \mathrm{~mm}$ there is minimal difference from the unactuated case. The noise in the actuated profile at $x_{p}=48 \mathrm{~mm}$ is due to experimental issues. The most substantial differences occur at $x_{p}=154$, where all of the profiles are pulled closer to the wall and the viscous sublayer thinned. This is similar to what was seen for the $607 \mathrm{~Hz}$ traveling 
wave case. Figure 7.7 shows the variance profiles at these streamwise positions. At $x_{p}=154 \mathrm{~mm}$, the peak variance is closer to the wall for all of the actuated profiles. This agrees with the thinning seen in the mean velocity profile. The spikes at $x_{p}=48 \mathrm{~mm}$ result from the experimental issues mentioned.

Table 7.2: The streamwise measurement locations for the $810 \mathrm{~Hz}\left(T^{+}=59\right)$ traveling wave along with the maximum and peak to peak displacement amplitude. All positions are at $z=0 \mathrm{~mm}$ and the three positions over the traveling wave surface $\left(a^{+} \neq 0\right)$ are shown in Figure 7.5.

\begin{tabular}{c|ccccc}
\hline Streamwise Position, $z[\mathrm{~mm}]$ & 316 & 268 & 154 & 48 & -19 \\
\hline$a_{\text {crest }}[\mu \mathrm{m}]$ & 0 & 69.7 & 25.2 & - & 0 \\
$a_{c r e s t}^{+}$ & 0 & 3.5 & 1.3 & - & 0 \\
$a_{p-p}^{+}$ & 0 & 6.9 & 2.5 & - & 0 \\
\hline
\end{tabular}

At $x_{p}=154 \mathrm{~mm}$, the wall displacement is quite low $\left(a^{+}=1.3\right)$. This is confirmed in the timeaveraged variance profile. If the amplitude were at all substantial, it would increase near the wall due to the oscillating relative $y$ effect (Section 6.3.3). Since the displacement is so low, it is surprising that the boundary layer is affected at all, either via thinning or thickening of the sublayer. The source could relate to the traveling wave pattern itself. In particular, the amplitude on either spanwise side of the measurement location (Figure 7.5). The large amplitude on either side could be pushing the turbulence vortices off the wall and funneling them into this lower amplitude region. As a result, there is a greater amount of turbulence and bursting events in this region which would thin the sublayer and increase the local skin friction coefficient. Thus, this suggests that the traveling wave pattern plays a role in how the turbulent boundary layer is affected. This is discussed further in Section 7.3.

\subsubsection{Standing Waves}

As explained in Chapter 4, the steady-state traveling waves are inherently connected to the structural properties of the surface. This is how the traveling waves in Section 7.1.1 could all be generated with $(5, n)$ mode shapes $(n=2-4)$. As a result, the traveling waves share a number of similarities with standing waves (mode shapes). This includes wall-normal displacement, and node lines along the streamwise direction. So, the standing waves may alter the turbulent boundary layer in a similar manner as the traveling waves. To investigate this, two different standing waves are generated here. These are at $385 \mathrm{~Hz}\left(T^{+}=122\right)$ and $630 \mathrm{~Hz}\left(T^{+}=74\right)$ and take the form of the $(2,3)$ and $(3,4)$ mode shapes, respectively. These frequencies were chosen since they coincide with natural frequencies and are within the same frequency range as the traveling waves. These standing waves are excited using piezoelectric actuators on the same plate as the traveling 


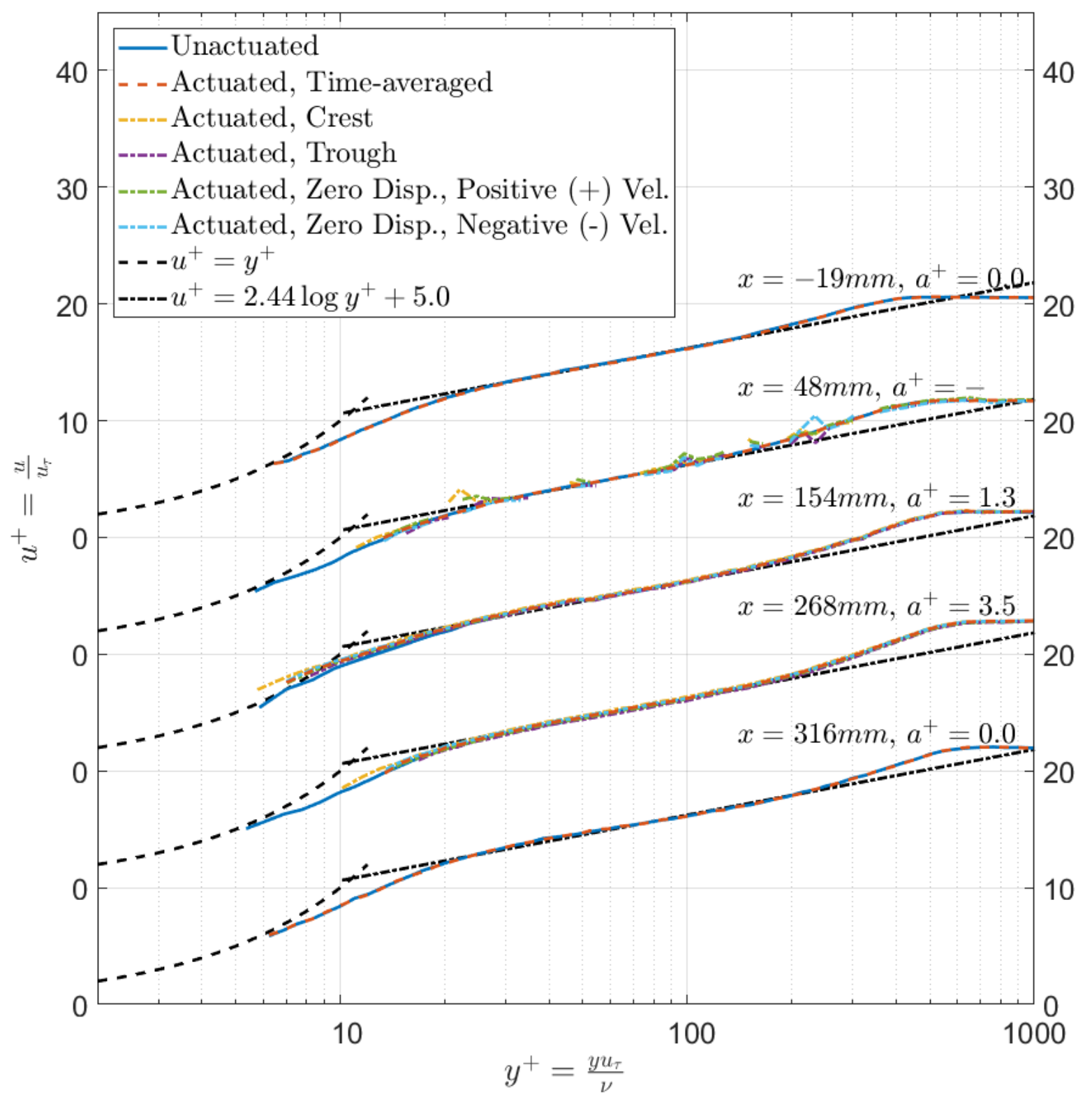

Figure 7.6: Experimentally measured phase-locked, turbulent boundary layers at five streamwise positions for the $810 \mathrm{~Hz}\left(T^{+}=59\right)$ traveling wave surface. At each location, the time-averaged actuated and unactuated profiles are shown. For the locations directly over the moving surface, the phase-locked actuated profiles are given.

waves; no phase difference, $\gamma$, is needed for the standing waves. In addition, the standing waves were excited to have roughly the same peak displacement as the traveling waves $a_{\text {crest }}^{+} \approx 10$. By exciting standing and traveling waves with similar conditions, a comparison can be made between the two wave types. 


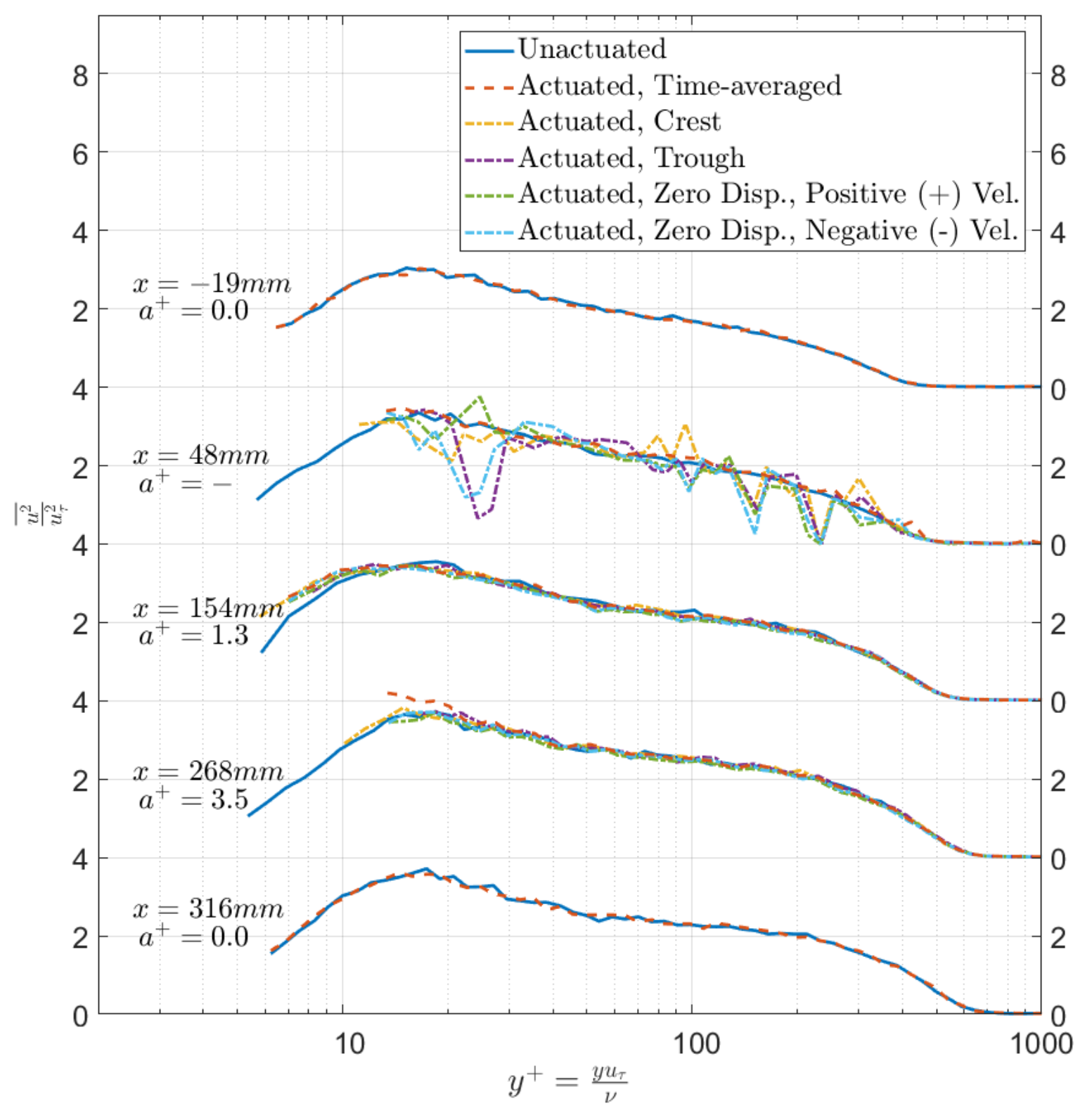

Figure 7.7: Variance/Streamwise Reynolds stress profile at streamwise positions over the $810 \mathrm{~Hz}\left(\mathrm{~T}^{+}=59\right)$ traveling wave surface. The first and last profiles are upstream and downstream of the traveling waves, respectively. The phase-locked extracted profiles are shown for the locations directly over the actuated surface.

The standing waves results are presented in the same format as the traveling waves in the previous section (Section 7.1.1). Each standing wave is consecutively discussed with the $385 \mathrm{~Hz}$ wave first. The standing wave pattern is presented and the excitation configuration presented. Then, the mean velocity profiles experimentally measured at five streamwise locations are shown. 
Three positions are directly over the actuated surface, while one each is upstream and downstream. Then, the variance/streamwise Reynolds stress is presented. All of these results are discussed in comparison with those for the traveling waves. This procedure is then repeated for the $630 \mathrm{~Hz}$ standing wave. Additional results and figures for these standing waves can be found in Appendix D.

\subsubsection{385Hz Standing Wave}

The first standing wave is generated at a frequency of $f=385 \mathrm{~Hz}\left(T^{+}=122\right)$ which coincides with the natural frequency of the $(2,3)$ mode shape shown in Figure 7.8. While a single actuator could excite the standing wave, multiple actuators are used to increase the out-of-plane displacement of the wave. The actuation configuration used to excite the $(2,3)$ mode shape is shown in Figure 7.9 and given in Equation 7.2. Unlike traveling waves, no phase difference $(\gamma)$ is needed. The four actuators on the left side of the plate lie on top of in-phase anti-nodes of the mode shape (Figure 7.8), so the actuators are also excited in-phase $\left(0^{\circ}\right)$. This is the same case for the right side of the plate, expect the four actuators are excited with a $180^{\circ}$ phase offset compared to the left side. The two MFC actuators in the middle of the plate are not used since they lie on a nodal line of the mode. The eight used actuators were excited at $f=385 \mathrm{~Hz}$ with $\pm 225 \mathrm{~V}$. Since the plate is excited at a natural frequency, less input voltage $( \pm 225$ vs. $\pm 350 \mathrm{~V})$ is necessary to produce a similar amplitude as the traveling waves $\left(a_{\text {crest }}^{+} \approx 10\right)$.

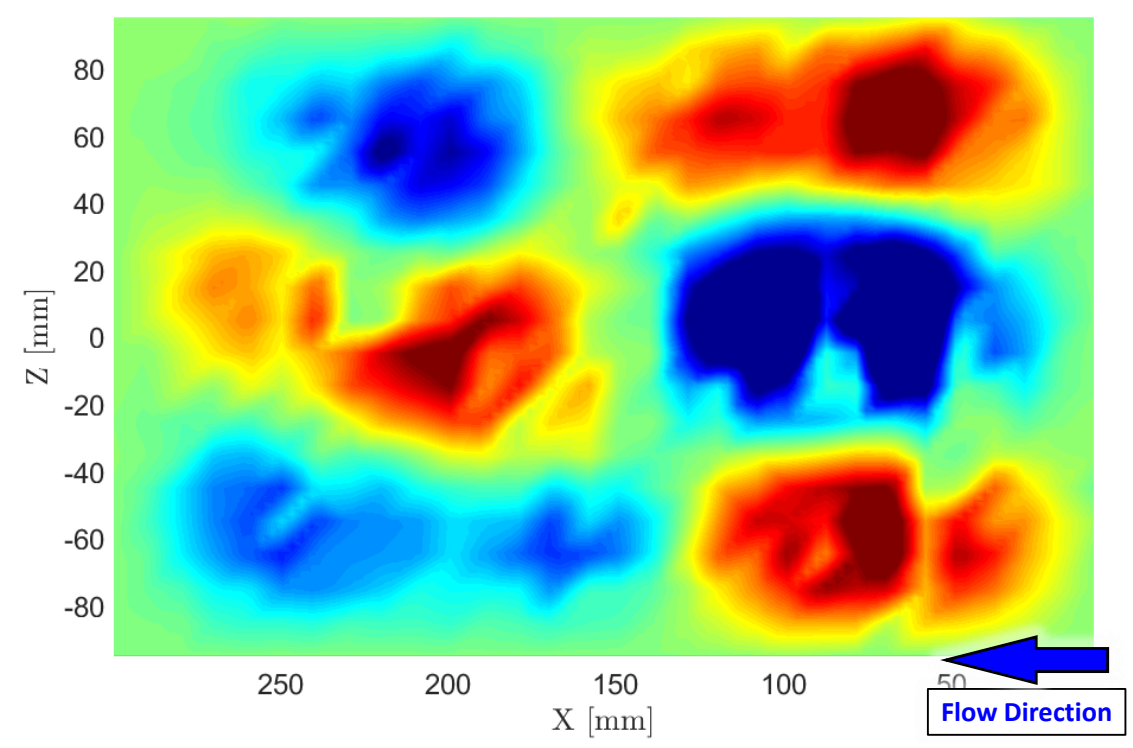

Figure 7.8: Operational deflection shape (ODS) of the $(2,3)$ mode shape at $385.2 \mathrm{~Hz}\left(\mathrm{~T}^{+}=122\right)$ excited using a single piezoelectric actuator 


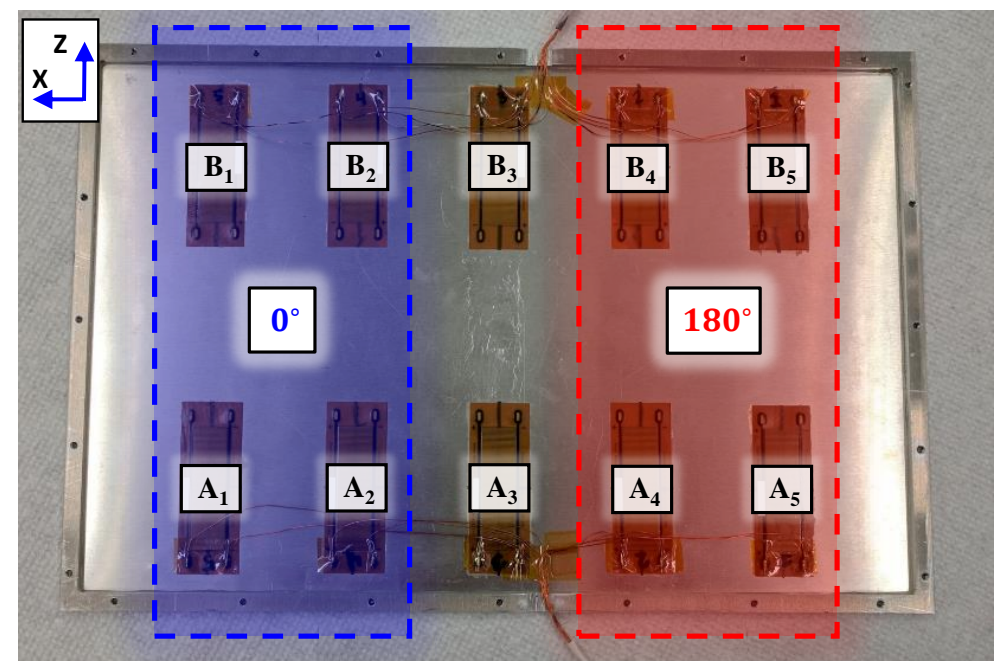

Figure 7.9: Actuation configuration to excite the $385 \mathrm{~Hz}\left(\mathrm{~T}^{+}=122\right)$ standing wave. The actuators highlighted in blue have $0^{\circ}$ phase and those in red have $180^{\circ}$. The $A_{3}-B_{3}$ actuators are not excited here

$$
\begin{array}{cl}
\phi_{A_{1}}=\phi_{A_{2}}=0^{\circ} & , \quad \phi_{B_{1}}=\phi_{B_{2}}=0^{\circ} \\
\phi_{A_{4}}=\phi_{A_{5}}=180^{\circ} & , \quad \phi_{B_{4}}=\phi_{B_{5}}=180^{\circ}
\end{array}
$$

The resultant standing wave pattern is shown in Figure 7.11, which gives the RMS of the experimentally measured out-of-plane velocities. By comparing this to the $(2,3)$ mode shape in Figure 7.8, it is visible that they are the same: 2 anti-nodes in the $x$ direction and 3 in the $z$. The time progression over a single period of the standing wave is shown in Figure 7.10. In each of these time-steps, the anti-nodes remain in the same location with no propagation across the node lines. The fixed location of a single anti-node is shown in the figure as a visual aid.

The turbulent boundary layer was measured at five different locations in the streamwise direction. These positions are listed in Table 7.3 and the three locations directly over the actuated surface are shown in Figure 7.11. Two of the measurement locations lie over anti-nodes of the standing wave, while one is over a node line. This is demonstrated by the displacements in Table 7.3 , where the node location has $a_{\text {crest }}^{+}=2.1$ while both anti-nodes have $a_{\text {crest }}^{+} \approx 11$.

The mean velocity profiles experimentally measured at these locations are shown in Figure 7.12. These profiles show that the standing waves effect the boundary layer in the same manner as the traveling wave (Section 7.1.1). Upstream and downstream of the plate, there is no difference between the unactuated and actuated profiles. Directly over the moving wall, the actuated profiles are pushed off of the surface and the viscous sublayer thickened. The profile over the crest of the wave is effected by the moving surface, but not as much as the time-averaged. The variance profiles shown in Figure 7.13 also confirm the same behavior between the traveling and standing 
$\mathrm{T} / 6$

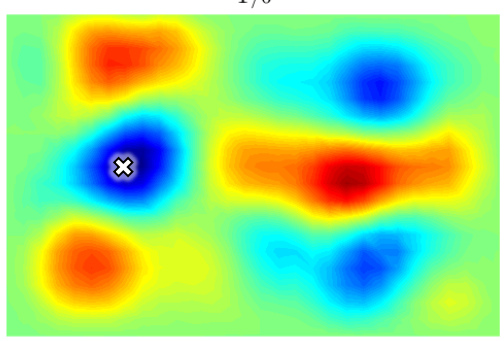

4T/6

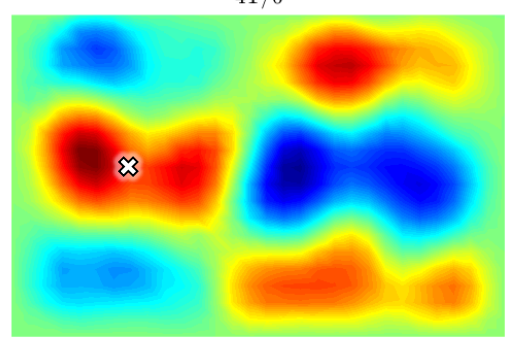

$2 \mathrm{~T} / 6$

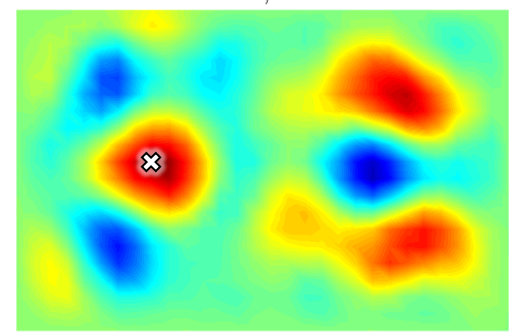

$5 \mathrm{~T} / 6$

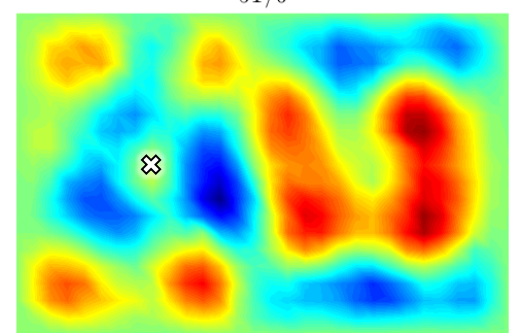

$3 \mathrm{~T} / 6$
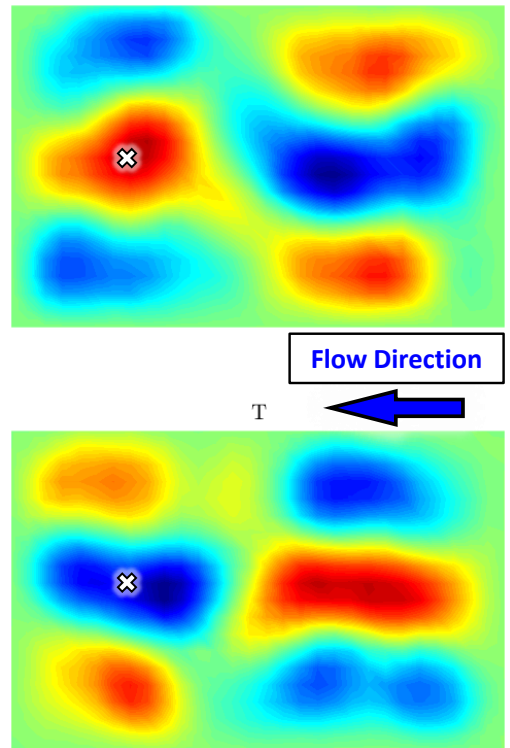

Figure 7.10: Instantaneous velocities of the $385 \mathrm{~Hz}\left(T^{+}=122\right)$ standing wave with $(2,3)$ mode shape. Six different time steps over the period $T$ of the wave are shown. There are no traveling waves, so each anti-node stays at a fixed location. The $\times$ symbol shows how an anti-node oscillates from positive (red) to negative (blue) over the period, but remains at the same location

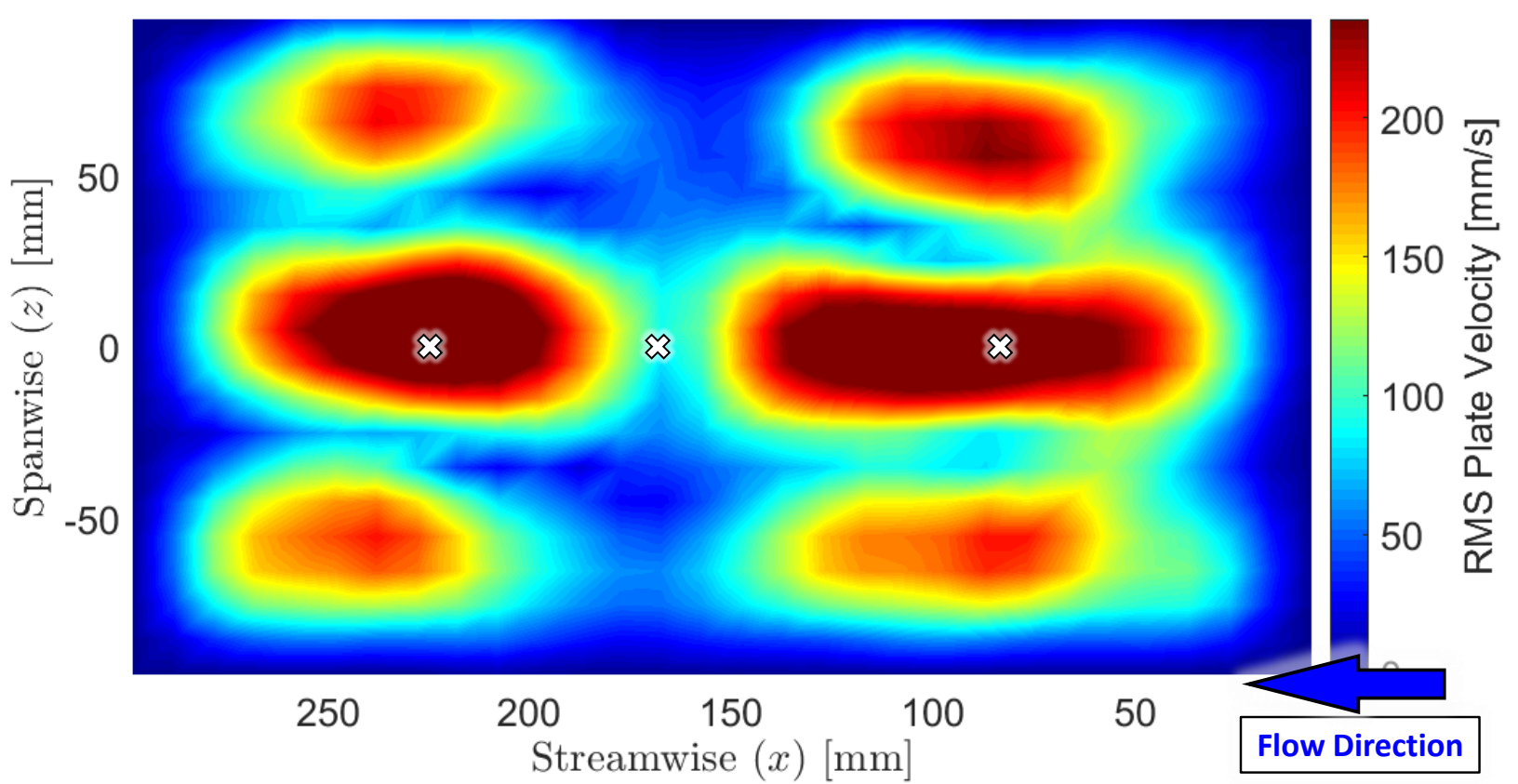

Figure 7.11: Root-mean-square (RMS) velocity of the standing wave at $f=385 \mathrm{~Hz}\left(T^{+}=122\right)$. The boundary layer measurement positions are highlighted. 
Table 7.3: The streamwise measurement locations for the $385 \mathrm{~Hz}\left(T^{+}=122\right)$ standing wave along with the maximum and peak to peak displacement amplitude. All positions are at $z=0 \mathrm{~mm}$ and the three positions over the standing wave surface $\left(a^{+} \neq 0\right)$ are shown in Figure 7.11.

\begin{tabular}{c|ccccc}
\hline Streamwise Position, $z[\mathrm{~mm}]$ & 316 & 224 & 169 & 84 & -19 \\
\hline$a_{\text {crest }}[\mu \mathrm{m}]$ & 0 & 219.1 & 42.7 & 223.0 & 0 \\
$a_{\text {crest }}^{+}$ & 0 & 10.8 & 2.1 & 11.2 & 0 \\
$a_{p-p}^{+}$ & 0 & 23.2 & 4.6 & 21.5 & 0 \\
\hline
\end{tabular}

waves. The time-averaged actuated profile has the same increase near the wall due to the oscillating relative $y$ position (Section 6.3.3). While the peak variance over the crest of the wave tends to be pushed off of the wall. The peak variance over the crest may indicate a slight decrease versus the unactuated, but no clear conclusion can be drawn.

Looking at the mean velocity profiles in Figure 7.12, the separation distance between the uanctuated and actuated profiles varies for each streamwise position. At $x_{p}=84 \mathrm{~mm}$ and $x_{p}=224 \mathrm{~mm}$ the wave displacement is $a^{+} \approx 11$ and both boundary layers are pushed off roughly the same amount. In comparison, the profile is minimally effected at $x_{p}=169 \mathrm{~mm}$ where the amplitude is quite low $\left(a^{+}=2.1\right)$. These same trends are confirmed by the variance profiles in Figure 7.13. Thus, just as for traveling waves, the displacement of the wall plays a significant role in how much the turbulent boundary layer is affected.

\subsubsection{630Hz Standing Wave}

The second standing wave is generated at $f=630 \mathrm{~Hz}\left(T^{+}=74\right)$, which is the natural frequency of the $(3,4)$ mode shape. Six of the ten MFC actuators are excited using the same procedure as described in Section 7.1.2.1. This actuation configuration is expressed in Equation 7.3 and shown in Figure 7.14. The resultant standing wave is shown in the RMS of the velocity in Figure 7.15. This is clearly the $(3,4)$ mode shape, with 3 anti-nodes visible in the streamwise $(x)$ direction and 4 in the spanwise $(z)$. The nodes lines also demonstrate that no traveling waves are propagating across the surface. Additional figures of the standing wave are shown in Appendix D.3. As with the previous standing and traveling waves, the turbulent boundary layer is measured at five streamwise locations. These are shown in Table 7.4 with the three locations directly over the standing wave shown in Figure 7.15.

$$
\begin{array}{cl}
\phi_{A_{1}}=\phi_{A_{5}}=0^{\circ} \quad, \quad \phi_{B_{1}}=\phi_{B_{5}}=0^{\circ} \\
\phi_{A_{3}}=180^{\circ} \quad, \quad \phi_{B_{3}}=180^{\circ}
\end{array}
$$

The turbulent boundary layers along the streamwise direction are shown in Figure 7.16. Once 


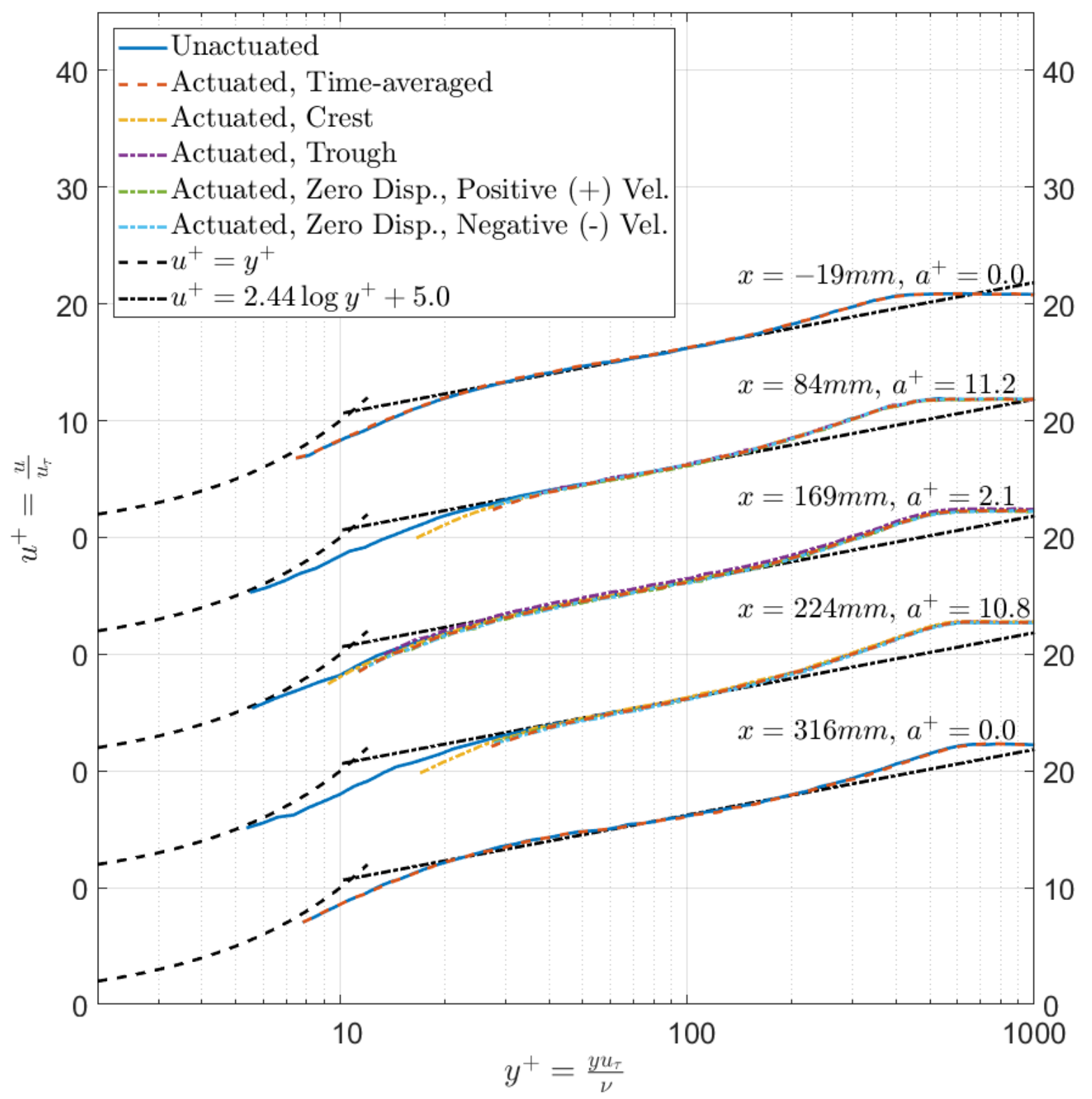

Figure 7.12: Experimentally measured phase-locked, turbulent boundary layers at five streamwise positions for the $385 \mathrm{~Hz}\left(T^{+}=122\right)$ standing wave surface. At each location, the time-averaged actuated and unactuated profiles are shown. For the locations directly over the moving surface, the phase-locked actuated profiles are given.

again, the moving wall pushes the mean velocity profile away from the surface and thickens the viscous sublayer. The phase-locked profiles also follow the same trend as seen for the $430 \mathrm{~Hz}$ wave. In particular, a clear difference between the two zero displacement positions is visible. When the surface is moving upwards into the flow (positive velocity), there is minimal change in 


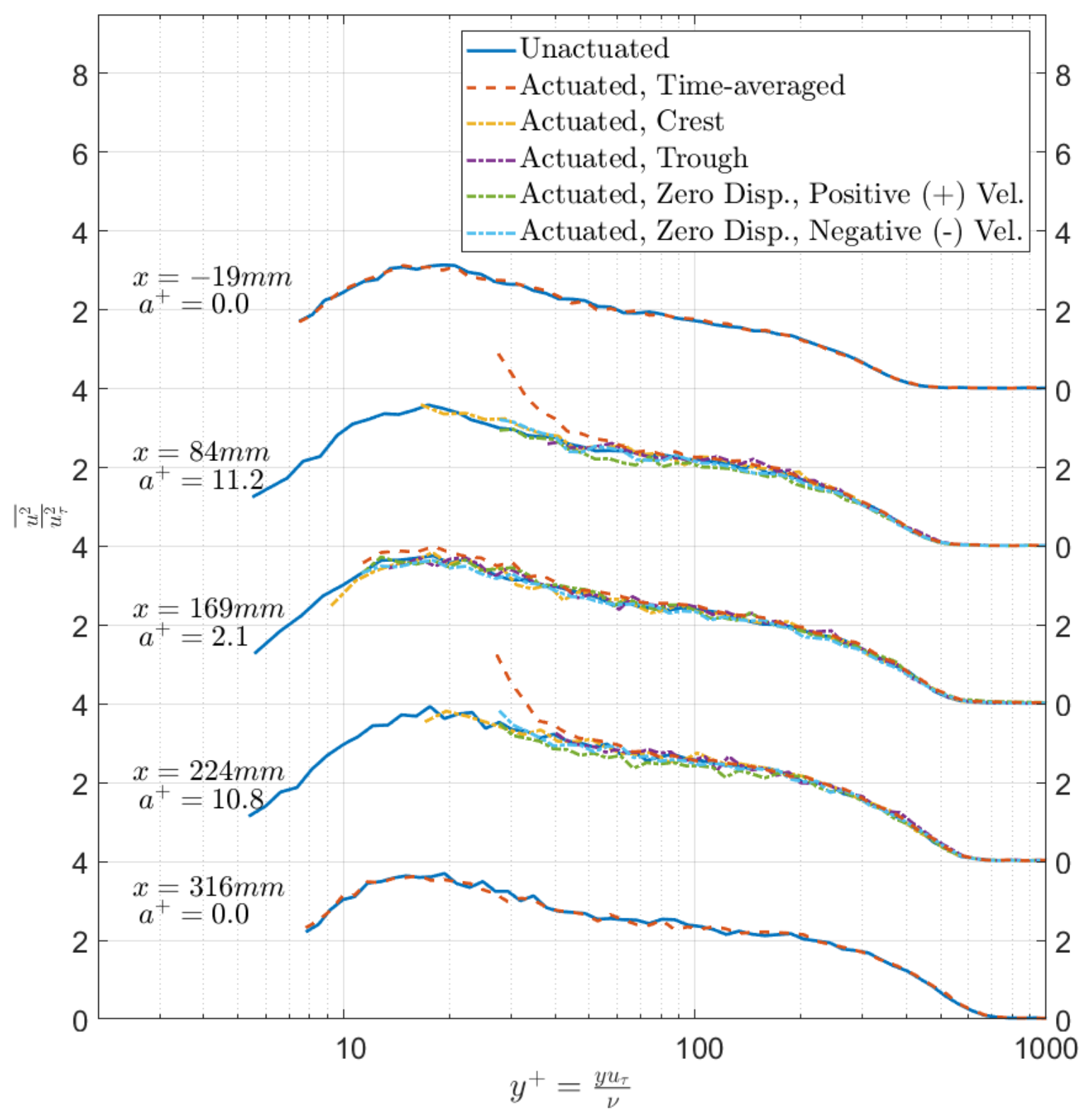

Figure 7.13: Variance/Streamwise Reynolds stress profile at streamwise positions over the $385 \mathrm{~Hz}\left(T^{+}=122\right)$ traveling wave surface. The first and last profiles are upstream and downstream of the traveling waves, respectively. The phase-locked extracted profiles are shown for the locations directly over the actuated surface.

the boundary layer. But, when the wall has a downward (negative) velocity, there is a significant thickening of the viscous sublayer, demonstrating the phase-lag effect. In addition, the dependence on wall amplitude is visible for the three profiles directly over the standing wave. The actuated profile at $x_{p}=65 \mathrm{~mm}$ with $a_{c r e s t}^{+}=10.1$ is affected more strongly than that at $x_{p}=234 \mathrm{~mm}$ where 


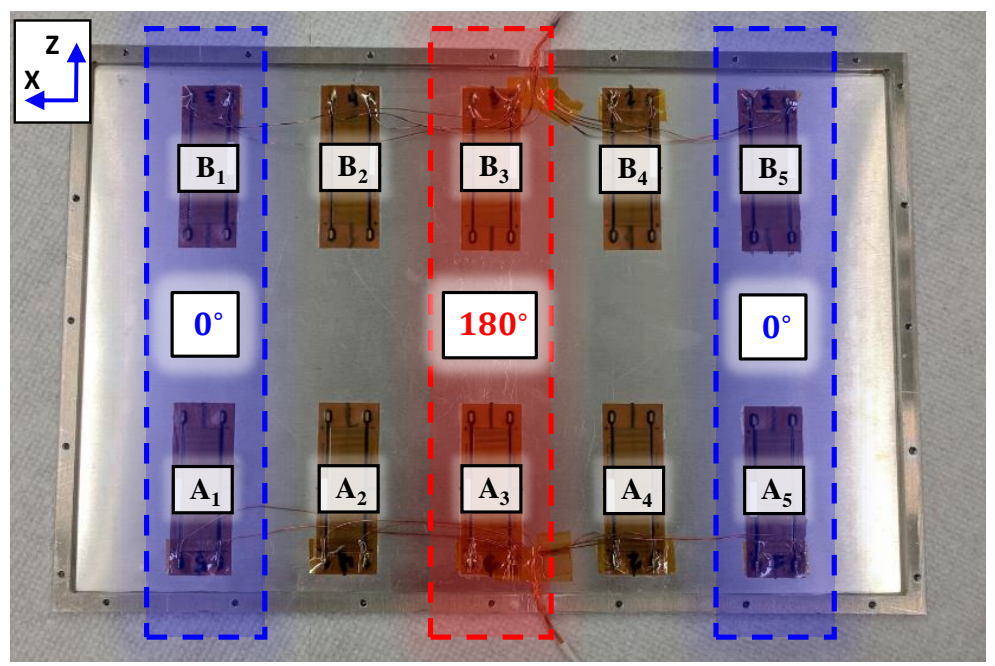

Figure 7.14: Actuation configuration to excite the $630 \mathrm{~Hz}\left(\mathrm{~T}^{+}=78\right)$ standing wave. The actuators highlighted in blue have $0^{\circ}$ phase and those in red have $180^{\circ}$. The $A_{2}-B_{2}$ and $A_{4}-B_{4}$ actuators are not excited here

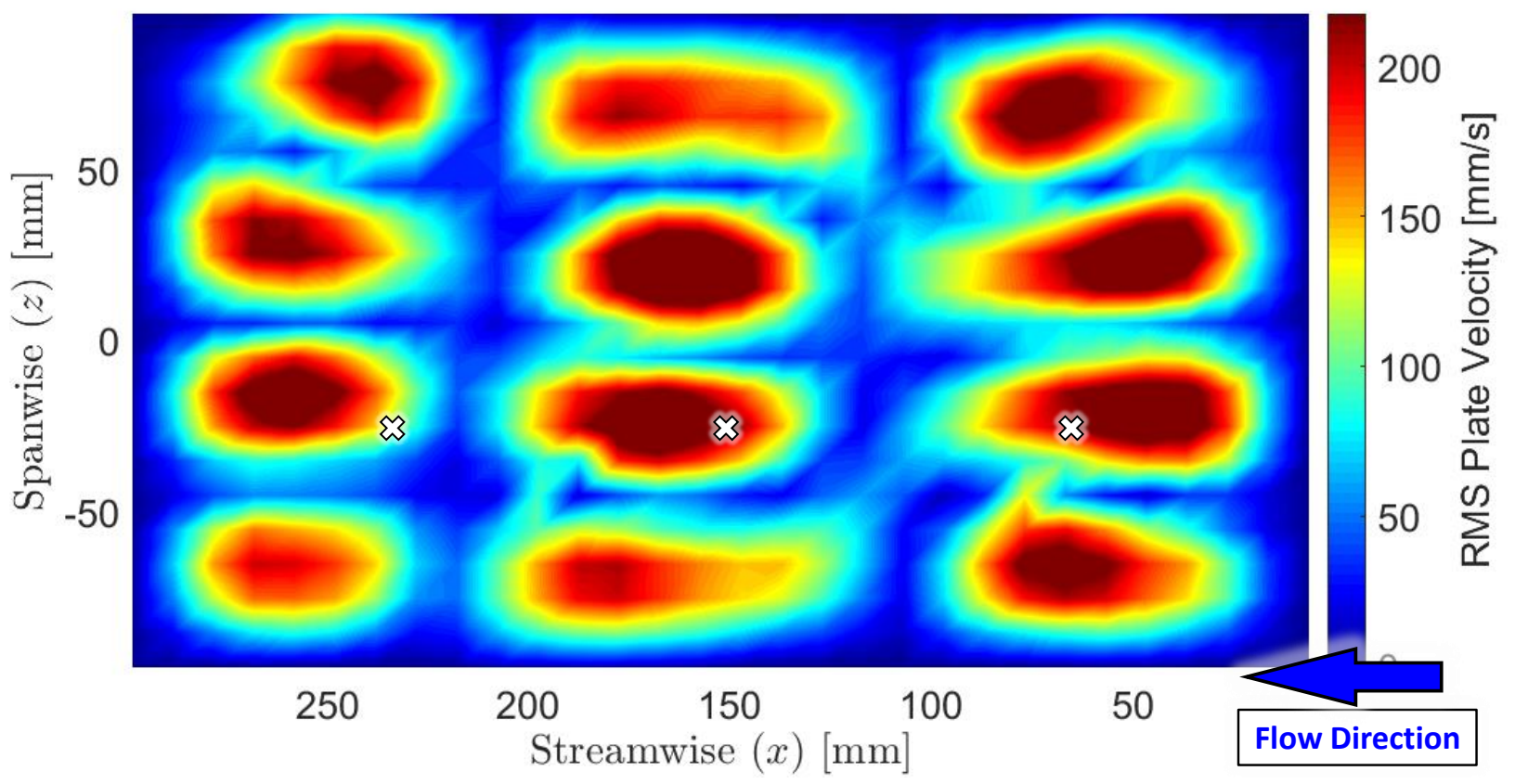

Figure 7.15: Root-mean-square (RMS) velocity of the standing wave at $f=630 \mathrm{~Hz}\left(T^{+}=74\right)$. The boundary layer measurement positions are highlighted.

the amplitude is $a_{\text {crest }}^{+}=4.7$. These results are further confirmed by the variance profiles, which are shown in Appendix D.3. 
Table 7.4: The streamwise measurement locations for the $630 \mathrm{~Hz}\left(T^{+}=74\right)$ standing wave along with the maximum and peak to peak displacement amplitude. All positions are at $z=0 \mathrm{~mm}$ and the three positions over the standing wave surface $\left(a^{+} \neq 0\right)$ are shown in Figure 7.15.

\begin{tabular}{c|ccccc}
\hline Streamwise Position, $z[\mathrm{~mm}]$ & 316 & 234 & 150 & 65 & -19 \\
\hline$a_{\text {crest }}[\mu \mathrm{m}]$ & 0 & 90.3 & 166.8 & 194.3 & 0 \\
$a_{\text {crest }}^{+}$ & 0 & 4.7 & 8.7 & 10.1 & 0 \\
$a_{p-p}^{+}$ & 0 & 9.7 & 17.7 & 21.2 & 0 \\
\hline
\end{tabular}

\subsubsection{Discussion}

The turbulent boundary layer was measured over a $430 \mathrm{~Hz}\left(T^{+}=112\right)$ wave in Chapter 6 and here over two travelings waves at $607 \mathrm{~Hz}\left(T^{+}=78\right)$ and $810 \mathrm{~Hz}\left(T^{+}=59\right)$. All of the traveling waves were based on the $(5, n)$ mode shape, such that the traveling wave patterns for all three frequencies were roughly the same. The $607 \mathrm{~Hz}$ and $810 \mathrm{~Hz}$ traveling waves did not have as strong of an effect on the boundary layer as the $430 \mathrm{~Hz}$ wave. This is mainly due to the smaller amplitudes excited at these frequencies. The maximum amplitudes for the $607 \mathrm{~Hz}$ and $810 \mathrm{~Hz}$ waves were $a_{\text {crest }}^{+}=5.9$ and $a_{\text {crest }}^{+}=3.5$, respectively; this compares with $a_{\text {crest }}^{+}=12.5$ for the $430 \mathrm{~Hz}$ wave. When the boundary layer was affected, the time-averaged profile was pushed off the wall. For the phase-locked profiles the effect was not as clear. In particular, for both the $607 \mathrm{~Hz}$ and $810 \mathrm{~Hz}$ cases, the crest profile was sometimes pulled closer to the wall, indicating the viscous sublayer was thinning. For the $810 \mathrm{~Hz}$ case, this was seen at a small amplitude between larger peaks in the spanwise direction. So, it is suggested that the traveling wave pattern plays a significant role in the alteration of the turbulent boundary layer.

The effect on the turbulent boundary layer was also measured over two standing waves. This was done to investigate whether traveling and standing waves alter the boundary layer in similar ways. Standing waves were generated at $385 \mathrm{~Hz}\left(T^{+}=122\right)$ and $630 \mathrm{~Hz}\left(\mathrm{~T}^{+}=74\right)$, with amplitudes in the same range as the traveling waves. The standing waves were found to effect the boundary layer in the same way as the traveling wave. The actuated profile is pushed off of the surface and the viscous sublayer thickened. For the phase-locked profiles, the crest is not effected as strongly as the time-averaged case, and the phase-lagged response of the boundary layer is also demonstrated. In addition, the boundary layer over the standing wave was seen to depend on the displacement of the surface. These results are identical to those of the $430 \mathrm{~Hz}$ traveling wave and thus suggest that there is minimal difference between traveling and standing waves. Despite the similar effects on the boundary layer, that does not mean they do so with the same strength. Although similar, the traveling and standing wave patterns themselves still have differences between them. So, the effect on the turbulent boundary layer should be quantified and compared for both wave types. 


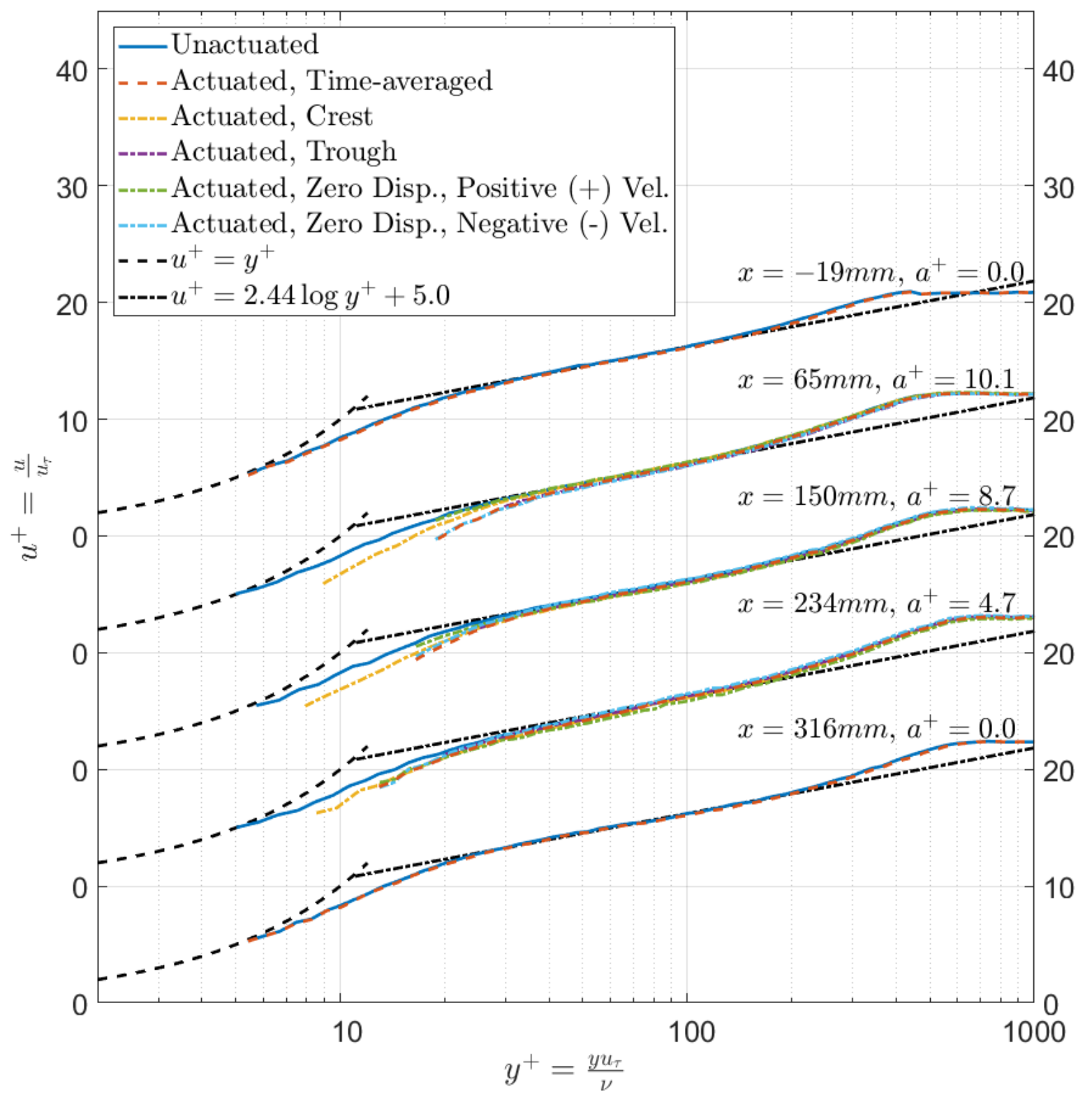

Figure 7.16: Experimentally measured phase-locked, turbulent boundary layers at five streamwise positions for the $385 \mathrm{~Hz}\left(T^{+}=74\right)$ standing wave surface. At each location, the time-averaged actuated and unactuated profiles are shown. For the locations directly over the moving surface, the phase-locked actuated profiles are given.

This discussed further via the parametric analysis in the next section. 


\subsection{Parametric Analysis}

The previous section showed that traveling and standing waves both effect the turbulent boundary layer, and both do so in similar manners. In particular, the mean velocity profile over the actuated surface is pushed off of the wall and the viscous sublayer is thickened. However, the parameters that control the strength of this effect are not clear. Previous results suggest that the amplitude plays a dominate role, but the effect of other parameters is not known. This includes the frequency and also the type of waveform itself: traveling versus standing waves. This section conducts a parametric analysis to correlate the boundary layer effect with the displacement amplitude and then determine the relationship with frequency and wave type (traveling and standing waves).

This section begins by quantifying the effect of the waves on the turbulent boundary layer. The $\Delta y$ offset between the unactuated and time-averaged actuated boundary layer profiles is found. This is conducted for 26 different cases with varying displacements, frequencies, and wave types (traveling and standing). Specifically, profiles over the three different traveling waves $(430 \mathrm{~Hz}, 607 \mathrm{~Hz}$, and $810 \mathrm{~Hz}$ ) and two standing waves $(385 \mathrm{~Hz}$ and $630 \mathrm{~Hz}$ ) presented in Chapter 6 and Section 7.1 are discussed. Then, the $\Delta y$ offsets from these 26 cases are correlated with the displacement amplitude of the wave. Finally, this displacement correlation is utilized to discuss the relationship with the frequency and wave type.

\subsubsection{Procedure}

The main effect of traveling and standing waves on the turbulent boundary layer is to thicken the laminar sublayer and push the mean velocity profile off of the surface. So, the offset distance between the unactuated and time-averaged actuated profiles is used to quantify the effect of the waves on the boundary layer. The time-averaged profile has an inherent non-linearity as discussed in Section 6.2.2 and may have a slight effect on the offset. However, the offsets are used more as a qualitative tool, so the time-averaged profile is deemed sufficient. Figure 7.17 shows a representative case of this offset, $\Delta y$. The unactuated and actuated mean velocity profiles are that over a $630 \mathrm{~Hz}$ standing wave at $x_{p}=65 \mathrm{~mm}$ and are plotted on a linear scale. The offset is found by first interpolating both the unactuated and actuated profiles with a spline fit; these are shown in the figure. Then the velocity, $u^{+}$, of the unactuated profile is found at $y_{\text {unact }}^{+}=23$. Next, the $y_{\text {act }}^{+}$ position of the actuated profile having the same $u^{+}$is determined. Finally, the difference between the actuated $y_{\text {act }}^{+}$position and $y_{\text {unact }}^{+}=23$ is the offset, $\Delta y$. In Figure 7.17, $y_{a c t}^{+}=28.3$ yielding $\Delta y=5.3$. The position $y_{\text {unact }}^{+}=23$ is used to accommodate the smallest measured $y^{+}$value for the actuated profile.

The result is a single $\Delta y$ for each unactuated and actuated boundary layer profile pair, which quantifies the effect of the waves on the turbulent boundary layer. Only the boundary layer profiles 


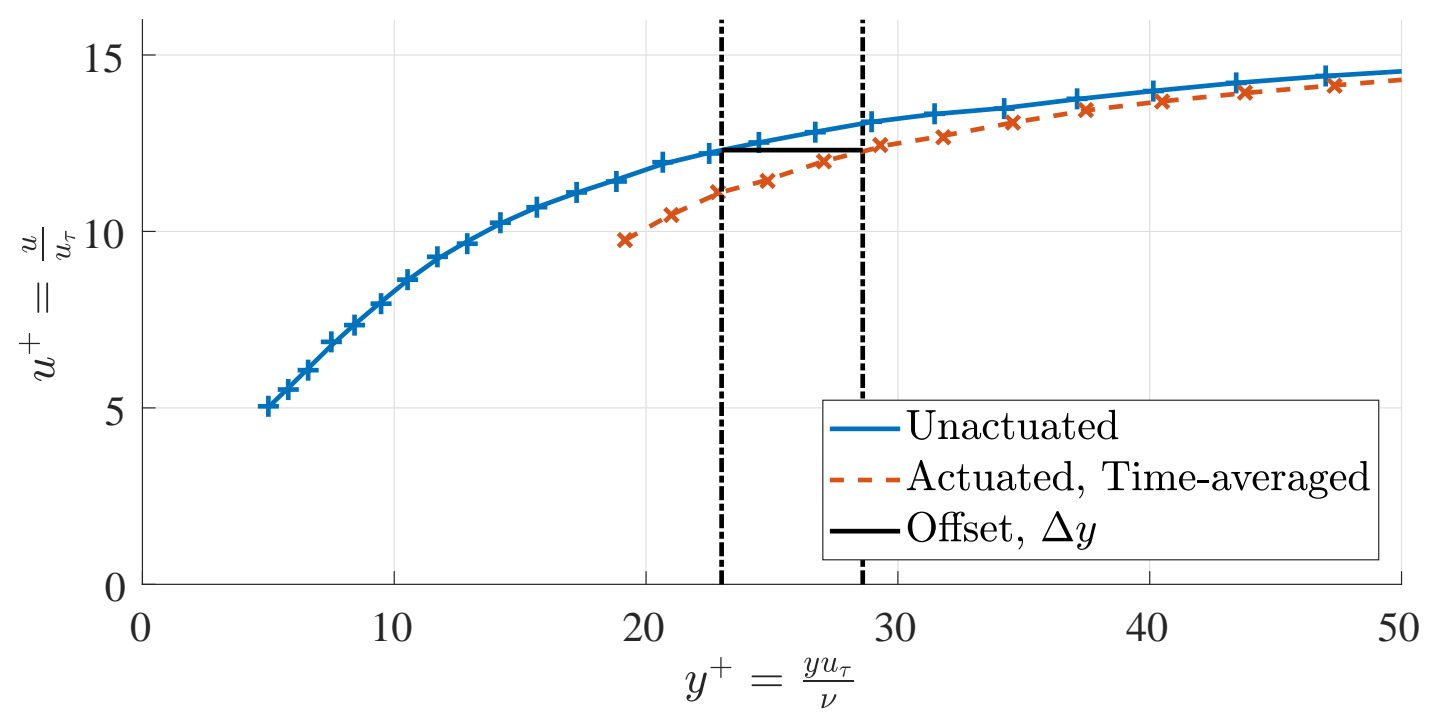

Figure 7.17: $\Delta Y$ offset between the unactuated and time-averaged actuated mean velocity profile at $x_{p}=65 \mathrm{~mm}$ over the $630 \mathrm{~Hz}$ standing wave. Linear scaling is used

directly over a wave surface are used, since there is no $\Delta y$ offset for the upstream and downstream positions. For the case of the $385 \mathrm{~Hz}$ standing wave, there are five boundary layer profiles (shown in Figure 7.12). Three of the profiles are over the actuated wall; thus, there are three separate $\Delta y$ values. Overall, there are 26 different cases pulling from the three traveling $(430 \mathrm{~Hz}, 607 \mathrm{~Hz}$, and $810 \mathrm{~Hz}$ ) and two standing waves $(385 \mathrm{~Hz}$ and $630 \mathrm{~Hz})$.

\subsubsection{Analysis}

The displacement amplitude plays a significant role in the effect on the boundary layer. This was seen in the results of both traveling (Section 6.3.5) and standing waves (Section 7.1.2). Thus, the relationship with displacement amplitude is the primary factor investigated here. This relationship is shown in Figure 7.18 for all the previously presented standing and traveling wave results. The $\Delta y$ for each actuated profile is plotted versus the peak to peak amplitude, $a_{p p}^{+}$, at the measurement location. The peak to peak amplitude is used here since the surface does not have symmetric displacement at every location. This can be seen in Figure 6.11b, where the crest and trough amplitudes are not the same. Thus, to limit any bias based on the crest or trough displacement, $a_{p-p}^{+}$is used.

Based on the data shown in Figure 7.18 there is a positive correlation between the $\Delta y$ offset and the displacement, $a_{p p}^{+}$. A linear least-squares fit is also shown in the figure and indicates the same positive relationship. Thus, as the amplitude of the wave increases, the boundary layer is pushed 


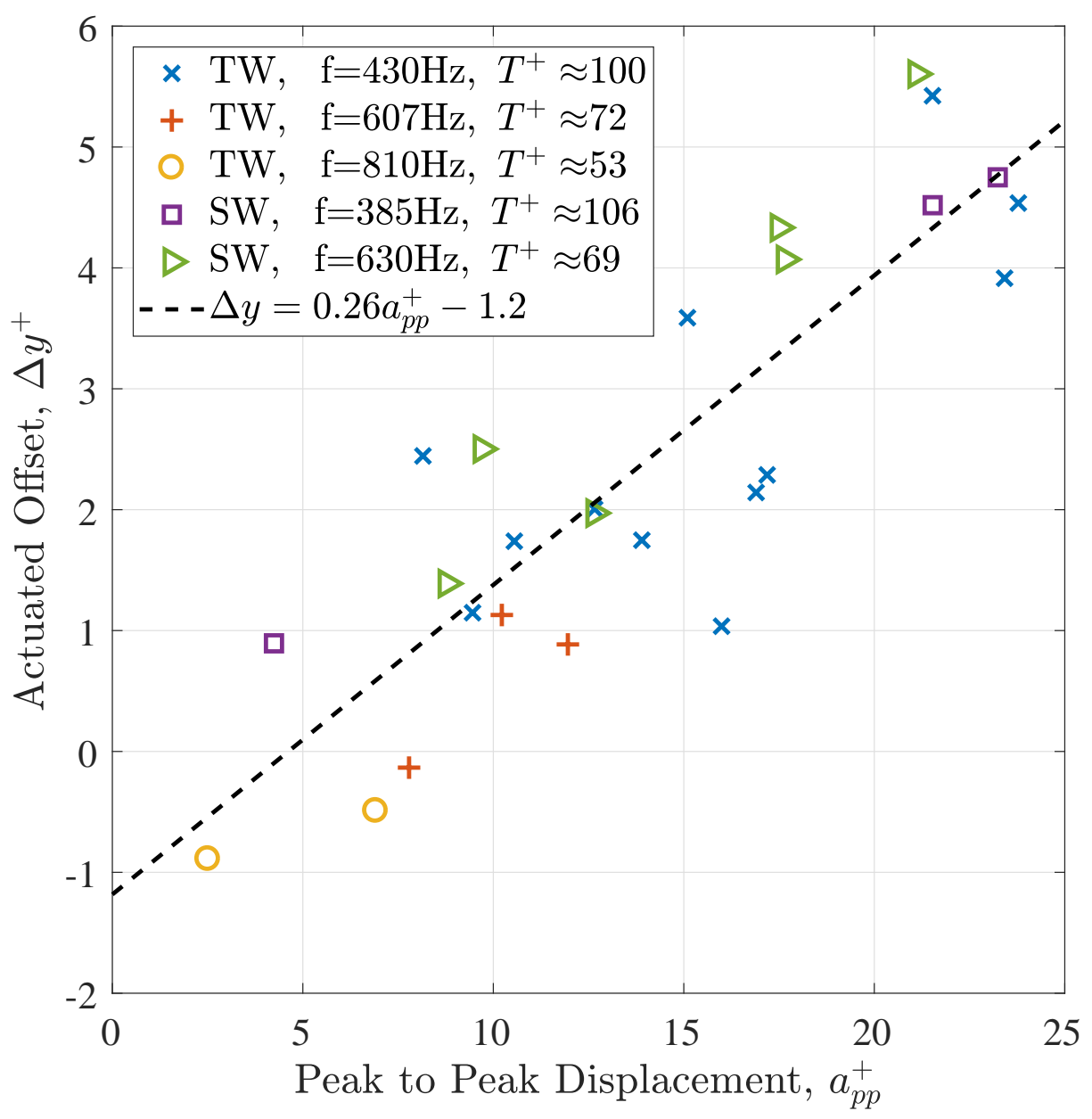

Figure 7.18: $\Delta y$ offset of the time-averaged actuated boundary layer profile as a function of the peak-to-peak, wall-normal displacement. Data from three different frequency traveling waves and two different standing waves is shown.

further off of the surface. A clear example of this is shown in Figure 7.19 for the $630 \mathrm{~Hz}$ standing wave. The standing wave was excited at four different amplitude levels by varying the voltage input to the piezoelectric actuators. Then, the unactuated and actuated boundary layer profiles were measured for each of these levels. The results in Figure 7.19 clearly show that as the wave amplitude increases, so too does the $\Delta y$ offset between the unactuated and actuated profiles. Thus, there is a clear dependence of the turbulent boundary layer on the displacement of the wave.

Looking back at Figure 7.18, a point of interest is that the the intercept of the linear line is not zero. Rather, at $a_{p p}^{+}=0$, the offset is negative $(\Delta y=-1.4)$. When the wave displacement is zero, the plate is not; thus, the actuated boundary layer should seemingly converge with the unactuated case $(\Delta y=0)$. However, the results state otherwise. In particular, the $607 \mathrm{~Hz}$ and $810 \mathrm{~Hz}$ traveling 


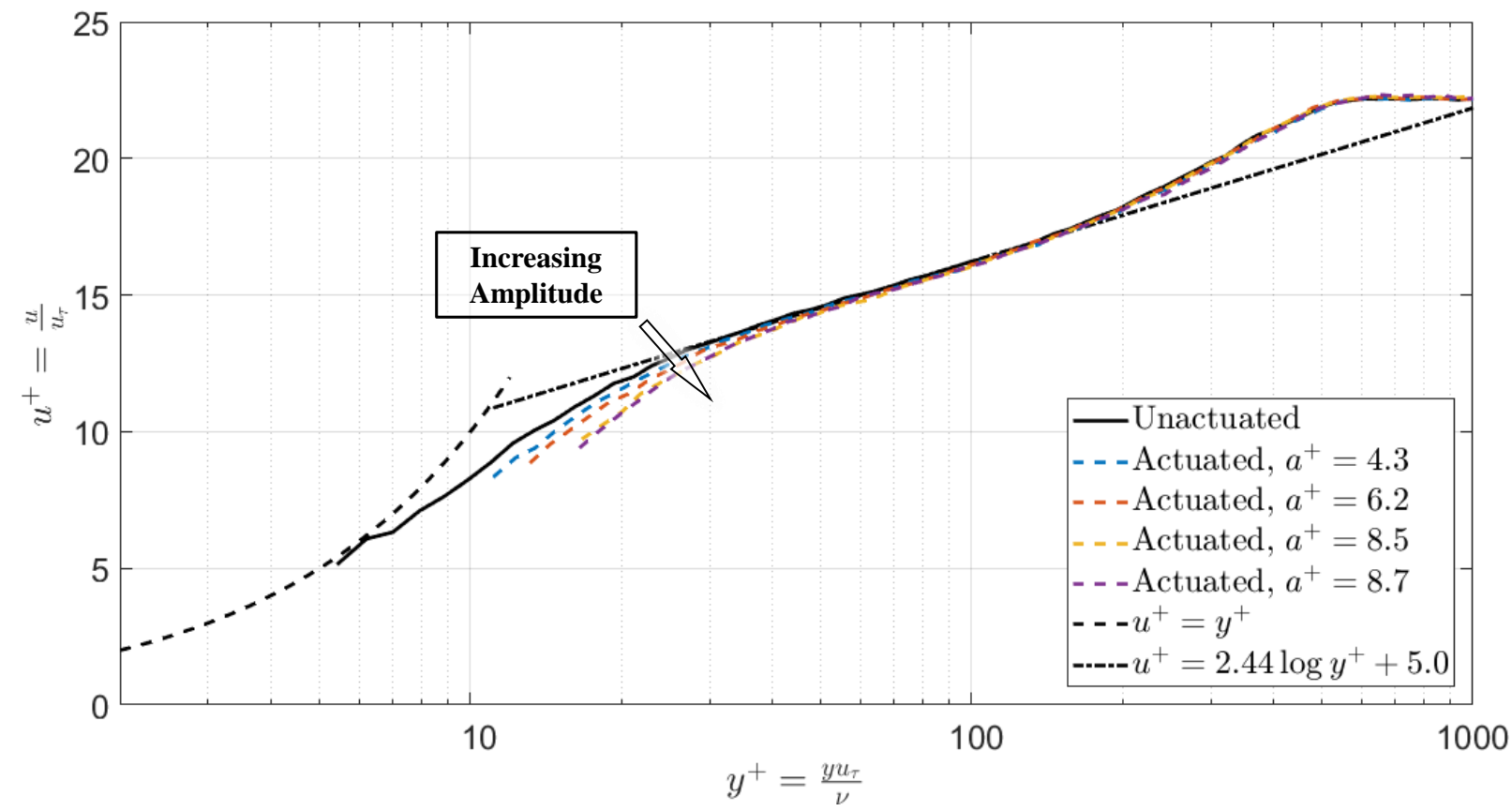

Figure 7.19: Time-averaged actuated mean velocity profiles over the $630 \mathrm{~Hz}$ standing wave at $x_{p}=150 \mathrm{~mm}$. Four different displacement amplitudes are shown

waves yield negative offsets. These are cases where the boundary layer is pulled closer to the wall and the viscous sublayer is thinned. As explained in Section 7.1.1, this thinning could relate to a dependence on the wave pattern itself. Even though the displacement is small at one location, it may be large at an adjacent one; thus, a zero displacement does not mean a flat, unactuated surface. It is suggested that the spanwise variation in wave displacement, funnels the turbulent vortices from higher amplitude regions to adjacent ones with smaller amplitudes. As a result, the boundary layer is pulled closer to the wall and the sublayer thinned. This would also mean an increase in the local skin friction. These results would indicate a dependence on the traveling (or standing) wave pattern.

Based on Figure 7.18, it is difficult to discern a difference between traveling and standing waves. So, the same data is replotted in Figure 7.20 to more clearly show the difference between the two wave types. In addition, new linear trends are fit to each of the wave types. From these trend lines, differences between the standing and traveling waves become apparent. The standing waves tend to yield larger $\Delta y$ offsets for a given displacement, $a_{p p}^{+}$. This suggests that the standing waves are more effective than the traveling waves at pushing the boundary layer off the wall. In addition, the intercept of the standing wave linear fit has a value much closer to zero. Assuming the same explanation as the traveling waves, this could suggest that the standing waves are not as dependent on the wave pattern. However, the boundary layer measurement locations for the 
$385 \mathrm{~Hz}$ and $630 \mathrm{~Hz}$ standing waves (Figures 7.11 and 7.15) are not at low points in the spanwise (z) amplitude. Rather, the locations tend to be at an anti-node (peak). The boundary layer should be measured over a streamwise node line of the standing wave: e.g. at $z=0 \mathrm{~mm}$ for the $630 \mathrm{~Hz}$ standing wave. It would then lie between two anti-nodes (peaks) in the spanwise $(z)$ direction. Thus, while standing waves appear more effective than traveling waves, this may result from the standing wave data not capturing the effect of wave pattern.

In addition, the difference between the standing and traveling wave in Figure 7.20 could stem from experimental uncertainties. As was discussed in Section 6.3.6 and Appendix C, there are experimental uncertainties in the boundary layer profiles. The offsets, $\Delta y$, calculated and shown in the figure do not account for any measurement errors. Looking at the data in Figure 7.20, there is fair bit of spread in the data such that the two linear lines do not distinctly represent just one wave type. Thus, when the both experimental uncertainty and spread in the data are considered, there is no clear difference between how standing and traveling waves affect the turbulent boundary layer.

In terms of frequency, Figure 7.20 may indicate a slight dependence, but this cannot be conclusively stated. The higher frequency traveling waves $(607 \mathrm{~Hz}$ and $810 \mathrm{~Hz}$ ) appear to have less effect on the boundary layer and even negatively effect it (sublayer thinning). But, these waves were only investigated at small amplitudes, $a_{p p}^{+}<12$, while the $430 \mathrm{~Hz}$ waves were tested at a larger range. So it is difficult to directly compare the different frequencies. In addition, the standing waves do not show a strong frequency dependence. The $385 \mathrm{~Hz}$ and $630 \mathrm{~Hz}$ standing waves seem to have similar levels of effectiveness and have even a greater frequency separation than the $430 \mathrm{~Hz}$ and $607 \mathrm{~Hz}$ traveling waves. Finally, any frequency dependence here cannot be disentangled from the relationship with wave pattern, since both involve the points of sublayer thinning. Thus, while there may be some frequency dependence it cannot be sufficiently isolated and explained.

Overall, there is a strong correlation between the $\Delta y$ offset and the wall-normal amplitude. In addition, no clear difference can be shown in how standing and traveling waves affect the boundary layer. It is is suggested that standing waves have a stronger effect, but this could relate to a lack of measurement locations and also the uncertainty in the experimental data. Finally, a slight frequency dependence was presented but could not be conclusively stated. The relationship with displacement amplitude was the overwhelming effect seen and it hindered correlation with other parameters such as wave type, wave pattern, and frequency. To more conclusively determine the relationship with these parameters, more targeted testing is required.

\subsection{Discussion}

Chapter 6 presented the results of a turbulent boundary layer flowing over a single frequency traveling wave. This traveling wave was generated using a novel implementation method involving 


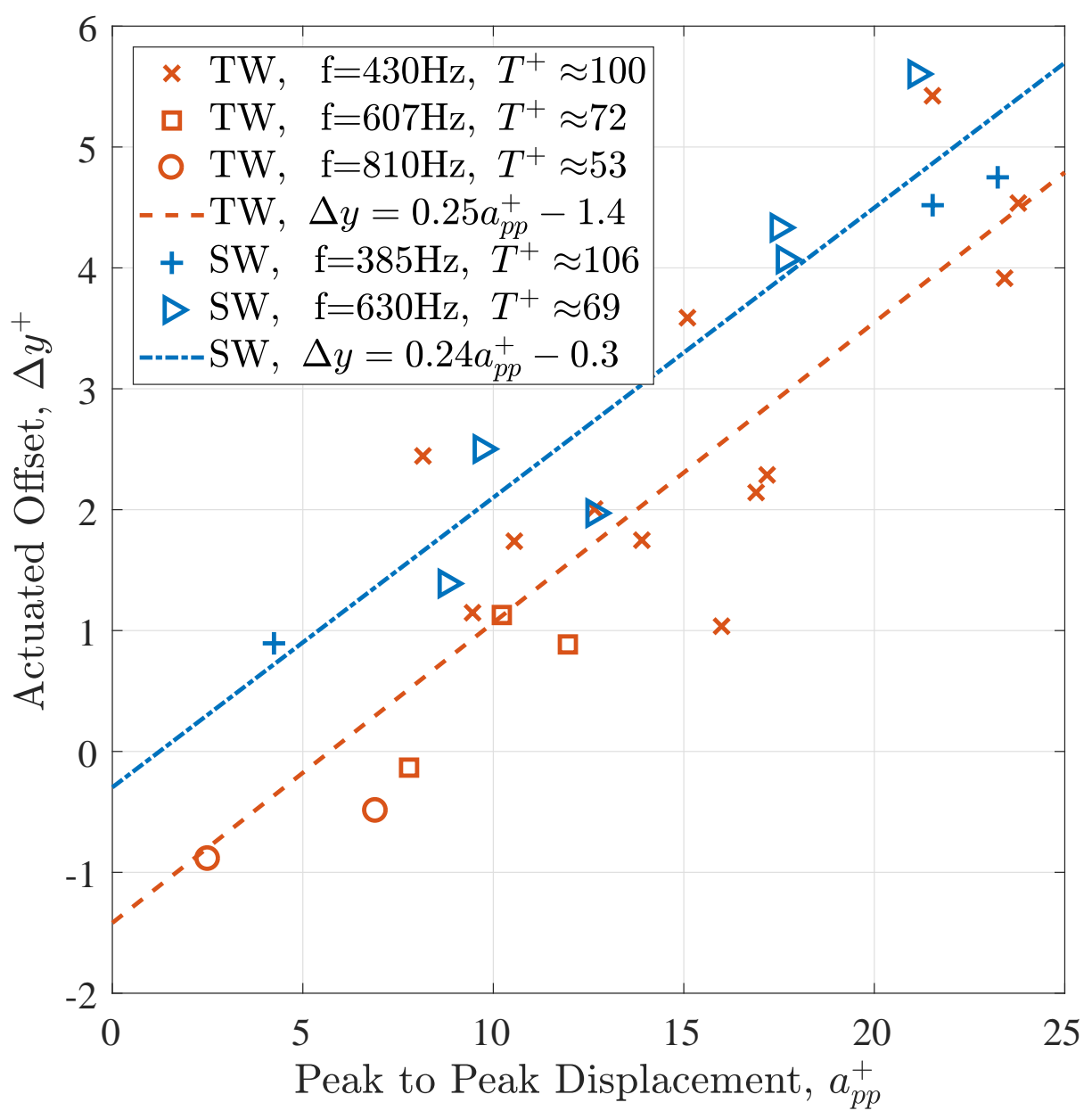

Figure 7.20: $\Delta y$ offset of the time-averaged actuated boundary layer profile as a function of the peak-to-peak, wall-normal displacement. Data from three different frequency traveling waves and two different standing waves is shown.

low-profile piezoelectric actuators. The turbulent boundary layer was experimentally measured over the actuated surface and the results demonstrated changes in the boundary layer profile consist a reduction in skin friction drag. Thus, this novel traveling wave generation method has promise as an implementable approach to reducing skin friction drag with traveling waves.

This chapter built upon the previous ones and investigated the turbulent boundary layer flowing over additional traveling waves and also standing waves. These results were presented in Section 7.1. The changes in the boundary layer profile were compared for the different frequencies and waveforms (traveling vs. standing) and with the results from Chapter 6. A parametric study was then conducted to quantify the change in the boundary layer and determine the variation among different parameters. This focused primarily on wave displacement since this was found to be the 
driving factor in altering the flow. These results were also analyzed to determine any difference and similarities between traveling and standing waves.

This section summarizes and discusses all of these results. First, the effects of the three different frequency traveling waves are discussed. This includes the overall effect of a traveling wave actuated surface on the turbulent boundary layer. Then the standing waves are analyzed and compared with traveling waves. The differences and similarities between the two wave forms are discussed.

\subsubsection{Traveling Waves}

The effects of three different traveling waves on the turbulent boundary layer have been investigated here. These are at frequencies of $430 \mathrm{~Hz}, 607 \mathrm{~Hz}$, and $810 \mathrm{~Hz}$ with normalized periods of $T^{+}=112, T^{+}=78$, and $T^{+}=59$, respectively. Each of these traveling waves takes the form of a $(5, n)$ mode shape with 5 anti-nodes along the streamwise direction and $n=2-4$ anti-nodes in the spanwise direction. Thus, despite the differences in frequency, the traveling wave pattern was roughly the same, with each having at least three wave-fronts propagating along the spanwise direction.

The main effect these traveling waves have on the turbulent boundary layer is to extend the viscous sublayer and push the buffer layer further off the wall. This was seen in the boundary layer profile measured directly over the actuated surface, while no changes were noticeable upstream and downstream. These same results were seen in previous studies [4, 5, 26, 30]. A phase-locked analysis showed that the boundary layer is affected differently depending on the instance in the wave oscillation. Over the crest of the wave, the boundary layer was generally pushed off the surface, but not as strongly as for the time-averaged. There is also a phase-lag effect such that when the wave oscillates and passes through zero displacement, the boundary layer is still effected. Thus, the boundary layer cannot instantaneously react to the flow and flow further from the surface lags behind. This was seen for the traveling wave at $430 \mathrm{~Hz}$, but was not clear for the other two waves $(607 \mathrm{~Hz}$ and $810 \mathrm{~Hz})$. However, the wave amplitude was much smaller for the higher frequencies, so the overall effect on boundary layer was small. In terms, of variance/streamwise Reynolds stress profiles, the peak variance of the crest profile is pushed off the wall. This indicates a shift in the buffer layer, which agrees with the thickening of the viscous sublayer seen in the mean velocity profiles. Even though the variance profile is pushed off the wall, the magnitude of the peak remains roughly the same as the unactuated case.

The general mechanism for the change in the turbulent boundary layer is the interaction of the traveling wave with the quasi-streamwise vortices (QSVs) in the near wall region. Klump [4] showed that the wall-normal vorticity was pushed off the wall, which in turn altered the QSVs. 
Tomiyama [3] suggested the QSVs were pushed off the wall by the spanwise induced flow. The QSVs are closely coupled with bursting events, which are in turn responsible for increases in the local skin friction in turbulent flow [23]. So by pushing the QSVs off the surface, the local skin friction is reduced, which manifests as a thickening of the viscous sublayer and lower velocity gradient in that region. In addition, the wall-normal velocity seems to play a strong role in the altering the boundary layer. Tomiyama [3] and Koh [28] suggested that the wall-normal velocity also contributes to pushing the vortices away from the wall. The results presented here also demonstrate a dependence on the wall-normal velocity which produces a phase-lagged effect of the flow. This phase-lagged effect conforms with the results of Li [30], who suggested that the inertia of the boundary layer is high enough so that it cannot respond to the downward motion of the wall. Overall, it is suggested that the decrease in skin friction relates to both the pushing of vortices away from the wall and the phase-lag of the fluid in reacting to the downward moving surface.

To quantify the effect on the turbulent boundary layer, the $\Delta y$ offset from the unactuated case was found for each actuated boundary layer profile. Plotting this versus the wave displacement amplitude showed a positive correlation (Figure 7.20). As the wave amplitude increased, the boundary layer was pushed further from the wall. At the lowest amplitudes, the boundary layer was actually pulled towards the wall. This indicates that the viscous sublayer is thinner for these cases, suggesting drag increases. This is interesting for a number of reasons. First off, zero amplitude means the surface isn't moving and it is essentially the unactuated case (flat wall). So the expectation would be that as the amplitude decreases, the change in the boundary layer would converge to zero. Secondly, at small amplitudes, the displacement is within the viscous sublayer $\left(y^{+}<5\right)$. For the case of a simple rough surface, this amplitude would constitute a hydraulically smooth surface such that there is no effect on the boundary layer. Granted, traveling waves are a dynamic method and thus have differences from roughness, but one would still expect minimal effect at small amplitudes. Therefore, the thinning of the viscous sublayer at small amplitudes seems to indicate an addition effect besides amplitude dependence.

The first suggestion is a dependence on frequency. The viscous sublayer was thinned for the higher frequency traveling waves: $607 \mathrm{~Hz}\left(\mathrm{~T}^{+}=78\right)$ and $810 \mathrm{~Hz}\left(\mathrm{~T}^{+}=59\right)$. The $430 \mathrm{~Hz}\left(\mathrm{~T}^{+}=112\right)$ traveling wave seemed the most effective. Previous studies have presented the optimal normalized period as $T^{+}=100[5,8,30]$; thus, the lower periods (higher frequencies) may be negatively effecting the turbulent boundary layer and thinning the viscous sublayer. These previous studies primarily investigated traveling waves at or above the optimal period $\left(T^{+}>100\right)$, so their conclusions may not directly here where lower periods were presented. In addition, the traveling wave amplitudes for the $607 \mathrm{~Hz}\left(T^{+}=78\right)$ and $810 \mathrm{~Hz}\left(T^{+}=59\right)$ traveling waves were much smaller than the $430 \mathrm{~Hz}$ case, so comparison between all frequency traveling waves is not conclusive. 
Another suggestion is the dependence on the traveling wave pattern itself. The largest case of viscous sublayer thinning occurs for the $810 \mathrm{~Hz}$ traveling wave (Figure 7.5). The point at $x_{p}=$ $154 \mathrm{~mm}$ has a small displacement, but has larger amplitudes to either spanwise side. The larger amplitude locations may be pushing the quasi-streamwise vortices (QSVs) off of the wall and into the adjacent low amplitude region. Thus, the QSVs and the accompanying bursting events are stronger at the measurement location and the viscous sublayer is thinned. Looking at a different effect, the traveling wave pattern likely also plays a role in the streamwise direction. Tamano [5] demonstrated that there is a streamwise recovery length over which the turbulent boundary layer returns to the unactuated profile. The traveling wave patterns in this study consist of three separate wave-fronts in the streamwise direction. Thus, there is a recovery length downstream of each wave-front. So the boundary layer profile downstream of a wave-front would be pushed further off of the surface than the displacement at that downstream location suggests. Overall, the pattern of the traveling wave likely plays a substantial role in varying the turbulent boundary layer.

In order to better understand the effects of spanwise traveling waves on the turbulent boundary layer, further experiments are necessary. First, traveling waves should be generated at a wider range of frequencies. This would provide a clearer indication of the frequency dependence. Each frequency wave should also be generated at a range of amplitudes. For the present study, the higher frequencies had small amplitude so it was difficult to determine the true dependence on frequency. Then, the relationship with the wall-normal velocity of the traveling should be investigated. Finally, the boundary layer should be measured at many locations over the traveling wave pattern. This includes measuring between two spanwise peaks to see if the viscous sublayer thins, and also downstream of a wave-front to determine the recovery length effect. In addition, measurements of the vorticty would provide knowledge of the QSVs and a better correlation with the underlying mechanism.

\subsubsection{Standing versus traveling Waves}

To determine the effects of a standing wave on the turbulent boundary layer, two standing waves were excited at $385 \mathrm{~Hz}$ and $630 \mathrm{~Hz}$ which correspond to the $(2,3)$ and $(3,4)$ mode shapes, respectively. When the frequencies are normalized to wall units, $385 \mathrm{~Hz}$ is $T^{+}=122$ and $630 \mathrm{~Hz}$ is $T^{+}=74$. These standing waves were chosen since their frequencies lie in the same range as those for the traveling waves. This, the goal was both to determine how standing waves alter the turbulent boundary layer and to compare against the traveling waves.

The results in Section 7.1.2 show that the standing wave adjusts the boundary layer in the same manner as the traveling waves. The actuated profile is pushed off of the surface and the viscous sublayer is thickened. The phase-locked analysis showed that the profile over the crest of the 
wave is not affected as strongly as the time-averaged. In addition, there is a lag in the response of the flow to the moving surface, similar to what is seen for the traveling waves. Comparing the variance/streamwise Reynolds stress plots to the traveling wave, the same effects were observed. The time-averaged actuated profile increases near the wall due to the oscillating relative $y$ position, while the variance over the crest is pushed off the wall. As a result, the standing and traveling waves appear to effect the turbulent boundary layer in the same way.

To determine whether the wave-types truly respond in the same way, the $\Delta y$ offset was quantified just as for the traveling wave. The offsets for the standing and traveling waves were then plotted together (Figure 7.18). First analysis of these results showed that they have roughly the same effect. There is a linear trend between displacement and offset and a given displacement will give approximately the same offset for both a standing and traveling wave. When the standing and traveling waves are plotted and clearly marked (Figure 7.20), some differences become clear. Linear trends fit to the wave types separately show that the standing wave tends to have a stronger effect on the boundary layer. In addition, the standing wave does not thin the viscous sublayer at small amplitudes as seen for traveling waves.

Despite the apparent differences between the standing and traveling wave, it is difficult to conclusively state that standing waves have a stronger effect than traveling waves. There is still significant overlap of the data in the parametric analysis, and the uncertainty was not accounted for in the parameteric analysis. Also, the standing wave data does not account for the potential wave pattern effect seen with the traveling waves. All of the boundary layers over the standing waves were measured at wave anti-nodes (peaks) or directly downstream of one. None of these measurement locations had large peaks to either spanwise side. As suggested in Section 7.3.1, a possible reason for viscous sublayer thinning is the diversion of quasi-streamwise vortices into the small amplitude region between two larger spanwise peaks. Thus, if the boundary layer was measured at such a position on the standing wave, the viscous sublayer might be thinned.

Another point to make is that there appears to be little difference between the standing wave frequencies. From the parametric plot (Figure 7.20) both the $385 \mathrm{~Hz}$ and $630 \mathrm{~Hz}$ standing waves seem to affect the boundary layer with roughly the same strength. For the traveling waves, it was suggested that the frequency might play a role in the viscous sublayer thinning, in particular the $607 \mathrm{~Hz}$ and $810 \mathrm{~Hz}$ waves being less effective than the $430 \mathrm{~Hz}$ wave. These are roughly the same frequencies as the $385 \mathrm{~Hz}$ and $630 \mathrm{~Hz}$ standing waves, which showed no difference. Since both wave types have similar effects on the boundary layer, it seems likely that they would share the same frequency dependence. Thus, it is suggested that there is not a large frequency dependence within this range.

In order to understand any difference between the standing and traveling waves, the wave patterns themselves must be compared. Both standing and traveling waves have displacement 
in the wall-normal direction. Both have multiple node lines extending the spanwise length of the plate resulting in separate peaks along the streamwise direction. The main difference is the traveling wave-fronts themselves. There are roughly three wave-fronts at each frequency, and they propagate in the spanwise direction. As a result, there are no points of zero displacement along the propagation path. In comparison, the standing wave has stationary anti-nodes with points of zero displacement along the spanwise direction (node lines). However, the traveling waves generated here are not ideal. The displacement is not constant along the propagation path. This is clear by looking at the traveling wave patterns (Figures 6.10,7.2, and 7.5). Overall, the differences between the traveling and standing waves are actually fairly minimal.

The traveling waves generated here can then be compared with those in literature. Several experimental papers $[8,29,30,32]$ have investigated the use of spanwise traveling waves all using the same experimental setup. In these studies, traveling waves are generated using a number of discrete electro-magnetic actuators. The result is a single streamwise wave-front propagating with uniform displacement in the spanwise direction. The studies showed that the buffer layer is pushed off the wall and the viscous sublayer is thickened. However, visual observation of the boundary layers shows that while the actuated profile is pushed off the surface, the $\Delta y$ is not as large as seen here in the present research. This is for roughly the same amplitudes and frequencies. Thus, the underlying mechanism may differ for the current study versus those in literature. This could relate to the variation in spanwise amplitude discussed above. The locations with larger displacement push the QSVs off the surface and funnel them into the adjacent small amplitude regions. The other studies have consistent spanwise amplitude, so the QSVs can only be pushed upwards. Thus, the present study is able to more easily push the QSVs away from the surface. However, this occurs only over the peaks of the traveling and standing waves. As previously discussed, the viscous sublayer can be thinned at small displacements. So, while the waves here may be effective over the anti-nodes, they are not at the nodes. If the goal is a reduction in skin friction drag, the net effect over the entire surface would be a combination of that over both the anti-nodes and nodes.

To better understand the effect of standing waves on the turbulent boundary layer, further testing is necessary. This includes tests similar to those recommended for the traveling waves: standing waves at various frequencies and amplitudes with many measurement locations over the surface. These can be used to determine the frequency and wave pattern dependence. In addition, more specific testing should be done to compare standing and traveling waves. A standing and traveling wave at the same frequency and roughly the same amplitude should be investigated. The frequency should be chosen with two factors in mind. First, so that it lies within the optimal range $\left(T^{+} \approx 100\right)$. Second the wave pattern of the standing and traveling wave should be roughly the same. As an example, if the standing wave were the $(5,2)$ mode shape, and the traveling wave were composed of the $(5,2)$ mode shape. Comparing these two wave types at the same frequency, 
amplitude, and pattern would allow for the most controlled conditions and thus the clearest conclusions. Finally, traveling and standing waves should be compared with an ideal traveling wave, where the displacement is constant along the propagation path. 


\section{Chapter 8}

\section{Conclusion}

The main focus of this research has been the development and testing of a low-profile traveling wave generation method that can be practically implemented to interact with the turbulent boundary layer with the aim of reducing skin friction drag. It was shown that steady-steady traveling waves can be generated on a two-dimensional surface using piezoelectric actuators, and that the wave pattern can be tailored by adjusting the number and location of actuators (actuation configuration). These traveling waves were then experimentally tested in a low-speed wind tunnel and their effect on the turbulent boundary layer measured. The results showed that the these traveling waves alter the boundary layer in a manner consistent with skin friction reduction. Thus, this novel traveling wave generation method is promising as an actual implementable technique for reducing skin friction drag. Finally, this wave generation method was used to compare the effects of standing and traveling waves on the turbulent boundary layer. The resultant parametric analysis showed that both standing and traveling waves affect the boundary layer in a similar manner. Overall, a novel traveling wave generation was developed and experimentally shown to alter the turbulent boundary layer in a manner consistent with skin friction drag reduction.

While the main goal for implementing traveling waves is to reduce skin friction drag, the underlying effect is a change in the turbulent boundary layer. To capture this more fundamental effect the experimental setup and approach focused on investigating the turbulent boundary layer instead of directly measuring the drag (discussed in Section 2). The overall results indicated that the traveling waves altered the boundary layer in a manner consistent with a reduction in skin friction drag. Due to the fundamental approach and the experimental setup, the actual reduction in skin friction could not be quantified. However, now that the implementable traveling wave generation method has been validated and shown to effect the turbulent boundary layer, further studies can be taken to quantify the skin friction reduction.

This section continues by providing a more in-depth summary of the research conducted. Chapters 4-7 contained the major findings of this study, so these four chapters are summarized below. 
Then, the novel contributions from this research are highlighted. Finally, recommendations for future work involving traveling waves, the effect on the turbulent boundary layer, and drag reduction are given.

\subsection{Summary of Results}

Chapter 4 focused on the development of the low-profile traveling wave generation method. These traveling waves were generated on a two-dimensional plate using flush-mounted piezoelectric actuators. The actuators were excited using the two-mode excitation method, which generates steadystate traveling waves in an open-loop fashion and with minimal frequency limitations. Previous use of this method on a two-dimensional surface was from a fundamental standpoint with most previous applications utilizing one-dimensional beams. The main goal was to further develop the two-mode excitation method in order to generate tailorable traveling wave patterns. Specifically, this was accomplished by understanding the relationship between traveling wave pattern and actuation configuration (location and number of actuators). Traveling waves could then be tailored for the specific application of drag reduction/turbulent boundary layer manipulation.

An in-house finite element model was developed to simulate a thin clamped plate with composite elements representing the piezoelectric actuators. Next, experimental modal analysis was conducted to extract the structural properties from the plate for different two boundary conditions: free and fixed. Then, the finite element model was manually updated to account for the material properties, boundary conditions, and internal stresses of the experimental plate. Traveling waves were then computationally and experimentally generated to validate the model. Finally, traveling waves were tailored by exciting the plate at various actuation configurations.

The results showed that different traveling wave patterns could be generated at the same frequency simply by varying the location and number of actuators (actuation configuration). The patterns were adjusted to produce propagating waves across the whole plate or only in specific regions. In effect, the actuation configuration alters the participation of nearby mode shapes. Thus, traveling waves can be tailored by adjusting the actuation configuration to take advantage of the structure's modal properties. This tailor-ability coupled with the low-profile nature of the piezoelectric actuators makes this traveling wave generation method a promising technique not just for reducing skin friction drag, but also for other applications including propulsion and solid-state locomotion.

Chapter 5 then explained the wind tunnel experimental setup along with the procedure for measuring and analyzing the turbulent boundary layer. Although the eventual application of these traveling waves is for drag reduction, a more fundamental approach was taken in this research. The underlying effect of the traveling waves on the turbulent boundary layer was studied instead 
of focusing on the skin friction. This was done in a small-scale, low-speed wind tunnel originally design for airfoil characterization. A significant amount of effort was spent refurbishing and heavily modifying this wind tunnel for the present research.

The same traveling wave plate from Chapter 4 was installed in the test-section of the wind tunnel. The setup was designed to develop a well-characterized turbulent boundary layer over this traveling wave plate. The flow quality in the wind tunnel was extensively tested to ensure it was properly defined for the experiments. The boundary layer was then measured via hotwire anemometry and a three-dimensional traversing system. The entire setup is controlled via a Labview interface with automated testing procedures for boundary layer measurement. Next, the analysis procedure for the boundary layer is outlined, describing statistical independence, nondimensionalization, and applying correction factors. The processed data is then converted into useable formats including mean velocity and variance boundary layer profiles. These experimental measured boundary layers are validated against accepted data from literature. This validation demonstrates that the turbulent boundary layer has been properly developed, accurately measured, and correctly analyzed.

Chapter 6 then tests the novel traveling wave generation method in the wind tunnel and validates its ability to effect the turbulent boundary layer. Since the same plate from Chapter 4 is installed in the wind tunnel, the frequency response is analyzed to observe changes due to the mounting conditions and air flow. Sufficiently large changes are present, so the developed finite element model is no longer applicable. Spanwise traveling waves are then tailored at a single frequency and used as a representative case for the effect on the boundary layer.

The turbulent boundary layer is measured at locations upstream, directly over, and downstream of the actuated traveling waves surface. The mean velocity and variance profiles are analyzed at these locations. Next, a phase-locked analysis is conducted in which boundary layer profiles are extracted over the crest, trough, and zero displacement positions of the wall-normal motion. The skewness and kurtosis are analyzed and then the boundary layers measured at several spanwise locations over the traveling wave surface are presented. Finally, the effect of experimental uncertainty on the results are discussed.

The results showed that the traveling waves in this research affected the turbulent boundary layer in a manner consistent with skin friction reduction. In addition, these results concurred with those seen in previous literature. The boundary layer is pushed off of the wall and the viscous sublayer is thickened. This indicates a decrease in the sublayer velocity gradient which represents a decrease in the local skin friction. This decrease could not be quantified since the boundary layer can not be physically measured close enough to the moving wall. The phase-locked analysis then showed that the affect on the boundary layer changes based on the phase-locked position. The boundary layer profile over the crest is pushed off the wall, but not as strongly as for the 
time-actuated. In addition, the viscous sublayer was thickened over the zero-displacement positions, despite their being no deformation at this location. This indicates that the flow cannot react quickly enough to the moving surface and it lags behind. In addition, the boundary layer was barely affected when the surface moves upwards into the flow, but is strongly affected for downwards motion. This suggests the phase-lag effect prevents the flow from adapting to the downward moving surface, and is the source of the skin friction reduction.

Additional results showed showed that the amount by which the boundary layer is affected, depends on the wall-normal amplitude. Next, the time-averaged variance profile increases in the near wall region. This was found to result from the experimental setup and measurement procedure. Thus, this increase is not due to a change in the turbulent boundary layer structure as previous reported in literature. Overall, the traveling waves affect the turbulent boundary layer in a manner consistent with a decrease in skin friction drag. This validates the low-profile, tailorable, traveling wave generation method developed in Chapter 4 as a implementable technique for reducing skin friction drag.

Chapter 7 investigates traveling waves at different frequencies and also the effects of standing waves on the turbulent boundary layer. The two-mode excitation method generates traveling waves by taking advantage of the plate's structural properties. As a result, the traveling wave patterns closely resemble the plate's mode shapes (standing waves). By measuring the turbulent boundary layer over the standing waves, it is found that the standing waves have roughly the same effect as the traveling waves. The data from these two wave types is then used to investigate the dependence on amplitude, frequency, and wave pattern.

Traveling waves at two additional frequencies to that in Chapter 6 are tested. These traveling waves were generated and tailored so as to have similar wave patterns as the single wave in the previous chapter. Next, two standing waves were generated at similar frequencies and amplitudes as the traveling waves. The turbulent boundary layer was then measured at multiple locations over all of these traveling and standing waves. The results showed that the standing waves affected the boundary layer in the same way as the traveling waves. The boundary layer was pushed off the wall and the viscous sublayer was thickened. The crest was not affected as strongly as the timeaveraged. And the zero-displacement profiles suggest a phase-lag effect and stronger effect on the downward motion of the actuated surface.

Given that both wave-types yielded similar effects, a parametric analysis was conducted on all the traveling and standing wave data together. Since the main effect is the pushing off of the boundary, this offset was quantified and used as the indicator for the change in boundary layer. This offset showed a distinct correlation with the wall-normal amplitude such that waves with larger amplitudes more strongly effect the boundary layer. This was seen for both wave types, and was found to be the dominant parameter affecting the turbulent boundary layer. For some traveling wave 
cases, the boundary layer was pulled closer to the wall and the viscous sublayer thinned, indicating a skin friction increase. This is likely a dependence on the traveling wave pattern. The sublayer was thinned when the wall-normal amplitude was small, so it is suggested that neighboring large amplitude regions funnel the turbulent vortices into the lower amplitude region. Overall, the results also showed that there is no clear difference in the effect of traveling and standing waves on the boundary layer. However, this could relate to the lack of measurement density over the standing waves and also the experimental uncertainty in the results. Future experiments may yet show a difference between the two wave-types.

\subsection{Contributions}

The first major contribution relates Chapter 4 and the development of the traveling wave method. Previous use of the two-mode excitation method primarily focused on one-dimensional beams. The method had been applied to two-dimensional surfaces, but it was done as a proof of concept and only two actuators were used. This research investigated the relationship between the traveling wave pattern and the actuation configuration (number and location of actuators). It was found that by using multiple sets of actuators and changing their location, traveling waves could be generated on the entire or only isolated portions of the surface. Thus, the resultant traveling wave pattern could be tailored. This was particularly useful in this research, where the aim was to generate spanwise traveling waves to affect the turbulent boundary layer. This relationship could also be utilized to tailor traveling waves for other applications. Such applications include propulsion, steady-state locomotion, and de-icing.

The next major contribution is demonstrating that this low-profile and implementable traveling wave generation method is able to affect the turbulent boundary layer, particularly in a manner consistent with skin friction reduction. Previous studies generated traveling waves using bulky actuation setups that placed significant limitations on bandwidth and wave pattern. Traveling waves were generated in this research using piezoelectric actuators that are low-profile and non-invasive. These actuators were excited using the two-mode excitation method which operates at most frequencies, is open-loop controlled, and allows tailorable traveling wave patterns. Although the effect on the skin friction was not directly quantified, these traveling waves were shown to alter the turbulent boundary layer in a manner consistent with skin friction reduction.

The next contribution is an explanation of an effect seen in literature. Previous studies showed that over a traveling wave actuated surface, the streamwise Reynolds stress (variance) increases near the wall. These studies stated that this results from the traveling wave altering the turbulent boundary layer structure. However, the present research showed that this near wall increase stems from the experimental setup. A stationary hot-wire probe over the moving wall produces an 
oscillation in the relative wall-normal (y) position. This manifests as an artificial increase in the streamwise Reynolds stress, and is not a change in the turbulent boundary layer structure.

Another contribution relates to the underlying effect of how traveling waves alter the turbulent boundary layer. Previous studies have conducted phase-locked analyzes, but only did so over the crest and trough of the wave. In this research, the phase-locked analysis was extended to look at the change in the turbulent boundary layer profiles over the zero-displacement positions of the wave oscillation. The results at these locations suggest that the flow responds in a phase-lagged manner to the oscillating wall. This lag, specifically during the downward motion of the wall, may contribute to the thickening of the viscous sublayer and thus the reduction in skin friction drag.

The final contribution is that standing and traveling waves affect the turbulent boundary layer in similar manners. The boundary layer is pushed off of the wall and the viscous sublayer is thickened. A parametric analysis showed that both are heavily dependent on the wall-normal amplitude. Both wave types also have roughly the same strength, in that a given wave displacement produces the same effect for each wave. It was also shown that the wave pattern plays some role in how the turbulent boundary layer is affected. Thus, differences between the two wave types may yet be seen when measuring the boundary layer over the entire surface.

\subsection{Future Work}

This research produced a number of notable contributions. As a result, it also set the ground work for further research. This includes further development of the traveling wave generation method, investigation into the effect of traveling and standing waves on the turbulent boundary layer, and application of this technique for drag reduction purposes.

The first recommendations for future work, relate to the traveling wave generation method. It was shown here that traveling waves could be tailored on a two-dimensional surface. However, this was primarily done using a case study. The underlying theory should be improved relating the actuation configuration (location and number of actuators) and resultant traveling wave pattern. In addition, a cost function should be developed for evaluating the quality of two-dimensional waves, as opposed to the visual inspection used here. Finally, the fluid structure interaction between the waves and the air flow should be investigated. In the present research, the quality of generated traveling waves decreased noticeably with exposed to airflow.

Next, the effect of traveling and standing waves on the turbulent boundary layer should be further investigated. In this research, the effects of three traveling and two standing waves were studied; however, no clear conclusion could be drawn regarding the effects of frequency. So, a wider range of frequencies and amplitudes should be tested. Also, further phase-locked analysis should be conducted to more clearly demonstrate any phase-lag effects and the dependence on 
the wall-normal velocity. Additional testing is also necessary to better understand the effects of wave pattern on the turbulent boundary layer. This would involve measuring the boundary layer at numerous locations over the actuated surface. The results could also be used to more conclusively state differences between standing and traveling waves. These future investigations would all benefit from the use of particle image velocimetry (PIV) and computational studies. By using these methods, the flow could be more easily studied at multiple locations while also providing a greater amount information that would aid in understanding the underlying mechanism and its dependence on phase-lag effects and the manipulation of turbulent vortices.

Finally, the applicability of this method for skin friction drag reduction should be studied. The skin friction drag should be quantified by measuring the viscous sublayer and calculating the velocity gradient. Once again, particle image velocimetry (PIV) and computational studies would be beneficial. The local skin friction should be evaluated at numerous locations over the traveling (and standing) wave surface, to determine the net drag effect of the wave. This could be compared with direct measurement of the skin friction drag via a force balance. In addition, the net energy savings should be investigated. This would study the power usage of the traveling waves and could be coupled with more efficient traveling wave generation. The energy balance in the fluid should also investigated. 


\section{References}

[1] Avirovik, D., Malladi, V. S., Priya, S. and Tarazaga, P. A., Theoretical and experimental correlation of mechanical wave formation on beams, Journal of Intelligent Material Systems and Structures, Vol. 27, No. 14, pp. 1939-1948, 2016. xiii, 2, 30, 31, 32

[2] Malladi, V., Avirovik, D., Priya, S. and Tarazaga, P., Characterization and representation of mechanical waves generated in piezo-electric augmented beams, Smart Materials and Structures, Vol. 24, No. 10, pp. 105026, 2015. xiii, 2, 31, 32, 33, 65

[3] Tomiyama, N. and Fukagata, K., Direct numerical simulation of drag reduction in a turbulent channel flow using spanwise traveling wave like wall deformation, Physics of Fluids (1994-present), Vol. 25, No. 10, pp. 105115, 2013. xiii, 2, 7, 35, 36, 41, 42, 43, 50, 51, 52, $53,130,155,156,186$

[4] Klumpp, S., Meinke, M. and Schröder, W., Drag reduction by spanwise transversal surface waves, Journal of Turbulence, , No. 11, pp. N22, 2010. xiii, 2, 7, 36, 38, 39, 40, 45, 50, $51,154,156,185$

[5] Tamano, S. and Itoh, M., Drag reduction in turbulent boundary layers by spanwise traveling waves with wall deformation, Journal of Turbulence, , No. 13, pp. N9, 2012. xiii, 2, 36, $40,41,43,44,47,48,50,51,52,53,130,154,155,156,157,185,186,187$

[6] Bai, H., Zhou, Y., Zhang, W., Xu, S., Wang, Y. and Antonia, R., Active control of a turbulent boundary layer based on local surface perturbation, Journal of Fluid Mechanics, Vol. 750, pp. 316, 2014. xiv, 2, 36, 43, 44, 45, 50, 51, 156

[7] Koh, S. R., Meysonnat, P., Meinke, M. and Schröder, W., Drag reduction via spanwise transversal surface waves at high Reynolds numbers, Flow, Turbulence and Combustion, Vol. 95, No. 1, pp. 169-190, 2015. xiv, 2, 7, 36, 45, 46, 47, 50, 51

[8] Roggenkamp, D., Jessen, W., Li, W., Klaas, M. and Schröder, W., Experimental investigation of turbulent boundary layers over transversal moving surfaces, CEAS Aeronautical 
Journal, Vol. 6, No. 3, pp. 471-484, 2015. xiv, 2, 36, 47, 48, 49, 50, 51, 52, 130, 156, 157, 186,189

[9] Hutchins, N. and Choi, K.-S., Accurate measurements of local skin friction coefficient using hot-wire anemometry, Progress in Aerospace Sciences, Vol. 38, No. 4, pp. 421-446, 2002. xvi, 96, 98, 99, 110

[10] Janke, G., Hot wire in wall proximity, pp. 488-498, Springer, 1987. xvi, 98, 99

[11] Polyakov, A. and Shindin, S., Peculiarities of hot-wire measurements of mean velocity and temperature in the wall vicinity, Letters in Heat and Mass Transfer, Vol. 5, No. 1, pp. 53-58, 1978. xvi, 98, 99

[12] Oka, S. and Kostic, Z., Influence of wall proximity on hot-wire velocity measurements, Disa information, pp. 29-33, 1972. xvi, 98, 99

[13] Ligrani, P. and Bradshaw, P., Subminiature hot-wire sensors: development and use, Journal of Physics E: Scientific Instruments, Vol. 20, No. 3, pp. 323, 1987. xvi, 98, 99

[14] Purtell, L., Klebanoff, P. and Buckley, F., Turbulent boundary layer at low Reynolds number, The Physics of Fluids, Vol. 24, No. 5, pp. 802-811, 1981. xvii, 118, 119

[15] Coles, D., The Turbulent Boundary Layer in a Compressible Fluid, 1962. xvii, 15, 108, 120,121

[16] ARMD Strategic Implementation Plan, 2017. 1

[17] IATA Resolution on the Implementation of the Aviation "CNG2020" Strategy. 1

[18] Green Aviation: A Better Way to treat the Planet, National Aeronautics and Space Administration, NF-2010-07-500-HQ, 2010. 1

[19] Walsh, M. J., Drag characteristics of V-groove and transverse curvature riblets, 1980. 1, 34

[20] Choi, K.-S., On physical mechanisms of turbulent drag reduction using riblets, Transport Phenomena in Turbulent Flows: Theory, Experiment, and Numerical Simulation, Vol. 1, pp. 185-198. 1, 34

[21] Perlin, M., Dowling, D. R. and Ceccio, S. L., Freeman Scholar Review: Passive and Active Skin-Friction Drag Reduction in Turbulent Boundary Layers, Journal of Fluids Engineering, Vol. 138, No. 9, pp. 091104, 2016. 1 
[22] Karniadakis, G. and Choi, K.-S., Mechanisms on transverse motions in turbulent wall flows, Annual Review of Fluid Mechanics, Vol. 35, No. 1, pp. 45-62, 2003. 1, 34, 35, 38, 51,52

[23] Robinson, S. K., Coherent motions in the turbulent boundary layer, Annual Review of Fluid Mechanics, Vol. 23, No. 1, pp. 601-639, 1991. 2, 51, 114, 130, 186

[24] Jung, W., Mangiavacchi, N. and Akhavan, R., Suppression of turbulence in wall bounded flows by high frequency spanwise oscillations, Physics of Fluids A: Fluid Dynamics (19891993), Vol. 4, No. 8, pp. 1605-1607, 1992. 2, 35, 130, 154

[25] Choi, K.-S., DeBisschop, J.-R. and Clayton, B. R., Turbulent boundary-layer control by means of spanwise-wall oscillation, AIAA journal, Vol. 36, No. 7, pp. 1157-1163, 1998. 2, 35

[26] Itoh, M., Tamano, S., Yokota, K. and Taniguchi, S., Drag reduction in a turbulent boundary layer on a flexible sheet undergoing a spanwise traveling wave motion, Journal of Turbulence, , No. 7, pp. N27, 2006. 2, 36, 38, 40, 47, 50, 154, 155, 156, 185

[27] Klumpp, S., Meinke, M. and Schröder, W., Friction drag variation via spanwise transversal surface waves, Flow, turbulence and combustion, Vol. 87, No. 1, pp. 33-53, 2011. 2, 36, $39,40,41,45,50,51$

[28] Koh, S., Meysonnat, P., Statnikov, V., Meinke, M. and Schröder, W., Dependence of turbulent wall-shear stress on the amplitude of spanwise transversal surface waves, Computers and Fluids, Vol. 119, pp. 261-275, 2015. 2, 36, 46, 47, 50, 51, 154, 155, 156, 186

[29] Li, W., Jessen, W., Roggenkamp, D., Klaas, M., Silex, W., Schiek, M. and Schröder, W., Turbulent drag reduction by spanwise traveling ribbed surface waves, European Journal of Mechanics-B/Fluids, Vol. 53, pp. 101-112, 2015. 2, 36, 49, 50, 189

[30] Li, W., Roggenkamp, D., Hecken, T., Jessen, W., Klaas, M. and Schröder, W., Parametric investigation of friction drag reduction in turbulent flow over a flexible wall undergoing spanwise transversal traveling waves, Experiments in Fluids, Vol. 59, No. 6, pp. 105, 2018. $2,130,154,155,156,157,185,186,189$

[31] Meysonnat, P. S., Koh, S. R., Roidl, B. and Schröder, W., Impact of transversal traveling surface waves in a non-zero pressure gradient turbulent boundary layer flow, Applied Mathematics and Computation, Vol. 272, pp. 498-507, 2016. 2, 36, 47, 50 
[32] Meysonnat, P. S., Roggenkamp, D., Li, W., Roidl, B. and Schröder, W., Experimental and numerical investigation of transversal traveling surface waves for drag reduction, European Journal of Mechanics-B/Fluids, Vol. 55, pp. 313-323, 2016. 2, 36, 48, 49, 50, 157, 189

[33] Loh, B.-G. and Ro, P. I., An object transport system using flexural ultrasonic progressive waves generated by two-mode excitation, IEEE transactions on ultrasonics, ferroelectrics, and frequency control, Vol. 47, No. 4, pp. 994-999, 2000. 2, 28, 29, 33

[34] Tomikawa, Y., Adachi, K., Hirata, H., Suzuki, T. and Takano, T., Excitation of a progressive wave in a flexurally vibrating transmission medium, Japanese journal of applied physics, Vol. 29, No. S1, pp. 179, 1990. 2, 28, 33

[35] Malladi, V. V. S., Albakri, M. and Tarazaga, P. A., An experimental and theoretical study of two-dimensional traveling waves in plates, Journal of Intelligent Material Systems and Structures, p. 1045389X16679284, 2016. 2, 33, 53

[36] Anderson, J. D. and Wendt, J., Computational fluid dynamics, Vol. 206, Springer, 1995. 8

[37] Driver, D., Application of oil-film interferometry skin-friction measurement to large wind tunnels, Experiments in Fluids, Vol. 34, No. 6, pp. 717-725, 2003. 8

[38] Barlow, J., Rae, W. and Pope, A., Low-speed wind tunnel testing, 1999, John Wiley and Sons, Canada. 9, 82, 83, 86

[39] Haritonidis, J. H., The measurement of wall shear stress, pp. 229-261, Springer, 1989. 9, 22

[40] Gouder, K., Potter, M. and Morrison, J. F., Turbulent friction drag reduction using electroactive polymer and electromagnetically driven surfaces, Experiments in fluids, Vol. 54, No. 1, pp. 1-12, 2013. 9

[41] Raffel, M., Willert, C. E., Wereley, S. and Kompenhans, J., Particle image velocimetry: a practical guide, Springer, 2013. 10

[42] Chue, S., Pressure probes for fluid measurement, Progress in aerospace sciences, Vol. 16, No. 2, pp. 147-223, 1975. 11, 80, 212, 213

[43] MacMillan, F., Experiments on Pitot-tubes in shear flow, Vol. 3028, HM Stationery Office, 1957. $11,212,213$

[44] Comte-Bellot, G., Hot-wire anemometry, Annual review of fluid mechanics, Vol. 8, No. 1, pp. 209-231, 1976. 12 
[45] Bruun, H. H., Hot-wire anemometry: principles and signal analysis, 1996. 12, 13, 17, 93, $96,97,98,146$

[46] Schetz, J. A., Boundary Layer Analysis, American Institute of Aeronautics and Astronautics, Reston, Virginia, 2010. 14, 21, 22, 77, 115, 116

[47] Tennekes, H. and Lumley, J. L., A first course in turbulence, MIT press, 1972. 14, 17, 18, $19,20,46,104,113$

[48] Davidson, P. A., Turbulence: an introduction for scientists and engineers, Oxford University Press, 2015. 14, 16, 50, 108, 156

[49] Zanoun, E.-S., Durst, F. and Nagib, H., Evaluating the law of the wall in two-dimensional fully developed turbulent channel flows, Physics of Fluids, Vol. 15, No. 10, pp. 3079-3089, 2003. 15

[50] Coles, D., The law of the wake in the turbulent boundary layer, Journal of Fluid Mechanics, Vol. 1, No. 2, pp. 191-226, 1956. 16

[51] Schlichting, H. and Gersten, K., Boundary-layer theory, Springer Science and Business Media, 2003. 16, 21, 22, 38, 78

[52] Clauser, F. H., The turbulent boundary layer, Vol. 4, pp. 1-51, Elsevier, 1956. 20

[53] Wei, T., Schmidt, R. and McMurtry, P., Comment on the Clauser chart method for determining the friction velocity, Experiments in Fluids, Vol. 38, No. 5, pp. 695-699, 2005. 21

[54] Anders, J., Outer-layer manipulators for turbulent drag reduction, Viscous Drag Reduction in Boundary Layers, Vol. 123, pp. 263-284, 1990. 22, 37

[55] Dutton, R., The Accuracy of Measurement of Turbulent Skin Friction by Means of Surface Pitot-Tubes and the Distribution of Skin Friction on a Flat Plate, HM Stationery Office, 1957. 22

[56] Kuribayashi, M., Ueha, S. and Mori, E., Excitation conditions of flexural traveling waves for a reversible ultrasonic linear motor, The Journal of the Acoustical Society of America, Vol. 77, No. 4, pp. 1431-1435, 1985. 25

[57] Mace, B., Wave reflection and transmission in beams, Journal of sound and vibration, Vol. 97, No. 2, pp. 237-246, 1984. 26 
[58] Hariri, H., Bernard, Y. and Razek, A., A traveling wave piezoelectric beam robot, Smart Materials and Structures, Vol. 23, No. 2, pp. 025013, 2013. 26, 30

[59] Minikes, A., Gabay, R., Bucher, I. and Feldman, M., On the sensing and tuning of progressive structural vibration waves, IEEE transactions on ultrasonics, ferroelectrics, and frequency control, Vol. 52, No. 9, pp. 1565-1576, 2005. 26, 27

[60] Tanaka, N. and Kikushima, Y., Active wave control of a flexible beam: proposition of the active sink method, JSME international journal. Ser. 3, Vibration, control engineering, engineering for industry, Vol. 34, No. 2, pp. 159-167, 1991. 26

[61] Tanaka, N. and Kikushima, Y., Active wave control of a flexible beam: fundamental characteristics of an active-sink system and its verification, JSME international journal. Ser. 3, Vibration, control engineering, engineering for industry, Vol. 35, No. 2, pp. 236-244, 1992. 27

[62] Gabai, R. and Bucher, I., Excitation and sensing of multiple vibrating traveling waves in one-dimensional structures, Journal of sound and vibration, Vol. 319, No. 1, pp. 406-425, 2009. 27

[63] Gabai, R. and Bucher, I., Spatial and temporal excitation to generate traveling waves in structures, Journal of Applied Mechanics, Vol. 77, No. 2, pp. 021010, 2010. 27

[64] Kim, G., Park, J. and Jeong, S., Analysis of dynamic characteristics for vibration of flexural beam in ultrasonic transport system, Journal of Mechanical Science and Technology, Vol. 23, No. 5, pp. 1428-1434, 2009. 29

[65] Dehez, B., Vloebergh, C. and Labrique, F., Study and optimization of traveling wave generation in finite-length beams, Mathematics and Computers in Simulation, Vol. 81, No. 2, pp. 290-301, 2010. 29, 31

[66] Bucher, I., Estimating the ratio between travelling and standing vibration waves under nonstationary conditions, Journal of sound and vibration, Vol. 270, No. 1, pp. 341-359, 2004. 31,65

[67] Malladi, V. V. S., Albakri, M. I., Gugercin, S. and Tarazaga, P. A., Application of projection-based model reduction to finite-element plate models for two-dimensional traveling waves, Journal of Intelligent Material Systems and Structures, p. 1045389X16679295, 2016. 33 
[68] Phoenix, A., Malladi, S. and Tarazaga, P. A., Traveling wave phenomenon through piezoelectric actuation of a free-free cylindrical tube, ASME Conference on Smart Materials, Adaptive structures and Intelligent Systems, Colorado Springs, Co, pp. 21-23. 34

[69] Walsh, M. J., Riblets as a viscous drag reduction technique, AIAA journal, Vol. 21, No. 4, pp. 485-486, 1983. 34

[70] Viswanath, P., Aircraft viscous drag reduction using riblets, Progress in Aerospace Sciences, Vol. 38, No. 6, pp. 571-600, 2002. 34

[71] Choi, K.-S., Near-wall structure of a turbulent boundary layer with riblets, Journal of fluid mechanics, Vol. 208, pp. 417-458, 1989. 34

[72] Szodruch, J., Viscous drag reduction on transport aircraft, AIAA paper, Vol. 685, 1991. 35

[73] Quadrio, M., Drag reduction in turbulent boundary layers by in-plane wall motion, Philosophical Transactions of the Royal Society of London A: Mathematical, Physical and Engineering Sciences, Vol. 369, No. 1940, pp. 1428-1442, 2011. 35

[74] Mito, Y. and Kasagi, N., DNS study of turbulence modification with streamwise-uniform sinusoidal wall-oscillation, International journal of heat and fluid flow, Vol. 19, No. 5, pp. 470-481, 1998. 35

[75] Quadrio, M., Ricco, P. and Viotti, C., Streamwise-travelling waves of spanwise wall velocity for turbulent drag reduction, Journal of Fluid Mechanics, Vol. 627, pp. 161-178, 2009. 35

[76] Kendall, J. M., The turbulent boundary layer over a wall with progressive surface waves, Journal of Fluid Mechanics, Vol. 41, No. 02, pp. 259-281, 1970. 35

[77] Nakanishi, R., Mamori, H. and Fukagata, K., Relaminarization of turbulent channel flow using traveling wave-like wall deformation, International journal of heat and fluid flow, Vol. 35, pp. 152-159, 2012. 35

[78] Du, Y. and Karniadakis, G. E., Suppressing wall turbulence by means of a transverse traveling wave, Science, Vol. 288, No. 5469, pp. 1230-1234, 2000. 35

[79] Du, Y., Symeonidis, V. and Karniadakis, G., Drag reduction in wall-bounded turbulence via a transverse travelling wave, Journal of Fluid Mechanics, Vol. 457, pp. 1-34, 2002. 35, $38,43,51$ 
[80] Swearingen, J. D. and Blackwelder, R. F., The growth and breakdown of streamwise vortices in the presence of a wall, Journal of Fluid Mechanics, Vol. 182, pp. 255-290, 1987. 35,51

[81] Zhao, H., Wu, J.-Z. and Luo, J.-S., Turbulent drag reduction by traveling wave of flexible wall, Fluid Dynamics Research, Vol. 34, No. 3, pp. 175-198, 2004. 35, 38

[82] Li, W., Roggenkamp, D., Jessen, W. and Klaas, M., PIV and $\mu$-PTV investigations of turbulent flow over a flexible wall undergoing traveling surface wave motions, 18th International Symposium on the Application of Laser and Imaging Techniques to Fluid Mechanics. 36, 49, 50, 51, 130, 156

[83] Schoppa, W. and Hussain, F., A large-scale control strategy for drag reduction in turbulent boundary layers, Physics of Fluids (1994-present), Vol. 10, No. 5, pp. 1049-1051, 1998. 39,50

[84] Jiménez, J. and Pinelli, A., The autonomous cycle of near-wall turbulence, Journal of Fluid Mechanics, Vol. 389, pp. 335-359, 1999. 39, 50

[85] Luchik, T. and Tiederman, W., Timescale and structure of ejections and bursts in turbulent channel flows, Journal of Fluid Mechanics, Vol. 174, pp. 529-552, 1987. 40, 130

[86] García-Mayoral, R. and Jiménez, J., Drag reduction by riblets, Philosophical Transactions of the Royal Society of London A: Mathematical, Physical and Engineering Sciences, Vol. 369, No. 1940, pp. 1412-1427, 2011. 42

[87] Choi, H., Moin, P. and Kim, J., Direct numerical simulation of turbulent flow over riblets, Journal of fluid mechanics, Vol. 255, pp. 503-539, 1993. 43

[88] El-Samni, O., Chun, H. and Yoon, H., Drag reduction of turbulent flow over thin rectangular riblets, International Journal of Engineering Science, Vol. 45, No. 2, pp. 436-454, 2007. 43

[89] Stille, S., Schiek, M., Silex, W., Beck, T., van Waasen, S. and Singheiser, L., Novel Approach for Endurance Testing of Riblet-Structured Thin Sheets Under Realistic Loading Conditions in Active Drag-Reduction Systems, Journal of Testing and Evaluation, Vol. 45, No. 4, 2016. 50

[90] Leo, D. J., Engineering analysis of smart material systems, John Wiley and Sons, 2007. 55

[91] MFC-Macro Fiber Composite. 55, 58, 133 
[92] Shahab, S. and Erturk, A., Electrohydroelastic Euler-Bernoulli-Morison model for underwater resonant actuation of macro-fiber composite piezoelectric cantilevers, Smart Materials and Structures, Vol. 25, No. 10, pp. 105007, 2016. 60

[93] Bilgen, O., Aerodynamic and electromechanical design, modeling and implementation of piezocomposite airfoils, 2010. 73

[94] Bailey, S., Hultmark, M., Monty, J., Alfredsson, P. H., Chong, M., Duncan, R., Fransson, J., Hutchins, N., Marusic, I. and McKeon, B., Obtaining accurate mean velocity measurements in high Reynolds number turbulent boundary layers using Pitot tubes, Journal of Fluid Mechanics, Vol. 715, pp. 642, 2013. 80, 212

[95] Dengel, P. and Fernholz, H., An experimental investigation of an incompressible turbulent boundary layer in the vicinity of separation, Journal of Fluid Mechanics, Vol. 212, pp. 615636, 1990. 120

[96] Tarazaga, P. A., Johnson, M. E. and Inman, D. J., Experimental validation of the vibroacoustic model of a pressurized membrane, Mechanical Systems and Signal Processing, Vol. 45, No. 2, pp. 330-345, 2014. 127

[97] Paidoussis, M. P., Fluid-structure interactions: slender structures and axial flow, Vol. 1, Academic press, 1998. 128

[98] Fu, Y. and Price, W., Interactions between a partially or totally immersed vibrating cantilever plate and the surrounding fluid, Journal of Sound and Vibration, Vol. 118, No. 3, pp. 495-513, 1987. 128

[99] Choi, K., Yang, X., Clayton, B., Glover, E., Atlar, M., Semenov, B. and Kulik, V., Turbulent drag reduction using compliant surfaces, Proceedings of the Royal Society of London A: Mathematical, Physical and Engineering Sciences, Vol. 453, pp. 2229-2240, The Royal Society. 154

[100] Ricco, P. and Wu, S., On the effects of lateral wall oscillations on a turbulent boundary layer, Experimental Thermal and Fluid Science, Vol. 29, No. 1, pp. 41-52, 2004. 154

[101] McKeon, B., Li, J., Jiang, W., Morrison, J. and Smits, A., Pitot probe corrections in fully developed turbulent pipe flow, Measurement science and technology, Vol. 14, No. 8, pp. 1449, 2003. 212, 213

[102] Zagarola, M. V. and Smits, A. J., Mean-flow scaling of turbulent pipe flow, Journal of Fluid Mechanics, Vol. 373, pp. 33-79, 1998. 212 
[103] Figliola, R. S. and Beasley, D., Theory and design for mechanical measurements, John Wiley and Sons, 2015. 214, 215, 217

[104] Coleman, H. W. and Steele, W. G., Engineering application of experimental uncertainty analysis, AIAA journal, Vol. 33, No. 10, pp. 1888-1896, 1995. 217

[105] Couto, P. R. G., Damasceno, J. C. and de Oliveira, S. P., Monte Carlo simulations applied to uncertainty in measurement, InTech, 2013. 220, 221

[106] Cox, M. G. and Siebert, B. R., The use of a Monte Carlo method for evaluating uncertainty and expanded uncertainty, Metrologia, Vol. 43, No. 4, pp. S178, 2006. 220 
Appendices 


\section{Appendix A}

\section{Finite Element Model Information}

\section{A.1 Terminology in Finite Element Model}

Elements of the reduced stiffness matrix for a linear-elastic material under the plane-stress assumption

$$
\begin{aligned}
& C_{11}=C_{22}=\frac{E}{1-v^{2}} \\
& C_{12}=C_{21}=\frac{v E}{1-v^{2}} \\
& C_{44}=C_{55}=C_{66}=\frac{E}{2(1+v)}
\end{aligned}
$$

Based on this, $C_{b}$ and $C_{s}$ stiffness matrices, introduced by Equation 4.3, are defined as follows

$$
\begin{aligned}
C_{b} & =\left[\begin{array}{ccc}
C_{11} & C_{12} & 0 \\
C_{21} & C_{22} & 0 \\
0 & 0 & C_{66}
\end{array}\right] \\
C_{s} & =\left[\begin{array}{cc}
C_{44} & 0 \\
0 & C_{55}
\end{array}\right]
\end{aligned}
$$

For the formulation of mass matrices, the effective volumetric mass density of the composite plate-piezo element is defined in terms of the density of each component and its corresponding volume fraction $\left(V^{F}\right)$ as follows

$$
\bar{\rho}=\rho_{\text {Plate }} V_{\text {Plate }}^{F}+\rho_{\text {Piezo }} V_{\text {Piezo }}^{F}
$$


The shape functions matrices in Equation 4.6

$$
\begin{aligned}
N_{t} & =\left[\begin{array}{cccccc}
N_{1} & 0 & 0 & N_{2} & \cdots & 0 \\
0 & N_{1} & 0 & 0 & \cdots & 0 \\
0 & 0 & N_{1} & 0 & \cdots & N_{8}
\end{array}\right] \\
N_{r} & =\left[\begin{array}{ccccc}
N_{1} & 0 & N_{2} & \cdots & 0 \\
0 & N_{1} & 0 & \cdots & N_{8}
\end{array}\right] \\
N_{d} & =\left[\begin{array}{llll}
N_{1} & N_{2} & \cdots & N_{8}
\end{array}\right]
\end{aligned}
$$

where $N_{i}$ is the second order shape function associated with the $i^{t h}$ node.

Element matrices in Equation 4.9

$$
\begin{aligned}
& M_{t t}^{e}=\bar{\rho} \iiint_{\bar{V}} N_{t}^{T} N_{t} \mathrm{~d} V \\
& M_{r r}^{e}=\bar{\rho} \iiint_{\bar{V}} z^{2} N_{r}^{T} N_{r} \mathrm{~d} V \\
& K_{t t}^{e}=\iiint_{V_{\text {Plate }}}\left(B_{b t}{ }^{T} C_{b} B_{b t}+B_{s t}{ }^{T} C_{s} B_{s t}+\sigma_{x x}^{0} B_{t n x}{ }^{T} B_{t n x}+\sigma_{y y}^{0} B_{t n y}{ }^{T} B_{\text {tny }}\right) \mathrm{d} V+ \\
& \iiint_{V_{\text {Piezo }}}\left(B_{b t}{ }^{T} C_{b} B_{b t}+B_{s t}^{T} C_{s} B_{s t}\right) \mathrm{d} V \\
& K_{t r}^{e}=K_{r t}^{e T}=\iiint_{V_{\text {Plate }}} B_{s t}{ }^{T} C_{s} B_{s r} \mathrm{~d} V+\iiint_{V_{\text {Piezo }}} B_{s t}{ }^{T} C_{s} B_{s r} \mathrm{~d} V \\
& K_{r r}^{e}=\iiint_{V_{\text {Palte }}} z^{2}\left(B_{b r}{ }^{T} C_{b} B_{b r}+B_{s r}{ }^{T} C_{s} B_{s r}+\sigma_{x x}^{0} B_{r n x}{ }^{T} B_{r n x}+\sigma_{y y}^{0} B_{r n y}{ }^{T} B_{r n y}\right) \mathrm{d} V+ \\
& \iiint_{V_{\text {Piezo }}} z^{2}\left(B_{b r}{ }^{T} C_{b} B_{b r}+B_{s r}{ }^{T} C_{s} B_{s r}\right) \mathrm{d} V \\
& K_{d d}^{e}=\iiint_{V_{\text {Piezo }}} N_{d}^{T} \beta_{33}^{\varepsilon} N_{d} \mathrm{~d} V \\
& K_{t d}^{e}=\iiint_{V_{\text {Piezo }}} B_{b t}{ }^{T} h_{b} N_{d} \mathrm{~d} V \\
& F_{t}^{e}=\iint_{A} N_{t}^{T} f_{t} \mathrm{~d} A \\
& F_{r}^{e}=\iint_{A} N_{r}^{T} f_{r} \mathrm{~d} A
\end{aligned}
$$


where

$$
\begin{aligned}
& B_{b t}=\left[\begin{array}{ccc}
\partial / \partial x & 0 & 0 \\
0 & \partial / \partial y & 0 \\
\partial / \partial y & \partial / \partial x & 0
\end{array}\right] N_{t} \\
& B_{s t}=\left[\begin{array}{lll}
0 & 0 & \partial / \partial x \\
0 & 0 & \partial / \partial y
\end{array}\right] N_{t} \\
& B_{b r}=\left[\begin{array}{cc}
\partial / \partial x & 0 \\
0 & \partial / \partial y \\
\partial / \partial y & \partial / \partial x
\end{array}\right] N_{r} \\
& B_{s r}=N_{r} \\
& B_{t n x}=\left[\begin{array}{ccc}
\partial / \partial x & 0 & 0 \\
0 & \partial / \partial x & 0 \\
0 & 0 & \partial / \partial x
\end{array}\right] N_{t} \\
& B_{\text {tny }}=\left[\begin{array}{ccc}
\partial / \partial y & 0 & 0 \\
0 & \partial / \partial y & 0 \\
0 & 0 & \partial / \partial y
\end{array}\right] N_{t} \\
& B_{r n x}=\left[\begin{array}{cc}
\partial / \partial x & 0 \\
0 & \partial / \partial x
\end{array}\right] N_{r} \\
& B_{r n y}=\left[\begin{array}{cc}
\partial / \partial y & 0 \\
0 & \partial / \partial y
\end{array}\right] N_{r}
\end{aligned}
$$




\section{Appendix B}

\section{Wind Tunnel Correction Factors}

\section{B.1 Pitot Tube Correction Factors}

Since a pitot tube is inserted into the wind tunnel, it disrupts the fluid flow introducing errors into the pressures. To account for these errors, four different types of correction factors can be applied: viscous, shear flow, near-wall, and turbulence. Since the magnitude of each depends upon the particular flow, the magnitude of each effect changes with flow condition or position of the tube within the flow. For an in depth discussion see Chue [42]; however, both McKeon [101] and Bailey [94] also provide concise discussions.

The correction factor for the viscosity on the pitot tube is shown in Equation B.1 [102]. The measured dynamic pressure is $q$, the local velocity is $u$, the pressure coefficient is $C_{P}$, and $R e_{\text {probe }}$ is the Reynolds number determined using the probe diameter and the local velocity. Using the measured dynamic pressure, the probe Reynolds number can be used to adjust the velocity. The viscous correction should be applied within the range $30<R e_{\text {probe }}<100$. In the current research, when $R e_{\text {probe }}=100$ the correction adjusted the velocity by only $0.5 \%$. The majority of measured velocities fell outside of this $R e$ range; thus, the viscous effects were considered negligible here.

$$
C_{P}=\frac{q}{\frac{1}{2} \rho u^{2}}=1+\frac{10}{R e_{\text {probe }}^{1.5}}
$$

The shear flow pitot tube correction factor accounts for the error resulting from the deflection of the streamlines by a pitot tube in a velocity gradient. It is also known as the velocity gradient correction. This factor can be used to modify the measured velocity, but it is more commonly used to modify the effective vertical position of the pitot tube in the flow. This correction takes the form as shown in Equation B.2, where $\Delta y$ is the shift in position, $d_{p}$ is the pitot tube diameter, and $\varepsilon$ is a constant. A value of $\varepsilon=0.15$ was used, which was determined by MacMillan [43]. More complex 
correction factors dependent on the velocity gradient itself can also be applied [101].

$$
\Delta y=\varepsilon d_{p}
$$

When the pitot tube is very near the wall, the streamlines which typically deflect around the probe tip are blocked by the wall. This causes the flow to only deflect away from the wall. The method used to fix this is known as the near-wall correction and takes the form as shown in Equation B.3, where $u$ is the local mean velocity. This equation was also determined by MacMillian [43], and it was found to be of importance when the probe is within two probe diameters of the wall.

$$
\frac{\Delta u}{u}=0.015 \exp \left[-3.5\left(\frac{y}{d_{p}}-0.5\right)\right]
$$

The final pitot tube correction is for turbulent effects, where the fluctuating velocities affect both the total pressure measurements and the static ports [42]. As a result, the correction can be applied as shown in Equation B.4, where $u$ is the local mean velocity, and $u^{\prime}, v^{\prime}$, and $w^{\prime}$ are the $x, y$, and $z$ fluctuating velocities, respectively. The difficulty of applying this correction is that independent measures of the turbulent intensity are required. For the current case, no additional turbulence correction was implemented. As pointed out by McKeon [101], turbulence effects were not removed from the MacMillan velocity gradient correction. Thus a turbulence effect is already accounted for in the velocity gradient correction (Equation B.2), meaning an additional turbulent correction factor is not necessary.

$$
\Delta P=\frac{1}{2} \rho\left(u^{2}+\overline{u^{\prime 2}}+\overline{v^{\prime 2}}+\overline{w^{\prime 2}}\right)
$$




\section{Appendix C}

\section{Uncertainty Analysis}

This appendix provides a discussion of uncertainty and how it affects the quantities in this research. This is particularly important for Chapters 6 and 7 where boundary layer profiles are visually compared to establish differences. Thus, to conclusively interpret the results and visually distinguish any differences, the uncertainties in the boundary layer profile (velocity and wall-normal position, y) are necessary.

This appendix begins by providing a brief review of uncertainty theory and how it applies to the present research. This is not intended to be a comprehensive review; so for further discussion, the reader is directed to Figliola [103] or another of the considerable sources on the subject. Then, the uncertainties in the current research are discussed. The boundary layer profile is of particular importance, so the procedures for determining the uncertainties in the velocity and $y$ position are explained. In addition, the factors contributing to the uncertainty in the momentum thickness are presented.

\section{C.1 A Brief Review of Uncertainty Theory}

There are two different categories of uncertainty: systematic and random. Systematic error remains constant in repeated measurements under fixed operating conditions [103]. Two main examples of systematic error are the resolution on a sensor and the manufacturer provided accuracy on said sensor. On the other hand, random errors refer to errors in the measured value due to statistics. Time variant random error results from the variation in multiple values measured at the same location. This error is defined as the standard deviation of the means and is shown in Equation C.1, where $S_{x}$ is the standard deviation in some quantity $x$ and $N$ is the number of $x$ values. In addition to time variant error, random error also arises from spatial variance. This results from measuring a quantity at different locations where the value should nominally be the same. The spatially variant 
random error is also quantified using the standard deviation of the means (Equation C.1).

$$
P=\frac{S_{x}}{\sqrt{N}}
$$

Since the random error is based upon the standard deviation of the data, it depends upon a Gaussian distribution. Within a Gaussian distribution, one standard deviation contains $68 \%$ of the possible values. Thus, if the random error is taken as shown in Equation C.1, the confidence level of the result is $68 \%$, but the general confidence level used in most engineering application is 95\%. A 95\% confidence interval corresponds to roughly two standard deviations in infinite statistics, meaning that multiplying the random error by two would give the error to $95 \%$ confidence. However, since these values are measured, finite statistics are required and the number of samples (degrees of freedom) measured changes the multiplication factor needed to achieve a confidence level. The random uncertainty in a value due to a single random error is shown in Equation C.2, where $u_{P}$ is the random uncertainty, $t_{v, 95}$ is the Student-t value for $v$ degrees of freedom at $95 \%$ confidence, and $P$ is the random error.

$$
u_{P}= \pm t_{v, 95} P
$$

A single quantity, can have multiple sources of systematic or random error; thus, multiple errors are combined. This is done via the root-sum-squares (RSS) method. Instead of simply summing the errors, it is assumed that all errors will not occur in their worst possible way for measurements [103]. As a result, the total systematic error for a quantity is described by Equation C.3, where each $B_{i}$ is a different systematic error and there are $\mathrm{K}$ in total. The total random error is described in a similar manner as shown in Equation C.4.

$$
\begin{aligned}
& B=\left(B_{1}^{2}+B_{2}^{2}+\ldots+B_{K}^{2}\right)^{1 / 2} \\
& P=\left(P_{1}^{2}+P_{2}^{2}+\ldots+P_{K}^{2}\right)^{1 / 2}
\end{aligned}
$$

The total uncertainty in a quantity is the combination of the total systematic and random errors (Equations C.3 and C.4). The total uncertainty in a quantity $x$ is shown in Equation C.5 where $u_{x}$ is the uncertainty and the random error is modified by the t-value with degrees of freedom, $v$. Important to note here is that the total random error is composed of multiple random errors (Equation C.4) which each may have different degrees of freedom. In this case, the degrees of freedom used for the t-value in Equation C.5 is determined via the Welch-Satterthwaite formula shown in Equation C.6, where $P_{i}$ and $v_{i}$ are the individual random errors and their degrees of 
freedom, respectively.

$$
\begin{gathered}
u_{x}=\left(B^{2}+\left(t_{v, 95} P\right)^{2}\right)^{1 / 2} \\
v=\frac{\left[\sum_{i=1}^{K} P_{i}^{2}\right]^{2}}{\sum_{i=1}^{K}\left(P_{i}^{4} / v_{i}\right)}
\end{gathered}
$$

When calculating a quantity using measured values, the uncertainty of the independent variables transfer to the dependent quantity. So, the uncertainty of the variable, $w$, in Equation C.7 is dependent on the uncertainties of $\left(x_{1} \ldots x_{N}\right)$. These uncertainties propagate as shown in Equation C.8, where $P_{w}$ is the random error in $w$ and $P_{x, i}$ are the random errors in $x_{i}$. This same procedure is applied to calculate the systematic error, $B_{w}$. The total uncertainty, $u_{w}$, would then be found using Equation C.5. An example of the uncertainty propagation in the wind tunnel is when calculating the density, $\rho$. The density is given in Equation C.9, where $P_{a t m}$ is the atmospheric pressure, $R$ is the ideal gas constant, and $T$ is the temperature. $R$ is a constant and is assumed to have zero error; however, both the pressure and temperature contribute to the total error in the density. Thus, the systematic and random density errors $\left(B_{\rho}\right.$ and $\left.P_{\rho}\right)$ are defined in Equations C.10 and C.11, respectively.

$$
\begin{gathered}
w=w\left(x_{1}, x_{2}, \ldots x_{N}\right) \\
P_{w}=\left[\sum_{i=1}^{N}\left(\frac{\partial w}{\partial x_{i}} P_{x, i}\right)^{2}\right]^{1 / 2} \\
\rho=\rho P_{a t m}, T=\frac{P_{a t m}}{R T} \\
B_{\rho}=\left[\left(\frac{\partial \rho}{\partial P_{a t m}} B_{P, a t m}\right)^{2}+\left(\frac{\partial \rho}{\partial T} B_{T}\right)^{2}\right]^{1 / 2} \\
P_{\rho}=\left[\left(\frac{\partial \rho}{\partial P_{\text {atm }}} P_{P, a t m}\right)^{2}+\left(\frac{\partial \rho}{\partial T} P_{T}\right)^{2}\right]^{1 / 2}
\end{gathered}
$$

These above equations for uncertainty propagation assume that the errors in the various quantities are independent of one another. This is indeed the case for the density, since the errors in the atmospheric pressure and temperature and are not correlated. In some cases, the errors are correlated with one another. An example of this is the momentum thickness (Section C.2.3), which numerically integrates over all $y$ positions in the boundary layer. All the $y$ positions share the same 
systematic error, so the uncertainties are correlated; random errors cannot be correlated [103]. To account for this, the uncertainty propagation is updated to the form shown in Equation C.12. The second term in the equation cycles through the variables that are correlated and adds their contribution to the total systematic error. Coleman [104] provides a more thorough discussion of correlated uncertainty.

$$
B_{w}=\left[\sum_{i=1}^{N}\left(\frac{\partial w}{\partial x_{i}} B_{x, i}\right)^{2}+\sum_{i=1}^{N-1} \sum_{j=i+1}^{N} \frac{\partial w}{\partial x_{i}} \frac{\partial w}{\partial x_{j}} B_{x, i} B_{x, j}\right]^{1 / 2}
$$

As mentioned, the total uncertainty for the propagated error is the same as in Equation C.5; except for the degrees of freedom (DOFs). The degrees of freedom of the total random error, $v$, is dependent upon the DOFs of the individual random errors (DOFs of $P_{a t m}$ and $T$ here). Similar to Equation C.6, the $v$ value for use in the propagation of uncertainty is calculated using Equation C.13, where $x_{i}$ are the independent variables ( $P_{a t m}$ and $T$ for density). If the DOFs of each independent variable are large $(N \geq 30)$, then it is reasonable to take $t_{v, 95}=2$ and Equation C.13 need not be implemented [103].

$$
v_{\rho}=\frac{\left[\sum_{i=1}^{K}\left(\frac{\partial \rho}{\partial x_{i}} P_{x, i}\right)^{2}\right]^{2}}{\sum_{i=1}^{K}\left[\left(\frac{\partial \rho}{\partial x_{i}} P_{x, i}\right)^{4} / v_{x, i}\right]}
$$

The final point to mention is how the uncertainty propagates when taking the mean of a quantity. Each of the values to be averaged has an uncertainty; thus, the mean has an uncertainty that is dependent upon the uncertainty of each term included in the average. If the values being averaged were collected at different physical locations, an additional term is required to quantify the spatial uncertainty. As before, the systematic and random errors should be kept separate until they are combined to find a total uncertainty for a quantity. The density can be once again used to illustrate this point. The mean density is defined as shown in Equation C.14, where $\bar{\rho}$ is the mean density, $\rho_{i}$ are densities at different positions, and $K$ is the number of positions. Each density, $\rho_{i}$, has a systematic and random error, which will then all contribute to the uncertainty of the mean density. Equations C.15 and C.16 show the total systematic and random errors in the mean density, where $B_{\rho_{i}}$ and $P_{\rho_{i}}$ are the systematic and random errors of each density at the different positions. This random error here defines the variation with time, represented by the subscript $t$.

$$
\bar{\rho}=\frac{1}{K} \sum_{i=1}^{K} \rho_{i}
$$




$$
\begin{aligned}
& B_{\bar{\rho}}=\left[\frac{1}{K^{2}} \sum_{i=1}^{K} B_{\rho_{i}}^{2}\right]^{1 / 2} \\
& P_{\bar{\rho}, t}=\left[\frac{1}{K^{2}} \sum_{i=1}^{K} P_{\rho_{i}}^{2}\right]^{1 / 2}
\end{aligned}
$$

In addition to the temporal random error, there is a spatial random error. This would be represented by $P_{\bar{\rho}, s}$, and is the same form as Equation C.1. The total random error would then be the RSS of the temporal and spatial random errors (Eqn C.4). The total uncertainty of the mean density is found by using Eqn C.5 to combine the systematic error (Eqn C.15) and the total random error. In the case of the density, there actually is no spatial error since the temperature and atmospheric pressure are not spatially dependent. However, when taking the mean of the free-stream velocity and momentum thickness the spatial random error must be accounted for.

\section{C.2 Uncertainty Calculations}

This section presents the calculation of uncertainties for the main variables of interest in this research. The main quantity of interest is the turbulent boundary layer profile, which is the flow velocity as a function of distance from the surface, $y$. The factors contributing to the uncertainties in both the velocity and $y$ position are discussed. The uncertainties of a representative turbulent boundary layer are then presented. When analyzing turbulent boundary layers, another important variable is the momentum thickness. So, this section also presents the procedure for calculating the uncertainty in the momentum thickness.

\section{C.2.1 Velocity}

As discussed in Section 5.1.4.3, the flow velocity is measured using hot-wire anemometry. The hot-wire probe outputs a voltage signal that is converted to flow velocity via a calibration curve. As a result, the uncertainty in the flow velocity stems almost entirely from the uncertainty in the hot-wire calibration. While there is some error in the voltage signal, it is minor in comparison to that from the calibration. Thus, to determine the uncertainty in the velocity, the errors must be propagated through the calibration procedure.

The hot-wire calibration is conducted by simultaneously measuring the free-stream velocity and the voltage signal from the hot-wire anemometer. The wind tunnel is cycled through various speeds to generate data at a range of free-stream velocities. A power law curve known as King's law is then fit to this data. This is the calibration curve is used during experimental testing to 
convert hot-wire voltages to flow velocity. A flow chart of this procedure is shown in Figure C.1. When conducting the hot-wire calibration, four separate quantities are measured: static pressure, temperature, atmospheric pressure, and hot-wire voltage. The atmospheric pressure is actually collected from local weather station. So, the error in all four of these quantities will contribute to the uncertainty in the calibration.

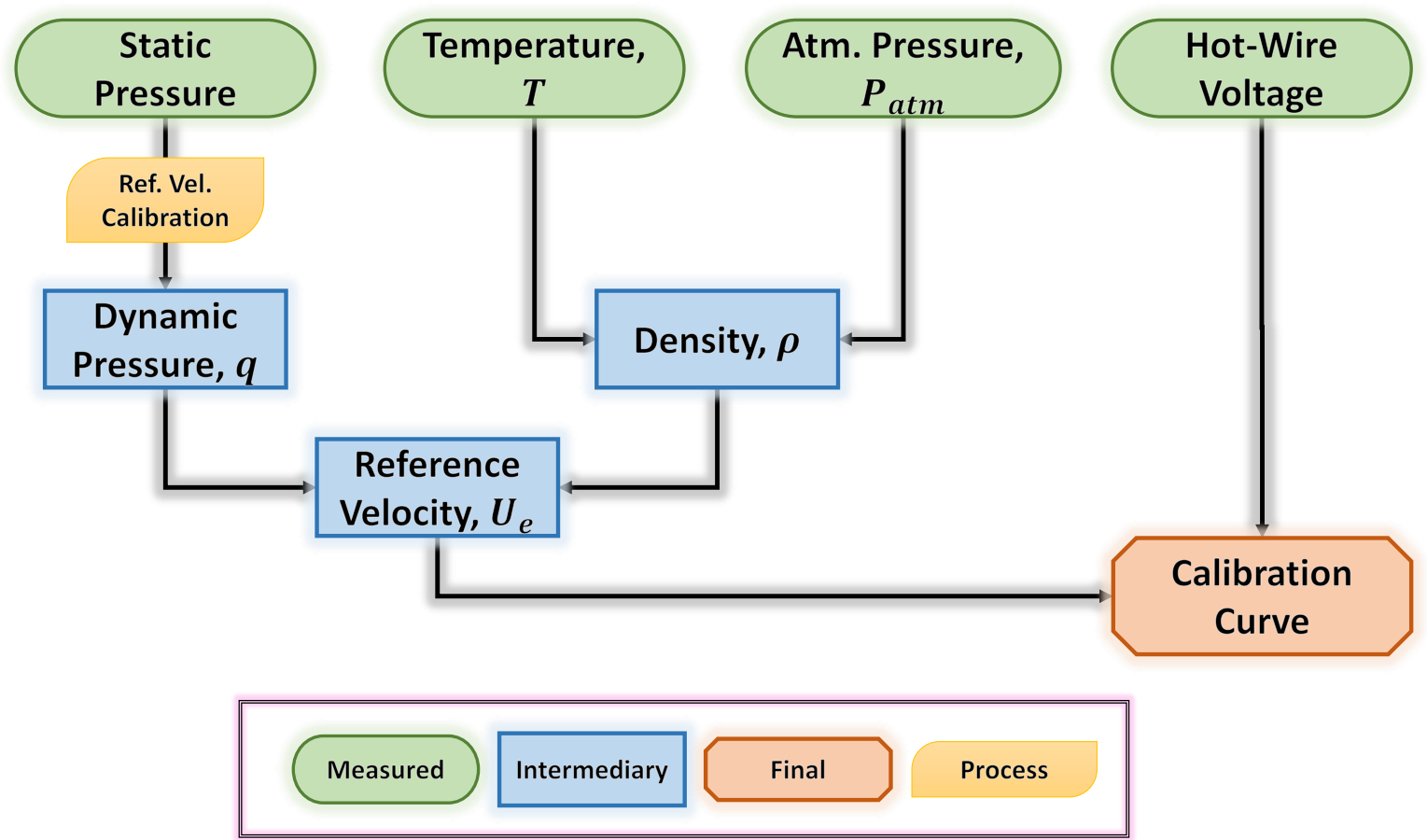

Figure C.1: Flow chart detailing the procedure for conducting the hot-wire calibration. The top row are measured quantities and the output is the calibration curve

The static pressure is used to measure the free-stream velocity in the tunnel. As described in Section 5.1.3.4, a reference velocity calibration is conducted to relate the static and dynamic pressure. The data is fit with a linear line and the static pressure can be mapped to the dynamic pressure using Equation C.17, where $q$ is the dynamic pressure, $P$ is the static pressure, and $a_{1}$ and $a_{0}$ are the slope and intercept. Each of these terms in the equation has error bounds. The static pressure has systematic errors from the pressure transducers and random errors from the data collection. While, the slope and intercept have error bounds from the linear fit, which can be determined from the least-squares fitting procedure. The uncertainty in the dynamic pressure is then calculated by applying Equation C. 8 to the linear fit and propagating the errors through.

$$
q=a_{1} P+a_{0}
$$

The velocity is calculated by using the dynamic pressure, $q$, and the density, $\rho$, in Equation 
C.18. As shown in Figure C.1, the density is dependent on the temperature, $T$, and the atmospheric pressure, $P_{\text {atm }}$, in the form shown in Equation C.9. The temperature has systematic errors from the thermocouple and also a random error from the data collection. The atmospheric pressure has systemic error relating to the precision of the measurement, but no random error since the value was taken from a local weather station. Using all of these errors, the uncertainty in the velocity is calculated. The temperature and atmospheric pressure errors are propagated through the density equation. Then the density and dynamic pressure errors are propagated through to the flow velocity.

$$
U_{e}=\sqrt{\frac{2 q}{\rho}}
$$

The calibration curve between the flow velocity and the hot-wire voltage signal can then be determined. Figure C. 2 shows an example case with the voltage plotted versus the velocity. For each data point, the $95 \%$ uncertainty in both the voltage and velocity is given. The first thing to point out is that the error in the voltage signal is much less than that from the velocity. This is because the velocity has numerous sources contributing to the overall uncertainty. Next, the uncertainty in the velocity is significantly larger at smaller velocities. One of the major error sources is the static pressure measurement, and in particular the systematic errors in the pressure transducers. The errors in the transducers are constant at all pressures. Thus, the percent error is larger for lower pressures (i.e. velocities). In addition, this already lop-sided error distribution is amplified as the uncertainty propagates through the equations.

Also shown in Figure C.2 is the calibration curve between the voltage and velocity. As discussed in Section 5.1.4.3, this power law curve is used to map experimentally measured hot-wire voltages to the flow velocity. The curve shown in Figure C.2 provides a strong fit to the data; however, it does not account for the errors in the velocity and voltage. Thus, when the curve is used to convert a voltage to a velocity, there must be an uncertainty on this velocity. Unfortunately, there is no straight-forward procedure for propagating the uncertainties through the calibration curve. The power law curve was fit to the data using a non-linear least squares fit, meaning the propagation techniques discussed in Section C.1 cannot be used. As a result, the uncertainty is propagated through using a Monte-Carlo simulation.

The Monte-Carlo method for uncertainty propagation involves using the distribution of the input data to generate new sets of data and then propagate that through the process to determine the output distribution $[105,106]$. In this case, the input are the data points in Figure C.2, and the output is the calibration curve. Each of the velocity and voltage values in the figure has a distribution, which is just the uncertainty. The error bars in the figure are the total uncertainties to $95 \%$ confidence. These were found by combining the random and systematic errors for each value. The random error follows a Gaussian distribution, while the systematic follows a uniform 


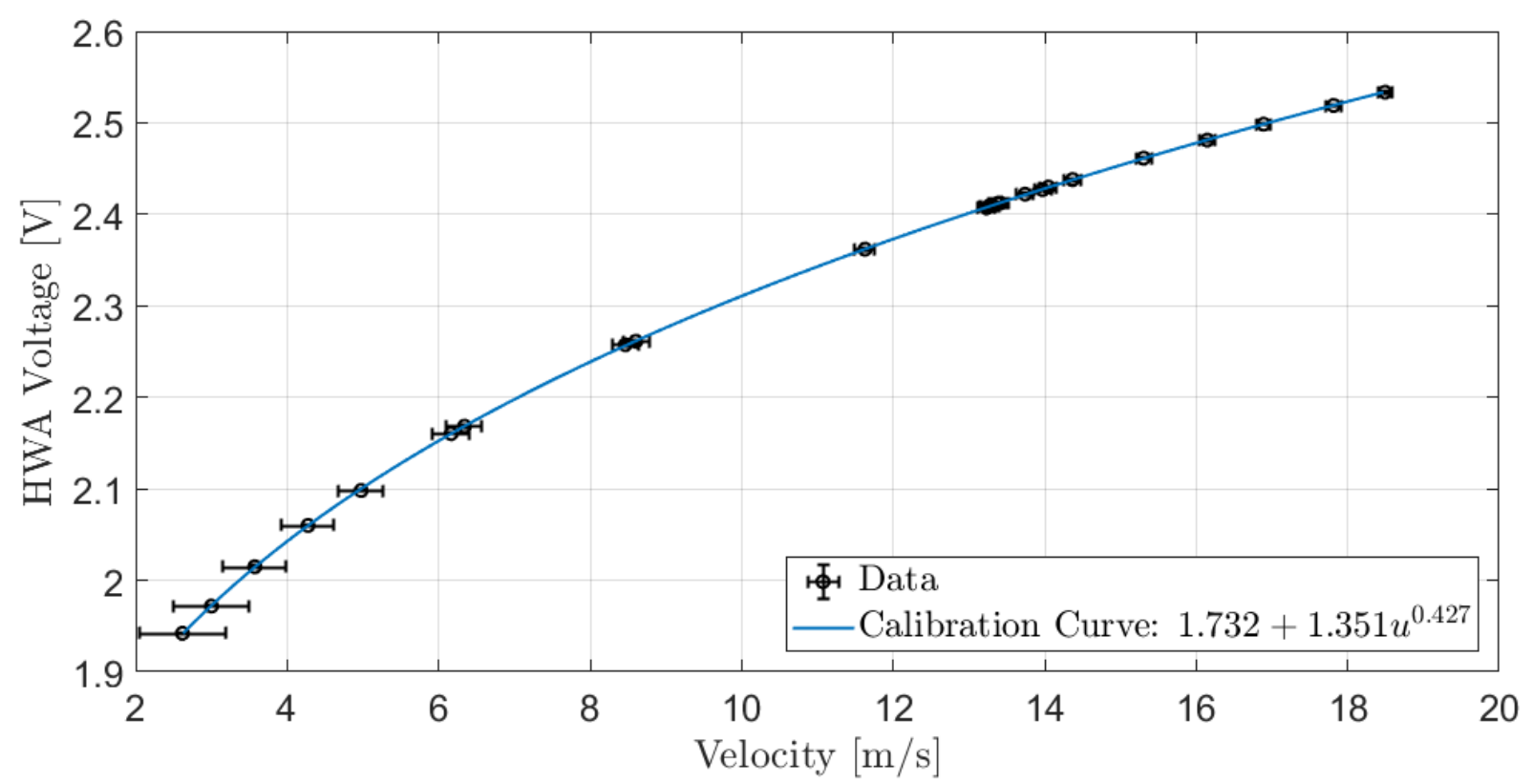

Figure C.2: Hot-wire anemometer (HWA) calibration data showing the voltage vs. velocity with the error on each data point. The calibration curve fit to the data is also shown

distribution. Thus, new data points can be generated using the error distribution around the each of the points in Figure C.2. There are 26 points in the figure, so a new set of calibration data involves generating 26 new points, each about one of the points in the figure. A power law curve is then fit to the new data set, which will be different from the initial calibration curve. This process is repeated a large number of times, yielding a distribution in the calibration curves. The uncertainty in the velocity is determined from the spread of these curves.

To provide a reasonable representation of the distribution in the calibration curves, 200,000 data sets are generated. This was chosen to provide $95 \%$ coverage probability [105]. Each of these data sets of 26 points is then fit with a power law curve. Figure C.3 shows the initial data and calibration curve along with the curves from three of the 200,000 data sets. At lower velocities, there is greater separation between the curves, which agrees with the larger uncertainty in the velocities. From these 200,000 calibration curves, there is in envelope in which all curves fall. Thus, the envelope describes the boundaries in which all possible velocities from the calibration curve will lie. The edges of this envelope are then used to define the uncertainty in the calibration curve.

Figure C. 4 shows the error bounds on the calibration curve fit through the original data. This curve is the 95\% confidence interval on the envelope bounding all the Monte-Carlo calibration curves. So, when a voltage is mapped to a flow velocity, the uncertainty on that velocity is defined by the $95 \%$ envelope at that location. Interestingly, at lower velocities the $95 \%$ confidence envelope 
is smaller than the error on each individual data point. This is because the curve is a fit of all the data points, so the uncertainty at the lower velocities is positively affected by the smaller uncertainties elsewhere. At higher velocities, the uncertainty from the calibration curve is actually larger than the uncertainty in the calibration data. In Figure C.4 at $u \approx 18.2 \mathrm{~m} / \mathrm{s}$, the $95 \%$ confidence envelope is larger than the error bounds for the data point; it is difficult to see since the calibration curve starts to level off. Thus, the larger uncertainty at the lower velocities is increasing the uncertainty at higher positions.

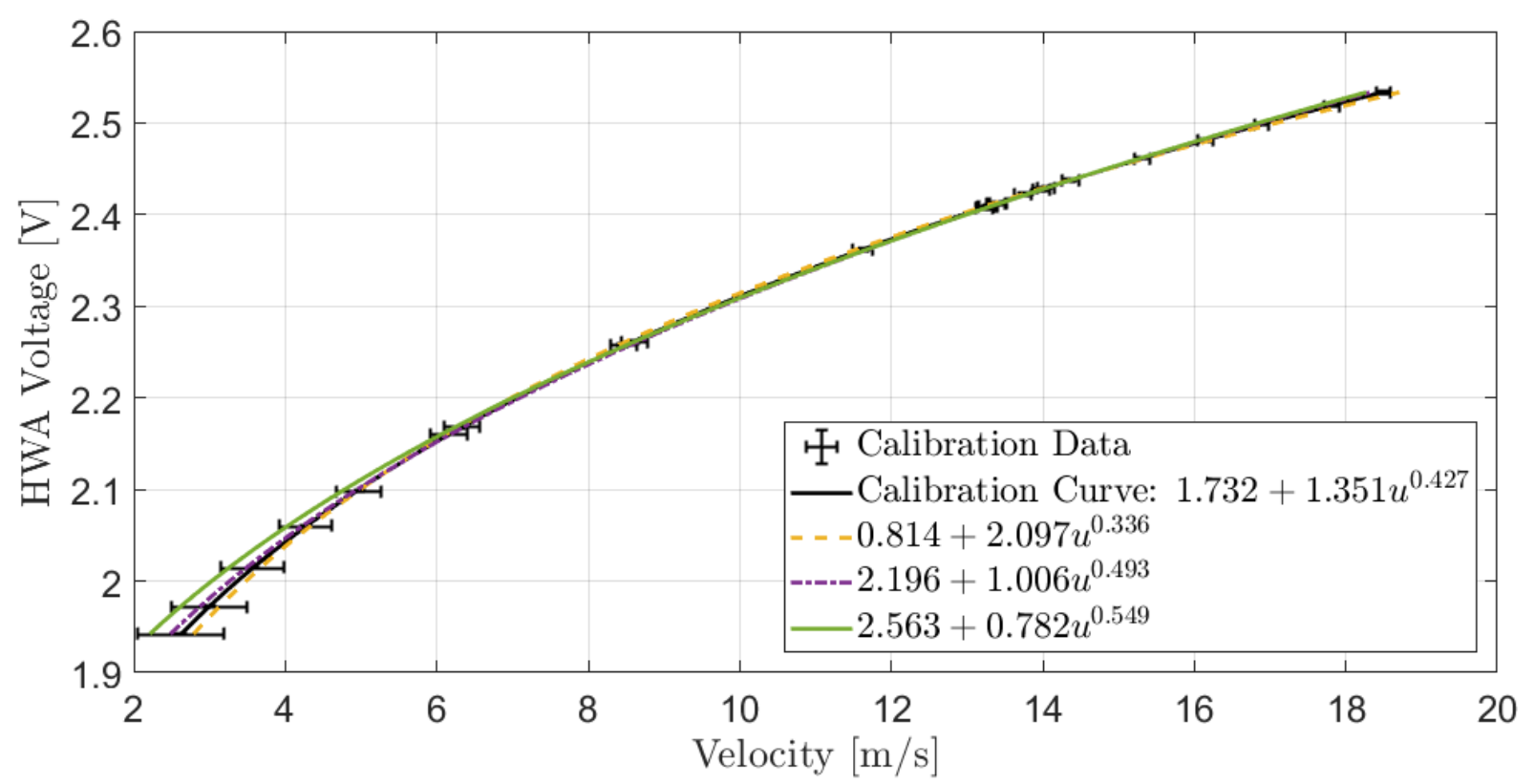

Figure C.3: Example calibration curves generated for the Monte-Carlo simulation along with the actual data and the main calibration curve

Figure C.5 shows turbulent boundary layer profiles measured over an unactuated and actuated surface. The uncertainties of both the velocity and $y$ position are shown in the figure; Section C.2.2 discusses the $y$ uncertainty. The velocity error is a minimum where the profile meets the log-law $\left(y^{+} \approx 70\right)$, but increases to either side. This corresponds with that seen in the calibration curve (Figure C.4) where the uncertainty was largest close to and far from the wall. The uncertainties are the same for both the unactuated and actuated profiles since the error sources are the same for each.

\section{C.2.2 Y Position}

The uncertainty in the $y$ position primarily stems from the error in the absolute $y$ position with respect to the surface. There is some uncertainty in the relative $y$ position as the linear stages 


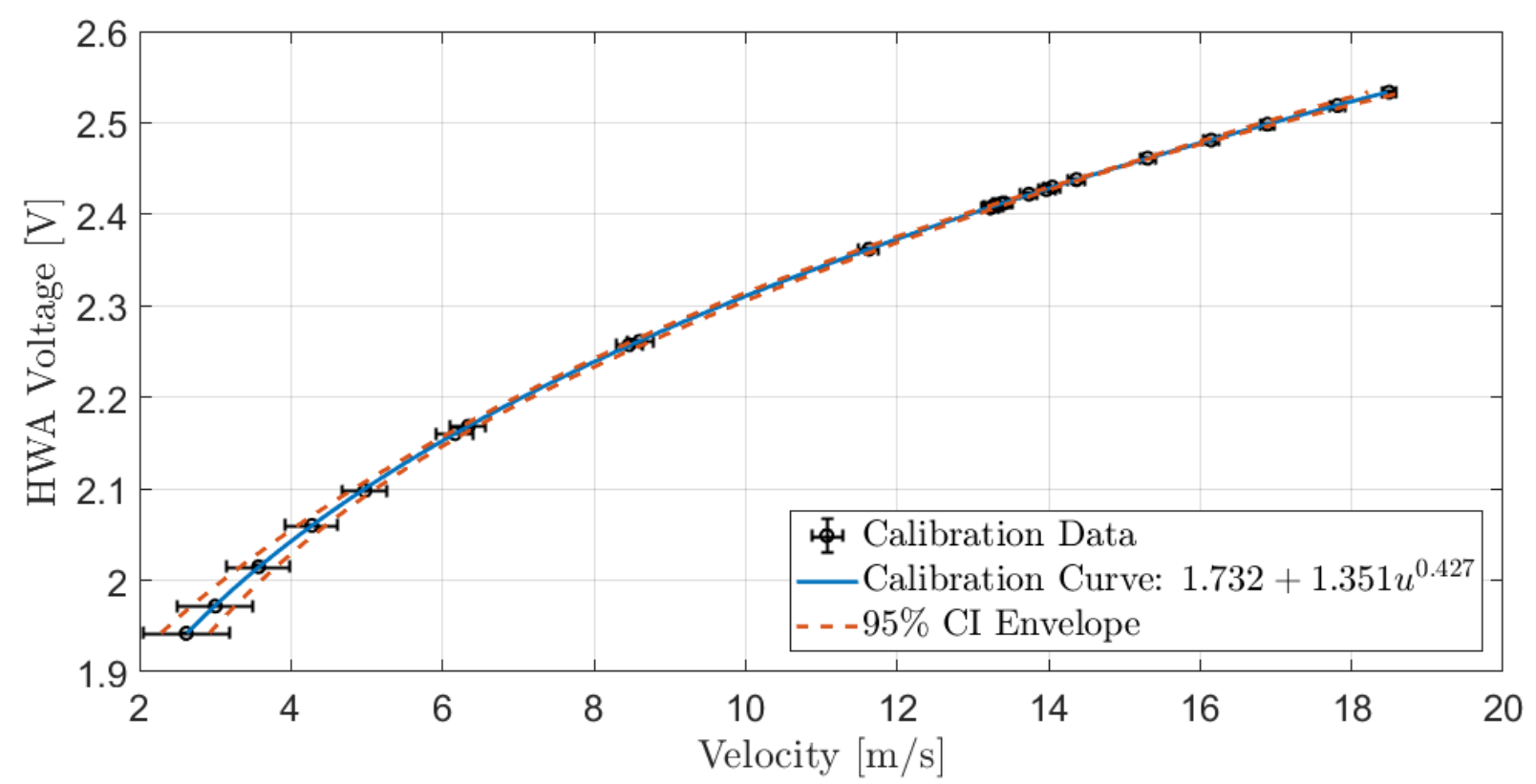

Figure C.4: 95\% confidence interval error bounds around the calibration curve determined by the Monte-Carlo simulation

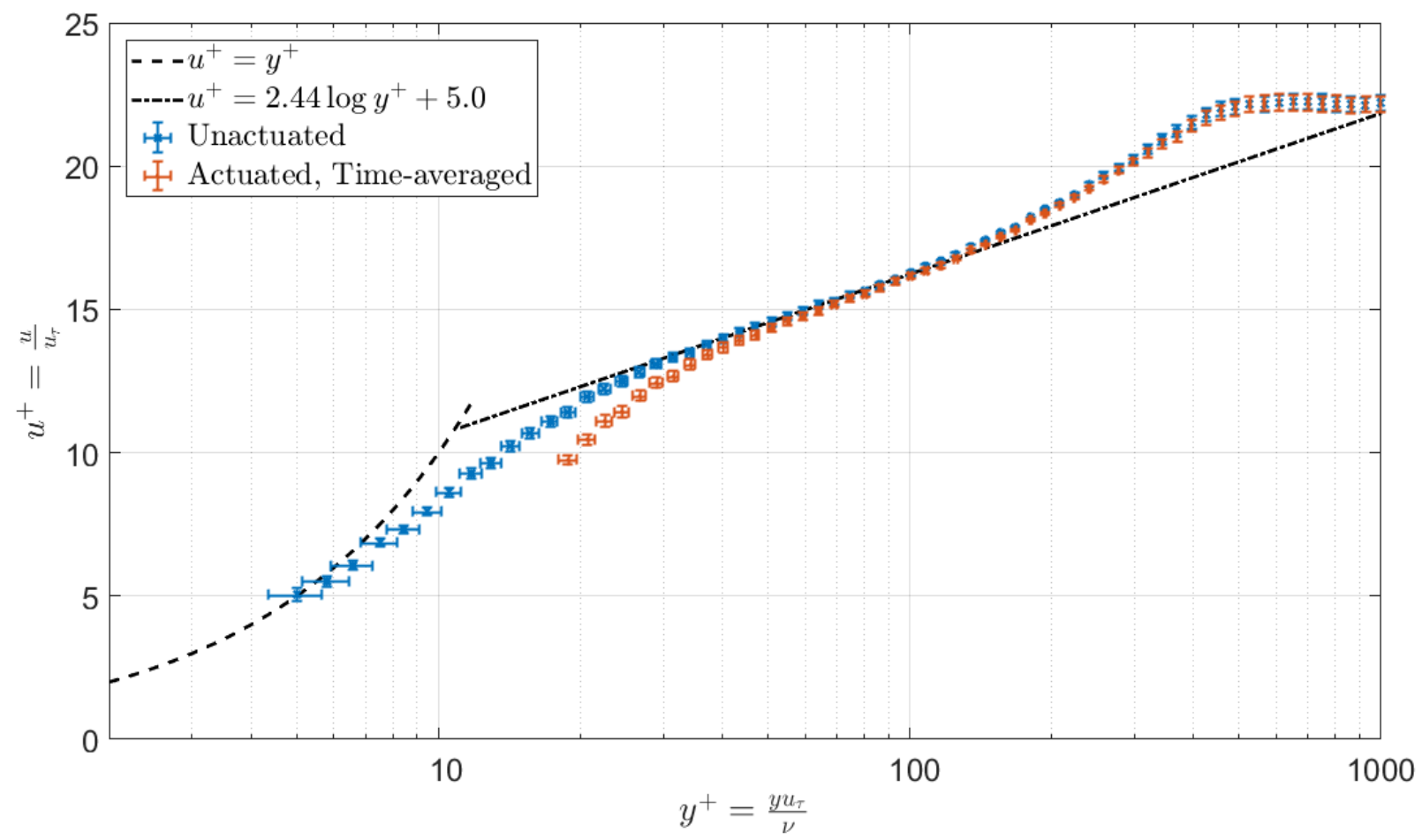

Figure C.5: Turbulent boundary layer over the unactuated and actuated surface with the velocity and $y$ position uncertainties shown. 
translate the hot-wire probe (Section 5.1.4.1, however, these are small when compared to the error in the absolute $y$ position. As discussed in Section 5.1.4.4, significant effort was spent in accurately placing the probe near the wall and finding the $y$ position. The lowest error occurs when the hotwire probe is touching the surface, then the error is related to the position of the sensor wire on the probe face. So when the probe is touching the surface, the $y$ position is $y=0.0025 \pm 0.0025 \mathrm{in}$ where the probe face is $0.005 \operatorname{in}(127 \mu \mathrm{m})$ tall. In post-processing, the $y$ positions were manually adjusted to reduce the error (Section 5.2.3). The boundary layer profile was adjusted so that it passed through $u^{+}=y^{+}$. As a result, the uncertainty on the $y$ positions is $\pm 0.000,5 \operatorname{in}(12.7 \mu m)$.

When the boundary layer is measured directly of the oscillating traveling wave surface, the $y$ positions are adjusted by the displacement of the surface (Section 6.2.2). The wall displacement is experimentally measured, but there is uncertainty in the values, primarily due to random errors. For the case shown in Figure C.5, time-averaged actuated profile was shifted by $y=-6.4 \pm 10.5 \mu m\left(y^{+}=-0.33 \pm 0.54\right)$ to account for the mean displacement of the surface. Prior to the shift, the $y$ uncertainty was $\pm 0.000, \sin (12.7 \mu \mathrm{m})$. So, the two uncertainties are combined using the methods discussed in Section C.1 and the resultant uncertainty for the actuated $y$ positions is $\pm 16.5 \mu m$ ( \pm 0.85 in wall units).

The total $y$ uncertainty on the boundary layer profile is shown in Figure C.5. The $y$ uncertainty appears to increase closer to the wall (smaller $y$ positions). The $y$ uncertainty is actually constant for all positions in the profile, but it appears to increase since the $y$ positions are plotted on a logarithmic scale. While $y$ uncertainty does remain constant, the percent uncertainty is larger for the smaller $y$ positions. Comparing the unactauted and actuated profiles, the $y$ uncertainty is larger for the actuated case (at the same $y$ value) due to the error in accounting for the displacement of the surface.

\section{C.2.3 Momentum Thickness}

The momentum thickness, $\theta$, was introduced in Section 5.2.4. The analytic equation integrates over the boundary layer profile, but experimentally it is calculated using the form shown in Equation C.19, which is the numerical sum over the profile. $y_{j}$ are the $y$ positions in the boundary layer profile, $u_{j}$ are the local velocities over the profile, and $U_{e}$ is the free-stream velocity. The uncertainty in $\theta$ can be calculated using the propagation methods discussed in Section C.1, but the process is more complicated. The momentum thickness is dependent on all the variables shown in Equation C.20, so the uncertainty from each term must be propagated through. In addition, all of the $y$ values have correlated errors, since the systematic errors arise from the same source. Thus, the correlated uncertainty propagation presented in Equation C.12 is used to find the total 
systematic error.

$$
\begin{gathered}
\theta=\frac{1}{2} \sum_{j=1}^{N-1}\left(y_{j+1}-y_{j}\right)\left[\frac{u_{j}}{U_{e}}\left(1-\frac{u_{j}}{U_{e}}\right)+\frac{u_{j+1}}{U_{e}}\left(1-\frac{u_{j+1}}{U_{e}}\right)\right] \\
\theta=\theta\left(y_{1}, \ldots, y_{N} ; u_{1}, \ldots, u_{N} ; U_{e}\right)
\end{gathered}
$$




\section{Appendix D}

\section{Additional Traveling and Standing Wave Results}

\section{D.1 607Hz Traveling Wave}

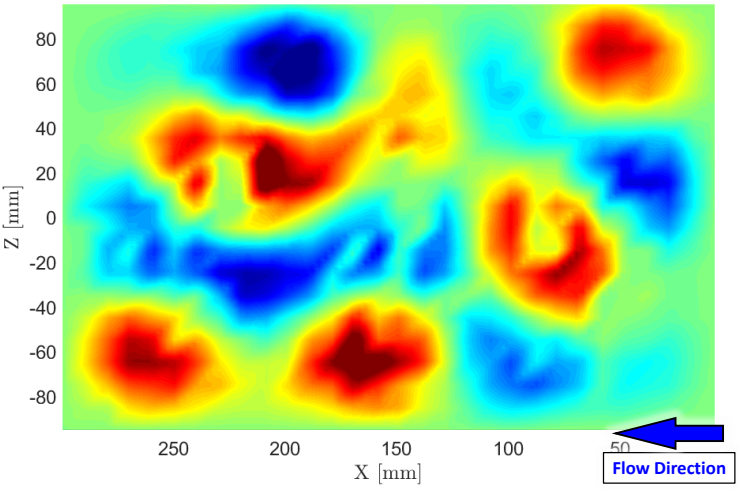

(a)

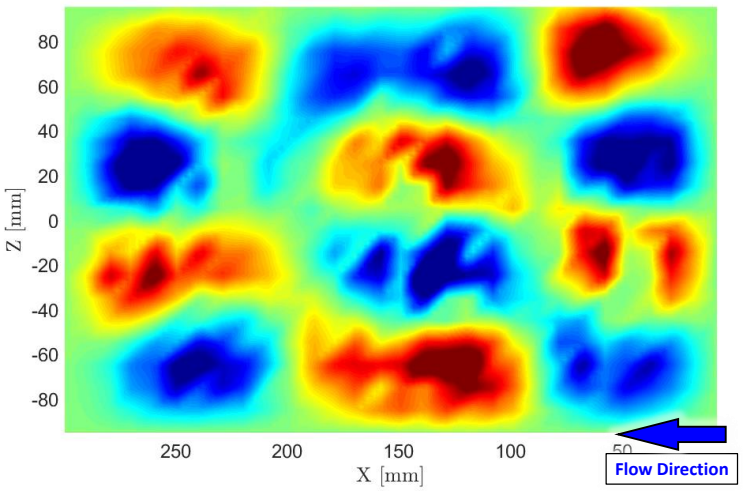

(b)

Figure D.1: Operational deflection shapes (ODSs) showing the (a) $(2,4)$ mode at $f=579 \mathrm{~Hz}$, $T^{+}=82$ and (b) the $(3,4)$ mode at $f=630 \mathrm{~Hz}, T^{+}=74$. These are the participating mode shapes in the $607 \mathrm{~Hz}\left(T^{+}=78\right)$ traveling wave 
$\mathrm{T} / 6$

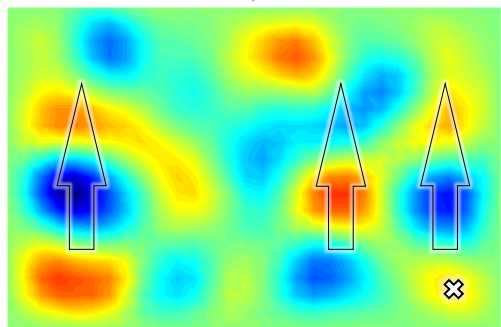

$4 \mathrm{~T} / 6$

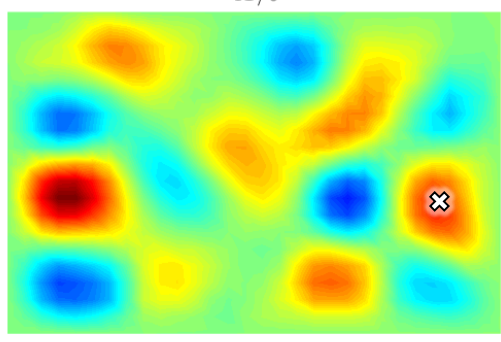

$2 \mathrm{~T} / 6$

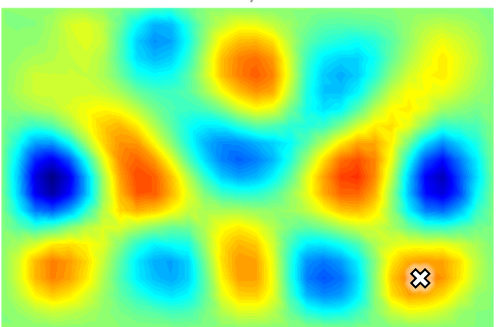

$5 \mathrm{~T} / 6$

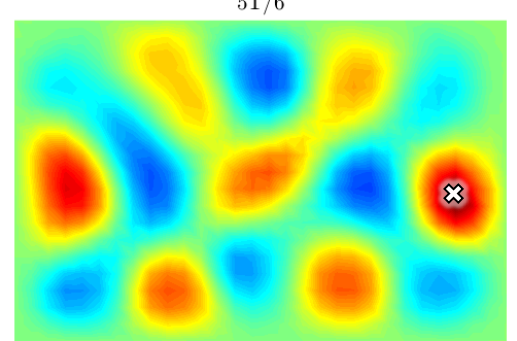

$3 \mathrm{~T} / 6$
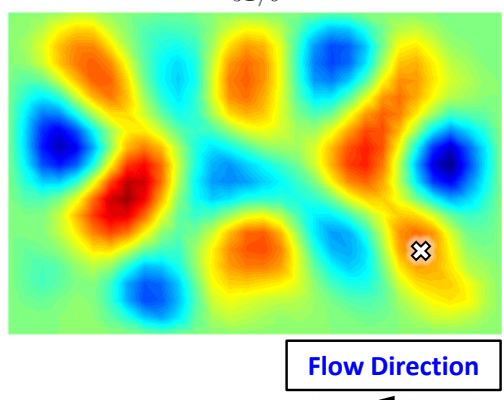

$\mathrm{T}$

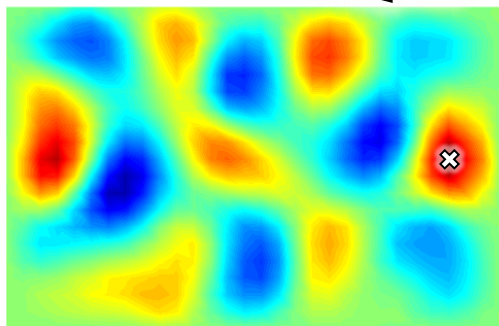

Figure D.2: Instantaneous velocities of the $607 \mathrm{~Hz}\left(T^{+}=78\right)$ traveling wave at six different time steps in the period $T$ of the wave. There are three wavefronts propagating in the spanwise direction. The propagation direction of these are shown in the first time-step. The $\times$ symbol follows the right wavefront traveling in the $+z$ direction.

\section{D.2 $810 \mathrm{~Hz}$ Traveling Wave}

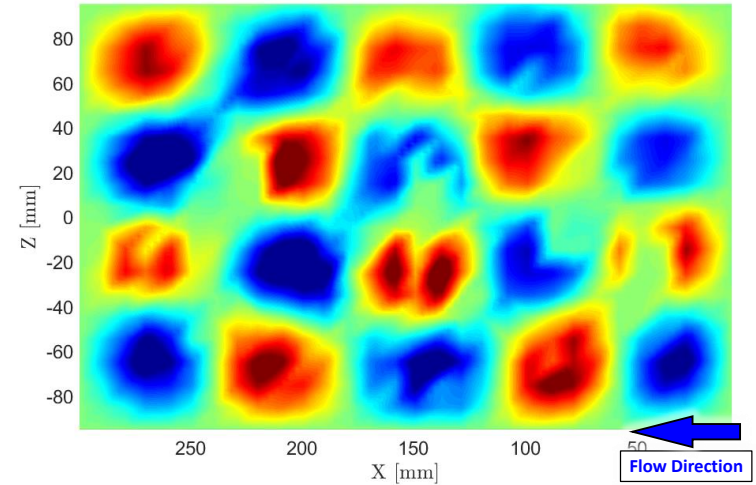

(a)

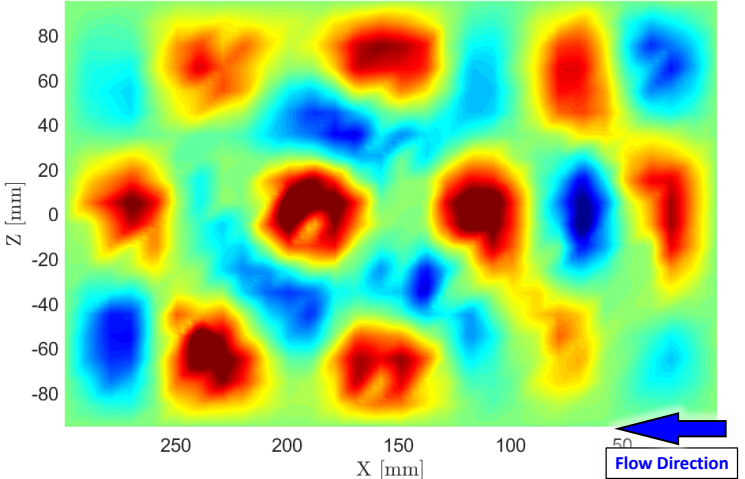

(b)

Figure D.3: Operational deflection shapes (ODSs) showing the (a) $(5,4)$ mode at $f=792.2 \mathrm{~Hz}$, $T^{+}=60$ and (b) the $(7,3)$ mode at $f=868.8 \mathrm{~Hz}, T^{+}=55$. These are the participating mode shapes in the $810 \mathrm{~Hz}\left(T^{+}=59\right)$ traveling wave 
$\mathrm{T} / 6$

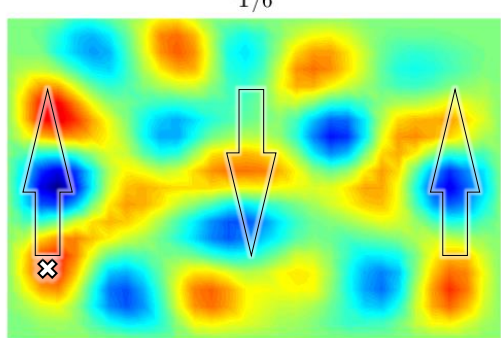

$4 \mathrm{~T} / 6$

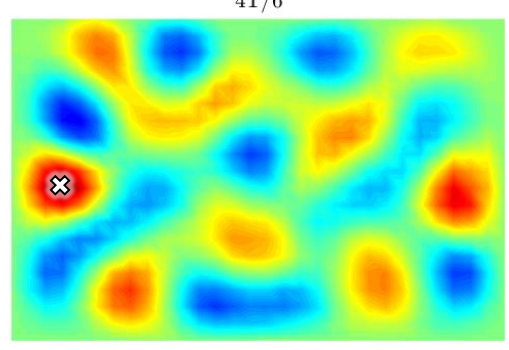

$2 \mathrm{~T} / 6$

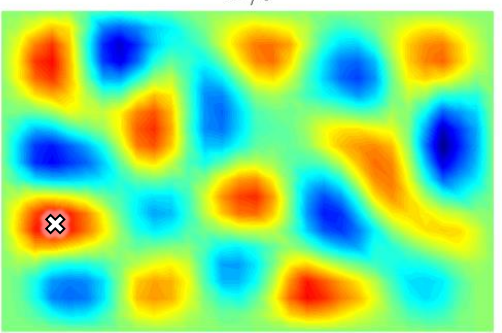

$5 \mathrm{~T} / 6$

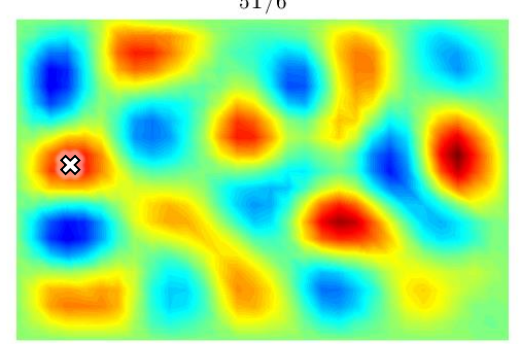

$3 \mathrm{~T} / 6$

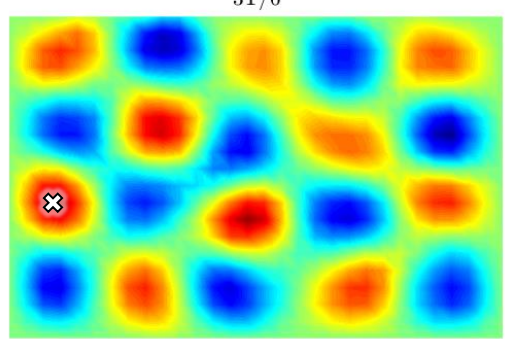

Flow Direction

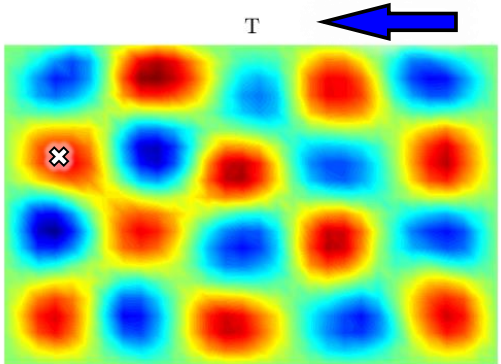

Figure D.4: Instantaneous velocities of the $810 \mathrm{~Hz}\left(T^{+}=59\right)$ traveling wave at six different time steps in the period $T$ of the wave. There are three wavefronts propagating in the spanwise direction. The propagation direction of these are shown in the first time-step. The $\times$ symbol follows the left wavefront traveling in the $+z$ direction.

\section{D.3 630Hz Standing Wave}




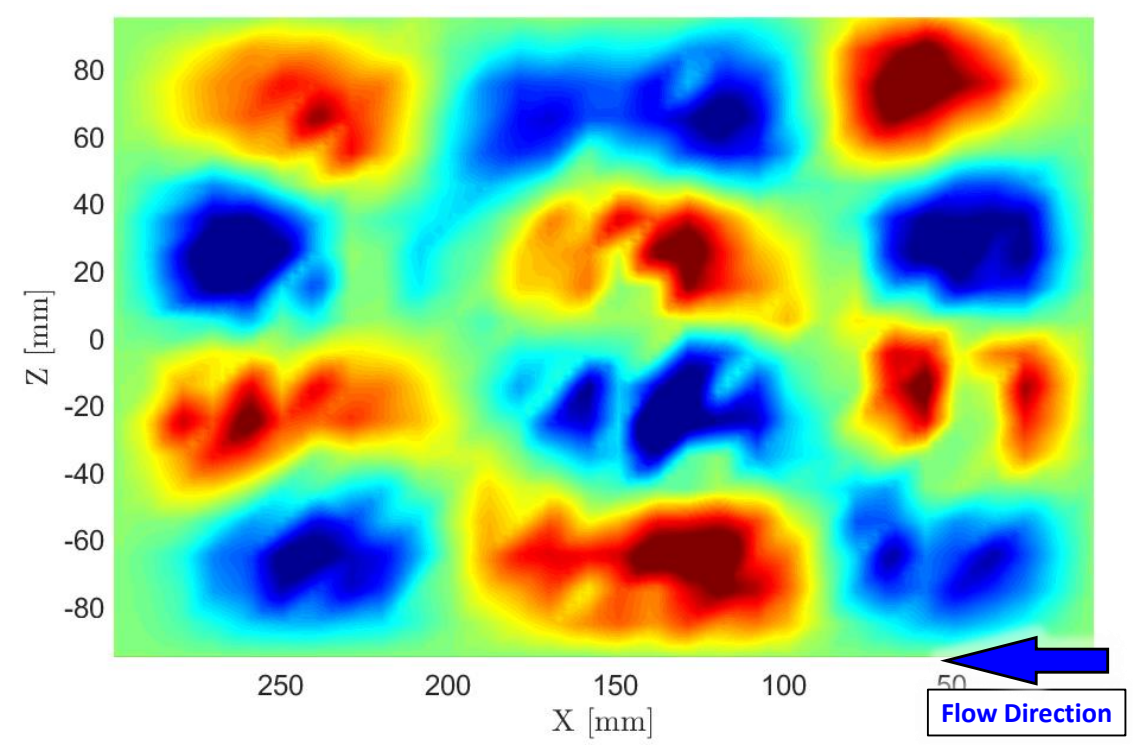

Figure D.5: Operational deflection shape (ODS) of the $(3,4)$ mode shape at $630.5 \mathrm{~Hz}\left(T^{+}=74\right)$. This is excited using a single actuator, while the standing wave in Section 7.1.2.2 is excited using six actuators

$\mathrm{T} / 6$

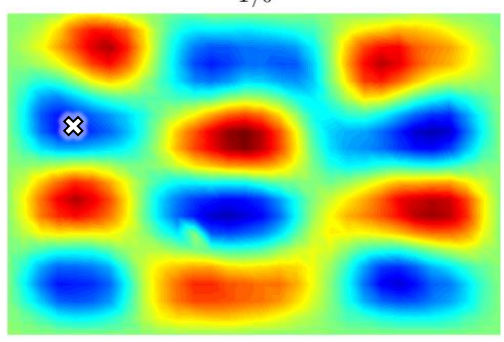

$4 \mathrm{~T} / 6$

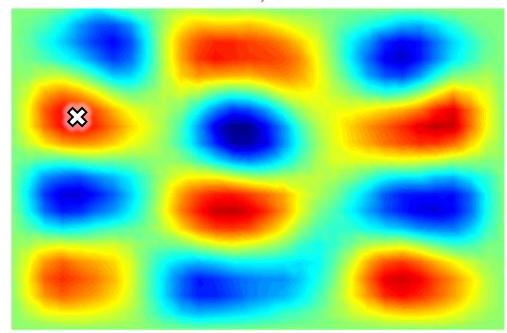

$2 \mathrm{~T} / 6$

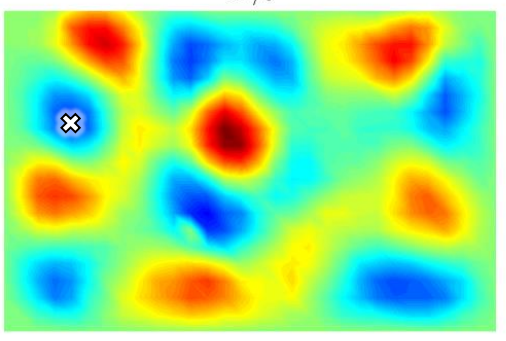

$5 \mathrm{~T} / 6$

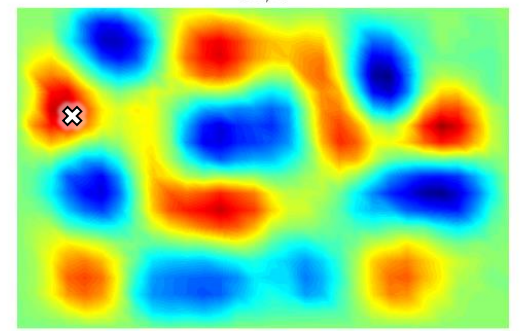

$3 \mathrm{~T} / 6$

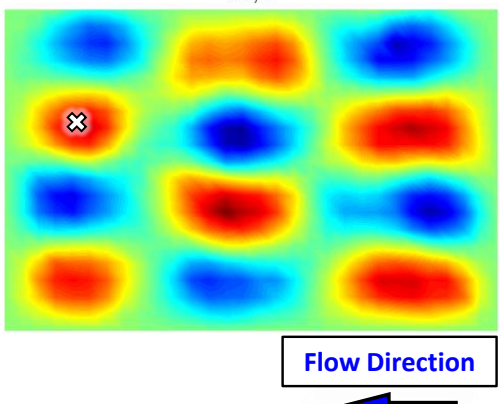

$\mathrm{T}$

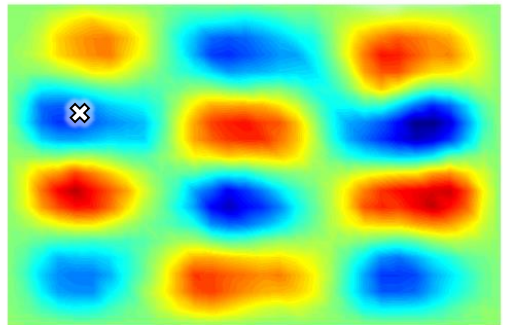

Figure D.6: Instantaneous velocities of the $630 \mathrm{~Hz}\left(T^{+}=74\right)$ standing wave with $(2,3)$ mode shape. Six different time steps over the period $T$ of the wave are shown. There are no traveling waves, so each anti-node stays at a fixed location. The $\times$ symbol shows how an anti-node oscillates from positive (red) to negative (blue) over the period, but remains at the same location 


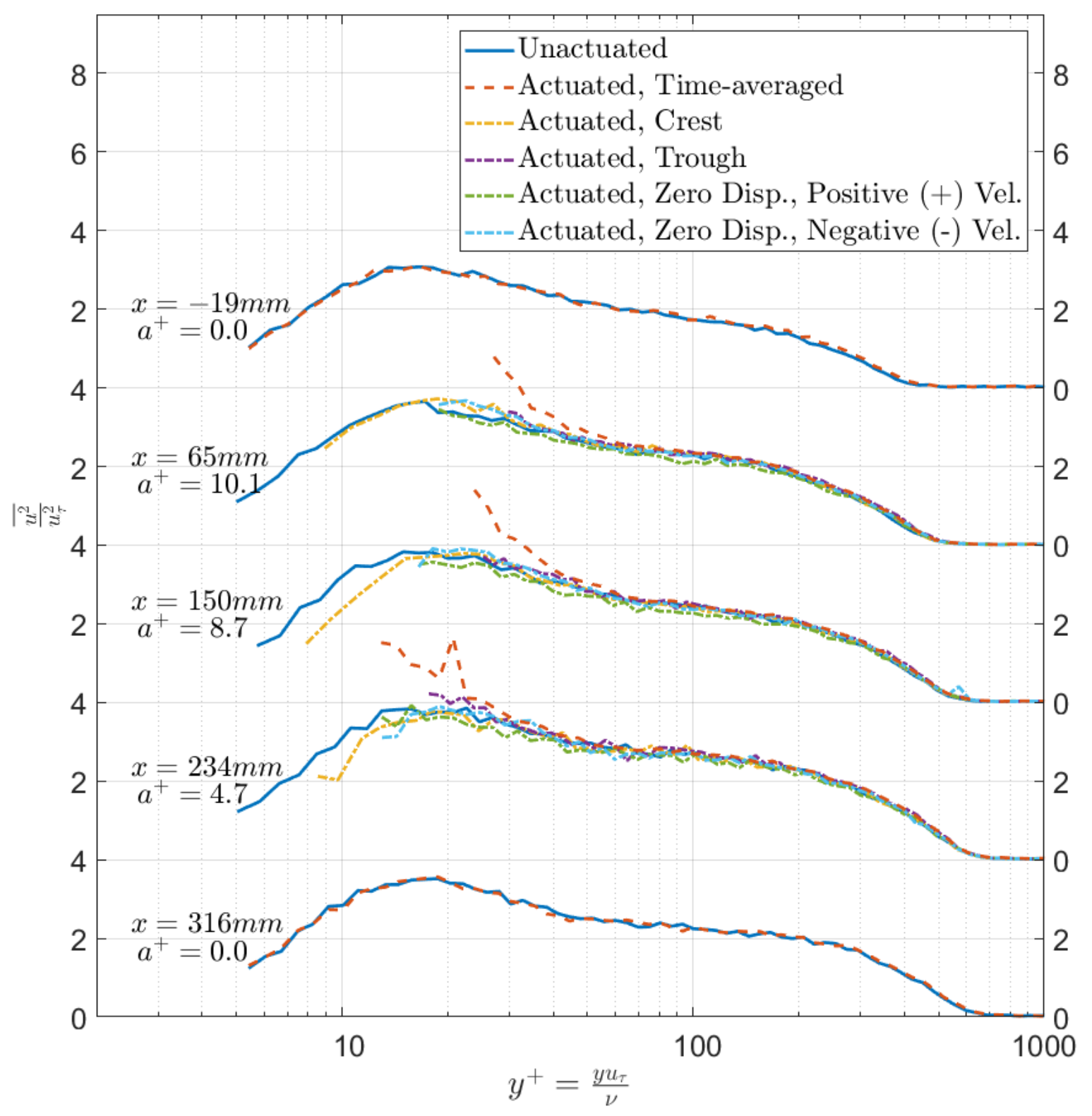

Figure D.7: Variance/Streamwise Reynolds stress profile at streamwise positions over the $630 \mathrm{~Hz}\left(\mathrm{~T}^{+}=74\right)$ traveling wave surface. The first and last profiles are upstream and downstream of the traveling waves, respectively. The phase-locked extracted profiles are shown for the locations directly over the actuated surface. 